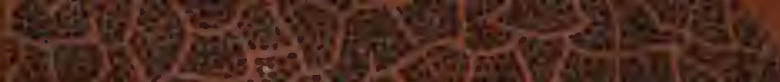

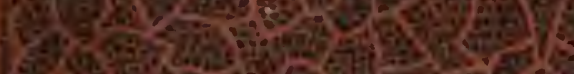

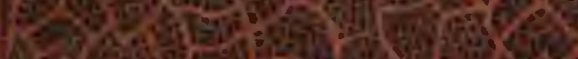

B.

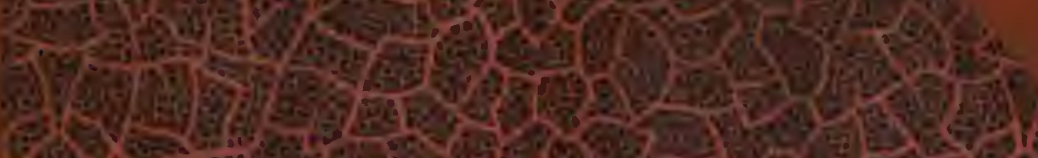

Why

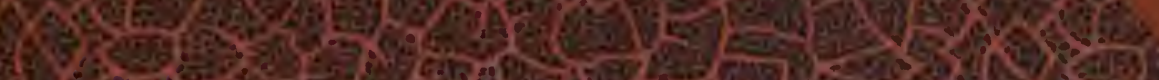
1.

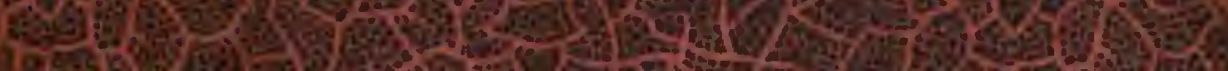

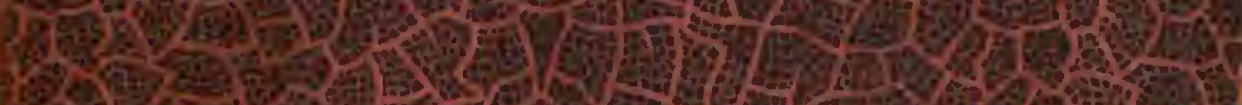

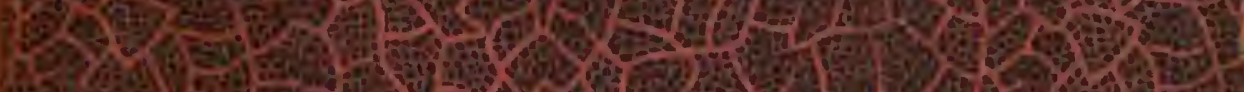

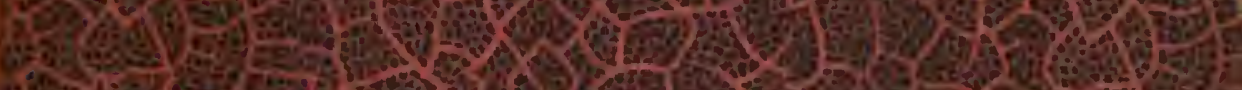

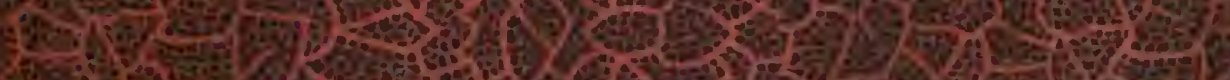
30 of

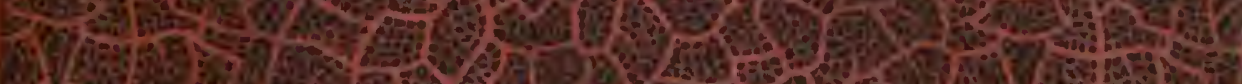

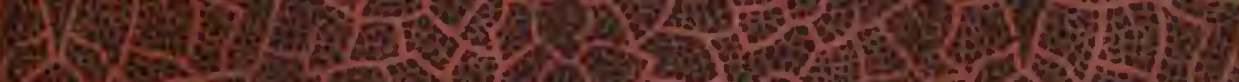

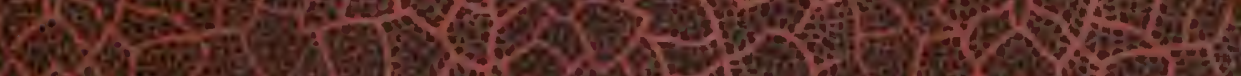
5.9. S.6.

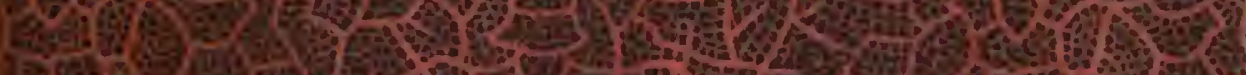

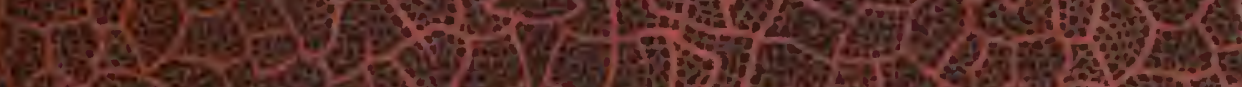
6.

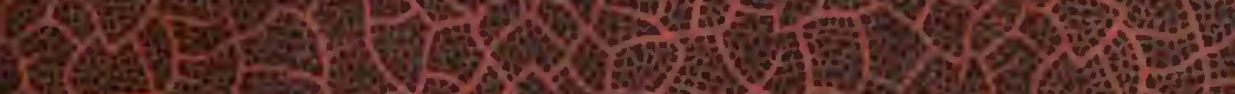

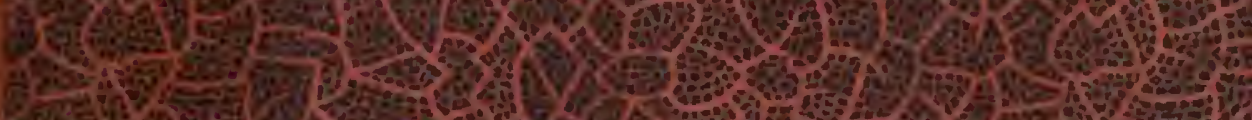

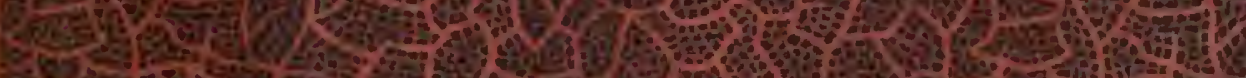

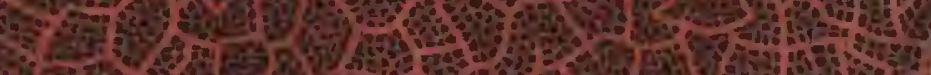

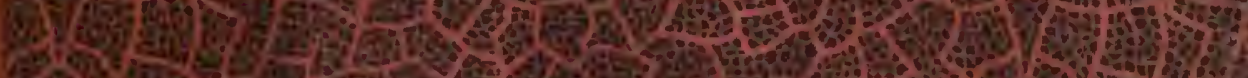

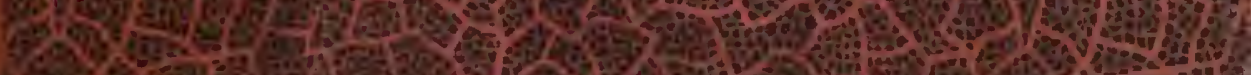

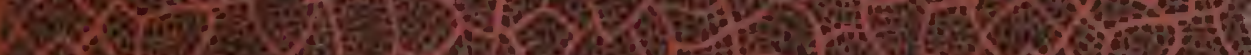
W. Q17. Q.5.

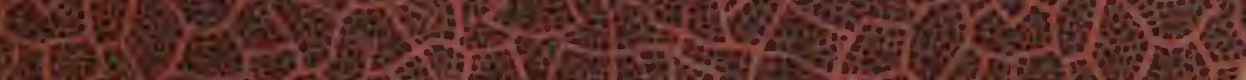

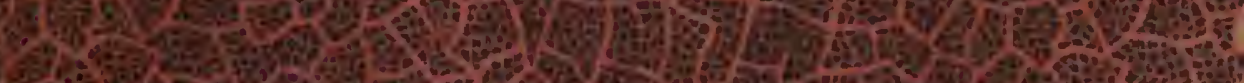
tos.

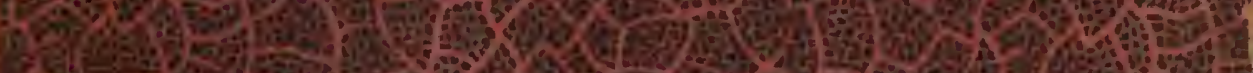

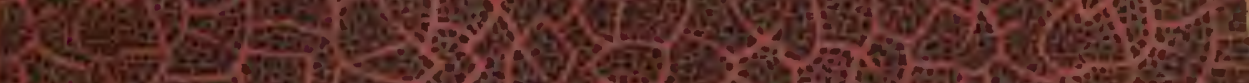

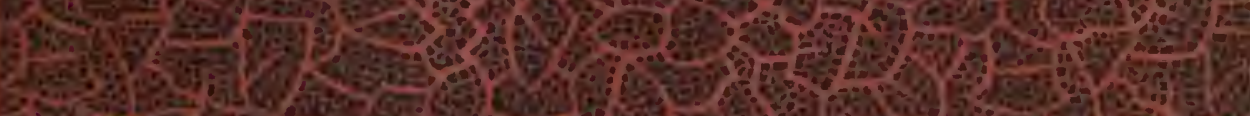
30
0

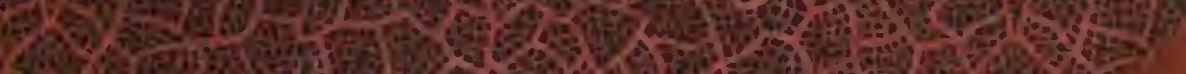

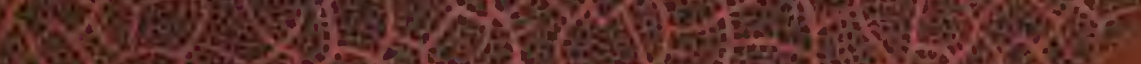
WHarm:

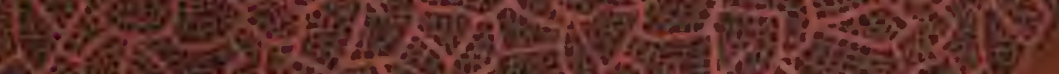
ine

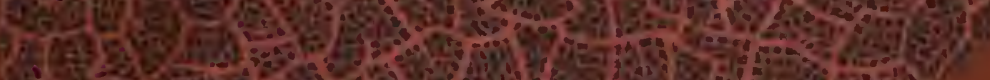
15.

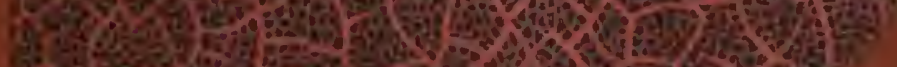



FUNIGATED

1 P D 01981

325.858 


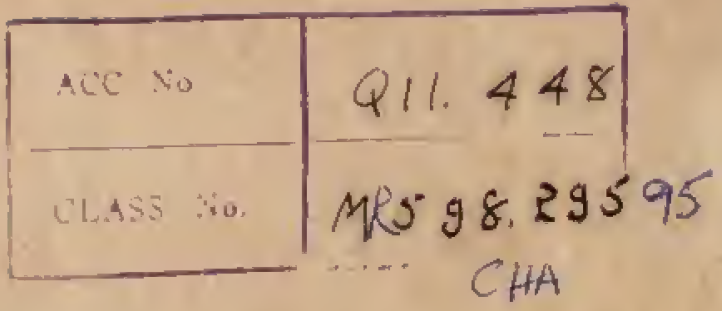




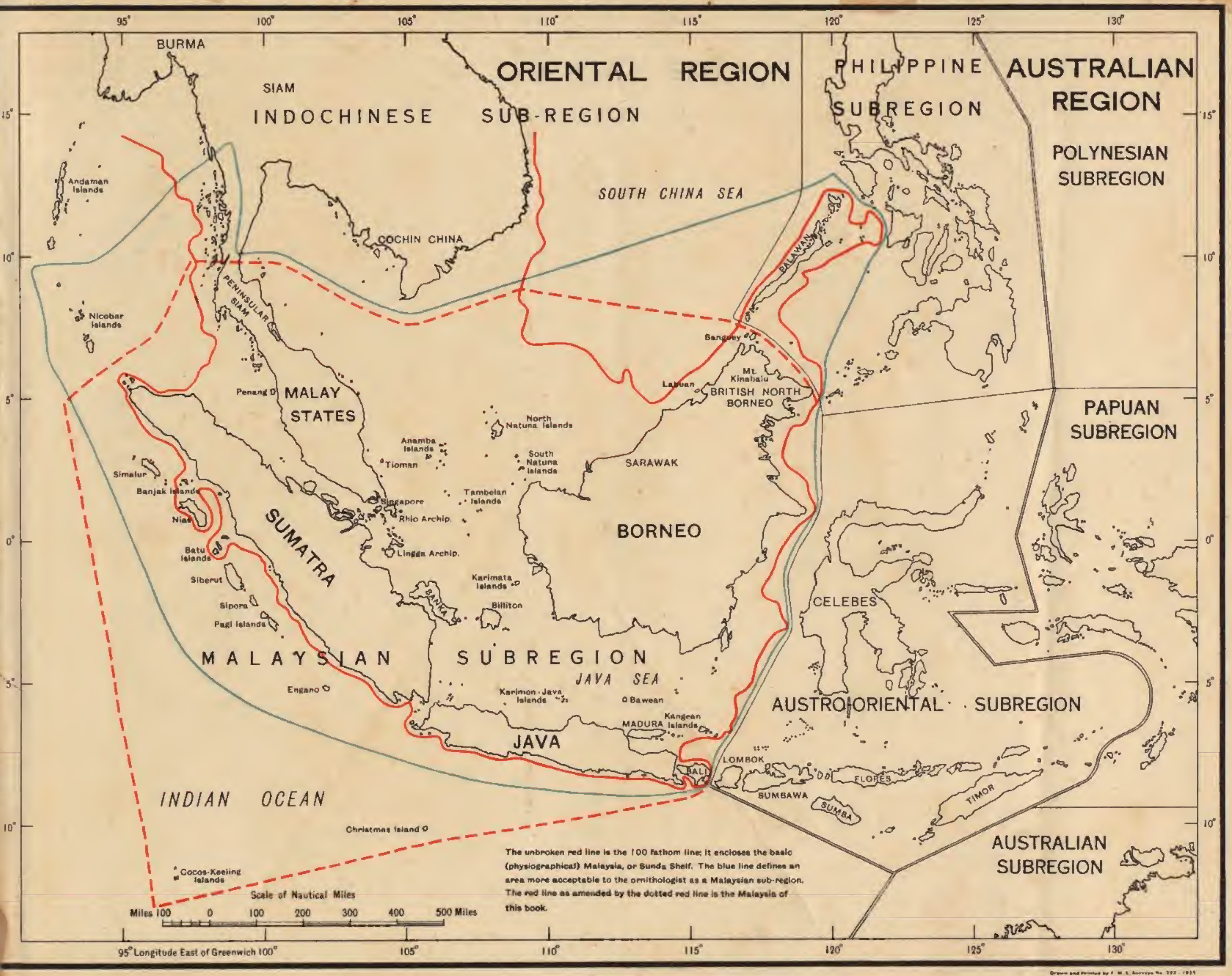



Bulletin of the Rafles Museum, Singapore, Straits Settlements No. Ir, December, 1935 .

\section{A HANDLIST OF}

\section{MALAYSIAN BIRDS}

A SYSTEMATIC LIST OF THE BIRDS OF THE MALAY PENINSULA, SUMATRA, BORNEO AND JAVA, INCLUDING THE ADJACENT SMALL ISLANDS

BY

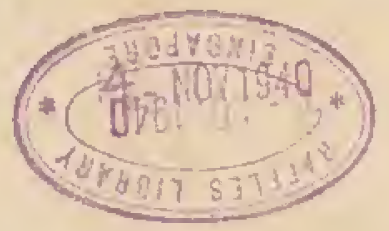

FREDERICK NUTTER CHASEN, c.M.Z.S., M.в.O.V., Director, Raffles Museum, Singapare $2-\frac{11}{445}$

Published by Authority.

SINGAPORE :

Printe at the Governsent Prastac Ofrice, Sungafore, eY W. T. Cherry, Governdenz Prister. 



\section{CONTENTS.}

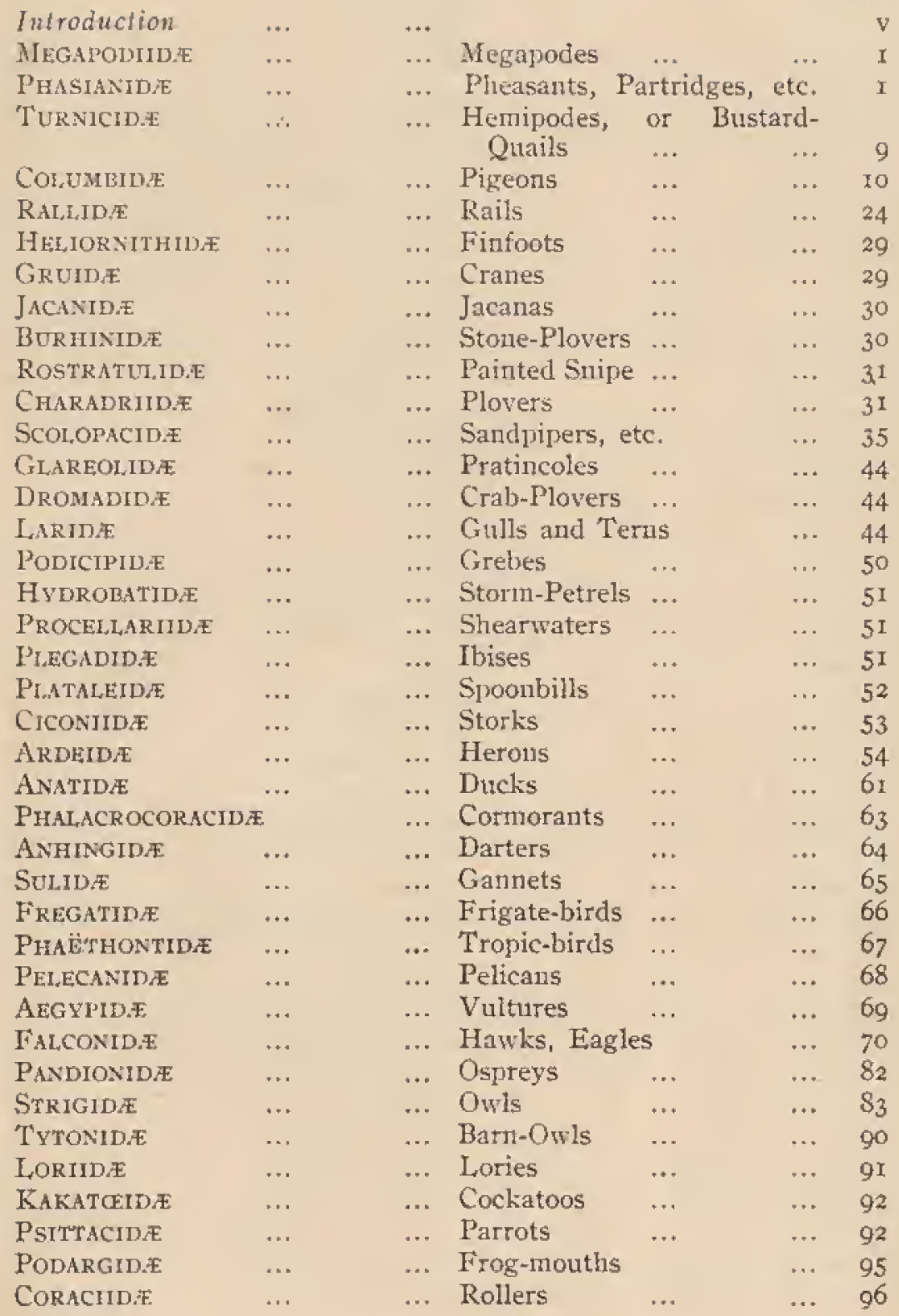


CONTENTS.

\begin{tabular}{|c|c|c|c|c|c|c|}
\hline ALCEDINIDE & $\cdots$ & $\ldots$ & Kingfishers & $\cdots$ & $\ldots$ & 97 \\
\hline BUCEROTIDE & $\ldots$ & $\ldots$ & Hornbills & $\cdots$ & $\ldots$ & 105 \\
\hline UPUPIDE & $\cdots$ & $\ldots$ & Hoopoes & $\cdots$ & ... & 109 \\
\hline MEROPIDE & $\cdots$ & $\ldots$ & Bee-eaters & ... & $\cdots$ & I09 \\
\hline CAPRIMULGIDE. & $\ldots$ & $\ldots$ & Nightjars & $\ldots$ & $\cdots$ & rio \\
\hline MYCROPODIDE & $\ldots$ & $\ldots$ & Swifts & $\ldots$ & $\ldots$ & I I 3 \\
\hline TROGONIDE & $\cdots$ & $\ldots$ & Trogons & ... & $\cdots$ & II \\
\hline CUCULIDE & $\ldots$ & $\ldots$ & Cuckoos & $\cdots$ & $\ldots$ & $\mathrm{r} 22$ \\
\hline INDICATORIDE & $\ldots$ & $\ldots$ & Honey-guides & $\ldots$ & $\cdots$ & 133 \\
\hline Capitonide & $\ldots$ & $\ldots$ & Barbets & $\ldots$ & $\ldots$ & I 34 \\
\hline PICIDE & $\cdots$ & $\cdots$ & Woodpeckers & $\ldots$ & $\ldots$ & 135 \\
\hline EURYI,AIMIDE & $\ldots$ & $\ldots$ & Broadbills & ... & $\ldots$ & $\mathrm{J} 53$ \\
\hline Pittide & $\cdots$ & $\ldots$ & Pittas & $\ldots$ & $\ldots$ & I 56 \\
\hline HIR UNDINIDE & $\ldots$ & $\ldots$ & Swallows & $\cdots$ & $\ldots$ & 160 \\
\hline MUSCICAPIDE & $\ldots$ & $\cdots$ & Flycatchers & $\ldots$ & $\ldots$ & 162 \\
\hline CAMPEPHAGIDE & $\ldots$ & $\ldots$ & Cuckoo-Shrike & & $\ldots$ & ISI \\
\hline PYCNONOTIDE: & $\ldots$ & $\ldots$ & Bulbuls & $\ldots$ & ... & I 88 \\
\hline TIMALIID $E$ & $\ldots$ & $\ldots$ & Babblers & $\ldots$ & $\ldots$ & 204 \\
\hline TROGLODYTIDE & $\ldots$ & $\ldots$ & Wrens & $\ldots$ & $\ldots$ & $23 \mathrm{I}$ \\
\hline TURDIDE & $\ldots$ & $\ldots$ & Thrushes & $\ldots$ & $\ldots$ & 232 \\
\hline SYLVIIDF & $\ldots$ & $\ldots$ & Warblers & .. & ... & 244 \\
\hline ARTAMIDE: & $\ldots$ & $\ldots$ & Swallow-Shrik & res & .. & 256 \\
\hline LANIIDE & $\ldots$ & $\ldots$ & Shrikes & ... & $\ldots$ & 256 \\
\hline PARIDA & $\cdots$ & $\ldots$ & Tits & $\ldots$ & ... & $26 \mathrm{I}$ \\
\hline SITTID & $\ldots$ & $\ldots$ & Nuthatches & $\ldots$ & $\ldots$ & 262 \\
\hline ZOSTEROPIDE & $\ldots$ & $\ldots$ & White-eyes & $\ldots$ & $\ldots$ & 263 \\
\hline DICÆIDE & $\ldots$ & .. & Flower-peckers & & $\ldots$ & 267 \\
\hline NeCtariNIIDE & ... & $\ldots$ & Sun-birds & ... & $\ldots$ & 273 \\
\hline MetLiPHAGID & $\ldots$ & $\ldots$ & Honey-sttckers & & $\ldots$ & 284 \\
\hline MoTACILIIDE & $\ldots$ & $\ldots$ & Wagtails and & Pipits & $\ldots$ & 284 \\
\hline $\mathrm{AL}_{f} A \mathrm{~L}^{\prime} \mathrm{D} \cap \mathrm{D} / \mathrm{E}$ & $\ldots$ & $\ldots$ & Larks & ... & $\ldots$ & 287 \\
\hline FRINGIILIDIE & $\ldots$ & $\ldots$ & Finches and $B$ & Buntings & $\ldots$ & 288 \\
\hline PLOCEIDE & $\cdots$ & ... & Weaver-Finch & & $\cdots$ & 289 \\
\hline STURNID.E & $\cdots$ & $\cdots$ & Starlings & $\ldots$ & $\ldots$ & 294 \\
\hline GRACULIDE & $\cdots$ & $\ldots$ & Tree-Starlings & & $\ldots$ & 296 \\
\hline ORIOLIDIE & $\ldots$ & $\cdots$ & Orioles & $\ldots$ & $\cdots$ & 299 \\
\hline DICRURIDF & $\ldots$ & $\ldots$ & Drongoes & ... & $\ldots$ & 302 \\
\hline CoRvidex & $\ldots$ & $\ldots$ & Crows & .. & ... & $30 S$ \\
\hline
\end{tabular}




\section{INTRODUCTION}

\section{ZOO-GEOGRAPHICAL.}

Although the term Malaysia was used by several zoologists in the nineteenth century only F. H. H. GOMLEMarD ${ }^{1}$ seems to lave employed it in a systematic sense, and he for the vast region extending from Sumatra to the Philippines and Moluccas, "the home of the true Malay race".

In $1918^{2} \mathrm{C}$. BoDkN KLoss introduced and defined Malaysia in a formal manner as a sub-region of the Oriental Region.-

"Malaysian-pertaining to the Malay Peninsula, Sumatra,

Borneo and Java. Malayan-pertaining to the Malay Peninsula, c.f. Sumatran, etc."

To the faunist this restricted Malaysia is more homogeneous than the larger sub-region, Indo-Malaya of Wallace, within which the Philippines ${ }^{3}$ provide a sharp division against the Malay Peninsula and Sunda Islands.

The exact boundaries of a zoo-geographical zone inust always be of an arbitrary nature and as it is obvious that such a zone is basically physiographical, rather than zoological, it is reasonable to try to fix its natura] boundaries with regard to geographical facts "and when zoological appearances do not agree with geographical ones to regard the former as anomalots and endeavour to explain them"."

A basic, or physiographical Malaysia can soon be defined as all land standing on the Stunda Shelf below about Lat. $10^{\circ} \mathrm{N}$. It is an area in which the sea-depths are less than one-hundred and usually less than forty fathoms : it leaps to the eye from a bathymetrical map.

As a zoo-geographical zone of any kind, lowever, the unmodified Sunda Shelf is not satisfactory and it needs modifications on zoological and geographical grounds, as well as on those of expediency.

For instance, the Malaysian avifauna extends on the zuestern side of the Peninsula to about the latitude of Tavoy and the northern boundary of the sub-region ought to be extended thence. A similar

"Australasia", ii, 1804, p. 2.

Journ, F.M.S, Mus, vii, 1018, p. 245.

3 Excluding the Palawan group and Sibutu, the Philippine Islands lie outside the roo fathom line. Their fauna is poor compared with that of Malaysia. It lacks many Malaysian genera, but on the other hand include? many peculiar forms.

4 C. Boden Kloss, "The Zoo-geograjhical Boundaries between Asia and Australia and some Oriental Sub-Regions", Bull. Raffles. Mus., 2, Ig29, p. I. 
faura is also found on the Nicobar Islands;' and on the deep-water islands of the West Sumatran Chain. It has been thought expedient however, to exclude Tenasserim and the Nicobars. They are fully covered by regional publications dealing with British India and the Philippine Islands; their inclusion would be undesirable bibliographically; and furtliermore, I have no collections or detailed knowledge of these areas. It can be noted that their omission is not inportant for they would only add to the list a few geographical races of species alreacly included and, in the case of Tenasserim, one or two continental species.

On the other hand the Sunda Slielf includes the Palawan groups of islands and Sibutu, of which the avifauna though strongly Bornean has a conspicuous Philippine element. As these islands are also politically Philippine and fully covered by other pubications they are excluded from the present work.

Lastly, purely on grounds of geographical proximity, the CocosKeeling Islands and Christmas Island in the Indian Ocean are included although the natural avifauna of the former is, in the main, Oceanic, and of the latter, Austro-Oriental.

\section{GENERAL FEATURES OF THE MALAYSIAN AVIFAUNA.}

Excluding migratory species (the great majority from the north and a small minority from the south-east) many of which in their non-breeding season are found everywhere in the subregion from its coasts to the tops of its highest mountains, we find that the avifauna is more or less sharply divided at an altitude of about 3,000 feet although such broad division excludes a small, but not unimportant, group of sub-montane forms. The montane forms may, or may not be represented in the lowlands by allied sub-species: usually they are not.

On the mountains of Sumatra, Java and Borneo, Sondaic species occur together with a geographically more widely spread HimalayoSondaic element. A third element, more purely Continental (as distinct from Himalayo-Sondaic) reaches the Malayau and Sumatra mountains. An outstanding feature of the mountain ornis is an isolated and imperfectly identified element on the Bornean mountains : it is either Philippine or Austro-Oriental."

In the lowlands the Himalayo-Sondaic association which is perhaps the palreonalaysian fauna, is lost in the Malaysian, or taking a rather broader view, the Indo-Malayan fauna. The Malaysian fauna is comparatively even, pointing to land connections geologically not

- The Andamans and Nicobars form a part of the mountain range that once extended from Cape Negrais in Lower Burma, a continuation of the Arakan Yoma, to Achin Head in Sumatra. The Andamans contain an impoverished Burmese fauna: that of the Nicobars approximates to the Sumatran type. Malcolm Smith, ig3i.

2 See Kobinson and Kloss, Journ. F.M.S. Mus. viii, :918, p. 97. 
renote although sub-species of varying degrees of distinctness are usually developed on each of the large land-masses. On the whole the avifauna of Java is the most distinct, indicating the early separation of that island. The birds of Sumatra and the Malay Peninsula are very similar although each area has some characteristic fonns, but more specialization is apparent in Borneo.

Java shares with the Indochinese sub-region, but not with the remainder of Malaysia, a small group of species. This is usually explaned by a theory which postulates a series of very complicated changes in land-surfaces for which, as in the case of some nuch simpler changes supposed by the zoo-geographer to have taken place in Malaysia, no geological evidence seems yet to have been brought forward." In this connection, however, another hypothesis demands consideration in which land-levels and connections are only of secondary importance. There is a tendency for many migratory birds to establish themselves, and to become differentiated as resident races in the southern parts, in many cases, on the absolute southern periphery, of their range.

In the case of tropical breeding races of Charadrius alexandrinus and Acrocephalus orientalis the range of the resident form is still invaded, in season, by the migratory subspecies. Among the passeres we sometimes find resident races in Sumatra, Borneo, and Java, or sone conbination within this grous, with migratory forms of the same species only ranging as far soutl as the Malay Peniusula. Here it is important to note that the present soutlern limit of the migrants varies with the species. These migrants (eg. Oriolus, Dicnurus) seem to dislike the water-crossing and there is a suggestion in all these species that a migratory labit, having established southern, differentiated groups, is dying out.

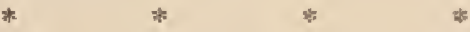

In the systematic part of this book the Malaysian localities for the various species are arranged in four rows corresponding to four Provinces, Malayan, Sumatran, Javan and Bornean, and althotigh this arrangement is largely natural, that is to say, in close agreement with both zoological and geographical facts it is also much tempered by a desire for a simple arrangement, and the four provinces are certainly not equal in value. Some of the small islands in particular require further study.

1 Visual evidence of minor coastal changes in Malaysia to-day suggests an interesting point in connection with the great changes in land-surface that must have taken place in order to produce the erratic distribution of many species. Separation of such land-masses as the Malay Peninsula and Surnatra was probably preceded by a long period during which the intervening ground was a barrier to some species and not to others. For instance, an extensive mangrove swamp in the south of the Straits of Malacca would prevent the passage of leopards but not of monkeys. 
THE MALAYAN PROVINCE.?

The Malay Peninsula; the small islands close to both ifs coasts; the Anamba and Tambelan Islands.

The northern boundary of this province and consequently that of the sub-region is here regarded as Lat. $10^{\circ} \mathrm{N}$. which is at the narrowest part of the Isthmus of Kra. Any further extension embraces territory within the political linits of British India, which is undesirable. The adopted dividing line, is, furthermore, not unnatural, for although on the west const, along the region of the lieavier rainfall and evergreen forests the Malayan avifauna extends through Tenasserim to about the latitude of 'Tavoy, on the lowlands of the east coast the Malayan element in the avifauna of Sian stops at the Pakchan Estuary ${ }^{2}$. A herpetologist would draw the southern line of his Indo-chinese fauna at about Lat. $12^{\circ} \mathrm{N}$. for by lifting it from to ${ }^{\circ} \mathrm{N}$. about a dozen Malayan species of amphibians and reptiles are excluded ${ }^{3}$. A botanist suggests that the division should be at about the latitude of Singgora in Peninsular Siam and that an intermediate area more Indo-chinese tlan Malayan lies between Lat. $7^{\circ} \mathrm{N}$. and $10^{\circ} \mathrm{N} .{ }^{4}$. So little is known of the avifatina of the ligh mountain range between Tenasserim and Sian that any discussion as to its affinities would be unprofitable.

Several faunal boundaries, that is lines either coincident with the geographical limits of climatic changes, past, or present, or indicating former breaks in the continuity of the land surface, and impeding the spread of species, can be appreciated in the Malay Peninsula. There is one in the north, stretching from, roughly, Alor Star in Kedah on the west coast across to Singgora in Peninsular Sian on the east coast. It is noticeable that certain continental species extend further south on the west than on the east side of the Peninsula. There is also reason to suppose that the Peninsula south of Malacca was once isolated, but the biological evidence for this is clearer in mammals than in birds. The shallow, and narrow strait between the mainland and Singapore Island also acts as a minor faunal boundary.

The distinctness of the mountain faunas throughout Malaysia has already been mentioned above. It remains to be added that the true mountain fauna of the Peninsula fades out in Selangor, and

"For the ornithological history, general outline, bibliography, etc, of this province, see Robinson, "The Birds of the Malay Peninsula", i, 1927, pp. xiji-xlviij (Peninsula); Chas, and Kloss, Journ. Mal, Br, Roy. Asiatic Soc., vi, pt. iii, I028, pp. 43-48 (Anamba Islands); Oberholser, Proc. U. S. Nat. Mus., 1v, 1919. p. r29 (Tambelan Islands).

2 Robinson and Kloss, Journ. Nat. Hist. Soc. Siam, v, 1921, p. 15.

3 Malcolm A. Smith, Faun. Brit. Ind., Reptiles and Amphibia, i, 1031, p. 13.

4 Ridley, Journ. Straits Br. Roy. Asjat. Soc. 59, Inrt, p. 55. 
that the birds on the hills of Negri Sembilan, Malacca and Jolsore are usually strbmontane, or purely lowland species. Certain isolated peaks, even in the north of the Peninsula, are similarly characterized.

Other outstanding features of the peninsular avifauna are that certain continental species occur in the north, but not in the south; that some characteristically Malaysian species only occur in the soutl;; that subspecific variation is north against south, and never west against east, which is interesting in view of the fact that the main physical feature of the Peninsula is a high range of mountains forming a backbone, running north and south; that there is a curious midpeninsular gap in the range of certain woodpeckers; that, although, like the other land-masses in Malaysia, the Peninsula has developed many subspecies of its own, it contains no species that is not more widely spreat, although the Malayan race may be well-differentiated; and tiat a cuckoo is found in the lowlands in the northern part of the Peuinsula and, normally, only in the mountains further south.

In a few species there is a racial similarity between the resident birds of Ceylon, on the one hand, and those of the southern part of the Malay Petinsula, or in rarer cases, other, more remote parts of Malaysia, on the other. This convergence at the extrenities of the Ceylon-Burma-Malayan arc is usually attributed to a striking similarity in the two habitats, although it nust be noted that the majority of the resident birds in the two areas are very distinct and that the similarities in the avifatuas have, at times, been over-emphasized.

The coastal islands in the Malayan Province are of little special interest ornithologically. Pheasants, partridges, trogons, barbets, woodpeckers, broadbills and most species of babblers are usually absent, or rare, althougth the Langkawi Islands, off the west coast, are ratlier unustual in this respect, and therefore perliaps of recent separation. Subspecific differention on these sulall coastal islands is very rare although a Langkawi woodpecker, and a Tionan babbler (Slachyris) have been dignified with racial names. Outstanding features are the presence of the argus pheasant on Pangkor in the Straits of Malacca and, nost interesting, of a wren-babbler (Napothera) otherwise only known from the mountains of the mainland, on Tionan. In the Tioman Archipelago, oft the east coast, there are signs of subspecific divergence in the direction of the insular subspecies found in the Anamba Islands.

The resident, purely land-birds of the Anamba Islands in the South China Sea are merely representatives of conmon Malayan species: most of the species diverge in the direction of large average size, although the differences are very small: as noted above these differences may be adumbrated on the eastern coastal islands of the Malay Peninstula. The Tambelan Islands, between Singapore and Borneo and situated nearer to the latter, have an impoverished avifanna of no special interest, the few resident land-birds apparently belonging to the Anamba subspecies. 


\section{THE SUMATRAN PROVINCE.}

Sumatra and the shallow water islands off its east coast with which the islands of the Rhio, and Lingga Archipelagos are intimately relaled. The large islands of Banka and Billiton. The whole of the chain of islands off the west coast of Sumatra, the most important of which from noth to south are, Simalur, the Banjak Islands, Nias, the Balu Islands, the Mentawi Islands (Siberut, Sipora, and the Pagi Islands), and Engano.

In spite of the much larger size of the island, the avifauna of Sumatra is less rich than that of the geographically nore favourably situated Malay Peninsula, but the two faunas are very closely allied. That of Sumatra shows rather more specialization: it lacks some continental forms, but on the other hand there is present a Sunda Island element. Sumatra has a few peculiar species and numbers of welldifferentiated subspecies, but as in the Malay Peninsula no genus, or highly peculiar form is found (Gymnocrotaphus and Chalcoionus are not here recognized), although the occurrence of Rimator, otherwise only known from the Himalayas, is renarkable. In the extrente south of the island there is a minor infiltration of Javanese forms.

Most subspecific differentiation within the island is north against south, but in a few cases races on the alluvial flats of the east coast are not the same as those occupying the older land in the west: In such cases the western race approximates to the Javan and the eastern to the Malayan subspecies.

An interesting item is that a brondbill (Serilophus) on the mouutains of Acheen in the north of Sumatra is inseparable from the Malay States subspecies, geographicaily near and opposite. whereas the bird occupying the remainder of Sumatra, that is to the south, is more like that found in the nortit of the Malay Peninsula.

The faunal boundary which cleaves the island from north to south is the Barisan Range in which the only major depression, that in the neighbourhood of Padang Siclempuar, Lat. I $30^{\prime} \mathrm{N}$., forms a second barrier, a number of subspecies being confined to the island north of this place ${ }^{2}$. The low-lying islands on the east coast of Sunatra call for no special remark.

The Rhio and Lingga Archipelagos contain a poor avifauna contposed of species common to the lowlands of central-east Sumatra and the south of the Malay Peninsula. The only degree of specialization seems to be an almost imperceptible divergence in the direction of races found in the Anamba and Natuna Islands. The avifaunas of

- The basic literature for this province is, or is summarized in, the following papers.-Sumatra: Rob. and Kloss, Journ. F.M.S. Mus., viii, pt. 2, $1918, \mathrm{j} .8 \mathrm{I}$; Kloss, Treubia, xiii, 1931 , p. 209. Rhio Archipelago: Chasen, Bull. Raftles Mus,, 5, 1031, p. 114. Lingga Archipelago: Dammerman, Treubia, viii, 1926, p. 325. Banka: Kloss, l.c.s. Billiton : Kloss, Treubia xiif, 1931, p. 203. Hest Sumatran Islands: Chas, and Kloss, Ibis, 1926, p. 306 ; Riley, Proc. U.S. Nat. Mus., Ixxr. Art. 4, 1929, J. I.

2 See Kloss, Treubia, xiii, 1931, p. 301. 
Banka and Billiton are closely allied but by no means exactly alike and include a few not very distinctive, subspecies. Fresh material on which a critical exanination of races in the modern way can be based is needed from Banka, but in the meantine it can be stated that the avifaunas are largely what could be expected from the map positions. Basically, Malay-Sunatran, there is a strong Bornean influence in Billiton which is less strongly marked in Banka.

The islands of the West Sumatra Chain' are of special interest for, collectively, they contain many well-marked bird-races, althougin taking the broad view followed in this handlist, no pectuliar sjecies? In their fauna the islands vary much among themselves, and sometimes from Sumatra. There is no proof that this island chain ever formed one land-mass, or that all the islands were ever joined to Sumatra and the whole archipelago cannot be treated as an entity. There is reason for regarding the deeper water islands, that is those outside the one-liundred fathom line (Simalur, the Mentawi Islands, and Engano) as forming a zoo-geographical province, small in area, but equal in value to the four other provinces here adopted. Those within the forty-fathom line, the Batu and Banjak Islands, have a few subspecies of their own, but these are not so distinct as some on the deeper water islands of Simalur and the Mentawi group where nost of the land birds are peculiar to the islands. Nias also has a number of subspecies of its own, but these are not so well marked as the most distinctive of the Mentawi races and quite a number of the Nias birds seent inseparable from Sumatran forms.

\section{THE BORNEAN PROVINCE.3}

Borneo and its coastal islands, the most important of which are Labuan, and Mantanani off the north-zest coast; Maratua, off the cast coast; and Pulau Laul off the south-east corner; the North Bornean Islands of Banguey, Balambangan, and Mallealle; the North and South Natuna Islands; the Karinala Islands.

The outstanding feature of the avifauna of Borneo is that althougls it has the general Malaysian facies it is more specialized than that of the Malayan and Sumatran, and if consideration is limited to the high-level fauna, even the Javan, Province.

The avifauna is by no means even, either in the mountains, or in the lowlands, and there are several obvious famal boumdaries in the island. The most striking, if not the most efficacious of these is

1 See Kloss, Proc. Zool. Soc., 1927, p. 797.

2 The mammals include an isolated genus of Monkeys (Simias), a very distinct langur ('Pygathrix potenziani), and other very peculiar forms.

3 The basic literature from the systematic view-point can be obtained by reference to.-Moulton, Journ. Straits Br. Roy. Asiat. Soc. 67, 1914. p. 125 (Borneo); Chasen and Kloss, Bull. Raffles Mus., A, ro3o (Borneo and the coasta! islands); Oberholser, Bull. U.S. Nat. Mus. I50, 1932 (Natuna Islands); Chasen and Kloss, Treubia, xiv, 1933. p. 155 (Karimata Istands). 
formed by the high montain ranges of the interior, the general strike of which is in a north-east and south-west direction' ${ }^{\prime}$. In sonte species, a race found east and south of these mountains is separable from that found, broadly speaking, in Sarawak. In many other cases races are distributed, broadly, as above but meet on the northern ("northwestern") coast in the neighbourhood of Baran where there is a most important faunal boundary. Occasionally, a northern race thus cut off extends only a short distance down the east coast.

Within the major divisions given above there are also tendencies for areas of faunal distinction to separate in the lowlands of south, and of west Borneo. Certain rivers running west and east act as boundaries in the case of some mammals.

A slight Javan influence is apparent in the south of Borneo.

Turning to the mountains we find that, within the island, the great massif of Mt. Kinabalu in the north has far fewer peculiar forms than was formerly thought, for recent collecting has shewn that it shares most of its birds with other high peaks in North Sarawak. On the neiglibouring Mt. Dulit there are signs of independent specialization although these are not so numerous as some recent authors would have us believe. It is interesting to note that a few nontane species also occur on the isolated peaks of Poi and Penrissen in the north-west corner of the island: the forn is usually that found on the northern mountains, but in one or two cases, distinctive subspecies have been developed.

The islands off the north-west coast of Borneo call for little comment beyond mention of an indigenous race of a small owl on Mantanani.

Mallewalle, Banguey and Balambangan, situated off the northern extremity of Borneo, outside the ten fathom line, but on a hank of less than twenty fathoms, have an avifauna almost entirely Bornean but with slight indications of internal differentiation, and there is also a small non-Bomean element, derived from the adjacent Palawan group of islands.

Maratua island, off the east coast, in the Celebes Sea, lias also produced a few subspecies: on it there is, too, a Phillippine influence unknown on the mainland of Borneo or in the Banguey group.

In some respects the avifauna of the Karimata Islands, of the west coast, is closer to that of Sunnatra than of Borneo: some of the species merely tend to large size as on other islands in the South China Sea, but within the gronp two birds seem to be represented by

- See a map and paper by E. Banks in Proc, Zool, Soc. 1033, D. 273: and the same author's detailed study of the distribution of Sciurus prevosti in Borneo, op.cit, 1031,1$\}$. 1336 , which also has a usefu! map. 


\section{INTRODUCTION"}

well-marked races, one of which, it is most interesting to note, is found on Panebangan Island which lies within the five fathom line and presumably within sight of the Bornean coast.

Many subspecies of birds have been described fron the Natuna Islands. The special affinities of the birds of the southern group are Sarawacian, but in the northern islands the Bornean influence is less marked. Only on Great Natuna Island is there anything but au impoverished avifauna.

\section{THE IAVAN PROVINCE."}

Java with Madura; Bali. Many small islands in the Java Sea, the most important of which are the Thousand Islands; the Watcher Islands off Batavia; the Karimon-Java Islands; Bawean; the Kangean Islands including Raas and Sapudi; various small islands betacen Balt and Bornco, the most important of tohich are Arends, Solombo, Kalambau, and Mata Siri. Christmas Island in the Indian Ocean. The Cocos-Keeling Islands.

The avifauna of Java is poorer than that of the three other large land-masses in Malaysia, a circumstance perhaps due to the smaller area of the island. It is, however, the most specialized, or distinct, only a minority of its resident land-birds being inseparable from those occuring in the Malay Peninsula, Sumatra and Borneo, whereas the majority are well-marked peculiar subspecies, or widely divergent forms often called "representative species". The genera Psaltria and Crocias are peculiar to Java which also shares with the Himalayas, and not witl the rest of Malaysia, Serinus. One of the most striking features of the Javan avifauna is that it shares a rumber of birds with the Indo-Chinese sulb-region, these being either absent from the rest of Malaysia, or only represented in the north of the Malay Peninsula. This fact bas already received notice in this introduction.

In the extreme west of the island is a minor Stunatran infiltration, perhajs of recent date. A marked penetration of Austro-Oriental (Lesser Sunda Island) forms is especially noticeable in the east of the island.

There seems to be a slight reduction in the number of species correlated with longitude, fewer species being represented in the west. Subspecific variation is mainly longitudinal and if a species is divided into western and eastern races, the latter may be found on Bali. In a few cases montane species are represented by different forms on several peaks.

Basic literature for the Tavan Province-"Birds of the Island of Java", Kuroda, 1933 (Java, Thousand Islands \&c.); Stresemann, Nov. Zool., xx, 1913, p. 325 and Rensch, Mitt. Zool. Mus., xvi, 1930, p. 530 (Bali): Chasen and Kloss, Treubia xiv, 1933, p. 165 (Karimon Java); Oberholser, Proc. U.S. Nat. Mus., lii, 1917, p. 183 (Bawean); Hartert, Nov. Zool., jx, 1902, p. 419 (Kangean Islands); Oberholser, Proc. U.S. Nat, Mus., liv, 1917, p, 1,77 (Solombo, Mata Siri \&c.); Chasen, Bull. Rafties Mus., 8, 1033, 1. 55 (Christmas Island): "Corals and Atolls", WoodJones, 1912 (Cocos-Keeling Islands). 
Little is known of the avifauna of Madura but it seems not to be specialized in any way. The avifatuna of Bali which is rich for the size of the island and includes some montane forms is closely allied to that of East Java and much more remotely to the very distinct AustroOriental ornis on the neighbouring island of Loubok. The two islands are, of course, separated by the narrow, but deep strip of water, forming part of the well-known "Wallace's Line", which can be regarded as the sotthern end of the zoo-geographical boundary between the Malaysian and Austro-Oriental sub-regions. Most, but not all, socalled Balinese forms are also found in East Java. One of the exceptions is a sturnine genus Leucopsar, peculiar to Bali. The Austro-Oriental influence is shewn by the presence of Trichoglossus, Lichmera and Plilinopus cinclus which reach their western limit in Bali.

A few subspecies have been described from various small islands in the Java Sea on which both Bornean and Javan species are found, but these islands are most remarkable for providing stepping stones for an infiltration to the west of Austro-Oriental forms which even reaches the Thousand Islands off the west point of Java and Billiton.

The birds of the Karimon-Java Islands sluow a close affinity to those of Java. A species represented in South I3orneo and Java by different subspecies, if it occurs in the Karimon-Java Islands, is either represented there by the Java race, or by a race which is, according to the present state of our knowledge confined to the islands, or else found in the same, or a very slightly altered state, on other islands in the Java Sea.

Bawean has a few indigenous subspecies: its avifauna is mixed Javan and Bornean, but the former element is dominant.

The Kangean Islands with sone well-marked endemic subspecies are also mostly Javan in their avifauna, but there is also a strong Austro-Oriental element.

The fauma of Christmas Island is highly specialized. The avifanna is Austro-Oriental in its present affinities which is surprising when the nearness of Java is considered. In more detail we find that of the Austro-Oriental subspecies the Christmas Island races are most like those found in the chain of islands stretching from Sumbawa to Timor Laut, and, furthermore, when, within this limited area, subspecific distinctions can be recognized in a slecies, the Christmas Island form is more like that found in Sumba, Savu, Timor \&c., than that inhabiting Sumbawa and Flores.

The birds of Cocos-Keeling are of no great interest. They include oceanic forms, introduced species, and stragglers from Java and elsewhere. There is no indigenous, peculiar, land-bird except Rallus philippensis andrewsi whose sub-specific status I have been unable to check.

In the future I hope to attempt a more detailed analysis of the Malaysian avifauna. 


\section{ARRANGEMENT, E'TC.}

The Order is that of Sharpe's "Handlist" adapted in a few places in accordance witl the views of some workers on various groups such as those of Dr. P. R. Lowe based on his investigations on the waders, aithougl in this case the result is not very obvious as the present lrandlist deals only with formations below tlie rank of Fanily. Some other, but minor, adjustments have also been made, when 1 have felt convinced that they could be justfied. Muscilrea, for instance, is transferred to the Laniida and merged in Pachycephala. A number of other changes recently proposed have not been accepted as they seem based on very superficial grounds and, to my mind, represent change rather than progress. Most ornithologists are aware that sone genera and species are probably nisplaced in the "Handlist", especially among the Pycnonotida, Timalide and Turdida but until the anatomy of these anomalous forms has been critically and comparatively examined I can appreciate no reason for disturbing an established order. Cases in point are Chalcoparia, Egithina and Irena.

The Synonymy is purely regional. I have tried to make it as complete as possible but the library in Singapore is incomplete and I have had to get many references and type-localities from Europe. The majority of the references have been checked in the original publication, but sonne from a secondary source, in which latter case an attempt has been made to avoid the works of mere copyists. I expect, however, that this syllonyny will prove to be a weak point in my work. A number of nomina nuda have been excluded as they seem never to lrave crept into literature.

Distribution. - The localities are arranged in four rows corresponding with the Malayan, Sumatran, Bornean and Javan Provinces as defined above. Continental distribution is indicated in three ways. Peninsular Siam implies the northern part of the Malay Peninsula down to about Lat. $6^{\circ}$ N. Malay Stales means roughly, the southeru half of the Peninsula: it is the area of British influence and includes the Unfederated Malay States, the Federated Malay States and the Straits Settlements of Penang, Malacca and Singapore. Malay Peninsula includes both the above divisions.

Literature.-The references given in the footnotes are, naturally, not complete but basic, or suggestive, and can be added to those given by Peters. Although at times they may seem, at first glance, relatively unimportant, it will be found, usually, that they contain, or lead to, the essential literature on the group.

The Asterisk indicates that topotypes of the race have not been examined. In the great majority of cases these races liave therefore been accepted. They are nostly based on islands off the west coast of Sumatra (excluding the Mentawi Islands) and Banka. 
Popular Names.-If any merit accrues from the introduction of these it devolves on Mr. N. B. KinNear who persuaded me, agaiust my original inclinations, to include them here. I have met him half-way and found, or invented, English names for the species: for the subspecies, names can always be made by prefixing the geographical adjective although in a few cases names have been found for subspecies when these are very distinctive in appearance as in the case of Christmas Island forms. In general, the principle of simplicity has been followed. Some names, such as "Vielllots" Fireback" for Lophura rufa seem to have been establisled for many years. I have also tried to use names already published by Rosinson and STUART BAKER but sometimes this has not been a reasomable course to follow. "Rosy Minivet" for Pericrocolus roseus is suitable for Indian representatives of the specieg but the Malaysian fornis are grey, not pink. It has also seemed to me ungracious to drop a name originally applied as a mark of honour, or respect, and Oriolus hosei therefore appears here as "Hose's Oriole".

\section{NOMENCLATORIAI.}

In the following pages an attempt is made to abide by the "International Rules of Nomenclature". One of the chief unsettled difficulties is the vexed question of how far one should preserve the original spelling of names when these are obviously, from the purist's point of view, incorrect. 'The excellent recommendation of the rules that the original orthography should be faithfully preserved, except in the case of printer's errors, lapsus calami, and errors of transcription, has, unfortunately, been negated by the broad interpretation given to these exceptions in the "opinions" following the rules. The modern practice of regarding a nomen nudum as unusable if it was, subsequent to its original publication placed as a synonym by a revisor, seens unreasonable, and it is surely better to establish these floating names under the name of the autlior who first associated them with a valid description.

In this handlist I have continued to use several old established names although some recent autliors have regarded these as indeterminable. I suggest that these authors should be required to indicate in what way the names are inapplicable rather than the reverse. A large percentage of the older descriptions are so generalized that they are, prima facie, indeterminable. In the case of Platsymurus recently rejected, I contend that the figures on which the name is based are not only recognizable, but excellent likenesses.

An outstanding difference anong systematists concerns the status of new names, introduced inadvertently, as a result of a mistaken identification. Alcippe cinerea Blyth, and Cyornis unicolor infuscala Hartert, are both new combinations, with good descriptions, applied in this manner, but the first in the mistaken notion that it was Malacopleron cinereus Eyton, and the second, Muscicapa infuscala Blyth. 


\section{SYSTEMATIC.}

There can be few more difficult areas for a systematist to deal with than Malaysia. With its four large land-nasses, its high mountains, compact archipelagos, abundant coastal islands, and numerous oceanic islands, the division of species widely spread in the sub-region often calls for a nicety of judgment in which the personal element must, in the present state of ornithological practice, play a large part. Througliout the subregion, as elsewhere, the smaller islands show a relatively greater tendency to produce subspecies than do the large land masses. On the former the "biological discipline" seems less severe and variation is perpetuated: on the latter it is absorbed. In tnany cases the subspecies are well-defined the differences being qualitative rather than quantitative in character and in some instances the Javan and Bornean representatives of species also occuring on the Continent are so well marked that only those workers, who like myself take a very broad view of a "species". wottld attempt to link them in a trinominal nomenclature.

The Anamba Islands in the South China Sea present the reverse state of affairs. They are inhabited by continental, lowland species, but the insular forms usually diverge in the direction of slightly larger average size. On paper the distinctions seem insignificant, in birds the size of a bulbul sometimes only amounting to two or three millimetres on the mean, but to ignore stich distinctions based or good serjes is to ignore one of the most important phenomena presented by the island avifauna.

An even more delicate development of this situation is presented by some species in which a tendency to modification in the same direction is expressed, whenever a group is isolated, over a large geographic range. Aplonis is a case in point. On all the small islands of the South China Sea and its ramifications the glossy starling varies in the direction of large size and a more robust bill. The first name applied to these insular birds was based on specimens from the Anamba Islands, but as the same subspecifie cliaracters are found in varying degrees on all small islands south to the coast of Sarawak and then along the east coast of Borneo the result is a race ill-defined in range and characters. An interesting plienomenon bearing on the origin of subspecies may here be noticed. In Malaysia, where a section of a species is segregated it not infrequently throws up an individual in which a subspecies seems to be adumbrated. Thus among sunbirds and flowerpeckers living on coastal islands occasional individuals have relatively large bills, a character on whicis subspecies in these groups are often based; certain species in the Tioman and Rhio Archipelagos produce large individuals which are approaching, or are inseparable from, large races of the species found in the remoter Anamba and Natuna Islands; and the large fruit-pigeon Ducula aenea, of which, in spite of large series, I cannot separate a Bornean subspecies, does occasionally show a very blue tail in that island. 
In these species it would seem that isolation and the correlated un. checked variation is a more important factor in the evolution of new forms than adaptation to a peculiar environment.

In this list a very broad view of a "species" is taken and there is an extentsive linking of geographical races and representatives in the German fashion as I feel that in this way the specific names can usefully be given a secondary function to express relationship. Many of the combinations adopted are novel. For instance, the monotypic Bornean genus Allocolops is reduced to the rank of a subspecies of Melanocichla lugubris and the rather distinct Rhipidura phoenicura of Java becomes $R$. perlata phoenicura. In the opposite direction species are split down to the lowest possible limit and many of the stuspecies accepted are based on very fine distinctions. In the matter of genera I have been very conservative and have "lumped" extensively, for I cannot yet appreciate, among birds, any natural generic criterion and have therefore employed the genus merely as a chapterheading. Perhaps the views of the advocates of the small genus, even though the implication is the creation of a large number of monotypic genera, are more logical for, on a superficial survey, there does seem a point below which some groups of species seem indivisible e.g. the more typical Zosterops, but consideration of this seems best left to workers with cosmopolitan collections at their disposal.

The day is probably not far distant when the systematist will extend the present system of trinominal nomenclature to embrace, for want of a better term, antediluvian representatives, or in other words to recognize among existing species, stbspecies in time as well as in space. That such is the relationship' between numerous groups of closely allied forms now found distributed over the same territory seems very probable. Anthreptes malacensis and $A$. rhodolaema; and Aegithina tiphia and $A$. viridissima are two sucl pairs. In each, the first named species is commoner than the second, more wide spread in Malaysia, has developed more subspecies, and is more adaptable in the matter of terrain being found commonly in cultivated areas. It sliows, in fact, all the manifestations one would expect an older stock to show in relation to a later wave of immigrants. Physiological intolerance must bere play a part. A system of nomenclature exemplified by Anthreptes malacensis [rhodolama] now suggests itself, but at this point I hasten to add that the system has not been adopted in this list.

* $\%$ * * * *

If in the following pages it is found that some names and views do not agree with those in papers previously pubtished it nust be remembered that opinions often alter as new material accumulates. Our former method of work was to write reports on general collections from specified localities. Although such papers are, primarily, of interest to the zoo-geographer and the famist the method has its disadvantage from the systematist's point of view for there is always 
a danger that the identifications in sucl a paper are less precise than in "revisions" when the whole of the available material of one species, or group, is reviewed at one time. Having dealt with a common sumbird in a dozen papers in several years there is a chance of hasty, or perfunctory examination when the species again turns up in a small collection from an island, the avifauna of which it is desirable to list.

I have, therefore, on the present occasion, surveyed anew all the material in Singapore; studied in detail much of the relevant material in the museums of London, Tring, Buitenzorg and Kuching; and examined a number of the more critical forms in hasty visits to the museums of Amsterdam, Leyden and Berlin. In the Raffles Museum, Singapore, there are now hotsed collections which, taken together, are probably the most representative of Malaysia in existence. Unfortunately, I have not been able to visit the United States National Museum wherein ate extensive Malaysian collections.

In a number of cases, subspecies based on alleged colour distinctions have not been accepted. Some of this difference of opinion depends no doubt on the observer's eye; some of it on lack of agreement anent the criterion of separation for it seems clear that some authors separate races when the extremes of series differ, but I suspect that most of it is due to lack of understanding of postmortem changes in colour. Very little seems to be known of this subject, but I an convinced that skins kept in museums in the tropics even in the shelter of protected cabinets usually fade soover than do those in museums in England.

The rapidly fugitive colours of Cissa and the more slowly fading pigments of Harpactes are well-known, but alnost as astonishing in their colour change are skins of some of the more brightly coloured timaliids such as Mesia and Siva. Less well-known are the receding reds of Aethopyga and the rapidity with whicl sone snall, dullcolotured timaliids and pycnonotids change colour after death. In these the olive element in the plumage soon disappears, the greyness fades away, the browns come up, deepen in colour, ("burn") and then a general "snuffiness" pervades the skin. In a recent test case I found that some skins of Stachyris naculatus lost their freshness in a few months and altered considerabiy in three years. On the other hand, in 1930, I found that some skins of various species well-known to fade, collected by Whitehead in 1888 , were practically identical in tone with others taken personally in 1929. Little seems to be known about even the superficial causes of these post-mortem changes. Ignoring such drastic treatment as total immersion in spirit prior to skinning. I have noted that in some cases, tow and grass used as stuffing material have altered the colour of manmal skins. Drying over a wood fire will also produce queer results at times and relaxing and remaking not infrequently darkens a skin. Perliaps some post-mortem changes are due to physiological causes stich as the age of the feather when the bird is collected. 


\section{ACKNOWLEDGEMENTS.}

I cannot conclude this introduction without acknowledging the great help I have received, over a period of years, from numerous ornithologists. First, and foremost, is Mr. NORMAN KINNEAR of the British Museum of Natural History, whose patience seentrs inexhaustible. Over a long period he has answered, pronptly, many requests for the checking of references and type localities.

In Europe my best thanks are also due to Lord RorHschin who allowed me to work at Tring; Dr. ERWIN STREsEmann of Berlin, who is always ready to examine critically a palearctic migrant for me; and Mr. G. C. H. JUNGE who has answered numerous queries about specimens in the Leyden Museum. In the East, Mr. E. Banks, the Curator of the Sarawak Museum, has always placed his collections at my disposal in a most generous manner and by doing so has added materially to our knowledge of the Bornean fauna. Time and time again, witlrout reserve, he has sent to me the results of his numerous collecting expeditions in Borneo. Dr. K. W. DAMMERMAN of the Zoological Museum at Buitenzorg, Java, has also kindly placed valuable collections at my disposal. Messrs. E. Mays, C. B. TICAHURST and J. H. Ril.ev are other valued correspondents.

Lastly, I am pleased to acknowledge the heavy debt I ove to my former chief, Mr. C. Bonen Kross. We originally planned to produce this list in collaboration, and in its early days used to discuss difficulties as they arose.

F. N. CHASEN.

Singapore, December, 1935. 


\section{A HANDLIST OF MALAYSIAN BIRDS.}

\section{Family MEGAPODIIDÆ. \\ Genus MEGAPODIUS Gaimard. ${ }^{2}$}

Megapodius reinwardt Dumont.

Megapode.

Megapodius reinwardt reinwardt Dumont.

Megapodius Reinwardt Dumont, Dict. Sci. Nat. (Levrault), xix, I823, p. 416: Amboyna error = Iombok.

Distr.-Kangean Islands (Kangean and Sepanjang); Java Sea Islands of Solombo Besar, Arends and (?) Mata Siri.

Megapodius reinwardt cumingii Dillwyn.

Megapodius Cumingii Dillwyn, Proc. Zool, Soc. I85I, p. II8, pl. 39 : Labuan.

Megapodius lowii Sharpe, Proc. Zool. Soc. 1875, p. 111: Labuan.

* Megapodius cumingit tolutitis Bangs and Peters, Occ. Pap. Boston Soc.

Nat, Hist, v, 1927, p. 235: Maratua Island, East Borneo.

Distr.-Bornean Islands of Labuan and its islets; Tiga, Mangalum, Mantanani, Balanbangan, Banguey, Mallewallé and ? Maratua.

\section{Family PHASIANID在.}

\section{Genus RHIZOTHERA Gray.}

Rhizothera longirostris (Temm.).

Long-billed Partridge.

Rhizothera longirostris longirostris (Temm.).

Perdix longirostris Temminck, Cat. Syst. Orn. 1807, p. I58: North Sumatra.

Tetrao curvirostris Raffles, Trans. Linn. Soc. xiii, 1822, p. 323 : Sumatra.

Distr.-Malay Peninsula.

Suniatra,

Borneo (lowlands).

1 There is justification for a wider linking of the forms of Megapodius than has hitherto been practised. We do not believe in the occurrence (unaided) of the genus on any of the large land masses in Malaysia, but megapodes will undoubtedly be discovered on other small islands in the sub-region. 
Rhizothera longirostris dulitensis O.-Grant.

Rhizolhera dulitensis Ogilvie-Grant, Bull. Brit. Orn. Cl. iv, I\$95, p. 27 : Mount Dulit, North Sarawak.

Distr.-Borneo (Mt. Dulit).

\section{Genus ARBOROPHILA Hodgson.}

Arborophila brunneopectus (Blyth). Brown-breasted Tree-Partridge. [Arborophila brunneopectus brunneopectus (Blyth).

Arboricola brunneopectus "Tickell," Blyth, Journ. Asiat. Soc. Bengal, xxiv, 1855, p. 276 : Tenasserim.

Distr.-Extra-limital.]

Arborophila brunneopectus campbelli (Rob.).

Arboricola campbelli Robinson, Bull. Brit. Orn. Cl. xv, 1904, p. 28 :

Telom, Perak-Pahang boundary, Malay States.

Distr.-Malay States.

Arborophila brunneopectus rolli (Rothsch.).

Arboricola rolli Rothschild, Bull. Brit. Oru. Cl. xxv, 1909, p. 7 : Battak Mts., North-east Sumatra.

Distr.-Sumatra (North).

Arborophila brunneopectus sumatrana O.-Grant.

A rborophila sumatrana Ogilvie-Grant, Ann. Mag. Nat. Hist. (6) viii, I 891, p. 297 : Kaba Mt, South-west Sunatra.

Distr.-Sumatra (Central and West).

Arborophila brunneopectus javanica (Gmel.).

Tetrao javanica Gmelin, Syst. Nat. I, pt. 2, 1789, p. 761 : West Java.

Distr.-Java (West).

Arborophila brunneopectus bartelsi Siebers.

Arborophila javanica bartelsi Siebers, Treubia, xi, 1929, P. I49: Mt. Cherinai, Central Java.

Distr.-Java (Central).

t The affinities of some of the Arboropkila forms of South-eastern Asia are difficult to discover as the genus shows an unusually strong tendency to produce well-marked geographical representatives, Robinson (Birds Mal. Pen. ii, 1028, p. 8 ) has stated that the nearest ally of campbelli is atrogularis, but we think that the former is the Malayan representative of brunneopectus. $A$, rolli, favanica, and hyperythra are other Sunda Island forms of the same "species": Javanica although very distinct in colour is linked to brtnneopectus by some richly coloured examples of campbelli. The presence of two very distinct sub-species in Java is awkward, but orientalis is only found in the east of the island. A. rubrirostris seems nearest to A. davidi of Cochin China. 
Arborophila brunneopectus orientalis (Horsf.).

Perdix orientalis Horsfield, Trans. Linn. Soc. xiii, $182 \mathrm{I}$, p. 184 : Blambangan, East Java.

Perdix personata Horsf., Zool. Res. part viii, I824, pl. and letter press: East Java.

Distr. - Java (East).

Arborophila brunneopectus hyperythra (Sharpe).

Bambusicola hyperylhra Sharpe, Ibis, I879, p. 266 : Lawas Mts., Nortil-west Borneo.

Distr.-Borneo.

Arborophila brunneopectus erythrophrys (Sharpe).'

Bambusicola erythrophrys Sharpe, Ibis, I890, p. I39, pl. 4 : Mount Kinabalu, North Borneo.

Distr-Borneo (North).

Arborophila rubrirostris (Salvad.). Red-billed Hill-Partridge. Peloperdix rubrirostris Salvadori, Ann. Mus. Civ. Gen. xiv, I879, p. 25I : Mt. Singalan, Padang, West-central Sunuatra,

Distr.-Sumatra.

Arborophila charltonii (Eyton). Chestnut-breastea Tree-Partridge.

Arborophila charltonii charltonii (Eyton).2

Perdix Charlonii Eyton, Ann. Mag. Nat. Hist. xvi, I\$45, p. 230 :

Territory of Malacca.

Disit.-Malay Peninsula.

Sumatra.

Arborophila charltoni graydoni (Sharpe and Chubb).

Arboricola graydoni Sharpe and Chubb, Ornis, xiii, I9o6, p. 133 : Lanug, North Borneo.

Distr.-Borneo (North).

\section{Genus CALOPERDIX Blyth.}

Caloperdix oculea (Tennin.).

Ferruginous Wood-Partridge.

Caloperdix oculea oculea (Temm.).

Perdix oculea Tenminck, Pig, et Gall. iii, I8I5, pp. 408, 732 :

India, error $=$ central parts of Malay Peninsula (Rob and Kloss, 1921).

Distr.-Malay Peninsula.

4. erythrophrys and A, hyperythre are distinct forms.

2 The presence of this bird in Sumatra needs confirmation. 
Caloperdix oculen ocellata (Raffles).

Tetrao ocellatus Rafles, Tran, Linn. Soc. xiii, I822, p. 322 : Bencoolen, West Sumatra.

Caloperdix sumalrana Ogilvie-Grant, Bull. Brit. Orn. Cl. ii, 1802. p. 5 :Padang, West Sumatra.

Distr. Sumatra.

Caloperdix oculea borneensis O.-Graut.

Caloperdix borneensis Ogilvie-Grant, Bull. Brit. Orı, Cl, ii, 1892 , p. 5: Mt. Dulit, North Sarawak.

Distr.-Borneo.

\section{Genus ROLLULUS Bonnaterre.}

Rollulus roulroul (Scop.).

Crested Wood-Quail.

Phasianus roulroul Scopoli, Del. Flor, et Faun. Insubr. ii, I786, p. 93: Malacca.

Columba cristata Gmel., Syst. Nat. i, pt. 2, 1788, p. 774: Malacca.

Phasianus cristatus Sparrm., Mus. Carls., fasc 3, 1788, pl. 64:Celebes error = Borneo.

Tetrao viridis Gmel., Syst. Nat. i, pt. 2, 1780 , p. 761 : Sumatra (Raffles, 1822).

Tetrao porphyrio Shaw and Nodd., Nat. Misc. iii, I70!, pl. 84 : Malacca.

Perdix coronata Lath., Suppl. Index Orn. 1801, p. 62. Malacca.

Disir.-Malay Peninsula.

Stmatra; Banka and Billiton.

Borueo.

\section{Genus MELANOPERDIX Jerdon.}

Melanoperdix nigra (Vig.).

Black Wood-Partridge.

Melanoperdix nigra nigra (Vig.).

Crypfonyx niger Vigors, Zool, Journ, iv, 1829, p. 349 : Bencoolen, West Sumatra (Rob, and Kloss I023).

Cryptonyx fermginens Vig., Zool. Journ. iv, 1829 , p. 349 : Bencoolen (female descr.h.

Cryptonyx dussumieri Less., Bélang. Voy. Ind. 1834, p. 275, Oiseaux, pl. 7 : Malacca.

Perdix aruginosus Eyton, Proc. Zool. Soc. 1839, p. to6: Malacca (female descr.).

Distr.-Malay States.

Suncatra.

Melanoperdix nigra borneensis Rothsch.

Melanoperdix nigra bomeensis Rothschild, Bull. Brit. Orn. Cl. xxxviii, I9I7, p. 3; Balingean, Sarawak.

Distr.-Borneo. 
Genus HAMATORTYX Slatpe.

Hematortyx sanguiniceps Sharpe. Crimson-l1eaded Wood-Partridge. Hematorlyx sanguiniceps Sharpe, Ibis, I879, p. 266: Lawas Mts.. North-west Borneo.

Distr.-Borneo.

\section{Genus EXCALFACTORIA Bonaparte.}

Excalfactoria chinensis (Linn,). ${ }^{2}$ Blue-breasted Button-Quail. Excalfactoria chinensis chinensis (Linn.).

Tetrao chinensis Linn., Syst. Nat, I2th. ed. 1, 1766, p. 277 : China.

Distr.--Malay Peninsula.

Sunnatra (? North and East).

\section{Excalfactoria chinensis palmeri Riley.}

Excalfactoria chinensis palmeri Riley, Proc. Biol. Soc Wasli. xxxii, 1919, 1. 93 : Daru, Java.

Distr.-Sumatra (?South and West); Billiton; ? Banka.

Java; North Watcher Island, Thousand Islands and Java Sea.

Excalfactoria chinensis lineata (Scop.).

Oriolus linealus Scopoli, Del. Flor. et Fant. Insubr, pt. 2, I786, p. 87 : Luzon, Philippine Islands.

Excaltactoria chinensis carulescens Hachisuka, Bult. Bri ${ }^{*}$ Orn. Cl, xlvii, 1926, p. 69 : Sarawak, Borneo.

Distr,-Borneo.

Genus HOUPPIFER Guérin-Méneville.

\section{Houppifer erythrophthalmus (Rafles). Fire-back Pheasant.}

Houppifer erythrophthalmus erythrophthalmus (Raffles).

Phasianus erythrophthalmus Raffles, Trans. Linn. Soc. xiii, I822, p. 32I: Bencoolen, West Sumatra.

Houppifer diazdii Guér.-Mén., Icon. Règ. Anim., Ois, 1828-38, p. 26 , pl. 43, fig. 2: Javà error = Sumatra.

Phasianus purpureus Gray, Ill. Ind. Zool. i, 1830-32, pl. 42 : Penang.

Distr.-Malay States.

Stunatra.

1 This division of the Malaysian Excalfactoria cannot be regarded as final and large series, especially of females, from Sumatra, South Borneo and Java are needed to define the racial boundaries: it is doubtful if such series exist. The North Bornean and Sarawak bird is not separable from topo-typical lineata, but the Javan race, palmeri, seems distinct. Birds from the south of Borneo are, perhaps, palmeri. There are, at least, two races in Sumatra, and in some respects birds from the north of the island are approaching trinkutensis, described from the Nicobars. The racial distinc. tions seen in the Sunda Islands are remarkable as it seems quite impossible to split continental chinensis in spite of its wide range, although birds from Hainan look rather different in series. 


\section{Houppifer erythrophthalmus pyronotus (Gray).}

Alectrophasis pyronotus G. R. Gray, List of Birds, I84I, p. 78 : Borneo.

Alectrophasis personatus Temm. MS., Bp., Compt. Rend, xlii, 1856 , p. 879 : Borneo.

Distr.-Borneo.

Houppifer inornatus (Salvad.).

Salvadori's Pheasant.

Acomus inornatus Salvadori, Ann. Mus. Civ. Gen. xiv, I879, p. 250 :

Padang, West Sumatra.

Distr.-Sumatra.

Genus LOPHURA F']eming.

Lophura ignita Sliaw and Nodd.

Bornean Fire-back Pheasant.

Lophura ignita ignita (Shaw and Nold.).

Phasianus ignilus Shaw and Nodder, Nat. Misc. ix, text to pl, 32I, I 797 : Borneo.

Gallus macartneyi Temm., Pig. et Gall. ii, 1813, p. 273: Sumatra error $=$ Borneo.

Euplocamus nobilis Sclat., Proc. Zool, Soc. 1863, p. 118, pl. xvi: Borneo.

Distr.-Banka.

Borneo.

Lophura ignita sumatrana (Dubois)."

Euplocanus sumatranus Dubois, Bull. Ac. Belg. ii, 47, 1879, p. 825 : Palembang, Sumatra (male).

* ¿Lophura sumatrana delacouri Ghigi, Oiseau vii, 1926, p. 51, col. pl.: Sumatra.

Distr.-Sumatra. (South).

Lophura rufa (Raffles).

Vieillots' Fire-back Pheasant.

Phasianus rufus Raffles, Trans. Linn. Soc. xiii, 1822, p. 321 : Bencoolen, Sumatra (female).

Phasiamus ignitus Raffles (nec. Shaw and Nodd.), Trans. Linn. Soc. xiii, 1822, D. 320: Sumatra (male).

Phasianus castanews Gray in Griff. ed. Cuv, iii, 1829, p. 28: Penang \{female\}. ${ }^{2}$

Euplocamus vieilloti Gray, List Gen. Birds, 1 $\$ 41$, p. 77 : Malacca.

* ?Lophura sumatrant albipennis Ghigi, Oiseau, vil, 1926, p. 51 : Sumatra.

Distr.-Malay Peninsula.

Sumlatra (North and Central).

- This remains an unsatisfactory race and most of our information is based on menagerie birds, the provenance of which is always doubtful. Considering the evidence as a whole it does seem likely that there is, in South Sumatra, a pheasant in which the female is very bright rufous, and the male more like L. ignita than L. rufa. But see Klos5, Treubia, xiii, 193I, p. 304.

2 From the locality, probably an imported bird. 


\section{Genus LOBIOPHASIS Sharpe.}

Lobiophasis bulweri Sharpe.

Bulwer's Pheasant.

Lobiophasis Bulweri Sharpe, Ann. Mag. Nat. Hist. (4) xiv, 1874 , p. 373: Lawas Mts., Sarawak, Borneo.

Lobiophasis castaneicaudatus Sharpe, Proc. Zool. Soc. 1877, p. 94 : Lawas River, Sarawak, Borneo.

Distr.-Borneo.

\section{Gemus GALLUS Brisson.}

Gallus gallus (Limn.).

Red Jungle-Fowl.

Gallus gallus gallus (Linn.).

Phasianus gallus Linn., Syst. Nat. Ioth ed. 1758, p. 158 : Pulau Condore, off the mouth of the Mekong, Cochin China.

Coturnix spadicea Bonnaterre, Tabl. Encycl. Méth. i, 1791, p. 218: Malacca (Kloss, 1932).

Distr.-Malay Peninsula. Sumatra (North only).

Gallus gallus bankiva Temm.

Gallus Bankiva Temminck, Pig. et Gall. ii, I $\&_{1} 3$, p. 87 : Java.

Gallus gallus robinsoni Rothschild, Nov. Zool. xxxiii, 1926, p. 206 : Sumatra.

Distr.-Sumatra (South), Java; Bali.

Gallus varius (Shaw and Nodd.). Green Jungle-Fowl.

Phasianus varius Shaw and Nodder, Nat. Misc. x, 1798, pl. 353: Java.

Gallus furcatus Temm., Cat. Syst. Cab. d'Ornith. etc. 1807, p. 146: Malay Arch. = Java (Mathews 10,32).

Gallus Javanicus Horsf., Trans. Linn. Soc. xiii, 1821 , p. 185 : East Java.

Distr._Java; Bali; Kangean Island; Bawean Island.

\section{Gerus POLYPLECTRON Temminck.}

Polyplectron bicalcaratum (Linn.).

Peacock-Pheasant.

[Polyplectron bicalcaratum bicalcaratum (Linn.).

Pavo bicalcaratus Linn., Syst. Nat. Ioth ed. 1758 , p. I56: China error = Thoungyah, Burma (Lowe, 1925).

Distr.—Extra-limital.] 
Polyplectron bicalcaratum malacensis (Scop.).

Phasianus malacensis Scopoli, Del. Flor. et Faun. Insubr. ji, $\mathbf{1 7 8 6 ,}$ p. 93 : Territory of Malacca.

Polyplectron hardwickii J. E. Gray, Ill. Ind. Zool. 1, 1831, pI. 37 : "India" error = Malacca.

Polyplectron lineatwm J. E. 'Gray, I11. Ind. Zool. i, 1831, pl. 38: Malacca.

Distr.-Malay Peninsula. Suntatra.

Polyplectron schleiermacheri Brïgg. Bornean Peacock-Pheasant.

Polyplectron Schleiermacheri Brüggemann, Zool. Gart. 1877, p. 213 : Muara Teweh, South-east Borneo.

Distr,-Borneo.

Polyplectron inopinatus (Rothsch.). Rothschild's Peacock-Pheasant.

Chalcurus inopinatus Rotbschild, Bull. Brit. Orn. Cl. xiii, I903, p. 4I: Ulu Pahang, Malay Peninsula.

Distr.-Malay States.

Polyplectron chalcurum Temm. Sumatran Peacock-Pheasant.

Polyplectrum chalcurum Temminck, in Cuv. Regn, An. i, I829, p. 474 : Sumatra.

Polyplectrum inocellatus Less., Traité d'Orn. 1831, p. 487 : Sumatra.

Distr.-Sumatra.

Genis ARGUSIANUS Gray.

Argusianus argus (Linn.).

Argus Pheasant.

Argusianus argus argus (Linn.).

Phasianus argus Linn., Syst. Nat. I 2th ed. 1, 1766, p. 272: Chinese Tartary error $=$ Malacca .

Argus giganteus Temm., Pig. et Gall, ii, 1813 , P. 410 : Malacca.

Argus pavonius Vieill., N. Dict. d'Hist. Nat, ii, 1816, p. 507 (new name).

Argur pavonimus Vieill., Gal, des Ois, ii, 1825, 1. 19, pl. 204 (new name).

Argus paeoninus J. E. Gray, Ill. Ind. Zool. i, 1831, pl. 36: China error = Malacca.

Distr.-Malay Peninsula. Sumatra.

Argusianus argus grayi (Elliot).

Argus grayi Ėlliot, Ibis, 1865, p. 423: Borneo.

Distr.-Borneo. 


\section{Genus 'RHEINARTIA Maingonnat.}

Rheinartia ocellata (Elliot.).

Ocellated Pheasant.

[Rheinartia ocellata ocellata (Elliot).

Argus ocellatus Elliot, Ann. Mag. Nat. Hist. viii, I87r, p. Ir9: Central Annam.

Distr._Extra-limital.]

Rheinartia ocellata nigrescens Rothsch.

Rheinardius ocellatus nigrescens Rothschild, Bull, Brit. Orn. Cl. xii, Ig02, p. 55: Ulu Pahang, Malay Peninsula.

Distr.-Malay States.

\section{Genus PAVo Linn.}

Pavo muticus Linn.

Peafowl.

Pavo muticus Linn., Syst. Nat. r2th ed. i, 1766, p. 268: Japan error= Java .

Pavo javanicus Horsf., Trans. Linn. Soc. xiii, 1821, p. 185 : Java.

Distr.-Malay Peninsula.

Java.

\section{Family TURNICIDÆ.}

\section{Genus TURNIX Bonnaterre.}

\section{Turnix suscitator (Gmel.).2}

Bustard-Quail.

Turnix suscitator suscitator (Gmel.).

Tetrao suscitator Gmelin, Syst. Nat. i, pt. 2, 1789, p. $76_{3}$ : Java.

Turnix javanica Rafin, Analyse, 1815 : Java.

Hemipodius pugnax Temm., Pig. et Gall. iii, 1815, pp. 612, 754: Java.

Dislr.-Sumatra (at least north to Medan and Deli); Rhio Archipelago; Banka; Billiton. Java; Bali.

Turnix suscitator atrogularis (Eyton).

Hemipodius atrogularis Eyton, Proc. Zool. Soc. $1 \$_{39}$, p. 107 : Malacca.

Disir.-Malay States.

? Stumatra (extreme North).

- Rheinardia and Maingounat are misprints. The genus is named after Commt. Rheinart.

2 Revisions: Stresemann, Mitt. Zool. Mus. Berl. xv, 1930, p. 644; and Rob. and Baker, Bull. Brit. Orn. Cl. xlviii, 1928, p. 58 . 
Tumix suscitator interrumpens Rob. and Baker.

Turnix suscitalor interrumpens Robinson and Baker, Bull. Brit. Orn. C1. xlviii, rg28, p. 60: Kossoom, Perinsular Siam.

Distr.-Perinsular Siam.

Turnix sylvatica (Desf.). Little Bustard-Quail. [Turnix sylvatica sylvatica (Desf.).

Tetrao sylvalicus Desfontaines, Mém. Ac. Roy. Soc. Paris, 1787, p. 500, pl. 13 : near Algiers.

Distr.-Extra-limital.]

* Turnix sylvatica bartelsorum Neum.

Bartel's Bustard-Quail.

Turnix sylvatica bartelsorum Neunann, Orn. Mon. 1929, p. I17: Cheribon, Java.

Distr.-Java.

Turnix tanki Blyth.

Indian Bustard-Quail.

[Turnix tanki tanki Blyth.

Turnix tanki Blyth, Journ. Asiat. Soc. Bengal, xii, I843, p. 180 : Bengal.

Distr.-Extra-linital.]

Turnix tanki blanfordi Blyth.

Turnix blanfordi Blyth, Journ. Asiat. Soc. Bengal, xxxii, I863, p. 8o: Pegu, Lower Burma.

Distr.-Peninsular Siam.

\section{Family COLUMBID $Æ .{ }^{ \pm}$}

\section{Genus SPHENURUS Swainson.}

Sphenurus seimundi (Rob.).

Seimund's Pintail Pigeon.

Sphenurus seimundi seimundi (Rob.).

Sphenocercus seimundi Robinson, Bull. Brit. Orn. Cl. $\mathrm{xxv}, 1910$, p. 98: Semangko Pass, Selangor-Pahang boundary, Malay Peninsula.

Distr.-Malay States.

Sphenurus oxyurus (Reinw.). ${ }^{3}$

Pintail Pigeon.

Columba oxyura Reinwardt in Temminck, Pl. Col. 240,1823 : Java.

Sphenurus semitorquatus Swains, Class. Birds, ii, 1837 , p. 348 : nom. nov.

$$
\text { Distr.-Simnatra. }
$$

- Oenopopelia tranquebarica humilus (Temm.) is found in the Malay Peninsula but we doubt if it is indigenous.

? Has also been recorded from Bormeo but we doubt its occurrence in that island. 
Sphenurus sphenurus (Gould).

Wedge-tailed Pigeon.

[Sphenurus sphenurus sphenurus (Gould).

Vinago sphenura Gould, Birds hitherto unfigured, from the Himalaya Mts. I 83 I, pl. 40 : Hinalayas.

Distr.-Extra-limital.]

Sphenurus sphenurus korthalsi (Bp.).

Sphenocercus korthalsi Bonaparte, Consp. Gen. Av. ii, 1854 , p. 9 : Mt. Gede, West Java.

Sphenocercus etorques Salvad., Ann. Mus. Civ. Gen. xiv, 1879, p. 244 : Sumatra.

Distr.-Sumatra.

Java.

Sphenurus sphenurus robinsoni (O.-Grant)."

Sphenocercus robinsoni Ogilvie-Grant, Bull. Brit. Orn. Cl. xix, 1906, p. I2: Gunong Tahan, Pahang, Malay Peninsula.

Distr,-Malay States.

Genus BUTRERON Bonaparte.

Butreron capellei (Temm.). Larger Thick-billed Green Pigeon.

Butreron capellei capellei (Temm.).

Columba capellei Temminck, Pl. Col. 143, 1823: Java.

Distr.,Java (West).

Lampongs, South Sumatra.

Butreron capellei magnirostris (Strick.).

Treron magnirostris Strickland, Ann. Mag. Nat. Hist. IS 44 , p. II6, footnote: Malay Penizsula.

Butreron capellei messopora Oberh., Journ. Acad. Sci. Wash. xiv, 1924, p. 209 : Klumpang Bay, South-east Borneo.

Butreron capellei panochra Oberb., Journ, Acad. Sci. Wash. xiv, r924, p. 300: Besitan River, East Sumatra.

Distr,-Malay Peninsula.

Sumatra (? except South).

Borneo.

*Butreron capellei passorhina Oberh.

Butreron capellei passorhina Oberholser, Proc. U.S. Nat. Mus. liv, I917, p. I92: Mata Siri Island, Java Sea.

Distr.-Mata Siri Island, Java Sea.

:A doubtful race: additional material from the Malay Peninsula is required before robinsoni can be finally accepted. 


\section{Genus TRERON Vieillot.}

Treron curvirostra (Gmel.). Smaller Thick-billed Green Pigeon.

Treron curvirostra curvirostra (Gmel.).

Columba curvirostra Gmelin, Syst. Nat. i, pt. 2, 1789, p. 777, n. 45 : Tanna error = Selangor, Malay Peninsula (Rob. and Kloss, I92I).

Treron nasica Schleg., Nederl. Tijd. Dierk i, i863, p. 67 : Banjermasin, South Borneo.

Distr. - South Peninsular Siam, Malay States.

Sunatra (part); Rhio Archipelago; Banka; Billiton.

Borneo; North Natuna Islands.

Treron curvirostra nipalensis (Hodgs.).

Toria nipalensis Hodgson, Asiat. Res. xix, $18_{36} 6$, p. 164, pl. 9 : Nepal.

Distr.-Peninsular Sian (except extrene South); Langkawi and Butang Islands off west coast; islands of the Bandon Bight off the east coast of the Malay Peninsula.

Treron curvirostra harterti Parrot.

Treron nipalensis harterti Parrot, Abh. Kon. Bay. Akad. Wiss. xxiv, 1907, p. 26r : Deli, Nortli-east Sumatra.

Distr, - Sumatra (North-east).

-Treron curvirostra haliploa Oberh.

Treron curvirostra haliploa Oberholser, Smith. Misc. Coll. Ix, 7, IgI 2, p. 4 : Sibabo Bay, Simalur Island.

Distr.-Simalur Island, West Sumatra.

* Treron curvirostra pega Oberh.

Treron curvirostra pega Oberholser, Smith. Misc. Coll. lx, 7, I9r2, p. 4 : Siaba Bay, Nias Island.

Distr.-Nias Island, West Sumatra.

Treron curvirostra smicra Oberh.

Treron curvirostra smicra Oberholser, Smiths. Misc. Coll. 1x, 7, I912, p. 3 : Tana Bala Island, Batu Islands.

Distr.-Batu Islands; Mentawi Islands (Siberut and Sipora), West Sumatra.

*Treron curvirostra hypothapsina Oberly.

Treron curvirostra hypothapsina Oberholser, Smith. Misc. Coll. 1x, 7, I9I2, p. 3 : Engano Island.

Distr.-Engano Island, West Sumatra.

Kelapa Island, West Java."

- Racial identity not certain. 


\section{Treron curvirostra erimaca Oberh.}

Treron curvirostra erimaca Oberholser, Journ. Wash. Acad. Sci, xiv, I924, p. 297 : Balabac Island, Philippine Islands.

Distr.-North Bomean Islands (Banguey and Balambangan).

\section{Treron pompadora (Gmel.).}

Pompadour Green Pigeon.

[Treron pompadora pompadora (Gmel.).

Columba pompadora Gmelin, Syst. Nat. i, pt. 2, 1789, p. 775 : Ceylon.

$$
\text { Distr.-Extra-limital.] }
$$

Treron pompadora pulverulenta Wall.

Treron pulverulenta Wallace, Ibis, $1 \$ 63$, 1. 319: East Java.

$$
\text { Distr.-Sunatra (South). }
$$

\section{Treron pompadora vordermani Finsch.}

Treron Vordermani Finsch, Notes Leyden Mus. xxii, Ig00, p. I62: Kangean Islands.

Distr.-Kangean Islands.

Treron fulvicollis (Wagl.). Cinnamon-headed Green Pigeon.

Treron fulvicollis fulvicollis (Wagl.).

Columba fulvicollis Wagler, Syst. Av. Columba, Sp. S, 1827: Java, error $=$ Sumatra .

Columba fermginea Wagl. (nec Forst.), Isis, 1829, p. 738: Java error = Sumatra.

Columba cinnamomea Temm., Pl. Col. Livr. 93, text, 1835: Pontianak, South Borneo.

Treron tenuirosire Eyton, Ann. Mag. Nat. Hist. xvi, 1845, p. 230 : Malacca.

Distr.-Malay Peninsula.

Sumatra; Rhio Archipelago; Banka; Billiton.

Borneo (except North).

Treron fulvicollis oberholseri Chas.

Treron fulticollis oberholseri Chasen, Bull. Raffles Mus, o, 1934, p. 92 : Bunguran, North Natuma Islands.

Distr.-North Natuna Islands.

*Treron fulvicollis melopogenys (Oberh.).

Dendrophassa fulvicollis melopogenys Oberholser, Smiths. Misc.

Coll. 1x, 7 , Igra, p. 3 : Nias Island, West Sumatra.

Distr.-Nias Island, West Sumatra. 
Treron fulvicollis baramensis Meyer.

Treron fulvicollis baramensis Meyer, Jonrn. f. Orn. I89., p. 73 : Baram, North-west Borneo.

Distr.-Borneo (North Sarawak and British North Borneo); North Bornean Islands.

Treron bicincta (Jerd.). Orange-breasted Green Pigeon.

[Treron bicincta bicincta (Jerd.).

Vinago bicincta Jerdon, Madras Jouru. Lit. Sci. xii, I 840, p. I 3 : Madras.

Distr.-Extra-limital.]

Treron bicincta praetermissa Rob. and Kloss.

Treron bisincta praelermissa Robinson and Kloss, Journ. Fed. Malay States Mus. x, I92 I, D. 203: Koln Lak, South-west Siam.

Distr.-Malay Peninsula.

Treron bicincta javana Rob, and Kloss.

Treron bisincta javana Robinson and Kloss, Journ. Fed. Malay States Mus. xi, I923, p. 53: Badjoelmati, East Java.

Distr.-Java (Central and East).

Treron vernans ${ }^{3}$ (Limn.).

Pink-necked Green Pigeon.

[Treron vernans vernans (I,inn.).

Columba vernans Linn., Mantissa Plant. I771, p. 526 : Luzon Island, Philippine Islands.

Distr.-Extra-limital.]

Treron vernans griseicapilla Schleg.

Treron griseicapilla Schlegel, Neder1. Tijd. Dierk. i, 1863, p. 70 :

Sumatra.

Dendrophassa vernans abbotti Oberholser, Journ. Wash. Acad. Sci xiv. 1924, p. 298: Trang, Peninsular Siam.

Distr.-Malay Peninsula; Tioman Archipelago (except Tioman Island).

Sumatra (except North-east); Rhio Archipelago; Banka; Billiton.

Borneo (except South-east); Mantanani and Mangalum Islands.

Java (West).

I Revision: Oberb., Bull. U.S. Nat. Mus. 159, t932, p. 32. 
Treron vernans purpurea (Gmel.).

Columba purpurea Gmelin, Syst. Nat. i, pt. 2, I739, p. 784 : Java.

Distr.-Java (Central and East); Bali; ? Kangean Islands.'

Borneo (South-east); Maratua Islands.

Treron vernans parva Kloss.

Treron vernans parva Kloss, Treubia, xiii, r931, p. 308: Deli, Northeast Sumatra.

Distr.-Sumatra (North-east).

Treron vernans adina (Obeth.)."

Dendrophassa vernans adina Oberholser, Bull. U.S. Nat. Mus. 98 , 1917, p. 20: Mata Island, Anamba Islands.

Dendrophassa vernans pellochlora Oberh., Bull. U. S. Nat. Mus. clix, 1932, p. 30 : Sirhassen Jsland, South Natuna Islands.

Distr.-Tionan Island, Anamba Islands; Nortls and South Natuna Islands.

* Treron vernans miza (Obeth.).

Dendrophassa vernans miza Oberholser, Smiths. Misc. Coll. 1x, 7 , I9I 2, p. 3 : Simalur Island.

Distr.-Simalur Island, West Sunatra; ? Banjak Islands.

*Treron vernans mesochloa (Oherh.).

Dendrophassa vernans mesochloa Oberholser, Sniths. Misc. Coll. Ix, 7, I9I 2, p. 2: Nias Island.

Dendrophassa vernans polioptila Oberholser, Smiths. Misc. Coll. 1x, 7, 1912, p. 3 : North Pagi Island, Mentawi Islands, West Sumatra.

Distr.-Nias Island; Tana Bala, Batu Islands; Mentawi Islands; Engano Island, West Sumatra.

Treron olax (Temm.).

Little Green Pigeon.

Columba olax Temninck, P1. Col. 24I, I823: Padang, Sumatra.

Osmotreron olax hageni, Parrot, Abh. Kon. Bay. Akad. Wiss. xxiv, 1907, p. 266 : Deli, North-east Sumatra.

Dendrophassa olax arismicra Oberholser, Journ. Acad. Sci. Wash. xiv, 1924, p. 298 : Segah River, North-east Borneo.

Distr.-Malay Peninsula.

Sumatra; Rhio Archipelago; Banka.

Borneo; North Natuma Islands.

Java.

t Birds from the Kangean Islands are, according to Hartert, like purpurea in colour, but the few published measurements show that they are larger.

- Visual records from the Tambelan Istands probably refer to this race : T. v. griseicapilla from Bomeo appears to visit the Natuna Islands at certain seasons. 


\section{Genus PTILINOPUS Swainson.}

Ptilinopus porphyreus ('Temm.).

Sunda Island Fruit-Dove.

Columba porphyrea "Reinw" Temuninck, Pl. Col. 106, IS23: Java. Columba roseicollis Wagl., Syst. Av. Columba, 1827 : Java.

Ptilinopus erythrocephalus Swains, Class. Birds, ii, 1837, p. 347 : new name for $C$. porphyrea

$$
\text { Distr.-Sumatra. }
$$

Java; Bali.

Ptilinopus cinctus (Temm.).

White-headed Fruit Dove.

[Ptilinopus cinctus cinctus (Temm.).

Columba cincta Temminck, in Tenm. and Knip, Pigeons, 18 ro, p. 58, pl. 23 : South Asia=Timor (Hartert, I904).

Dislr.-Eixtra-limital.]

\section{Ptilinopus cinctus albocinctus Wall,}

Ptilinopus albocinctus Wallace, Proc. Zool. Soc. I 863, p. 496, pl. 39 : Flores.

Ptilinopus albocinctus baliensis Hart., Nov. Zool. iii, 1896, p. 553 : Bali. Distr.-Bali.

Ptilinopus jambu (Gmel.).

Pink-headed Fruit-Dove.

Columba jambu Gmelin, Syst. Nat, i, pt. 2, $17 \delta_{9}$, p. $7 S_{4}$ : Java, error = Sumatra (Hartert, 1002).

Distr.-Malay Peninsula; Tioman Archipelago.

Sunatra; Rhio Archipelago; Banka; Billiton.

Borneo.

Ptilinopus melanocephalus (Penn.).

Black-naped Fruit-Dove.

Ptilinopus melanocephalus melanocephalus (Penn.).

Columba melanocephala Pennant, Ind. Zool, I769, p. 6, pl. 6 : Java. Columba Indica Müll., Natursyst. Suppl. 1776, p. 133 : Batavia.

Hamatana richmondena Math, Bull, Brit. Orn. Cl, xlvi, 1926, p. 60 : nom. nov.

Distr.-Java; Bali; Kangean Islauds.

*Ptilinopus melanocephalus massopterus (Oberh.).

Hacmataena melanocephala massoptera Oberholser, Proc. U.S. Nat.

Mus. liv, I917, p. IgI : Mata Siri Island, Java Sea.

Distr.-Mata Siri Island, Java Sea. 
Ptilinopus melanocephalus bangueyensis (Meyer).

Ptilopus bangueyensis Meyer, Journ. f. Ornith. I89r, p. 7o : Bangtey Island, North Borneo.

Distr.-Nortl Bornean Islands of Banguey and Balambangan; Maratua Islands.

Karimon-Java Islands.

\section{Gents DUCULA Hodgs.}

Ducula aenea ${ }^{1}$ (Linn.).

Green Imperial Pigeon.

Ducula zenea (Lintr.).

Columba anea Linn., Syst. Nat. 12th ed. i, I766, p. 283 : Moluccas, error =Flores (Hart , and Goodson, I9I8).

Colomba Moluccensis Müll., Natursyst. Suppl. 1776, p. 133: Moluccas error = Borneo (Oberh. 1932).

Muscadivores aeneus polius Oberh., Bull. U. S, Nat. Mus. 98, 1917, p. 18: Siantan Island, Anamba Ișlánds.

Muscadivores aeneus arhadius Oberh., Journ. Wash. Acad. Sci. xiv, 1924, p. 296 : Kateman River, East Sumatra.

Muscadivores aenews diatropurus Oberh. , Bull. U. S. Nat. Mus. 159, 1932, p. 25: Pulau Midai, Natuna Islands.

Distr.-Malay Peninsula; Tionan Archipelago; Anamba Islands; Tambelan Islands.

Sumatra; Rhio Archipelago; Banka; Billiton.

Borneo; Natuna Islands; Karimata Islands.

Java; Karimon-Java Islands.

Ducula zenea palawanensis (Rlas.).

Carpophaga enea nov. var. palawanensis Blasius, Ornis, iv, I888, p. 316 : Palawan.

Distr.-Banguey Island, North Borneo.

*Ducula senea mista (Oberh.).

Muscadivores aneus mistus Oberholser, Smiths. Misc. Coll. 1x, 7, rgr2, p. 2 : Simalur Island, West Surcatra.

Disir.-Simalur Island, West Sumatra.

Ducula anea babiensis (Richm.).

Muscadivores consobrina babiensis Richmond, Proc. Biol. Soc. Wash. xxv, I9I2, p. I03: Babi Island, West Sumatra.

Distr.-Babi and Lasia Islands, West Sumatra.

1 Revision: Oberh., Bull. U.S. Nat. Mus. 1591, D. 27. 
Ducula senea consobrina (salvad.).

Carpophaga consobrina Salvadori, Ann. Mus. Civ. Gen. (2) 4, I887, p. $55^{8}$ : Nias Island, West Sumatra.

Carpophaga vandepolli Büttikofer, Notes Leyd. Mus. xviii, I $\$ 06$, p. Igo: Nias Island.?

Distr.-Nias Island; Banjak Islands, West Sumatra.

Ducula senea vicina (Riley).

Muscadivores aneus ricinus Riley, Proc. Biol. Soc. Wasl. xl, I927, p. 95: Sipora Island, Mentawi Islankls, West Sumatra.

Distr.-Batu Islands; Mentawi Islands, West Sumatra.

Ducula anea cenothorax (Salvad.).

Carpophaga enothoras Salvadori, Anz. Mus. Civ. Gen. (2) I2, I892, p. I 39 : Engano Island, West Sumatra.

Dislr.-Eingano Island, West Sumatra.

Ducula rosacea (Tentm.).

Island Imperial Pigeon.

Ducula rosacea rosacea (Temm.).

Columba rosacea Tenminck, P1. Col. 578, I835: 'Timor.

Distr. ${ }^{2}$-Thousand Islands, Java Sea (West); Kangean Islands; Karimon-Java Islands.

*Ducula rosacea zamydra (Oberh.).

Muscadivores rosaceus zamydrus Oberholser, Proc. U.S. Nat. Mus. liv, 1917, p. 179: Solombo Besar Island, Java Sea.

Distr.-Islands of Solombo Besar and Arends, Java Sea (East).

Ducula rosacea whartoni (Sharpe). Chrismas Island Pigeon.

Carpophaga whartoni Sharpe, Proc. Zool. Soc. 1887, p. 515, p1. 43: Christmas Island, Indian Ocean.

Distr.-Christnuas Island, Indian Ocean; Cocos Keeling Islands (introduced).

Ducula pickeringii (Cass.). Pickering's Imperial Pigeon.

Ducula pickeringii pickeringii (Cass.).

Carpophaga pickeringii Cassin, Proc. Acad. Nat. Sci. Philad. vii, 1854, p. $22 \mathrm{~S}$ : Mangsi Islet, North Borneo.

Carpophaga everetti O.-Grant, Ann. Mag. Nat. Hist. (6) ii, 1888, p. 351 : Mantanani Island.

Distr.-North Bornean Islands of Mangsi, Mangalum and Mantanani; Maratua Islands.

- Fide G. C. A. Junge (Mus. Leyden) in litt.

= Birds from all these localisies may of course be $D . r$. samydra which we have not seen. 
Ducula badia (Raffles).

Mountain Imperial Pigeon.

Ducula badia badia (Raffles).

Columba badia Raffles, Trans. Linn. Soc. xiii, 1822 , D. 317 : Bencoolen, West Sumatra.

* Columba capistrata Temm., Pl. Col. 165, 1823: Batavia, West Java.

Distr.-Malay Peninsula.

Sunuatra.

Borneo.

Java (West only)."

Ducula badia lacernulata (Temm.).

Columba lacernulata Temminck, Pl. Col. 164, 1823: Java.

\$Ducula concolor Bp., Compt. Rend. xliii, 1856, p. 836 : Java.

Distr.-Java (West and Central).

Ducula badia williami (Hart.).

Carpophaga williami Hartert, Nov. Zool. iii, I\&g6, p. 552 : Bali.

Distr._Java (East); Bali.

Genus MYRISTICIVORA Reichenbach.

Myristicivora bicolor (Scop.).

Pied Imperial Pigeon.

Myristicivora bicolor bicolor (Scop.) .

Columba bicolor Scopoli, Del Flor. et Fauna Iusubr. ii, $x 786$, D. 94 : New Guinea.

Disir.-Malay Peninsula; Tioman Archipelago; Anamba Islands; Tambelan Islands; Saddle Island.

Sumatra; Rhio Archipelago; Lingga Archipelago; Billiton; Simalur Island; Flat Islands; Nias Island; Sipora and Pagi Islands, Mentawi Islands; Engano Island.

Borneo; Mangalum Island; Mantanani Island; Maratua Islands; Natuna Islands; Karimata Islands.

Java; Kangean Islands; Christmas Island.

\section{Gents COLUMBA Linn."}

\section{Columba argentina $\mathrm{B} \mathrm{p}$.}

Grey Wood-Pigeon.

Columba argentina Bonaparte, Consp. Gen. Avium ii, 1854, p. 36 :

Borneo (Robinson and Kloss, 1923 ).

[Myristicivora] Carpophaga grisea (not Columba grisea Bonnaterre 179o), B]3., Consp. Gen. Av, ii, 1855, p. 36 (Gray MS.) : Indian Archipelago.

- Very few examples of badia have been recorded from West Java and it seems likely that it either occupies a very restricted area in the island, or that it is a casual visitor from the Lampongs.

* A skin of Columba livia intermedia Strickl, in the British Museum. from Salanga (Puket), Peninsular Siam, is probably not that of a wild bird.

Revision: Snouck v. Schaub., Org. Cl. Ned. Vog. iii, 1930-31, p. 13. 
Columba phasma Richmond, Proc. U. S. Nat. Mus. xxvi, 1903, p. 490 : nom. nov.

Distr.-? Jarak Island, Straits of Malacca; ${ }^{2}$ Anamba Islands. Sumatra; Karinon, Bintang and Batam Islands, Rlio Archipelago; Taya Island, Lingga Archipelago; Simalur Island; Pagi Islands.

Borneo; Karimata Islands; North Natuna Islands.

Columba punicea (Tick.).

Purple Wood-Pigeon.

C. (Alsocomus) punicens Tickell in Blyth, Journ. Asiat. Soc. Bengal, xi, I842, p. 461: Singhbhum, Chota Nagpur.

Distr.-Peninstilar Siam.

Columba vitiensis Quoy and Gaim.

[Columba vitiensis vitiensis Quoy and Gaim.

Calumba ritiensis Quoy and Gainard, Voy. "Astrolabe", Zool. i, I 830, p. 246 , pl. 28 : Fiji.

Dislr.-Extra-liunital.]

Columba vitiensis griseogularis (Wald. and Layard). Grey-throated Wood-Pigeon.

Ianthanas griseogularis Walden and Layard, Ibis 1872 , 1\%. I04, pl. 6: Guimaras, Plilippine Islands.

Distr.-Islands of Tiga and Mantanani, North Borneo; ${ }^{2}$ Maratua Islands.

\section{Genus MACROPYGIA Swainson.}

Macropygia unchall (Wagl.).

Large Cuckoo-Dove.

Macropygia unchall unchall (Wagl.).

Columba Unchall Wagler, Syst. Av. Columba no. 38,1827 : Java. Columba leptogrammica Temm., Pl. Col. 560, I 835 : Java.

Macropygia walik-mehra Reichb., Tauben, I862, p. 86, pl. 3: Java.

Distr.-Malay States.

Sumatra.

Java.

Macropygia phasianella (Temm.). Sunda Island Cuckoo-Dove.

[Macropygia phasianella phasianella (Temm.).

Columba phasianella Temminck, Trans, Linn. Soc. xiii, I\$2 T, p. 129: near Port Jackson, New South Wales.

Distr.-Extra-limital.]

Visual records by H. C. Robinson.

a Has also been recorded from the mainland of North Borneo but this needs confirmation. 


\section{Macropygia phasianella emiliana Bp.}

Macropygia eniliana Bonaparte, Consp. Gen. Avium ii, 1855, p. 58 : Java.

Macropygia phasianella banussa Siebers, Treubia, xi, 1929, p. 152 : Palembang, Sumatra.

$$
\text { Distr. -Java; Bali. }
$$

Suniatra.

Macropygia phasianella megala Siebers.

Macropygia phasianella megala Siebers, Treubia, xi, 1929, p. I5I: Ardjasa, Kangean Island.

Distr.-Kangean Island.

Macropygia phasianella borneensis Roh. and Kloss.

Macropygia emiliana borneensis Robinson and Kloss, Journ. Fed. Malay States Mus. X, I921, P. 203: Saribas, Sarawak, Borneo.

Distr.-Borneo.

* Macropygia phasianella hypopercna Oberh.

Macropygia emiliana hypopercna Oberholser, Suiths. Misc. Coll. lx, 7, I912, p. 2 : Simalur Island, West Sumatra.

Distr.-Simalur Island, West Sumatra.

Macropygia phasianella modiglianii Salvad.

Macropygia modiglianit Salvadori, Ann. Mus. Civ. Gen. (2) 4 , I887, p. 559, pl. 8: Nias Island, West Sumatra.

Disir.-Nias Island, West Sumatra.

Macropygia phasianella elassa Oberh.

Macropygia emiliana elassa Oberholser, Smiths. Misc. Coll. 1x, 7, I912, p. 2 ; North Pagi Island, Mentawi Islands, West Sumatra.

Distr-Siberut, Sipora, North and South Pagi Islands, West Sumatra.

Macropygia phasianella cinnamomea Salyad.

Macropygia cinnamonea Salvadori, Ann. Mus, Civ, Gen, (2) 12, I892, p. I40: Engano Island, West Sumatra.

Distr.-Engano Island, West Sumatra.

Macropygia ruficeps (Temm.).1

Macropygia ruficeps ruficeps (Temm.).

Columba ruficeps 'Temminck, PI. Col. 56r, 1835: Java.

Distr.-Java; Bali.

Revision: Stres., Nov, Zool. xx, 1913, p. 3rt. 
Macropygia ruficeps malayana Chas. and Kloss.

Macropygia ruficeps malayana Chasen and Kloss, Bull. Raftes Mụs., 5. r931, p, 82 : Semangko Pass, Perak-Pahang Boundary, Malay States.

Distr.-Malay States.

Macropygia ruficeps sumatrana Rob. and Kloss.

Macropygia ruficeps sumatrants Robinson and Kloss, Jouru. Straits Brauch, Roy. Asiat. Soc. 1xxx, I9r9, p. 77 : Ophir District, West Sumatra.

Distr.-Sumatra.

\section{Macropygia ruficeps nana Stres.}

Macropygia ruficeps nana Stresemann, Nov. Zool. xx, I913, p. 3II : Mt. Kina Balu, North Borneo.

Distr.-Borneo.

- Macropygia ruficeps simalurensis Richm.

Macropygia simalurensis Richmond, Proc. Biol. Soc. Wash. xv, r902, p. r87 : Simalur Island, West Sumatra.

Distr.-Sinalur Istand, West Sumatra.

Genus STREPTOPELIA Bonaparte.

Streptopelia bitorquata (Tenun.).

Island Turtle-Dove.

Streptopelia bitorquata bitorquata (Temm.).

Columba bitorquata Temminck in Temm. and Knip, Pigeons, i, ISII, p. 86, pl. 40: India error=Timor (Hellmayr, I914).

Distr.-Sumatra.' Java; Bali.

Streptopelia bitorquata dusumieri (Temm.).

Columba dusumieri Temminck, P1. Col. I823, pl. I88: Manila, Philippine Islands.

Distr.-Borneo*

Streptopelia chinensis (Scop.).

Spotted Dove.

[Streptopelia chinensis chinensis (Scop.).

Columba chinensis Scopoli, Del, Flor. et Fauna Insubr. I786, p. 94 : China.

Distr.-Extra-limital.]

- Doubtfully indigenous in Sumatra.

2 Aimost certainly introduced into Borneo as a cage-bird. 
Streptopelia chinensis tigrina (Temm.)."

Columba Tigrina Temminck in Tenm. and Knip, Pigeons, i, 1811, p. 94 , tab. 43 : Java.

Metriopelia inornata $\mathrm{Bp}$, Consp. Gen. Av. ii, 1855 : Brazil error = Java (Mathews, 1927).

Distr._Malay Peninsula; Tambelan Islands.

Sumatra (part); Rhio Archipelago; Banka; Billiton.

Borneo; North Bortiean Islands.

Java; Bali; Kangean; Bawean Island.

Streptopelia chinensis minor (Parrot).

Turtur tigrinus minor Parrot, Abh, Kon. Bay. Akad, Wiss. 2,

K1. 24, Bd. i, I907, p. 275 : Deli, North-east Sumbtra.

Distr,-Sumatra (North).

\section{Getus GEOPELIA Swainson.}

Geopelia striata (Linn.).

Barred Ground-Dove.

Geopelia striata striata (I.inn.).z

Columba striata Linn., Syst. Nat. Izth ed, i, 1766, p. 282 : "East Indies" = Malacca.

Columba malaccensis Gmel., Syst. Nat. i, t788, p. 788: Kedah ex Sonnerat.

Columba bantamensis Sparrm., Mus. Carls. fasc. 3, 1788, pl. 67: Java.

Geopelia lineata Swains., Class Birds, ii, 837 , p. 348: New name for bantamensis $\mathrm{Sp}$.

Distr.-Malay Peninsula; Anamba Islands.

Sumatra; Billiton; Banka.

Borneo; Natuna Islands.

Java; Bali; Kangean Islands; Bawean Island; Karimon-Java Islands.

\section{Genus CHALCOPHAPS Goull.}

Chalcophaps indica (Linñ.).

Emerald Dove.

Chalcophaps indica indica (Linn.).

Columba indica Linn., Syst. Nat. Ioth ed. 1758, p. 164: Calcutta (Baker, r92S).

Columba jazanensis Müll, Natursyst. Suppl. 1776, p. 133: Java.

1 This is a favourite cage-bird in Malaysia and the range given may be artificially large.

- Has almost certainly been introduced into some of the islands included in the range. We are not satisfied that Columba sinica Linn., Syst. Nat. roth ed. 1758, p. 164 applies to this species. 
Columba Javanica Gmel., Syst. Nat. 1789, p. 781 : Java.

Peristera bornensis Bp., Consp. Av. ii, $1855 . \mathrm{p} .91$ : as syn. of $C$. javanica. Distr.-Malay Peninsula; Tionan Archipelago; Anamba Islands; Tambelan Islands.

Sunatra; Rhio Archipelago; Banka; Billiton; West Sumatran Islands of Simalur, Babi, Lasia, Nias, Siberut, North Pagi and Eingano.

Borneo; Nortly Bornean Islands; Mangalum Island; Natuna Islands.

Java; Bali; Kangean Islands; Bawean Island.

\section{Chalcophaps indica natalis I.ister.}

Chalcophaps natalis Lister, Proc. Zool. Soc, 1888 , p. 522 : Christmas Island.

Distr.-Christmas Island, Indian Ocean

\section{Gerus CALCENAS Gray.}

Calcenas nicobarica (Linn,). Nicobar Pigeon.

Caloenas nicobarica nicobariea (Linn.).

Columba nicobarica Linn., Syst. Nat. Ioth ed. 1758, P. I64 : Nicobar Islands.

Distr. - Small islands almost everywhere in Malaysia but apparently never on the large land masses of the Malay Peninstrla, Sumatra, Borneo, Java, Bali, Banka and Billiton.

\section{Family RALLID后.}

Rallus striatus Linn.

Blue-breasted Banded Rail.

Rallus striatus striatus (Linn.).

Rallus striatus Linn., Syst. Nat. I2th ed. r, I766, p. 262: Philippine Islands = Manila (Oberh., 1924).

Distr.-Borneo (perhaps not in the South).

\section{Rallus striatus gularis Horsf. ${ }^{1}$}

Rallus gularis Horsfield, Trans. Linn. Soc. xiii, 1821, p. 196: Java. Distr.-Malay Peninsula.

Sunnatra; Billiton.

? South Borneo.

Java.

t Birds from the Malay Peninsula and Sumatra belong to the same race: good series of gularis are not available for comparison. The Malayan bird does not attain the large size of the Indian albiventer Sw. 
Rallus striatus jouyi (Stejn.).2

Hypotanidia jouyi Stejneger, Proc. U.S. Nat. Mus, ix, I8S6, p. 362: Shanghai.

Distr.-Malay States.

*Rallus striata reliquus (Oberh.).

Hypolanidia striala reliqua Oberholser, Proc. U.S. Nat. Muss. 1v, I919, p. 476: Simalur Island, West Sumatra.

Distr.-Simalur Island, West Sumatra.

\section{Rallus philippensis Linn.}

Philippine Rail.

[Rallus philippensis philippensis Linn.

Rallus philippensis Linn., Syst. Nat. I 2th ed. I, I766, p. 263 : Philippine Islands.

Distr._Extra-limital.]

Rallus philippensis andrewsi (Math.).

Eulabeomis philippensis andreu'si Mathews, Bds. Austr. I, I911, p. 199: Cocos-Keeling Islands.

Distr.-Cocos-Keeling Islands.

Rallus fasciatus Raffles.

Malay Banded Crake.

Rallus fasciatus Raffles, Trans. Linn. Soc. xiii, I822, 1, 328 : Bencoolen, West Sumatra.

Gallinula eurizoma Temm., Pl. Color. 417, i 826 : Java.

Rallus ruficeps Less., Traité d'Orn. 1831, p. 537 : Java.

Distr.-Malay Peninsula; Anamba Islands.

Sunatra; Rhio Archipelago; Banka; Billiton; West Sumatran Islands of Batu group, Sipora and Engano.

Borneo; South Natuna Islands.

Java; Bali.

Rallus eurizonoïdes (Lafres.).

Banded Crake.

[Rallus eurizonoĩdes eurizonoïdes (Lafres.).

Gallinula eurizonö̈des Lafresnaye, Rev. Zool. I845, p. 368: Phillipines.

Distr._Extra-Limital.]

Rallus eurizonoides nigrolineatus (G. R. Gray).2

Zapomia nigrolineata G. R. Gray, Cat. Bds. Nepal, 1846, p. 143 : Nepal.

Rallus superciliaris Eyt. (not Vieill.), Ann. Mag. Nat. Hist. xvi, 1845, p. 230 : Malacca.

Rallina telmalophila Hume, Stray Feath. vii, 1878 , p. 142 : Malacca.

Disit.-Malay Peninsula. Sumatra.

- We refer certain large birds from Singapore and Johore to jouyi, but do not know how this bird differs from albiventer $\mathrm{Sw}$.

a Fide J. L. Peters (1034). 
Rallus paykullii Ljungh.

Chinese Banded Crake.

Rallus Paykullii Ljungh, Kongl. Vet. Acad. Handl. 1813, p. 258 :

Batavia, Java.

Porsana rutgenis Wall., Proc. Zool. Soc. 1865, p. 481 : Sarawak, Borneo. Rallina (Euryzona) zonaventris Cab., Journ. f. Orn. I88r, p. 425: Malacca.

Distr.-Malay States.

Sunatra.

Borneo.

Java.

Genus PORZANA Vieillot.

Porzana pusilla (Pall.).

Eastern Little Crake.

Porzana pusilla pusilla (Pall.).

Rallus pusillus Pallas, Reise Prov. Russ. Reichs. iii, 1776, p. 700 * Dauria.

Distr.-Malay Peninsula.

Sumatra.

Borneo.

Java (West).

Porzana cinerea (Vieill.).

Grey-bellied Crake.

Porzana cinerea cinerea (Vieill.).

Porphyrio cinereus Vieillot, Nouv. Dict. xxviii, I8r9, p. 29: Java (Mathezes, tort).

Rallus quadristrigatus Horst., Trans. Linn. Soc. xiii, 1821, p. 196: Java. Distr,-Malay Peninisula.

Sumatra,

Borneo.

Java; Kangean Islands.

Porzana fusca (Linn.).

Ruddy Crake.

Porzana fusca fusca (Linn.).

Rallus fuscus Linn., Syst. Nat. I 2th ed. I, I766, p. 262 : Philippine Islands.

Rallus rubiginosus Temm., P!. Col. 357, 1825 : Java.

Distr.-Malay States.

Sumatra.

Borneo.

Java; Christmas Island.

Porzana fusca erythrothorax (Temm. and Schleg.).

Gallinula erythrothorax Temminck and Schlegel, Faun. Japon. Aves, I 849 , p. I2I, pl. 78 : Japan.

Distr,-Peninsular Sian. 
Porzana bicolor Wald.' Elwe's Crake.

Porzana bicolor Walden, Ann. Mag. Nat. Hist. (4) 9, IS72, p. 47: Darjiling, Sikkim.

Distr.—? Malay States.

Genus AMAURORNIS ${ }^{2}$ Reichenbach.

Amaurornis phanicurus (Penn.). White-breasted Water-Hen.

[Amaurornis phoenicurus phœnicurus (Pentn.).

Gallinula phanicurus Pennant, Ind. Zool. 1769, p. 10: Ceylon.

Distr.-Extra-linital.]

Amaurornis phoenicurus chinensis (Bohll.).

Fulica chinensis Boddaert, Tab1, Planch, En1lun, $17 S_{3}$, p. 54 : China $=$ Hongkong (Stresemann, 1913).

Distr.-Malay Peninsula. Sumatra. ${ }^{3}$

Amaurornis phœenicurus javanicus (Horsf.).

Gallinula javanica Horsfield, Trans. Linn. Soc. xiii, IS21, p. 196: Java.

Gallinula sumatranus Raffles, Trans. Linn. Soc. xiii, 1822, pp. 70, 328 : Sumatra.

Amanrornis phanicura cleptea Oberh., Smiths Misc. Coll. 1x, 7 1912, p. 2 : Nias Island.

Distr.-Malay States (extreme south); Singapore Island.

Sumatra; Banka; Rhio Archipelago; West Sumatran Islands of Simalur, Nias, Batı Islands and the Mentawi Islands. Borneo; South Natura Islands; Billiton.

Java; Bali; Thousand Islands, Java Sea; Kangean Islands.

Genus GALLINULA Brisson.

Gallinula tenebrosa Goujd.

Black Moor-Heis.

[Gallinula tenebrosa tenebrosa Gould.

Gallinula tenebrosa Gould, Birds Austr. I $\$_{4} 6$, pt. xxiii, pl. 73 : South Australia,

Disir.-Extra-limital.]

* Gallinula tenebrosa frontata Wall.

Gallinula frontata Wallace, Proc. Zool. Soc. I863, P. 35: Buru.

Distr._Borneo (South-east). doubt.

J The authenticity of a single specimen recorded from Johore is open to

af. Stres., Nov. Zool. xx, 1913, p. 303; Chas. and Kl., Journ. Siam Soc. (Nat. Hist. Suppl.) vii, 1928 , p. 157. race.

3 Long-winged birds occurring in Sumatra are, no doubt, migrants of this 
Gallinula chloropus (Linn.).

Moor-Hen.

[Gallinula chloropus chloropus (I,inn.).

Fulica chloropus Linn., Syst. Nat. Ioth ed. 1758, p. 152: England.

Distr.-Extra-limital.]

\section{Gallinula chloropus orientalis Horsf.}

Gallinula orientalis Horsfield, Trans. Lim. Soc. xiii, I82 I, D. I95: Java.
Distr.-Malay States.
Sumatra.
Borneo.

Java; Bali; Kangean Islands.

\section{Genus GALLICREX Blyth.}

Gallicrex cinerea (Gmel.). ${ }^{1}$

Water-Cock.

[Gallicrex einerea cinerea (Gmel.).

Fulica cinerea Gmelin, Syst. Nat. i, pt. 2, r789, p. 702 : China.

Distr.-Extra-linital.]

Gallicrex cinerea plumbea (Vieill.).

Gallinula plumbea Vieillot, Nouv. Dict. d'Hist, Nat. ed. 2, xii, 1817, p. 404 : Java.

Gallinula gularis Horsf., Trans. Linn. Soc. xiii, 1821, p. 195: Java.

Gallinula lugubris Horsf., Trans. Linn. Soc, xiii, 1821, p. I95: Java.

Distr.-Malay Peniusula.

Sumatra; Rhio Archipelago.

Borneo.

Java (West only); Karimon-Java Islands and Kalambau Islands, East Java Sea.

\section{Genus PORPHYRIO Brisson.}

Porphyrio albus (White).

Purple Moor-Hen.

[Porphyrio albus albus (White).

Fulica alba alba White, Journ. Voy. New South Wales, I790, 1. 238 : Lord Howe Island.

Distr.-Eixtra-linital.]

Porphyrio albus viridis Begbie.

Porphyrio viridis Begbie, Malayan Peninsula, I834, 1. 5 I5: Malacca.

Distr.-Malay States.

1 Judging from the few available measurements the birds occurring in Malaysia seem rather smaller than more northern representatives of the species. 


\section{Porphyrio albus indicus Horsf.}

Porphyrio Indicus Horsfield, Trans, Linn. Soc. xiii, I82r, p. 194 : Java.

Porphyrio smaragdinus Temm., Pl. Col. 421, 1826 : Java.

Porphyrio bemmeleni Buttik., Notes Leyd. Mus. xi, 1889 . p. I0t: Lake Toba, North Sumatra.

Distr.-Java; Kangean Islands.

Sumatra; ? Nias Island, West Sumatra.

Borneo.

\section{Genus FULICA Linn.}

Fulica atra Linn.

Coot.

\section{"Fulica atra atra Livn.*}

Fulica alra Linn., Syst. Nat. Iotil ed, 1758, p, 152: Sweden.

Fulica lugubris S. Müll., Verh. Nat. Gesch. Land en Volk. 1847, p. 454 : Java.

Distr. - Sumatra.

\section{Family HELIORNITHIDÆ.}

\section{Gents HELIOPAIS Sharpe.}

\section{Heliopais personata (Gray).}

Masked Finfoot

Podica personata Gray, Proc. Zool. Soc. I848, p. 90: Malacca.

Distr,-Malay Peninsula (ircluding island of Koh Pennan in Bandon Bight).

Sumatra.

\section{Family GRUIDÆ.}

\section{Genus GRUS Pallas.}

Grus antigone (Linn.).

Sartis Crane.

[Grus antigone antigone (Lintr.).

Ardea Antigone Linn., Syst. Nat. roth ed. I758, P. I42: Further India.

Distr, -Extra-linital.]

Grus antigone sharpii Blanford.

Grus (Antigone) sharpit Blanford, Bull. B.O.C. v, I895, p. 7 ; Burma. Distr.-Malay Peniusula (not south of Perak).

: We do not believe in the occurrence of two species of Porphyrio in Sumatra, but have seen no topotypes of bemmeleni which is perhaps a recognizable northern race.

- We have never examined a Malaysian specimen of the coot. 


\section{Family JACANIDÆ.}

\section{Genus HYDROPHASIANUS Wagler.}

Hydrophasianus chirurgus (Scop.). Pheasant-tailed Jacana.

Tringa Chirurgus Scopoli, Del. Flor, et Faum. Insubr, 2, 1786, p. 92 : Luzon, Philippine Islands.

Distr,-Malay States.

Sumatra.'

Borneo.

Java.

\section{Genus METOPIDIUS Wagler}

Metopidius indicus (Lath.).

Bronze-winged Jacana.

Parra indica Latham, Index Orn. ii, I790, p, 765: India.

Parra superciliosa Horsfield, Trans. Linn. Soc, xiii, 1821, 1. 194: Java.

Distr.-Malay Peninsula."

Sumatra.

Java.

Genus IREDIPARRA Mathews.

Irediparra gallinacea (Temm.).

Lotus-Bird.

*Irediparra gallinacea gallinacea (Temm.).

Parra gallinacea Temminck, P1. Col. 464, IS28: Menado, Celebes. Dist.,-Borneo (South-east).

\section{Family BURHINIDA.}

\section{Genus ESACUS Lesson.}

Esacus magnirostris (Vieill.).

Stone-Plover.

[Esacus magnirostris magnirostris (Vieill.).

Oedicnemus magnirostris Vieillot, Nouv. Dict. Hist. Nat. xxiii, 1818, p. 231: Australia (Berlepsch, 1911).

Distr.-Eixtra-limital. ]

-We have recently examined a specimen from Kateman, East Sumatra.

additional locality, Bandon, Peninsular Siam (Mus, Aagaard). 


\section{"Esacus magnirostris scommophorus (Oberh.)."}

Orthorhamphus nagnirostris scommophorus Oberliolser, Proc. U.S.

Nat. Mus, Iv, 19I9, p. I33: Wai Island, Tambelan Islands, South China Sea.

Distr.-Peninsular Siam; Tambelan Islands.

Sumatra; Banka; Rhio Archipelago.

Borneo.

Java; Bali; Kangean Islands.

\section{Family ROSTRATULID王.}

\section{Genus ROSTRATULA Vieillot.}

\section{Rostratula benghalensis (Linn.).}

Painted Snipe.

\section{Rostratula benghalensis benghalensis (Linn.).}

Rallus benghalensis Linn., Syst. Nat. Ioth ed. 1758, p. 153: Asia. Rhynchata orientalis Horsf., Trans. Linn. Soc, xiii, 8821, p. 193: Java.

Distr.-Malay States.

Sunjatra.

Borneo.

Java.

\section{Family CHARADRIIDA.}

Genus HOPLOPTERUS Bonaparte.

Hoplopterus spinosus (Linn.).

Spur-winged Plover.

[Hoplopterus spinosus spinosus (Lint1.).

Charadrius spinosus Linn, Syst. Nat, Ioth ed. I75S, p. 151: Egypt.

Distr.,Eixtra-limital.]

Hoplopterus spinosus duvaucelii (Lress.).

Charadrius Duvaucelii Lesson, Dict. Sci. Nat. Hist. ed. Levr. xlii, I 826 , p. 38 : Calcutta.

Distr.-Peninsular Siam.

There are also visual records from the Anamba Islands; Simalur, Babi Island and the Banjak Islands, West Sumatra; and the North Bornean Islands. Oberholser and Mathews think that extra-limital specimens differ from the Australian race: Mrs. Meinertzhagen and Stuart Baker lump them all but consider that the Tambelan and Andaman birds may possibly be separated as scommophorks. This latter action is, prima facie, inadmissible. We have not seen scommophorus and use the name for all Malaysian birds. 


\section{Genus XIPHIDIOPTERUS Reichenbach.}

Xiphidiopterus tricolor (Horsf.). Black-thighed Wattled Lapwing. Vanellus tricolor Horsfield, Trans, Linn. Soc. xiii, I821, p. I86: Java.

Vanellus cucullatus Temm., P1. Col. 1830, pl. 505 : Java.

Distr,-Java.

Surnatra.

Genus LOBIVANELLUS ${ }^{`}$ G. R. Gray,

Lobivanellus indicus (Bodd.).

Wattled Isapwing.

[Lobivanellus indicus indicus (Bodd.).

Tringa Indica Boddaert, Tabl. Planch. Erslum. I $7 \delta_{3}$, p. 50 : Goa, India.

Distr.-Extra-limital.]

Lobivanellus indicus atronuehalis Jerd.

Lobivanellus alronuchalis "Blyth" Jerdon, Bircls of India, iii, I864, p. 648 : Burma.

Distr.-Malay Peninsula.

? Sumatra. ${ }^{7}$

\section{Genus SQUATAROLA Cuvier.}

Squatarola squatarola (Linn.).

Grey Plover.

[Squatarola squatarola squatarola (Lin11.).

Tringa Squalarola Linn., Syst. Nat. Ioth ed. 1758, p. 149: Sweden.

Distr.-Extra-limital.]

Squatarola squatarola australis Rejch.

Squatarola helvelica australis Reichenbach, Novit. Sys. Av., No. v. 1851, p. 3 : Australia.

Distr.-Malay Peninsula.

Sumatra; Rhio Archipelago.

Borneo; North Natuna Islands.

Java.

- Peters (1934) mentions Microsarcops cinereus (Blyth) from the Malay States, but we know of no reliable record from our area.

- The presence of this species in Sumatra needs confirmation. 


\section{Genus CHARADRIUS Linn.}

Charadrius apricarius Linn.

Golden Plover.

[Charadrius apricarius apricarius Linn.

Charadrius apricarius Linn., Syst. Nat. Ioth ed. 1758, p. I50: Lapland.

Distr.-Extra-limital.]

\section{Charadrius apricarius fulvus Grmel.}

Charadrius fulvus Gmelin, Syst. Nat. i, pt. 2, I789, p. 687: Tahiti.

Distr.-Malay Peninsula; Anamba Islands; Tambelan Islands.

Sumatra; Banka; West Sumatran Islands of Simalur, Nias, Batu Islands; Mentawi Group.

Borneo; Natuna Islands; Karimata Islands.

Java; Bali; Kangean Islands; Christmas Island.

\section{Charadrius leschenaultii Less.}

Large Sand-Plover.

Charadrius leschenaultii leschenaultii I,ess.

Charadrius Leschenatlii Lesson, Dict. Sci. Nat. ed. Levr., xlii, 1826, p. 36 : India.

Distr.-Malay States; Anamba Islands.

Sumatra; Banka; West Sumatran Islands of Sinalur, Nias and Mentawi Group.

Borneo; North Natuna Islands.

Java; Bali; Kangean Islauds.

Charadrius mongolus Pallas.

Small Sand-Plover.

Charadrius mongolus mongolus Pall."

Charadrius nongolus Pallas, Reise Prov. Russ. Reichs iii, I776, p. 700 : Siberia.

Distr.-North Bornean Islauds of Banguey and Balambangan. Java.

Charadrius mongolus atrifrons Wagl.

Charadrius atrifrons Wagler, Isis, I829, col. 650: Bengal.

Charadrius sanguineus Less., Dict. Sci. Nat, ed, Levr. xlï, 1826: Java.

Charadrins cirhepidesmos Wagl., Syst. Av., Charadr. sp. 18, 1827: Java.

Distr.-Malay Peninsula; Anamba Islands.

Sumatra; Rhio Archipelago.

Borneo; North Natuna Islands.

Java,

' Within Malaysian limits we have only seen undoubted $C$. m. mongolus from Balambangan Island: one from Banguey Island also bas the wing rather long for C. m. atrifrons, 
Charadrius asiaticus Pall.

Eastern Dotterel.

[Charadrius asiaticus asiaticus Pall.

Charadrius asiaticus Pallas, Reise Prov. Russ. Reiclis, ii, I773, p. 7 I5: South Tartary Steppes.

Dislr._Extra-limital.]

Charadrius asiaticus veredus Gould.

Charadrius veredus Gould, Proc, Zool. Soc. 1848, p. 38 ; North Australia.

Distr.-Malay States.

Borneo; North Natuna Islands. Java.

Charadrius dubius Scop.

Little Ringed Plover.

Charadrius dubius curonicus Gmel."

Charadrius curonicus Gmelin, Syst. Nat. i, pt. 2, 1789, p. 692 :

Curonia $=$ Baltic Prov. of Russia.

Charadrius pusillus Horsf., Trans. Linn. Soc. xiii, 1821, p. 187: Java.

Distr.-Malay Peninsula; Anamba Islands.

Sumatra; Nias Island, West Sumatra.

Bormeo.

Java; Kangean Islands.

Charadrius dubius jerdoni (Legge).

Aegialitis jerdoni Legge, Proc. Zool. Soc. 1880, p. 29 :Ceylon.

Distr.-Malay Peninsula.

Sumatra.

Borneo.

Charadrius alexandrinus ${ }^{2}$ Linn.

Kentish Plover.

Charadrius alexandrinus alexandrinus Linn.

Charadrius alexandrinus Linn., Syst. Nat. Ioth ed. 1758, p. I50: Eigypt.

Distr.-Malay Peninsula.

Borneo; North Natuna Islands.

Charadrius alexandrinus dealbatus (Swinh.).

Aegialitis dealbatus Swinhoe, Proc. Zool. Soc. 1870, p. 138 : Hainan.

Distr.-Malay States.

Sumatra.

Borneo.

t The typical race has been recorded from Mergui and may therefore occur in Malaysia.

"Revision: Neum., Nov. Zool. xxxv, 1929, p. 212. 
Charadrius alexandrinus seebohmi Hart. and Jackson.

Charadrius alexandrinus seebohmi Hartert and Jackson, Ibis, IgI5, P. 529: nom. nov. for $C$. cantianus minutus Seeb. not Pallas

("Southern shores of Red Sea, and island of Ceylon".).

Distr.-Java. ${ }^{1}$

Charadrius peronii Schleg.

Malay Sand-Plover.

Charadrius peronii Schlegel, Mus. Pays-Bas, I865. Cursores, p. 33 :

Borneo (Harterl, 1902).

Distr.-Malay Peninsula; Tioman Archipelago.

Sumatra; Rhio Archipelago.

Borneo; Labuan; North Bornean Islands of Mangalum, Tiga and Balambangan.

Java; Bali; Kangean Islands.

\section{Genus HIMANTOPUS Brisson.}

Himantopus bimantopus (Linn.).

Black-winged Stilt.

Himantopus himantopus himantopus (Linn.)."

Charadrius Himantopus Linn., Syst. Nat. roth ed. 1758, p. I5I: Southern Europe.

Distr.-Malay States.

Borneo.

Himantopus himantopus leucocophalus Gould.

Himantopus leucocephalus Gould, Synops. Birds Austr. pt. 2, I837, pl. 34 : New South Wales.

Distr.-Borneo."

Java.

\section{Family SCOLOPACIDÆ.}

Genus NUMENIUS Brisson.

Numenius arquata (Linn.).

Curlew.

[Numenius arquata arquata (Linn.).

Scolopax Arquata Linn., Syst. Nat. Ioth ed. 1758, p. I 45 : Sweden. Distr.-Extra-limital.]

" Two specimens (Feb., May). Wings $100-103 \mathrm{~mm}$. "Breeding in Java" (fide the collector). Not $C$. ruficapillus.

* Has been recorded from Borneo by Blasius (1884) but we have not seen the paper. The identification needs confirmation (? leucocephalus).

3 We do not admit the locality "Sumatra" for this form. The Suada Islands bird is, of course, timorensis Mathews if tais race is good. 
Numenius arquata orientalis Brehm.,

Numenius orientalis C. L. Brehm, Handb. Naturg. Vög. Deutschl. $1831, p .610$ : East Indies.

Numenius nasicus Temm., Man. d'Orn. 2nd ed. iv, 1840, p. 393: Borneo and Sumatra.

Distr.-Malay Peninsula.

Sumatra; Rhio Archipelago; Banjak Islands, West Sumatra.

Borneo.

Java.

Numenius madagascariensis (Linn.)." Australian Curlew.

Scolopax madagascariensis Linn., Syst. Nat. I2th ed. i, I766, p. 242 : Madagascar error = Macassar, Celebes.

Distr.-Sumatra; Rhio Archipelago.

Borneo.

Java,

Numenius phaeopus (Linn.).

Whimbrel.

[Numenius phaeopus phaeopus (Linn.).

Scolopax Phaeopus Linn, Syst. Nat. roth ed. I758, p. I46: Sweden. Distr._Extra-limital.]

Numenius phacopus variegatus (Scop.).3

Tantalus variegatus Scopoli, Del. Flor. et Faun. Insubr, ii, 1786, p. 92 : Luzon, Philippine Islands.

Distr.-Malay Peninsula.

Sumatra; Rhio Archipelago; Banka; Billiton; Sinalur Island,

Batu Islands and Mentawi Islands, West Sumatra.

Borneo; North Natuna Islands.

Java; Bali (vis. rec.); Kangean Islands.

Christmas Island.

\section{Numenivs minutus Gould.}

Numenius minutus Gould, Proc. Zool. Soc. for 1840 , 1841 , p. 176 : New South Wales.

Distr.-Borneo. ${ }^{4}$

?Java.

- Replaces $N$. lineatus Cuv. (nom, nud.).

- Replaces Numenius cyanopus.

3 A few specimens from the Malay Peninsula seem exactly like typical phacopus: many others are true variegatus; others again seem intermediate.

4 Chasen and Kloss, Journ. Malay Branch Roy. Asiat. Soc. vi, pt. 3. 1928 , p. 68. Meyer and Wiglesworth include Java in the range, apparenty on the authority of von Schierbrand, but the record nceds confirmation : it is omitted by Bartels and Stresemann in their list of Java birds. 
Genus LIMOSA Brisson.

Limosa limosa (Linn.).

Black-tailed Godwit.

[Limosa limosa limosa (Linn.).

Scolopax Limosa Linn., Syst. Nat, roth ed, I758, p. I47 : Sweden. Distr._Extra-limital.]

Limosa limosn melanuroides Gould.

Limosa melanuroides Gould, Proc. Zool. Soc. I846, p. 84 : Port Essington, Northern Territory, Australia.

Distr.-Malay States.

Sumatra.

Borneo.

Java.

Limosa lapponica (Linn.).

Bar-tailed Godwit.

[Limosa lapponica lapponica (I.inn.).

Scolopax lapponica Linn., Syst. Nat. Ioth ed. 1758, p. 147: Lapland. Distr.-Extra-limital.]

Limosa lapponica baueri Naum.

Limosa Baueri Naumann, Naturg. Vög. Deutschl. viii, 1836, p. 429: New Holland = Victoria.

Distr.-Malay Peninsula.

Sumatra.

Borneo; North Natuna Islands. Java.

\section{Genus PHALAROPUS Brisson.}

Phalaropus lobatus (Linn.).

Red-necked Phalarope.

Tringa tobata (typ. err.) Linn., Syst. Nat. roth ed. 1758 , p. 148: Hudson Bay, North America.

Distr.-Borneo.

\section{Genus SCOLOPAX Linn.}

Scolopax rusticola Linn.

Woodcock.

Scolopax rusticola rusticola Linn.

Scolopax Rusticola Linn., Syst. Nat. Ioth ed. I758, p. I45: Sweden. Disir.-Malay States.

Scolopax saturata Horsf.

Scolopax saturata saturata Horsf.

Scolopax saturata Horsfield, Trans. Linn. Soc. xith, I $\$ 21$, p. I91 : Java.

Distr._Java.

Sumatra.

- There is a dubious record from the Cocos-Keeling Islands 


\section{Genus CAPELLA Frenzel.}

Capella stenura (Bp.).

Pintail Snipe.

Scolopax stenura "Kuhl" Bonaparte, Ann. Stor. Nat. Bologna, iv, 1830, p. 335 : Sunda Islands.

Scolopax pectinicauda Peale, U.S. Expl. Exped. Birds, 1848, p. 227 : Singapore.

Disfr.-Malay Peninsula; Anamba Islands.

Sumatra; Banka; West Sumatran Islands of Simalur, Nias and Mentawi Group; Billiton.

Borneo,

Java; Bali; Karimon-Java Islands.

Christmas Island.

Capella megala (Swinh.).

Swinhoe's Snipe.

Gallinago megala Swinhoe, Ibis, r86r, p. 343 : Pekin.

Dislr.-Malay States.

Borneo.

Java; Bali.

Capella gallinago (Linn.).

Common or Fantail Snipe.

Capella gallinago gallinago (Linn.).

Scolopax Gallinago Linn., Syst. Nat. Ioth ed. I758, p. I47 : Sweden.

Distr.-Malay Peninsula.'

Sumatra.

Borneo.

Java; Cocos-Keeling Islands.

\section{Genus LIMNODROMUS Wied.}

Limnodromus griseus (Gmel.).

Snipe-billed Godwit.

[Limnodromus griseus griseus (Gmel.).

Scolopax grisea Gmelin, Syst. Nat. i, pt. 2, 1789, p. 658: Coast near New York.

Distr,-Extra-limital.]

Limnodromus griseus semipalmatus (Blyth).

Macrorhamphus semipalmatus "Jerdon" Blyth, Journ. As. Soc. Bengal, xvii, i848, i, p. 252 : near Calcutta.

Distr.-Malay Peninsula.

Sumatra."

Borneo.

Java.

t We have one or two pale birds from the Malay Peninsula which may be raddef Buturlin.

"Sumatra" is here given on the authority of a manuscript note left by Robinson. 


\section{Genus ARENARIA Brisson.}

Arenaria interpres (Linn).

Turnstone.

Arenaria interpres interpres (Litin.).

Tringa Interpres Linn., Syst. Nat. Ioth ed. I758, p. I48: Sweden. Distr.-Malay Peninsula.

Sunatra; Banka; Simalur Island and Mentawi Islands, West Sumatra.

Borneo; North Natuna Islands.

Java; Bali.

\section{Genus CALIDRIS Merrem.}

Calidris canutus (Linn.).

Knot.

Calidris canutus canutus (Linn.)."

Tringa Canutus Linn., Syst. Nat, roth ed. I758, p. I49: Sweden.

Distr.-Banka Island.

North Natuna Islands.

Java.

Calidris tenuirostris (Horsf.).?

Great Knot

Tolanus tenuirostris Horsfield, Trans. Linn. Soc. xiii, 1821, p. 192 : Java.

Distr.-Malay States.

Borneo; North Natuna Islands.

Java.

Genus CROCETHIA Billberg.

Crocethia alba (Pall.).

Sanderling .

Tringa alba Pallas, Vroeg's Cat. Coll., Adumb. 1764, p. 7 : Coast of North Sea, Holland.

Distr.-Malay States.

Borneo.

Java.

Christmas Island.

1C. c. rogersi (Maths.) if that form is really distinct,

- The occurrence of this species in the east of Sumatra is almost certain but we cannot trace a record from the island. 


\section{Genus LIMICOLA Koch.}

Limicola falcinellus (Pont.). Broad-billed Sandpiper.

[Limicola falcinellus falcinellus (Pont.).

Scolopax Falcinellus Pontonpidan, Danske Atlas, 1763, p. 623: Denmark.

Distr.-Extra-limital. ]

Limicola falcinellus sibirica Dress. ${ }^{\text {t }}$

Limicola sibirica Dresser, Proc. Zool. Soc. I876, p. 674: Siberia and China.

Distr.-Malay Peninsula.

Sumatra.

Java.

Genus PHILOMACHUS Merrem.

Philomachus pugnax (Linn.). Ruff (male); Reeve (female).

Tringa Pugnax Linn., Syst. Nat. Ioth ed. $175^{8}$, p. 148 : Sweden.

Distr.-Malay States.

Borneo.

\section{Genus EROLIA Vieillot.}

Erolia ruficollis (Pall.).

Red-necked Stint.

Trynga ruficollis Pallas, Reise Prov. Russ. Reichs, iii, 1776, p. 700 : South Transbaikalia.

Totanus damacensis Horsf., Trans. Linn. Soc. xiii, 1821 , p. 192 : Java. Distr.-Malay Peninsula; Anamba Islands.

Sumatra; Rhio Archipelago; Simalur Island, West Sumatra. Borneo; North Natuna Islands.

Java.

Christmas Island.

Erolia minutilla (Vieill.).

Long-toed Stist.

Erolia minutilla minutilla (Vieill.).

[Tringa minutilla Vieillot, Nouv. Dict. Hist. Nat, xxxiv, I8i9, p. 446: North America.]

Disir.-Extra-limital.

- We can trace no record for Borneo although the species no doubt occurs there. 
Erolia minutilla subminuta (Middend.).

Tringa subminuta Middendorf, Reise Nord. und Ost. Siberien, ii, 1853, p. 222 : Siberia.

Distr.-Malay Peninsula."

Banka Island.

Borneo.

Java.

Erolia temminckii (Leisler) .

Temminck's Stint.

Tringa temminckii Leisler, Nacht, zu Bechst. Nat. Deutsch. I812, p. 63 : Germany.

Distr.-Malay States.

Borneo."

*Erolia acuminata (Horsf.).

Siberiar Pectoral Sandpiper.

Tolanus acuminatus Horsfield, Trans. Linn. Soc. xili, 1821, p. 192 : Java.

Distr.-Java.

Erolia testacea (Pail.).

Curlew-Sandpiper.

Scolopax lestacea Pallas, in Vraeg's Cat. Adumbr. 1764, p. 5 : Holland.

Distr.-Malay Peninsula.

Sumatra.

Borneo; North Natuna Islands.

Java.

Erolia alpina (Linn.).

Dunlin.

[Erolia alpina alpina (Linn,).

Tringa alpina Linn., Syst. Nat. Ioth. ed. 1758, p. I49: Lapland.

Distr.-Extra-limital.]

\section{Erolia alpina sakhalina (Vicill.) , 3}

Scolopax sakhalina Vieillot, Nouv. Dict. Hist. Nat. iii, I816, p. 359: Russia.

Distr.-Borneo. Java.

This species is of course tolerably certain to occur in Sumatra but we cannot find a record: it is scarce in the Malay Peninsula but has been taken on an is!and in the Straits of Malacca.

"Seebohm and Vorderman both give "Borneo" for this species apparently on the strength of a specimen in the Leyden Museum collected by Schwaner. Vorderman also gives "Java" which is not accepted by Bartels and Stresemann,

3 At this distant date Schlegel's records (1864) need confirmation as to locality. The species has not since been recorded from Malaysia and old specimens labelled as "alpina" in the Rafles Museum from the Natuna Islands ate Erolia testacea. 


\section{Genus XENUS Kaup.}

Xenus cinereus (Güld.).

Avocet-Sandpiper.

Scolopax cinerea Güldenstadt, Nov. Conm. Petrop. xix, 1774, p. 473 : Shores of Caspian Sea.

Totanks javanicus Horsf., Trans. Linn. Soc. xiii, 1821, p. 193 : Java.

Scolopax sumatrana Raffles, Trans. Linn. Soc. xiii, 1822, p. 327 : Sumatra.

Distr.-Malay Peninstla,

Sumatra.

Borneo; North Natuna Islands.

Java.

\section{Genus TRINGA Linn.}

Tringa totanus (Linn.).

Redshank.

Tringa totanus totanus (Linn.).

Scolopax Totanus Linn., Syst. Nat. Ioth ed. 1758, p. 145 : Sweden.

Distr.-Malay Peuinsula; Anamba Islands.

North Natuva Islands.

Tringa totanus eurhinus (Oberh.).

Totanus totanus eurhinus Oberholser, Proc. U.S. Nat. Mus. xxii, 1900, p. 207 : Ladak.

Distr.-Malay Peninsula; Tambelan Islands.

Sumatra; Sinalur Island, West Sumatra.

Borneo; North Natuna Islands.

Java; Bawean Island.

Tringa stagnatilis (Bechst.).

Marsh Sandpiper.

Totanus stagnatilis Bechsteia, Or11. Taschenb. 1803, p. 292: Germany.

Distr.-Malay States.

Borneo.

Java.

Tringa ochropus Linn.

Green Sandpiper.

Tringa Ocrophus Linn., Syst. Nat. Ioth ed. 1758, p. I49: Sweden.

Distr._Borneo.'

Karimon-Java Islands.

Tringa incana (Gmel.).

Grey-rumped Tattler.

[Tringa incana incana Gmel.

Scolopax incana Gmelin, Syst. Nat. i, pt. 2, 1789, p. 658: Eimeo and Palmerston Islands, Pacific Ocean.

Distr._Extra-limital.]

1 We have seen a recent specimen from Sarawak. 
Tringa incana brevipes (Vieill.).

Totanus brevipes Vieillot, Nouv. Dict. Hist. Nat. vi, I8I6, p. 410 : Timor.

Distr.-Malay States.

Sumatra.

Borneo; North Natuna Islands.

Java; Kangean Islands; Thousand Islands, Java Sea.

Christmas Island.

Tringa hypoleucos Linn.

Common Sandpiper.

Tringa Hypoleucos Linn., Syst. Nat. roth ed. I758, p. 149 : Sweden.

Distr.-Malay Peninsula; Anamba Islands; Tambelan Islands.

Sunatra; Rhio Archipelago; Banka; West Sumatran Islands of Simalur, Nias and Mentawi grotnp; Billiton.

Borneo; Natuna Islands; Karinata Islands.

Java; Bali; Kangean Islands; Bawean Island; Karimon-Java Islands.

Christnas Island.

Tringa guttifer (Nord.).

Armstrong's Sandpiper.

Totanus guttifer Nordmann, in Ërman's Reise, Naturh. At1. I835, p. 17: near Ochotsk.

Distr.-Malay States.

Borneo.

Tringa nebularia (Gunn.).

Greenshank.

Scolopax nebularia Gunnerus, in Leem, Beskr. Finm. Lapp. I767, p. 251 : Norway.

Distr._Malay Peninsula.

Sumatra.

Borneo; North Natuna Islands.

Java.

Christmas Island; Cocos-Keeling Islands.

Tringa glareola Linn.

Wood Sandpiper.

Tringa Glareola Linn., Syst. Nat. Ioth ed. 1758, p. 149: Sweden.

Totanus aftris Horsf., Trans. Linn. Soc. xiii, i8zi, p, 191 : Java,

Totawus kuhlii Brehm, Handb. Naturg. Vög, Deutschl, 1831, P, 64l: Java.

Distr.-Malay Peninsula; Anamba Islands.

Sumatra.

Borneo; North Natuna Islands.

Java; Bali. 


\section{Family GLAREOLID $\not$.}

\section{Genus GLAREOLA Brisson.}

Glareola pratincola (Linn.) .

Collared Pratincole.

[Glareola pratincola pratincola (Linn.).

Hirundo Pratincola Linn., Syst. Nat. I2th ed. i, I766, p. 145: Austria.

Distr.-Extra-linital. ]

Glareola pratincola maldivarum Forst.

Glareola (Pratincola) Maldivarum J. R. Forster, Faun. Indica, 1795. p. II : Maldive Islands.

Glareola orientalis Leach, Trans. Linn. Soc. xiii, 1821, p. 132: Java.

Distr.-Malay States; Anamba Islands.

Sumatra; Banka; West Sumatran islands of Nias and Mentawi Group; Billiton.

Borneo; Natuna Islands.

Java; Thonsand Islands, Java Sea; Karimon-Java Islands.

Christmas Island.

Glareola isabella (Vieill.).

Long-legged Pratiucole.

Glareola isabella Vieillot, Aralyse, г8 6 , p. 69 : Australia.

Distr._Borneo; Billiton.

Java. $^{\text {: }}$

\section{Family DROMADID无.}

\section{Genus DROMAS Paykull.}

Dromas ardeola Payk.

Crab-Plover.

Dromas Ardeola Paykull, Konge. Vet. Akad. Handl. Stockholm, xxvi, I805, p. 182 ; India.

Distr.-Malay States.

Family LARIDE.

Genus CHLIDONIAS Rafinesque.

Chlidonias leucoptera (Tenun.). White-winged Black Tern. [Chlidonias leucoptera leucoptera (Temm.).

Sterna leucoptera Tennninck, Mau. d'Orn. I8I5, p. 483: Mediterranean Sea.

Distr._Extra-linital.]

- The evidence for the occurrence of this species in Java is not good but it is very likely to occur there. 
Chlidonias leucoptera grisea (Horsf.)."

Slema grisea Horsfield, Trans. Linn. Soc. xiii, 1821, p. 199: Java.

Distr._Malay Peninsula (West Coast).

Sumatra (Straits of Malacca).

Borneo.

Java.

Chlidonias hybrida (Pall.).

Whiskered Tern.

[Chlidonias hybrida hybrida (Pall.).

Sterna hybrida Pallas, Zoogr. Rosso-Asiat. ii, I8II, p. 338 : Russia Distr.-Eixtra-limital.]

Chlidonias hybrida javanica (Horsf.). ${ }^{2}$

Sterna javanica Horsfield, Trans. Liun. Soc. xiii, 182I, p. 198 : Java.

Distr.-Borneo.
Java.

\section{Genus GELOCHELIDON Brehm.}

Gelochelidon nilotica (Gmel.).

Gull-billed Tern.

[Gelochelidon nilotica nilotica (Gmel.).

Sterna nilotica Gmelin, Syst. Nat. i, pt. 2, I789, p. 606: Egypt.

Disir.-Extra-limital.]

Gelochelidon nilotica affinis (Horsf.).

Sterna affinis Horsfield, Trans. Linn. Soc. xiii, I8zI, p. I99: Java.

Distr.-Malay Peninsula.

Sumatra.

Borneo; Natuna Islands.

Java.

\section{Genus STERNA Linn.}

Sterna hirundo Linn.

Common Tern.

[Sterna hirundo hirundo Linn.

Stcrna Hirundo Limn., Syst. Nat. roth ed. 1758, p. I37: Sweden. Distr._Extra-limital.]

Sterna hirundo longipennis Nordm.3

Nordmann's Tern.

Sterna longipennis Nordmann in Erman's Ver\%. Thieren u. Pflatizen, I835, p. 17: Ochotsk, Siberia.

Distr.-Malay Peninsula (both coasts).

Stminatra.

Java; Borneo.

1 We have only recognized an eastern race for this tern after much hesitation.

" " $H$. hybrida" has been recorded from Nias by Rosenberg.

3 Records of Awviatilis (=hirthdo), and tibelana from Malaysia all seem to apjly to this form: a specimen has been examined from Sarawak. 
Sterna dougallii Mont.

Roseate Tern.

[Sterna dougallii dougallii Mont.

Sterna Dougallii Montagu, Ornith. Dict. Stuppl. 1813, no pagination :

Scotland.

Disir.-Extra-limital.]

Sterna dougaltii bangsi Math.:

Sterna dougallii bangsi Mathews, Birds Austr. ii, rgr2, p. 364 :

Foochow, China.

Distr.-Malay Peninsula (both coasts).

Sumatra.

Borneo.

Java.

Sterna bengalensis Less.

Smaller Crested Tern.

Sterna bengalensis bengalensis Less.?

Sterna bengalensis Lesson, Traité d'Orn. 1831, p. 621 : Coasts of India.

Sterna media Horsf. (nec Vieill. 1820) Trans. Linn. Soc. xiii, 1821, p. 199 : Java.

Distr.-Malay Peninsula (Straits of Malacca).

Sumatra.

Java,

Sterna bergii Licht. ${ }^{3}$

Large Crested Tern.

[Sterna bergii bergii Licht.

Sterna bergii Lichtenstein, Verz. Doubl. Zool. Mus. Berlin, 1823 , p. 80: Cape of Good Hope.

Distr.-Extra-limital.]

Sterna bergii edwardsi (Math.).

Thalasseus bergii edwardsi Mathews, Birds Austr. ii, I912, p. 347 :

Ceylon.

Distr.-Malay Peninsula (Straits of Malacca). ? Sumatra.4

- Occurring as it does in the South China, Sulu and Java Seas this tern must be included in the avifauna of Borneo although we know of no actual record from that island. Specimens from Luzon and Palawan also appear to be bangsi (fde H.C. Robinson in litt.). S.d. korustes Hume (Andamans), is said to visit the Mergui Archipelago and may therefore occur in the Straits of Malacca, but our few specimens from this latter locality seem to be bangsi.

$\triangle$ Although recorded from Celebes this tern is not known from Borneo, or the Philippines, but as it is found in Java the south of Borneo is also possibly within the range,

3 Revision: Oberh., Proc. U.S. Nat. Mus. xlix, 1015, p. 315.

4 There is no definite record for Sumatra, but it is almost certain that this race must occur within sight of the coast.

Rosenberg records a form of this species from Nias: it may be edwardsi or cristalus. 


\section{Sterna bergii cristata Steph.}

Sterna cristatus Stephens, Gen. Zool. xiii, 1826, p. I46: China (Mathews, Ig/2).

Distr.-Malay Peninsula (East Coast south to Singapore).

Sumatra; Rhio Archipelago; Anamba Islands.

Borneo; Natuna Islands.

Java; Thousand Islands, Java Sea; Bali; Kangean Islands; Karimon-Java Islands.

Sterna bergii halodrama (Oberh.).:

Thalasseus bergii halodramus Oberholser, Proc. U.S. Nat. Mus. xlix, 1915, p. 522: Pata Island, near Sulu Island, South Philippine Islands.

Distr.—?Borneo (North-west).

Sterna ankethetus Scop.

Panayan or Bridled Tern.

Sterna anzthetus anzethetus Scop.

Sterna Ancthetus Scopoli, Del. Flor. et Faun. Insubr. 1786, p. 92 : Panay Island, Philippine Isiands.

Distr.-Malay Peninsula (both coasts); Anamba Islands; Tambelan Islands.

Sumatra; Taya Island, Lingga Archipelago; Billiton.

Borneo; Natuna Islands.

Java.

Sterna anæthetus antaretica Less.

Stema anlarclica Lesson, Tratté d'Orn. 1831, p. 631 : Matrititus.

Distr.-Cocos-Keeling Islands.

Sterna fuscata Linn.

Sooty Tern.

Sterna fuscata fuscata Lintr.

[Sterna fuscala Linn., Syst. Nat, 12th. ed. i, 1766, p. 228: Santo Doningo, West Indies.

Distr._Extra-linital.]

Sterna fuscata nubilosa Sparm.2

Sterna nubilosa Sparrman, Mus. Carls. fasc. 3, I788, No. 63 "India Orientalis'".

Sterna infuscata Licht., Verz. Doubt, Zool, Mus, Berlin, 1823, p. 8t : East Indies.

Distr.-Borneo. Java.

- We have a skin from Baram, Sarawak which is remarkably paler than any other bird in the collection: it is tentatively referred to halodramus which according to its describer occurs almost within sight of the north-east coast of Borneo.

2 The occurrence of this species in Sumatra, although recorded by: Vorderman needs confirmation. 
Sterna albifrons Pall."

Little Tern or Ternlet.

[Sterna albifrons albifrons Pall.

Stema albifrons Pallas in Vroeg's Adunb. 1764 , p. 6: Holland.

Distr.-Extra-limital. ]

Sterna albifrons sinensis Gmel.

Sterna sinensis Gmelin, Syst. Nat. i, pt. 2, 1789, p. 608 : China.

Distr.-Malay Peninsula (both coasts but the east especially).

Borneo; Natuna Islands.

Java.

?Sumatra (Straits of Malacca).

Sterna albifrons pusilla Temm.2

Sterna pusilla Temminck, Man. d'Orn., ed. 2, $18_{40}$, p. 465: Java.

Distr.-Malay Peninsula (Straits of Malacca). ${ }^{3}$

?Sumatra (Straits of Malacca, therefore almost certainly the Sumatran coast).

?Java ("apparently wintering in Java", H, C. Robinson in litt.).

Sterna albifrons saundersi Hume.

Sterna saundersi Hume, Stray Feathers, v, I877, pp. 324-6: Karachi, India.

Distr,-Malay Peninsula (Straits of Malacca).

Sumatra.

Sterna sumatrana Raffles.

Black-naped Tern.

Sterna sumatrana sumatrana Raffles."

Sterna Sumatrana Raffles, Trans, Linn, Soc, xiii, I822, p. 329 : Sumatra.

Distr.-Malay Peninsula (both coasts); Anamba Islands.

Sumatra; Taya Island, Lingga Archipelago; West Sumatran; Islands of Nias and Engano.

Borneo; Natuna Islands; Karimata Islands.

Java; Bali; Kangean Islands.

I See Baker, Bull. B. O, C. xlix, 1028, p. 37 and Ticehurst, t.c. p. 66.

- Seems to be a good race, formerly known as gouldi Hume (nec Reichb.), but the use of pusilla is perhaps not certain for it.

3 Certain specimens from the Straits of Malacca and North-east Sumatra can be plausibly referred this form.

4 Probably found throughout Malaysia, along all coasts and on all small islands. 


\section{Genus ANOUS Stephens.}

Anoüis stolidus (Linn.).

Noddy.

[Anoüs stolidus stolidus (Linn.).

Sterna stolida Linn., Syst. Nat. Ioth ed. 1758, p. I37: West Indies. Distr.-Extra-linital.]

Anoüs stolidus pileatus (Scop.). ${ }^{x}$

Sterna pileata Scopoli, Del. Flor. et. Faun. Insubr, ii, I786, p. 92 : Philippine Islands.

Distr.-Malay Peninsula (Straits of Malacca); Anamba Islands; Tambelan Islands.

Sumatra; Nias Island, West Sumațra.

Borneo; Natuna Islands.

Java; Karimon-Java Islands.

Christmas Island; Cocos-Keeling Islands.

Anoüs minutus Boie.

White-capned Noddy.

[Anoüs minutus minutus Boie.

Anous minutus Boie, Isis, I844, col. I 88 : Australia.

Distr._Extra-limital.]

Anoüs minutus worcesteri (McGreg.).

Micranous worcesteri McGregor, Phil. Journ, Sci, D, vi, I9II, p. I83: Cavilli Island, Sulu Sea.

Distr.-Malay Peninsula (Straits of Malacca). ${ }^{2}$

? Strmatra (Straits of Malacca); Billiton.

Borneo.

\section{Genus GYGIS Wagler.}

Gygis alba (Sparrin.).3

White Tern.

[Gygis alba alba Sparrm.

Sterna alba Sparrman, Mus. Carl. 1, 1786, no. xi : Ascension Island (Mathews, 1927).

Distr.-Eixtra-linital.]

- Rare in Malayan waters near the coasts and apjarently also rare on the Javan coasts. Breeds on Barren Island between Singapore and Borneo.

7 The single specimen on record from Malacca (Maingay) is referred in this form purely on geographical grounds.

3 This tern is known from the Bay of Bengal, but we doubt if it ever occurs in Malayan seas. Vorderman's Sumatran record is a dubious one and the presence of the bird on the coast of Java needs confirmation. 
Gygis alba monte Math.

Gygis alba monte Mathews, Birds Austr. ii, 1912, p. 443 : Seychelles. Distr.-Sumatra.

Java.

Cocos-Keeling Islands.

\section{Genus LARUS Linn.}

Larus ridibundus Linn.

Black-headed or Laughing Gull.

Larus ridibundus ridibundus Lin11.

Larus ridibundus Linn., Syst. Nat, I 2 th ed. i, 1766, p. 225 : England.

Distr.-Singapore Straits.

*Larus brunnicephalus Jerd.

Brown-hooded Gull.

Larus brunnicephalus Jerdon, Madr. Journ. Lit. Sci. xii, 1840 , p. 225 : India.

Distr.-Peninsular Siam.

\section{Family PODICIPIDÆ.}

\section{Genus PODICEPS Latham.}

Podiceps ruficollis (Pall.).

Little Grebe.

[Podiceps ruficollis ruficollis (Pall.).

Colymbus ruficollis Pallas in Vroeg's Cat. Adumb. I764, p. 6: Holland.

Distr,-Extra-limital.]

Podiceps ruficollis philippensis (Bonn.).

Colymbus Philippensis Bonnaterre, Tabl, Encycl. Méth. i, r79o, p. 58: Philippine Islands.

Distr.-Peninsular Siam.

Stumatra.

Borneo.

*Podiceps ruficollis vulcanorum (Rensch).

Podicebs ruficollis vulcanorum Rensch, Journ. f. Ornith. I929, 2, p. 205 : Lombok.

Distr._Java; Bali. 


\section{Family HYDROBATID压.}

Genus OCEANODROMA Reichenbach."

Oceanodroma leucorhoa (Vieill.). Leach's Fork-tail Petrel.

[Oceanodroma leucorhoa leucorhoa (Vieil].).

Procellaria leucorhoa Vieillot, Nov. Dict. Hist. Nat. xxy, 1818 ,

p. 422: Maritime parts of Picardy.

Distr._Extra-limital.]

Oceanodroma leucorhoa monorhis (Swinh.).

Swinhoe's Petrel.

Thalassidroma monorhis Swinlroe, Ibis, I867, p. 386: near Amoy, China.

Distr.-Straits of Malacca; South China Sea; Java Sea.

\section{Family PROCELLARIIDE.}

\section{Genus PUFFINUS Brisson}

*Puffinus leucomelas (Temml.). ${ }^{2} \quad$ White-fronted Shearwater. Procellaria leucomelas Temminck, P1. Col. 1835, pl. 587: Seas of Japan.

Distr.-Borneo (North).

\section{Family PLEGADID压.}

\section{Genus THRESKIORNIS G. R. Gray.}

Threskiornis athiopicus (Lath.).

White Ibis. [Threskiornis athiopicus athiopicus.

Tantalus athiopicus Latham, Ind. Orn. ii, 1790, p. 706: Ethiopia. Distr.—Extra-limital.]

Threskiornis athiopicus melanocephalus (Lath.) .3

Tantalus melanocephalus Latham, Ind. Orn. ii, 1790, p. 709 : India.

lois leucon Temm., Pl. Col. 481, 1829: Java.

Distr._Malay Peninsula

Sumatra; Batam Island, Rhio Archipelago.

Borneo.

Java.

' A white-rumped species, probably a form of Occanites oceanicus (Kuhl), has been observed in the Straits of Majacca.

a A form of Puffinus is said to breed in the Cocos-Keeling Islands.

3 Robinson (1927), excludes the species from Borneo and Sumatra, but there are well authenticated specimens from Sarawak in the Kuching Museum. We have seen it on the east coast of Sumatra. Specimens examined from Java. 
Genus PSEUDIBIS Hodgson.

Pseudibis papillosa (Temm.).

Black Ibis.

[Pseudibis papillosa papillosa (Temm.).

Ibis papillosa Temm., Planch. Col. 304, 1824: India.

Distr.-Extra-lituital.]

Pseudibis papillosa davisoni (Hume).

Geronticus Davisoni Hume, Stray Feathers, iii, I875, p. 300 :

Pakchan, Tenasserim

Distr,-Peninsulat Siam.

Bortieo:

Genus THAUMATIBIS Elliot.

Thaumatibis gigantea (Oust.).

Giant Ibis.

Ibis gigantea Oustalet, Bull. Soc. Philom. (7) i, I877, p. 25: Cochin China.

Distr.-Peninsular Sian.

\section{Genus PLEGADIS Kaup.}

*Plegadis falcinellus (Linn.).

Glossy Ibis.

[Plegadis falcinellus falcinellus (Linn.).

Tantalus Falcinellus Linn., Syst. Nat. I2th ed. i, I766, P, 241 : Austria.

Distr._Extra-limital.]

Plegadis falcinellus peregrinus $(\mathrm{Bp} .)^{2}, 2$

Ibis peregrina Bonaparte, Consp. Gen. Av. it, 1855, p. I59: Java.

Distr.—Borneo.

Java.

\section{Family PLATALEIDA.}

Genus PLATALEA Linn.

Platalea leucorodia (Linn.).

Spoonbill.

[Platalea leucorodia leucorodia Linn.

Platalea Leucorodia Linn., Syst. Nat., roth ed. 1758, p. I39: Europe.

Distr.-Eixtra-linnital.]

- Bornean records of papillosa we refer to davisont.

- For the present we have referred all Sunda Island birds to the smal! eastern race but the possibility of migrants from the north $[P$. $f$. falcivellus $)$ must not be overlooked. We can find no actual record for Sumatra: Dammerman jecords it with a query from Batam Island in the Rhio Archipelago. P. f. peregrimis breeds in Java. 
*Platalea leucorodia regia Gould.

Plalalea regia Gould, Syn. Bds. Austr. pt. iv, $18_{3} 8$, App. [. 7 : New South Wales.

Distr.-Borneo.

\section{Family CICONIIDÆ.}

\section{Genus IBIS Lacépède."}

Ibis cinereus (Raffles).

Southern Painted Stork.

Tantalus cinereus Raffles, Trans. Linn. Soc. xiii, 1822, p. 327 : Bencoolen, Sumatra.

Tantalus lacteus, Temm., Pl. Col. 352, 1825 : Java.

Distr.-Malay Peninsula (north to Selangor). Sumatra. Java.

Ibis leucocephalus (Penn.). Painted Stork or Pelican-Ibis. Tantalus leucocephalus Pennant, Ind. Zool. I769, p. II, pl. Io: Ceylon.

Distr.-Malay Peninsula (south to Selangor).

\section{Genus DISSOURA Cabanis.}

Dissoura episcopus (Bodd.).

White-headed Stork.

Dissoura episcopus episcopus (Bodd.).2

Ardea Episcopus Boddaert, 'Tabl. Planch. Enlun. 1783, p. 54, 906 : Coromandel Coast.

Dissoura neglecta Finsch, Orn, Mon. xii, 1004, p. 94 : Java.

Distr.-Malay Peninsula (north).

Sumatra.

Java; Bali (vis.).

Dissoura stormi (Blas.). .

Storm's Stork.

Mclanopelargus episcopus stomi W. Blasius, Mitt. Geogr. Ges.

Nat. Mus. Lübeck, I896, p. I20: Poutianak, Borneo.

Dissura mortoni O.-Grant, Bult. Brit. Orn. Cl. xiii, เgo2, p. 26 : Lamag. British North Borneo.

Distr. - Malay States (Perak).

Sumatra.

Borneo.

1 Little is known about the distribution of the species of this genus in the Malay Peninsula.

a We cannot separate $D$. e. nerlecta.

3 This species may only be a southern form of $D$. episcopus, but the birds look very different in the flesh and until we know more of their ranges in the Malay Peninstula and Sumatra it seems best to keep them separate. 
Genus XENORHYNCHUS Bonaparte.

Xenorhynchus asiaticus (Lath.).

Black-necked Stork.

Xenorhynchus asiaticus asiaticus (Lath.).

Mycteria asiatica Latham, Ind. Orn. ii, 1790, p. 670: India.

Distr.-Peninsular Siam."

\section{Genus LEPTOPTILOS: Lesson.}

Leptoptilos javanicus (Horsf.). Lesser Adjutant Bird. Ciconia Javanica Horsfield, Trans. Linn. Soc, xiii, 1821, p. I88: Java.

Ciconia capillata Temm., Pl. Col. 312, 1824: Java.

Distr.-Malay Peninsula.

Sumatra; Billiton.

Borneo,

Java; ? Bali.

\section{Family ARDEID无.}

Genus ARDEA Linn.

Ardea purpurea Linn.

Purple Heron.

[Ardea purpurea purpurea Linn.

Ardea purpurea Linn., Syst. Nat. 12th ed. i, 1766, p. 236: France (Siresemann, 1920).

Distr._Extra-limital.]

\section{Ardea purpurea manillensis Meyen}

Ardea purpurea var, manillensis Meyen, Acta Acad. Leop. Carol. xvi, Suppl., 1834, p. 102: Manila, Philippine Islands.

Distr.-Malay Peninstrla.

Sumatra; West Sumatran islands of Nias and Engano.

Borneo.

Java; Bali; Kangean Islands.

Ardea sumatrana Raffles. ${ }^{3}$

Dusky+gray Heron.

Ardea sumatrana sumatrana Raftles.

Ardea Sumatrana Raffles, Trans. Linn. Soc. xiii, 1822, p. 325 :

Bencoolen, West Sumatra.

Ardea typhon Temm., Pl. Col. 475, 1829: Sumatra (Baker, 1929).

"We do not believe in the locality "Penang" (Brit. Mus.) for this species.

a Leptoptilos dubius $(G m$.), thas also been recotded from Malaysia but we think in error.

"This is the "Lepterodius gularis asha" of Moulton's Bornean hist. 
Ardea robusta Bp., Consp. Gen. Av, ii, 1855, p. 110 : in 5yn. A. typhon, Typhon Temminckii Reichenb., Syst. Av. Nat. 1852, p. xvi : new name for A. typhon.

Distr.-Malay Peninsula.

Sumatra; Rhio Archipelago; Billiton; West Sumatran Islands of Babi, Banjak Islands and Pagi Islands.

Borneo; North Bornean islands of Bangtey and Balambangan.

Java; Bali; Bawean Island.

Ardea cinerea Linn.

Common Heron.

[Ardea cinerea cinerea Linn.

Ardea cinerea Linn., Syst. Nat. Ioth ed. 1758, p. 143: Sweden.

Distr.-Extra-limital.]

Ardea cinerea rectirostris Gould.

Ardea rectirostris Gould, Proc. Zool. Soc. 1843 , p. 22 : New South Wales error $=$ South India.

Distr.-Malay Peninsula.

Sumatra.

Borneo ${ }^{1}$.

Java.

Genus EGRETTA T. Forster.

Egretta intermedia (Wagl.).

Smaller Egret.

Egretta intermedia intermedia (Wagl.).

Ardea intermedia Wagler, Isis, I 829 , P. 659 : Java.

Ardea melanopus Wagl., Isis, 1829, p. 650: Java.

Distr.-Malay Peninsula.

Sumatra; Billiton; Nias Island.

Borneo.

Java; Kangean Islands; Thousand Islands.

Egretta alba (Lint.).

Larger Egret.

[E gretta alba alba (1,inn.).

Ardea alba Linu., Syst. Nat. roth. ed. 1758, p. 144: Europe.

Distr.-Extra-limital.]

- We have examined a fresh specimen collected in Sarawak. 
Egretta alba modesta (Gray).

Ardea modesta J. E. Gray, Zool. Misc. I831, p. 19: India.

Distr.-Malay Peninstula.

Sumatra.

Borneo.

Java.

Egretta eulophotes (Swinh.).

Chinese Egret.

Herodias eulophotes Swinhoe, Ibis, I860, p. 64: Amoy, China.

Disir._Peninsular Siant."

Sipora, Mentawi Islands, West Sumatra.

Borneo.

Christmas Island.

Egretta garzetta (Linn.).

Little Egret.

Egretta garzetta garzetta (Linn.).

Ardea Garzetta Linn., Syst. Nat. 12th ed. i, I766, p. 237 : "Oriente".

Distr,-Malay Peninsula.

Sumatra; Nias Island, West Sumatra.

Borneo.

Egretta garzetta nigripes (Temm.).

Ardea nigripes Temminck, Man. d'Ord, ecl. 2, iv, 1840, p. 376 : Sunda Islands=Java (Mathews, 1927).

Distr.-Java; Bali; Kangean Islands.

Cocos-Keeling Islands, ${ }^{2}$

\section{Genus DEMIGRETTA Blyth.}

Demigretta sacra (Gmel.).

Reef Heron.

Demigretta sacra sacra (Gmel.).

Ardea sacra Gmelin, Syst. Nat. i, pt. 2, I789, p. 640: Tahiti.

Distr.-Malay Peninsula; Anamba Islands; Tambelan Islands. Sunatra; Rhio Archipelago; Lingga Archipelago (Nyamok Islets), West Sumatran Islands of Simalur, Banjak Islands, Nias, Mentawi Islands, Engano Billiton. Borneo; Natuna Islands; Karimata Islands. Java; Bali; Kangean Islands; Karimon-Java Islands. Christmas Island; Cocos-Keeling Islands.

I See Hume, Stray Feathers, viii, 1879, p. 158, and Sharpe, Cat. Birds Brit. Mus., xxvi, $1 \$ 08$, p. 141 .

- We have associated birds from the above localities (except Java) with nigripes putely on geographical grounds: the only specimens we bave seen are from the type locality. 


\section{Genus NYCTICORAX ${ }^{1}$ T. Forster.}

Nycticorax nycticorax (Linn.).

Night Heron.

Nycticorax nycticorax nycticorax (Linn.).

Ardea Nycticorax Linn., Syst, Nat. Ioth ed. 1758, p. I42: South Europe.

Distr.-Malay Peninsula.

Sunatra; Banka; Nias Island, West Simatra.

Borneo.

Java; Kangean Islands.

Nycticorax caledonicus (Gmel.). ${ }^{2} \quad$ Australian Night Heron.

[Nycticorax caledonicus caledonicus (Gmel.).

Ardea caledonica Gmelin, Syst. Nat. I, pt. 2, 1789, p. 626: New Caledonia.

Disir.-Extra-linital.]

Nycticorax caledonicus manillensis Vig.

Nycticorax Manillensis Vigors, Proc. Comn, Zool, Soc. I83I, p. 98: Manila, Philippine Islauds.

Distr.-Borneo (north).

* Nycticorax caledonicus hilli Math.

Nycticorax caledonicus hilli Mathews, Nov. Zool. xviti, I912, p. 233: Parry's Creek, North-west Australia.

Distr.-Cocos-Keeling Islands.

Genus GOISAKIUS ${ }^{3}$ G. R. Gray.

Goisakius melanolopus (Raffles).

Tiger Bittern.

Goisakius melanolophus melanolophus (Raftles).

Ardea melanolopha Raffles, Trans. Linn. Soc. xiii, 1822, p. 326 : West Sumatra.

Nycticorax limnophilax Temm., Pl. Col. 581,1836 : Java.

Disir.-Malay Peninsula.

Sumatra; Banka.

Borneo.

Java; Thousand Islands.

- Revision: Peters, Bost, Soc. Nat. Hist. 39, 1930, p. 263.

- A form of this bird is said to breed in Java, but no specimens have been examined.

3 C. . Hach, Ibis 1926, p. 5\$5; Kloss, Ibis, 1927, p. 526. 


\section{Genus BUTORIDES' Blyth.}

Butorides striatus (Linn.).

Little Green Heron.

[Butorides striatus striatus (Linn.).

Ardea striata Linn., Syst. Nat. Ioth ed. 1758, p. 144: Surinam, South America.

Distr.—Extra-limital.]

\section{Butorides striatus javanicus (Horsf.).}

Ardea Javanica Horsfield, Trans. Linn. Soc. xiii, I82I, p. I9o: Java.

* Butorides javanicus carcinophonts Oberh., Journ. Wash. Acad. Sci. xiv, 1924, p. 294 : Alanga Island, Maratua Group, East Borneo.

Butorides javanicus abbotti Oberb., Bull. U.S. Nat. Mus. 159, 1932, p. 14: Langkawi Island, Straits of Malacca.

Distr.-Malay Peninsula; Anamba Islands.

Sumatra; Rhio Archipelago; Nias Island; Billiton.

Borneo; Maratua Islands; Natuna Islands.

Java; Bali; Kangean Islands; Bawean Island,

Butorides striatus sipora Chas. and $\mathrm{Kl}$.

Butorides striatus sipora Chasen and Kloss, Ibis, I926, p. 277 : Sipora Island, West Sumatra.

Distr.-Sipora and Siberut Islands (Mentawi Islands), West Sumatra.

Butorides striatus amurensis Schrenck.

Ardea (Bulorides) virescens var amurensis Schrenck, Reise Amur Lande, i, pt. 2, I860, p. 44I: Anurland.

*Butorides striatus actophilus Oberh., Smiths. Misc. Coll. 1x, 7, 1912, p. 1 : North Pagi Island, West Sumatra.

*Butorides striatus icasopterus Oberh., Smiths. Misc. Coll. Ix, 7, 1912, p. 1: Simalur Island, West Sumatra.

Distr. ${ }^{2}$ - Malay Peninsula.

Borneo; Natuna Islands.

Java.

Christmas Island.

- Revisions: Oberh., Proc. U.S. Nat. Mus. xlii, 1912, p. 529; Oberh., Bull. U.S. Nat. Mus. 159, 1932, p. 14.

- There is much difference of opinion concerning the Malaysian races of this heron. A long-winged form visits the sub-region in winter. The wing-range of specimens examined is $180-202 \mathrm{~mm}$. which is nearer to that given for connectens Stres., Orn. Mon. xxxviii, 1930, p. 48 (Kwangsi) than to true amurensis. 


\section{Genus ARDEOLA Boie.}

Ardeola ralloides (Scop.).

Pond-Heron.

[Ardeola ralloides ralloides (Scop.).

Ardea ralloides Scopoli, Ann. i, Hist. Nat. I 769, p. 88: Krain.

Distr.-Extra-limital.]

Ardeola ralloides bacchus (Bp.)."

Buphus bacchus, Bonaparte, Consp. Gen. Av. ii, 1855, p. I27: Malay Peninsula.

?Cancroma leucoptera Bodd., Tabl. PI, Enl, 1783, p. 54: Malacca.

?Ardea malaccensis Gmel., Syst. Nat. i, 2, 1789, p. 643: Malacca.

Distr.-Malay Peninsula.

Borneo; Natuna Islands.

Ardeola ralloides speciosa (Horsf.).

Ardea speciosa Horsfield, Trans. Linn. Soc. xiii, 1821 , p. I89: Java.

Buphus psendo-ralloides Brehm, Naumannia, 1855, p. 200: "India orientali" = Java.

Distr.-Borneo.

Java; Bali; Thousand Islands; Karimon-Java Islands; Kangean Islands. ${ }^{2}$

Ardeola ibis (Linn.).

Cattle Egret.

[Ardeola ibis ibis (Linn.).

Ardea Ibis Linn., Syst. Nat. Ioth ed. I758, p. I44: Egypt.

Distr.-Extra-limital.]

Ardeola ibis coromanda (Bodd.).

Cancroma Coromanda Boddaert, Tabl. Pl. Enlum. I783, p. 54 : Coromandel Coast.

Ardea affris Horsf., Trans. Linn. Soc. xiii, 1821, p. 189 : Java.

Distr.-Malay Peninsula; Anamba Islands.

Sumatra.

Borneo.

Java; Bali.

t Probably visits Sumatra: has been recorded from the Andamans and "leucoptera" is recorded from Nias Island, West Sumatra. It is impossible to say what this latter is. We cannot distinguish between bacchus and grayi in winter, or immature plumage and as all birds of this genus in breeding. or moulting plumage, examined by us from the Malay Peninsula are bacchus, grayi is omitted from the list. A specimen of speciosa said to be from Singapore, is in the Berlin Museum but the provenance is doubtful.

2 There seems to be no trustworthy record for Sumatra. See Salomonson, Bull. Mus. Hist. Nat. Paris, 1929, D. 347. 
Genus IXOBRYCHUS Billberg.

Ixobrychus sinensis ${ }^{1}$ (Gmel.).

Yellow Bittern.

Ixobrychus sinensis sinensis (Gme].).

Ardea Sinensis Gmelin, Syst. Nat. i, pt. 2, 1789, p. 642: China.

Ardea lepida Horsf., Trans, Linn. Soc. xiii, 1821 , p. 190: Java.

Distr.-Malay Pensinsula.

Sumatra; Nias Island, West Sumatra; Billiton.

Borneo; Natuna Islands.

Java; Bali; Karimon-Java Islands.

Ixobrychus cinnamomeus (Gmel.) . Chestnut Bittern

Ixobrychus cinnamomeus cinnamomeus (Cimel.).

Ardea cinnamomea Ginelin, Syst. Nat. i, pt. 2, I789, p. 643: China. Ardea nebulosa Horsf., Trans. Linn. Soc. xiii, 1821 , p. 190 : Java.

Botaurus assimilis Brehm, Isis, 1842, p. 582: Java.

Distr.-Malay Peninsula.

Sumatra.

Borneo.

Java; Bali; Kangean Islands.

Ixobrychus eurhythmus (Swinh.). Schrenck's Bittern.

Ardetta eurhythma Swinhoe, Ibis, 1873, p. 74, pl. 2 : Amoy, South China.

Distr.-Malay States.

Sumatra.

Borneo.

Java; Mata Siri Island, Java Sea; Karimon-Java Islands.

Genus DUPETOR Heine and Reichenow.

Dupetor flavicollis (Lath.) .

Black Bittern.

Dupetor flavicollis favicollis (Lath.).

Ardea flavicollis Latham, Ind. Orn, ii, 1790, p. $70 \mathrm{r}$ : India.

Ardea picata Raffles, Trans. Linn. Soc. xiii, 1822, p. 326 : Sumatra.

Ardea bilineata Less., Traité d'Orn. 1831, p. 574 : Java.

Distr._Malay Peainsula.

Sumatra; Nias Island, West Sumatra.

Borneo.

Java.

1 Revision: Oberh., Bull. U.S. Nat. Mus. 159, 1932, p. 17.

$[60]$ 


\section{Genus BOTAURUS Stephens.}

Botaurus stellaris (Linn.).

Bittern.

Botaurus stellaris stellaris Linn.

Ardea stellaris Linn., Syst. Nat. 1oth ed. 1758, p. I44: Sweden.

Distr.-Malay Peninsula.

Botneo.

\section{Family ANATIDE.}

\section{Genus ASARCORNIS Salvadori.}

Asarcornis scutulata (Mï1l.).' White-winged Wood-Dutck. Anas scutulata S. Müller, Verh. Nat. Gesch. Land en Volk. I812, P. I59: Java.

Distr.-Malay Peninsula.

Sumatra.

Java.

Genus NETTAPUS Brandt.

Nettapus coromandelianus (Gmel.). Cotton Teal.

Nettapus coromandelianus coromandelianus (Gmel.).

Anas coromandeliana Gmelin, Syst. Nat. i, pt. 2, I789, p. 522 : Coromandel, India.

Distr.-Malay Peninsula.

Sumatra; Rhio Archipelago; Banka.

Eorneo.

Java.

Genus DENDROCYGNA Swainson.

Dendrocygna javanica (Horsf.)

Whistling Teal.

Dendrocygna javanica javanica (Horsf.).

A nas Javanica Horsfeld, Trans. Linn. Soc. xiij, I821, p. 200: Java.

Distr.-Malay Peninsula.

Sunnatra.

Borneo.

Java; Kangean Islands.

The status of birds occurring in Java and Sumatra seems uncertain. Specimens in the Raffles Museum from Korinchi, Sumatra seem to be domesticated hybrids, but free-flying birds certainly occur in Sumatra although we cannot say if in colour they show signs of domestication. If Müller's types are crossbred ducks this species should be known as A. leucoptera Blyth (Tenasserim). 
Dendrocygna javanica arcuata (Horsf.).

A nas arcuata Horsfield, Zool. Res. in Java, 1824, pl. 65 and text : Java.

Distr.-Sumatra (South); ? Nias Island, West Sumatra. Borneo.

Java; Bali.

\section{Genus ANAS Linn.}

Anas platyrhynchos Linn.

Mallard.

*Anas platyrhynchos platyrhynchos Linn.

Anas platyrhynchos Linn., Syst. Nat. Ioth ed. 1758, p. 125: Sweden.

Distr.-Borneo.

Anas superciliosa Gmel.

Australian Wild Duck.

[Anas superciliosa superciliosa Gmel.

Anas superciliosa, Gmelin, Syst. Nat. i, pt. 2, I789, p. 537 : New Zealand.

Distr._Extra-limital.]

Anas superciliosa percna Riley.

Anas superciliosa percna Riley, Proc. Biol, Soc. Wash. xxxii, I9r9, p. 93 : Celebes.

Distr.-Sumatra.

Java; Bali; Kangean Islands.

*Anas penelope Linn.

Wigeon.

Anas Penelope Linn., Syst. Nat. roth ed. 1758, p. I26: Sweden. Distr.-Borneo.

Anas crecca Linn.

Teal.

Anas crecca crecea Linn.

Anas Crecca Linn., Syst. Nat. Ioth ed. I758, p. I25: Sweden. Distr.-Malay Peninsula.

Anas querquedula Linn.

Garganey.

Anas Querquedula Linn., Syst. Nat. roth. ed. 1758, p. 126 : Sweden.

Anas (Querquedula) humeralis Müll., Verh. Nat. Gesch, Land en Volk. 1842, p. 159: Java.

Distr.-Malay Perinsula.

Stumatra.

Borneo.

Java. 
Anas gibberifrons gibberifrons S. Müll.

Anas (Mareca) gibberifrons S. Müller, Verh. Nat. Gesch. Land ent Volk. I842, p. I59: Celebes.

Distr._Java.

Christmas Island.

Anas acuta Linn.

Pintail.

*Anas acuta acuta Linn.

Anas acuta Limn., Syst. Nat, Ioth ed. J758, p. 126: Sweden.

Distr.-Borneo.

Genus SPATULA Boie.

Spatula clypeata (Linn.).

Shoveller.

Anas clypeata Linn., Syst. Nat. Ioth ed. 1758, p. 124 : Sweden.

Distr.-Malay Peninsula.

Borneo.

Genus NYROCA Fleming.

- Nyroca fuligula (Linn.).

Tufted Duck.

Anas Fuligula Linn., Syst. Nat. Ioth ed. 1758, p. I28: Sweden.

Distr.—? Malay Peninsula.

Borneo.

\section{Family PHALACROCORACID蚱.}

Genus PHALACROCORAX Brisson.

Phalacrocorax carbo (Linn.).

Cormorant.

[Phalacrocorax carbo carbo (Linn.).

Pelecanus Carbo Linn., Syst. Nat. roth ed. 1758, p. 133 : Europe. Distr.-Extra-limital.]

Phalacrocorax carbo sinensis (BI11m.).

Pelecanus sinensis J. F. Blumenbach, Abb. Nat. Gegen. No. 25, 1798: China.

Distr.-Malay Peninsula.

Sumatra.

- Ducks reported as occurring in the Cocos-Keeling Islands are perhaps of this species. 
Phalacrocorax sulcirostris (Brandt).

Little Black Cormorant.

[Phalacrocorax sulcirostris sulcirostris (Brandt).

Carbo sulcirostris Brandt, Bull. Sci. Imp. Acad. Sci. St. Petersb. iii, 1837 , p. 56 : New South Wales.

Distr._Extra-limital.]

Phalacrocorax sulcirostris territori (Math.). ${ }^{9}$

Mesocarbo ater territori Mathews, Birds Austr. iv, 1915, p. I76: Northern Territory of Australia.

Distr.-Borneo.

Java.

Phalacrocorax niger (Vieill.).

Little Cormorant.

Hydrocorax niger Vieillot, Nouv. Dict. d'Hist. Nat. viii, $1817, \mathrm{p}$. 88: Bengal.

Carbo javanica Horsf., Trans. Linn. Soc. xiii, 1821, p. 197: Java.

Distr.-Malay Peninsula.

? Nias Island, West Sumatra.

Borneo.

Java."

Phalacrocorax melanoleucos (Vieill.).

Pied Cormorant.

[Phalacrocorax melanoleucos melanoleucos (Vieill.).

Hydrocorax melanoleucos Vieillot, Nouv. Dict. d'Hist. Nat. viii, IS17, p. 88: New South Wales.

Distr,_Extra-linital.]

*Phalacrocorax melanoleucos melvillensis (Math.).

Carbo melanoleucos melvillensis Mathews, Austr. Av. Rec. i, Igra,

p. 74: Melville Island, Northern Territory, Australia.

Distr.-Bali.

\section{Family ANHINGIDÆ.}

\section{Genus ANHINGA Brisson.}

Anhinga rufa (Lacép, and Daud.).

Darter or Snake Bird.

[Anhinga rufa rufa (Lacép. and Daud.).

Plotus rufus Lacépède and Daudin, Buffon Hist. Nat. ed. Didot, Quadr. xiv, 1802, p. 319: Senegal.

Distr._Extra-limital.]

- Breeding birds examined but no comparison of subspecies possible.

2 Peters includes Sumatra in the range of this species but we know of no reliable record from that island. 
Anhinga rufa melanogaster Penuant.

Anhinga melanogaster Pennant, Ind. Zool. I769, p. I3, pl. I2: Ceylon.

Distr._Malay States,"; Langkawi Islands.

Sumatra.

Borneo.

Java.

\section{Family SULID王.}

Genus SULA Brisson.

Sula dactylatra Less.

Masked Gannet.

[Sula dactylatra dactylatra Less.

Sula dactylatra Lesson, Traité d'Orn, 1831, p. 601: Ascension Island, Atlantic Ocean.

Distr.-Extra-limital.]

Sula dactylatra bedouti Math.:

Sula dactylatra bedouti Mathews, Austr. Av. Rec., i, I9I3, p. I8g: Bedottt Island, South-west Australia.

Distr.-Coast of Sarawak. Java.

Cluristmas Island, Indian Ocean.

Sula abbotti Ridgw.

Abbott's Gannet.

Sula abbotii Ridgway, Proc. U.S. Nat. Mus. xvi, I893, p. 599: Assumption Island, Indian Ocean.

Distr.-Christmas Island; Cocos-Keeling Islands.

Sula leucogaster (Bodd.) . ${ }^{3}$

Booby or Brown Gannet.

[Sula leucogaster leucogaster (Bodd.).

Pêlecanus Leucogaster Boddaert, Tabl. P1. Ënl. I7 $S_{3}$, p. 57 : Cayenne.

Distr.-Extra-limital.]

- Almost certain to occur in Peninsular Siam.

- Little is known of the distribution of $S$. dactylatra in Malaysia. Records from Java and Sumatra may tefer to $S$, abbotti. Christmas I 1 land is here included because Mathews (1927) specifically extends the range of bis S. . bedouti to that island whence we have only seen $S$, abbothi. A form of the species identified by Bangs as $S$. $d$. personata breeds in the Sulu Sea: some authors suggest that bedowti is inseparable from personata. The only specimens we have seen from Malaysia are from the coast of Sarawak.

3 Ct. Rob. and Kinn., Bull, B. O. C. xlviii, 1928, p. 63 . 
Sula leucogaster plotus (I'orst.),

Pelecanus Plotus Forster, Descr. Aninn, ed. Licht., IS44, P. 278 : near New Caledonia.

Distr.-Malay Peninstila (both coasts).

Sumatra.

Borneo; Tiga Island, North-west Coast of Borneo.

Java.

Christmas Island; Cocos-Keeling Islands

Sula sula (Linn.).

Red-footed Gannet.

[Sula sula sula (Linn.).

Pelecanus Sula Limn, Syst. Niat, izth ed., i, г766, p. a IS : Barbados, West Indies.

Distr.-Extra-limital.]

Sula sula rubripes Gould.

Sula rubripes Gould, Synops. Birds Austr. pt. iv, I 838, App. P. 7 : Australia.

Distr,-Pagi Islands, West Sumatra.

Coast of Nortli Borneo.

Java; Bali.

Christmas Island; Cocos-Keeling Islands.

\section{Family FREGATIDæ.}

Genus FREGATA Lacépède.

Fregata andrewsi ${ }^{3}$ Math.

Andrews' Firigate Bird.

Fregata andrewsi Mathews, Austr. Av. Rec, ii, 19r4, p. r20:

Christmas Island, Indian Ocean.

Distr.-Ananba Islands.

? Sull islands near Billiton ("aquila").

Coast of Sarawak.

Java.

Christmas Island; Cocos-Keeling Islands.

- We rather doubt if it is correct to place all Malaysian birds undet one sub-species.

Revisions: Lowe, Nov. Zool. xxxi, 1924, 1). 299 ; Chas, and Kil., Journ. Mal. Br. Roy. Asiat. Soc. ii, r924, p. 62.

3 A specimen of $F$ a aquila said to be from the Straits of Malaccn is in the British Museum. We believe that a mistake in labelling has occurred. 
Fregata ariel (G. R. Gray).

Lesser Frigate Bird.

Fregata ariel ariel (G. R, Gray).x

Atagen Ariel G. R. Gray, List. Gen. Birds, iii, I845, p. 669, pl. 183: Raine Island, North Queensland.

Distr.-Malay Peninsula (both coasts); Tioman Archipelago; Anamba Islands.

Sumatra (Straits of Malacca near the Aroa Islands).

Coast of Sarawak; Labuan; Balambangan Island, North Bormeo; Maratua Islands; Karimata Islands.

Java; Kangean Islands.

Cocos-Keeling Islands.

Fregata minor (Gme1.).

Firigate Bird.

Fregata minor minor (Gmel.).

Pelecanus minor Gmelin, Syst. Nat, i, pt. 2, 1789, p. 572 : Christmas Island, Indian Ocean.

Fregata minor listeri Mathews, Austr. Av. Rec. ii, 1914, p. 119 : Christmas Island, Indian Ocean.

Distr. C Coast of Sarawak ${ }^{2}$; Sulu Sea.

Java; Bawean Island.

Christmas Island.

\section{Family PHAÉTHONTID王.}

\section{Genus PHAËTHON Linn.}

Phaëthon rubricauda Bodd.

Ked-tailed Tropic Bird.

[Phaèthon rubricauda rubricauda Bodd.

Phaeton rubricauda Boddaert, Tabl. Pl. Eul, I $-\varepsilon_{3}$, p. 57 : Manritius.

Distr.-Eixtra-limital.]

\section{Pheëthon rubricauda westralis Math.}

Phathon rubricanda uestralis Mathews, Austr. Av. Rec. i, I9I2, p. 88: West Australia.

Distr.-Christmas Island; ? Cocos-Keeling Islands. ${ }^{3}$

- A frigate bird is also found far inland on Korinchi Lake, West Sumatra. Dr. P. R. Lowe lists birds from the Cocos-Keeling Islands as $F$, ariel sub$\mathrm{sp}$. We have never seen specimens from that locality. Has also been recorded from Christmas Island but we doubt if the bird occurs there and suspect a mistake in labelling.

2 Formerly, but I think wrongly, identified by Mr. C. Boden Kloss and myself as $F$. $m$. aldabrensis Maths.

3 $P$. rubricanda is included in Vorderman's Sumatran list without sufficient authority. 
Phaëthon lepturus Datid.

White-tailed Tropic Bird

*Phaëthon lepturus lepturus Daud.

Phaëton lepturus Daudin, Buffon's Hist. Nat. ed. Didot, Quadr. xiv, 1802, p. 319 : Mauritius.

Distr.—? Cocos-Keeling Islands.'

Phaëthon lepturus fulvus Braudt. Christmas Island Tropic Bird.

Phaëthon fulvus Brandt, Mém. Acad. Imp. Sci. St. Petersb. (6) 5 , 1840 , p. 269 : No type loc, =Christmas Island, Indian Ocean.

Phaëton flovo-aurantius Laur, Ann. Lyc. N. York, vii, 1862, p. 142:

Typ. loc. unknown =Christmas Island, Indian Ocean.

Distr.-Christmas Island, Indian Ocean; Cocos-Keeling Islands.

Phaëthon æthereus Linn.

Short-tailed Tropic Bird.

[Phaëthon athereus athereus Linn.

Phaêthon athereus Linm., Syst. Nat. roth ed. 1758, p. I34: Ascension Island.

Distr._Extra-limital.]

Phaëthon æthereus indicus Hutne.

Phaeton indicts Hume, Stray Feathers, iv, 1876, p. 481 : Mekran Coast.

Distr.-Straits of Malacca.

\section{Family PELECANID瓜.}

\section{Genus PELECANUS Linn.}

Pelecanus onocrotalus Linm.?

White Pelicant.

Pelecanus Onocrotalus Lintı, Syst. Nat. Ioth ed. I758, p. 132: Caspian Sea, Russian Asia.

Pelecanus javanicus Horsf,, Trans. Linn. Soc, xiii, 1821, p. I97: Java.

Distr.-Malay Peninsula.

Sumatra.

Java,

- There is some confusion about the forms of Phathon found in the Cocos-Keeling Islands. Ogilvie-Grant (Cat. Birds Brit. Mus, xxvi, 1898, p. 454) placed Nicholson's record (Ibis, 1882, p. 70) of " $P$. candidus" relating to a specimen obtained by Forbes in the Cocos-Keeling Islands, under $P$. lepturus, but wrongly quoted Java as the locality. Wood-Jones (P.Z.S. 1909, p. 141) however, records the Cocos breeding bird as rubricauda, but judging from a photograph given to me by Mr. C. Clunies Ross, the former author's view seems corfect.

a In our treatment of the pelicans we have followed Messrs. C. H. B. Grant and C. W. Mackworth-Praed, 1934. 
Pelecanus roseus Gmelin, Syst. Nat. i, pt. 2, I789, y. 570: Manila, Philippine Islands.

Disir.-Malay Peninsula.

Sumatra.

Java.

\section{Family ÆGYPIIDÆ.}

\section{Genus GYPS Savigny.}

Gyps indicus (Scop.).

Long-billed Vulture.

[Gyps indicus indicus (Scop.).

Vultur indicus Scopoli, Del. Flor. et Faun. Insubr. ii, I786, p. S5: Pondicherry.

Distr.—Extra-limital.]

\section{Gyps indicus nudiceps Baker.}

Gyps indicus nudiceps Stuart Baker, Bull. B.O.Cl. xlvii, I927, p. I5I. New name for G. tenuirostris Hume: Nepal.

Distr.-Malay States.

\section{Genus PSEUDOGYPS Sharpe.}

Pseudogyps bengalensis (Gmel.). White-backed Vulture.

Vultur bengalensis Gnelin, Syst. Nat. i, I788, p. 245 : Bengal.

Distr.-Malay Peninsula. ${ }^{3}$

\section{Gentus TORGOS Kaup.}

Torgos calvus (Scop.).

King Vulture.

Vultur calvus Scopoli, Del. Flor. et Faun. Insubr, if, r786, p. 85 : Pondicherry.

Distr.-Malay Peninsula.*

Replaces $P$. philippensis Gmel.

- Although nudiceps occurs as far south as Perak and is almost without doubt therefore found in Peninsular Siam we can find no record for that territory.

3 Now only found as far south as Perak, but formerly extending further down the Malay Peninsula perhaps even to Singapore whence, however, there are only some old visual records.

4 South to Perak, but formerly further down the Peninsula. 


\section{Gents NEOPHRON Savigny.}

Neophron percnopterus (Linn.). Egyptian or White Vulture.

[Neophron percnopterus percnopterus (Iinn.).

T'ultur Perenopterus Linn., Syst, Nat. Joth ed. 3758, p. 87: Egypt. Distr.-Extra-linital.]

- Neophron perenopterus ginginianus (Lath.).

Vultur ginginianus Lathan, Ind. Orn. i, 1790, P. 7 : Coromandel.

Distr.-Peninsular Siann.'

\section{Family FALCONIDÆ.}

\section{Gent1s CIRCUS Lacépéde.2}

Circus aruginosus (Linn.).

Marsh Harrier.

Circus eruginosus eruginosus Linn.

Falco aruginosus Linn., Syst. Nat. Ioth ed. 1758, p. 91 : Sweden.

Distr.-Malay Peninsula. Sumatra.

Circus spilonotus Kaup.

Eastern Marsh Harrier.

Circus spilonotus spilonotus Kanp.

Circus spilonotus Katp, Isis, I847, p. 953: East Siberia.

Distr.-Malay States. ${ }^{3}$ Borneo.

Circus melanoleucus (Penn.).

Pied Harrier.

Falco melanoleucus Pennant, Ind. 2ool. 1769, p. 12, pl. it, Ceylon.

Distr.-Malay Peninsula. Bоглео.

\section{Genus ACCIPITER Brisson.}

Accipiter trivirgatus (Temm.).

Crested Goslawk.

Accipiter trivirgatus trivirgatus (Temm.).

Falco trivirgatus Temm., PI. Col. 303, IS24: Sunatra.

Astur anceps Bp., Consp. Gen. Av. i, 1850, p. 31: as syn. of trivirgatus.

Distr.-Sumatra; Nias Island, West Sumatra.

Borneo.

Java.

- According to Dr, W. L. Abbott extends to Penang but we have never seen it there.

"We regard Kelham's record of $C$. pygargus ("cineraceus") from the Malay States as too uncertain for inclusion.

3 Almost certainly occurs in Peninsular Siam. 
Accipiter trivirgatus indicus (Hodgs. or Pear.)."

Astur indicus Hodgson or Pearson, Bengal Sporting Mag. vii, 1836,

p. 177: India.

Distr.-Malay Peninsula.

North Natuna Islands.

Accipiter badius (Gmel.).

Siskra.

[Accipiter badius badius (Gmel.).

Falco badius Gmelin, Syst. Nat. i, pt. I, 1788, p. 280 : Ceylon.

Distr._Extra-linital.]

Accipiter badius poliopsis (Hume).

Micronisus poliopsis Hume, Stray Feathers ii, I874, p. 325: North Pegu.

Distr., Malay Peninsula."

Sumatra.

Accipiter soloënsis (Horsf.). Clinese Gosllawk.

Falco Soloënsis Horsfield, Trans. Linn. Soc. xiii, I821, p. 137 : Central Java.

Falco cuculaides Temm., Pl. Col. 110 and 120,1822 : West Java.

Distr.-Malay Peninsula.

Sumatra; West Sumatran Islands of Simalur and Nias.

Borneo.

Java; Thousand Islands, Java Sea.

Accipiter fasciatus (Vig. and Horsf.).

Australian Goshawk.

[Accipiter fasciatus fasciatus (Vig. and Horsf.).

Astur Fasciatus Vigors and Horsfield, Trans. Linn. Soc. xv, I827, p. I8I: New South Wales.

Distr.-Euxtra-linital.]

Accipiter fasciatus natalis (Lister).

Christmas Island Goshawk.

Urospizias natalis Lister, Proc. Zool. Soc. for $18 \delta \$$, p. 523 : Christmas Island.

Distr,-Christmas Island, Indian Ocean.

- Judging from published measurements birds from the Malay Peninsula are rather smaller than typical indicus : they are certainly not the smaller and brighter $t$, trivirgatus and should nerhaps be indicated by trivirgatus $>$ indicts.

"The provenance of "Singapore" specimens is a little doubtful. Buttiköfers "poliopsis" from Nias is stated to have the cere yellow and is therefore probably $A$. soloentusis.

3 See Stres., Journ. F. Orn. 1925, p. 319. 
Accipiter virgatus ('T'emm.).

Small Sparrow-Hawk.

Accipiter virgatus virgatus (Temm.).

Falco virgatus "Reinw." Temminck, P1. Col. I09, 1823, : Java.

Accipiter rufotibialis Sharpe, Ibis, 1887, p. 437: Mt. Kinabalu, North Borneo.

Distr.-Malay States.

Sumatra. '

Borneo.

Java; Bali.

Accipiter virgatus gularis (Temm. and Schleg.),

Astur (Nisus) gularis Temminck and Schlegel, Faun. Japon. Aves, I 844, p. 5, pl. 2 : Japan.

PAccipiter nisoides Blyth, Journ. Asiat. Soc. Bengal, xvi, 1847, p. 727 : Malacca. ${ }^{2}$

Distr.-Malay Peninsula.

Sumatra; Rhio Archipelago; Billiton.

Borneo; Nortl Natuna Islands.

Java.

Accipiter nisus (Linn.).

Sparrow-Hawk.

[Accipiter nisus nisus (Linn,).

Falco Nisus Linn., Syst. Nat. Ioth ed. 1758, p. 92: Sweden.

Distr.-Extra-limital.]

Accipiter nisus nisosimilis (Tick.).

Falco Nisosimilis Tickell, Journ. Asiat. Soc. Bengal, ii, I833, p. 57 I : Borabham, Bengal.

Distr,-Borneo. ${ }^{3}$

Genus BUTEO Lacépede.

Buteo burmanicus Hume.

Desert-Buzzard.

Buteo burmanicus burmanicus Hume.

Buteo burmanicus Hume, Stray Feathers, iii, I875, p. 30 : Thayetmyo, Burma.

Distr.-Malay States.

" "virgatus" has also been recorded from the West Sumatran Islands of Simalur, Lasia, Siumat, and Nias, but as in all these localities the migratory gularis no doubt occurs the identifications are not certain.

a . visoides is more likely to apply to a migratory form than to $a$ resident race which, in the Malay Peninsula, would probably only be obtained in the mountains of the interior. Swann's remarks in Mon. Bds. Prey, 1926, p. 327 concerning $A$, afhwis in tbe Malay Peninsula and Borneo seem to refer to A. $v$. gularis: South-west Siam appears to be the southern limit of affnis. Wintering adults taken in the Malay Peninsula are very variable in colour. Most males are inseparable from Japanese birds, but paler individuals may be referable to $A$. v. stevensoni Gurney.

3 A female in the Sarawak Museum, taken at Kuching, Sarawak on 3 otb March, 1805 makes a noteworthy extension of range for this species. 


\section{Genus HIERAAËTUS ${ }^{x}$ Kaup.}

Hieraaëtus pennatus (Gmel.).

Booted Eagle.

\section{Hieraaëtus pennatus pennatus (Gmel.).a}

Falco pennatus Gmelin, Syst. Nat. i, pt. I, 1788, p. 272: Spain (Stuart Baker, 1930).

Distr.-Malay States.

Hieraaëtus kienerii ( $\mathrm{E}$. Geoff.). Rufous-bellied Hawk-Eagle.

[Hieraaětus kienerii kienerii (E. Geoff.).

Astur Kienerii G. S. [=E. Geoffroy], Mag. Zool. I835, cl. 2, pl. 35: Hinlalayas.

Dislr.-Extra-linital.]

Hieraaëtus kienerii formosus Stres.

Hierraëtus kiencri formosus Stresemann, Orn. Monatsb. xxxii, I924, p. IoS: Celebes.

Distr.-Malay States.

Sumatra.

Borneo.

Java.

\section{Genus ICTINÄ̈TUS Blyth.}

Ictinaètus malayensis (Temm.). ${ }^{3}$

Black Eagle.

Falco malayensis Temminck, Pl. Col. II7, I824: Java.

Distr. - Malay Peninsula.

Sumatra; Nias Island, West Sumatra; Billiton.

Borneo.

Java; Bali.

\section{Genus SPIZAËTUS Vieillot.}

Spizaëtus cirrhatus" (Gmel.).

Changeable Hawk-Eagle.

[Spizaëtus cirrhatus cirrhatus (Gmel.).

Falco cirrhatus Gnelin, Syst. Nat. I, 1788, p. 274 : India.

Distr.-Extra-limital.]

I Revisions: Stres., Journ, f. Orn. Ixxii, 1924, p. 432; Orn. Monatsber, 1924, p. 108; Chas. and K1., Journ. Siam Soc. (Nat. Hist. Suppl.) vii, Igz", p. 162 .

2 A very rate bird in the Malay Peninsula.

3 Peniger Hodgs. from Nepal seems unrecognizable. Doherty is responsible for the Bali record, but his specimen does not appear to have been critically examined.

4C7. Stres., Journ. f. Orn. Ixxii, Ig24, p. 430. 
Spizaëtus cirrhatus limnzetus Horsf.

Falco Limneelus Horsfield, Trans, Linn, Soc. xiii, 1821, p. 138 : Java.

Falco caligatus Raffes, Trans. Linn. Soc. xiij, 1822, p. 278: Sumatra.

Falco nivens Temm., PI. Col. 127, 1823 : Java.

Limunétus horsfieldii Vig., Mem. Raffles, 1830, p. 649 : new name for limuaêtus.

Distr.-Malay Peninstrla.

Sumatra; Pagi Islands, West Sumatra.

Borneo.

Java.

Spizaëtus nipalensis' (Hodgs.).

Hodgson's Hawk-Eagle.

Spizaëtus nipalensis nipalensis (Hodgs.).

Nisaëtus Nipalensis Hodgson, Journ. Asiat. Soc. Bengal, v, I836, p. 229 : Nepal.

Disir,-Peninsular Sianı.

Spizaëtus nipalensis alboniger (Blyth). Blyth's Hawk-Eagle.

Nisaëtus alboniger Blyth, Journ. Asiat. Soc. Bengal, xiv, I845, p. $17 \hat{3}$ : Malacca.

Spiaaêtus borneonensis Gray, Cat. Acciptr. 1848, p. I6: Borneo.

Spizaêtus nanus Walt., Ibis, 1868, p. 14, pl, 1: Borneo.

Distr._Malay Peninsula; Anamba Islands.

Sunatra; Banjak Islands and Nias Island, West Sumatra.

Borneo.

-Spizaètus nipalensis bartelsi Stres.

Spizačlus nipalensis bartelsi Stresentann, Journ. f. Orn. 1xxii, 1924, p. 431 : West Java.

Distr.-Java. ${ }^{2}$

\section{Genuts CIRCAËTUS Vieillot.}

Circaëtus gallicus (Gmel.).

Short-toed Eagle.

*Circaëtus gallicus gallicus (Gmel.).

Falco gallicus Comelin, Syst. Nat, i, 10t. 1, 1788, p. 259: France.

Distr.-Malay States.

' Cf. Stres., Journ. f. Orn. 1xii, 1924, p. 432.

- Swann has suggested that the Javan and Bornean sub-species are inseparable in which case other names, now quoted as synonyms of alboniger, would have priority over bartelsi, but Swann's figures for the wing-length of typical alboniger are too small (our males run up to $348 \mathrm{~mm}$. which is also about the maximum for the Bornean birds we have seen : we have never seen a bird from Borneo as large as the type of bartelsi, $358 \mathrm{~mm}$. in wing-lengtb. 
Genus SPILORNIS ${ }^{t}$ G. R. Gray.

Spilornis cheela" (Lath.).

Serpent Eagle.

[Spilornis cheela cheela (Lath.).

Spilormis Cheela Latham, Ind. Orn. i, I790, p. I4 : Lucknow, India. Distr. Extra-limital.]

Spilornis cheela bassus (Forst.).

Falco Bassus J. R. Forster, in Levaillant, Naturg. Afr. Vög, r79\$, p. 55, pl. I5: Africa error =Sumatra.

Falco bacha Daudin, 'Traité d'Oru, ii, rSoo, 43 : the same.

Spilornis cheela malayensis Swann, Syn. List. Accip. 1920, p. 83: Raub, Pahang, Malay States.

Distr.-Malay Peninsula; Anamba Islands.

Sunatra: Rhio Archipelago.

\section{Spilornis cheela pallidus Wald.3}

Spilomis pallidus Waklen, Ibis, i8 72 : p. 363 : Sarawak.

Spilornis raja Sharpe, Bull. B. O. C1, i, 1803, D. 59 : Sarawak.

? Spilornis cheela richmondi Swann, Syn. List. Accip. 1922, p. 135: South-west Borneo.

Distr.-Borneo (lowlands).

\section{Spilornis cheela kinabaluensis Scl.}

Spilornis cheela kinabaluensis W. I. Sclater, Bull. B.O.Cl. xI, rgr9, p. 37 : Mt. Kinabalu, British Nortl Borneo.

Distr.-The mountains of North Borneo.

Spilornis cheela bido (Horsf.).

Falco bido Horsfield, Trans. Limu. Soc. xiii, I82 I, D. I37: Java.

Distr.-Java; Bali.

*Spilornis cheela baweanus Obert.

Spilornis bassus baneanus Oberholser, Proc. U.S. Nat. Mus. lii, 1917,1 . $18_{5}$ : Bawean Island.

Distr.-Bawean Ișland.

2 There seems to be some doubt as to whether or not Hacmatomis Gould antedates Hamatornis Vigors (March, 1832 ) the use of which for this genus is invalidated by Hematoryis Swains. (Feb. 1832 ).

2 Revision : Oberh., Bull. U.S. Nat. Mus. 159, 1932, D. 19.

${ }^{3} \mathrm{~A}$ thin race standing on average small size and pale colour only. North of the Baram River it is probably not separable from bassus: birds agreeing with richmondi occur in Sarawak. S. raja is merely an immature bird of pallidus: the type and unique specimen has been regarded as representing a Bornean race of rufpectus of Celebes. 


\section{Spilornis cheela natunensis Chas.}

Spilornis cheela nalunensis Chasen, Bull. Raffles Mus. ix, 1934, p. 93 : Bunguran Island, North Natuna Islands.

Distr.-North Natuna Islands.

Spilornis cheela abbotti Richn.

Spilomis abbotti Richmond, Proc. U.S. Nat. Mus., xvi, I903, p. 492: Simalur Island, West Sumatra.

Distr.-Simalur Island, West Sumatra.

Spilornis cheela salvadorii Betl.

Spilornis cheela salvadorii, Berlepsch, Nov. Zool. ii, I895, p. 73 : Nias Island, West Sumatra.

Distr.-Nias Island, West Sumatra.

Spilornis cheela sipora Chas, and Kloss.

Spilornis elgini sipora ${ }^{1}$ Chasen and Kloss, Ibis, I926, p. 278, pl. 3 : Sipora Island, West Sumatra.

Distr.-Sipora Island, Mentawi Group, West Sumatra.

\section{Genus BUTASTUR Hodgson.}

*Butastur liventer (Temm.). Fulvous-winged Buzzard-Eagle.

Falco liventer 'Temminck, Pl. Col. 438, I827: Celebes.

Falco pallidus Lesson, Traité d'Orn. 1830, p. 82: Java.

Distr.-Borneo. ${ }^{2}$
Java.

Butastur indicus (Gmel.). Grey-faced Buzzard-Eagle.

Falco indicus Gmelin, Syst. Nat. i, pt. I, I788, p. 264: Java.

Buteo (Butastur) fascialus Hay, Madr. Journ. Lit. Sci. xiii, pt. 2, 1845. p. I46: Malacca.

Astur barbatus Eyton, Ann. Mag. Nat. Hist. xvi, 1846, p. 227: Malay Peninsula.

Distr.-Malay Peninsula.

Borneo.

Iava.

I A serpent-eagle also occurs in the Pagi Islands, West Sumatra. I now think that Kloss and I were wrong in linking this race to $S$, elgini.

- The evidence for the occurrence of this species in Borneo is very slight, but as it occurs in Celebes we have retained it in the Bornean list for the present. 


\section{Genus HALIAEETUS Savigny. ${ }^{1}$}

Halizetus leucogaster (Gmel.).

White-bellied Sea-Eagle.

Falco leucogaster Gmelin, Syst. Nat. I, pt. I, 1788, p. 257 : Java (Hart. I902).

Haliaetus fulviventer Vieill., Nouv. Dict. d' Hist. Nat. ed. 2, xxviii,

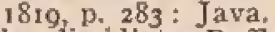

Falco dimidiatus Raffles, Trans. Linn. Soc. xiii, 1822, p. 277 : Sumatra. Distr.-Malay Peninsula; Anamba Islands; Tambelan Islands.

Sumatra; Rhio Archipelago; West Sumatran Islands of Simalur, Banjak group, Nias and Mentawi group.

Borneo; North Bornean Islands; Nortls Natuna Islands; Maratua grottp; Karimata Islands.

Java; Thousand Islands; Bali; Karimon-Java Islands; Kangean Islands.

\section{Genus ICTHYOPHAGA Lesson.}

\section{Icthyophaga ichthyatus (Horsf.).}

Larger Fishing-Eagle.

lethyophaga ichthyæetus ichthyetus (Horsf.).

Falco Ichthycetus Horsfield, Trans. Linn. Soc. xiii, 1821, p. 236 : Java.

Pandion Horsfieldii Selby, Cat. Gen. Sub-gen. Types Aves, 1840, p. 2 : new name.

Iehthyaêtus bicolor Gray, List. Gen. Birds, 2nd ed., 1841, p. 4: new name.

Distr.-Malay Peninsula.

Sumatra; Rhio Archipelago; Taya Island, Lingga Archipelago (viz.).

Borneo.

Java.

Icthyophaga nana (Blyth.).

Smaller Fishing-Eagle.

Icthyophaga nana nana (Blyth).

Ichthyäetus nanus Blyth, Journ. Asiat. Soc. Bengal, xi, I842, p. 202 : Singapore Island.

Falco (Pandion) humilis S. Müll., Verh. Nat. Gesch. Land en Volk. $1845, \mathrm{pp} .47 \mathrm{I}, 48$, pl. 6 : Sumatra.

Distr.-Malay Peninsula.

Sumatra.

Borneo; North Natuna Islands.

\section{Gentis HALIASTUR Selby}

Haliastur indus (Bodd.).

Brahminy Kite.

[Haliastus indus indus Bodd.

Falco Indus Boddaert, Tabl. Pl. Enlunn. 1783 , p. 25 : Pondicherry, India.

Distr.,Extra-linital.]

" " $H$. leucoryphus" for Borneo is based on a misidentification. 
Haliastur indus intermedius Gurney.

Haliastur intermedius Gurreyr, Ibis, 1865, p. 28 : Java.

Distr.-Malay Peninisıla.

Sumatra; Rlio Archipelago; Banka; Billiton and Mendanau; West Sumatran Islands of Sinalur, Banjak group, Nias and Pagi Islands.

Borneo; North Bornean Islands.

Java; Bali; Kangean Islands.

Gentis MILVUS' lacépède.

Milvus migrans (Bodd.).

Kitè.

[Milvus migrans migrans (Bodd.).

Falco migrans Botdaert, Tabl. Pl. Enlutu, I7 $83,0.28$ : France.

Distr.-Extra-linital.]

Milvus migrans govinda Sykes.

Milvus Gowinda Sykes, Proc. Comm. Zool. Soc. I832, p. 8 I : Deccan. Distr.-Malay States."

*Milvus migrans lineatus (Graỹ).

Haliätus lineatus Gray in Hardwiche's Illustr. Ind. Zool. I, I83I, p. I, pl. I8: China.

Distr.-Borneo.

\section{Genus ELANUS Savigny.}

Elanus creruleus (Desfont.).

Black-winged Kite.

Elanus cæruleus cæruleus (Lesfont.).

Falco caruleus Desfontaines, Hist. (Mén.) Acad. Paris for 1787 , $(x-89)$ p. 503 : Algiers.

Distr.-Malay States. ${ }^{3}$

Elanus cæruleus hypoleucus Gould.

Elanus hypoleucus Gould, Proc. Zool. Soc. 1859, p. I27: Celebes. Elants intermedius Schleg., Mus. Pays-Bas, Milvi, 186z, p. 7 : Java.

Distr.-Sumatra.

Borneo.

Java.

r Revision: Snouck. v. Schaub., Alauda, 1932, p. 156.

= On the continent only known from as far south as Kob Lak in Southwest Siam.

3 Not yet definitely recorded from Peninsular Siam. 
Gemus MACHARHAMPHUS Westerman,

Macharhamphus alcinus Westerman.

Slender-billed Pern.

Machaerhamphus alcinus alcinus Wester.

Macharhamphus alcinus Westerman, Bijd. tot de Dierk. I, I 848 (? 1851), p. 29, pl. 12: Malacca.

Distr.-Malay Peninsula.

Sunuatra.

Borneo.

\section{Gentis PERNIS ${ }^{1}$ Cuvier.}

Pernis apivorus (Linn.).

Honey-Buzzard.

[Pernis apivorus apivorus ( $\mathrm{Lint}$.).

Falco apivonus Linn., Syst. Nat. Ioth. ed. I758, p. 9I: Sweden.

Distr.—Extra-limital.]

Pernis apivorus ptilarhyncus (Temm.).

Falco ptilorityncts Temminck, PI. Col. 44, IS2I : Java.

Pernis torquata Less., Traité d'Orn. 1830, p. 76 : Sumatra.

Pernis tavedalii Hume, Stray Feathers, ix, isso, p. 446: Malacca.

Distr.-Malay Peninsula.

Sumatra; Siberut Island, West Sumatra.

Borneo.

Java.

Pernis apivorus japonicus Kurola.

Pernis apivorus japonicus Kuroda, Dobuts. Zasshi, 37, I925, p. 226

(English version); Hondo, Japan: also Fornosa and China.

Distr.-Malay Peninsula; Auamba Islands.

Sumatra; Rhio Archipelago; Banka.

Borneo.

Java.

\section{Pernis apivorus ruficollis Less."}

Pernis nuficollis Lesson, Traite d'Orn. $1 \$ 30$, p. 77 : Bengal.

Distr.-Malay Peninsula.

\section{Gents AVICEDA Swainson.}

Aviceda leuphotes (Dumont).

Black-crested Baza.

Falco leupholes Dumont, Dict. Sci. Nat. xvi, IS20; p. 217 : Pondicherry.

Distr.-Malay Peninsula.

ICf. Kloss, Treubia, xiit, 1931, 13. 314 .

= Botb $P$, a, japonicus and rufcollis migrate to Malaysia. 
Aviceda jerdoni (Blyth).

Jerdon's Baza.

Aviceda jerdoni jerdoni (Blyth).

Pernis Jerdoni Blyth, Journ. Asiat. Soc. Bengal, xi, r842, p. 464 : Malacca.

Aviceda sumatrensis Lafr., Rev. Zool. 1848, p. 210 : Sumatra.

Distr.-Malay Peninsula.

Sumatra.

Aviceda jerdoni borneensis (Sharpe).

Baza borneensis Slarpe, Ibis, I893, p. 557: Pontianak, Borneo.

Distr.-Borneo.

\section{Gentus MICROHIERAX Sharpe.}

Microhierax cærulescens (Linn.). Falconet.

[Microhierax carulescens cæerulescens (Liun.).

Falco carulescens Linn., Syst. Nat. Ioth ed. I758, p. S8: Bengal.

Distr.-Extra-linital.]

Microhierax carulescens fringillarius (Drap.).

Falco fringillaritus Drapiez, Dict. Class. d'Hist. Nat. vi, I 824, p. 4I2, pl. 5: Sumatra (Hartert, 1002).

Hierax malayensis Strick., And. Nat. Hist, xiii, t844, p. 33: "Malay species" = Malacca.

Distr.-Malay Peninsula.

Sumatra.

Borneo.

Java.

Microhierax latifrons Sharpe.

White-fronted Falconet

Microhierax latifrons Sharpe, Ibis, I879, p. 237, pl. 7 : Lawas River, North-west Borneo.

Distr,-Borneo (North).

\section{Genus FALCO Linn.}

Falco peregrinus Tunst.

Peregrine Falcon.

[Falco peregrinus peregrinus Tunst.

Falco Peregrinus Tunstall, Orn. Brit. I77I, D. I: Great Britain.

Distr.-Extra-limital.] 
Falco peregrinus calidus Lath.

Falco calidus Latlam, Ind. Oru. 1, 1790, 1). 41: India.

Distr.-Malay States.

Sumatra; Banka; Billiton (? subsp.).

Borneo.

Java; Thousand Islands.

Faleo peregrinus ernesti Sharpe.

Falco emesti Sharpe, Ibis, 1S94, p. 545: Mt. Dulit, Sarawak.

Distr...-Sumatra,

Borneo,

Java; Bali.

Falco subbuteo Linn.

Hobby.

Falco subbuteo subsp.

Falco Subbuteo Linn., Syst. Nat. Ioth ed. 1758, p. 89 : Sweden. Dislt.-Java.

Falco severus Horsf.

Horsfield's Hobby.

Falco severus severus Horst.?

Falco severus Horsfield, Trans. Linn. Soc. xiii, I82x, p. I35: Java. Falco aldrowandrii "Reinw," in Temm, Pl. Col, 128, 1822: Java.

Distr.-Sumatra.

Borneo.

Java.

Falco tinnunculus Linn. ${ }^{3}$

Kestrel.

[Falco tinnunculus tinnunculus Litn.

Falco Tinnunculus Linn., Syst. Nat. Ioth ed. 1758, p. 90: Sweden. Distr-Extra-limital.]

Falco tinnunculus japonensis Ticeh.

Falco timunculus japonensis Ticehurst, Bull. B. O. Cl. I, I929, p. Io: new nante for $F$. japonicus Temn, and Schleg. (Japan).

Distr.-Malay States.

Borneo.

1. A falcon, no dotrbt of this species, was seen by Dr. Abbott on Simalur Island, West Sumatra. Not yet recorded from Peninsular Siam, but almost certain to occur there.

s $F$. severus has been obtained at Bangkok in Siam but there are no satisfactory records from the Malay Peninsula.

3 All kestrels of this species are rare in Malaysia. The few we have seen together with several from Siam seem to represent two races. "tinnunculus" has also been recorded from Nias Island, West Sumatra. 
Falco tinnunculus saturatus Blyth.

Falco saturatis Blyth, Journ. Asiat. Soc. Bengal, xxviit, I859, D. 277 : Tenasserin.

Distr.-Malay States.

Falco moluccensis (Bp.).

Moluccan Kestrel.

[Falco moluccensis moluccensis $(\mathrm{Bp}$.).

Tinnunculus moluccensis "Schleg." Bonaparte, Consp. Gen. Av. I, I 850, p. 27 : Moluccas.

Dist.r._Extra-linital.]

Falco moluccensis occidentalis (Mey, and Wigl.).

Tinnunculus nolticcensis occidentalis Meyer and Wiglesworth, Abh. Ber, Mus, Dresden, vi, No. 2, I896, p. 8: Celebes.

* Cerchneis moluccensis microbalia Oberh., Proc. U.S. Nat. Mus. liv, 1917, p. 179: Solombo Besar Island, Java Sea.

Distr.-Borneo.

Java; Bali; Kangean Islands; Solombo Besar Island, Java Sea.

*Falco cenchroides Vig. and Horsf.

Nankeen Kestrel.

Falco Cenchroides Vigors and Horsfield, Trans. Iinn. Soc. xv, I $827, \mathrm{p}$, I $8_{j}$ : New South Wales.

Distr.-Java.

\section{Family PANDIONIDÆ.}

Genus PANDION Savigny.

Pandion haliatus (Linn.).

Osprey.

Pandion halizetus halizetus (Linn.). ${ }^{1}$

Falco Haliatus Linn., Syst. Nat. Ioth ed. 1758, p. 91: Europe.

Distr.-Malay Peninsula.

Sumatra; Rlio Archipelago.

Borneo; North Natuna Islands.

Java; Bawean Island.

Pandion halizetus cristatus (Vieill.).

Buteo cristalus Vieillot, Nouv. Dict. d'Hist. Nat. iv, I8I6, p. $48 \mathrm{I}$ : Tasmania.

Distr.-Malay Peninsula; Billiton.

Java; Bali; Kangean Island and Karimon-Java Islands.

3 Some specimens from the Malay Peninsula and the Natuna Islands are $P .7$. halifits: the other localities are extracted from literature and may, therefore, refer to $P$. $h$. cristatus which also occurs in Malaysia. We have only listed as cristatus specimens examined, or mentioned in, apparently, critical records. 


\section{Family STRIGIDE.}

\section{Genus ASIO Brisson. ${ }^{1}$}

Asio flammeus (Pontopp.).

Short-eared Owl.

Asio flammeus flammeus (Pont.).

Strix flammea Pontoppilan, Danske Atlas, r, 1763, p. 617, pl, 25 : Denmark.

Distr.-Malay States.

Borneo.

Genus BUBO: Duméril.

Bubo zeylonensis (Gmel.).

Cerlon Fish Owi.

[Bubo zeylonensis zeylonensis (Gmel.).

Strix zeylonensis Gmelin, Syst. Nat. I, pt. 1, 1788, P. $287:$ Ceylon.

Distr.-Extra-limital.]

Bubo zeylonensis leschenault (Temm.).

Strix leschenawlt Temuninck, Pl. Col. 20, 1824 : Chandanagore, East India (Baker, I 930 ).

Distr.-Peninstuar Sian.

Bubo ketupu (Horsf.).

Fish Ow!

Bubo ketupu ketupu (Horsf.).

Strix Ketupu Horsfield, Trans. Limn. Soc. xiii, I821, p. I4 I : Java.

Ketupa javanensis Less., Traité d'Orn. $1830, \mathrm{p} .1 \mathrm{t} 4$ : new name for S. ketupu.

Distr.-Malay States.

Sunlata; Rhio Archipelago; Billiton; Banka.

Borneo (part).

Java; Bali.

Bubo ketupu aagaardi Neum.

Bubo ketupu aagaardi Neumann, Bull, Brit, Orn, Cl. Iv, 1935, p. $13^{8}$ : Bang Nara, Peninsular Sian.

Distr.-Peninsular Sianı. ${ }^{3}$

*Bubo ketupu pageli Neum.4

Bubo ketupu pageli Neumann, Bull. Brit. Orn. Cl. Iv, 1935, p. I38: Marudu Bay, British North Borneo.

Dist. - North-east Borneo.

1 A, otws was inciuded in Robinson's Handlist of birds of the Matay Peninsula (igro) on the strength of a locally obtained specimen in the Raftes Museum, Singapore, but the specimen is no longer extant and we think that perhaps $A$. flamiters was intended.

a Cf. Meise, Orn. Monatsber, xli, r033, p. I6g.

3 Birds from the Malay States are nearer to ketupu than to a agardi.

- Exact topotypes not seen and the race is therefore maintained here. but birds from Sarawak and Java seem inseparable. 
*Bubo ketupu büttikoferi nom, nov.

Ketupa minor Buttikofer, Notes Leyd. Mus., xviii, I896, p. I65:

Nias Island, West Sumatra (not B. minor Schleg. 1862).

Distr.-Nias Island, West Sumatra.

Bubo coromandus (Lath.).

Dusky Horned Owl.

[Bubo coromandus coromandus (Lath.).

Strix coromanda Latham, Ind. Orn, i, 1790, p. 53: Coromandel Coast.

Distr._Extra-limital.]

Bubo coromandus klossii Rob.

Bubo coromandus klossii Robinson, Journ. Fed. Mal. States Mus. iv, IgIr, p. 246: Gunong Semanggol, North Perak, Malay States.

Distr.-Malay Peninsula.

Genus HUHUA Hodgson.

Huhua sumatrana (Raftles).

Eagle-Owl.

Huhua sumatrana sumatrana (Raffles).

Strix sumatrana Raffles, Trans. Linn. Soc. xiii, I822, p. 279 : Sumatra.

Bubo orientalis minor Schleg., Mus. Pays-Bas, Oti, r862, p. 13: Banka.

Distr.-Malay Peninsula.

Sumatra; Banka.

Huhua sumatrana strepitans (Tenm.).

Strix strepitans Temminck, P1. Col. I74, I823: Batavia, Java.

Strix orientalis Horsf. (nec Shaw), Trans. Linn. Soc. xiii, 1821, p. 140 : Java.

Distr.-Borneo. ${ }^{t}$

Java; Bali.

Genus OTUS` Pennant.

Otus scops (Linn.).

Scops Owl.

[Otus scops scops (Linn.).

Strix Scops Linn., Syst. Nat. Ioth ed. 1758, p. 92: Italy.

Disir.-Extra-limital.]

1 We bave never been able to compare birds from Borneo and Java, but those from the former locality are less boldly barred below than specimens from the Malay Peninsula and Sumatra.

a Revision: Stres., Mitt. Zool. Mus. 1925, p. 191. 
Otus scops malayanus (Hay).

Scops Malayanus Hay, Madr. Jour. Lit. Sci. xiii, pt. 2, 1845, p. 147: Malacca.

Distr.-Malay Peninsula."

Otus scops mantananenșis Sharpe.

Scops mantananensis Sharpe, Bull. B.O. Cl. i, I892, p. 4 : Mantanani

Island, North-west Borneo.

Distr.-Mantanani Island, Borneo.

Otus spilocephalus (Blyth).

Mountain Scops Owl.

[Otus spilocephalus spilocephalus (Blyth).

Ephialtes spilocephalus Blyth, Journ. Asiat. Soc. Bengal, xv, I840, p. 8: Darjeeling.

Distr._Extra-limital.]

Otus spilocephalus siamensis Rob. and Kloss.

Otus luciae siamensis Robinson \& Kloss, Journ. Fed. Mal. States Mus. x, 1922, p. 26r : Kao Nong, Bandon, Peninsular Sian.

Distr.-Peninsular Siam.

Otus spilocephalus vulpes $O$.-Grant."

Heteroscops vulpes O.-Grant, Bull. Brit. Orn. Cl. xix, 1906, p. I I :

Gunong Tahan, Pahang, Malay States.

Distr.-Malay States.

Otus spilocephalus stresemanni Rob.

Athenoptera spilocephalus stresemanni Robinson, Bull. Brit. Orn.

Cl. xiviii, I927, p. I27: Korinchi, West Sumatra.

Distr.-Sumatra.

Otus spilocephalus lucire (Sharpe).

Scops lucia Sharpe, Ibis, I888, p. 478 : Mt. Kinabalu, North Borneo. Distr.--Borneo.

Otus spilocephalus angelinze (Finsch).

Pisorhina angelinae Finsch, Orn. Monatsber. xx, 1912, p. I56: West Java.

Distr.-Java.

"We cannot confirm the locality "Sumatra" given by Robinson (1927).

- Stresemann unites lucie, vulpes, stresemanni and vandewateri, but although the species is very variable and the series examined small we are prepared to accept the first three forms as fair average subspecies. In the case of vandewateri we are not yet convinced that it is a form of spilocephalus. 
Otus vandewateri (Rob. and Kloss). ${ }^{1} \quad$ Vandewater's Scops Ow. Pisorhina vandewateri Robinson and Kloss, Journ. Strs. Br. Roy. Asiat. Soc. Lxxiii, 1916, p. 275: Korineini Peak, Sunatra. Distr.-Sumatra.

Otus umbra (Rich1n.). ." Bartussan Scops Owl.

* Otus umbra umbra Richm.

Pisorhina unbra Richmond, Proc. U.S. Nat. Mus. xxvi, 1903, p. 494 : Simalur Island, West Sumatra.

Distr.-Sinnalur Islaud, West Sunatra.

* Otus umbra enganensis Riley.

Otus umbra enganensis Riley, Proc. Biol. Soc. Wash. xl, r927, p. 93: Engano Island, West Sunatra.

Distr.-Eingano Island, West Sumatra.

Otus bakkamoena (Penı.). Collared Scops Owl. [Otus bakkamoena bakkamoena (Penin.).

Otus Bakkamoena Pennant, Ind. Zool. 1769, p. 3, pl. 3 : Ceylon. Distr.-Extra-linital. ]

Otus bakkamoena condorensis Kloss.

Otus bakkamoena condorensis Kloss, Journ. Sian Soc., Nat. Hist. Suppl. viii, I930, p. 8r: Condore Island.

Distr.-Peninsular Siant.

Otus bakkamoena lempiji (Horsf.).

Sirix Lempiji Horsfield, Trans. Linn. Soc. xiii, 1821, p. 140: Java. Scops javanicus Less., Traité d'Orn. $8_{3} \mathrm{O}_{\mathrm{o}}$ p. to : Java.

Strix noctula Temm., PI. Col. 99, 1 SzI : Java, Sumatra, Banda.

Distr.-Malay Peninsula.

Sumatra; Banka; Bílliton.

Borneo; North Natuna Islands.

Java; Bali; Kangean Islauds. ${ }^{4}$

Otus bakkamoena mentawi Chas. and Kloss.

Olus bakkamana mentawi Chasen and Kloss, Ibis, I926, p. 279: Sipora Island, West Sumatra.

Distr.-Siberut and Sipora Islands, Mentawi Group, West Sumatra. ${ }^{3}$

- Stresemann places this owl as a synonym of $O$. spilocephalus lucie (=stresemanni Rob.), but we are not yet convinced.

- Can almost certainly be lumped with one of the other more widely spread Malaysian species, but as we have not seen either umbra or enganeusis we cannot attempt a wider nomenclature.

3 A small owl, possibly this torm, also occurs in the Pagi Islands, Mentawi Group.

$\$$ The Kangean form is possibly separable: Robinson seemed to think that a Bornean race was separable, but we cannot see any peculiarity in ten specimens from Sarawak. 
Otus brookii (Sharpe). Rajah's Scops Owl.

Otus brookii brookii (Sharpe).

Scops brookii Sharpe, Bull. B. O. Cl. i, 1892, p. 4: Mt. Dulit, North Sarawak, Borneo.

Distr.-Borneo. Java."

Otus brookii solokensis (Hart.).

Pisorhina solokensis Hartert, Bull. Brit. Cl. i, 1893, p. 39 : Pađang Highlands, Sunatra.

Distr.-Sumatra.

Otus sagittatus Cass.

Large Malay Scops Ow1.

Ephialtes sagittalus Cassin, Journ. Acad. Nat. Sci. Philad. iv, r850: Malacca.

Distr.-Malay Peninsula.

Otus rufescens (Horsf.). Reddish Scops Owl.

Otus rufescens rufescens (Horsf.).

Strix rufescens Horsfield, Trans. Linn. Soc, xiij, r821, p. 140: Java.

Scops mantis "Müll.", Bp., Consp. Gen. Avium, i, 1850, p. $47:$ Borneo. Distr.-Sumatra; Banka. Borneo. Java.

Otus rufescens malayensis Hach, $=$

Otus rufescens malayensis Hachisuka, Birds of Phil., iii, I934, p. 52 : Mt. Oplir, Malacca.

Distr.-Malay States.

\section{Genus NINOX Hodgson. ${ }^{3}$}

Ninox scutulata (Raffles). Hawk-Owl.

Ninox scutulata scutulata (Raffles).

Strix scutulata Raffles, Trans. Linn. Soc. xiii, 22, p. 280 : Sumatra. Distr.-Malay Peninsula.

Sunatra.

Borneo.

Java.

The Javan form is probably sejarable.

"We only have four specimens of this owl. Two from the Malay States are rather paler and redder than two from Borneo.

3 C/. Kloss, Treubia, xiii, 1931, p. 316. 
Ninox scutulata burmanica Hurue,

Ninox burmanica Hume, Stray Feathers, iv, 1876, p. 285: Pegu.

Distr.-Malay Peninsula.

Ninox scutulata malaccensis (Eyton).

Athene malaccensis Eyton, Ann. \& Mag. Nat. Hist. xvi, 1845, p. 228 : Malacca.

Noctua hirsuta minor Schleg., Mus. Pays-Bas, Av, Noct. 1873, p. 24 : Banka (by elimination).

Distr.-Malay Peninsula.

Sumatra; Rhio Archipelago; Banka.

Ninox scutulata borneensis (Bp.).

Athene borneensis Bonaparte, Consp. Gen. Aviun, I850, p. $4 \mathrm{I}$ : Borneo.

Ninox labwanensis Sharpe, Cat. Bds. Brit. Mus. ii, 1875, p. 165 : Labuan Island.

Distr.-Borneo; North Natuna Islands.

Ninox scutulata javanensis Stres.

Ninox scululata javanensis Stresemann, Orn. Monatsber. xxxvi, I928, p. 54: West Java.

Distr.-Java (West).

Ninox forbesi Scl.

Island Hawk-Owl.

INinox forbesi forbesi Scl.

Ninox forbesi P. L. Sclater, Proc. Zool. Soc. I883, p. 52, pl. 'xi : Timor Laut.

Distr.-Extra-limital.]

Ninox forbesi natalis Lister.

Christmas Island Hawk-Owl.

Ninox natalis Lister, Proc. Zool. Soc. I88S, p. 525: Christmas Island, Indian Ocean.

Dislr.-Christmas Island.

\section{Genus STRIX Linn.}

Strix orientalis Shaw. ${ }^{3}$

Wood-Owl.

Strix orientalis orientalis Shaw.

Strix orientalis Shaw, Gen. Zool. vii, I809, p. 257 : China, error $=$ Cochin China.

Distr.-Malay Peninsula.

IFor the present we regard japonica Schleg., and ussuriensis Buturlin, as synonyms of scutulata, a migratory form in Malaysia. " $N$. scululata" has also been recorded from the Lingga Archipelago.

- Sporadic as far south as Singapore.

3 Cf. Stres., Orn. Monatsber. xxxii, t924, p. Ir I. 
Strix orientalis seloputo Horsf.

Strix Selo-puto Horsfield, Trans. Linn. Soc. xiii, IS2I, p. I40: Java.

Strix pagodorum Temm., Pl. Col. 230, 1823: Java (Stresemann, 1924). Distr.-Java.

*Strix orientalis baweana Oberil.

Sirix bawcana Oberholser, Proc. U.S. Nat. Mus, lii, I917, p. Igo: Bawean Island, Java Sea.

Distr.-Bawean Island.

Strix leptogrammica' Temn.

Malayan Wood-Owl.

Strix leptogrammica leptogrammica Temm.

Strix leptogrammica Temminck, Pl. Col. 525, I831 : Borneo.

Distr.-Borneo; Billiton.

Strix leptogrammica maingayi (Hume).

Symium maingayi Hume, Stray Feathers, vi, I878, p. 27 : Malacca.

Distr.-Malay Peninsula.

Strix leptogrammica myrtha (Bp.).

Ciccaba myrtha Bonaparte, Consp. Gen. Aviun, i, I850, p. 44 : Sumatra.

Distr.-Sumatra.

*Strix leptogrammica bartelsi (Finsch).

Syrnium Bartelsi Finsch, Bull. Brit. (Irn. Cl. xvi, I906, p. 63 : West Java.

Distr_-Java (west and central).

* Strix leptogrammica nyctiphasma Oberh.

Strix leptogrammica nyctiphasma Oberholser, Journ. Wash. Acad.

Sci. Xiv, I924, p. 302 : Bangkaru Island, Banjak Islands, West Simatra.

Distr,-Banjak Islands, West Sumatra.

*Strix leptogrammica niasensis (Salvad.).

Symium niasense Salvadori, Ann. Mus. Civ. Gen. (2), iv, I887, p. 526: Nias Island.

Distr.-Nias Island, West Sumatra.

'Cf. Stres, Orn. Monatsber. xxxii, 2924, p. two. 


\section{Genus GLAUCIDIUM Boie."}

Glaucidium brodiei (Burt.).

[Glaucidium brodiei brodiei (Burt.).

Noctua Brodici Burton, Proc. Zool. Soc. for $1835,18_{3} 6$, p. I52 : Himalayas

Distr._Extra-limital.]

Glaucidium brodiei tubiger (Hodgs.).

Noctwa tubiger Hodgson, Asiat. Res. xix, r836, p. I75: Nepal.

Disfr._Malay Peninsula.

Glaucidium brodiei sylvaticum ( $\mathrm{Bp}$.).

Alhene syltalica Bonaparte, Consp. Gen. Av. 1850, p. 40 : Padang Highlands, Stumatra.

Distr.-Sumatra.

Glaucidium brodiei borneense Slarpe.

Glaucidium borneense Slarpe, Bull. Brit. Orn. Cl. i, I893, p. 55 : Mt. Kalulong, Sarawak, Borneo,

Distr.-Borneo.

Glaucidium cuculoides ( Vig.).

Barred Owlet.

[Glaucidium cuculoides cuculoides (Vig.).

Noctua cuculoides Vigors, Proc. Zool. Soc. 1830-3I, I83I, p. S: Himalayas.

Distr._Eixtra-linital.]

Glaucidium cueuloides castanopterum (Horsf.).

Strix castanoptera Horsfield, Trans. Linn. Soc. xiï, IS21, p. I4o: Java.

Distr.-Java; Bali.

\section{Family TYTONID在.}

Genus TYTO: Billberg.

Tyto alba (Scop.).

Barn Owl.

\section{[Tyto alba alba Scop.}

Strix alba Scopoli, Annus, i, Hist. Nat. 1769, p. 2 I : North Italy. Distr.-Extra-limital.]

1 G. radialum (Tick.), is supposed to have been obtained by Cantor in Kedah, but we believe a mistake in labelling has occurred and do not admit the species to the Malayan list.

Revision: Hart., Nov, Zool. xxxix, t034, p. I5r.

$$
[90]
$$


Tyto alba javanica (Gmel.).

Strix javanica Gmelin, Syst. Nat. I, 17t. I, I788, p. 295: Java.

Distr.-Malay States."

Java; Thousand Islands; Kangean Islands.

\section{Genus PHODILUS Geofir. St. Hilaire.}

Phodilus badius² (Horsf.). Bay Owl.

Phodilus badius badius (Horsf.).

Strix badia Horsfeld, Trans. Linn. Soc. xiii, I821, p. 139: Java.

Phodilus badins abhotli Oberh., Proc. Acad. Sci. Wash. xiv, 1024, j. 302 : Province Wellesley, Malay Peninsula.

Distr. - Malay States.

Sumatra; Billiton; Nias Island.

Borneo.

Java; Bali.

*Phodilus badius arixuthus Oberh.

Phodilus badius arixuthus Oberholser, Bull. U.S. Nat. Mus, I59, I932, 1). 40: Bunguran Island, North Natuna Islands.

Distr.-Bunguran Island, Natuna Islands.

\section{Family LORIIDE.}

\section{Genus TRICHOGLOSSUS Stephens.}

\section{Trichoglossus ornatus (Lin11.).}

Lorikeet.

[Trichoglossus ornatus ornatus (Li111.).

Psittacus omalus Linn., Syst. Nat, Ioth ed, 1758, 1. 98: America error $=$ Celebes.

Distr._Extra-limital.]

*Trichoglossus ornatus mitchellii Gray.

Trichoglossus mitchellii Gray, List. Psitt. Brit. Mus. I859, p. 62 ; Lombok.

Distr.-Bali.

tNo definite record for Peninsular Siam: of very doubtful occurrence in Sumatra and Borneo.

2Revision: Oberh., Bull. U.S. Nat. Mus. 159, 1932, p. 40; Robinson, Bull. Brit, Orn. Cl. xlvii, 1927, p. 121. 
Family KAKATEIDÆ.

Genus KAKATOË Cuvier.

Kakatoë sulphurea (Grnel.).

White Cockatoo,

[Kakatoë sulphurea sulphurea (Gmel.).

Psiltacus sulphureus Gmelin, Syst. Nat. I, pt. 1, I788, p. 330 : Celebes.

Distr.-Extra-limital.]

*Kakatoë sulphurea abbotti Oberl.,

Kakatoe parvulus abbotti Oberholser, Proc. U.S. Nat. Mus. liv, I9I7, p. I8I : Solombo Besar Island, Java Sea.

Distr.-Solombo Besar Island; Java Sea.

\section{Family PSITTACIDÆ.}

\section{Genus TANYGNATHUS Wagler.}

Tanygnathus lucionensis (Linn.). Phillipine Paroquet.

Tanygnathus lucionensis lucionensis (Lint.).

Psillacus lucionensis Linn., Syst. Nat. I2th. ed. I, I766, p. I46: Philippine Islands.

Tanygnathus salvadorii O.-Grant, Ibis. 1896 , p. 562 : Mantanani Island. Tanygnathus lucionensis horrisonus Bangs and Peters, Occ. Pap. Boston Soc. Nat, Hist. v, 1927, p. 236 : Maratua Island.

Distr.-Mantauani Island, North-west Borneo; Maratua Island, East Borneo."

\section{Genus PSITTACULA ${ }^{3}$ Cuvier.}

Psittacula alexandri (Linn.).

Red-breasted Paroquet.

Psittacula alexandri alexandri (Lint.).

Psittacus Alexandri Linn., Syst. Nat, roth. ed. I, 1758, p. 97 : Java (Hartert, 1902).

Psittacus javanicus Forst. in Osb, Voy, China, i, 1771 , p. 156: Java.

Psittacus bimaculatus Sparrm., Mus. Carls, ii, 1787 , No, xxx: Java.

Psittacus Osbeckii Lath., Ind Orn. i, 1790, p. 87: Java.

Distr.-Java, Bali, Kangean Islands; Borneo (south).

I A Cockatoo of this group is also found in Bali where it has no doubt been introduced : Stresemann identifies it as occidentalis Hartert of Lombok. A comparison between occidentalis and abbotti is needed.

- We cannot separate birds from Maratua and Mantanani Islands from lucionensis of Luzon. This parrot has been introduced into many parts of its present range. A specimen from Lawas on the mainland of N. Borneo, in the British Museum, we regard as an introduced bird.

3 Revision: Snouck. v. Schaub., Alauda, 1933, p. 307. 


\section{PSITTACIDE.}

Psittacula alexandri dammermani Clas. and $\mathrm{K}$ loss.

Psillacula alexandri dammermani Chasen and KIoss, Treubia, xiv, I933, p. I68: Karinon-Java Island, Java Sea.

Distr.-Karimon-Java Island.

Psittacula alexandri fasciata (Müll.).

Psitlacus fasciatus P. L. S. Müller, Natursyst. Suppl. I 776, p. 74 : Pondicherry.

Distr.-Peninstulat Sian.

* Psittacula alexandri cala (Oberh.).

Conurus fasciatus calus Oberholser, Smiths. Misc. Coll. 1x, 7, I9I2, p. 4 : Simalur Island, West Sumatra.

Distr,-Simalur Island, West Sumatra.

*Psittacula alexandri major (Richm.).

Paleomis major Richmond, Proc. Biol. Soc. Waslı. xv, I902, p. I88: Babi Island, West Sumatra.

Distr.-Babi and Lasia Islands, West Sunatra.

*Psittacula alexandri perionca (Oberh,).

Conurus fasciatus perioncus Oberholser, Smiths. Misc. Coll. 1x, 7, IgI 2, P. 4: Nias Island, West Sumatra.

Distr,-Nias Island, West Sunatra.

Psittacula longicauda (Dodd.).

Long-tailed Paroquet.

Psittacula longicauda longicauda (Bodd.).

Psittacus longicauda Boddaert, Tabl. Planch. Enlum, 1783, p. 53 : Malacca.

Psiltacus malaccensis Gmel., Syst. Nat. i, 1788, p. 325 : Malacca.

Psitlacus erubescens Shaw, Gen. Zool. viit, 2,1812, p. 437 : Malacca.

Psittacus barbatulatis Bechst. in Lath, Vögel, iv, 1,1811, p. 77 : Malacca.

Conurus erythrogenys Lesson, Traité d'Orn. $1 \$ 30$, p. 215 : no loc. = Malacca.

Palconis viridimystax Blyth, Journ. Asiat. Soc. Bengal, xxv, 1856, p. 446 : no loc, = Malacca.

Distr.-Malay States; Anamba Islanis.

Sunatra; Banka; Billiton.

Bortieo.

Psittacula longicauda defontainei Chas.

Psittacula longicauda defontainei Chasen, Bulł. Raffles Mus. ix, 1934, p. 93: Bunguran Island, Nort! Natuna Islands.

Distr.-Bintang Island; Rhio Archipelago.'

Natuna Islands; Karimata Islands.

- This parrot occurs on otber islands in the Rhio Archipelago, but we have no specimens and cannot give the exact range of the large race, defontaines. 
Paittacula longicauda modesta (I'raser).

Palconis modestus Fraser, Proc. Zool. Soc. I845, p. 16: no loc. =Engano Island.

Palaormis luciance Verr., Rev. Mag. Zool. 1850, p. 598, pl. 13: no loc = Engaso.

Palaornis erythrogeny's (nec Lesson), Proc. Zool. Soc. 1850, p. 245: no loc. = Engano.

Paleornis traseri Moore, Cat. Birds Mus. East Ind. Coy, 2, 1856-58, p. 621 (note) : new name for $P$. erythrogenys Fraser.

Distr._Engano Island, West Smmatra.

\section{Genus PSITTINUS Blyth.}

Psittinus cyanurus (lorst.).

Little Malay Parrot.

Psittinus cyanurus cyanurus (Forst.).

Psitiacts cyanurus Forster, Indische Zool, 1795, p. 6: Malacca.

Psittacus malaccensis inec Gmel.\}, Lath., Ind. Orn. i, 1790, p. 130: Malacca.

Psittacus incertus Shaw and Nodd., Nat. Misc. xix, t807, pl. 760: India errore $=$ Malacca.

Psittacus macropterus Kuhl, Nova Acta Phys. Acad. Leop. Carol. $\mathbf{x}$, 1820, p. 6: as syn. of $P$. malaccensis Lath.

Psittacula reticulata Less., Traité d'Orn. 1830, p. 204 : no loc.

Distr.-Malay Peninsula.

Sumatra; Rhio Archipelago.

Borneo.

*Psittinus cyanurus abbotti Richm.

Psitinus nbbotti Richmond, Proc. Biol. Soc. Wash. xv, Igoz, p. ISS : Simalur Island, West Sumatra.

Distr.-Simalur and Siumat Island (near Simalur), West Sumatra.

Psittinus cyanurus pontius Oberh.

Psittinus cyanurus pontius Oberholser, Smiths. Misc. Coll. $1 \mathrm{x}, 7$, I912, p. 5: South Pagi Island, West Sumatra.

Distr.-Mentawi Islands (Siberut, Sipora, Sonth Pagi), West Sumatra.

\section{Genus LORICULUS Blyth.}

Loriculus vernalis (Sparrm.).

Indian Loriquet.

Loriculus vernalis vernalis (Sparr.).

Psitlacus vernalis Sparman, Mus. Carls. 1787 , no. xix : no locality. Distr.-Peninsular Sian.'

- Of doubtful occurtence in the north of the Malay States. 


\section{Loriculus vernalis pusillus $5 \mathrm{chleg}$.}

Loriculus pusillus "G. R. Gray" Schlegel, Mus. Pays-Bas, iii, Psittaci, 1864, p. 132 : Java.

Coryllis javanica Finsch. Papag. ii, 1868, p. 64: Java.

Distr.Java; Bali,

Loriculus galgulus ( (іпл.).

Malay Loriquet.

Loriculus galgulus galgulus (Linn.).

Psitlacus Galgulus I,imn., Syst. Nat. Ioth ed. 1758, p. I03 : India errot $=$ Malacca.

Psitacus pumilus Scop., Del. Flor. et Faun. Insubr. 1786, p. 87: Malacca.

Psitlacula cyaneopileata Bourj., Perr. 1837-38, pl. 88: Malacca.

Loriculus galgmlus lamprochlorus Oberh., Smiths. Misc. Coll. 1x, 7 , 1912, D. 5: Nias Island, West Sumatra.

Distr,-Malay Peninsula (rare in north); Anamba Islands (vis.): Sumatra; Rhio Archipelago; Banka; Billiton; West Sumatran Islands of Banjak group, Nias and Mentawi group.

Borneo; Nortl Bornean Islands; Maratua Islands.

*Loriculus galgulus dolichopterus Oberh.

Loriculus galgulus dolichopterus Oberholser, Sniths. Misc. Coll Ix, 7. I912, P. 5: Eugano Island, West Sumatra.

Distr.-Engano Island, West Sumatra.

\section{Family PODARGID压.}

\section{Genus BATRACHOSTOMUS Gould.}

Batrachostomus auritus (Gray).

Large Frogmoutli.

Podargus auritus J. E. Gray, in Griffith ed. Cuv. Anim. King. ii, I829, 1). II 4 : Sumatra.

Bombycistomas fullertonii Hay, Joum. Asiat. Soc. Beng. i\$4r, p. 574, woodcut: Sumatra.

Distr.-Malay States.

Sumatra.

Borneo; North Natuna Islands.

Batrachostomus poliolophus Hartert. Hartert's Frogmonth.

Batrachostomus poliolophus Hartert, Notes Leyd. MLus. I892, p. 63 :

Padang, West Sumatra.

Distr.-Sunatra.

Batrachostomus harterti Sharpe." "Dulit Frogmouth.

Batrachostomus harterti Sharpe, Ibis, IS92, p. 323: Mt. Dulit, Sarawak.

Distr.-Borueo. 
Batrachostomus stellatus (Gould).

Gould's Frogmouth.

Podargus stellatus Gould, Proc. Zool. Soc. IS37, p. 43: Malncca.

Podargus parvulus Schleg., Journ. f. Ornith. 1856, p. 460 : Borneo.

Batrachostomus stictopterus Cab. and Heine, Mus. Hein, ii, 1860, p. 124 : Malacca.

Distr.-Malay Peninsula.

Sumatra; Rhio and Lingga Archipelagos.

Borneo; North Natuna Islands.

Batrachostomus mixtus Sharpe.' Sharpe's Frogmouth.

Batrachostomus mixtus Sharpe, Bull. Brit. Orn. Cl. i, IS92, p. 4: Mt. Dulit, Sarawak.

Distr-Borneo.

Batrachostomus javensis (Horsf.) . Horsfield's Frogmouth.

Podargus Javensis Horsfield, Trans. Linn. Soc, xiii, I\$2I, p. I4I : Java.

Podargus corntus Temm., Pl. Col. 159, 1823: Sumatra.

Balrachostomus adspersus Brügg., Ann. Mag. Nat. Hist. (4) xx, 1877 , p. $178:$ Borneo.

Distr.-Stmatra.

Borneo.

Java.

Batrachostomus affinis Blyth.

Blyth's Irogmouth.

Batrachostomus affinis Blyth, Journ. Asiat. Soc. Beug., 1847, p. IISo: Malacca.

Distr.-Malay Peninsula.

Sumatra; Rhio Archipelago.

Borneo; Banguey Island, North Borneo.

\section{Family CORACIIDÆ.}

\section{Genus CORACIAS Linn.}

Coracias benghalensis (Linn.).

Indian Roller.

[Coracias benghalensis benghalensis (Linn.).

Corvus benghalensis Linn,, Syst. Nat. Ioth ed. 175S, p. I06: Bengal.

Distr._Extra-limital.]

Coracias benghalensis affinis Horsf.

Coracias affinis Horsfield, Proc. Zool. Soc. for 1839 , 1840 , p. 164 : Ašsam.

Distr.-Malay Peninsula. ${ }^{2}$

I Perbaps only a phase of B. affinis.

- I have seen this roller in Kelantan near the Trengganu border (June). 
Gents EURYSTOMUS' Vieillot.

Eurystomus orientalis (Linn.).

Broad-billed Roller.

\section{Eurystomus orientalis orientalis (Lin1.).?}

Coracias orientalis Linn., Syst. Nat. I2th ed. I, 1766, p. I59: Java. Eurystomus fuscicapillus Vieill, Nouv. Dict. d'Hist. Nat, xxix, $18 \mathrm{ig}$, p. 426 : India.

Distr.-Malay Peninsula; Anamba Islands.

Sumatra; Billiton; Simalur Island, West Sumatra.

Borneo; North Borneo Islands.

Java; Bali.

Eurystomus orientalis calony $\mathbf{x}$ Sharpe.

Eurystomus calonyx Sharpe, Proc. Zool. Soc. I89o, p. 55I: Nepal. Distr.-Malay Peninsula.

Sunatra; Rhio Archipelago; West Sumatran Islands of Simalur and Sipora; Billiton.

Borneo; North Bornean Islands; North Natuna Islands. Java; Bahi.

\section{Eurystomus orientalis connectens Stres.}

Eurystonus oricntalis connectens Stresemant, Nov. Zool. $\mathrm{xx}_{1}$ 1913, p. 302: Moa Island.

Distr.-Kangean Islands.

\section{Family ALCEDINID $\mathbb{A}^{3}$}

Genus RAMPHALCYON* Reichenbach.

Ramphalcyon amauroptera (Pears.). Brown-winged Stork-billed. Kingfisher.

Halcyon Amauropterus Pearson, Journ. Asiat. Soc. Beng. x, $184 \mathrm{I}$, p. 635 : Calcutta.

Distr.-Malay Peninsula (south to Langkawi Islands).

\section{Ramphalcyon capensis (Linn). Stork-billed Kingfisher.}

\section{Ramphalcyon capensis capensis (Linn.).}

Aledo capensis Linn., Syst. Nat. 12th. ed. I, 1766, p. I80: Java.

Pelargopsis fraseri Sharpe, Proc. Zool. Soc. 1870, p. 65: Java.

Distr.-Java.

: Stres., Nov. Zool. xx, 1013, p. 297.

a The species has also been recorded from Nias, the Thousand Islands and the Karimon-Java Islands. Birds from these localities might belong to either calonyz or orientalis.

3 Cf. Laub., Arch. f. Naturgesch. 1924, p. 55.

4 Revision: Oberb., Proc. U.S. Nat. Mus. Xxxv, 1909, p. 657. 
Ramphalcyon capensis malaccensis Slatpe.t

Pelargopsis malaccensis Sharpe, Proc. Zool. Soc. IS70, p. 67: Malacca.

Ramphalcyon capensis hydrophila Oberholser, Proc. U.S. Nat. Mus. xxxv, 1909, p. 677: Singapore [sland.

Distr.-Malay Peniusula.

Rhio Archipelago; Lingga Archipelago.

\section{Ramphalcyon capensis cyanopteryx Oberh.:}

Ramphalcyon capensis cyanopleryx Oberholser, Proc. U.S. Nat. Mus. xxxv, I909, p. 676: Tapanuli Bay, Nortly-uest Sumatra. Ramphalcyon capensis arignota Oberh., Proc. Biol. Soc. Wash. xxxvii, 1924 , p. 136 : Indragiri River, South-east Sumatra.

Distr.-Sunatra; Banka; Billiton; Mansalar Island, west coast of Sumatra.

\section{*Ramphalcy on capensis simalurensis (Riclim1.).}

Pelargopsis simalurensis Richmond, Proc. U.S. Nat. Mus. xxvi, 1903, p. 498 : Simalur Island, West Sumatra.

Distr:-Simalur Island, West Sumatra.

*Ramphalcyon capensis sodalis (Ricluti.).

Pelargopsis sodalis Richnond, Proc. U.S. Nat. Mus. xxvi, I903. p. 499: Tuangku Island, Banjak Islands, West Sunatra.

Distr.-Banjak Islands, West Sumatra.

*Ramphalcyon capensis nesoeca Oberh.

Ramphalcyon capensis nesoeca Oberholser, Proc. U.S. Nat. Mus. xxxv, 1909, p. 674: Nias Island.

Distr.-Nias Istand and Batu Islands, West Sumatra.

Ramphalcyon capensis isoptera Oberly.

Ramphalcyon capensis isoptera Oberholser, Proc. U.S. Nat. Mus. xxxv, 1909, p. 67I: Sikakap Strait, Pagi Islands, West Sunatra.

Distr.-Mentawi Islands (Siberut, Sipora, Pagi Islands), West Sumatra.

It is difficult to draw a line between burmanica as represented by specimens from Siam and malaccensis and there is a large intermediate zone. With fair series before us it seems just possible to separate the two forms at the Isthmus of Kra.

2 In both North and South Sumatra birds inseparable from both malaccensis and javana occur. The Sumatran race seems in fact exactly intermediate and we recognize it because we have seen no ochraceous headed birds from the Malay Peninsula, or dark headed birds from Borneo. 


\section{ALCEDINIDE.}

\section{Ramphalcyon capensis javana (Bodd.).}

Acedo javana Boddaert, Tabl. Planch. Enlum. ${ }_{7} S_{3}$, p. 47 : Java error $=$ Borneo.

Alcedo lencocephala Gmel., Syst. Nat. i, pt. 1, 1788, p. 456: Java error $=$ Borneo.

Alcedo javanica Shaw, Gen. Zool. viii, $18 \mathrm{12}$, p. 67: Java error = Borneo. Ramphalcyon capensis innominata van Oort, Notes Leyd. Mus. xxxii, 1910, p. 126 : Borneo.

Distr.-Bormeo.

Ramphalcyon capensis floresiana (Sharpe).

Pelargopsis florisiana Sharpe, Proc. Zool. Soc. 1870, p. 68: Flores. Distr-Bali.

\section{Gerus ALCEDO Linn.}

Alcedo atthis ${ }^{1}$ (Linn.).

Common Kingfisher.

[Alcedo atthis atthis Linn.

Gracula Alltis Linn., Syst. Nat. Ioth ed. I758, p. Io9: Eigypt. Distr,-Extra-linital.]

Alcedo atthis bengalensis Gmel.

Alcedo bengalensis Gmelin, Syst. Nat, I, pt. I, I788, p. 450 : Bengal.

Alcedo bengalensis a, indica, $b$, sondaica Reichb., Handb. spec. Orn. Invest. Alcedinex, 1851, p. $3:$ loc. of b. Java, etc.

Distr.-Malay Peninsula; Anamba Islands.

Sumatra; Rhio Arclipelago; West Sumatran islands of Simalur, Banjak Group, Nias and Siberut.

Borneo; Natuna Islands; Karimata Islauds.

Java.

Alcedo atthis floresiana Sharpe.

Aleedo floresiana Sharpe, Cat. Birds Brit. Mus. xvii, 1892, p. I5I: Flores.

Diștr.-Bali.

Alcedo euryzona Temus.

Broad-zoned Kingfisher.

Alcedo euryzona euryzona Temm,

Alcedo cryzona (sic) Temminck, text to P1. Col. 508, is 30 : Java.

Distr.-Java.

Sunatra.

Borneo.

${ }^{1}$ Cf. Stres, Nov, Zool. $x x, 1013$, p. 313. 


\section{ALCEDINIDE.}

Alcedo euryzona nigricans Blytt."

Alcedo nigricans Blyth, Journ, Asiat. Soc. Beng, xvi, I847, p. I 80 : Malacca.

Distr,-Malay Peninsula.

\section{Alcedo meninting ${ }^{2}$ Horsf.}

Deep-Blue Kingfisher.

\section{Alcedo meninting meninting Horsf.}

Alcedo meninting Horsfield, Trans. Linm. Soc. xiij, 182r, p. r7z: Java.

Alcedo megarhynchus Hart., Vog. Mus. Senck. Gesell, t\&ot, p. 134 : as syn. of meninting.

Distr.-Java; Bali.

\section{Alcedo meninting verrauxii De La Berge, 3}

Alcedo Verreauxii De La Berge, Rev, Mag. Zool. 185i, p. 305, pl. 9 : Borneo.

Distr.-Malay Peninsula.

Sunnatra; Rlıio Archipelago; Banka; Billiton.

Borneo.

*Alcedo meninting subviridis Oberh.

Alcedo meninting subviridis Oberholser, Sniths. Misc. Coll. Ix, 7, 1912, p. 7 : Nias Island, West Sumatra.

Distr.-Banjak Islands and Nias Island, West Sumatra.

*Alcedo meninting callima Oberh.

Alcedo meninling callima Oberholser, Smiths. Misc. Coll. $1 x, 7$, 1912, p. 7 : Tana Bala Island, Batu Islands, West Sumatra.

Distr.-Batu Islands, West Sumatra.

Alcedo meninting proxima Richn.

Alcedo meninling proxima Richmond, Proc. Biol. Soc. Wash. xxv, Igr2, p. ro4: North Pagi Island, West Sumatra.

Distr.-Mentawi Islands (Sipora; North Pagi) West Sumatra.

I Males from the Malay Peninsula seem to have the underparts more washed with rufous than those from Java and Borneo.

a Kevisions: Laub., Orn. Monatsber, xxiii, I9ı5, p. I67; Baker, Bull. B. 0. Cl. xxxix, $1919,0.36$.

3 A. m. scintillans Baker is said to occur in Peninsular Siam, but a specimen from Junk Zeylon is certainly not scintillans. Verrawit is rather smaller than typical menimting which, in the east of Java, is also rather less blue. 


\section{ALCEDINIDE.}

Alcedo coerulescens ${ }^{x}$ Vieill.

Small Blue Kingfisher.

Alcedo coerulescens Vieillot, Nouv. Dict. d'Hist. Nat. xix, I8I8,

p. 40I : Timor error = Java (Laubmann, IgI6).

Alcedo beryllina Vieill., Nouv. Dict. d'Hist. Nat. xix, 1818, p. 414 : Java

Alcedo biru Horsf., Trans. Linn. Soc. xiit, I821, p. 172 : Java.

Distr._Java, Bali and Kangean Islands.

\section{Genus CEYX z Lacépède.}

Ceyx erithacus (Linn.).

Three-toed Kingfisher.

Ceyx erithacus erithacus (Linn.).

Alcedo erithaca Linn., Syst, Nat. Ioth ed. 1758 , 1. I I5: Bengal.

Ceyx enopopygitus Oberh., Smiths. Misc. Coll. Ix, 7. 1912, p. 7 : Aru Bay, East Sumatra.

Distr.-Malay Peninsula.

Sumatra; Aroa Islands (Struits of Malacca).

Ceyx erithacus motleyi Chas. and Kkloss.

Ceyx erithacus molleyi Chasen and Kloss, Journ. f. Orn. rg29, ii, p. I06: Bettotan near Sandakan, British North Borneo.

Distr,-Borneo; North Bomean Islands; Nias Island, West Sumatra.

Ceyx rufidorsus Strickland. Red-backed T'liree-toed Kingfisher.

Ceyx rufidorsa Strickland, Proc. Zool. Soc. I846, p. 99 : Malacca.

Cey $x$ dillwynni Sharpe, Proc. Zool. Soc. 1868, p. 593: Labuan, Northwest Borneo.

Ceyx innominatus Salvad., Att. R. Accad. Sci. Torino, iv, 1869 , p. 465 : Java.

Ceyx sharpei Salvad., Att. R. Accad. Sci. Torino, iv, 1860, p. 463 : Sarawak.

Ceyx enerythra Sharpe, Cat. Bds. Brit. Mus. xvii, 1802, p. $179 \div$ Malacea.

Ceyx rufidorsus robusta Parrot, Abh. Kon. Bay. Akad, Wis5, xxiv, igo7,

p. 208 : Sumatra.

Distr.-Malay Peninsula.

Sumatra; Rhio Archipelago; Lingga Archipelago; Banka; Billiton; West Sumatran Islands of Batu group; Siberut and Sipora in Mentawi group. ${ }^{3}$

Borneo; North Natuna Islands.

Java; Bali; Kangean Islands; Bawean Island.

' Cf. Laub., Orn. Monatsber., xxiv, 1916, p. 6.

- Cf. Oberh., Bull. U.S. Nat. Mus. 98, 1917, p. 23; Laub., Orn. Monatsber. $x \times x i, 1923$, B. 80; Chas. and KI., Journ. f. Ornith. 1929, p. 106. Sumatra.

3 Perhaps also on Simalur and islands of the Banjak group, West 
Genue LACEDO Reichenbach.

Lacedo pulchella (Horsf.).

Banded Kingfisher.

Lacedo pulchella pulchella (Horsf.).

Dacelo pulchella Horsfield, Trans. Linn. Soc. xiii, IS2T, P. I75: Java.

Dacelo buccoides Temm., Pl. Col. 586,1835 : Sumatra (Lanbmann, 1024). Distr.-Malay States.

Sumatra; Rhio Archipelago.

North Nattma Islands.

Java.

Lacedo pulchella amabilis (Hume).

Carcineutes amabilis Hume, Stray Feath. 1, I873, p. 474: Pegu.

Distr.-Peninsular Siam.

Lacedo pulchella melanops (Bp.).

Halcyon melanops Bonaparte, Consp). Gen. Av. I, I 850, p. I54: Borneo.

Distr,-Banka Island.

Borneo.

Genus HALCYON Swainson.

Halcyon coromanda ${ }^{1}$ (Lath.).

Ruddy Kingfisher.

Halcyon coromanda coromanda (Lath.).

Alcedo coromanda Latham, Ind. Orn. i, 1790, D. 252: Rangoon (Oberholser, I9I5).

Distr.-Malay Peninsula (south to Johore).

Suniatra (north-east).

Halcyon coromanda minor Temm, and Schleg.

Alcedo (Halcyon) coromanda minor 'Temminck and Schlegel, Siebolds Fauna Japon., Aves, 1848 , p. 76 ; Pontianak, Borneo (Oberholser, 1915).

Entomophora coromanda neophora Oberholser, Proc. U.S. Nat. Mus. xlviii, 1015, p. 646: Tapanuli Bay, North-west Sumatra.

Entomophora coromanda pagana Oberholser, Proc. U.S. Nat. Mus, xlviii, tg15, p. 648: North Pagi Island, West Sumatra.

Distr.-Borneo.

Malay Peninsula (extreme south); Tioman Archipelago; Rhio Arclipelago; Banka; Bíliton; Batu and Mentawi Islands, West Sumatra. ${ }^{2}$

Sumatra (south and west).

Java.

I Revision: Oberh., Proc. U.S. Nat. Mus. xlviji, 1915, p. 639; Kloss, Journ. F.M.S. Mus. x, 1921 , p. 216.

* This is no doubt the form occurring on Simalur, Lasia and Nias Islands, West Sumatra. We have also included the Tioman race under minor, bu: we bave only seen young birds and their identification is doubtful. 
Halcyon smyrnensis (Linn.).

White-breasted Kingfisher.

[Haleyon smyrnensis smyrnensis (Linn.).

Alcedo smymensis Linn., Syst. Nat, Ioth ed. 1758, p. II6 Smyrna. Distr._Extra-linnital.]

Halcyon smyrnensis fusca (Boddl.).

Alcedo fusca Bođdaert, Tabl. Planch. Enlum. 1783, p. 54; based on

P1. Enlum. 894: Malabar.

Halcyon perpulchra Madarász, Ann. Mus. Hung. ii, 1904, p. 85 : Singapore.

Distr,-Malay Peninsula,

Halcyon cyanoventris (Vieill.).

Javan Kingfisher.

Alcedo cyanoventris Vieillot, Nouv, Dict. d' Hist. Nat, xix, I8I8, p. 4I2: Java.

Alcedo melanoptera Horsf., Trans. Linn. Soc. xiii, 1821 , p. 174: Java. Alcedo omnicolor "Reinw"', Temm., Pl. Col. 135, IS22 : Java.

Distr,-Java; Bali.

Halcyon pileata (Bodd.).

Black-capped Kingfisher.

Alcedo pileata Boddaert, Tabl. Planch. Enlun. 1783, p. 41 : China. Disir.-Malay Peninsula.

Sumatra; Rhio Archipelago; West Sumatran Island of Simalur.'

Borneo; North Natuna Islands.

Halcyon sancta Vig. and Horsf.

Sacred King fisher.

Halcyon sancta sancta Vig. and Horst.

Halcyon sanctus Vigors and Horsfield, Trans. Linn. Soc. $x v, 1827$, p. 206 : Australia.

Dislr.-Sumatra; Billiton; Mendanau.

Borneo (south).

Java; Bali; Kangean Islands; Karimon-Java Islands.

Halcyon chloris ${ }^{2}$ (Bodd.).

White-collared Kingfisher.

[Halcyon chloris chloris (Bodd.).

Alcedo Chloris Boddaert, Tabl. Pl. Ennlum. I783, p. 49 : Buru Island, Moluccas.

Distr.-Extra-limital.]

- Also visual records from West Sumatran Islands of Babi and the Mentawi group.

2 Revision : Oberh., Proc. U.S. Nat. Mus, 1v, I9!9, p. 351 ; Kloss, Journ. F.M.S. Mus, x, I921, p. 214 ; Laub., Verh. Ornith. Bay, xv, 1923, p. 383. 


\section{Halcyon chloris humii Sharpe.}

Halcyon humii Sharpe, Cat. Bds. Brit. Mus, xvii, I892, p. 281 , pl.

8: Selangor, Malay Peninsula.

Distr.-Malay Peninsula; Tioman Archipelago.

Sumatra (north-east).

Halcyon chloris cyanescens (Oberh.).

Sauropatis chloris cyanescens Oberholser, Proc. U.S. Nat. Mus. lii, I9I7, p. I89: Taya Island, off south-east coast of Sumatra.

Sauropatis chloris palmeri Oberb., Proc. U.S. Nat. Mus. Iv, Jgrg. p. 369 : Java.

Distr.-Anamba Islands; Tambelan Islands; Saddle Island.

Sumatra (south-east and south); Rhio Archipelago; Lingga Archipelago; Banka; Billiton; Mendanau.

Borneo; North Bormean Islands; Maratua Islands; North and South Natuna Islands; Karimata Islands.

Java; Bali; Bawean Island; Thousand Islands; Karimon-Java Islands; Solombo Besar Island.

Halcyon chloris chloroptera (Oberh.).

Sauropatis chloris chloroptera Oberholser, Proc. U.S. Nat. Mus. 1919, p. 379 : Simalur Island, West Sumatra.

Sauropatis chloris amphiryta Oberh., Proc. U.S. Nat. Mus. Iv, 1919, p. 382 : Nias Island, West Sumatra.

Distr.-Sumatra (north-west); West Sumatran islands of Simalur, Nias, Siberut and the Pagi Islands.

Halcyon chloris azela (Oberh.).

Sauropatis chloris azela Oberholser, Proc. U.S. Nat. Mus, Iv, I9r9, p. 377 : Engano Island, West Sumatra.

Distr.-Engano Island, West Sumatra.

Halcyon concreta (Temun.). Chestrut-collared Kingfisher.

Halcyon concreta concreta (Temm.).

Dacelo concreta Temm., P1. Col. 346, IS25: Sumatra.

Halcyon varia Eyton, Proc. Zool. Soc. 1839, p. ıor: Malaya = Malace a restr.

Distr,-Malay Peninsula.

Sumatra; Banka; Billiton.

Holcyon concreta borneana Chas, and kloss.

Halcyon concreta borneana Chasen and Kloss, Bull. Raff. Mus, 4. 1930, p. 24 : near Sandakan, North Borneo.

Distr.-Borneo. 


\section{Family BUCEROTID瓜.}

\section{Genus BUCEROS Linn.}

Buceros rhinoceros Litu.

Rhinoceros Hornbill.

Buceros rhinoceros rhinoceros Linin.

Buceros Rhinoceros Lint., Syst. Nat. I, 1758 , D. 104: India = Malacca.

Buceros sumatrana Schleg, and Müll., Verh. Nat. Gesch. Zool, 1840, Aves, p. 22 : Sumatra.

Buceros indica Schleg. and Müll., Verb. Nat. Gesch, Zool. 1840, Aves, p. 22 : Seringapatum error = Malacca.

Buceros sublunatus "Temm",, Bp., Consp. Gen. Av., i, 1850 , p. go: new name for $B$. indica.

Distr.-Malay Peninsula.

Sumatra; ?Rhio Archipelago; Billiton.

Buceros rhinoceros borneoensis Schl. and Müll.

Buceros Borneoensis Schlegel and Müller, Verh. Nat. Gesch. Zool. I840, Aves, P. 22: Barito River, Soutly-east Borneo (restr. Chasen and Kloss, 1930).

Bucerns thinoceroides Bp., Consp. Gen. Av. i, 1950, p. 89 : new name fot B. borneoensis.

Distr.-Borneo.

Buceros rhinoceros silvestris Vieill.

Buceros silvestris Vieillot, Nouv. Dict. d'Hist. Nat. iv, ISI7, p. 592: Moltuccas error = Java (Stres.).

Buceros diadematus Dumont, Dict. Sc. Nat. vi, I817, p. 203 : Java.

Buceros lunatus Temm., P1. Col. 546, 1834 : Java.

Buceros javanica Schl, and Muill., Verh. Nat. Gesch. Zool. 1840, Aves, p. 22: Java.

Distr.-Java.

\section{Genus DICHOCEROS Gloger.}

Dichoceros bicornis (Lin11.).

Great or Homrai Hornbill.

Buceros bicornis Linn., Syst. Nat. roth ed. 1758, p. I04: China error $=$ Sumatra. ${ }^{3}$

- Buceros cristatus Vieili, Nov. Dict. d'Hist. Nat. iv, 1816, p. 591: Batavia error $=$ Sumatra.

Distr.-Malay Peninsula.

Sumatra.

- The type locality is neither Travancore, selected by Baker in 1022, not the Malay Peninsula selected by Robinson and Kloss in 1923. The species does not occur in China as originally stated but an additional locality "Strat Sunda" was provided in Syst. Nat, 1766, i, p. 153 : since the bird is not found in Java on the east side of the Sunda Straits the type locality is therefore "Sumatra". If, as Baker states (F.B.I. Birds, 2nd ed. 4, 1927 , p. 284) the Sumatran and continental races are separable the few we have seel from Sumatra seem like Malavan birds) then the latter must bear the name cavatus Shaw : Philippines error=India (Baker, 1030).

[ 105$]$ 


\section{Gienus ANTHRACOCEROS Reichenbach.}

Anthracoceros coronatus (Bodd.).

Pied Hornbill.

[Anthracoceros coronatus coronatus (Bodd.).

Buceros coronatus Boddaert, Tabl. P1. Enlum. 175,3, p. 53 : Malabar.

Distr._Extra-limital.]

Anthracoceros coronatus leucogaster ([1lyt]1).

Buceros leucogaster Blyth, Journ. Asiat. Soc. Bengal, $x_{1}$, j 84 , p. 922: Tenasserim.

Distr.-Malay Peninsula (south to Perak).'

Anthracoceros coronatus convexus (Temm.).

Buceros convexus Temminck, Pl, Col. 530,1832 : Java.

Buceros intermedius Blyth, Journ. Asiat. Soc. Bengal, xvi, 1847, D. 995 : Penang.

Distr.-Malay Peninsula (north to Patani); Tambelan Islands.

Stmmatra; Rhio Archipelago; West Sumatran Islands of Mansalar, the Mentawi group and Nias.

Borneo.

Java; Bali.

Anthracoceros coronatus zamelaenus (Oberh.).

Hydrocissa convexa zamelaena Oberholser, Bull. U.S. Nat. Mus. I59, I932, p. 38 : Liugung Island, Nortl Natuna Islands.

Distr.-North Natuna Islands.

Anthracoceros coronatus barussensis (Oberh.).

Hydrocissa convexa barussensis Oberholser, Journ. Wash. Acad. Sci. xiv, 1924, p. 30I: Tana Bala Istand, Batı Islands, West Sunatra.

Distr.-Batu Islands, West Sumntra.

Anthracoceros malayanus (Raffles) . 2

Black Hornbill.

Buceros malayanus Raffles, 'Trans. Linn. Soc, xiii, ISzz, P. $29 z$ : Sumatra.

Buceros antracicus Temm., Pl. Col. 529, 1832 : Borneo.

Buceros bicolor Eyton, Proc. Zool. Soc. 1839, p. 104: Malacca.

1 Although there is a slight overlap in the range of leucogaster and convexus in the north of the Malay Peninsula both forms can very well be regarded as races of coronatus.

2 We believe that white and black bills are characteristic of male and female respectively and that Grant (Cat Birds, xvii, ISg2, p. 360) was wrong in attributing the black bill to a subadult stage. The meaning of the colour of the superciliary stripe is less clear : it is white or dark grey independent of age and sex. 
Buceros ellioti Hay, Madr. Journ, xiii, pt, 2, 1844, p. 152: Malacca.

Buceros nigrirostris Blyth, Journ. As. Soc. Bengal. xvi, 1847, p. 995 : Malacca.

Distr.-Malay Peninsula.

Sunatra; Banka; Billiton.

Borneo.

\section{Genus CRANORRHINUS Cabanis and Heine.}

Cranorrhinus corrugatus (Temm.). Wrinkled Hornbill.

Cranorrhinus corrugatus corrugatus (Temtu.).

Buceros corrugatus Temminck, Pl. Col. 531, I832: Borneo.

Buceros gracilis Temm., Pl. Col. 535, 1832 : Pontianak, Borneo.

Buceros rugosus Begbie, Malay Peninsula, 1834, D. 513: Malay Peninsula = Malacca.

Hydrocissa migratorius Maingay, Proc. Asiat. Soc. Bengal, I\$68, p. 196 : Malacca.

Distr.-Malay Peninsula.

Sumatra.

Borneo.

* Cranorrhinus corrugatus megistus Oberlh.

Cranorhinus corrugatus megistus Oberholser, Smiths. Misc. Coll.

1x, 1912, p. 6: Tana Bala Island, Batu Islands, West Sumatra.

Distr.-Batu Islands, West Sumatra.

\section{Gent1s RHYTICEROS Reichenbach.}

Rhyticeros plicatus (Forst.).

Wreathed Hornbill.

[Rhyticeros plicatus plicatus (Forst.).

Buceros plicalus Forster, in Pennant, Ind. Zool. 178r, p. 40: Ceram. Distr.-Eixtra-limital.]

\section{Rhyticeros plicatus subruficollis (Blyth),}

Buceros subruficollis Blyth, Journ. Asiat. Soc. Bengal, xii, I843. p. 177 : Teuasserim.

Distr.-Malay Peninsula.

Sumatra.

Borneo.

'Bartels and Stresemann (Treubia, xi, 1929, p. 112) regard $R$. undulatus as a subspecies of plicatus but it is open to question whether subruficollis is not more nearly related to that bird. Of it and the three more western forms, undulatus, subruficollis and narcondami the most distinct is undulatus in having the base of the bill in boti sexes covered with transverse ridges which are absent in the others. Therefore we would regard undulatus as a distinct species and treat the others as races of plicatus. 
Rhyticeros undulatus (Shaw)." Malaysian Wreatled Hornbill.

Buceros undulatus Shaw, Gen. Zool. viii, 18I1, p. 26: Java.

Buceros javanicus Shaw, Gen. Zool. viii, $18 \mathrm{iI}$, p. 28 : Java.

Buceros niget Vieill., Nov. Dict. d'Hist. Nat. iv, $1816,0.502$ : Java,

Buceros anmulatus Dumont, Dict. Sc. Nat, vi, 1817, p. 210 : Java.

Distr.-Malay Peninsula.

Sumatra; Mansalar Island, West Sumatra.

Borneo.

Java; Bali.

\section{Genus ANORRHINUS Reichenbach.}

Anorrhinus galeritus (Tenm.). Bushy-crested Hornbill.

Buceros galeritus 'Temminck, P1. CoI. 520, 1831 : Sumatra.

Buceros carinatus Blyth, Journ. Asiat. Soc. Bengal, xiv, 1845. p. 187. Malacca.

Distr,-Malay Peninsula.

Sumatra.

Borneo; North Natuna Islands.

\section{Genus BERENICORNIS Bonaparte.}

Berenicornis comatus (Rafles). Long-crested Hormbill.

Buceros comatus Raffles, Trans. Linn. Soc. xili, I822, p. 3,30 : Sumatra.

? Buceros lugubris Begbie, Malay Peninsula, 1834 , p. 513: Malay Penin. sula $=$ Malacca.

Distr._Malay Peninsula.

Sumatra.

Borneo.

\section{Genus RHINOPLAX Gloger.}

Rhinoplax vigil (Forst.).

Helmeted Hornbill.

Buceros vigil Forster, in Pennant, Ind. Orn. I78I, p. 40: Tenasserim.

Buceros scutatus Bodd., Tabl. Planch. Enlum. 1783, p. 55: on P1. Enlum. 933.

Buceros galeatus Gmel., Syst. Nat. i, pt. 2, 1788, p. 360: on Pl. Enlum. 933.

Distr.-Malay Peninsula.

Sumatra.

Borneo.

- Buceros pusaran Raffles, Trans, Linn. Soc, xiii, t822, p. 293 (Sumatra) is indeterminable. 


\section{Family UPUPID无.}

Gents UPUPA Linn.

Upupa epops Linn.

Hoopoe.

[Upupa epops epops Ling.

Upupa epos Lintu., Syst. Nat. whth exl. I758, p. II7: Sweden.

Distr._Extra-limital.]

\section{Upupa epops saturata Lönn.}

Upupa cpops saturata Lönnberg, Arkiv. f. Zool, v, 1909, p. 29: Mongolia.

$$
\begin{gathered}
\text { Distr.-Malay States. } \\
\text { Borneo. }
\end{gathered}
$$

\section{Upupa epops longirostris Jerd.}

Upupa longirostris Jerdion, Birds of India, i, 1862, p. 393 : Burma.

Distr.-Malay Peninsula.

Sunlatra.

\section{Family MEROPID瓜.}

\section{Genus MEROPS Linn.}

Merops leschenaulti ${ }^{2}$ Vieill.

Bay-headed Bee-eater.

Merops leschenaulti leschenaulti Vieill.

Merops leschenaulti Vieillot, Nov. Dict. d'Hist. Nat. xiv, I8I7, P. I7: Java, error = Ceylon.

Distr.-Malay Peninsula.

Sumatra. ${ }^{3}$

Merops leschenaulti quinticolor Vieill.

Merops quinticolor Vieillot, Nov. Dict. d'Hist, Nat, xiv, I8r7, p. 21: Ceylon, error = Java.

Metops urica Horsf., Trans. Linn. Soc. xiii, 1821, p. 172: Java.

Distr.-Java; Bali.

-Also occurs in South-west Siam and therefore almost certainly in Peninsular Siam. See Kozlova, Ibis, 1932, p. 530 .

* Nerops erythrocephalus Gmel. has been used for this species, but see Whistler, Journ. Bomb. Nat. Hist. Soc. xxxvii, 1935, p. 758.

3 Dr. W. L. Abbott considers that he saw this species on Simalur Island. West Sumatra. 
Merops viridis Linn.

Chestnut-headed Bee-eater.

Merops viridis viridis Linn.

Merops viridis Linn., Syst. Nat, 12th. ed. i, 1766, p. 182: Java.

Merops sumatramus Raffles, Trans. Linn. Soc. xiii, i\$22, p. 294 : Sumatra.

Merops cyanopygitus Less, Tratté d'Orn. r831, p. 238: Sumatra.

Merops swmatranus caligewus Bangs and Penard, Proc. New England Zool. Cl. viii, 1023, D. 43: West Java.

Distr.-Malay Peninsula.

Sumatra; Rhio Archipelago; Banka; Billiton; Nias Island, West Sumatra.

Borneo; North Natuna Islands; Karimata Islands. Java.

Merops superciliosus Lint.

Brown-breasted Bee-eater.

[Merops superciliosus superciliosus Lin11.

Merops superciliosus Linu., Syst. Nat. rath, ed, I, I766, p. IS $S_{j}$ :

Madagascar.

Distr.-Extra-linital.]

Merops superciliosus javanicus Horsf.

Merops jazanicus Horsfield, Trans, Linn. Soc. xiii, I821, p. I7I: Java.

Distr.-Malay Peninsula.

Sumatra; Rhio Archipelago; West Sumatran Islands of Sintalur, Nias, Siberut and Pagi.

Borneo.

Java; Bali; Kangen Islands.

Gentls NYCTIORNIS Swainson.

Nyctiornis amicta ('Tenum.).

Red-breasted Bee-eater.

Merops amtctus 'Temminck, Pl. Col. 3 IO, 1824 : Bencoolen, Sumatra.

Nyctiorns malaccensis Cab. and Heine, Mus. Hein. ii, 1850, p. 133 : Malacca.

Distr.-Malay Peninsula.

Sumatra.

Borneo.

\section{Family CAPRIMULGID $Æ$.}

Genus LYNCORNIS Gould.

Lyncornis cerviniceps Gould.

Giant Nightjar.

Lyncomis cerviniceps cerviniceps Gould.

Lyncomis cerviniceps Gould, Icon. Av. pt. 2, 1838 , pl. 14: China, error=Trang, Peninsular Siam (Rob, and Kloss, 1922).

Distr.-Malay Peninsula."

Apparently as far south as Penang. 
Lyncomis Tenminckii Gould, Icon. Av. pt. 2, 18,38: Borneo.

Caprimulgus pulcher Hay, Madras Journ, xiji, pt. 2, 1844, p. 161 : Malacca.

Distr.-Malay States.

Sumatra; Banka.

Borneo.

\section{Genus CAPRIMULGUS Linn.}

\section{Caprimulgus concretus $\mathrm{Bp}$.}

Bornean Nightjar.

Caprimulgus coneretus concretus $\mathrm{BP}$.

Caprimulgus concrelus Bonaparte, Consp. Gen. Av, i, I850, p. 60 : Bormeo.

Caprimulgus borneensis "Wall.," Salvad., Ann. Mus. Civ. Gen. v, 1874 , D. 117: S. Borneo.

*Caprimulgus mirificus Oberholser, Smiths. Misc. Coll. 1x, 7, 1012, p. 7: Siak kiver, East Sumatra.

Dist t, -Bortieo.

Sumatra; Billiton.

Caprimulgus pulchellus Salvad.

Salvadori's Nightjar.

Caprimulgus pulchellus pulchellus Satvad.

Caprinulgus pulchellus Salvadori, Ann. Mus. Civ. Gen. xiv, 1879 , p. 195: Padang, West Sumatra.

Distr.-Sumatra,

- Caprimulgus pulchellus bartelsi Finsch.

Caprimulgus Barielsi Finsch, Notes Leyd. Mus, xxiii, I902, p. I48: West Java.

Distr.-Java.

Caprimulgus affinis Horsf.

Allied Nightjat.

Caprimulgus affinis affinis Horsf,

Caprimuigus affinis Horsfield, Trans. Linn. Soc. xiii, I821, P. I42: Java.

Caprimulgus bisignatus Boic, Mus. Lugd., teste Cass, Cat. Capr. Mus. Philad. 1851:

Caprimulgus arundinaceus Jacq. and Puch., Voy, au Pôle Sud, Ois. p. 03,1853 : Borneo.

Caprimulgus faberi Mever, Sitzungsber. Ges. Isis, i, 1884, p. 20: W. Sumatra.

Distr.-Sumatra; Billiton; Nias Island, West Sunatra." Borneo.

Java; Bali; Karimon-Java Islands.

- Dr. Dammerman includes affinis in the avifauna of the Rhio Archipelago but no specimen has yet been obtained. 
Caprimulgus macrurus' Horsf.

Long-tailed Nightjar.

Caprimulgus macrurus macrurus Horsf,

Caprimulgus macrurus Horsfeld, Trans, Linn. Soc xiii, I82I, p. I42: Java.

Caprintlgus salvadorif Sharpe, Proc. Zool. Soc. 1875. p. 99, pl. wh Labuan.

Distr.-Borneo; Banguey Island, North Borneo.

Java; Bali.

\section{Caprimulgus macrurus bimaculatus Peale.}

Caprimulgus bimaculatus Peale, U.S. Exploring Expedn. vii, Mannln. and Onith. I848, p. I7o: Singapore Island.

Caprimulgus macrurns anamesus Oberholser, Proc. U.S. Nat. Mus. Xlviii, 1915, P. 593 : Singapore Island.

Distr.-Malay States (notth to Penang).

Sumatra; Rhio Archipelago.

Caprimulgus macrurus ambiguus Hartert.

Caprimulgus macrurus ambiguus Hartert, Ibis, ISg6, p. 373: Sonth Tenasserim ( $K$ loss, $197 S$ ).

Distr.-Peninsular Siann.

Caprimulgus indicus Lath.

Migratory Nightjar.

[Caprimulgus indicus indicus Lath.

Caprimulgus indicus Latham, Ind. Orı. ii, 1790, p. 588: India.

Distr.-Extra-linital.]

Caprimulgus indicus jotaka Temm, and Schleg.

Caprimulgus jolaka Temminck and Schlegel, Siebold's Fanna Japon.

Aves, 1847, p. 37, pl, 12: Japan.

Distr.-Malay Peninsula.

Sumatra.

Borneo; at sea off the Naturta Islands.

Java.

Caprimulgus indicus innominatus Hume.?

Caprimulgus innominala Hume, Stray Feathers, iif, I875, p. 3IS :

I'enasserim.

Dislr.-Peninsular Sian.

I Revision: Oberh., Proc. U.S. Nat. Mus. xlviit, 1915, p. 587.

- This race needs confirmation but there is some evidence for the recognition of a breeding race, smaller and darker than jotaka, in Indo-China and to it we tentatively refer specimens from the island of Terutau in the Straits of Malacca and from South Annam. 


\section{Family MICROPODID平。 \\ Genus HEMIPROCNE Nitzsch.}

\section{Hemiprocne longipennis (Rafin.).}

Crested Tree-Swift.

\section{Hemiprocne longipennis longipennis (Rafin.).}

Hirundo longipennis Rafinesque, Bull. Soc. Philom. Paris, iii, ISoz, p. 153 : Java.

Hirundo Klecho Horsf., Trans, Linn, Soc, siii, 180r, p. 143: Java. Distr.-Java; Bali.

\section{Hemiprocne longipennis harterti Stres.}

Homiprocne longipennis harterti Stresennann, Nov. Zool. xx, I913. p. 339: Deli, North-east Simatra.

Hemiprocne longipennis anochra Oberh., Bull. U.S. Nat. Mus. 159, 1932, p. 42: Bunguran Island, North Natuna Islands.

Distr.-Malay Peninsula; Anamba Islands.

Sunatra; Rhio Arclipelago; Banka; Billiton.

Bormeo; North Bormean Islands; North Natuna Islands; Karinata Islands.

"Hemiprocne longipennis perlonga (Richm.).

Macropteryx perlonga kichmond, Proc. U.S. Nat. Mus. xxvi, I903. p. 502: Sintalur Island, West Sumatra.

Distr.-Sinalur Island, West Sumatra.

*Hemiprocne longipennis ocyptera Oberh.

Hemiprocne longipennis ocyplera Oberholser, Smiths. Misc. Coll.

$1 \mathrm{x}, 7, \operatorname{Ig} 2$, p. 7 : Nias Island, West Sumatra.

Distr. $\rightarrow$ Nias Island, West Sumatra.

"Hemiprocne Iongipennis thoa Oherh.

Heniprocne longipennis thoa Oberholser, Smiths. Misc. Coll. $1 \mathrm{x}, 7$, I9:2, 1. 8: Pinie Island, Batu Islands, West Sumatra.

Distr._Batu Islands; Pagi Islands; Eingano, West Sunatra .

Hemiprocne comata ('Temmil.).

Hemiprocne comata comata (Temm.). Tufted Tree-Swift Cypselus comatus Temminck, P1. Col. 268, I824: Sumatra.

Distr.-Malay Peniusula; Auanza Islands (vis.).

Sumatra; Rhio Archipelago; Banka; West Sumatra Islands of Nias, Batu Islauds, Siberut and Pagi Islands.

Borneo; North Natuna Islands.

1 We have referred the Engano bird to the nearest race geographically but have never seen specimens from the island. 
Genus COLLOCALIA' Gray.

Collocalia francica (Gmet.).

Grey-rumped Swiftlet.

[Collocalia francica francica (Gmel.).

Hirundo francica Gmelin, Syst. Nat. I, pt. 2, I7S9, p. 1017: Maturitius.

Distr.—Extra-limital.]

\section{Collicalia francica germani Oust.2}

Collocalia germani Oustalet, Bull. Soc. Phil. Paris, I\$76, p. I: Condor Istand, off Cochinchina.

Distr.-Malay Peninsula.

Coasts of Sarawak and Northi Borneo.

Collocalia francica amechana Oberh.t

Collocalia francica amechana Oberholser, Proc. U.S. Nat. Mus. 1xii, 1912, 1. 13: Jimaja Island, Anamba Islands.

Distr.-South Malay Peninsula; Anamba Islands.

Collocalia francica perplexa Riley.

Collocalia francica perplexa Riley, Proc. Biol. Soc. Wash, xl, I927, P. I40: Maratua Island, East Borneo.

Distr.-Maratua Islands, East Borneo.

* Collocalia francica javensis Stres.

Collocalia francica javensis Stresemann, Bull. Raffles. Mus., 6, 1931, p. 89: Cheribon, Java.

Distr.-Java; Kangean Islands.

* Collocalia francica bartelsi Stres.

Collocalia francica bartelsi Stresentann, Orn. Monatsber, xxxv, I927, p. 46: North coast of West Java.

Distr. 一Java. ${ }^{5}$

I Revision: Strese., Bull. Raffles Mus. 6, I931, p. 83.

- In North Borneo C. francica germani nests on Mantanani Island and on Berbaja islet in Sandakan Harbour. Only a few miles away and within sight of Berhala, there are nesting colonies of $C$. vestita maralua on Gomantong Hill. From the Maratua Islands, E. Borneo, Riley records both C. $t$. perplexa and $C$. $v$, maratua, and we therefore regard francica and vestita as "species", but otherwise, in our arrangement of this very difficult genus follow the latest reviewer, Dr. E. Stresemann in Bull. Raffles Mus., 6, to 31, p. $\delta_{3}$.

3 We have now examined large series of birds from these Bornean localities: they seem absolutely inseparable from true germani.

4 This is the earliest name of which we are certain but inexpectata may be applicable to both this and perplexa. The rump is atways at least slightly paler than the back but darker than in germani.

3" "Breeding places probably off the North Coast of West Java," (Stresemann). 
Collocalia vestita (Less.).

Brown-rumped Swiftlet.

Collocalia vestita vestita (Less.).

Salangana vestita Lesson, Echo du Monde Savant, $x$, IS 43, p. 134 : Sumiatra.

Collocalia milifica Gray, Gen. Birds, i, I845, f. 55: Sumatra.

Collocalia francica aerophila Oberh., Proc. U.S. Nat. Mus. xlii, 1912. D. I6: Nias Island.

Collocalia francica natuma Stres, Orn. Monatsber, xxxvi1i, 1930, p. I8r : Bunguran Island, N. Natuna Islands.

Dislr.-South Malay Peninstula,

Sumatra; Billiton; West Sumatran Islands of Sipora, Nias, and ? Simalur.

Natıua Islands.

Collocalia vestita maratua Riley.

Collocalia s'estita maratua Riley, Proc. Biol. Soc. Wash. xl, r927. p. I4I : Maratua Island, East Borneo.

Distr.-Borneo; Maratua Islands, East Borneo, ${ }^{1}$

Collocalia fuciphaga (Thun.) .

Thunberg's Swiftlet.

Collocalia fuciphaga fuciphaga (Thunb.).

Hinudo Fuciphaga Thumberg, Kongl. Vet, Acad, Haudl, xxxiii, IS2I, P. I53: Java.

Hemiprocne salangana Streubel, Isis, 1S48, p. 368: Java.

Disir.-Java.

Cóllocalia (? fuciphaga) innominata Hume.

Hume's Swiftlet.

Collocalia innominala Hume, Stray Feathers, i, 1873, p. 294 : South Andaman Islands.

Distr.-Malay States.

? Sumatra (West).

Collocalia lowi (Sliarpe).

Low's Swiftlet.

Collocalia lowi lowi (Sharpe).

Cypselus lowi Sharpe, Proc. Zool. Soc. I879, p. 333 : Labuan Island, Borueo.

Distr-Sumatra; Nias Island, West Sumatra.

Borneo (west and north).

\section{Collocalia lowi robinsoni Stres.}

Collocalia lowi robinsont Stresemann, Bull. Rafles MLus., 6, I931, p. 98: Bẽlitung Island, south-west of 'Terutau Island, Straits of Malacca.

Distr.-Malay Peninsula; Anamba Islands. "

- Perhaps the Natuna race should be included under maratua which is slightly darker than typical vestita.

- We have not seen specimens from the Anamba Islands but as robinsoni breeds in the Tioman Archipelago the Anamba form is more likely to be robinsoni than lonvi. 


\section{Collocalia lowi tichelmani Stres.}

Collocalia brevirostris tichelmani Stresemann, Orn. Monatsber. xxxiv, 1926, p. I04: Tamaloeang, Soutli-east Borneo.

Distr._-Borneo (south-east).

*Collocalia (? lowi) vulcanorum Stres.

Collocalia brevirostris vulcanorum Stresemann, Mitt. Zool. Mus. Berlin xii, 1926, p. 352 : West Java.

Distr,-Java (west).

Collocalia gigas Hart. and Butl.

Giant Swiftlet.

Collocalia gigas Hartert and Butler, Bull. Brit. Orn. Cl. xi, Igor, p. 65: Selangor, Malay States.

Disir.-Malay States.

Sumatra.

Java.

Collocalia esculenta (Iinn.).

White-bellied Swiftlet.

[Collocalia esculenta esculenta (Linn.).

Hirundo esculenta Linn,, Syst. Nat. Ioth. ed. 1758, p. I91: Amboyna.

Distr._Extra-liunital.]

Collocalia esculenta linchi Horsf. and Moore.

Collocalia linchi Horsfield and Moore, Cat. Bds. Mus. East Ind. Coy. i, 1854 , p. 100 : Java.

Disir.-? Sumatra (sontli-east). Java; Bali; Kangean Islauds; Bawean Island.

\section{Collocalia esculenta cyanoptila Oberli.}

Collocalia linchi cyanoptila Oberholser, Proc. Acad. Nat. Sci. Philarlelplia, Igo6, 1\%. 205: Bunguran Island, North Natura Islands.

Disir. - Malay States.

? Sumatra (east);' Lingga Arrchipelago.

Borneo; North Natuna Islands.

\section{Collocalia esculenta oberholseri Stres.}

Collocalia linchi oberholseri Stresemann, Nov, Zool. xix, Igrz, p. 348: North Pagi Island, West Sumatra.

Distr.-Sumatra (west); Nias Island and Mentawi Islands, West Sumatra. ${ }^{2}$

- Specimens from North-east Sumatra are small and are possibly nearer to $C$. l. affuis Beavan of the Andaman Islands than to cyanoptila: we have not seen affinis. C. l, elachyptera Oberh. (Mergui Arch.) probably occurs in Peninsular Siam.

- We have not seen specimens from Nias and place them with obcrholseri purely on geographical grounds. 


\section{Collocalia esculenta dodgei Richm.}

Collocalia dodgei Richmond, Smiths. Quart. ii, 1905, D. 431 : Mt. Kinabalu, North Borneo.

Distr.-Borneo (monntains of the North). Sumatra (Korinchi Peak).

\section{Collocalia esculenta natalis Lister.}

Collocalia natalis I,ister Proc. 2001. Soc. 1888, p. 520: Christmas Island, Indian Ocean.

Distr.-Christmas Island, Indian Ocean.

\section{Genus CHETURA Stepliens.}

Chetura caudacuta (Iath.). White-throated Spinetail Swift.

[Chatura caudacuta caudacuta (Latis.).

Hirundo caudacula Latham, Index Orn. Suppl. I8or, p. 57 : New South Wales.

\section{Distr.-Lixtra-1imital.]}

Chatura caudacuta nudipes Hodgs.

Chatura Nudipes Holgson, Journ. Asiat. Soc. Bengal, v, 1836, p. 779 : Nepal.

$$
\text { Distr.-Java. }
$$

\section{Chretura caudacuta cochinchinensis Oust.}

Chetura cochinchinensis Oustalet, Bull. Soc. Philon. Paris, I878, p. 52: Saigon1, Cochin China.

Hirurdinapus haesii Büttikofer, Notes Leyd. Mus. ix, 1887, p. 40: Padang Highlands, West Sumatra.

Distr.-Malay States.

Sumatra.

Java.

Chatura gigantea ('I'emm.). Spinetail Swift.

Chetura gigantea gigantea ('Temm.).

Cypselus giganteus Temminck, P1. Col. 364, 1825: Java.

Distr. - Malay States.

Sumatra; Rhio Archipelago.

Borneo; North Natuma Islands.

Java.

\section{Chatura gigantea indica Hume.}

Chatura indica Hume, Stray Feathers, i, 1873, p. 47I : Travancore.

Distr.-Malay States. 


\section{Chætura celebensis Scl.}

\section{[Chatura celebensis celebensis Scl.}

Chatura giganlea, var. celebensis P. L. Sclater, Proc. Zool, Soc. I865, p. 608: Menado, Celebes.

Distr.-Extra-linital.]

"Chæetura celebensis ernsti Bartels.t

Bartel's Spinetail Swift.

Chatura emsti Max Bartels Jr., Orn. Monatsber. xxxix, I93I. p. 54: Mt. Halinoen, West Java.

Distr.-Java (west).

Chactura leucopygialis (Blyth) .

Grey-rumped Spinetail Swift.

Acanthylis letucopygialis Blyth, Jonru. Asiat. Soc. Bengal, xviii, I 849, p. 809 : Penang.

Acanthylis coracimus Bp., Consp. Gen. Avium, i, 1850 , p. 64 : Borneo.

Disir.-Malay Peninsula.

Sumatra.

Borneo; North Natuna Islands.

Java.

\section{Genus CYPSIURUS I esson.}

Cypsiurus batasiensis (J. E. Gray).

Palm Swift.

[Cypsiurus batasiensis batasiensis (J. E. Gray).

Cyp. Balasiensis J. E. Gray, Griffith's Anim. Kingd, vii, I829, P. 60 : India.

Distr.-Eixtra-linital.]

Cypsiurus batasiensis infumatus (Sclat.).

Cypselus infumatus Sclater, Proc. Zool. Soc, I 865, p. 602: Borneo.

Distr. - Malay Peninsula; Tambelan Islands (vis.).

Sumatra.

Borneo.

Java; Bali.

Gent1s MICROPUS Wolf.

Micropus pacificus (Lath.).

White-rumped Swift.

Micropus pacificus pacificus (Lath.).

Himudo pacifica Latham, Index Orm. Suppl. 1So1, p. 58: New South Wales.

Distr.-Malay Peninsula.

Sumatra; Rlio Archipelago (vis.).

Java. 
Micropus pacificus cooki (Hat.).

Cypselus pacificus cooki Harington, Bull. Brit. Orn. Cl. xxxi, 1913, p. 57 : North Shan States.

Distr,-Malay States.

Micropus affinis (J. E. Gray).

House-Swift.

[Micropus affinis affinis Gray.

Cypselus affuis J. E: Gray, in Gray and Hardwicke's I1l, Ind. Zool. i, 1832, pl. 35 : Ganges.

Distr.-Dixtra-[imital.]

Micropus affinis subfurcatus (Blyth).

Cypselus subfurcatus B1yth, Asiat. Soc. Bengal xviii, I 849, D. So7: Penang.

Cypselus lencopygialus Cass., Proc. Ac. Phil. v, 1852 , p. 58, pl. 13: Sumatra.

Distr.-Malay Peninsula; Anamba Islands (vis.).

Sunnatra; Rhio Archipelago (vis.); Billiton.

Borneo; North Natuna Islands.

Java.

\section{Family TROGONIDÆ.}

\section{Gents HARPACTES Swainson.}

Harpactes diardii (T'emu1.).

Diard's Trogon.

Harpactes diardii diardii (Temm.).

Trogon diardii Temmincl, P1. Col. 541, iS32: Borneo.

Distr.-Banka.

Borneo.

\section{Harpactes diardii sumatranus Blas.}

Harpacles diardi sumatranus Blasius, Mitt. Geogr. Ges. Nat. Mus.

lubeck, 2 Reihe, Heft. I0, ISg6, p. 95: Sumatra.

pyotrogan neglectus Forbes and Robinson, Bull. Liverpool Mus, ii, Igoo, p. 34 : Malacca.

Distr.-Malay Peninsula.

Sumatra.

Harpactes kasumba (Raffles).

Red-naped Trogon.

Harpactes kasumba kasumba (Raffles).

Trogon Kasumba Raffles, Trans. I,inu. Soc. xiii, I822, p. 282: Bencoolen, Sumatra.

Trogon Coutea Cuv., Règ. An. i, 1829, 1., 459 : Sumatra.

Trogon Temninckit Gould, Proc. Zool. Soc, 1835, p. 29 : Sumatra.

Distr.-Malay Peninsula.

Sumatra. 
Harpactes kasumba impavidus (Chas, and Kloss).

Pyrotrogon fasciatus impavidus Chasen and Kloss, Bull. Rafles Mus., 5, I93I, p. 84: Bettotan near Sundakan, British Norti Borneo,

Pyratrogon kasumba usa Harrisson and Hartley, Bull. B. O. Cl. liv. 1034, p. 150: Mt. Kalutong, Sarawak.

Distr, -Borneo.

Harpactes whiteheadi Sharpe.

Whitehead's 'Trogon.

Harpacles whiteheadi Sharpe, Ibis, r888, p. 395, pl. rz: Mount Kinabalu, British North Borneo.

Distr.-Borneo (mountains).

Harpactes erythrocephalus (Gould).

Red-headed Trogon.

[Harpactes erythrocephalus erythrocephalus (Gould).

Trogon erythrocephalus Gould, Proc. Zool. Soc. 1834, p. 25 : Rangoon.

Distr.-Extra-limital.]

Harpactes erythrocephalus chaseni Riley.r

Harpactes erythrocephalus chaseni Riley, Proc, Biol. Soc. Wasli. xlvii, I934, p. I15: Semangko Pass, Selangor-Pahang boundary, Malay States.

Distr.-Malay States.

Harpactes erythrocephalus flagrans (Mü1l.).

Trogon fagrans Sal. Müller, Tijd. Nat. Ges, Phys, $1 S_{35}, 13.338$. pl. 8, fig. 2 : Sumatra.

Distr.-Sumatra.

Harpactes duvaucelii ('Temm.). Red-rumped Trogon.

Trogon duvaucelii Temminck, Pl. Col. 29I, I824: Sumatra.

Distr.-Malay Peninsula.

Sunatra; Rhio Archipelago; Banka; Billiton.

Borneo; North Natuna Islands.

I This race needs confirmation: as some recent authors have remarked the bird from the Malay States ought to be separable, but Robinson who worked through the species in $1927 \mathrm{saw}$ no reason for separation and I have a recollection that he once told me that he could not separate a Malayan subspecies. I have never seen fresh skins of true erythrocephalus. 
Harpactes orrhophaeus Cab. and Heine. Cinnamon-rumped Trogon. Harpactes orthophaeus orrhophaeus (Cab. and Heine).

Pyrotrogon orrophaeus Cabanis and Heine, Mus. Hein. iv, I863, p. ${ }_{5} 6$ : Malacca.

Distr,-Mralay States."

Sumatra.

Harpactes orrhophaeus vidua 0 - Grant.

Harpactes vidua O.-Grant, Cat. Bds. Brit. Mus. xvii, I892, P. 50 : Mt. Dulit, North Sarawak, Borneo.

Distr.-Borneo.

Harpactes oreskios (Temm.). Orange-breasted Trogon.

Harpactes oreskios oreskios (Temin.).

Trogon oreskios Termminck, P1. Col, I8r, 1823: Java,

Harpactes gouldii Swains., Class. Birds, ii, 1837 , p. 337 : New name for Pl. Col. "121" $\{=181\}$.

Distr.-Java.

\section{Harpactes oreskios uniformis Rob.}

Pyrotrogon oreskios uniformis Robinson, Journ. Fed. Malay States Mus, vii, 19I, p. 149 : Trang, Peninsular Siam.

Disir.-Malay Peninsula.

Suniatra.

Harpactes oreskios dulitensis O.-Grant.

Harpactes dulitensis O.-Grant, Cat. Bds. Brit. Mus. xvii, I892, T. 502 : Mt. Dulit, North Sarawak, Borneo.

Distr-Borneo.

Harpactes reinwardtii ('Temm.). Reinwardt's Trogon.

Harpactes reinwardtii reinwardtii (Temm.).

Trogon Reinwardti Temminck, P1. Col. 124, I823: Java.

Distr.-Java.

Harpactes reinwardtii mackloti (S. Müll.).

Trogon Mackloti Sal. Müller, Tijd. Nat. Gesch. Phys. I835, p. 336 , pl. 4, fig. I : Sumatra.

Distr._Sumatra.

T The specimen recorded by Muller from the Malay Peninsula probably came from the neighbourhood of Junk Seylon, Penirsular Siam. T, nutilus Vieill. and T. cinnamomers Temm. are too uncertain for inclusion here. 


\section{Family CUCULIDE.}

\section{Gents CLAMATOR Kaup.}

Clamator coromandus (Linn.). Red-winged Crested Cuckoo.

Cuculus coromandus Linn., Syst. Nat. I2th. ed. $i, 1766$, P. I 7 : Coromandel Coast.

Distr.-Malay Peninsula.

Sumatra; Lingga Arclipelago.

Bonieo.

Java.

\section{Gemus SURNICULUS' Lesson.}

Surniculus lugubris (Horsf.).

Black Drongo-Cuckoo.

Surniculus lugubris lugubris (Horsf.).

Cuculus lugubris Horsfield, Trans. Linw. Soc. xiii, t821, p. 179: Java.

Cuculus albopunctulatus Drap., Dict. Class. Hist. Nat. iv, 123, p. 570 : Java.

Distr.-Java; Bali.

\section{Surniculus Iugubris brachyurus Stre5.}

Surniculus lugubris brachyurus Stresemann, Nov. Zool. xx, I9I3, 1. 340: Pahang, Malay States.

Distr.-Malay Peninsula.

Sumatra; Rhio and Lingga Archipelagos; Banka.

Borneo.

Surniculus lugubris dicruroides (Hodgs.).

Pseudomis dicrurotdes Hodgson, Journ. Asiat. Soc. Bengal, viit, 1839 , p. 136: Nepal.

*Surniculus lugubris massorhinus Oberholser, Journ. Wasb. Acad. Sci. xiv, 1924, 0. 300: Siak River, East Sumatra.

Distr.-Malay Peninsula.

Sumatra. ${ }^{2}$

North Natuna Islands.

* Surniculus lugubris barussarum Oberh.

Surniculus lugubris barussarum Oberholser, Sniths. Misc. Coll. Ix, 7 , 1912, p. 5 : Tana Bala Island, Batu Islands.

Disir.-Batu Islands, West Sumatra; Nias (? subspecies).

I Revisions: Stres., Nov, Zool, xx, 1913, p. 340 ; Baker, Nov, Zool, xxvi, 1919, p. 291.

- Specimens examined from N. E. Sumatra. 
Genus HIEROCOCCYX S. Mïller.

Hierococcyx sparverioïdes (Vig.).

Large Hawk-Cuckoo.

Hierococcyx sparverioïdes sparverioïdes (Vig.).

Cuculus sparverioides Vigors, Proc. Comm. Zool. Soc. i, I832, p. 173: Simla.

Distr.-Malay Peninsula.

Borneo.

Sumatra.

Java.

Hierococcyx sparverioïdes bocki Wardl, Raws.

Hierococcyx bocki Wardlaw Ramsay, Ibis, I886, p. I57: Mt. Sago, West Stunatra.

Distr.-Malay States.

Sumatra.

Borneo.

Hierococcyx fugax (Horsf.). Malayan Hawk-Cuckoo.

Hierococcyx fugax fugax (Horsf.).

Cuculus fugax Horsfield, Trans. Linn. Soc, xiii, I821, P. I78: Java.

Dis!r.-Malay Peninsula.

Sunatra; Rhio Archipelago; Iilliton.

Borneo; Karimata Islands.

Java.

Hierococcyx fugax nisicolor (Blyth).

Cuculus nisicolor Blyth, Journ. Asiat. Soc. Bengal xil, I 843 , p. 943 : Nepal.

Distr.-Malay Peninsula.

Sumatra; Billiton; Siberut Island, West Sumatra.

Java.

Hierococcyx fugax hyperythrus (Gould).

Cuculus hyperythrus Gould, Proc, Zool, Soc. 1856, p. 96 : Shanghai.

Distr.-Borneo.

Although we have recently recognised fugax and wisicolor as distinct and bave given characters for their recognition there remains an element of doubt in our minds as to whether or not they should be united as one immigrant race in which case $H$. vegans could be regarded as a small southern resident form of the same species. Most of the fugax examined by us are females and most of the nisicolor males.

2 A form of $H$. flugax also occurs in Banka. 
Hierococcyx vagans (S. Müll.)."

Small Hawk-Cuckoo.

Cuculus vagans S. Müller, Verh. Nat. Gesch. Land en Volk. 1845 , p. 233, note: Java.

Distr.-Malay Penirsula.

Borneo.

Java.

\section{Genus CUCULUS Linn.}

Cuculus micropterus Gould.

Indian Cuckoo.

Cuculus micropterus micropterus Gould.

Cuculus micropterus Gould, Proc. Zool. Soc. I 837, 'p. 137 : Himalayas.

Cuculus affnis "Hay", Blyth, Journ. Asiat. Soc. Bengal, xy, 1846, p. 18 : Malacca.

Distr.-Malay Peninsula.

Sumatra; Banka.

Borneo.

Java; Thousand Islands.

Cuculus micropterus concretus $\mathrm{S}$. Müll.

Cuculus concretus S. Müller, Verlt. Nat. Gesclı., Land en Volk. I845, p. 236 : Borneo.

Distr.-Borneo.

Sunatra.

Java.

Cuculus canorus Linn.

Common Cuckoo.

[Cuculus canorus canorus Linn.

Cuculus canorus Linn., Syst. Nat, roth ed. I758, p. I Io: Sweden.

Distr.-Extra-limital.]

Cuculus canorus telephonus Heiue, ${ }^{3}$

Cuculus telephonts Heine, Journ, f. Orn, 1863, p. 352: Japan.

Distr.-Malay States.

Stunatra; Lingga Archipelago.

Borneo.

Java; Bawean Island,

1. This is Hierococcyx nanus Hume, Stray Feathers, y, 1877 , p. 490 : South Tenasserim.

- The following names are uncertain but may apply to this bird. C. assimilis Brehm, Isis, 1843, p. S93 (Java): C. striatus Drap., Dice. Class, d'Hist, Nat. iv, 1843, p. 570 (Java); $C$. canoroides S. Müll,, Verh. Nat. Gesch. Land en Volk. 1845, p. 235 (Java). 


\section{CUCULIDE.}

Cuculus optatus Gould.'

Gould's Cuckoo.

Cuculus optalus Gould, Proc. Zool. Soc. 1845, p. Is: North Australia.

Cuculus horsteldi "Moore", Horsfield and Moore, Cat. Bds. Mus. East Ind. Coy. $\mathrm{ii}_{1} 1858$, p. 703 : Java.

Distr,-Malay States.

Sumatra; Nias Island, West Sumatra.

Borneo.

Java; Bali.

Cuculus poliocephalus Lath.

Sinall Cuckoo.

[Cuculus poliocephalus poliocephalus Lat]s.

Cucults poliocephalus Lathan, Index Orn. i, 1790, p. 214 : India. Dislr.-Eixtra-limital.]

Cuculus poliocephalus insulindae Hartert.

Cuculus internedius insulindae Hartert, Vög. pal. Fauna, ii, I9г2, p. 952 : Mt. Kinabalu, North Borneo.

Distr.-Borneo. ${ }^{2}$

Cuculus poliocephalus lepidus S. Müll.

Cuculus lepidus S. Müller, Verlı. Nat. Gesch. Land ez Volk. I\$45, p. 236 : Timor.

Distr.-Malay States.

Sumatra. ${ }^{3}$

Java; Bali.

Genus PENTHOCERYX ${ }^{4}$ Cabanis and Heine.

Penthoceryx sonneratii (Lath.).

Banded Bay Cuckoo.

[Penthoceryx sonneratii sonneratii (Lath.).

Cuculus Sonneratii Lathan, Index Orn. i, I790, D. 2 I5: Bengal.

Distr.-Extra-limital.]

Penthoceryx sonneratii malayanus Chas, and Kloss.

Penthoceryx sonnerati nalayanus Clias. and Kloss, Bull. Raffles Mus., 5, r.931, P. 84 : Kuala Lumpur, Selangor, Malay States.

Distr.-Malay Peuinsula (not extreme soutl).

1C. saluratus Blyth, Journ. As. Soc. Bengal, xif, 1843, p. 942 (India) is possibly the name for this bird, but optatus is the first name of certain application. Also occurs in South-west Siam and therefore almost certainly in Peninsular Siam.

:The Bornean form is separable from lepidus of Java on account of the darker grey parts of the plumage.

3 The few birds from Sumatra seen by us (together with those from the Lesser Sunda Islands! seem nearer to lepidus thau to insulindae. Some are lepidus $>$ insulindae.

4 Cf. Baker, Bull. B. O. Cl. xxxix, 1919, D. 45. 


\section{CUCULIDE.}

Penthoceryx sonneratii fasciolatus (S. Müll.).

Cuculus fasciolatus S. Müller, Verh, Nat, Gesch., Land en Volk, I 843, p. 177 : Sumatra.

Distr.-Malay States (extrene south).

Sumatra; Rhio Archipelago.

Borneo.

Penthoceryx sonneratii musicus (Ljungh).

Cuculus musicus Ljungh, Kongl. Vet. Acad. Handl, xxiv, I804, p. 309 , Taf. 6: Batavia, Java.

Cuculus pravata Horsf., Trans. Linn. Soc. xiii, 1821, p. 179: Java.

Cuculus rufovitlatus Drap., Dict. Class. d'Hist. Nat, jv, 1823, p. 568 : Java.

Distr.-Java.

Genus CACOMANTIS ${ }^{\wedge}$ S. Müller.

Cacomantis merulinus (Scop?.).

Brain-fever Bird.

[Cacomantis merulinus merulinus (Scop.).

Cuculus merulinus Scopoli, Del Flor. et Fann. Insubr. ii, I786, p. 89 : Panay Island, Philippines.

Distr.-Extra-limital.]

Cacomantis merulinus querulus Heine.

Cacomantis querulus Heine, Journ, f. Orn, $1 \$ 63$, p. 352: Nepal.

Dislr.-Malay Peninsula. ${ }^{2}$

Cacomantis merulinus threnodes $\mathrm{Cab}$. and Heine.

Cacomantis threnodes Cabanis and Heine, Mus. Hein. iv, I862, p. I9: Malacca.

Cacomantis dysonymus Heine, Journ. f. Orn. 1863. p. 352 : Borneo.

Distr.-Malay States.

Sumatra; West Sumatran Islands of Siberut and Iingano.

Borneo.

Cacomantis merulinus lanceolatus (S. Müll.).

Cuculus lanceolatus S. Müller, Verh. Nat. Gesch., I,and en Volkenk. I 843, p. 178 : Java.

Distr.-Java,

*Cacomantis merulinus subpallidus Oberl1.

Cacomantis merulinus subpallidus Oberholser, Snths. Misc. Coll. 1x, 7, IgI2, p. 4: Nias Island, West Sumatra.

Distr_-Nias Island, West Sumatra.

1Revisions: Stres., Nov. Zool. xix, 1913, p. 332; Hart., Nov, Zool. xxxii, 1925, p. J64.

2C. Me ihrenodes and C. m. querulus inosculate in the north of the Malay States. 
Cacomantis variolosus (Vig. and Horsf.). Fian-tailed Cuckoo.

[Cacomantis variolosus variolosus (Vig. and Horsf.).

Cuculus variolosus Vigors and Horsfield, Trans. Linn. Soc. $x v, ~ 1827$, p. 300 : New South Wales.

Distr.-Extra-linnital.]

Cacomantis variolosus sepulcralis (S. Müll.).

Cuculus sepuleralis S. Müller, Verh. Nat. Gesch. Land en Volk. I843 P. 177 : Java.

Distr.-Malay Peninsula.

Sumatra; Simalur Island, West Sumatra; Billiton.

Borneo.

Java; Bali.

\section{Genus CHALCITES Lesson.}

Chalcites xanthorhynchus (Horsf.). Violet Cuckoo.

Chalcites xanthorhynchus xanthorhychus (Horsf.).

Cuculus xanthorhynchus Horsfeld, Trans. Linn. Soc. xit, I825, p. 179 : Java.

Distr. - Malay Peninsula. ${ }^{2}$

Stunlatra; Lingga Archipelago.

Borneo; North Natuna Islands.

Java.

Chalcites xanthorhynchus bangueyensis Chas, and Kloss.3

Chalcites (Chalcococcyx) xanthorhychus bangueyensis Chasen and Kloss, Journ. f. Ornith, I929, ii, p. rog: Bangtiey Island, North Borneo.

Distr-Banguey Island.

Chalcites maculatus (Ginel.).4

Emerald Cuckoo.

Trogon maculalus Gmelin, Syst. Nat. i, 17S8, p. 404: Ceylon.

Distr.-Malay Peninsula. Sumatra.

Revision: Hart, and Stres, Now, Zool, xxxii, 1925, p. 158.

- The largest specimen we have is a female with id wing-length of to6 $\mathrm{mm}$. from Trang, Peninsular Siam. If there is a large northern race it will stand as $C$. $x$, limborgi Tweeddale, Proc. Zool. Soc. 1877, p. 366 : Tenasserim.

3 This is a poor race and we bave now seen one or two specimens like topotypes from elsewhere. For the present it can stand on average characters: it seems likely that the name will eventually have to be used to cover a non-Javan Malaysian race. Good series of topotynical zanhorhynchus seem not to exist.

4 This is an extremely rare bird in the Malay States and furthermore only a seasonal visitor: Mr. Stuart Baker's remarks on Malayan birds in Fauna Brit. Ind., Bds., and ed. iv, 1927 , p. 162 , seem to apply to anothe: species. For a discussion concerning the type locality for which "Pegu" was proposed by Robinson and Kloss in ro23. see Whistler. Journ. Bomb. Nat. Hist. Soc, xxxvii, 1934, p. 521 . 
Chalcites basalis (Horsf.).

Bronze Cuckoo.

Cucultus basalis Horsfield, Trans. Linn. Soc. xiii, IS2I, D. I79: Java.

Cuculus chalcites Temm,, PI. Col, 102, fig. 2, 1823: l'Océanie= Java (Mathews).

Cuculus neglectus Schleg., Mus. Pays-Bas, Cuculi, 1804, p. $35=$ Borneo Disir.-Malay Peninsula.

Sumatra.

Borneo; North Natuna Islands.

Java; Bali; Kangean Islands; Christmas Island.

Chalcites malayanus (Raffles).

Malay Cuckoo.

Chalcites malayanus malayanus (Raffies).

Cuculus malayanus Raffes, Trans. Linn. Soc. xiii, I822, p. 286 :

Malay Peninsula.

Distr.-Malay Peninsula.

Sumatra.

Borneo; Maratua Islands, Last Bormeo.

Java.

Gentus EUDYNAMYS Vigors and Horsfield.

Eudynamys scolopaceus (Linn1.).

Kंoel.

Eudynamys scolopaceus scolopaceus (Linn.).

Cuculus scolopaceus Limn., Syst. Nat, 10th, ed, I7.58, p. In : Bengal.

Distr.-Malay Peninstrla.

South Natuna Islands.

Eudynamys scolopaceus malayanus Cab, and Heine.'

Eudynamis malayana Cabanis and Heine, Mus. Hein. iv, I862, p. 52 : Sumatra.

Distr.-Malay Peninsula.

Smmatra; Rhio Archipelago; Banka; West Sumatran Islands of Sinalur, Babi, Lasia (vis.), Nias and Pagi.

Borueo; Karimata Islands; North Natuna Islands.

Java; Thousand Islands; Karimon-Java Islands; Kangean Islands; Bawean Island.

- Excluding obviously immature, doubtful or moulting birds the adult females of our large series of this cuckoo are readily divisible into two well defined groups. One of these consists of comparatively large rufous birds with the bill relatively robust: these we regard as malayana. The other section includes rather smaller birds in which the martings on the plumage are white and the bill less robust. These we have listed as scolopacea, but the wing-range of these birds is very large for one race and perhaps specimens of chinensis $\mathrm{Cab}$. are included. All our records of localities based on literature and not skins are included above under malayona as this is the common form: our material comes from the Malay Peninsula and Natuna Islands only. 


\section{Genus CENTROPUS Illiger.}

Centropus rectunguis Strick1.

Malaysian Crow-Pheasant.

Cenlropus rectunguis Strickland, Proc. Zool. Soc. I846, p. I04: Malacca.

Distr.-Malay States.
Sumatra,
Borneo.

Centropus sinensis ${ }^{\mathrm{s}}$ (Steph.).

Crow-Pheasant or Concal.

[Centropus sinensis sinensis (Steph.).

Polophilus sinensis J. F. Stephens in Shaw, Gen. Zool. (Aves), ix, I815, p. 51: Ningpo, China (restr. Stresemann, 1913).

Distr._Extra-limital.]

Centropus sinensis intermedius (Hume).

- Centrococcyx intermedius Hume, Stray Feathers, $i$, I873, p. 454: Thayetmyo, Burma.

Distr.-Peninsular Siam.

\section{Centropus sinensis bubutus Horsf.}

Centropus Bubutus Horsfield, Trans. Linn. Soc. Lond. xiii, I\$2I, p. 180: Java.

Centropus philippensis javanica Horsfield, Zool. Res. Java, 1823, text to pl. 60 : new name for $C$. bubutus.

Distr.-Java; Bali; Kangean Islands.

\section{Centropus sinensis eurycercus Hay.}

Centropus eurycercus Hay, Journ. Asiat. Soc. Bengal, xiv, 1845 , p. 551: Malacca.

Centrococcyx acheenensis Hume, Stray Feathers, vi, 1878, p. 17t: Acheen, North Sumatra.

Distr.-Malay Peninsula.

Sumatra; West Sumatran Islands of Nias and Siberut.

Borneo; North Natuna Islands.

Centropus bengalensis ${ }^{2}$ (Gmel.). Lesser Crow-Pheasant or Coucal.

[Centropus bengalensis bengalensis (Gmel.).

Cuculus bengalensis Gmelin, Syst. Nat. i, pt. I, 1788, p. 412: Bengal.

Distr.-Extra-linital.]

1 Revision: Stres., Nov, Zool. $x x, 1913$, p. 321.

- Revision: Stres., Nov. Zool, xix, 1912, p. 336. 


\section{CUCULIDE.}

Centropus bengalensis javanicus (Dum.).

Cuculus javanicus Dumont, Dict. Sci. Nat. II, I818, p. 144 : Java. Centropus lepidus Horsf., Trans. Linn. Soc. xiii, 1821, p. 180: Java. Centropus athnis Horsf., Trans. Linn. Soc. xiit, 1821, p. J80: Java, Centropus pumilus Less., Traité d'Orn, 1831, D. 136: Java, Sumatra = Sumatra.

Distr.-Malay Peninsula.

Sumatra; Banka; Rlio Archipelago.

Borneo; North Natuna Islands.

Java; Bali.

* Centropus nigrorufus (Cuv.).

Sunda Island Crow-Pheasant.

Cuculus nigrorufus Cuvier, Règne An. i, I8I7, p. 426: Java.

Centropus purpureus Shelley, Cat. Bds. Brit. Mus. xix, 1801, p. 348 : Sumatra.

Distr.-Sumatra.

Centropus celebensis Qtoy and Gaimard. Celebes Crow-Pheasant.

ICentropus celebensis celebensis Quoy and Gaim.

Centropus celebensis Quoy and Gaimard, Voy. Astrol, i, I830, p. 230, pl. 20: Minahassa, North Celebes.

Distr.-Extra-limital.]

*Centropus celebensis kangeangensis Vorderm.

Centropus kangeangensis Vorderman, Nat. Tijidschr. Ned. Ind. lii, 1893, p. 190: Kangean.

Distr.-Kangean Islands.

\section{Genus ZANCLOSTOMUS Swainson.}

\section{Zanclostomus javanicus (Horsf.). Red-billed Malkoha.}

Zanclostomus javanicus javanicus (Horsf.).

Phanicophaus Javanicus Horsfield, 'Trans. Linn. Soc. xiii, I82I, p. 178 : Java.

Coccyzus rubirostris Drap., Dict. Class. Hist. Nat, iv, 1823, p. 558 : Java. Piaya chrysogaster Less., Traité d'Orn. 1830, p. 140 : La Guiane error= Java.

Piaya erythrorhyncha Less., Traité d’Orn. 183o, p. 141: Java.

Distr.-Java.

Zanclostomus javanicus pallidus Rob. and Kloss.

Zanclostomus javanicus pallidus Robinson and Kloss, Journ. Fed.

Mal. States Mus. x, I921, p. 203 : Kedah Peak, Malay States.

Distr.-Malay Peninsula.

Sumatra.

Borneo. 
Zanclostomus javanicus natunensis Clias.

Zanclostomus javanicus ratunensis Chasen, Bull. Raffles Mus. 9, I934, p. 94 : Bunguran Island, North Natuna Islands.

Distr.-North Natuna Islands.

Genus RHOPODYTES Cabanis and Heine.

Rhopodytes tristis (Less.). Large Green-billed Malkoha.

[Rhopodytes tristis tristis (Less.).

Melias tristis Lesson, Traité d'Orn. 1830, p. I32: Bengal.

Distr.-Extra-limital.]

Rhopodytes tristis longicaudatus (Blyth).

Phenicophaus longicaudatus Blyth, Journ. Asiat. Soc. Bengal, x, I84I, p. 923 : Moulmein.

Distr.-Malay Peninsula.

Rhopodytes tristis elongatus (S. Müll.).

Phoenicophaus elongatus Sal. Müller, Tijd. Nat. Ges. Phys. I 835 , p. 342, pl. 4, fig. 5 : West Sumatra.

Distr.-Sumatra.

\section{* Rhopodytes tristis kangeangensis Vord.}

Rhopodytes kangeangensis Vorderman, Nat. Tijdschr. Ned. Ind. lii, I893, D. I85: Kangean.

Distr.-Kangean Islands.

Rhopodytes diardi (Less.) .

Small Green-billed Malkoha.

Rhopodytes diardi diardi (L ess.).

Melias diardi Lesson, 'Traité d'Orn. 1830, p. I32: Sumatra.

Phanicophans nigriventris Peale, U.S. Explor. Exped. viii, 1848, p. 140 : Singapore.

Distr.-Malay Peninsula.

Sumatra.

Rhopodytes diardi borneensis Salvad.

Rhopodytes borneensis Salvadori, Ann. Mus. Civ. Gen. v, I874, p. 72 : Borneo.

Distr.-Borneo.

Rhopodytes sumatranus (Raffles).

Rufous-bellied Malkoha.

Cuculus sumatranus Raffles, Trans. Linn. Soc, xiii, I822, p. 287 :

Sumatra.

Distr.-Malay Peninsula.

Sumatra; Rhio and Lingga Archipelago; Billiton and Mendanau Islands; Banka.

Borneo; North Natuna Islands. 


\section{Genus RHINORTHA Vigors.}

Rbinortha chlorophaa (Raffles) . Raffles' Green-billed Malkoha,

Cuculus chlorophcus Raffles, Trans. Linn. Soc. xiii, I822, p. 288 : Sumatra.

Phanicophans cantceps Vig., Mem. Rafles. App. 1830, p. 671: Sumatra.

Bubutus Duvaucelii Less., Traité d'Orn. 1830 , p. 143: Sumatra.

Bubutus /sidori Less., Traité d'Orn, 1830, p. 143: no loc, = Sumatra.

Cucultus sumatrensis Less., Traité d'Orm. $1830, \mathrm{p}, 143$ : as synonym.

Phavicophaus chlorocephalus Eyton, Proc. Zool. Soc. 1839, p. 105: new name for $C$. chlorophat.

Phanicophaus viridirostris Eyton, Proc. Zool. Soc. 1839, p. 105 : Malaya $=$ Malacca.

Phanicophans lacidus Blyth, Journ. Asiat, Soc. Beng. xiii, 1844, p. 390 $=$ Malacca.

Anadanus rufcauda Peale, U.S. Explor. Exp. 1848, p. 142, pl, 39 : Singapore.

Rhinortha chlorophaea fuscigularis Baker, Bull. Brit. Orn. Cl. xxxix, 1919, p. 77 : Borneo.

Distr.-Malay Peninsula.

Sumatra; Banka.

Borneo; North Bornean Islands.

\section{Genus PHCENICOPHAUS Vieillot.}

Phcenicophaus curvirostris (Shaw and Nodd.). Chestnut-breasted

Phonicophaus curvirostris curvirostris (Shaw).

Malkoha.

Cuculus curvirostris Shaw, Nat. Misc. I8ro, pl. 905: West Java.

Phanicophaus tricolor Steph., Gen. Zool. ix, 1815, p. 61, pl. 14: Africa error = Java.

Phrenicothaus viridis Vieill., Nov, Dict. d'Hist. Nat, xviii, 1818 , p. 462 : Java.

Phevicophaus melanognathus Horsf., Trans, Linn. Soc. xiii, 1821, p. I78: Java.

Phanicophaus viridirufus Bp., Consp. Gen. Av., i, 1850, p. 98: Java.

Distr.-Java (west and central).

\section{Phonicophaus curvirostris deningeri Stres.}

Phoenicophaës curviroslris deningeri Stresemann, Nov. Zool. xx, I9I3, p. 347 : Bali.

Distr.-Java (east); Bali.

Phoenicophaus curvirostris borneensis (Blas, and Nehr.).

Rhamphococcyx erythrognathus var. borneensis Blasius and Nehrhorn, Jahresbr. des Vereins Nat. Braunschweig, i881, p. 19: Sarawak.

Phoenicophaes microrhinus Berlepsch, Nov. Zool. ji, 1895, p. 70 : Borneo.

Distr.-Borneo; North Bornean Islands; North Natuna Islands. 


\section{CUCULIDE.-INDICATORIDE.}

Phonicophaus curvirostris erythrognathus $\mathrm{Bp}$.

Phaenicophaeus erythrognathus Bonaparte, Consp. Gen. Avium, i, I 850, p. 98 : Sumatra.

Rhamphoceccyx curvirostris singularis Parrot, Abh. Kon. Bay, Akad. Wiss. xxiv, 1007, D. 186: Sumatra.

Distr.-Malay Peninsula; Anamba Islands. Sumatra; Banka.

Phoenicophaus curvirostris aneicaudus J. and E. Verr.

Phanicophaus aneicaudus J. and E. Verreaux, Rev. Mag. Zool. 1855, p. 357 : Ceylon error $=$ Sipora Island, West Sumatra; (Chas, and Kloss, 1026.).

Distr,-Siberut, Sipora, North and South Pagi Islands, Mentawi Group, West Sumatra.

Genus CARPOCOCCYX G. R. Gray.

Carpococcyx radiatus ('Temmn.).

Pheasant-Cuckoo.

Carpococcyx radiatus radiatus (Temm.).

Calobates radiceus Temminck, P1. Col. 538, 1832: Borneo.

Distr.-Borneo.

Carpococcyx radiatus viridis Salvad.

Carpococcyx viridis Salvadori, Ann. Mus. Civ. Gen. xiv, 1879, p. 187 : Sumatra.

Distr. - Sumatra.

\section{Family INDICATÓRID丑.}

\section{Genus INDICATOR Stephens.}

Indieator archipelagicus Temm.

Honey-Guide.

Indicalor archipelagicus Tenminck, Pl. Col. 542, fig. I, I832: Borneo.

Indicator malayanus Sharpe, Proc. Zool. Soc. 1878, p. 794 : Malacea.

Indicator archipelagicus itornatus Neumann, Bull. Brit. Orn. Cl. xxi, 1908, p. 97 : Klang, Selangor, Malay Peninsula.

Distr-Malay Peninsula.

Sumatra,

Borneo. 


\section{Family CAPITONID在.}

\section{Genus CALORHAMPHUS Lesson.}

Calorhamphus fuliginosus: (Temm.).

Brown Barbet.

Calorhamphus fuliginosus hayi (J. E. Gray).

Bucco hayi J. E. Gray, Zool. Misc. I832, p. 33 : Malacca.

Caloramphus sanguinolentus Lesson, Rev. Zool. 1839, p. 139 : Sumatra. Megalorhynchus spinosus Eyton, Proc. Zool. Soc. 1839, p. I06: Malacca

Distr. - Malay Peninsula.

Sumatra.

Calorhamphus fuliginosus fuliginosus (Temm.).

Mycropogon fuliginosus Temminck, Pl. Col, text, livr. 83, 1830 : West Borneo (restr. Pontianak).

Distr.-Borneo (not north).

Calorhamphus fuliginosus tertius Chas. and Kloss.

Calorhamphus fuliginosus tertius Chasen and Kloss, Journ. f. Ornith. 1929, ii, p. Iro: Bettotan near Sandakan, British North Borneo.

Distr.-Borneo (north).

Genus CHOTOREA Bonaparte.

Chotorea corvina (Temm.).

Brown-throated Barbet.

Bucco corvinus Temminck, Pl. Col. 522, I $8_{30}$ : Java.

Distr.-Java (west and central).

Chotorea chrysopogon (Temm.).

Gold-whiskered Barbet.

Chotorea chrysopogon chrysopogon (Temm.).

Bucco chrysopogon Temminck, Pl. Col. 285, I824: Sumatra.

Distr.-Sumatra.

Chotorea chrysopogon laeta Rob. and Kloss.

Chotorhea chrysopogon laetus Robinson and Kloss, Journ. Fed. Malay States Mus. viii, p. 2, 1918, p. I41: Bukit Tangga, Negri Sembilan, Malay States.

Distr.-Malay Peninsula.

- Bucco lathami Gmel. may apply to a form of this species, but Latham's "Buff-faced Barbet" on which lathami was founded is described and figured with long rictal bristles: there is consequently a strong element of doubt about its identification, and we think it is more likely to be Telragonops frantsi Scl., than Xantholama hamatocephala P. L. S. Müll, in the synonomy of which it has been placed. 
Chotorea chrysopogon chrysopsis (Goff.).

Megalaima chrysopsis Goffin, Mus. Pays-Bas, Buccones, 1863, p. 15: Borneo.

Distr.-Borneo.

Chotorea javensis (Horsf.).

Black-banded Barbet.

Bucco Javensis Horsfield, Trans. Linn. Soc. xiii, 182I, p. 18I : Java.

Bucco kotorea Temm., Pl. Col. livr. 88, 1831 : Java.

Bucco tristis Drap., Dict. Class. Hist. Nat, ii, 1822, p. 195 : Java.

Distr.-Java.

Chotorea mystacophanes ('Temm.).

Gaudy Barbet.

Chotorea mystacophanes mystacophanes (Temm.).

Bucco mystacophanos Temminck, P1. Col. 315, I824: Sumatra.

Bucco quadricolor Eyton, Proc. Zool. Soc. I839, p. 105: Malacca.

Cyanops mystacophanes aurantityons Rob, and Kloss, Journ. Nat. Hist. Soc. Siam, iii, 1919, p. 100: Ghirbi, Peninsular Siam.

Distr.-Malay Peninsula.

Sumatra.

Chotorea mystacophanes humei ( $\mathrm{C}$. and G. Marshall).

Megalaema humei C. and G. Marshall, Ibis, I870, p. 536: Sarawak.

Disit,-Borneo.

* Chotorea mystacophanes ampala Oberh.

Chotorea mystacophanes ampala Oberholser, Smiths. Misc. Coll. Ix, 7, Igr2, p. 6: Tana Bala Island, Batu Islands, West Sumatra.

Distr.-Batu Islands, West Sumatra.

Chotorea rafflesii (Less.).

Many-coloured Barbet.

Chotorea rafflesii rafflesii (Less.).

Bucco Rafflesii Lesson, Rev. Zool. I839, p. I37 : Sumatra.

Bucco versicolor (nec P. L. S. Muller, 1776) Raffes, Trans, Linn. Soc. xiii, 1820, p. 284 : Sumatra.

Distr.-Sumatra; Banka.

Chotorhea rafflesii malayensis Chas.

Chotorhea rafflesii malayensis Chasen, Oruith. Monatsber. I935, p. I47: Pulau Ubin near Singapore.

Distr.-Malay Peninsula.

Chotorhea rafflesii billitonis Chas.

Chotorhea rafflesii billitonis Clasen, Ornith. Monatsber. 1935, P. I47:

Billiton Island.

Distr,-Billiton; Mendanau. 
Chotorea rafflesii borneensis Blas.

Chotorea versicolor bormeensis Blasius, Verh, z. b. Ges. Wien, xxxiii, I883, p. 25: South-east Borneo.

Distr.-Borneo.

\section{Genus CYANOPS Bonaparte.}

Cyanops asiatica (Lath.).

Blue-throated Barbet.

[Cyanops asiatica asiatica (Lath.).

Trogon asiaticus Latham, Index Orn. i, I790, p. 20I: India.

Distr._Extra-limital.]

Cyanops asiatica chersonesus Chas. and Kloss.

Cyanops asialica chersonesus Chasen and Kloss, Bull. Brit. Orn.

Cl. xlviii, 1927, p. 46: Khao Luang, Nakawn Sritarmarat, Peninsular Sian.

Distr.-Peninsular Siam.

Cyanops armillaris (Teinm.).

Blue-crowned Barbet.

Cyanops armillaris armillaris (Temm.).

Bucco armillaris Temminck, Pl. Col. 89, I823: Java : restr. Province of Bantam, West Java.

Distr.-Java (west and central).

Cyanops armillaris baliensis Rensch.

Cyanops armillaris baliensis Rensch, Orn. Monatsber. xxxvi, 1828, p. 80 : Bali.

Distr,-Java (extreme east); ${ }^{1}$ Bali.

Cyanops armillaris henricii (Temm.).

Bucco henricii Temminck, P1. Col. 524, I831 : Sumatra.

Bucco malaccensis Hartl., Rev. Zool. 1842, p. 336: Malacca.

Distr.-Malay States.

Sumatra.

Cyanops armillaris brachyrhyncha Neum.

Cyanops henricit brachyrhyncha Neumann, Bull. Brit. Orn. Cl. xxiii, 1908, p. 30: Baram, Sarawak.

Distr.-Borneo. ${ }^{3}$

A large series from Banjoewangi, East Java, seems just separable on average characters, from armillaris of West Java.

${ }^{2} C$. henricii seems to be rare in its type locality and we have never been able to compare a series of topotypes with birds from the Malay States and Borneo. 
Cyanops armillaris pulcherrima (Sharpe). Yellow-headed Barbet.

Megalama pulcherrima Sharpe, Ibis, I885, p. 393; Mt. Kinabalu, North Borneo.

Distr,-Borneo.

Cyanops franklinii (Blyth).

Golden-throated Barbet.

[Cyanops franklinii franklinii (Blyth).

Bucco Franklinii Blyth, Journ. Asiat. Soc. Beng. xi, I842, p. I67:

Darjeeling.

Distr.-Extra-limita1.]

Cyanops franklinii minor Chas. and Kloss.

Cyanops franklini minor Chasen and Kloss, Bull. Brit. Orn. Cl. xivi, 1926, p. 57 : Mt. Ijau, Perak, Malay States.

Distr.-Malay States.

*Cyanops franklinii trangensis Riley.

Cyanops franklini trangensis Riley, Proc. Biol. Soc. Wash. xlvii, 1934, p. II6: Trang, Peninsular Siam.

Distr.-Peninsular Siain.

Cyanops oorti (S. Müll.).

Müller's Barbet.

Cyanops oorti oorti (S. Mijll.).

Bucco Oorti Sal. Müller, Tijd. Nat. Ges. Phys. ii, I835, p. 34I, pl. iv, fig. 4: Sumatra.

Distr.-Malay States.

Sumatra.

Cyanops oorti monticola Shatpe.

Mountain Barbet.

Cyanops monticola. Sharpe, Ann. Mag. Nat. Hist. (6) iii, I889, p. 424: Mt. Kinabalu, North Borneo.

Distr.-Borneo.

Cyanops australis" (Horsf.). Little Barbet.

Cyanops australis australis (Horsf.).

Bucco australis Horsfield, Trans. Linn. Soc. xiii, I821, p. IOI: Java.

Distr.-Java.

"If "Bucco cyanocephalus" Reinw., Batav, Courant, Feb, 1820 , is a valid description it is, perhaps, the species name for this group of small barbets, but as in the case of other references to the "Batav. Courant" given in Cat. Bds. xix, l cannot trace the original. 
*Cyanops australis hebereri (Rensch)."

Xantholaema australis hebereri Rensch, Mitt. Zool. Mus. Berlin, xvi, 1930, p. 532 : Bali.

Distr.-Bali.

Cyanops australis duvaucelii (Less.).

Bucco duvaucelii Lesson, Traite Ornith. 1830, p. 164 : Sumatra.

Megalama duvauceli borneonensis Parrot, Abh. Kon. Bay. Akad. Wiss., Klass 2, 24, i, 1007: Borneo.

Cyanops duvaceli robinsoni Baker, Bull, B. O. Cl. xxxix, 1918, p. 20 : Klang, Selangor, Malay States.

Bucco frontalis Temm., Pl. Col. 536, fig. 1, 18,32: Sumatra.

Distr.-Malay Peninsula (north to Perlis).

Sumatra; Banka.

Borneo.

Cyanops australis stuarti (Kob. and Kloss).

Mesobucco duvauceli stuarti Robinson and Kloss, Journ. Nat. Hist. Soc. Sian, iii, I9I8, p. I0o: Junk Seylon.

Distr.-Peninstitar Siam.

-Cyanops australis gigantorhina (Oberh.).

Mezobucco duvaucelit gigantorhinus Oberholser, Smiths. Misc. Coll. Lx, 7, 1912, p. 6: Nias Island, West Sumatra.

Distr.-Nias Island, West Sumatra.

* Cyanops australis tanamassae (de Schau.).

Mesobucco duvauceli tanamassae de Schauensee, Proc. Acad. Nat.

Sci. Phil. Ixxxi, 1929, p. 52 I : Tana Masa Island, Batu Islands, West Sumatra,

Distr.-Batu Islands, West Sumatra.

Cyanops australis eximia (Sharpe).

Mesobucco eximius Sharpe, Ibis, I892, p. 324 : Mt. Dulit, Sarawak.

Distr.-Borneo (part).

Cyanops australis cyanea (Hart. and Hart.).

Mesobucco duvaucelii cyanews Harrisson and Hartley, Bull. Brit.

Orn. C1. liv, I934, P. I5I : Mt. Kinabalu, N. Borneo.

Distr.-Mt. Kinabalı, North Borneo.

- We have not seen hebereri, but the difference in size between it and australis must be very slight the maximum wing length of our series of the latter form trom West Java is $77 \mathrm{~mm}$, and from East Java $78 \mathrm{~mm}$.

2 The type is a female, wrongly sexed on the label; other than this the race seems to rest on one male and an unsexed juvenile (measurements reversed in the original description) in the Singapore Museum. 


\section{Genus THEREICERYX Blanford.}

Thereiceryx zeylanicus (Gmel.).

Dull Barbet.

[Thereiceryx zeylanicus zeylanicus (Gmel.).

Bucco Zeylanicus Gmelin, Syst, Nat. i, pt. i, 1788, p. 408: Ceylon. Distr._Extra-limital.]

Thereiceryx zeylanicus intermedius Haker.r

Thereiceryx lineatus intermedius Stuart Baker, Bull. B.O. Cl. xxxix, I9I8, p. I9: North Tenasserim.

Distr.-Malay Peninsula (not in the Federated Malay States).

Thereiceryx zeylanicus lineatus (Vieill.), 2

Capito lineatus Vieillot, Nov. Dict. Hist. Nat, iv, I8I6, p. 500 : Java (Stresemann).

Bucco fuscicapillus Drap., Dict. Class, ii, 1822, p. 195: Java.

Distr.-Java; Bali.

\section{Genus XANTHOLAMA Bonaparte.}

Xantholæma hamacephala (P. L.: S. Müil.). Coppersmith Barbet.

[Xantholsema hremacephala hemacephala (P. L. S. Müll.).

Bucco hamaccphalus P. L. S. Müller, Natursyst. Suppl., I776, p. 88: Philippine Islands.

Distr.-Extra-limital.]

Xantholzema hsemacephala indica (Lath.).

Bucco indicus Latham, Index Ort, i, r790, p. 205 : India.

Distr.-Malay Peninsula (not in the south).

Xantholæema hæmacephala delica (Parrot).

Megalaema haemacephala delica Parrot, Abh. Kon. Bay. Akad. Wiss. 2. K1. 24. Bd. 1. Abt., I907, p. 169: Deli, Sumatra.

Disir.-Sutnatra.

I We do not agree with C. B. Ticehurst (Journ. Bomb. Nat. Hist. Soc. xxxiv, 1930, p. 470) that this race is unrecognizable $(119-139 \mathrm{~mm}$. which is the range given by him for northern birds seems a very large range for one race) but we think it is probably the same as McClellandi Horsf. and Moore, Cat. Birds. Mus. East Ind. Coy. ii, 1858, p. 637 (North-east Bengal).

"Although it is possible that this race occurs in Cochin China "lineatus" cannot be used to cover the large birds occurring in other parts of French Indo-China. 
Xantholema hemacephala rosea (Dum.), ,

Bucco rosetus Dumont, Dict. Sci. Nat. iv, I806, p. 52 : Java.

Capito rasaceicollis Vieill., Nov. Dict. Hist. Nat. iv, 1816, p. 500: Java.

Distr.-Java; Bali.

Sumatra (south).

Genus PSILOPOGON S. Müller.

Psilopogon pyrolophus S. Müill.

Fire-tufted Barbet.

Psilopogon pyrolophus S. Müller, Tijd. Nat. Gesch. Phys, ii, 1835, p. 339, P1. iv, fig. 3: Sumatra.

Buccotrogon torquatus Von Kreling, Jahr. Nat. Gesch. Emden, 1852, p. 20 (not seen; fide Cat. Bds. xix, p. 98.).

Distr.-Malay States.

Sumatra.

\section{Family PICID瓜.}

\section{Genus PICUS Linn.}

Picus vittatus ${ }^{3}$ Vieill.

Bamboo Green Woodpecker.

Picus vittatus vittatus Vieill.

Picus vittatus Vieillot, Nov. Dict. d'Hist. Nat. xxvi, I818, p. 9 I :

Java (Hartert, 1902; restr. West Java, Stresemann, 1921).

Picus affnis Raffles, Trans. Linn. Soc, xiii, 1822, p. 288 : Sumatra.

Picus dimidiatus Valenc, (ex Horsf. MS.), Dict. Nat. Sci. xl, 1826, p. 174 : Java.

Gecinus xanthopygius Bp., Consp. Gen. Av, i, 1850, p. 127: Java.

Distr.-Malay States.

Sumatra; Iringga Archipelago.

Java (west).

Picus vittatus limitans Stres.

Picus vitlatus limilans Stresemann, Arch, f. Naturgesch. 1xxxvii, I921, Ab. A, Heft 7, p. 74: East Kangean Island.

Distr.-Java (east); Bali; Kangean Islands.

- We follow Bartels and Stresemann and treat rosea as a subspecies of hiemacephala but it must be noted that both forms have been recorded from the Lampongs : the only record for rosea seems to be that of the two birds obtained by Buxton and recorded by Walden (Ibis, I377, p. 487) and it is time that the presence of rosea in Sumatra, if correct, was confirmed.

"Cf. Stres, "Die Spechte der Insel Sumatra", Archiv. f. Naturg. tgzt, p. 64: Hesse, Kritische Untersuchungen über Piciden im Berlin p. 64; Hessé, Kritische Untersuchungen über Piciden im Berlin Mus., Mitt. Zool. Mus. Berl. vi, 1912 , p. 133 .

3 Ct. Kloss, Ibis, 1926, p. 684 . 
Picus vittatus connectens (Rob. and Kloss).

Gecinus vittatus connectens Robinson and Kloss, Bull. Brit. Orn.

Cl. xl, I9I9, D. 13: Langkawi Islands, Straits of Malacca.

Dist\%.L_angkawi Islands, Straits of Malacca.

Picus viridanus' Blyth.

Scaly-bellied Woodpecker.

[Picus viridanus viridanus B]yth.

Picus viridanus Blyth, Journ. Asiat. Soc. Bengal, xii, I843-44, p. 1000: Arracan.

Distr._Extra-limital.]

\section{Picus viridanus weberi Müll.}

Gecintus weberi Müller, Orn. Ins. Salanga, I882, p. 69 (sep.) : Junk Seylon.

Picus viridanus meridianus Kloss, Ibis, 1926, p. 689: Trang, Peninsular Siam.

Distr.-Peninsular Siam.

Picus canus² Gmel.

Black-raped Green Woodpecker.

[Picus canus canus Gmel.

Picus canus Gijelin, Syst. Nat. I, pt. I, I 788, p, 434 : Norway.

Distr._Extra-limital.]

Picus canus robinsoni (O.-Grant).

Gecinus robinsoni Ogilvie-Grant, Bull. Brit. Orn. Cl. xix, Igo6, p. Io: Gunong Tahan, Pahang, Malay States.

Distr.-Malay States.

Picus canus dedemi (van Oort).

Gecinus dedemi van Oort, Notes Leyden Mus, xxxiv, 1911, p. 59 : Battak Mountains, North-east Sumatra.

Distr.-Stumatra.

Picus chlorolophus Vieill. ${ }^{3}$

Small Yellow-raped Woodpecker.

[Picus chlorolophus chlorolophus Vieill.

Picus chlorolophus Vieillot, Nouv. Dict. Hist. Nat. xxvi, 1818 , p. 78 : Bengal.

Distr. -Extra-limital.]

Picus chlorolophus rodgeri (Hart, and Butlr.).

Gecinus rodgeri Hartert and Butler, Nov. Zool. v, I 898 , p. 508 :

Mt. Ijau, Perak, Malay States.

Distr.-Malay States.

I Cf. Kloss, Ibis, 1926, p. 684 .

- Revision: Snouck. v. Schaub.; Org. Cl. Ned. Vog. v, 1932, p. 4.

з Cf. Meinert2., Bull. B. O.. C. Xliv, 1924, p. 53 . 
PICIDA.

Picus chlorolophus vanheysti (Rob, and Kloss).

Brachylophus chlorolophus vanheysti Robinson and Kloss, Journ.

Straits Branch Roy. Asiat. Soc. 1xxx, I9r9, p. 97: Sumatra.

Distr. - Sumatra.

Picus puniceus Horsf. Crimson-winged Woodpecker.

Picus puniceus puniceus Horsf.

Picus puntceus Horsfield, Trans. Linn. Soc. xiii, I821, p. I76: Java.

Picus gularis Wagl., Syst. Av. Add., Picus, sp. no. 6, (nec no.8g) \& 1827.

Distr,_Java,

Picus puniceus continentis (Rob. and Kloss).

Brachylophus puniceus continentis Robinson and Kloss, Journ.

Fed. Malay States Mus. x, 1921, p. 204: Tapli, Pakchan

Estuary, Релinsular Siam.

Distr.-Malay Peninsula.

Picus puniceus observandus Hart.

Gecinus puniceus observandus Hartert, Nov. Zool. iii, I8g6, p. 542 : Sumatra.

Distr.-Sumatra; Banka; Nias Island, West Sumatra. Borneo.

\section{Genus CALLOLOPHUS Salvadori.}

Callolophus miniaceus (Penn.).

Banded Red Woodpecket.

Callolophus miniaceus miniaceus (Penr.).

Picus mineaceus Pennant, Ind. Zool. $1769, \mathrm{p} 1.4$ : India, error = Java.

Distr.-Java (west and central).

Callolophus miniaceus malaccensis (Lath.).

Picus malaccensis Latham, Index Orn. i, r790, p. 24I: Malacca.

Distr.-Malay Peninsula.

Sumatra; Banka; Billiton.

Borneo (north-west). ${ }^{3}$

Callolophus miniaceus dayak Stres.

Callolophus miniatus dayak Stresemann, Archiv. f. Naturgesch. lxxxvii, I921, Ab. A, Heft 7, p. 82: Landak, West Borneo.

Distr,-Borneo.

' C. $m$. dayak is a good race apparently occupying most of Borneo but this larger winged form occurs on the lower slopes of Mt. Kinabalu. 
PICIDF.

Callolophus miniaceus niasensis (Bittt.).

Chrysophlegma niasense Büttikofer, Notes Leyden Mus. xviii, I896, p. I69: Nias Island, West Sumatra.

Disly.-Nias Island, West Sumatra.

\section{Genus CHRYSOPHLEGMA Gould.}

Chrysophlegma mentale (Temm.). Chequered-throated Woodpecker. Chrysophlegma mentale mentale (Temm.).

Picus mentalis Temminck, Pl. Col. 384,1825 : Java.

Picus squamicollis Le55. Traité d'Ornith. 1831, p. 229: Java.

Distr.-Java.

Chrysophlegma mentale humii Harg.

Chrysophlegma humii Hargitt, Ibis, I889, p. 231 : Malacca.

Distr.-Malay Peninsula. Sumatra; Banka.

Borneo (western half).

Chrysophlegma mentale saba Chas. and Kioss.

Chrysophlegma mentale saba Cliasen and Kloss, Journ. f. Ornith. 1929, ii, p. II : Sandakan, British North Borneo.

Distr.-Borneo (east).

Chrysophlegma flavinucha (Gould). Large Vellow-naped Woodpecker. [Chrysophlegma flavinucha flavinucha (Gould).

Picus flavinucha Gould, Proc. Zool. Soc. for 1833 , 1834, p. I20: East Himalayas.

Distr.-Eixtra-limital.]

Chrysophlegma flavinucha wrayi Sharpe.

Chrysophlegma wrayi Sharpe, Proc. Zool. Soc. I888, p. 279 : Mts. of Perak, Malay States.

Distr.-Malay States.

Chrysophlegma ftavinucha mystacale Salvad.

Chrysophlegma mystacalis Salvadori, Ann. Mus. Civ. Gen. xiv, 1879, p. I82: Stumatra.

Distr.-Sumatra. 


\section{Genus CHLOROPICOIDES Malherbe.}

Chloropicoides rafflesii (Vig.).

Three-toed Woodpecker.

Chloropicoides rafflesii rafflesii (Vig.).

Picus Rafflesii Vigors, Raff. Mem. I8 3 I, App. p. 669 : Sumatra.

Tiga amictus Gray, Gen. Birds, ii, 1846, p. 441 : nom, nud.

Picus (Tiga) labarum Less, Ois. réc. découv, 1847, p. 199 : Sumatra (Duvaucel).

Distr.-Sumatra; Banka.

Chloropicoides rafflesii peninsularis Hesse.

Gauropicoides raffesi peninsularis Hesse, Ornith. Monatsber. xix, I911, p. I92: Malacca.

Distr.-Malay Peninsula.

Chloropicoides rafflesii borneonensis Hesse.

Gauropicoides raffesi borneonensis Hesse, Orn. Monatsber. xix, I9II, p. I92: Mt. Dulit, North Sarawak, Borneo.

Distr.-Borneo.

\section{Genus GECINULUS Blyth.}

Gecinulus grantia (Horsf.).

Pale-headed Woodpecker.

[Gecinulus grantia grantia (Horsf.).

Picus Grantia Horsfield, Proc. Zool. Soc. for I839, I840, p. I65: Assam.

Distr.-Extra-limital. ]

Gecinulus grantia robinsoni Kloss.

Gecinulus viridis robinsoni Kloss, Ibis, I9I8, p. I05: Ginting Bidei, Selangor-Pahang boundary, Malay States.

Distr.-Malay Peninsula.

\section{Genus DRYOBATES Boie.}

Dryobates analis ( $\mathrm{Bp}$.$) .$

Spotted-breasted Pied Woodpecker.

Dryobates analis analis (Bp.).

Picus analis Bonaparte, Consp. Ger. Av. i, 1850, p. I37: Java, restr. East Java.

Dendrocophus a. brevipenus Hesse, Ornith. Monatsber. 1011, p. $18 z$ : Java.

Distr.-Sumatra. * Java; Bali.

- Specimens from Peninsular Siam are intermediate between robinsoni and viridis Blyth (Tenasserim).

"The locality "Sumatra" rests on Walden's record of birds collected by E. G. Buxton in the Lampongs (Ibis, 1877, B. 200). As in the case of Xintholama rosea confirmation is needed. Buttikofer considers that the "Picus percussus" of Nieuwenhuisen and Rosenberg from Nias is also D. analis (Notes Leyd. Mus. xviti, i806, p. 195). 
Dryobates analis montis Rob. and Kloss.

Dryobatis analis montis Robinson and Kloss, Journ. Fed. Malay States Mus. xi, 1923, p. 53: Tjibodas, West Java.

Distr.-Java' (mourtains of west).

Dryobates hardwickii (Jerd.).

Pigmy Woodpecker.

[Dryobates hardwickii hardwickii (Jetd.).

Picus (Dendrocopus) hardwickii Jerdon, Madras Journ. Lit. Sci. xiii, I844: South India.

Distr. - Extra-limital.]

Dryobates hardwickii canicapillus (Blyth).

Picus canicapillus Blyth, Journ. Asiat. Soc. Beng. xiv, I845, p. I97: Arracan.

Distr,-Peninsular Siam,

Dryobates hardwickii auritus (Eyton).

Tripsurus auritus Eyton, Ann. \& Mag. Nat, Hist. Xvi, I 845, p. 229 : Malacca.

Dryobates canicapillus suffusus Rob. and Kloss, Bull. Brit. Orn, Cl, xl, 1919, p. 14: Kuala Lumpur, Selangor, Malay States.

Distr.-Malay States.

*Dryobates hardwickii volzi Stres.

Dryobates canicapillus volzi Stresemann, Verh. Orn. Ges. Bayern, xiv, 1920, p. 288 : Laut Tawar, North Sumatra.

Distr.-Sumatra.

Dryobates hardwickii aurantiiventris (Salvad.).

Picus (Bcopipo) hardwickit aurantiventris Salvadori, Afti $\mathrm{R}$. Ac. Sci. Torino, iii, 1868, p. 524 : Sarawak.

lyngipicus picatus Hargitt, Ibis, 1882, p. 41: North-west Borneo. ,

Distr.-Bormeo. ${ }^{2}$

Dryobates moluccensis (Gmel.).

Lesser Pigmy Woodpecker.

Dryobates moluccensis moluccensis (Gmel.).

Picus moluccensis Gmelin, Syst, Nat. I, pt. 1, I788, p. 439 : Malacca (Oberholser).

Picus variegalus Wagl, (nec Lath.), Syst. Av. Gen. Picus, sp. 26, 1827: Java.

1 This race does not inhabit the whole of West Java but only the hills in that part of the island: birds from Wynkoops Bay on the south coast are like typical analis. Our series consists of eleven montis against eighteen analis

"Kuroda has recorded "picatus" from Tawao on the east coast of British North Borneo and there is just a possibility that it is a good race with a very limited range. 
Iyngipicus tusco-albidus Salvad, Ucc. Born. 1874, p. 42: Java (new name for $P$. variegatus Wagl.).

Picus sondaicus Salvad. Ucc. Born. 1874, p. 43: Java.

fyngipicus /rater Salvad, and Gigl., Atti della R. Accad. Sc. Tor, xx, 1885, p. 824 : Malacca.

Dryobates moluccensis tantulus Riley, Proc. Biol. Soc. Wash. xl. tg27, p. 140: Tanggarong, N. E. Borneo.

Distr.-Malay States.

Sumatra; Rhio Archipelago; Billiton.

Borneo; North Borneo Islands.

Java; Bali.

\section{Genus BLYTHIPICUS Bonaparte.}

Blythipicus rubiginosus (Swains.).

Maroon Woodpecker.

Blythipicus rubiginosus rubiginosus (Swains.).

Hemicircus rubiginosus Swainson in Jardine's Nat. Libr., Birds West Africa, ii, 1837 , p. 150 : West Africa error $=$ Malacca (Harilaub).

Picus melanogastey Hay, Madr, Journ. 1844, p. 153: Malacca.

Distr.-Malay Peninsula.

Blythipicus rubiginosus parvus Chas. and Kloss.

Blythipicus rubiginosus paryus Chasen and Kloss, Journ. f. Ornith.

I929, ii, p. II2: Bettotan near Sandakan, British North Borneo.

Distr.-Sumatra.

Borneo.

Blythipicus pyrrhotis (Hodgs.). ${ }^{1}$

Bay Woodpecker.

[Blythipicus pyrrhotis pyrrhotis (Hodgs.).

Picus pyrrhotis Hodgson, Journ. Asiat. Soc. Bengal, vi, I837, p. ros: Nepal.

Distr._Extra-linital.]

Blythipicus pyrrhotis cameroni Rob.

Blythipicus pyrrholis cameroni Robinson, Bull. Brit. Orn. Cl, xlviii, 1928, p. 57 : Mt. Mengkuang Lebah, Selaugor, Malay States.

Distr.-Malay States.

\section{Genus MEIGLYPTES Swainson.}

Meiglyptes tristis (Horsf.). Fulvous-rumped Barred Woodpecker. Meiglyptes tristis tristis (Horsf.).

Picus trislis Horsfield, Trans. Linn. Soc. xiii, I82I, p. 177 : Java.

Picus poicilophus Temm., Pl. Col. 197, 1823 : Java.

Distr.-Java.

' Cf. Kinn., Bull. B. O. C. xlvi, 1926, D. 72. 
Meiglyptes tristis micropterus Hesse.

Meiglyples tristis micropterus Hesse, Orn. Monatsber. xix, IgI, p. $18 z$ : Borneo.

Distr. - Sumatra,

Borneo; North Bornean Islands; North Natuna Islands.

Meiglyptes tristis grammithorax (Malh.).

Phaiopicus grammithorax Malherbe, Monogr. Pic. ii, p. I2, I862, pl. 48: Malay Peninsula.

Distr.-Malay Peninsula.

* Meiglyptes tristis microterus Oberh.

Meiglyptes granmithorax microterus Oberholser, Smiths. Misc. Coll.

$1 \mathrm{x}, 7$, 1912, p. 6: Nias Island, West Sumatra.

Distr.-Nias Island, West Sumatra.

Meiglyptes tukki (Less.). Buff-necked Barred Woodpecker.

Meiglyptes tukki tukki (Less.).

Pictus tukki Lessor, Rev. Zool. I 839, p. 167 : Sumatra.

Hemicircas brunneus Eyton, Proc. Zool, Soc. 1839, p. 106 : Malacca.

Picus luridus Nitzsch, Pterylog, 1840, p. 137: Sumatra.

Meiglyptes fuscus Peale, U.S. Expl, Exped, viii, 1848, p. 132: Singapore.

Meiglyptes tukki araleus Oberh., Bull. U.S. Nat. Mus. 159. 1932, p. 46 :

Bunguran Island, North Natuna Islands.

Distr.-Malay Peninsula.

Sumatra; Billiton.

Borneo; North Natuna Islands.

-Meiglyptes tukki percnerpes Oberh.

Meiglyptes tukki percnerpes Oberholser, Journ. Wasl. Acad. Sci. xiv, I3, I924, p. 30I: Batu Jurong, South-west Borneo.

Distr-Borneo (South-west).

Meiglyptes tukki pulonis Chas. and Kloss.

Meiglyptes tukki pulonis Chasen and Kloss, Journ. f. Ornith. I929, ii, p. II2: Banguey Island, North Bormeo.

Distr.-Banguey Island, North Borneo.

\section{*Meiglyptes tukki calceuticus Oberh.}

Meiglyptes tukki calceuticus Oberholser, Smiths. Misc. Coll. 1x, 7, Igr2, p. 6: Tuanku Island, Banjak Islands, West Sumatra.

Distr.-Banjak Islands, West Sumatra.

* Meiglyptes tukki infuscatus Salvad.

Miglyptes infuscatus Salvadori, Ann. Mus. Civ. Stor. Nat. Genov.

(2) iv, I887, p. 53 : Nias Island, West Sumatra.

Meiglyptes tukki hylodromus Oberh., Journ. Wash. Acad. Sci. xiv.

No. 13,1924, p. 301 : Nias Island, West Sumatra.

Dislr,-Nias Island, West Sumatra. 
PICIDE.

\section{Genus MICROPTERNUS Blyth.}

Micropternus brachyurus (Vieill.).

Rufous Woodpecker.

Micropternus brachyurus brachyurus (Vieill.).

Picus brachyurus Vieillot, Nouv, Dict. Hist. Nat. xxvi, I8I 8 , D. 103 : Java.

Distr, - Java (not east).

Micropternus brachyurus badius (Raffles).

Picus badius Raffles, Trans. Linn. Soc. xiii, 1822, p. 289 : Smmatra.

Distr.-Sumatra; Banka; Billiton and Mendanau Islands.

* Micropternus brachyurus celaenephis Oberh.

Microptemus phaioceps celaenephis Oberholser, Smiths. Misc. Coll. $1 \mathrm{x}, 7,1912$, p. 6: Nias Island, West Sumatra.

Distr.-Nias Island, West Sumatra.

Micropternus brachyurus squamigularis (Sundev.).

Picus squamigularis Sundevall, Consp. Av. Picin. I866, p. 89: Malacca.

Distr.-Malay Peninsula."

Micropternus brachyurus badiosus (Bp.).

Meiglyptes badiosus Bonaparte, Consp. Gen. Avium, i, I850, p. II3 : Borseo.

Distr.-Borneo; North Natuna Islands.

\section{Genus DINOPIUM Rafinesque.}

Dinopium javanense (Ljungh). Golden-backed Three-toed WoodDinopium javanense javanense (Ljungh). pecker.

Picus javanensis Ljungh, Kon. Vet. Akad. Handl, xvii, I797, D. I34, pl. 6: Batavia, West Java.

Picus tiga Horsf., Trans. Linn. Soc. xiii, 1821 , 1), 177 : Java.

Chrysonotus tridactylus Swains., Class. Birds, ii, 1937, p. 309 : new name.

Tiga rufa Stoliczka, Journ. Asiat. Soc. Bengal, xxxix, 187o, p. 280 : irenaming of tridactylus.

Dinopim javanense palmarum Stres., Arkiv, f. Naturgesch. 102r,

Ab. A, Heft 7, p. 93: Fort de Kock, West Sumatra.

Distr.-Malay Peninsula.

Sumatra; Rhio Archipelago.

Java (west and central).

: Birds from Peninsular Siam are intermediate between squamigularis and williamsoni Ḱloss (Koh Lak; S. W. Siam). 
Dinopium javanense exsul Hart.

Tiga javanensis exsul Hartert, Nov. Zool. viii, 1901, p. 5t: Bali.

Distr._Java (east); Bali.

Dinopium javanense intermedium (Blyth).

Picus (Tiga) intermedius Blyth, Journ. Asiat. Soc. Bengal, xiv, I 845 , p. I93: Ramree Island, Arracan.

Distr.-Peninsular Siam (extreme north).

Dinopium javanense borneonensis (Dub.).

Tiga borneonensis Dubois, Proc. Zool. Soc. I897, p. 782: Borneo.

Disir.-Borneo.

*Dinopium javanense raveni Riley.

Dinopium raveni Riley, Proc, Biol. Soc. Wash. xl, 1927, p. I39: Eraban Island, North-east Borneo.

Distr.-Ëraban Island and ? Eastern Borneo.

\section{Genus CHRYSOCOLAPTES Blyth.}

Chrysocolaptes lucidus: (Scop.). Golden-backed Four-toed Wood[Chrysocolaptes lucidus lucidus (Scop.). pecker.

Picus lucidus Scopoli, Del. Flor. et Faun. Insubr. ii, I786, p. 89 : Mindanao, Philippine Islands.

Distr.-Extra-limital.]

Chrysocolaptes lucidus gutticristatus (Tick.).

Picus guttacristatus Tickell, Journ. Asiat. Soc. Bengal, ii, I833, P. 578 : Borabhum.

Chrysocolaptes gutticristatus indo-malayicus Hesse, Orn. Monatsber. xix, I91I, p. 182: Junk Seylon.

Distr.-Peninsular Siam (south to Langkawi Islands).

Chrysocolaptes lucidus chersonesus Kloss.

Chrysocolaptes strichus chersonesus Kloss, Ibis, IgI8, p. II3:

Sikarang, Sotuth Johore.

Distr.-Malay States (extreme south and Singapore Island).

Sumatra; Rhio Archipelago.

Java (west and central).

I Cf. Rob., Journ. F.M.S. Mus. vii, 1917, p. 161. 
Chrysocolaptes Iucidus strictus (Horsf.)."

Picus strictus Horsfield, Trans. Linn. Soc. xiii, $1821, p .176$ : Java.

Distr.-Java; Bali; Kangean Islands.

Chrysocolaptes validus ('Temm.).

Chrysocolaptes validus validus (Temtn.).

Picus validus Temminck, P1. Col. 378,1825 : Java.

Distr.-Java (west and central).

Chrysocolaptes validus xanthopygius Finsch.

Chrysocolaples xanthopygius Finsch, Notes Leyden Mus. xxvi, 1905, p. 34: Upper Mahakam River, West Borneo.

Distr.-Malay Peninsula.

Sumatra.

Borneo; North Natuna Islands.

\section{Genus HEMICIRCUS Swainson.}

Hemicercus concretus (Temm.). Grey-and-Buff Woodpecker.

Hemicercus concretus concretus (Temm.).

Picus concretus Temminck, Pl. Col, 90, I $8_{24}$ : Java.

Distr.-Java (west and central).

Hemicercus concretus coccometopus (Reichb.).

Hemicercus coccometopus Reichenbach, Scans. Picinae, I854, p. 401, pl. 656: Sumatra.

Hemicercus brookeanus Salvad., Atti. R. Ac. Sc. Tor, iii, I868, Sarawak, Borneo.

Disir.-Sumatra; Banka Island.

Borneo.

Hemicircus concretus sordidus (Eyton).

Dendropicus sordidus Eyton, Ann. Mag. Nat. Hist, xvi, I845, p. 229: Malacca.

Distr.-Malay Peninsula.

Hemicircus canente (Less.). Heart-spotted Woodpecker.

Hemicircus canente canente (Less.).

Picus canente Lesson, Cent. Zool. IS32, p. 2I5: Pegu.

Distr.-Peninsular Sian.

I There is broadly speaking a large overlap in the range of gutticristatus and strictus in Java but at present we prefer to retain both forms as subspecies of lucidus: strictus seems to be found only in the mountains: commonly in East Java and very rarely in West Java. C. L. gutticristatus is rare in Java and according to the information at our disposal seems to occur only on the coastland in the western balf of the island: it is perhaps a recent arrival from Sumatra. 


\section{Genus MÜLLERIPICUS Bonaparte.}

Mülleripicus pulverulentus (Temm.).

Great Slaty Woodpecker.

\section{Mülleripicus pulverulentus pulverulentus (Temm.).}

Picus pulverulentus Temminck, P1, Col. 389, I826: Java.

Picus gutturalis Valenc., Dict. Sc. Nat. xl, 1826, p. 178: Sumatra.

Pictus mackloti Wagl., Syst. Av. Picus, 1827, 5p. 4 : Sumatra.

Picus horsheldi Wagl., Syst. Av. Picus, 1827, sp. 5: Java.

Hemilophus mülleri Bp., Consp. Gen. Av. i, 1850, p. 13i: Borneo.

Hemilophus fischeri Brügg., Abhandl. Nat. Ver. Brem. 1877, D. 454: Borneo.

Distr.-Malay Peninsula.

Sumatra; Rhio Archipelago.

Borneo; North Natuna Islands.

Java (not in the east).

\section{Genus DRYOCOPUS* Boie.}

Dryocopus javensis (Horsf.).

Great Black Woodpecker.

Dryocopus javensis javensis (Horsf.).

Picus javensis Horsfield, Trans. Linn. Soc. xiii, I82I, p. I75 : Java.

Picus leneogaster Valenc., Dict, Sci. Nat. xl, 1827, p. 178 : Java (Stresemann, $\mathrm{IOH}_{3}$ ).

Picus horsfeldi Wagl., Syst. Av. 1827 , Picus, $5 p .5$ : new name for $P$. javensis.

Picus fulvigaster Drap., Dict. Class. Hist, Nat. xiii, 1828 , p. 503 : Java.

Picus maximus malayensis Bland, Journ. Asiat. Soc. Bengal, vi, 1837 , p. 952: Johore.

Distr.-Malay Peninstla.

Sunnatra; Rhio Archipelago; Banka; Mansalar Island, West Sumatra.

Borneo; North Natuna Islands; North Bornean Islands.

Java; Bali.

Dryocopus javensis parvus (Richm.).

Thriponat parvus Richmond, Proc. Biol. Soc, Wash. xv, Igo2, p. I89: Simalur Island, West Sumatra.

Distr.-Simalur Island, West Sumatra.

*Dryocopus javensis büttikoferi (Richm.).

Thriponax javensis büttikoferi Richmond, Proc. Biol. Soc. Wash. xxv, Igr2, p. Io4: Nias Island, West Sumatra.

Distr.-Nias Island, West Sumatra.

1. We have never been able to compare good series of this species from the Sunda Islands. A single bird from Borneo is darker than a fair series from the Malay States. Birds from the Langkawi Islands northwards are pulverulentus $>$ harterti.

= Cf. Stres., Nov, Zool. xx, 1913, p. 317. 
Genus VIVIA Hodgson.

Vivia innominata (Burt.). Speckled Piculet.

[Vivia innominata innominata (Burt.).

Picumnus innominatus Burton, Proc. Zool. Soc. for 1835,1836 , p. 154: Himalayas.

Distr.-Extra-limital.]

Vivia innominata malayorum (Hart.).

Picumnus innominatus malayorum Hartert, Vög. pal. Faun. Heft vii, rgr2, p. 937 : Perak, Malay States.

Distr.-Malay States.

Sumatra.

Borneo.

Genus SASIA Hodgson.

Sasia abnormis (Temm.).

Malaysian Rufous Piculet.

Sasia abnormis abnormis (Temm.).

Picumnus abnormis Tenminck, P1. Col. 371, fig. 3, 1825: Java.

Sasia everetti Hargitt, Ibis, 1881 , p. 235: Borneo.

Distr--Malay Peninsula.

Sumatra; Billiton.

Borneo.

Java (not the east).

Sasia abnormis magnirostris Hart.

Sasia abnormis magnirostris Hartert, Nov. Zool. viii, rgor, p. 5I : Nias Island, West Sumatra.

Distr.-Nias Island.

Sasia ochracea Hodgs. Indian Rufous Piculet.

[Sasia ochracea ochracea (Hodgs.).

Sasia ochracea Hodgson, Journ. Asiat. Soc. Bengal, v, 1836, p. 778 : Nepal.

Distr.-Extra-limital.]

Sasia ochracea reichenowi Hesse,

Sasia ochracea reichenowi Hesse, Orn. Monatsber. xix, I9II, p. I8I : Burma.

Distr._Peninsular Siam. 


\section{Family EURYLAIMIDA.}

Genus CALYPTOMENA Raffles.

Calyptomena viridis Raffl.

Green Broadbill.

Calyptomena viridis viridis Raffl.

Calyptomena viridis Raffles, Trans. Linn. Soc. xiii, 1822, p. 295 : Sumatra.

Calyptomina Raffesia Swains., Anim, Menag. 1838, p. 296 (new name for $C$. viridis).

Calyptomina candacuta Swains., Class. Birds, ii, 1837, p. 252 (nom. nud); Anim. Menag. 1838, p. 296 : India, error $=$ Singapore.

Distr.-Malay States.

Sumatra; Lingga Archipelago; Nias Island, West Sumatra. Borneo; North Natuna Islands.

Calyptomena viridis continentis Rob, and Kkloss.

Calyptomena viridis continentis Robinson and Kloss, Journ. Fed.

Mal. States Mus. xi, r923, P. 54: Chumporn, Penjnsular Siam. Distr.-Peninsular Sian.

Calyptomena viridis siberu Chas. and Kloss.

Calyptomena viridis siberu Chasen and Kloss, Ibis, I926, p. 285 :

Siberut Island, Mentawi Islands, West Sumatra.

Distr.-Mentawi Islands (Siberut and Pagi), West Sumatra.

Calyptomena hosii Sharpe.

Hose's Broadbill.

Calyptomena Hosii Sharpe, Ann. Mag. Nat. Hist. (6) ix, I892, p. 249 : Mt. Dulit, North-west Borneo.

Distr.-Borneo.

Calyptomena whiteheadi Sharpe.

Whitehead's Broadbill.

Calyptomena whiteheadi Sharpe, Proc. Zool. Soc. 1887, p. 558: Mt. Kinabalu, North Borneo.

Distr.-Borneo.

Genus PSARISOMUS ${ }^{2}$ Swainson.

Psarisomus dalhousiz (Jameson). Long-tailed Broadbill.

[Psarisomus dalhousite dalhousiz (Jatneson).

Eurylaimus dalhousice Jameson, Edinb. New Phil. Journ, xviii, $18_{35}$, p. 389 : North India,

Distr._Extra-limital.]

I We cannot separate the Bornean Bird (c/. Robinson, 1927).

2 Cf. Chas. and Kl., Journ. Nat. Hist. Soc. Siam (Nat. Hist. Suppl.) viii, 1932, p. 236 . 
Psarisomus dalhousize psittacinus (S. Müll.).

Eurylaimus psittacinus Sal. Müller, Tijd. Nat. Ges. Phys. 1835. p. 349, pl. 5, fig. 6: West Sumatra.

Distr.-Malay States.'

Sumatra.

Psarisomus dalhousize borneensis Hart.

Psarisomus dalhousice borneensis Hartert, Gen. Av, pt, I, I905, P. 7 : Mt. Kinabalu, North Borneo.

Distr.-Borneo.

\section{Genus SERILOPHUS Swainson.}

Serilophus lunatus (Gould). Silver-breasted Broadbill.

Serilophus Iunatus lunatus (Gould).

Eurylainus lunatus Gould, Proc. Zool. Soc. for 1833,1834, p. 133 : Rangoon.

Distr.-Peninsular Siam (north).

Serilophus lunatus stolidus Rob. and Kloss.

Serilophus lunatus stolidus Robinson and Kloss, Bull. Brit. Orn.

Cl., xl, 1919, p. 16: Khao Nawng, Bandon, Peginsular Sian.

Distr.-Peninsular Siam (central).

Serilophus lunatus rothschildi (Hart. and Butlr.).

Serilophus rothschildi Hartert and Butler, Bull. Brit. Orn. Cl. vii, 1898, p. 50: Mt. Ijau, Perak, Malay States.

Distr.-Malay States.

Sumatra (north).

Serilophus lunatus intensus Rob. and Kloss.

Serilophus lunatus intensus Robinson and Kloss, Journ, Fed. Mal.

States Mus. viii, pt. 2, I9I8, p. I50: Mt. Korinchi, West Sumatra.

Distr.-Sumatra (excluding north).

\section{Genus EURYLAIMUS Horsfield}

Eurylaimus javanicus Horsf.

Banded Broadbill.

Eurylaimus javanicus javanicus Hotsf.

Eurylaintus Javanicus Horsfield, Trans. Linn. Soc. xiii, I821, p. I70: Java.

Eurylaimus horsfieldii Temm., Pls. Col. 130, 131, 1823: Java,

Distr.-Java. ${ }^{2}$

* Birds from the mountains of the Malay States are not quite the same as typical psiltacinus and in some respects are approaching assimilis Hume (Burma) : the races of $P$. dalhousia are all rather thin.

a The Lampong form is not the typical race as has been suggested. 
Eurylaimus javanicus harterti van Oort.

Eurylaimus javanicus harterti van Oort, Notes Leyd. Mus. xxxi, 1909, p. 209: Deli, North-east Sumatra.

Distr.-Sumatra; Rhio Archipelago.

\section{Eurylaimus javanicus pallidus Chas.}

Eurylaimus javanicus pallidus Clasen, Bull. Raffl. Mus. Io, I935, p. 43: Kao Nawng, Peninsular Siam.

Distr.-Malay Peninsula.

\section{Eurylaimu javanicus billitonis Kloss.}

Eurylaimus javanicus billitonis Kloss, Treubia, xiii, I93I, p. 295 : Billiton Island.

Distr.-Biliton Island.

Eurylaimus javanicus brookei Rob. and kloss.

Eurylaimus javanicus brookei Robinson and Kloss, Bull. Brit. Orn.

C1. x1, I919, p. I5: Saribas district, South-west Sarawak.

Distr.-Borneo; Nortl Natuna Islands. ${ }^{1}$

Eurylaimus ochromalus Rafh.

Black-and-Yellow Broadbill.

Eurylaimus ochromalus ochromalus Raff.

Eurylaimus ochromalus Raffles, Trans. Linn. Soc, xiii, 1822, p. 297 : Singapore.

Eurylaimus cucullatus Temm., Pl. Col, 261, t\$24, Sumatra.

Eurylaemus raffesii Les5., Compl. Buff. viii, p. 463, 1837: Sumatra.

Distr.-Malay Peninsula.

Sumatra; Rhio and Lingga Archipelagos; Billiton

North Natuna Islands.

Eurylaimus ochromalus kalamantan Rob. and Kloss.

Eurylaimus ochromalus kalamantan Robinson and Kloss, Bull. Brit.

Orn. Cl. xl, I9I9, p. I5: Saribas district, South-west Sarawak.

Distr.-Borneo.

*Eurylaimus ochromalus mecistus Oberh.

Eurylaimus ochromalus ntecistus Oberholser, Smiths, Misc. Coll. lx, No. 7, Igr2, p. 8: Tuanku Island, Banjak Islands, West Sumatra.

Distr,-Banjak Islands, West Sumatra.

- The Natuna form has not been examined and is placed here purely on geographic grounds. 


\section{Genus CORYDON Lesson.}

Corydon sumatranus (Rafti.).

Dusky Broadbill.

Corydon sumatranus sumatranus (Raff.).

Coracias Sumatranus Raffles, Trans. Lirn, Soc. xiii, IS22, p. 303 :

Sumatra.

Eurylaimus corydon Temm., Pl. Col. 297, 1824: Sumatra.

Corydon temminckii Less., Man. d'Orn. i, 1828, p. 177: Sumatra.

Distr.-Malay Peninsula.

Sumatra.

Corydon sumatranus brunnescens Hart.

Corydon sumalranus brunnescens Hartert, Bull. Brit. Orn. Cl. xxxvii, 1916, p. 4 : Baram, Sarawak.

Distr.-Borneo; North Natuna Islands.

\section{Genus CYMBIRHYNCHUS Vigors.}

Cymbirhynchus macrorhynchos (Gmel.). Black-and-Red Broadbill. Cymbirbynchus macrorhynchos macrorhynchos (Gmel.).

Todus macrorhynchos Gmelin, Syst. Nat. i, pt. I, I788, p. 446 : Borneo.

Todtis nasutus Lath., Index Orn. i, 1790, p. 268 : nom, nov.

Erolla nasica Less., Traité d'Orn. 1830, D. 260: nom, nov.

Etwylaimus lemniscatus Raff., Trans, Linn. Soc. xiit, iS22, p, 206 : Bencooler, West Sumatra.

Distr.-Sumatra (west).

Borneo.

Cymbirhynchus macrorhynchos malaccensis Salvad.

Cymboritynchus malaccensis Salvadori, Att. R. Accad. Sci. Torino, ix, 7874 , p. 425 : Malacca.

Distr.-Malay Peninsula.

Sumatra (east); Banka; Billiton.

\section{Family PITTID瓜.}

Pitta cæerulea (Raffi.)

Giant Pitta.

Pitta cerulea cerulea (Raffi.).

Myiothera carulea Raftles, Trans. Linn. Soc. xiii, I822, p. $30 \mathrm{r}$ : Sumatra.

Pitta gigas Temm., Pl. Col. 217, 1823: Sumatra.

Brachyurus davisoni Hume, Stray Feathers, iii, 1875, p. 321: hills dividing Siam and Tenasserim.

Distr.-Malay Peninsula.

Sumatra. 
Pitta caerulea hosei Baker.

Pitta cerulea hosei Baker, Bull. Brit. Orn. Cl. xxxix, I9I8, p. 20 : Mt. Dulit, Sarawak.

Distr.-Borneo.

\section{Pitta nipalensis (Hodgs.).}

[Pitta nipalensis nipalensis (Hodgs.).

Paludicola Nipalensis Hodgson, Journ. Asiat. Soc. Bengul, vi, I837, p. I03: Nepal.

Distr._Extra-limital.]

Pitta nipalensis schneideri Hart.

Schneider's Pitta.

Pitta schneideri Hartert, Bull. Brit. Orn. Cl. xxv, 1909, p. 9 : Battak Mts., North Sumatra.

Distr.-Sumatra.

Pitta brachyura (Linn.).

Lesser Blue-winged Pitta.

Pitta brachyura brachyura (Liñ.).

Corvus brachyurus Linn., Syst. Nat. I2th ed. 1, 1766, p. I58: Ceylon.

\section{Pitta brachyura cyanoptera Temm.:}

Pitta cyanoptera Temminck, P1. Col. 218, I823: Sumatra.

Pitta Malaccensis Blyth, (nec Müll, and Schleg.), Journ. Asiat. Soc. Beng, xii, 1843 , p. 960 : Malacca.

Pitta molnccensis lepta Oberh., Smiths. Misc. Coll. Ix, No. 7, 1912, p. 8: Nias Island, West Sumatra.

Distr.-Malay Peninsula.

Sumatra; Rhio Archipelago; West Sumatra islands of Banjak group, Nias and Mentawi group.

Borneo; North Natuna Islands.

Java.

Pitta megarhyncha Schleg ${ }^{2}$.

Larger Blue-winged Pitta.

Pilla megarhyncha Schlegel, Vög. Ned. Ind. 1863, Pilta, p. 11, pl. 4, fig. 2: Banka.

Distr.-Malay Peninsula.

Sumatra; Rhio Archipelago; Banka.

- We consider that Turdus moluccensis Müll, is only doubtfully applicable to this bird. Small birds resembling lepta occur in other parts of Malaysia.

serhaps a southern breeding race of $P$, brachyura. 
Pitta nympha Tenmm. and Schleg.

Chinese Blue-winged Pitta.

*Pitta nympha nympha Temm. and Schleg.

Pitta nympha Temminck and Schlegel, Fauna Japon., Aves, 1850, p. I35. Suppl. Tab. A: Korea.

Pitta berta Salvad., Att. Sci. Tor. iii, 1868, p. 527 : Borneo. Distr.-Borneo.

Pitta sordida P. I. S. Müll. Green-breasted Pitta.

[Pitta sordida sordida (P. L. S. Miill.).

Turdus sordidus P. L. S. Müller, Natursyst. Suppl. I776, p. I43: Philippine Islands.

Distr.-Extra-limital.]

Pitta sordida mulleri Bp.

Pitta mulleri Bonaparte, Consp. Gen. Avium, i, r850, p. 256 : Celebes, errot $=$ Sotth

Pitla sordida sumatrana Kloss, Treubia, xiit, 1931, p. 332 : Palembang, Sumatra.

Pilta sordida jazana K̈loss, Treubia, xii, 1031, p. 332 : Bandjar, Java.

Distr._Sumatra.

Borneo.

Java (west).

Pitta sordida cucullata Hartl.

Pilta cucullata Hartlaub, Rev. Zool. I843, p. 65 : Malacca.

Pitta malaccensis Mìll. and Schl., Verk. Nat, Gesch., Zool. Pitta, 1840, p. 19: Malacca.

Distr.--Malay Peninsula.

Sumatra; Nias Island, West Sttmatra.

Java.

Pitta sordida bangkana Schleg.

Pilla bangkana Schlegel, Vög. Ned. Ind., Pitta 1863, p. 8, pl. 2, fig. 5: Banka Island.

Distr.-Banka; Billiton.

Pitta venusta Sal. Müll.

Black-and-Scarlet Pitta.

Pitta venusta venusta S. Müll.

Pitta venusta Sal. Müller, Tijd. Nat. Ges. Phys. ii, I835, p. 348 , pl. 5, fig. 4: West Sumatra.

Distr.-Sumatra.

Pitta venusta ussheri Sharpe.

Pitta ussheri Sharpe, Proc. Zool. Soc. IS77, p. 94 : Lawas River, North Borneo.

Distr.-Borneo. 
Pitta granatina Temm.'

Red-headed Pitta.

Pitta granatina granatina Temm.

Pitta granatina Temminck, Pl. Col. 506, I830: Borneo.

Pitta granatina borneensis Ell., Auk, ix, 1892, P. 218 : Borneo.

Distr,-Borneo.

Pitta granatina coccinea Eyton.

Pilta coccinea Eyton, Proc. Zool. Soc. I839, p. 104 : Malacca.

Pitla granatina vanheurni Kloss, Journ. Fed. Mal. States Mus. x, 192r,

p. 212: Langkat, North-east Sumatra.

Distr.-Malay Peninsula.

Sumatra.

Pitta arcuata Gould. ${ }^{2}$

Blue-banded Pitta.

Pitta (Phonicocichla) arquata Gould, Ann. Mag. Nat, Hist. (4), vii, I87x, p. 340: Borneo.

Distr.-Borneo.

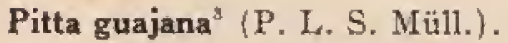

Banded Pitta.

Pitta guajana guajana (P. L. S. Müll.).

Turdus guajanus P. I. S. Müller, Natursyst. Suppl. 1776, p. I45: Banjoewangi, East Java, (restr. Kloss, 1026).

Turdus cyanurus Bodd., Tabl. Pl. Enlum. 1783, p. 21 : Java.

Distr.—Java (East); Bali.

Pitta guajana affinis (Horsf.).

Myiothera affinis Horsfield, Trans. Linn. Soc. xiii, I821, p. 154:

Bantam Province, West Java (restr. Kloss, 1926).

Distr.,Java (West).

Pitta guajana schwaneri Bp.

Pitta schwaneri Bonaparte, Consp. Gen. Avium I, I850, p. 256 : Borneo.

Disit.-Borneo.

Pitta guajana irena Temm.

Pitta irena Temminck, P1. Col. text opposite pl. 597, 1836: Sumatra. Pitta boschis Müll. and Schleg., Verh. Nat, Gesch., Zool., Pitta, 1840, p. 5, pl. 1: Sumatra.

Disir.-Malay Peninsula.

Sumatra.

1 All the Malaysian black-and-scarlet pittas may be forms of granatina. but we need to know more of the ranges of the various forms in Sumatra and Borneo before linking them. Sce BuIl. Raffl. Mus. 4, 1930, p. 45.

- This species seems to occupy an intermediate position between the black-and-scarlet pitas of Malaysia and the non-Malaysian forms of the celebensis-mackloti association.

3 Cf. Kloss, Journ. Mal. Br. Roy. Asiat. Soc, iv, If 26, p. 161 . 
Pitta gurneyi Hume.

Gurney's'Pitta.

Pitta Gurneyi Hume, Stray Feathers, iii, 1875 , p. 296, pl. 3 : South Tenasserim.

Distr.-Peninsular Siam.

Pitta baudi Müll. \& Sčhleg. ${ }^{1}$

Blue-headed Pitta.

Pitta baudi Müller and Schlegel, Verh. Nat. Gesch., Zool., Pitta, I840, pp. 10, 15, pl. 2 : Borneo.

Distr,-Borneo.

\section{Family HIRUNDINID在.}

\section{Genus DELICHON Horsfield and Moore.}

Delichon urbica (Ling.).

House-Martin.

[Delichon urbica urbica (Linn.].

Hirundo urbica Linn., Syst. Nat. Ioth. ed. I758, p. I92: Sweden, Europe.

Distr._Extra-limital.]

Delichon urbica dasypus (Bp.).

Chelidon dasypus Bonaparte, Consp. Gen. Avium, i, I850, p. 343 : Borneo.

Distr.-Malay States.

?Rhio Arch. (vis.).

Borneo.

Java.

\section{Genus HIRUNDO Linn.}

Hirundo rustica Linn. ${ }^{2}$

Common Swallow.

[Hirundo rustica rustica Linn.3

Hirundo rustica Linn., Syst. Nat. Ioth. 1758, p. 19: : Sweden.

Distr,-Extra-linital.]

Hirundo rustica gutturalis Scop.

Hirundo gutturalis Scopoli, Del. Flor, et Faun. Insub. ii, 1786, p. 96: Philippine Islands.

Distr.-Malay Peninsula; Anamba Islands.

Sunatra; Rhio Archipelago; West Sumatran Islands of Simalur and Siberut.

Borneo; Bornean Islatıds; North Natuna Islands. Java; Bali; Christmas and Cocos-Keeling Islands.

t The nearest relative of this strangely isolated form seems to be P. gurneyi of Burma and Siam !

C C. Oberh., Butl. U.S. Nat. Mus. 98, 1917, p. 28.

3 There seems to be no satisfactory record for the occurrence of the typical form of the swallow in Malaysia.

$$
\text { [ } 160]
$$


HIRUNDINIDA.

Hirundo tahitica Gmel. ${ }^{1}$

Const-Swallow.

[Hirundo tahitica tahitica Gu].

Hirundo tahitica Gmelin, Syst. Nat. i, pt. 2, 1789, p. I016: Tahiti.

Distr.—Extra-timital.]

Hirundo tahitica javanica Sparrm.

Hirundo javanica Sparruan, Mus. Carls. iv, I7S9, pl. roo: Java.

Distr.-Sumatra (Lampongs).

Java; Kangean Islands.

Hirundo tahitica abbotti (Oberh.).

Hypurolepis jasanica abbotti Oberholser, Bull. U.S. Nat. Mus. 98, I917, p. 32: Manguan Island, Anamba Islands.

Hyfurolepis javanica mesata Oberh, Bull. U.S. Nat. Mus. 159, 1932, p. 51 : Seraia Island, Natuna Islands.

Distr.-Malay Peninsula; Anamba Islands; Tambelan Islands.

Sumatra (not Lampongs); Rhio Archipelago; Billiton; Mendanau.

Borneo; Nortl Bornean Islands; Natuna Islands; Karimata Islatids.

*Hirundo tahitica hypolampra (Oberh.) . ${ }^{a}$

Hypurolepis javanica hypolampra Oberholser, Jouru. Wash. Acad. Sciences, xvi, 1926, p. 5I5: Nias Island, West Sumatra.

Distr,-Nias Istand, West Sumatra.

Hirundo daurica Linn.

[Hirundo daurica daurica Linn.

- Hinudo daurica Linn., Mantissa Plant., ii, 1771, p. 528: Siberia.

Dislr.-Eixtra-limital.]

*Hirundo daurica striolata Temn. \& Schleg.

Striated Swallow.

Hirundo striolata T'mminck and Schlegel, Fauna Japon., Aves, I847, p. 33 : Java.

Distr.-Borneo.

Java.

Hirunda daurica badia (Cass.).

Cliff-Swallow.

Cecropis badia Cassin, Proc. Plilad. Acad. Sci. 1853, p. 37 I : Malacca.

Cecropis archetes Hume, Stray Feathers, v, 1877. p. 266: Malacca.

Distr.-Malay Peninsula.

- Cf. Oberh., Bull. U.S. Nat. Mus. 159, 1932, p. 51.

a We have riever seen topotypes of this race but do not consider that birds from Sumatra which island was included in the range of hypolampra are separable from abboti. 
Genus RIPARIA Forster.

Riparia riparia (Linn.).

Sand-Martin.

[Riparia riparia riparia (Linn.).

Hirundo riparia Linn., Syst. Nat, Ioth. ed. 1758, p. 192: Sweden. Distr.-Extra-1imital.]

*Riparia riparia ijimse (Lünnb.).

Clivicola riparia ijmce Lönnberg, Journ. Coll. Sci. 'Tokyo, xxiii, art 14,1908, p. 38 : Sachalin.

Distr.-Borneo.

\section{Family MUSCICAPID无:}

\section{Genus HEMICHELIDON Hodgson.}

Hemichelidon sibirica (Gme\}.).

Sooty Flycatcher.

Hemichelidon sibirica sibirica (Gmel.).2

Muscicapa sibirica Gmelin, Syst. Nat. i, pt. 2, I7S9, p. 936: Lake Baikal,

Distr.-Malay Peninsula; Ananba Islands.

Sumatra.

Borneo; Natuna Islands.

Java.

Hemichelidon sibirica fuliginosa Hodgs.

Hemichelidon fuliginosa Hodgson, Proc. Zool. Soc. 1845, p. 32 : Nepal.

Distr.-Peninsular Siam.

- It is certain that more genera are recognized here than is necessary, but although several authors have made bold attempts to lump the palaarctic genera, the tropical groups have not been included in their reviews. We have, therefore, chosen to be conservative rather than inconsistent but it may be noted that of the genera recognized here Hartert has already merged Hemichelidon, Alseonix, Siphia, Cyoruis, Poliomyias, Zanthopygia, and Cyanopila. "Muscitrea" is here placed in the Laniide for we do not see how it can be separated from Pachycephala.

- We have seen several supposed examples of $H$. griseisticta Swinh., from Borneo but they are all really $H$. sibirica. Some Malaysian birds may be $H$. s. incerta La Touche (North China) which we have not seen.

3 Cl. Baker, Bull. B. O. C. xliii, 1923, p. 155. 
Hemichelidon ferruginea Hodgs.

Ferruginous Flycatcher.

Hemichelidon ferruginea Hodgson, Proc. Zool. Soc. I845, p. 32 : Nepal.

Hemichelidon cinereiceps Sharpe, Ibis, 1887, p. 441: Mt, Kinabal's, Borneo.

Distr.-Malay Peninsula.

Sunatra.

Bonneo.

Java.

Genus ALSEONAX Cabanis.

Alseonax latirostris (Raffl.).

Brown Flycatcher.

Alseonax latirostris latirostris (Raffl.).

Musicapa latirostris Raffles, Trans. Linn. Soc. xiii, I822, p. 3I2:

Sumatra.

Distr.-Malay Penimsula.

Sunnatra; Siberut Island, West Sumatra.

Borneo; Natuna Islands.

Java.

Genus SIPHIA Hodgson. ${ }^{1}$

Siphia parva (Beclist.).

Red-breasted Flycatcher.

[Siphia parva parva Bechst.

Muscicapa parva Bechstein, Getreue Abbild. Heft 2, I793, p. 26 : Holland.

Distr._Extra-linital.]

Siphia parva albicilla (Pall.).

Muscicapa Albicilla Pailas, Zoogr. Rasso-Asiat. i, I8ti, p. 462: Dauria.

Distr.-Peninsular Sian.

\section{Genus CYORNIS Blyth. ${ }^{2}$}

Cyornis vivida Swinh.

Blue-and-Orange Flycatcher.

[Cyornis vivida vivida Swinh:

Cyornis vivida Swinhoe, Ibis, 1864, p. 363 : Formosa.

Dislr.-Extra-limital.]

Siphia obscura Sharpe, Proc. Zool. Soc. 1881 , p. 789 (Borneo), is an American bird.

- Revisions : Stres., Orn. Monatsber, xxxiit, 1025, p. 45; Rob. and Kinn., Nov, Zool. Xxxiv, 1928, p. 23I; Chas. and KI., Bull. Raff, Mus. 2, 1929, p. 23. 
Cyornis vivida sumatrana (Salvad.).

Nillava sumalrana Salvadori, Ann. Mus. Civ. Gen. (I), xiv, IS79, p. 20 I : Mt. Singalan, West Sumatra.

Cyornis peninsularis Robinson, Journ. Fed. Mal. States Mus., ii, 1909, p. $\mathrm{I}_{3}$ : Telom, Perak, Malay Peninsula.

Distr.-Malay States. Sumatra.

Cyornis concreta (S. Müll.).

Cyornis concreta concreta (S. MüH.). White-tailed Blue Flycatcher.

Muscicapa concreta Sal. Müller, 'Tijd. Nat. Ges. Phys. ii, I835, p. 351: West Sumatra.

Distr.-Malay States.

Sumatra.

Cyornis concreta everetti (Sharpe). Everett's Blue Flycatcher.

Siphia everetti Sharpe, Ibis, I89o, p. 360: Mt. Penrissen, West Sarawak, Borneo.

Distr,-Borneo.

*Cyornis ruecki Oust.

Rueck's Blue Flycatcher.

Cyornis ruecki Oustalet, Bull. Soc. Philonath. (7) v, I8SI, D. 7S: Malacea.

Cyornis vanheysti Robinson and KJloss, Journ. Straits Branch Roy, Asiat. Soc. 8o, 1919, p. 104: North-east Sumatra.

Disir.-Malay States.

Sumatra (north-east).

Cyornis unicolor Blyth.

Pale Blue Flycatcher.

[Cyornis unicolor unicolor Blyth.

Cyomis unicolor Blyth, Journ. Asiat. Soc. Bengal, xii, I843, p. I007: Darjeeling.

Distr.-Eixtra-linital.]

Cyornis unicolor infuscata Hart.

Cyornis unicolor infuscata Hartert, Nov. Zool. ix, I902, p. 550: Java.

Cyornis wnicolor harterti Rob. and Kinn., Nov. Zool. xxxiv, 1928, p. 256 : Java.

Distr.-Malay Репiusula.

Sumatra.

Borneo.

Java. 
Cyornis rubeculoides (Vigs.).

Blue-throated Flycatcher.

[Cyornis rubeculoides rubeculoides (Vigs.).

Phanicura rubeculoides Vigors, Proc. Zool. Soc. I8 3 x, p. 35 : N.W. Himalayas.

Distr. - Extra-linital.]

\section{Cyornis rubeculoides dialilaema Salvad.}

Cyornis dialilaema Salvadori, Ann. Mus. Civ. Gen. (2a), vii, r889, p. 387 , North Tenasserim.

Cyornis rubeculoides chersonites Oberh., Proc. Biol, Soc. Wash. xxxiii, 1920, p. 85 : Trang, Peninsular Siam.

Distr.-Peninsular Sianı.

Cyornis rubeculoides glaucicomans Thayer \& Bangs.

Cyornis lickelliae glaucicomans Thayer and Bangs, Bull. Mus. Comp. Zool, lii, 1909, p. I4I: Hupeh, Central China.

Cyornis anak Robinson and Kloss, Journ. Fed. Mal. States Mus., x, 1922, p. 261: Trang, Peninsular Siam.

Distr._Peninsular Siam?.

Cyornis turcosa (Brüggm.). Malaysian Blue Flycatcher.

Cyornis turcosa turcosa (Brüggg.).

Muscicapa turcosa Brüggeman, Ahhandl. Nat. Ver. Bremen, v, I877, p. 457: Muara Tewah, South-east Borneo.

Muscicapa elegans Temm., Pl. Col. 596, fig. 2, 1836: Sumatra (nec M. elegans Less.).

Cyornis elegans antelia Oberh., Proc. Biol. Soc. Wash. xxxiii, 19zo, p. 87 : Long Iram, North-east Borneo.

Distr.-Borneo (sonth-east).

Cyornis turcosa rupatensis Oberh.

Cyornis elegans rupatensis Oberholser, Proc. Biol. Soc. Wash. xxxiii, 1920, P. 87 , Rupat Strait, South-east Sumatra.

Distr.--Malay States.

Sumatra.

Borneo (west).

Cyornis rufigastra (Raffl.).

Common Blue Flycatcher.

Cyornis rufigastra rufigastra (Raffl).

Muscicapa rufigastra Raffles, Trans. Linn. Soc. xiii, I822, D. 312: Sumatra.

Siphia beccariana Salvad., Atti. R. Acad. Torino, iii, 1868, p. 533: Sarawak. 
Cyornis frenatus Hume, Stray Feathers, ix, I880, p. II4: Jeram, Selangor.

Cyorris hosei Finsch, Notes Leyd. Mus., xxiii, Igor, p. 48: Borneo.

Cyornis erythrogaster Sharpe, Handlist of Birds, iif, 19or, p. 216 ; nom, emend, for rufigastra.

Cyornis banymas calocephala Oberh., Proc. Biol. Soc. Wash. xxxiii. 1920, p. 86 : Banka.

Distr.-Malay States,

Sumatra; Rhio Archipelago; Lingga Archipelago; Banka.

Borneo; North Bornean Islandis; Maratua Islands.

*Cyornis rufigastra rhizophorae Stres.

Cyomis rhizophorae Stresemann, Orn. Monatsb. xxxiii, I925, p. 50:

Coast of Nortli-west Java.

Distr.-Java (west); Sebesi Island, Sunda Strait. ${ }^{\text {I }}$

Cyornis rufigastra sumatrensis (Sharpe),

Siphia sumalrensis Sharpe, Cat. Birds, Brit. Mus. iv, 1879, p. 45 I : Sumatra.

Distr.-Malay Peninsula (not soutli). Sumatra.

Cyornis rufigastra lampra Oberh.

Cyornis banyumas lampra Oberholser, Bull. U.S. Nat. Mus. @S, I917, p. 35: Pulau Jimaja, Anamba Islands.

Distr.-Anamba Islands.

Cyomis rufigastra longipennis Clas, and Kloss,

Cyomis rufigastra longipennis Chasen and Kloss, Treubia, xii, I930, p. 271 : Karimon-Java Island, Java Sea.

Distr.-Karinon-Java Islands.

Cyornis rufigastra karimatensis Oberh.

Cyornis banyumas karimatensis Oberholser, Proc. U.S. Nat. Mus. lxiv, I924, Art. 22, p. 3: Karinata Island.

Distr.-Karimata Islands, West Borneo.

Cyornis banyumas (Horsf.).

Hill Blue Flycatcher.

Cyornis banyumas banyumas (Horsf.).

Muscicapa Banyumas Horsfield, 'Trans. Linn. Soc. xiii, ISz1, p. 146: Province of Banyimas, Java.

Cyornis banyumas limitans Rob., Bull. B. O. Cl. xlviii, 1927, p. 44 : Tamansari, East Java.

Distr.-Java (east and central).

1 We have never seen topotypical higophora, but the Sebesi birds are rather lighter blue above than most examples of rufigastra and we therefore refer them to the Javan form. 
Cyornis banyumas cantatrix (Tewm.).

Muscicapa cantalrix Temminck, Pl. Col. 226, I823: Bantam, West Java.

Distr._Java (west).

Cyornis banyumas coerulifrons Baker.

Cyornis magnirostris corulifrons Baker, Bull. Brit. Orn. Cl. xxxix, Igr8, p. 8: Peninsular Sian1.

Distr.-Malay Peninsula.

Cyornis banyumas magnirostris Myth.

Cyornis magnirostris Blyth, Journ. Asiat. Soc. Bengal, xviii, I849, p. 8.4: Darjeeling.

Distr.-Peninsular Siam.

Cyornis banyumas montana Rob. and Kinn.

Cyomis whitei montana Robinson and Kinnear, Nov. Zool. xxxiv, I928, p. 244 : Mt. Liang Kubang, Central Borneo.

Distr.-Borneo.

Cyornis cærulata (Bp.).

Large-billed Blue Flycatcher.

Cyornis cerulata cærulata $(\mathrm{Bp}$.$) .$

Schwaneria ccerulata Bonaparte, Rev. Mag. Zool. (2) ix, 1857, p. 54 : Samibarajan, South Borneo.

Distr.-Borneo (south, east and north).

Cyornis carulata rufifrons Wall.

Cyonis rufifrons Wallace, Proc. Zool. Soc. 1865, p. 476: Sarawak.

Cyornis nigrigularis Everett, lbis, 1891 , j. 45 : Mt. Penrissen, Sarawak.

Disit.-Borneo (west, and Sarawak).

Cyornis caerulata albiventer Junge.

Cyomis cacrulata albiventer Junge, Ardea, xxii, 1933, p. 105: Deli, North Sumatra.

Distr.-Sumatra.

Cyornis superba Stres.

Bornean Blue Flycatcher.

Cyomis superba Stresemann, Orı. Monatsb. xxxiii, 1925, p. 52 :

Mt. Penrissen, Sarawak.

Distr.-Borneo. 


\section{Genus POLIOMYIAS Sharpe}

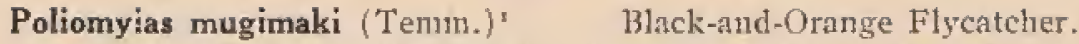

Muscicapa Mugimalai Temminck, Pl. Col. 577, fig. 2, I835: Japan.

Siphia erythaca Blyth, Journ. Asiat. Soc. Beng. xvî, 1\$47, p. I26: Malacca.

Distr.-Malay Peninsula.

Sumatra.

Borneo.

Java.

Gentis MUSCICAPELLA Bianchi.

Muscicapella hodgsoni (Horsf. and Moore). Pygmy Blue Flycatcher. [Muscicapella hodgsoni hodgsoni (Horsf. and Moore).

Nemura hodgsoni Horsfield and Moore, Cat. Birds. Mus, East Ind.

Coy. i, 1854, p. 300 : Nepal.

Distr._Extra-limital.]

Muscicapella hodgsoni sondaica (Rob. and Kloss).

Nitidula hodgsoni sondaica Rohinson and Kloss, Journ. Fed. Malay

States Mus., xi, 1923, p. 54: Korinchi Peak, West Sumatra.

Distr., Malay States.

Sumatra.

Borneo.

Genus MUSCICAPULA Blyth.

Muscicapula melanoleuca Blyth. Little Pied Flycatcher.

[Muscicapula melanoleuca melanoleuca 13lytir.

Muscicapula melanoleuca Blyth, Jonrn. Asiat. Soc. Bengal, xii, I 843 , p. 490 : Nepal.

Distr._Extra-limital.]

Muscicapula melanoleuca westermanni Slırpe.

Muscicatula westermanni Sharpe, Proc. Zool. Soc. ISS8, p. 270:

Gunong Ulu, Perak, Malay Peninsula.

Distr.-Malay Peninsula.

Suntatra (not soutli).

Borneo.

Muscicapula melanoleuca hasselti (Finscli).?

Muscicapa Hasselli Finsch, Notes Leydl. Mus. xx, ISg8, p. 94 : Java.

Disir.-Sunatra (south only).

Java; Bali.

'It is possible that Muscicapa rufigula first mentioned by Miill., Tijd. Nat. Gesch. Phy5. ii, 1835, p. 35 r (nom. nud. here) and probably from Sumatra applies to this bird. See Sharpe, Notes Leyd. Mus. i, I879, p. 27.

a This name has been ascribed to Robinson and Kloss, but Finsch's remarks seem to constitute a valid description. 


\section{Genus DENDROBIASTES Sharpe."}

Dendrobiastes hyperythra (Blyth).. Rufous-breasted Flycatcher.

[Dendrobiastes hyperythra hyperythra (Blyth).

Muscicapa hyperythra Blyth, Journ. Asiat. Soc. Bengal, xi, I842, p. $\$ S_{5}$ : Nepal.

Disir.-Eixtra-linital.]

Dendrobiastes hyperythra malayana (Grant).

Muscicapula malayana O.-Grant, Bull. Brit. Orn. Cl. xix, 19o6, p. Io: Mt. Tahan, Malay Peninstila.

Dendrobinstes hyperythrus sumatranus Hachisuka, Bull. Brit. Orn. Cl. xlvii, Ig26, p. 52: Mt. Korinclii, West Sumatra.

Distr.-Malay States.

Sumatra.

Borneo.

Dendrobiastes hyperythra mjöbergi Hart.

Dendrobiastes hyperythrus mjöbergi Hartert, Sarawak Mus. Journ. iii, I925, p. I: Mt. Poi, West Sarawak.

Distr.-Borneo (west). ${ }^{3}$

Dendrobiastes hyperythra vulcani Rob.

Dendrobiastes hyperythra tulcani Robinson, Journ. Fed. Mal. States Mus., vii, I9IS, p. 235: Tjibodas, West Java.

Distr._Java; Bali.

Genus ANTHIPES Blyth.

Anthipes solitaris (S. Müll.). White-gorgeted Filycatcher,

Anthipes solitaris solitaris (S. Müll.).

Muscicapa solitaris Sal. Müller, Tijd. Nat. Gesch. Phys. I835, p. 351: Padang, West Sumatra.

Distr.-Sumatra.4

- Revisions :Stres., Nov. Zool. xix, tgrz, p. 330; Snouck. v. Schaub., Org. Cl. Ned. Vog. i1, 1929, p. 52.

2We have no material to decide whether Muscicapa superciliaris Jerdon, 1840 , and Dimorpha superciliaris Blyth, 1842, are congeneric and have accordingly used Blyth's substitute name Muscicapa hyperythra published later in 1842 . Siphia hyperythra Cabanis, 1866 , must also be kept in mind in the case of any extensive lumping of Oriental genera.

3 Kinnear (Ibis, I929, P. 134) bas suggested that mjobergi is a species because it appears to occur side by side with malayana on Mt. Kinabalu in North Borneo: we have seen many skins of this species from Kinabalu and all are malayana except one male in the British Museum : as this bird bears no field label except a number, which if a record of altitude does not agree with its new label $(2,100 \mathrm{~m}$. against 8,000 feet $)$ we feel at liberty to doubt its provenance.

4 The north Sumatran race is perhaps $A$. s. malayana, but we have only examined one not fully adult bird. 
Anthipes solitaris malayana (Shatpe).

Digenea malayana Sharpe, Proc. Zool. Soc. 1888, p. 247 : Larut, Perak.

Distr.-Malay States.

Anthipes solitaris submoniliger Husne.

Anthipes submoniliger Hume, Str. Featl. v, I877, p. 105 : Mt. Muleyit, Tenasserim.

Distr.-Peninsular Sian.

\section{Genus NILTAVA Hodgson.}

Niltava grandis (Blyth).

Malaysian Niltava.

[Niltava grandis grandis (Blyth).

Chaitaris grandis Blyth, Jourת. Asiat. Soc, Bengal, xi, I842, p. I89: Darjeeling.

Distr.-Extra-linital.]

Niltava grandis decipiens Salvad.

Niltava decipiens Salvadori, Ann. Mus. Civ. Gen. (2) xii, I892, p. 49 : Suniatra.

Distr. - Sumatra.

Malay States.

Genus OREICOLA Bonaparte.

Oreicola dumetoria (Wall.).

Short-tailed Flycatcher.

Oreicola dumetoria dumetoria (Wall.).

Saxicola (?) dumetoria Wallace, Proc. Zool. Soc. I\$63, p. 490 : Lombok.

Siphia vordermani Sharpe, Ibis, 1890, p. 206 : Mt. Gede, West Java. Distr._Java.

Oreicola dumetoria muelleri (Blyth).

Muscicapa muelleri Blyth, Ibis, 1870, p. 166: Sumatra.

Siphia elopurensis Sharpe, Ibis, 1890, p. 206 : Sandakan, North Borneo.

Distr.-Malay Peninsula.

Sumatra

Borneo. 
Genus GERYGONE Gould.

Gerygone fusca (Gould).

Fily-eater.

[Gerygone fusca fusca (Gould).

Psilopus fuscus Gould, Synopsis Birds Australia, iv, 1838 , pl. 6 I :

Swan River, West Australia.

Distr.-Extra-limital.]

\section{Gerygone fusca sulphurea Wall.}

Gorygone sulphurea Wallace, Proc. Zool. Soc. for 1863,1864 , p. 490 : Solor.

Gerygone modiglianit Salvad., Ann, Mus. Gen. (2) xii, 18gr, p. 52 : Toba Lake, North-east Sumatra.

Gerygone pectoralis: Davison, Ibis, 1802 , p. 99 : Pahang coast, Malay States.

Gervgone salvadorii Buittik, Notes Leyden Mus, xv, 1893, p. 175 : South Borneo.

Gerygone modiglianii jacobsoni van Oort, Notes Leyden Mus., xxxi. $1009, \mathrm{p}, 207$; islet near Batavia, W. Java.

Gerygone modiglianii muscicapa Oberh., Smiths, Misc. Coll. 1x, No. 7, 1912, p. II : Engano Island.

Gerygone sulphurza plesseni Stres., Ornith. Monatsber. xxxiv, 1926, p. 22 : Bali.

Distr.-Malay Peninsula.

Sumatra; Engano Island, West Sumatra.

Borneo; Maratua Islands.

Java; Bali; Karimon-Java Istands.

\section{Genus ZANTHOPYGIA Blyth.}

Zanthopygia narcissina (Temm.). Black-and-Yellow Flycatcher.

"Zanthopygia narcissina narcissina (Temm.) .

Muscicapa narcissina Temminck, P1. Col. 577, fig. I, I835: Japan. Distr.-Borneo.

Zanthopygia narcissina zanthopygia (Hay).

Muscicapa Zanthopygia Hay, Madras Journal xiii, I845, p. 162 : Malacca.

Muscicapa (Mnscicapula) tricolor Hartl., Rev. Zool. 1845, p. 406: "Malacca?".

Dislr.-Malay Peninsula; Anamba Islands; Sumatra; Java.

- We have followed Meise (Zool. Nov. xxxv, 1931, p. 371) in our treat ment of this species.

2 The type of pectoralis is not in Singapore. 
Genus CYANOPTILA Blyth.

Cyanoptila cyanomelana (Temm.). Blue-and-White Filycatcher.

*Cyanoptila cyanomelana cyanomelana ('Temm.).

Muscicapa cyanomelana Temminck, Pl. Col. 470, 1829: Japan.

Distr.-Java."

Cyanoptila cyanomelana cumatilis Thayer and Batgs.

Cyanoptila cumatilis Thayer and Bangs, Bull. Mus. Contp. Zool.

lxii, 1909, p. I4I : Hupeh, China.

Distr.-Malay Peninsula.

Sintratra.

Java; Karimon-Java Islands.

Cyanoptila cyanomelana intermedia (Weig.).

Muscicapa cyanomelana intermedia Weigold, Abl. Ber. Zool. Mus.

Dresden, xv, 1922,3, p. 30 : Vladivostok area.

Distr,-Borneo.

Genus HYPOTHYMIS Boie. ${ }^{2}$

Hypothymis azurea (Bodd.).

Black-11aped Flycatcher.

[Hypothymis azurea azurea Bodd.

Muscicapa azurea Boddaert, Tabl. Planch. Enlum., I783, p. 4I : Philippine Islands.

Distr._Extra-limital.]

Hypothymis azurea styani (Hartl.).

Siphia styani Hartlaub, Abh. Nat. Ver. Bremen, xvi (2), 1898, p. 248: Hainan.

Distr.-Peninsulat Siant.

Hypothymis azurea prophata Oberh.

Hypolhymis azurea prophata Oberholser, Proc. U.S. Nat. Mus. xxxix, 1911, p. 597: Karimon Island near Singapore.

Distr.-Malay States.

Sumatra; Rhio Archipelago; Lingga Archipelago; Banka; Billiton.

Borneo; North Bornean Islands; Maratua Islands.

* Messrs. Bartels and Stresemann consider that this race occurs in Java : our black-breasted birds from Borneo answer to the description of inlermedia and are less blue above than some topotypical males of cyanomelana. Kuroda (1933) mentions C. c. cyanomelana as occurring in winter in the Malay Pentnsula, Sumatra, Borneo, and Java, but this extensive Malaysian range may be based on uncritical records in literature and in our list we have, therefore, only included birds we have examined with a view to subspecific identification with the exception of typical cyanomeland which is accepted for Java on published authority.

2 Revisions: Stres., Nov. Zool. xx, 1013, p. 280 ; Oberh., Proc. U.S. Nat. Mus. xxxix, 1911, p. 585 ; Oberh., Bull. U.S. Nat. Mus. 98, 1917, p. 38; Snouck. v. Schaub., Org. Cl. Ned. Vog. ii, 1929, p. 61. 
Hypothymis azurea javana Chas. and Kloss.

Hypothymis azurea javana Chasen and Kloss, Bull. Raftles Mus. 2, 1929, p. 22 : Badjoelmati, East Java.

Distr.-Java; Karimon-Java Island.

"Hypothymis azurea symmixta" Stres.

Hypoltymis azurea symmitta Stresemann, Journ. f. Ornith. I926, p. 554 : Alor.

Distr.-Bali.

Hypothymis azurea karimatensis Chas, and Kloss.

Hypothymis azurea karimatensis Clasen and Kloss, 13ull. Raffles Mus. 7, 1932, p. 8: Serutu Island, Karimata Islands.

Distr.-Karimata Islands, West Borneo.

Hypothymis azurea opisthocyanea Oberli.

Hypothymis azurea opisthocyanca Oberholser, Proc. U.S. Nat. Mus. xxxix, I911, p. 602 : Piling Island, Anamba Islands.

Distr-Anamba Islands; Tambelan Islands.

Hypothymis azurea gigantoptera Oberh.

Hypothymis azurea gigantoptera Oberholser, Proc. U.S. Nat. Mus. xxxix, IgIr, p. 600 : Bunguran Island, North Natuna Islands. Distr.-Natuna Islands.

Hypothymis azurea aeria Bangs and Peters.

Hypothymis aeria Bangs and Peters, Occ. Papers Boston Soc. Nat. Hist. v, I927, p. 237 : Maratua Island.

Distr.-Maratua Island, East Borneo.

*Hypothymis azurea consobrina Richm.

Hypolhymis consobrina Richmond, Proc. Biol. Soc. Wash. xv, 1902, p. I89: Simalur Island.

Distr.-Simalur Island, West Sumatra.

Hypothymis azurea abbotti Richm.

Hypothymis abbotti Richmond, Proc. Biol. Soc. Wash. xv, Igo2, p. I8g: Babi Island, West Sumatra.

Distr.-Islands of Babi and Lasia, West Sumatra.

*Hypothymis azurea isocara Oberl.

Hypothymis azurea isocara Oberholser, Proc. U.S. Nat, Mus. xxxix, I9II, p. 606; Bangkaru Island, Banjak Islands, West Sunatra. Distr._Banjak Islands, West Sumatra.

I Rensch considers that the Bali bird is only prophata $><$ symmixta. 
"Hypothymis azurea amelis Oberh.

Hypothymis azurea amelis Oberholser, Proc. U.S. Nat, Mus, xxxix, I9II, p. 608 : Nias Island.

Distr.-Nias Island, West Sumatra.

*Hypothymis azurea ponera Oberh.

Hypolhymis azurea ponera Oberholser, Proc. U.S. Nat. Mus. xxxix, I9I I, p. 604: Tana Masa Island, Batu Islands.

Distr.-Batu Islands, West Sumatra.

\section{Hypothymis azurea leucophila Oberh.}

Hypothymis azurea leucophila Oberholser, Proc. U.S. Nat. Mus. xxxix, IgI , p. 607 : North Pagi Island, West Sumatra.

Hypothymis azurea sipora Chasen and Kloss, Ibis, I926, p. 287 : Sipora Island, West Sumatra.

Distr.-Siberut, Sipora and Pagi Islands, Mentawi Islands, West Sumatra,

\section{Hypothymis azurea richmondi Obeth.}

Hypothymis azurea richmondi Oberholser, Proc. U.S. Nat. Mus. xxxix, 1911, p. 6r3: Engano Island.

Distr.-Engano Island, West Sumatra.

Gent1s RHIPIDURA Vigors and Horsfield.

Rhipidura albicollis (Vieill.).

Momitain Fantail Flycatcher.

Rhipidura albicollis albicollis (Vteill.).

Platyrhynchos albicollis Vieillot, Nouv. Dict. d'Hist. Nat. ed. 2, xxvii 1818, p. 13 : Bengal.

Distr.-Mt. Kinabalu, North Borneo.

Rhipidura albicollis atrata Salvad.s

Rhipidura alrata Salvadori, Ann. Mus. Civ. Gen. xiv, 1879, p. 203 : Padang, West Sumatra.

Rhipidura vidua (nec Salvad.), Schleg, in Snelleman, Midden Sumatra, iv, 1884 , pl. 2 : Sumatra.

Rhipidura schlegeli Sharpe, Zool. Rec, 1883 , Aves, p. 2 i : new name for $R$. vidua Schleg.

Distr,-Malay Peninsula.

Sumatra.

Borneo (not Mt. Kinabalı).

1 $R$. a. atrata is a thin race just recognizable by reason of the comparatively large white tips to the tail feathers but in birds from Kinabalu these tips are very small. 
Rhipidura phoenicura Müll. Javan Motntain Fantail Flycatcher. Rhipidura phoenicura S. Müller, Verh. Nat. Gesch. Land en Volk. I 443 , p. I85: Java.

Distr.-Java."

Rhipidura perlata S.Müll.

Spotted Fantail I'lycatcher.

Rhipidura perlata perlata S. Müll.

Rhipidura perlata Sal. Müller, Verh. Nat. Gesch. Land en Volk. I843, p. I85: Sumatra.

Leucocerca rhombiter Cab., Mus. Hein, i, 1850, p. 57: Sunda Islands. Rhipidura guttata "Tem." Gray, Handl, Bds, i, 869, p. 330 : Borneo (nom. nud.).

Distr.-Malay Peninsula.

Sumatra.

Borneo.

Java.

Rhipidura perlata euryura (S. Mï1\}.).

Rhipidura euryura S. Mïller, Verh. Nat. Gesch. Land en Volk. I 843, p. 185 , footnote: Java.

Distr.-Java*

Rhipidura javanica (Sparrm.).

Fantail Flycatcher.

Rhipidura javanica javanica (Sparrm.).

Muscicapa javanica Sparman, Mus. Carl. iii, 1788, pl. 75 : Java.

Platyrhyuchos perspicillatus Vieill., Nouv. Dict. d'Hist. Nat. ed. 2, xxxvii, 1818 , p. 14 : Africa error= Java.

Muscicapa umbellata Sundev., Phys, Sallsk. Jedskr. Lund, 1837, D. 72 : East Java.

Distr.-Java; Bali. ${ }^{3}$

Rhipidura javanica longicauda Wall.

Rhipidura longicauda Wallace, Proc. Zool. Soc. I865, p. 476 : Sumatra.

Lencocirca infumata Hume, Stray Feathers, i, 1873, p. 455: Acheen., North Sumatra.

Distr.-Malay Peninsula.

Sunatra; Rhio Archipelago; Banka; Billiton.

Borneo; North Bornean Islands; Maratua group.

- Has also been recorded from Borneo but we think in error. In spite of its very different colour, phavicura is, perhaps, only the Javan form of $R$. albicollis but as it lacks the white tips to the tail feathers, and we are unfamiliar with the many forms of this genus occurring in the regions to the east of Malaysia, we have, for the moment left phanicura as a species.

- Has also been recorded from Sumatra and Borneo but we consider that its presence in both these islands requires confirmation. $R$. ewryora is linked to $R$. perlata by incipient (or obsolete) spots on the under surface.

${ }_{3}$ The Bali bird is perhaps not javanica. 


\section{Genus TERPSIPHONE Gloger.'}

Terpsiphone paradisi (Lint1.).

Paradise F*lycatcher.

[Terpsiphone paradisi paradisi (I,in11.).

Corvus paradisi Linn., Syst. Nat. Ioth, ed. I758, p. 107: Ceylon

(Lim. 1766).

Dist.,-Extra-linital.]

Terpsiphone paradisi affinis (Blyth).?

Tchilrea affinis Blyth, Journ. Asiat. Soc. Bengal, xv, I846, p. 292 : Malacca.

Distr. - Malay States.

Sumatra (part); Rhio and Lingga Archipelagos; Billiton.

Terpsiphone paradisi indochinensis (Sal.).

Tchilrea affinis indochinensis F. Salomonsen, Ibis, I933, p. 734 : Cambodia.

Distr.-Peninsular Siam.

Sumatra (north).

Terpsiphone paradisi saturatior (Sal.).

Tchitrea affinis saluratior F. Salomonsen, Ibis, r933, P. 732 : Bhutan.

Distr.-Malay Peninsula. ${ }^{3}$

Terpsiphone paradisi borneensis (Hart.).

Tchilrea paradisi borneensis Hartert, Bull. Brit. Orn. Cl. xxxvi, I9I6, p. 75 : Sarawak.

Distr,-Borneo.

Terpsiphone paradisi australis Cluas.

Terpsiphone paradisi australis Chasen, Ornith. Monatsber, I935,

p. I47 : Lampongs, Sumatra.

Distr,-South (south). Java.

r Ct. Chas. and K1., Journ. Mal. Br. Roy. Asiat. Soc. vi, (3), 1928, p. 65 ; Salomon., Ibis, 1933 , p. 730 .

We have a large number of these birds from the Malay Peninsula and it seems impossible to fix any definite boundary between the northern irdochinensis and the southern afhnis for not only is there a lasge area in which intermediate specimens occur, but the two forms inosculate. Birds from the territory of Malacca southwards are invariably affuis: those from Peninsular Siam are either indochinensis, or nearer to that race than to affinis. Some birds from Selangor and Pahang are also affinis. We cannot agree with Salomonsen (1033) that Sumatra is inhabited by borneensis. In that island the situation appears to be rather like that in the Malay Peninsula. From the north we have a pale male in brown plumage indistinguishable from indochinensis of Siam. Other specimens from further south are nearer to afhnis which is the form occurring in the Rhio Archipelago. We bave, however, seen very few specimens from Sumatra.

3 Occurs as far south as Taiping in Perak, Malay States.

$$
[176]
$$


*Terpsiphone paradisi procera (Riclum.).

Tchitrea procera Richmond, Proc. U.S. Nat. Mus. xxvi, rgo3, p. 510: Simalur Island.

Distr-Simalur Island, West Stunatra.

Terpsiphone paradisi insularis Salvad.

Terpsiphone insularis Salvadori, Ann. Mus. Gen. (2a), iv, IS86, p. 539: Nias.

Distr.-Nias Island, West Sumatra.

Terpsiphone paradisi incei (Gould).

Muscipela Incei Gould, Birds of Asia, ii, I852, pl. I9: Shanghai. Dist.r.-Malay Peniusula.

Sumatra; Rlio Archipelago; Nias Island, West Sumatra.

Terpsiphone atrocaudata (Eyt.). Japanese Paradise Flycatcher. Terpsiphone atrocaudata atrocaudata (Eyt.).

Muscipela alrocaudata Eyton, Proc. Zool. Soc. I839, p. 102: Malay Peninstla = Malacca.

Distr.-Malay States.

Sumatra.

Gentus DRYMOPHILA Temminck.

Drymophila velata (Tetum.). Maroon-breasted Flycatcher. Drymophila velata velata (Temm.).

Drymophila velala Temminck, P1. Col. 334, I825: Java; Hartert, 1902.

Disir.-Java.

Drymophila velata casia (Less.).

Monarcha casia (sic) Lesson, Rev. Zool. I839, p. 167: Sumatra.

Muscicapa pectoralis Hay, Madr. Journ. Lit. Sci. xiii, 1844, p.161: Malacca.

Philenloma unicolor Blyth, Ibis, 1865, D. 46: Borneo.

Distr.-Malay Peninsula.

Sunatra.

Borneo.

Drymophila pyrhoptera ('Temm.). Chestnut-winged Flycatcher.

Drymophila pyrhoptera pyrhoptera (Temm.).

Muscicapa pyrhoplera Temminck, P1. Col. 596, 1836 : Borneo.

Muscipeta plumosa Blyth, Journ. Asiat. Soc. Beng, xi, 1842, p. 791 : Malacca.

Philentoma castaneum Eyton, Ann. Mag. Nat. Hist. xvi, 1845, p. 229 : Malacca.

x Ct. Kloss, Journ. F.M.S. Mus. x, 1921, p. 217. 


\section{MUSCICAPIDAE.}

Philentoma intermedius Hume, Stray Feathers, ix, tSSo, p. I13: Johore, Philentoma maxwelli Bartlett, Journ. Straits Br. Roy. Asiat. Soc. xxviii, 1895, p. 96: Sarawak.

Philentoma saravacense Bartlett, Sarawak Note-book, pt. 9, isg6, p. 8o: Sarawak.

Distr.-Malay Peninsula.

Stumatra.

Borneo.

Drymophila pyrhoptera dubia (Hart.).

Philenloma dubium Hartert, Nov. Zool. i, I894, p. 477 : Bunguran, North Natuna Islands.

Distr. -Natuna Islands.

\section{Genus RHINOMYIAS Sharpe.}

\section{Rhinomyias olivacea (Hume)." \\ Hume's Jungle-Flycatcher.}

Rhinomyias olivacea olivacea (Hume) , *3

Cyomis oltwaceus Hume, Stray Feathers, v, 1877, p. 338 : Sonth T'enasserin.

Rhinomyias pecloralis baliensis Hart., Nov. Zool, iii, 1806, p. 549 : Bali.

Distr.-Peninsular Siam.

Simatra; Billiton.

Borneo; Nortlı Natuna Islands.

Java; Bali.

Rhinomyias olivacea perolivacea Cliss. and Kloss.

Rhinomyias olivacea perolivacea Chasen and Kloss, Journ. f. Oraith. I929, ii, p. II3: Balambangan Island.

Distr,-Balambangan and Banguey Islands, North Borneo.

Rhinomyias brunneata (H. H. Slater). Migratory Jungle-Flycatcher.

Siphia brunneata H. H. Slater, Ibis, I897, p. I75: Fokhien.

Rhinomyias tardus Rob. and Kloss, Journ. Fed. Mal. States Mus. vi, 1915, p. 29: Selangor, Malay States.

Distr.-Malay States.

* Cf. Chas. and Kl., Bull. Raff. Mus. 4, 1930, p. 56.

- Stuart Baker has proposed a new genus, Olcyornis for this species. It is clearly out of place in Anthipes, but is, obviously, one of the Rhinomyias association. Two other forms, poliogenys and saturatior, linked to olivacea as stbspecies by Stuart Baker are not, in our opinion, even congeneric!

3 Myloterpe brumeicauda Salvad., Ann. Mus. Civ. Stor. Nat. Gen. (I), xiv, 1879 , p. 210 : West Sumatra, may apply to this species, but it also seems probable that it is either Alcippe cinerea Blyth (1844), or Muscitrea cinerea Blyth ( $1 \$ 47)$.

4 La Tonche ( 1925 ) linked $R$. brunneata and olivacea as subspecies, but we doubt if they are thus closely related. 
Rhinomyias umbratilis (Strickl.). White-throated Jungle-Flycatcher. Rhinomyias umbratilis umbratilis (Strick.).

Trichostoma umbratile Strickland, Contr. Orn., 1849, p. I26, pl. 31 : Borneo.

Alcippe pectoralis Salvad, Atti. R. Accad. Sci. Torino, iii, 1868, p. 530 : Borneo.

Muscicapa infuscata Blyth. Ibis, I87o, p. I65: Sumatra.

Siphia albo-oliwacea Hume, Stray Feathers, v, 1877, p. 488: Malacca.

Rhinomyias umbratilis richmondi Stone. Proc. Acad. Sci. Phil., Ixiv, 1902, p. 686: Mansalar Island, West Sumatra.

Distr,-Malay Peninsula.

Sumatra; Lingga Archipelago; Mansalar Island, West Sumatra; Billiton.

Borneo (lowlands); North Natuna Islands; Karimata Islands.

Rhinomyias umbratilis eclipsis Oberh.

Rhinomyias umbratilis eclipsis Oberholser, Snitlus. Misc. Coll. 1x, No. $7,1912,1$ \%. 12 : Tana Masa Island, Batu Islands.

Distr.-Batu Islands, West Sumatra.

Rhinomyias ruficrissa' Sharpe. Grey-breasted Jungle-Flycatcher.

Rhinomyias ruficrissa ruficrissa Sharpe.

Rhinomyias nuficrissa Sliarpe, Ibis, IS87, D. 44I: Mt. Kinabalu, North Borneo.

Distr,-Borneo.

Rhinomyias ruficrissa isola (Hach.) =

Rhinomyias ruficauda isola Hachisuka, Bull. Brit. Orn. Cl. lii, I932, p. IIo: Mt. Dulit, Sarawak.

Distr.-Borneo.

Rhinomyias gularis Sharpe.

Kinabalu Jungle-Filycatclier. Rhinomyias gularis Sharpe, Ibis, 1888 , p. 385 : Mt. Kinabalu, North Borneo.

Distr.-Borneo.

Is with other members of this genus we can, in the absence of material, attempt no further linking of subspecies: both $R$, nuficrissa and $R$. gularis, however, seem to have very near relatives in the Philipine Islands.

a This form is only accepted after much hesitation. To it the describer refers birds from Mt. Dulit, and Mt. Kalulong in Sarawak. In our collection five $\$$ kins from Dulit are rather more rufous above than a topotype of ruffrissa from Mt. Kinabalu, but a sixth skin from Dulit, and three from Mt, Temabo in Baram are inseparable from ruficrissa. 


\section{Crenus CULICICAPA Swinhoe."}

Culicicapa ceylonensis (Swains.).

Grey-headed Flycatclier.

Culicicapa ceylonensis ceylonensis (Swains.).

Platyrhynchus ceylonensis Swainson, Zool. Illus. Ser. I, I820, pl. I3: Ceylon.

Culicicapa ceylonensis amphiala Oberh., Smiths. Misc. Coll. Ix, No. 7. 1912, p. 12: North Pagi Island, West Sumatra.

Culicicapa ceylonensis antioxantha Oberh., Smiths. Misc. Coll. Ixxvi. No. 6, July, 1923, p. 9: Trang, Peninsular Siam.

Culicicapa ceylonensis pellopira Oberh., Smiths. Misc. Coll. Ixxvi, No. 6, 1923, p. 9: Java.

Culicicapa ceylonensis meridionalis Baker, Bull. Brit. Orn. Cl. xliv, Oct. 1923, p. 12: Peninsular Siam.

Culicicapa ceylonensis eophila Oberh., Bull. U.S. Nat. Mus. I59, 1932, p. $58:$ Bunguran Island, Natuna Islands,

Distr.-Malay Petuinsula.

Sumatra; Lingga Archipelago; Mentawi Islands, West Sumatra.

Borneo; North Natuma Islands.

Java; Bali.

* Culicicapa ceylonensis perenocara Obeth."

Culicicapa ceylonensis percnocara Oberholser, Sniths. Misc. Coll. 1x, No. 7, I912, p. I2 : Simalur Island.

Culicicapa ceylonensis pellonota Oberholser, Smiths. Misc. Coll, lx, No. 7,1912, p. 12: Nias Island.

Distr.-Sinalur and Nias Islands, West Sumatra.

\section{Genus STOPORALA Blyth."}

Stoporala indigo (Horsf.).

Sunda Island Blue Flycatcher.

Stoporala indigo indigo (Horsf.).

Muscicapa indigo Horsfield, Trans. Linn. Soc. xiii, I 821 , p. I46 : Java.

Dislr.-Java.

Stoporala indigo ruficrissa Salvad.

Stoparola ruficrissa Salvadori, Ann. Mus. Civ. Gen. xiv, I879, p. 202 : Padang district, West Sumatra.

Distr.-Sumatra.

' Cf. Chas. and Kloss, Journ. F.M.S. Mus. xiii, 1927, D. 5; Oberh., Bull. U.S. Nat. Mus. 159, t932, p. 58.

- We have never seen skins from Simalur and Nias and therefore follow Riley in regarding birds from these islands as belonging to one race and in being distinct from typical ceylonensis.

3 Cf. Spouck, v, Schaub, Org, Cl, Ned, Vog, i', Ig29, p. 40.

$$
[180]
$$


Stoporala indigo cerviniventris Sharpe.

Stoparola cerviniventris Sharpe, Ibis, IS87, p. 444: Mt. Kiuabalu, North Borneo.

Distr.-Borneo.

Stoporala thalassina (Swains.).

Verditer Flycatcher.

[Stoporala thalassina thalassina (Swains.).

Muscicapa thalassina Swainson, Nat. Libr. xxi, Flycatchers, $18_{3} 5$, p. 252 : India.

Distr.—_ixtra-linita1.]

Stoporala thalassina thalassoides (Cab.).

Glaucomyias thalassoides Cabanis, Mus. Hein. i, IS5I, p. 53, footnote; new name for Muscicapa thalassina Bp. (nec Swains.) : Sumatra.

Distr.-Malay Peninsula.

Sumatra.

Borneo.

\section{Family CAMPEGHAGIDA.}

Genus CORACINA Vieillot.

Coracina javensis (Horsf.).

Javan Cuckoo-Slurike.

Coracina javensis javensis (Horsf.).

Ceblepyris Javensis Horsfield, Trans. Linn. Soc, xiii, I82I, P. I45: Java.

Distr.-Java; Bali.

Coracina javensis larutensis (Sharpe).

Artamides larulensis Slarpe, Proc. Zool. Soc. I887, p. 435: Larut Hills, Perak.

Distr.-Malay States.

Coracina sumatrensis (S. Müill.)." Large Barted Ctrckoo-Shrike.

Coracina sumatrensis sumatrensis (S. Müll.).

Ceblypyris stumatrensis S. Müller, Verh. Nat. Gesch., Land en Volk. 1843 , p. 190: West Sumatra.

Grancalus concretus Hartl., Journ. f. Orn. 1864, p. 445: Borneo.

Artamides sumatrensis messeris Oberh., Journ. Wash. Acad. Sci, xvi, 1926, p. 517 : Trang, Peninsular Siam.

Arlamides sumatrensis nesiarchus Oberb., Journ. Wash. Acad. Sci. xvi, 1926, p. 517 : Parit Island, near Karimon Island, Rbio Archipelago.

Distr.-Malay Peninsula.

Sumatra; Rhio Archipelago.

Borneo; Nortî Bornean Islands.

1 Revision: Oberh., Bull. U.S. Nat. Mus. 159, 1932, p. 103. 


\section{Coracina sumatrensis bungurensis (Hart.).1}

Graucalus bungurensis Hartert, Nov. Zool. i, IS94, p. 477: Bunguran Island, North Natuna Islands.

Artamides sumatrensis calopolius Oberh., Bull. U.S. Nat. Mus. 98 , 1917, p. 34 : Mata Island, Anamba Islands.

Distr.-Anamba Islands.

Natuna Islands.

Coracina sumatrensis vordermani (Hatt.).

Graucalts vordermani Hartert, Bull. Brit. Orn. Cl. xii, I901, p. 32 : Kangean Island.

Distr.-Kangean Islands.

Coracina sumatrensis simalurensis (Richm.).

Gratecalus simalurensis Richmond, Proc. U.S. Nat. Mus. xxvi, igo3, p. $5 I_{3}$ : Simalur Island.

Distr.-Simalur Island, West Sumatra.

* Coracina sumatrensis babiensis (Richm.).

Grancalus babiensis Richmond, Proc. U.S. Nat. Mus. xxvi, I903, p. 5ז3: Babi Island, West Sumatta.

Distr.-Babi Island, West Sumatra.

Coracina sumatrensis kannegieteri (Bütt.).

Artamides Kannegieteri Büttikofer, Notes Leyd. Mus. xviii, I897, p. 175: Nias Island.

Distr.-Nias Island, West Sumatra.

Coracina sumatrensis crissalis (Salvad.).

Graucalus crissalis Salvadori, Ant. Mus. Civ, Gen. (2), xiv, 1894 , P. 592 : Sipora Island.

Artamides sumatrensis halistephis Oberb., Smiths. Misc. Coll. 1x, No. 7, I912, p. 14: South Pagi Island.

Distr.-Siberut, Sipora and Pagi Islands, West Sumatra.

Coracina sumatrensis enganensis (Salvad.).

Graucalus enganensis Salvadori, Ann. Mus. Civ. Gen. (2) xii, I892, p. I42: Ëngano Island.

Distr.-Engano Island, West Sumatra.

Individual birds from Tioman Island, east coast Malay Peninsula, and some of the Rhio Islands are also large and can be known as sumatrensis $>$ butugrensis $\{=$ nesiarchus Oberh. $)$. 
Coracina personata (S. Müll.).

Timor Cuckoo-Slirike.

[Coracina personata personata (Mäil.).

Ceblepyris larvata S. Müller, Verh. Nat. Gescli. Land en Volk. 1843, p. 190: Timor.

Distr.-Extra-linital.]

Coracina personata larvata (S. Miill.).

Ceblepyris larvata S. Muller, Verh. Nat. Gescli. Land en Volk. 1843 , p. Igo: Java.

Distr.-Java.

Coracina personata normani (Sharpe).

Graucalus normani Sharpe, Ibis, I887, p. 438: Mt. Kinabalu, North Borneo.

Distr.-Borneo.

Coracina personata melanocephala (Salvad.).

Graucalus melanocephalus Salvadori, Ann. Mus. Civ. Gen, (2) xiv, I879, p. 206 : Padang Highlands, Sumatra.

Distr.-Sumatra.

\section{Genus CHLAMYDOCHERA Sharpe}

Chlamydochera jefferyi Sharpe.

Whitehead's Cuckoo-Shrike.

Chlamydochera jefferyi Sharpe, Ibis, I887, p. 439, pl. I3: Mt. Kinabalu, North Borneo.

Distr,-Borneo.

\section{Genus LALAGE Boie.}

Lalage melanoptera (Rüpp.).

Large Grey Cuckoo-Shrike.

Ceblepyris melanoplera Rüppell, Mus. Senckenlb. iii, 1839, p. 25 , pl. 2, fig. i : New Holland, error=Burma.

Distr,-Peninsular Siam.

Lalage fimbriata ('Temm.).

Stwall Grey Cuckoo-Shrike.

Lalage fimbriata fimbriata (Temm.).

Ceblephyris fimbriatus Temminck, Pl. Col. 249, 250, I824: Java.

Volvocivora vidua Hartl., Journ. f. Orn. 1865, 13. 163: Java.

Distr._Java; Bali.

1 Cf. Kloss, Journ. F.M.S. Mus. x, 1921, p. 219. 
Lalage fimbriata culminata (A, Hay).

Ceblepyrts Culminatus A Hay, Madras Journ. xiii, I845, pt. 2, p. 157: Malacca.

Drymonax niger1 Less., Rev. Mag. Zool. 1830, p. 167: Sumatra.

Volvocivora schierbrandii Pelz., Reis. Novara, Vög. I865, p. 80, pl, 2, fig. I: Borneo.

Campephaga boneensis Salvad., Atti. R. Acc. Sci. Torino, iii, I868, p. 532 : Borneo.

Campophaga minor Davison, Ibis, 189z, p. 99 : Pahang.

Distr.-Malay Peninstila (north to South Peninsular Sianr).

Sumatra; Siberut Island, West Sumatra.

Borneo.

Lalage fimbriata neglecta (Hume).

Volvocivora neglecla Hume, Stray Feathers, v, I877, p. 203 : South Tenasserim.

Distr.-Peninsular Sian.

Lalage fimbriata compta Richmond.

Campephaga compta Richmond, Proc. U.S. Nat. Mus, xxvi, 1903, p. 514: Simalur Island.

Distr,-Simalur Island, West Sumatra.

Lalage nigra (Forst.).?

Pied Cuckoo-Shrike.

\section{Lalage nigra nigra (Forst.).}

Turdus niger Forster, Ind. Zool. $178 \mathrm{r}$, p. $4 \mathrm{I}$ : Singapore Island (Bangs, J022).

Turdus terat Bodd., Tabl. Pl. Enlum. 1783, p. 17: Singapore (Kloss, 1920).

Turdus orientalis Gmel., Syst. Nat. i, 1788, p. 821: Singapore (Kloss, 1026).

Ceblephyris striga Horsf, Trans, Linn, Soc, xiii, 1821, p. 145: West Java.

Pycnonotus humeraloides Less., Echo du Monde Savant, 1844, p. 733 : West Java.

Lalage nigra brimnescens Stuart Baker, Bull. Brit. Orn. Cl. xliv, Igzz. p. 13 : Selangor, Malay States.

Distr.-Malay Peninsula.

Sumatra.

Java (not east); Karimon-Java Islands.

Lalage nigra sueurii (Vieilt.).

Turdus Suerii Vieillot, Nov. Dict. d'Hist. Nat. ed. 2, xii, I8I8, p. 270: Timor.

Distr._-Java (east); Bali.

1 "Corpora" is almost certainly an error: otherwise the description is meaningless. In it the first phrase seems to refer to the head.

2 Cf. Kloss, Journ. Mal. Br. Roy. Asiat. Soc. iv, 1926, p. 158 . 
*Lalage nigra chalepa (Ober11.).

Perissolalage chalepa Oberholser, Proc. U.S. Nat. Mus. liv, I9I7, p. I 82 : Solombo Resar Island.

Distr.-Solombo Besar Island, East Java Sea.

\section{Lalage nigra schisticeps Neum."}

Lalage nigra schisticeps Nemmann, Journ. f. Ornith, lxvii, I9rg, p. 333 : Culion Island, Philipjine Islands.

Distr.-Borneo; Noth Bornean Islands; Maratua Islands.

* Lalage nigra empheris Oberh.

Lalage nigra empheris Oberiolser, Stnitls. Misc. Coll. 1x, No. 7 , I912, p. I5: Nias Island.

Distr.-Nias Island, West Sumatra.

\section{Genus PERICROCOTUS Boie, ${ }^{2}$}

Pericrocotus flammeus (Forst.).

Scarlet Minivet.

[Pericrocotus flammeus flammeus (Forst.).

Muscicapa flammea Forster in Penuant's Ind. Zool. I78r, p. 25: 'Travancore.

Distr,-Extra-imital.]

Pericrocotus flammeus flammifer Hume.

Pericrocotus flammifer Hume, Stray Feathers, iii, I875, p. $32 \mathrm{I}$ : Pakchan, South Tenasserim.

Distr.-Malay Peninsula.

Pericrocotus flammeus xanthogaster (Raffles).

Lanius xanthogaster Raftes, Trans, Linn, Soc, xiï, 1822, p. 309 : Bencoolen, West Sumatra.

Phaenicomis ardens Bp. Consp. Gen. Avium, i, 1850, p. 357 : Sumatra. Pericrocotus subardens Hume, Stray Feathers, $v_{1} 1877$, p. 196: Sumatra.

Disir,-Malay States. ${ }^{3}$

Sumatra; Banka; Billiton.

Borneo.

*Pericrocotus flammeus modiglianii Salvad.

Pericrocotus modiglianii Salvadori, Ann, Mus, Civ, Gen. (2) xii, I892, p. 130: Engano Island.

Distr.-Eingano Island, West Sumatra.

t The type of schisticeps is an artifact (Stres., Orn. Monatsber. xxx, I 922, p. 88 ).

2 Revisions: Rob., Journ. F.M.S. Mus. vi, 1915, p. $3^{1}$ : Stres., Mitt. Zool. Mus, xv, 1930, P. 635 .

3 In the Malay Peninsula, broadly speaking, xanthogaster occurs mainly in the south: it spreads northwards in the lowlands and flammi/er has extended its range into the Malay States along the mountain ranges. 
*Pericrocotus flammeus minythomelas Oberh.

Pericrocotus andamanensis minylhomelas Oberholser, Stmiths. Misc. Col1. Ix, No. 7, 1912, p. 14: Simalur Island.

Distr-Simalur Island, West Sumatra.

\section{Pericrocotus flammeus siebersi Renscli,}

Pericrocolus speciosus siebcrsi Rensch, Orn. Monatsber. xxxvi, I928, p. 47 : Mt. Gede, West Java.

Distr.-Java; Bali.

Pericrocotus montanus Saivad.

Wray's Minivet.

Pericrocotus montanus montanus Salrad.

Pericrocotus montanus Salvadori, Ann. Mus. Civ. Gen. xiv, I879, p. 205: Mountains of West Sumatra.

Pericrocotus qurayi Sharpe, Proc. Zool. Soc. 1888, p. 269, pl. 15 : South Perak.

Pericrocotus croceus Sharpe, Proc. Zool. Soc. 1888, D. 260 : South Perak. Distr.-Malay States.

Sumatra.

Pericrocotus montanus cinereigula Sharpe.

Pericrocolus cinereigula Sharpe, Ibis, I889, p. Ig2: Mt. Kinabalu, North Borneo.

Distr.-Borneo.

Pericrocotus igneus Blyth.

Fiery Minivet.

Pericrocotus igneus igneus Blyth.

Pericrocotus igneus Blyth, Journ. Asiat. Soc. Bengal, xv, I846. p. 309: Malacca.

Pericrocotus minutus Strickland in Jardine's Contr. Orn. 1849, p. 94, pl. 32 : Borneo.

Pericrocolus breviroslris minor "Verr." Strickland in Jardine's Contr. Orn. 1849 , p. 94: Borneo.

Pericrocotus flagrans $\mathrm{B}$., Consp. Gen. Avium, i, 1850, p. 357 : Sumatra.

Distr.-Malay Peninsula.

Sumatra; Billiton; Nias Island, West Sumatra.

Borneo.

*Pericrocotus igneus trophis Oberl1.

Pericrocolus igneus trophis Oberlolser, Smiths. Misc. Coll. 1x, No. 7, IgI2, p. I4 : Simalur Island.

Distr.-Simalur Island, West Sumatra.

- Birds from East Java and Balj are not exactly like topotypical siebersi, but are approaching exsul Wallace (Lombok). 
Pericrocotus cinnamomeus (Linu.). Small Minivet

[Pericrocotus cinnamomeus cinnamomeus (I,inn.).

Motacilla cinnamomea Linn., Syst. Nat. I2th. ed. i, 1766, p. 335 : Ceylon.

Disir._Extra-limital.]

Pericrocotus cinnamomeus vividus Baker.

Pericrocolus peregrinus vividus Stuart Baker, Butl. Brit, Oril, Cl, xl, I920, P. II4: Attaran River, Tenasserim.

Distr.-Peniusular Sianu.

Pericrocotus cinnamomeus saturatus Baker.

Pericrocolus peregrimus saturatus Baker, Bull. Brit. Orn. Cl. xi, I920, p. II5: West Java.

$\begin{aligned} & \text { Distr.-Sumatra. } \\ & \text { Borneo. } \\ & \text { Java; Bali. }\end{aligned}$

Pericrocotus roseus (Vieill.).

Pericrocotus roseus (Vieill.).

Rosy Minivet.

Muscicapa rosea Vieillot, Nov. Dict. d'Hist. Nat., ed. 2 , xxi, ISIS, p. 486 : Bengal.

Distr.-Peninsular Siam.

Pericrocotus roseus cantonensis Swinh.

Swinloe's Minivet.

Pericrocotus cantonensis Swinhoe, Ibis, 1861, p. 42 : Canton, South Clina.

Distr.-Peninsular Siam.

Pericrocotus roseus divaricatus (Raftles).

Asliy Minivet.

Lanius divaricatus Rafles, Trans. Linn. Soc. xiii, 1822, p. 306 : Sumatra.

Pericrocotus modestus Strickland, Proc. Zool. Soc. for 1846,1847 , p. 102: Malacca.

Distr,-Malay Peninsula.

Sumatra.

Borneo.

Pericrocotus miniatus (Temm.).

Sunda Minivet.

Pericrocotus miniatus miniatus (Temm.).

Muscicapa miniata Temminck, P1. Col. 156 , I822: West Java.

Distr.-Sumatra.

Java.

- Revision: Snouck, v, Schaub, Treubia, xi, 1930, p. 309. See also Whistler, Journ. Bomb. Nat. Hist, Soc. xxxvi, 1933, p. 342 .

" The localities Borneo and Sumatra need confrmation. 


\section{Family PYCNONOTIDÆ.'}

Gents EGITHINA Vieillot.

Egithina viridissima (Bp.).

Green Iora.

Agithina viridissima viridissima (Bp.).

Iora viridissima Bonaparte, Consp. Gen. Aviun, i, 1850, p. 397 : Sumlatra.

lora chloroptera Salvad., Ann. Mus. Civ. Gen. 1874, D. 192: Sarawak. Distr.-Malay Peninsula; Tioman Island.

Sumatra; Rhio and Lingga Archipelagos; Banka; Billiton. Borneo; North Natuna Islands.

Egithina viridissima thapsina Oberh.

Aegilhina viridissima thapsina Oberholser, Bu1l. U.S. Nat. Mus. 98,1917, p. 40: Siantan Island, Anamba Islands.

Distr.-Anamba Islands.

\section{Egithina viridissima nesiotica Oberl?.}

Aegithina viridissima nesiotica Oberholser, Smith. Misc. Coll. ix, No, 7, 1912, P, I0: Tana Bala Island, Batu Islands.

Distr.-Batu Islands, West Sumatra.

Egithina tiphia (Lirn).).

Common Iora.

[E Egithina tiphia tiphia (Linn.).

Motacilla tiphia Linn., Syst, Nat. 1oth. ed, I\$58, P. I86: Bengal.

Distr,-Eixtra-linital.]

Egithina tiphia micromelaena Oberh.

Aegithina tiphia micromelaenta Oberholser, Smiths. Misc. Coll. 1xxvi, No. 6, 1923, p. 7 : Banka Island.

Distr.-Peninsular Sian; Malay States (north).

Sumatra; Banka Island.

Egithina tiphia singapurensis Chas. and Kloss.

Aegithina tiphia singapurensis Chasen and Kloss, Bull. Raffl. Mus. 5, I931, p. 85: Singapore Island.

Distr.-Malay States (south).

There seems little point in removing from this family such genera as lrena, Chloropsis, etc. and creating specia! families for their reception until this and allied groups have received attention at the hands of a qualified anatomist.

Revisions: Snouck. v. Schaub., Org. Cl. Ned. Vog. vi, 1934, p. 140 and vii, 1935, p. 103 . 
Egithina tiphia viridis $(\mathrm{Bp}$, ).

Iora viridis Bonaparte, Consp. Gen. Avium, i, I850, p. 397 : North Borneo.

Distr.-Borneo (northern half); North Bormean Islands.

Egithina tiphia damicra Oberh.

Acgithina tiphia damicra Oberholser, Smiths. Misc. Coll. Ixxvi,

No. 6, I923, p. 7 : Smitau, Kapuas River, South-west Borneo. Aegithina tiphia zophonota Oberhoiser, Smiths. Misc. Coll. 1xxvi, No. 6 , 1023, p. 8: Taham, Central East Borneo.

Distr.-Borneo (southern half); Maratua Islands, East Borneo.

Egithina tiphia scapularis (Horsf.).

Jora scapularis Horsfield, Trans. Linn. Soc. xiii, I82I, p. I52: Java.

Distr.-Java; Bali.

*Egithina tiphia horizoptera Oberh.

Aegithina liphia horizoptera Oberholser, Smiths. Misc. Coll. 1x,

No. 7, Igr 2, 1. 9: Nias Island, West Sumatra.

Distr.-Nias Island, West Sumatra.

\section{Genus AËTHORHYNCHUS Sundevall.}

Aëthorhynchus lafresnayei (Hart1.).

Great Iora.

Aëthorhynchus lafresnayei lafresnayei (Hartl.).

Iora Lafresnayci Hartlaub, Rev. Mag. Zool. I844, p. 40I: Malacca.

Phanicomanos iora Sharpe, Proc. Zool. Soc. 1874, p. 427, pl. 54 : Jamaica, error = Malacca.

Distr.-Malay Peninsula.'

Genus CHLOROPSIS Jardine and Selby.

Chloropsis hardwickii Jardine and Selby. Orange-bellied Leaf-Bird. [Chloropsis hardwickii hardwickii Jard. and Selby.

Chloropsis hardwickii Jardine and Selby, Ill. Orn., sign. C. 3, I830: Nepal.

Distr.-Extra-limital.]

Chloropsis hardwickii malayana Rob. and Kloss.

Chloropsis hardwickii malayana. Robinson and Kloss, Journ. Fed.

Mal. States Mus. xi, r923, p. 55 : Mt. Ijau, Perak.

Distr.-Malay States.

Chloropsis venusta $(\mathrm{Bp}$.$) .$

Suntratran Leaf-Bird.

Phyllomis venusta Bonaparte, Consp. Gen Avium, I, IS50, P. 396 : Sunatra.

Distr.-Sumatra.

- Birds from Peninsular Siam are really A. lafresnayei $>$ innotata Blyth. 
Chloropsis sonnerati Jard, and Selby. Malachite-shouldered Leaf-Bird. Chloropsis sonnerati sonnerati Jard, and Selby.

Chloropsis sonnerati Jardine and Selby, Illus. Orn. $1 \$_{26} 6$, sign, B. 4 : Java.

Turdus viridis Horsf., Trans. Linn. Soc. xiii, 182!, p. 148: Java (not $T$. viridis P.L.S. Müll. 1776).

Phyllornis mullerit Temm., P1. Col., 1820 , livr. Sr : Java.

Dislr, -Java.

Chloropsis sonnerati zosterops Vig.

Chloropsis zosterops Vigors, Men. Life Raffles, I830, p. 674: Sumatra.

Chloropsis viridis viriditectus Hart., Nov. Zool. ix, 1go2, p. 2t2, Baran, Sarawak.

Distr.-Malay Peninstila.

Sumatra; Rhio Archipelago; Billiton.

Borneo; North Natuna Islands.

\section{Chloropsis sonnerati parvirostris Hart.}

Chloropsis zoslerops parvirostris Hartert, Orn. Monatsber, I 898. p. 93 : Nias Island.

Distr.-Nias Island, West Sumatra.

Chloropsis cochinchinensis (Gmel.). ${ }^{1}$ Green Leaf-Bird.

Chloropsis cochinchinensis cochinchinensis (Gm.).

Turdus cochinchinensis Gnelin, Syst. Nat. i, I788, p. 825: Cochin China.

Distr,-Peninsular Siam.

Chloropsis cochinchinensis icterocephala Less.

Chloropsis icterocephala Lesson, Rev. Mag. Zool. I840, p. I64: Paletnbang, Sumatra.

Phyllortis moluccensis J. E. Gray, Zool. Misc. 1832, p. 33: Malacca (Strickl. 1840 ).

Distr.-Malay States.

Sumatra; Rhio and Lingga Archipelagos; Banka; Billiton.

North Natuna Islands.

Chloropsis cochinchinensis viridinucha (Sliarpe).

Phyllomis viridinucha Sharpe, Ibis, I877, p. I5: Sarawak, Borneo.

Distr.-Borneo.

Chloropsis cochinchinensis nigricollis (V'eill.).

Philemon nigricollis Vieillot, Nov. Dict. d'Hist. Nat. ed. 2, xxvii, I8I8, p. 432 : Java.

Meliphaga javensis Horsf., Trans. Linn. Soc. xiii, $1 \$ 21$, p. 152: Java. Distr,-Java,

: Cf. Kloss, Journ. Mal. Br. Roy. Asiat. Soc. iv, 1926, p. 162. 
Chloropsis cochinchinensis flavocincta Sharpe.

Chloropsis flavocincta "Sharpe, Ibis, 1887 , p. 445 : Mt. Kinabalu, North Borneo.

Chloropsis kinabaluensis Sharpe, Ibis, 1887, p. 445: Mt. Kinabalu, North Borneo.

Distr.-Borneo.

Chloropsis cyanopogon (Temm.). Blue-whiskered Leaf-Bird. Chloropsis cyanopogon eyanopogon (Temm.).

Phyllornis cyanopogon Temminck, P1. Col. 512, x832: Palembang, Sumatra.

Chloropsis mysticalis Swains., Anim. in Menag. 1838, p. 296: India. егтот = Malacca.

Distr.-Malay States.

Sumatra.

Borneo; Banguey Island, North Borneo.

Chloropsis cyanopogon septentrionalis Rob. and Kloss.

Chloropsis cyanopogon seplentrionalis Robinson and Kloss, Journ.

Nat. Hist. Soc. Siam, iii, I9I8, p. I07: Ghirbi, Peninsular Siam.

Distr.-Perinsular Siam.

Chloropsis aurifrons (Termm).

Gold-fronted Leaf-Bird.

[Chloropsis aurifrons aurifrons (Temm.).

Phyllomis aurifrons 'Temminck, P1. Col. 484,1829 : Sumatra error = Cachar (Baker).

Distr.-Extra-linital.]

Chloropsis aurifrons media (Bp.).

Phyllonis media Bonaparte, Consp. Gen. Avium, i, 1850, p. 396 : Sumatra.

Disit.-Sumatra.

Genus IRENA Horsfield, ${ }^{+}$

Irena puella (Latls.).

Fairy Bluebird.

Irena puella puella (Latli.).

Coracias Puella Latłan, Index Orn, i, I790, p. I7I: Travancore, India.

Distr.-Peninsular Siam.

Irena puella malayensis Horsf, and Moore.

Irena malayensis Horsfield and Moore, Cat. Bds. Mus. East InoJ. Coy. 1, 1854, P. 274: Malacca.

Muscicapa cyanea Begbie, (not $M$. cyanea Müll., 1776), Malayan Peninsula, $1 \$_{34}$, D. 517 : Malay Peninsula.

Distr.-Malay States.

'Cf. Oberh., Wash. Acad. Sci. vii, 1917, p. 537. 
Irena puella criniger Sharpe.

Irena criniger Sharpe, Cat. Bds. Brit. Mus. iii, 1877, p. 267 : Borneo. Glauconympha cyanea megacyanea Oberholser, Journ. Wash. Acad. Sci. vii, 1917, p. 540: Tuanku Island, Banjak Islands, West Sumatra.

Distr.-Sumatra; Mendanau Island; West Sumatra Islands of Banjak, Nias, Batu, Siberut, Sipora and Pagi. Borneo.

Irena puella turcosa Walden.

Irena lurcosa Walden, Ann. Mag. Nat. Hist. (4) v, I87o, D. 4r7: Java.

Distr.-Java."

\section{Genus IXOS Tenminck.}

Ixos cinereus (Blyth).

Ashy Bulbul.

Ixos cinereus cinereus (Blyth).

Iole cinerea Blyth, Journ. Asiat. Soc. Bengal, xiv, I845, p. 573 : Malacca.

Trichophonus pulverulentus Bp., Consp. Gen. Avium, i, I850, p. 262: Sumatra.

Iole terricolor Hume, Stray Feathers, vii, 1878, p. 141: Malacca.

Disir.-Malay Peninsula.

Sumatra.

Ixos cinereus connectens (Sharpe).

Hemixus connectens Shatpe, Ibis, 1887, p. 446 : Mt. Kinabalu, North Borneo.

Distr.-Borneo.

Ixos virescens 'T'enm.

Java Streaked Bulbul.

I xos virescens virescens Temu.

Ixos virescens Temminck, Pl. Col. 382, I825: Java.

Distr.-Java,

Ixos virescens sumatranus (Wardl.-Rams.).

Hemixus sumatranus Wardlow-Ramsay, Ann. Mag. Nat. Hist. (5), $\mathrm{x}, 1882, \mathrm{p}, 431$ : Sumatra.

Trichophorus striolatus Bp., Consp. Gen. Avium, i, $1 \$ 50$, p. 262 : Sumatra.

Disir.-Sumatra.

Ixos malaccensis (Blyth).

Streaked Bulbul.

Hypsipetes malaccensis Blyth, Journ. Asiat. Soc. Bengal, Xiv, I845, p. 574 : Malacca.

Distr.-Malay Peninsula,

Sumatra; Lingga Island.

Borneo.

Not yet recorded from East Java. 
[Ixos mcclellandii meclellandii (Horsf.).

Hypsipetes Meclellandii Horsfield, Proc. Zool. Soc. for 1839 , I840, D. I59: Assam.

Distr.-Extra-linital.]

Ixos mcclellandii peracensis (Hart. and Butl.).

lole tickelli peracensis Hartert and Butler, Nov. Zool. v, r898, p. 506 : Mt. Ijau, Perak.

Distr.-Malay Peninsula.

\section{Gerus IOLE Blyth.}

Iole olivacea Blyth'.

Crested Olive Bulbul.

Iole olivacea olivacea Blyth.

Iole olivacea Blyth, Journ. Asiat. Soc. Bengal, xiii, 1844 , p. 386 : Singapore Island.

Trichophorus brunescens Finsch, Journ. f. Orn., 1867, p. 19: Sumatra (nom. nud.).

Distr.-Malay Peninsula.

Sumatra; Rhio Archipelago; Banka; Billiton.

North Natuna Islands.

\section{Iole olivacea cinnamomeoventris Baker.}

Iole virescens cinnanomeoventris Stuart Baker, Bull. Brit. Orn. Cl xxxviii, 1917, p. I6: Tenasserim.

Disir.-Peninsular Siam. *

* Iole olivacea crypta Oberh.3

Iole olivacea crypla Oberholser, Proc. Biol. Soc. Wash. xxxi, 1918, p. 197 : Jimaja Island, Anamba Islands.

Distr.-Anamba Islands.

Iole olivacea charlotte (Finscl).

Criniger Charlotte Finsch, Journ. f. Ornith, xv, 1867, p. Ig: Borneo.

Distr.-Borneo; Banguey Island, North Borneo.

We do not accept the lumping of /xacincla and /ole and resulting invalidation of lole olinaced Blyth.

2 Trang forms the meeting place of this race and 1 . 0 . olivacea: both forms occur in the district.

3 We have never seen exact topotypes of this race and in series it may prove to be rather larger than typical olvacea as most Anamba forms vary in the direction of large size. We cannot however admit crypta for Sumatra from which island we have seen large series. 


\section{Genus EUPTILOSUS Gray,}

Euptilosus eutilotus (Jard. and Selb.). Crested Brown Bulbul.

Brachypus eutilohs Jardine and Selby, Ill. Orn. (new ser.) I836, pl. 3 : Singapore Island.

Brachypodins tristis Blyth, Journ. Asiat. Soc. Beng. xiv, 1845, p. 576 : Penang.

Brachypus tympanistrigus Bp., (not Muill.), Consp. Gen. Avium, i, 1850, p. 264 : Sumatra.

Microtarsus cantori Horsf. and Moore, Cat. Bds. Mus. East Ind. Coy. i, $1854,13,409$ : Malacca.

Trichophorus susanwi $\mathrm{B}$. . Notes Coll. Delattre, in Comptes Rendus, 1854, P. 59: Bomeo.

Distr. Malay Peninsula.

Sumatra; Banka.

Botneo.

Euptilosus urostictus (Salvad.).

Wattled Bulbul.

[Euptilosus urostictus urostictus (Salvad.).

Brachypus urostictus Salvadori, Atti R. Accad. Sc. Torino, v; r87o,

p. 509: Philippine Istands.

Distr, -Extra-limital.]

Euptilosus urostictus nieuwenhuisii (Finsch).

Poliolophus Nieutenhuisii Finsch, Notes Leyd. Mus. xxiii, I9o2,

p. 95: Upper Kajan River, Bulongan, Central Borneo.

Distr.-Borneo.

Genus BRACHYPODIUS Blyth.

Brachypodius atriceps (Temm.). ${ }^{\mathrm{t}}$

Black-headed Buibul.

Brachypodius atriceps atriceps (Temm.).

Turdus alriceps Temnninck, Pl. Col. I47, I823: Java.

Lanius metanocephulos Gmel., Syst. Nat, i, 1788, 1) 300 : Sumatra.

Ixos chalcocephalus Temm, PI. Col. 453, fig. i, 1828 : Java.

Ixos metallicus Eyton, Ann. Mag. Nat. Hist. xvi, 1845. p. 228 : Malacca.

Brachypodius immaculatws Sharpe, Ibis, 1876, p. 39: Sumatra.

Brachypodius atriceps chrysophorus Oberh., Smiths. Misc. Coll. Ix,

No. 7 , 1912, p. 10: South Pagi Island, West Sumatra.

Distr.-Malay Peninsula.

Sumatra; Bauka; Billiton; West Sumatran Islands of Nias and Mentawi group.

Borneo.

Java; Bali.

I In the north of Peninsular Siam this species is more properly represented by $B$. a. $>$ cinereoventris Blyth (Tipperah) of which major Rob. and Kloss (North Cachar) seems to be a synonym.

₹ Not L. melanocephalus id., ib. p. 301. 
*Brachypodius atriceps baweanus (Finsch).

Brachypodius baweanus Finsch, Notes Leyd. Mus. xxii, rgor, p. 209: Bawean Island.

Microtarsus melanocephalos abbotti Oberh., Proc. U.S. Nat. Mus. lii, 1917, p. 193 : Bawean Island.

Distr.-Bawean Island.

*Brachypodius atriceps hyperemnus (Oberh.).

Microtarsus melanocephalos hyperemus Oberholser, Suiths. Misc. Coll. 1x, No. 7, Igr2, p. 10: Simalur Island.

Distr.-Simalur Island, West Sumatra.

Brachypodius atriceps hodiernus Bangs and Peters.

Microlarsus hodiernus Bangs and Peters, Occ. Papers Boston Soc.

Nat. Hist. v, 1927, p. 238 : Maratua Island.

Distr,-Maratua Islands, East Borneo.

\section{Genus MICROTARSUS Ėyton.}

Microtarsus melanoleuces Eyton. Black-and-White Bulbul.

Microtarsus melanoleucos Eyton, Proc. Zool. Soc. I\$39, p. I02: Malacca.

Brachypus vidua Bp. Consp. Gen. Avium, 1850, i, p. 264 : Borneo.

Microtarsus melanoleveos proximus? Riley, Proc. Biol. Soc. Wash. $\mathrm{xl}$, 1927, p. 96: Siberut Island, West Sumatra.

Distr.-Malay States.

Sumatra; Sibertt Island, West Sumatra.

Borneo.

\section{Genus CRINIGER Temminck.3}

\section{Criniger gutturalis $\left(\mathrm{B} \mathrm{p}_{\mathrm{f}}\right)$.}

Crested Bulbul.

Criniger gutturalis ochraceus Horsf. and Moore.

Criniger ochracets Horsfield and Moore, Cat. Bds. Mus, East Ind.

Coy. i, 1854 , p. 252: Tenasserin.

Criniger sordidus Richmond, Proc. U.S. Nat. Mus. xxii, 1900, p. 320 : Trang, Peninsular Siam,

Distr.-Peninsular Siani; Malay States (extrene north).

- We regard the grey forms of Brachypodius as common mutations, but it is curious that they are unknown in some areas occupied by the species.

: There is no difference in colour between many fresh skins of melanoleucos and "proximus".

3 The Malaysian subspecies of Criniger are very difficult to combine into species and the present attempt is, frankly, little more than suggestive. A significant feature is that only in the Malay Peninsula does one meet with two forms in any one place and that even here they are largely mutually exclusive. C. tephrogenys is common in the lowlands throughout the whole length of the Peninsula. C. sacculatus only occurs in the south of the Peninsula at high elevations. C. ochraceous occurs only in the north, but it averlaps the range of tephrogenys. Were it not for this it would be possible to regard all Malaysian forms as representatives of one widely-spread species. 
Criniger gutturalis sacculatus Rob.

Criniger ochraceus sacculalus Robinson, Ibis, t9r5, p. 746: Ginting Bidei, Selaugor.

Distr.-Malay States.

Criniger gutturalis sumatranus Wardl.-Rams.

Criniger sumatranus Wardlaw-Ramsay, Ann. Mag. Nat. Hist. (5) $\mathrm{x}, \mathrm{I} 8 S_{2}, \mathrm{p} .43 \mathrm{I}$ : Sumatra.

Distr.-Simatra (nountains).

Criniger gutturalis gutturalis (Bp.).

Trichophorus gutturalis Bonaparte, Consp. Gen. Avium, i, I850, p. 266 : Borneo.

Distr._Borneo (lowlands).

Criniger gutturalis ruficrissus Sharpe.

Criniger ruficrissus Slarpe, Proc. Zool. Soc. I879, p. 248: Mt. Kinabalu, North Borneo.

Distr.-Borneo (mountains).

Criniger tephrogenys (Jard. and Selb.). Scrub Bulbul.

Criniger tephrogenys tephrogenys (Jard, and Selb.).

Trichophorus tephrogeny's Jardine and Selby, Ill. Orn. iii, 1833. pl. I 27 : Malacca.

Distr.-Malay Peninsula.

Sumatra (lowlands).

Criniger tephrogenys barkelsi Coll, and Hart.

Criniger balicus barfelsi Collin and Hartert, Nov. Zool. xxxiv, 1927. P. 51: noln. nov. for Turdus gularis Horsf. (not Lath. ISor) : West Java.

Turdus gularis Horsf., Trans. Linn. Soc. xiti, 1822, p. 150: Java.

Distr.-Java (not east).

\section{Criniger tephrogenys balicus Stres.}

Criniger gularis balicus Stresemann, Nov, Zool. xx, I9ז3, p. 358: Bali.

Distr.-Java (east); Bali.

Criniger finschii Salvad.'

Finsch's Bulbul.

Criniger finschii Salvadori, Atti R. Accad, Torini, vi, 1871, p. 128 : Borneo.

Criniger theiodes Hume, Stray Feathers, iv, I876, D. 214 : Johore, Malay Peninsula.

Distr.-Malay States.

Sunlatra.

Borneo.

- This is not a common bird and we have never seen a good series of topotypes. 
PYCNONOTIDE.

\section{Genus ALOPHOIXUS Oates.}

Alophoixus phæocephalus (Hartl.).

White-throated Bulbul.

Alophoixus phrocephalus pheocephalus (Hattl.).

Ixos phcocephalus Hartlaub, Rev. Mag. Zool. I844, p. $40 \mathrm{r}$ : Malacca.

Trichophorus caniceps Lafr., Rev. Mag. Zool. 1845 , D. 367: "de 1'Inde" = Malacca,

Pycnonoths rufocaudatus Eyton, Ann. Nat. Hist. xvi, 1845, p. 228: = Malacca.

Trichophorus sulphuralus Bp., Consp. Gen. Avium, 1850, p. 262: Borneo error = Sumatra.

Criniger cantori Moore, Proc. Zool. Soc. 1854 , p. $279=$ Malacca.

Distr,-Malay Peninsula.

Sumatra; Rhio and Lingga Archipelagos; Billiton.

Nortl Natuna Islands.

Alophoixus phreocephalus diardi (Finsch).

Criniger diardi Finsch, Journ, f. Orn. I867, p. I8: Pontianak, South-west Borneo.

Distr.-Borneo (part).'

Alophoixus phæocephalus connectens Chas. and Kloss.

Alophoixus pheocephalus connectens Chasen and Kloss, Journ. $\mathrm{f}$. Orn. 1929, Bd. 2, p. I44: Sandakan, North Borneo.

Distr.-Borneo (part).

\section{Alophoixus phaecephalus medius Kloss.}

Alophoixus phaocephalus medius Kloss, Treubia, xii, 1930, p. 412: Loug Petah, Telen River, East Borneo.

Distr,-Borneo (part).

\section{Genus SETORNIS Lesson.}

Setornis criniger Less.

Van Bemmelea's Bulbul.

Setornis criniger Lesson, Rev. Mag. Zool. I839, p. I67: Sumatra error $=$ Borneo.

Trichophoropsis typus Bp., Compt. Rend, xxxviii, 1854, p. 59 : Borneo.

Criniger bemmeleni Finsch, Journ. f. Orn. 1867, p. 29: Borneo.

Distr.-Banka Island.

Borneo.

The ranges of the Bornean races of $A$. phaocephalus are rather complicated: See Kloss, Treubia, xii, 1930, p. 413. 
Genus TRICHOLESTES Salvadori.

Tricholestes criniger (Blyth).

Hairy-backed Bulbul.

Tricholestes eriniger criniger (Btyth).

Brachypodius ? criniger "Hay", Blyth, Journ. Asiat. Soc. Bengal, xiv, 1845 , p. 577 : Malacca.

Trichophorus minutus Hartl., Journ. f. Orn. 1853, p. 156: Malacca.

Distr.-Malay Peninsula; Tionan Island.

North Natuna Islands.

Tricholestes criniger sericea' Rob, and Kloss.

Tricholestes criniger sericea Robinson and Kloss, Jottrn. Fed. Mal. States Mus., xi, 1924, p. 288 : West Sumatra.

Distr.-Sunatra; Mansalar Island, West Stmmatra.

Tricholestes criniger viridis $(\mathrm{Bp}$.$) .$

Trichophoropsis viridis Bonaparte, Compt. Rent, is54, p. 59 : Borneo.

Distr.-Borneo.

\section{Genus ALCURUS Blyth.}

Alcurus leucogrammicus (S. Müll.).

Striated Green Bulbul.

Alcurus leucogrammicus leucogrammicus (S. Müll.).

Pycnonotus leucogrammicus Sal. Müller, T'jid. Nat. Gesch. Phys. 1835, p. 352 : Sumatra.

Distr.-Sumatra.

\section{Genus TRACHYCOMUS Cabanis.}

Trachycomus zeylanicus (Gmi.).

Yellow-crowned Bulbul.

Sturnus zeylanicus Gnelin, Syst. Nat. i, pt. 2, I789, p. So4: Ceylon error= Java (Rob. and Kloss, 1924).

Turdus ochrocephalus Gmel., Syst. Nat. i, pt. 2, 1789, p. 821 : Ceylos error = Java.

Tricophorus crispiceps Blyth, Journ. Asiat. Soc. Beng. xi, 1842, p. 204 : Malacca.

Distr.-Malay Peninsula.

Sumatra; Nias Island; West Sunatra.

Borneo.

Java.

Criniger sericea Müll, in Blyth, Ibis, IS65, p, 48 is nom, nud. and although the name was later treated as a synonym of $T$. criniger I consider that a subsequent description is valid. 


\section{Genus PYCNONOTUS Boie.}

Pycnonotus bimaculatus (Horsf.).

Orange-spotted Bulbul.

Pycnonotus bimaculatus bimaculatus (Horsf.).

Turdus bimaculalus Horsfeld, Trans. Linn. Soc. xiii, I82I, p. I47: East Java.

Crocopsis bimaculatus tenggerensis van Oort, Notes Leyden Mus. xxxiv, 1018, p. to: Mt. Tengger, East Java.

Distr.-Java (east); Bali.

Pyenonotus bimaculatus barat Rob. and Kloss.

Pycnonolus bimaculatus barat Robinson and Kloss, Journ. Straits Branch Roy. Asiat. Soc. Ixxxi, I920, p. I03: Korinchi, West Sumatra.

Disir.-Java (west).

Sumatra (part).

Pycnonotus bimaculatus snouckaerti Siebers.

Pycnonolus snouckaerli Siebers, Treubia, x, I928, p. 395 :

Takengon Lake, Acheen, North Sumatra.

Disir.-Sumatra (north).

Pycnonotus finlaysoni Strickl.

Stripe-throated Bulbul.

Pyenonotus finlaysoni finlaysoni Stricke.

Pycnonotus Finlaysoni Strickland, Ann. Nat. Hist. xiit, 1844, p. 411 : Malacca (Hantert).

Distr-_Malay Peninsula.

Pycnonotus goiavier (Scop.).

Yellow-vented Bulbul.

[Pycnonotus goiavier goiavier (Scop.).

Muscicapa goiavier Scopoli, Del. Faun. et Flor. Insubr. ii, I786, p. 96: Philippine Islands.

Distr. Extra-linital.]

Pycnonotus goiavier analis (Horsf.).

Turdus analis Horsfield, Trans. Linn. Soc. xiii, I8zI, p. 147: Java.

Distr.-Java; Bali; Karimon-Java Islands.

Pycnonotus goiavier personatus (Hume).

Otocompsa personala. Hume, Stray Feathers, i, 1873 , p. 457 :

Aclieen, North Sumatra.

Distr.-Malay Peninsula.

Sumatra; Rhio Archipelago; Banka; Billiton.

- Perhaps the continental form of $P$. bimaculatus. 
Pycnonotus goiavier gourdinii (Jacq. and Puch.).

Ixos gourdinii Jacquinot and Pucher, Voy. Pôle Sud, texte, Zool. iii, 1853, 1. 79: Bandjermasin, South Borneo.

Distr.-Borneo; Maratua Islands, East Borneo.

Pycnonotus leucops (Sharpe). ${ }^{1} \quad$ White-faced Mountain-Bulbul. Oreoctistes letucops Sharpe, Ibis, I888, p. 388 , pl. 9. fig. I : Mt. Kinabalu, North Borneo.

Distr.-Borneo.

Pycnonotus blanfordi Jerd.

Blanford's Bulbul.

[Pycnonotus blanfordi blanfordi Jerdon.

Pycnonolus blanfordi Jerdon, Ibis, IS62, p. 20: Thayetnyo, Burma.

Distr.-Extra-linital.]

Pycnonotus blanfordi robinsoni O.-Grant.

Pycnonolus robinsoni Ogilvie-Grant, Fasc. Malay. iii, 1906, p. 85 :

Patani, Peninsular Siam.

Distr.-Peninsular Siann.

Pycnonotus plumosus Blyth."

Pycnonotus plumosus plumosus Blyth.

Pycnonotus plumosus Blyth, Journ. Asiat. Soc. Bengal, xiv, I845, p. 567 : Singapore.

Distr.-Malay Peninsula.

Sumatra (east); Rhio Archipelago; Banka.

Java; Bawean Island.

Pycnonotus plumosus billitonis Chas.

Pycnonotus plumosus billitonis Chasen, Ornitl. Monatsber. I935, p. I4s : Billiton.

Distr.-Billiton Island.

\section{Pycnonotus plumosus porphyreus Oberh.}

Pycnonotus plumosus porphyreus Oberholser, Sniths. Misc. Coll.

1x, No. 7, 1912, p. 11: North Pagi Island, West Sumatra.

Picnonotus inornatus Bp. (not /xos inornatus Fraser, 1843), Consp. Gen. Avium, 1850, p. 263: Sumatra, restr. Padang, West Sumatra.

Distr.-Sumatra (west); West Sumatran Islands of Banjak group;

Mansalar; Nias; Batu group; Mentawi grotıp;

Pycnonotus plumosus insularis Chas, and Kloss.

Pycnonotus plumosus insularis Chasen and Kloss, Journ. f. Ornith. I929, Bd. 2, p. II5: Banguey Island, North Borneo.

Distr.-Borneo; ${ }^{3}$ North Bornean Islands.

- Although this bird bears a strong superficial resemblance to $P$. analis we believe that its nearest relative is the continental "Xanthixus" favescens.

* Cf. Chas, and Kl., Bull. Raff. Mus. 4 , 1030, p. 66.

3 Perhaps not the whole of Borneo: birds from areas other than the nortb and east may be $P$. pltmosus or plumosus $>$ instclaris: on the available material we cannot decide. 


\section{Pyenonotus plumosus chiroplethis Oberh.}

Pycnonotus plumosus chiroplethis Oberholser, Bull. U.S. Nat. Mus. 98, 1917, 0. 41 : Siantan Island, Anamba Islands.

Distr.-Anamba Islands; Tinggi Island, east coast Malay Peninsula.

Pycnonotus simplex Less.

Moore's Olive Bulbul.

Pycnonotus simplex simplex Less.'

Pycnonotus simplex Lesson, Rev. Mag. Zool. 1839, p. 167: Sunatra.

Microtarsus olinacens Horsf, and Moore, Cat. Bds. Mus. East Ind. Coy", 1854, p. 240: Malacca.

Pycnonotws olivacens chloeodis Oberh., Smiths. Misc. Coll. $1 \mathrm{x}$, No. 7 , 1912, p. II: Tapanuli Bay, North-east Sumatra.

Distr.-Malay Peninstula.

Sumatra; Rhio and Lingga Archipelagos; Banka; Nias Island, West Sunnatra.

Pycnonotus simplex perplexus Chas. and Kloss.

Pycnonotus simplex perplexus Chasen and Kloss, Journ. f. Orn. 1929, Bd. 2, p. II6: Balambangan Island, North Borneo.

Distr,-Borneo; South Natuna Islands; Billiton.

Pyenonotus simplex halizonus Obert.

Pycnonotus simplex halizonus Oberholser, Bull. U.S. Nat. Mus, 98,1917, p. 43 : Jinaja Island, Anamba Islands.

Pycnonotus simpler axanthizus Oberholser, Bull. U.S. Nat. Mus. 159, 1928, p. 78 : Bunguran Island, North Natuna Islands.

Distr._Anamba Islands.

North Natuna Islands.

Pycnonotus simplex prillwitzi Hart.

Pycnonolus prillwilzi Hartert, Nov. Zool, ix, 1902, p. 56I: Karangbolong, South Java.

Distr.-Java.

Pycnonotus brunneus Blyth.

Brown Bulbul.

Pycnonotus brunneus brunneus Blyth.

Pycnonotus brunneus Blyth, Journ. Asiat. Soc. Bengal, xiv, I\$45, p. 568: Malacca.

Pycnonotus brumneus zaphaens Oberh., 2 Proc. U.S. Nat. Mus. liv, 19:7, p. 194 : Mata Sireh Island, Java Sea.

Distr.-Malay Peninsula.

Sumatra; Banjak and Nias Islands, West Sumatra.

Borneo; Banguey Island, North Borneo; Karimata Islands.

1 $P$. brunneus is, of course, not a synonym of simplex lof. F.B.I. and ed. vii, p. 87) but a distinct species: modestus Blyth is a nomen nudum but it refers to bromeus and not to simplex.

$\Rightarrow$ We have never seen exact topotypes of $P . b$. aaphaeus but we cannot separate Bornean birds which are said by Oberholser to be eaphacus. 


\section{PYCNONOTIDA.}

Pycnonotus brunneus zapolius Oberh.

Pycnonotus brunneus zapolius Oberholser, Bull. U,S. Nat. Mus. 98 , 1917, p. 45 : Siantan Island, Anamba Islands.

Distr.-Tioman Island, east coast Malay Peninsula. Anauba Islands.

Pycnonotus erythropthalmos (Hume). Small Olive Bulbul

Pycnonotus erythropthalmos erythropthalmos (Hume).

Ixos erythropthalmos Hume, Stray Feathers, vi, 1878, p. 314: Pakchan, South Tenasserim.

Pycnonotus erythropthalmos cyanochris Oberb., Smiths. Misc. Coll. lx, No. 7, 1912, p. 10: Rupat Strait, East Sumatra.

Distr.-Malay Peninsula.

Sumatra; Billiton.

Pycnonotus erythropthalmos salvadorii Sharpe.

Pycnonotus salvadorii Sharpe, Cat. Bds. Brit. Mus, vi, I88r, p. 40r, (nom. nov. for $P$. pusillus Salvad., not Hamatornis pusillus Blyth, I84I): Sarawak, Borneo.

Pycnonolus pusillus Salvadori, Ann. Mus. Civ. Gen. 1874, p. 200: Sarawak.

Distr.-Borneo.

*Pycnonotus erythropthalmos isus Oherh.

Pycnonolus erythropthalmos isus Oberholser, Smiths. Misc. Coll. ix, No. $7,19 \mathrm{I2}, \mathrm{p}$. Io: Tuanku Island, Banjak Islands.

Distr.-Banjak Islands, West Sumatra.

*Pycnonotus erythropthalmos pammicrus Oberh.

Pycnonotus erythropthalmos pammicrus Oberholser, Smiths. Misc.

Coll. 1x, No. 7, 1912, p. I : Nias Island.

Distr.-Nias Island, West Sumatra.

Pycnonotus cyaniventris Blyth.

Grey-bellied Bulbul.

Pycnonotus cyaniventris cyaniventris Blyth.

Pycnonotus (?) cyaniventris Blyth, Journ. Asiat. Soc. Bengal, xi, 1842, p. 792 : Singapore Island.

Malacopteron aureum Eyton, Ann. Nat. Hist. xxvi, 1845, p. 229 : Malacca.

Brachypus poliopsis Bp., Consp. Gen. Avium, 1850, p. 264 : Sumatra.

Distr.-Malay Peninsula.

Sumatra.

Pycnonotus cyaniventris paroticalis (Sharpe).

Ixidia paroticalis Sharpe, Ibis, 1878, p. 418 : Sarawak, Borneo.

Distr.-Borneo. 
Pycnonotus tympanistrigus' (Müll.). Small White-streaked Bulbul.

- Ixos tympanistrigus S. Müller, Tijds. Nat. Gesch. Phys. 1835, p. 353 : Sumatra.

Brachypus tigus $\mathrm{B}$ p., Consp. Gen. Avium, i, 1850, p. 264 : Sumatra. Distr.-Sumatra.

Pycnonotus flaviventris (Tick.). Black-crested Yellow Bulbul. [Pycnonotus flaviventris flaviventris (Tickel1).

Vanga flaviventris Tickell, Journ, Asiat. Soc. Beng. ii, I833, p. 573: Chota Nagpur.

Distr._Extra-limital.]

Pycnonotus flaviventris minor (Kloss).

Otocompsa flaviventris minor Kloss, Ibis, IgrS, p. 200: Koh Lak, South-west Siam.

Distr.-Malay Peninsula.

Pycnonotus flaviventris montis (Sharpe).

Rubigula montis Sharpe, Proc. Zool. Soc. 1879 , p. 247 : Mt. Kinabalu, North Borneo.

Distr.—Borneo.

Pycnonotus dispar (Horsf,).

Ruby-throated Bulbu1.

Pyenonotus dispar dispar (Horsf.).

Turdus dispar Horsfield, Trans. Linn. Soc. xiii, I820, p. I50: Java.

Distr.-Sunatra. Java.

Pycnonotus squamatus (Temm.). Scaly-breasted Bulbul.

Pycnonotus squamatus squamatus (Temm.).

Turdus squamatus Temminck, Pl. Col. 453, I828: Java.

Distr.-Java.

Pycnonotus squamatus webberi (Hume).

Ixidia webberi Hume, Stray Feathers, viii, 1879, p. 40: Tongka (Junk Zeylon) Island, North Malay Peninsula.

Distr.-Malay Peninsula.

- Sumatra.

Borneo.

- Sharpe, Cat. Bds. vi, p. 62 (footnote) appears to have been mistaken in stating that the type of /xos Lympanistrigus Müll. is a specimen of Euptilosus eutilotus Jard, and Selby, for according to Mr. G. C. H. Junge (in litt.) who has kindly examined the material in the Leyden Museum the type specimen of tympanistrigus is the bird later described as $B$. tigus $\mathrm{Bp}$. : Finsch noted this in Notes Leyd. Mus. xxvi, p. 95 . 
Pycnonotus aurigaster (Vieill.).

Golden-vented Bulbul.

Pycnonotus aurigaster aurigaster (Vieill.).

Turdus aurigaster Vieillot, Nov. Dict. d'Hist. Nat. ed. 2, xx, I8I8, p. 258: Java.

Brachypus chrysorrhaus Stephs, Gen. Zool. xiii, 3826, p. 189.

Pycnonotus crocorrhous Strickl., Ann. Nat. Hist. xiii, 1844, p. 412 : Java. Pycronotus aurigaster martini Parrot, Abh. Kon. Bay. Acad. Wiss. xxjy, 1907, P. 239 : Sumatra.

Distr. - Singapore Island (introduced).

Sumatra (introduced).

Java.

Pycnonotus jocosus (Linn.).

Red-whiskered Bulbul.

[Pycnonotus jocosus jocosus (Linn.).

Lanius jocosus Linn., Syst. Nat, roth. ed. 1758, p. 95 : China.

Distr.-Extra-linital.]

Pycnonotus jocosus erythrotis (Bp.).1

Ixos erythrotis Bonaparte, Consp. Gen. Avium, 1850, p. 265 : Java, error $=$ Tavoy.

Distr.-Malay Peninsula. Sumatra (? introduced). Java (? introduced).

\section{Family TIMALIIDE.}

\section{Genus EUPETES Temminck.}

Eupetes macrocerus Tenm. Eupetes macrocerus macrocerus Ternm.

Rail-Babbler.

Eupetes macrocerus Temminck, P1. Col. 516, i $\delta_{31}$ : Padang, West Sumuatra.

Eupetes macrocercus griseiventris Stuart Baker, Bult. Brit. Orn. Cl. xxxviii, 1917, p. \&: Tung Song, Peninsular Siam.

Distr.-Malay Peninsula.

Sumatra.

North Natuna Islands.

Eupetes macrocerus borneensis Rob. and Kloss.

Eupetes macrocenus borneensis Robinson and Kloss, Journ. Fed. Malay States Mus, X, I921, p. 204 : Samaralıan, South Sarawak.

Eupetes macrocercus subrufus Hach., Bull, B. O. Cl., xlii, r926, p. 54 : Mt. Dulit, Sarawak.

Eupetes macrocereus stubrefus Hach., Bull, B. O. Cl., xlvii, 1926, p. 54 : Mt. Dutit, Sarawak.

Distr._Borneo.

- Records from the south of the Malay Peninsula, Sumatra and Java are no doubt founded on cage birds and it is very doubtful if the species naturally ranges further south than about the latitude of Kelantan. 


\section{Gentus GARRULAX Lesson."}

Garrulax erythrocephalus (Vig.) . Red-headed Laughing-Thrush. [Garrulax erythrocephalus erythrocephalus (Vig.).

Cinclosoma erythrocophalum Vigors, Proc. Zool. Soc. for $18_{31}$, I8 32 , p. I 7 I : Simla.

Dislr._Extra-limital.]

Garrulax erythrocephalus peninsulæ (Sharpe).

Trochalopterum peninsulce Sharpe, Proc. Zool. Soc. IS87, D. 436, pl. 37 : Mt. Ijat, Perak, Malay States.

Distr.-Malay Peninsula.

Garrulax leucolophus (Hardw.). White-crested Laughing-Thrush. [Garrulax leucolophus leucolophus (Hardw.).

Corrus leucolophus Hardwicke, Trans. Linn. Soc. xi, I8I5, p. 208, pl. I5: Kunaun.

Disir.-Extra-linital. ]

Garrulax leucolophus bicolor Hartl,

Garralax bicolor Hartlaub, Rev. Mag. Zool. IS44, P. 402: Sumatra. Distr.-Sunatra.

Garrulax rufifrons Less.

Plain-brown Laughing-Thrush.

Garrulax rufifrons rufifrons Less.

Garmlax mifrons Lesson in Bélanger, Voy. Inde, Zool, I834, p. 26, tab. 5: West Java.

Distr._Java (west).

Garrulax rufifrons slamatensis Siebers.

Garrulax rufifrons slamatensis Siebers, Treubia, xi, 1929, p. I50:

Mt. Slamat, Mid Java.

Distr.-Java, (Central).

Garrulax palliatus (Bp.). Grey-and-brown Laughing-Thrush.

Garrulax palliatus palliatus (Bp.).

Ianthocincla palliata Bonaparte, Consp. Gen. Avium i, I850, p. 37 I : Sumatra.

Distr, -Simuatra.

Garrulax palliatus schistochlamys Sharpe.

Garmiax schistochlamys Sharpe, Ibis, I888, p. 479: Mt. Kinabalu, Nortls Borneo.

Distr.-Borneo.

- Revisions: Meinertz., Ibis, 1928, p. 507 ; Berlioz, Rev. Hist. Nat. xi, 1930 , p. I. 
Genus MELANOCICHLA Shatpe.

Melanocichla lugubris (S. Müll.).

Black Babbling-Thrush.

Melanocichla lugubris lugubris ( $\mathrm{S} . \mathrm{M}$ ïll.).

Ianthocincla lugubris Sal. Müller, Tijd. Nat. Ges. Phys, I\$35, p. 344, pl. 5, fig. 2: West Suntratra.

Melanocichla peninsularis Sharpe. Proc. Zool. Soc. 1888, p. 274 : Mt.

Batu Puteh, Perak, Matay States.

Distr.-Malay States.

Sumatra.

Melanocichla lugubris calvus (Sharpe) $\mathrm{t}^{\mathrm{t}}$

Allocolops caluus Sharpe, Ibis, I8S8, p. 359 : Mt. Kinabalu, North Borneo.

Distr.-Borneo.

\section{Genus RHINOCICHLA Sharpe.}

Rhinocichla mitrata (S. Müll.). Cliestnut-capped Babbling-Thrusl. Rhinocichla mitrata mitrata (S. Müll.).

Timalia milrata Sal. Müller, Tijd. Nat. Ges. Phys. 1835, p. 345, pl. 5, fig. 3: Sumatra.

Garrulax viltatus de Filippi, Mus. Mediol, Anim. Vert, $1 \$ 47$, p. 31 : Sumatra (restr.).

Distr.-Sunatra.

Rhinocichla mitrata major Rob. and Kloss.

Rhinocichla milrata major Robinson and Kloss, Bull. Brit. Orn. Cl. xl, Igr9, p. I6: Mt. Ijau, Larut Hills, Perak.

Distr.-Malay States.

Rhinocichla mitrata treacheri (Sharpe).

Ianthocincla treacheri Sharpe, Proc. Zool. Soc. 1879 , p. 24S, pl. 23 : Mt. Kinabalu, North Borueo.

Distr.-Borneo (north).

Rhinocichla mitrata damnata Harr. and Hartley.

Rhinocichla milrata damnala Harrisson and Hartley, Bull. Brit.

Orn. Cl. liv, I934, p. I54: Mt. Dulit, Sarawak, Borneo.

Distr.-Borneo (part).

T The genus Allocotops Sharpe was made for calvus and the bird is of ten quoted as the monotypic representative of a highly specialized Bornean genus. The above action in reducing it to the rank of a subspecies may therefore be regarded as drastic, but I think it is the right one. I have noted incipient baldness in ligubris from the Malay Peninsula. 


\section{Genus POMATORHINUS Horsfield.}

Pomatorhinus montanus Horsf. Chestnut-backed Scimitat-Babbler.

Pomatorhinus montanus montanus Horsf.

Pomatorhinus montanus Horsfield, Trans, Lin11. Soc. xiii, I82I, p. I65: West Java.

Distr.-Java (not east).

\section{Pomatorhinus montanus ottolanderi Rob.}

Pomatorhinus nontanus ottolanderi Robinson, Journ. Fied. Mal. States Mus. vii, I918, 13. 235: Idjen Massif, East Java.

Distr._Java (east); Bali.

Pomatorhinus montanus bornensis Cab.

Pomatorlinus bornensis Cabanis, Mus. Heine, i, I\$50, p. 84 : Bormeo.

Distr.-Borneo.

Pomatorhinus montanus occidentalis Rob. and Kloss.

Pomatorhinus monianus occidentalis Robinson and Kloss, Journ.

Fed. Mal. States Mus. xi, I923, p. 51 : Selangor, Malay States.

Distr.-Malay States.

Sumatra.

Pomatorhinus schisticeps Hodgs. Brown-backed Scimitar-Babbler.

[Pomatorhinus schisticeps schisticeps Hodgs.

Pomatorlinus schisticeps Hodgson, Asiat. Res. xix, 1836 , p. I8I : Nepal.

Distr._Extra-limital.]

Pomatorhinus schisticeps fastidiosus Hart.

Pomatorhinus schisticeps fastidiosus Hartert, Bull. Brit. Orn. Cl. xxxvi, I9ı6, p. 81: Trang, Peninsular Sianı.

Distr.-Peninsular Siam.

Pomatorhinus hypoleucos (Blyth). I,arge Scimitar-Babbler.

[Pomatorhinus hypoleucos hypoleucos (Blyth).

Orthorhinus hypoleucos Blyth, Journ. Asiat. Soc. Beng. xiii, I844, p. 37 I : Arakan.

Distr._Extra-limital.]

Pomatorhinus hypoleucos wrayi Sharpe.

Ponatorhinus arayi Sharpe, Proc. Zool. Soc. I887, p. 437: Mt. Ijau, Larut Hills, Perak.

Distr.-Malay States. 


\section{Genus TIMALIA Horsfield.}

Timalia pileata Horsf.

Red-capped Babbler.

Timalia pileata pileata Horsf.

Timalia pileata Horsfield, Trans: Liun. Soc. xiii, I 82 I, p. I5I: Java.

Distr. - Java (not east).

Timalia pileata intermedia Kinn.

Timalia pileata intermedia Kinnear, Bull. Brit Orn. Cl. xlv, 1924, p. 9: Tautighoo, Burtaa.

Distr.-Peninsular Sian."

\section{Genus GAMPSORHYNCHUS Blyth.}

Gampsorhynchus rufulus Blyth. White-headed Babbler.

[Gampsorhynchus rufulus rufulus Blyth.

Gampsorhychus rufulus Blyth, Jouru. Asiat. Soc. Bengal, xiii, I $\$_{44}$, p. 37 I : Darjeeling.

Distr.-Eixtra-linital.]

Gampsorhynchus rufulus saturatior Sharpe.

Gampsorhynchus saluratior Sharpe, Proc. Zool. Soc. I888, p. 273 : Mt. Batu Puteh, Perak, Malay States.

Distr.-Malay States.

\section{Genus ANDROPHILUS Sharpe.}

Androphilus accentor Sharpe.

Friendly Babbler. Androphilus accentor Slarpe, Ibis, IS88, p. 390: Mt. Kinabalu, Nortl Bormeo.

Distr.-Borneo.

\section{Genus PELLORNEUM Swainson.}

Pellorneum ruficeps Swains.

Striped Babbler.

[Pellorneum ruficeps ruficeps Swains.

Pellorneum ruficeps Swainson, Fauna Boreal. Aner. I832, p. 487 : India.

Distr._Extra-limital.]

- The presence of this bird in the Malay Peninsula south of the Isthmus of Kra needs confirmation. 
Pellorneum ruficeps subochraceum Swinh.

Pellorneum subochraceum Swinloe, Ann. Mag. Nat. Hist. (4) vii, I87 I, P. 257: Tenasserint.

Distr.-Malay Peninsula.

Pellomeum capistratum (Temm.).

Black-capped Babbler.

Pellorneum capistratum capistratum (Temm.).

Myiothera capistrala Temminck, Pl. Col. $18_{5}, 18_{23}$ : Java.

Distr.-Java.

Pellorneum capistratum nigrocapitatum (Eyton).

Brachypteryx nigrocapitata Eyton, Proc. Zool. Soc. I839, p. I0\}: Malacea.

Bessethera barbata Cab., Mus. Hein, i, 1850, p. 76 : Malacca lOberh. 1022.).

Distr.-Malay Peninsula; Billiton.

North Natuna Islands.

Pellorneum capistratum nyctilampe (Oberh.)."

Drymocataphus nigrocapitalas nyclilampis Oberholser; Smiths. Misc. Coll. Ixxiv, 2, I922, D. ro: Banka.

Distr._Sunıatra; Banka.

Pellorneum capistratum capistratoides (Strickl.).

Goldana capistratoides Strickland, Coutr. Orn. I849, p. I28, pl. 36 : Soluth Borneo.

Distr.-Borneo (not nortli).

Pellorneum capistratum morrelli Chas. and Kloss.

Pellorneum capistratum morrelli Chasen and Kloss, Journ. f. Oruith. I929, Bd. 2, p. II8: Kudat, North Borneo.

Distr.-Borneo (north); Banguey Island, North Borneo.

Pellorneum tickelli Blyth.

Tickell's Babbler.

Pellorneum tickelli tickelli Blyth.

Pellorneum tickelli Blyth, Journ. Asiat. Soc. Bengal, xxviii, 1859 , p. 4 I4 : Amherst, Tenasserim.

Distr.-Perinsular Sian (north).

Pellorneum tickelli australe (Rob, and Kloss).

Drymocataphus tickelli australis Robinson and Kloss, Journ. Fed.

Mal. States Mus. x, I92I, p, 205 : Selangor, Malay States.

Distr.-Malay Peninsula.

- A rather doubtful race. The skins fade very quickly in the tropics, but some birds from Sumatra certainly seem darker than specimens trom the Malay Peninsula. 


\section{Genus MALACOCINCLA Blyth."}

Malacocincla abbotti ${ }^{2}$ Blyth.

Abbott's Brown Babbler.

Malacocincla abbotti abbotti Blyth.

Malacocincla Abbolti Blyth, Journ. Asiat. Soc. Bengal, xiv, I 845 , p. 601: Arakan.

Distr.-Malay Peninsula (south to about Penang).

Malacocincla abbotti olivacea (Strick.).

Malacopteron olivaceum Strickland, Proc, Zool. Soc, for $1 \$_{46} 6$, I $\$_{47}$, p. 102: Malacca.

Distr.-Malay Peninsula (south from Penang). Stmatra.

Malacocincla abbotti büttikoferi Finsch.

Malacocincla Bullikoferi Finsch, Notes Leyd. Mus. xxii, 19oI, p. 2I \&: South-east Borneo.

Distr.-Borneo.

* Malacocincla abbotti eritora Oberl.

Malacocincla abbotti eritora Oberholser, Smiths, Misc. Coll. 1xxiv, 2, 1922, p. II: Billiton Island.

Distr.,-Billiton Island.

Malacocincla abbotti baweana Oberh.

Malacocincla abbotti bazeana Oberholser, Proc. U.S. Nat. Mus. 1ii, $1917,19.194$ : Bawean Island.

Distr:-Bawean Island, Java Sea.

* Malacocincla abbotti sirensis Oberh.

Malacocincla abboth sirensis Oberholser, Proc. U.S. Nat, Mus. liv, $1917, \mathrm{p} .195$ :

Distr.-Mata Siri Island, Java Sea.

Malacocincla sepiaria (Horsf.). ${ }^{3}$ Horsfield's Brown Babbler.

Malacocincla sepiaria sepiaria (Horsf.).

Brachypteryx sepiaria Horsfield, Trans. Linn. Soc. xiit, I8zI, p. I58: West Java.

Distr,-Java (not east).

t A form of Malacocincla is said to occur in the Rhio Archipelago. It is most likely $M_{*} a$, olivacea (Strick.). Short revision: Chas, and KL., Bull. Raffles Mus. 4, 1930, p. 71.

: Several names (hypoides, concreta, pyca, schtwaneri) originally applied to specimens in the Leyden Museum, refer either to this species, or to M. sepiaria. Fortunately they seem-to be nomina mida in all citations.

${ }^{3}$ This distinct species is confused with $M$. abbotti in Faun. Brit. Ind. (and ed.). 
Malacocincla sepiaria minor (Meyer).

Turdinus sepiaria var. minor A. B. Meyer, Zeitsclir, f. gesanite Orn. i, ISS4, p. 210 : East Java.

Distr.-Jara (east); Bali.

Malacocincla sepiaria tardinata Hart.

Malacocincla sepiaria tardinala Hartert, Bull. Brit. Orı. Cl. xxxvi, I9I 5, D. 35: Mt. Tahan, Malay States.

Distr.-Malay Peninsula.

Sunatra (north-east).

Malacocincla sepiaria barussana Kob. and Kloss.

Malacocincla sepiaria barussana Robinson and Kloss, Jotru. Fed. Malay States Mus. X, I921, p. 205: Korinchi, West Sumatra.

Distr.-Sumatra (west and south).

Malacocincla sepiaria rufiventris Salvad.

Malacocincla mfizentris Salvadori, Ann. Mus. Civ. Gen. v, 1S74, P. 229 : Saranak.

Turdimus tephrops Sharpe, Bull, Brit. Orn. Cl. i, 1893, p. 54 : Mt. Kalulong, Sarawak.

Distr.-Borneo (Sarawak and Dutch Borneo).

Malacocincla sepiaria harterti Chas, and Kioss.

Malacocincla sepiaria harteri Chasen and Kloss, Jonrn. f. Ornith. 1929, Band 2, D. I 16 : near Sandakan, British North Borneo.

Dists.-Borneo (north).

Malacocincla perspicillatus (Bp.).: Large Ashy-throated Babbler.

Cacopilla perspicillata Bonaparte, Consp. Gen. Avium, i, IS50, 1. 257: Java etror $=$ Borneo (Billikofer, 180.5$)$.

Distr.-Borneo.

\section{Genus AETHOSTOMA Sharpe.}

Aethostoma rostratum (Blyti1).

Blyth's Babbler.

Aethostoma rostratum rostratum (Blytly).

Trichasloma rostratum Blyti, Journ. Asiat. Soc. Bengal, xi, IS4?, p. 795 : Singapore Island.

Brachypteryx buxtoni Walden, Proc. Zool. Soc. 1877 , p. 367 : Lampongs, Sumatra.

Ptitocichla leucogastra Davison, Ibis, 1892, p. 100: Pahang, Malay" Peninsula.

I A most mysterious bird of which little is known. 
Aethostoma rostrata aethalea Oberh., Smiths. Misc. Coll. Ixxiv, 2, 1922, p. 12: Karimon Anak Island, Rhio Archipelago.

Aethostoma rostrata paganica Oberh., Smiths. Misc. Coll. 1xxiv, 2, I922, p. 12: Upper Siak River, East Sumatra.

Distr.-Malay Perizisula.

Stumatra; Rhio and Lingga Archipelagos; Billiton.

\section{Aethostoma rostratum macropterum (Salvad.).}

Brachypteryx macroptera Salvadori, Atti. R. Acad. Sci. Torino, iii, IS68, p. 528: Sarawak.

Aethostoma witmeri Sharpe, Handlist of Birds, iv, 1903, p. 358: Sarawak. Distr.-Borneo; Banguey Island, North Borneo.

Aethostoma pyrrhogenys ('Temm.).

Temmick's Babbler.

Aethostome pyrrhogenys pyrrhogenys (Temm.).

Myiolhera pyrrhogenys Temminck, P1. Col. 442, IS27: West Java.

Disir. - Java (not east).

Aethostoma pyrrhogenys besuki Kloss.

Acthostoma pyrogenys besuki Kloss, Treubia, I3, I93I, p. 346 : Tamansari, East Java.

Distr,-Java (east).

Aethostoma pyrrhogenys büttikoferi (Vorderm.).

Trichostoma Buttikoferi Vorderman, Nat. Tjids. Ned. Ind. I892, p. 230: Lampongs, South Sumatra.

Distr.-Suntratra.

Aethostoma pyrthogenys canicapillum (Sharpe).

Turdinus canicapillus Sharpe, Ibis, 1887 , p. 450: Mt. Kinabalu, North Borneo.

Distr.-Mt. Kinabalu, North Borneo.

Aethostoma pyrrhogenys longstafi (Harr. and Hart.).

Malacocincla canicapillus longstaffi Harrisson and Hartley, Bull.

B.O.C. liv, 1934, P. 152: Mt. Dulit, Sarawak.

Distr.-Sarawak (not west).

Aethostoma pyrrhogenys erythrote (Sharpe).

Malacopterum erythrote Sharpe, Cat. Bds. Brit, Mus. vii, I883, p. 567, pl. 13, fig. 2: "Borneo".

Disir.-Sarawak (west). ${ }^{\mathrm{I}}$

1 On the mountains of West Sarawak (Poi and Penrissen) there is a race of A, pyrhogenys very variable in general colour, some specimens being as pale as $A$. p. canicapilla and others as dark as $A$. $p$. longstafh, but with the forehead and crown paler than in these two races. A faded specimen of this race is rather more rufous above than the old type of erythrote, but the birds are sufficiently alike to justify the adoption of the name as proposed above. 


\section{Gentus TURDINUS Blyth.}

Turdinus macrodactylus (Strick1.). Large-footed Babbler

Turdinus macrodactylus macrodactylus (Strick1.).

Malacopteron macrodactylum Strickland, Anu. Mag. Nat. Hist. xiii, 1844 , p. 417 : Malacca.

Brachypteryx albognlaris Hartl, Rev, Mag. Zool. 1844 , p. 401 : Malacca. Distr.-Malay States.

Turdinus macrodactylus bakeri Hach.

Turdinus macrodactylus bakeri Hachisuka, Bull. Brit. Oro. Cl. xlvii, 1926, p. 54: Trang, Peninsular Sian.

Distr..-Peninstiar Siam.

Turdinus macrodactylus lepidepleurus ( $\mathrm{Bp}$.$) .$

Cacopitta lepidopletra Bonaparte, Consp. Gen. Aviun i, 1850, p. 257: Java.

Distr.-Java."

Turdinus macrodactylus rufipectus Salvad.

Turdinus rufipectus Salvadori, Ann. Mus. Civ. Gen. xiv, I879, p. 224 : Sumatra.

Distr.-Sumatra.

Turdinus macrodactylus atrigularis (Bp.).

Cacopitia atrigularis Bonaparte, Consp. Gen. Av, i, 1850, p. 257 : Borneo.

Disir, -Bormeo.

Turdinus marmoratus S. Müll.

Müller's Babbler.

Turdinus marmoratus Wardlaw-Ramsay, Proc. Zool. Soc. I880, p. I5: West Sumatra.

Myiothera lonicata Müller (nec Licht.), Tjid. Nat. Ges. Phys. 1835, p. 348, pl. $v$, fig. 5 : West Sumatra.

Distr.-Malay States.

Sumatra.

Gentus ERYTHROCICHLA Sharpe.

Erythrocichla bicolor (Less.).

Ferruginous Babbler.

Brachypteryx bicolor Lesson, Rev, Zool, 1839, p. 138 : Sumatra.

Malacopteron ferruginosum Blyth, Journ. Asiat, Soc. Bengal, xiii, 1844, p. 383 : Singapore Island.

Napothera ribicawda Bp., Consp. Gen. Av. i, 1850, p. 359 : Sumatra.

Erythrocichla bicolor whiteheadi Hart., Bull. B. O. Cl. xxxvi, 1915, p. 36 : Britisb North Borneo.

Distr.-Malay Peniısula.

Sumatra.

Borneo.

- Has been recorded from Sumatra but we think in ertor. 


\section{Genus MALACOPTERON ${ }^{\perp}$ Eyton.}

Malacopteron magna Eyton. Larger Red-headed Tree-Babbler. Malacopteron magnum magnum Exton.

Malacopteron magnum Eyton, Proc. Zool. Soc, I839, P. IO3: Malacca.

Malacopleron majus Blyth, Journ. Asiat. Soc. Benga!, xvi, 1847, p. 461 ; Malacca.

Napothera pileata Bp., Consp. Gen. Avium, 1850, p. 359 : Sumatra.

Distr.-.Malay Peninsula.

Sunnatra; Nias Island, West Sunatra.

Borneo (not north); North Natuma Islands.

Malacopteron magnum saba Chas, and Kloss.

Malacopteron magnum saba Cliaset1 and Kloss, Bull. Rafles Mus. 4, r930, p. 75 : Sandakan, British Nortlı Borneo.

Distr.-Borneo (nortli).

Malacopteron cinereum Eyton. Smaller Red-headed 'Tree-Babbler. Malacopteron cinereum cinereum Eyton.

Malacopteron cinereus Eyton, Proc. Zool. Soc. I839, p. 103: Malacca.

Napothera coronata B\}., Consp. Gen. Avium, 1850 , p. 358 : Sumatra.

Distr.-Malay Peninsula.

Suntatra; Rhio and Lingga Archipelagos.

Borneo.

\section{Malacopteron cinereum rufifrons Cab.}

Malacopteron rufifrons Cabanis, Mus. Heine, i, IS50, p. 65: Java.

? Timalia ruficapilla Jaca. and Puch., Voy. Pôle Sud. Ois. iii, 1853. p. 80: Pulau Laut, S. E. Borneo (? error).

? Timalia squamifrons Bp., Comptes Rend. xxxviii, 1854, p. 59 (ex Puch. MS).

Distr,-Java. ${ }^{2}$

\section{Malacopteron cinereum bungurense Hart.}

Malacopteron cinereum bungurense Hartert, Nov. Zool. i, I894. p. 470: Bungurun, Nortl Natuma Islands.

Distr.-North Natuna Islands.

- If Malacopteron is considered preoccupied by Malacopterus Serv. 1833 Malacornis Gistel, $1 \$ 3 ̧ 0$ must be used. See Riley 1933.

2 The accurrence of this form in Sumatra, or Borneo, is doubtful. The name lepidocephalus seems to be nom. nud. in all citations. 
Malacopteron magnirostre (Moore). Brown-headed Tree-Babbler. Malacopteron magnirostre magnirostre (Moore).

Alcippe magnirostris Moore, Proc. Zool. Soc. for $1 \$_{54}, 1855, \mathrm{p} .277$ : Malacca.

Distr.-Malay Peninstula; Tioman Island.

Sumatra; Lingga Archipelago; Mansalar Island, West Sumatra.

Malacopteron magnirostre flavum Chas, and Kloss.

Malacopteron magnirostris favum Chasen and Kloss, Journ. Mal. Br. Roy. Asiat. Soc. vi, pt. 3, I928, p. 58: Siantan Island, Anamba Islands.

Distr.-Ananba Islands.

Malacopteron magnirostre cinereocapillum (Salvad.).

Alcippe cincreocapilla Salvadori, Atti. R. Accad. Sci. Torino iii, IS6S, p. 530: Sarawak, Borneo.

Turdinus kalnionga Sharpe, Bull. Brit. Orn. Cl. i, 1893, p. 54 : Mt. Kalulong, Sarawak.

Distr.-Borneo.

Malacopteron affine (Blyth).

Small Tree-Babbler.

Malacopteron affine affine (Blyth).

Trichastoma affine Blytil, Jourı. Asiat. Soc. Bengal, xi, I842, p. 795: Singapore Island.

Napothera atricapilla Bp. Consp. Gen. Avium. i, 1850, p. 359: Sumatra.

Alcippe cantori Moore, Proc. Zool. Soc. I854, p. 277 : Penang.

Malacopterum nelanocephalum Davison, Ibis, 1892, p. 101: Pahang.

Distr.-Malay Peninsula.

Suntatra.

Borneo.

? Java. ${ }^{7}$

*Malacopteron affine notatum Richm.

Malacopteron notatum Richmond, Proc. Biol. Soc. Wash. xv, 1902, p. I20: Bangkaru Island, Banjak Islands.

Distr.-Banjak Islands, West Sumatra.

\section{Genus OPHRYDORNIS Büttikofer.}

Ophrydornis albogularis (Blyth).

White-tlrroated Babbler.

Ophrydornis albogularis albogularis (Blyth).

Selaria albigularis Blyth, Journ. As. Soc. Bengal, xiii, IS44, p. 385 : Singapore Island.

Distr.-Malay States. Sumatra.

- The record for Java is unsatisfactory and needs confirmation. 
Ophrydornis albogularis moultoni Rob. and Kloss.

Ophrydornis albogularis moultoni Robinson and Kloss, Bull. Brit. Orn. Cl. xl, I919, p. 17: Saribas, Sarawak, Borneo.

Setaria albigularis lencogastra Hach., Bull. Brit. Orn. Cl., xlvii, 1926, p. 54 : Sarawak.

Distr.-Bогдео.

Gerus PTILOCICHLA Sharpe.

Ptilocichla leucogrammica' (Bp.). Bornean Streaked Babbler.

Cacopitta leucogrammica Bonaparte, Cons1). Gen. Avium, i, 1850, p. 257 : Borneo.

Distr.-Borneo.

\section{Genus ANUROPSIS Sharpe.}

Anuropsis malaccensis (Hartl.)."

Hume's Short-wing.

Anuropsis malaccensis malaccensis (Hart1.).

Brachypteryx malaccensis Hartlaub, Rev. Mag. Zool. IS44, p. 402 : Malacca.

Amiropsis malacensis drymodrama Oberk., Smiths. Misc. Coll. Ixxiv, 2, 1922, p. 9: Mandau River, East Sumatra.

Anuropsis malaccensis driophila Oberh., Smiths. Misc. Coll. Ixxiv, 2, 1922, p. 9: Trang, Peninsular Siam.

Distr.-Malay Peninsula; Anamba Islands.

Sumatra; Rhio and Lingga Archipelagos.

North Natuna Islands.

*Anuropsis malaccensis docima Oberh.

Anuropsis malaccensis docima Oberholser, Suniths. Misc. Coll. 1xxiv, 2, I922, p. Io: Banka Island.

Distr.-Banka Isłand.

Anuropsis malaccensis saturata Rob. and Kloss.

Anuropsis malacensis saturata Robinson and Kloss, Bull. Brit. Orn.

Cl. xl, ז920, p. 68 : Baram, Sarawak.

Distr.,Borneo (west); Billiton.

Anuropsis malaccensis poliogenis (Strick-1.).

Brachypteryx poliogenis Strickland, in Jardine's Contr. Otn. ii, I849, p. 93, pl. 31: Karau River, South-east Borneo.

Anuropsis malaccensis sordidus Chasen and Kloss, Journ, f. Orn. 1929.

Bd, 2, p. rig: near Sandakan, North Borneo.

Distr.-Borneo (north and east).

- The only Malaysian representative of a Pbilippine group.

- Cf. Oberh., Bull. U.S. Nat. Mus. 159, 1937, p. 61. 
Anuropsis malaccensis feriatus Chas. and Kloss.

Anuropsis malaccensis feriatus Chasen and Kloss, Nov. Zool, xxxvi, 1931, p. 279 : Mt. Mulu, North Sarawak.

Distr.-Borneo (part).

*Anuropsis malaccensis exsanguis Oberh.

Anuropsis malaccensis exsanguis Oberholser, Siniths. Misc. Coll.

$1 \mathrm{x}, 7,1912$, p. S: Tuanku Island, Banjak Islands.

Distr.-Banjak Islands, West Sumatra.

*Anuropsis malaccensis nesitis Oberh.

Anuropsis malaccensis nesitis Oberholser, Sniths. Misc. Coll. 1x,

7. I912, p. S: Tana Masa Island, Batu Islands.

Distr.-Batu Islands, West Sumatra.

\section{Genus NAPOTHERA Gray.}

Napothera brevicaudata (Blyth). Streaked Wren-Babbler.

[Napothera brevicaudata brevicaudata (B1ytl)] .

Turdinus brevicaudatus Blyth, Journ. Asiat. Soc. Bengal, xxiv, I855, p. 272: Tenasserim.

Distr.-Eixtra-limital.]

Napothera brevicaudata leucosticta (Sharpe).

Corythocichla leucosticta Sharpe, Proc. Zool. Soc. I887, p. 438 : Larut Hills, Perak.

Corythocichla brevicandata herberti Stuart Baker, Bull. Brit. Orn. Cl. xxxviii, 1917, p. 10: Tung Song. Peninsular Siam.

Distr. - Malay Peninsula; Tioman Island, east coast Malay Peninsula.

Napothera brevicaudata crassa (Sharpe).

Corythocichla crassa Sharpe, Ibis, I888, p. 391: Mt. Kinabalu, North Borneo.

Distr.-Borneo.

Napothera epilepidota ('Temun.).

Small Wren-Babbler.

Napothera epilepidota epilepidota (Teum.).

Mviothera epilepidola Temminck, P1. Col. 448, I827: Java (Hartert, 1902).

Myothera mutina Blyth, Ibis, 1865, p. 47 : Java.

Distr, 一Java.

Napothera epilepidota diluta (Rob. and Kloss).

Turdinulus epilepidotus dilutus Robinson and Kloss, Journ, Straits Branch, Roy. Asiat. Soc. No. 73, 1916, p. 276: Korinchi, Sumatra.

Distr.-Sumatra. 
Napothera epilepidota granti (Richm.).

Turdinulus granti Richnond, Proc. U.S. Nat. Mus. xxii, 1900, p. 320: Trang, Peninsular Sianı.

Turdinulus humei Hart., Nov, Zool. ix, 1002, p. 564: Mt. Tahan, Pahang.

Distr,-Malay Peninsula.

Napothera epilepidota exsul (Sharpe).

Turdinulus exsul Sharpe, Ibis, I888, I, 479 : Mt. Kinabalu, Borneo.

Distr.-Borneo.

\section{Genus RIMATOR Blyth.}

Rimator malacoptilus Blyth.

Long-billed Wren-Babbler.

[Rimator malacoptilus malacoptilus B]ytl.

Rinator malacoptilus Blyth, Journ. Asiat. Soc. Betrgal, xvi, I\$47, p. I54: Darjeeling.

Distr.--Extra-limital.]

\section{Rimator malacoptilus albostriatus Salv.}

Rimalor albostriatus Salvadori, Ann. Mus. Civ. Gen. xiv, IS79, p. 224: Padang Highlands, Sumatra.

Distr.-Sumatra.

\section{Genus ALCIPPE' Blyti}

Alcippe nipalensis (Hodgs). Mountain Nun-Thrush. [Alcippe nipalensis nipalensis (Holgs.).

Siva nipalensis Hodgson, Indian Review, rs ${ }_{3} S$, p. 89 : Nepral.

Distr.-Extra-limital.]

Alcippe nipalensis peracensis Sharpe.

Alcippe peracensis Sharpe, Proc. Zool. Soc. 1887, p. 439 : Perak.

Distr. -Malay States.

Alcippe poioicephala (Jerd.) .

Indian Nun-Thrush.

[Alcippe poioicephala poioicephala (Jetd.).

Thimalia poioicephala Jerdon, Madras Journ. Lit. Sci. xiii, I\$44, 1. 169 : Nilgiris.

Distr.-Extra-linital.]

Alcippe poioicephala davisoni Hat.

Alcippe phacocephala davisoni Harington, Journ. Bombay Nat. Hist.

Soc. xxiii, I9I5, p. 453 : Tavoy.

Distr.-Peninsular Sian.

I For continued use of this name see Riley 1933. 
Alcippe poioicephala pyrrhoptera (Bp.).

Napothera pyrrhoptera Bonaparte, Consp. Cen. Avium, i, IS50, p. 358 : Java.

Alcippe solitaria Cab., Mus. Hein, i, $1 \$_{50}$, p. $\$ 7$ : Sumatra error $=$ Java. Alcippe dumetoria Cab., Mus. Hein. i, 1850 , p. 88 : Java.

Mixornis erythronota Reichn., Journ. f. Orn. 1895, p. 356 : Java.

Distr.-Java.

Alcippe cinerea Blytl. ${ }^{1}$

Nun-Thrush.

Alcippe cinerea cinerea Blyth.:

Alcippe cinerea Blyth, Journ. Asiat. Soc. Bengal, xiii, 1844 , p. 384 :

Singapore Island,

?Hyloterpe brmneicauda Salvad., Ann. Mus. Civ. Gen. (1), xiv, 1879, p. z10: Padang, West Sumatra.

Alcippornis Ununeicanda eviphaea Oberh., Smiths Misc. Coll. Ixxiv, 2, 1922 , p. 2: Borneo.

Alcippornis brwneicauda eripolia Oberh., Bull. U.S. Nat. Mus. I59, 1932, p. 63: Bunguran Island, North Natuna Islands.

Distr.-Malay Peninsula.

Sumatra.

Borneo; North Natuna Islands.

* Alcippe cinerea hypocneca Oberh.

Alcippe cinerea hypocneca Oberholser, Suiths. Misc. Coll. 1x, No. 7 , I91 2, ग. S: Pinie Island, Batu Islands.

Distr.-Batu Islands, West Sumatra.

\section{Genus PSEUDOMINLA Oates.}

\section{Pseudominla castaneiceps (Hodgs.).}

Tit-Babbler.

[Pseudominla castaneiceps castaneiceps (Holgs.).

Minla castanciceps Hodgson, Ind. Review, 1538, p. 33: Nepal.

Distr.-Extra-limital.]

Pseudominla castaneiceps soror (Sharpe).

Minla soror Sharpe, Proc. Zool. Soc. $\mathrm{IS}_{7}, \mathrm{p}, 439, \mathrm{pl} .3{ }^{8}$, fig. I : Mt. Ijau, Perak.

Disir.-Malay States.

'Cf. Oberh., Bull. U.S. Nat. Mus. 159, 1932, p. 63.

: Blyth's name is, as Oberholser (1032) points out, a new combination of generic and specilic terms. as a result of a mistaken identification and it is questionable whether it constitutes a new and valid designation for the species so misidentified. 


\section{Genus STACHYRIS Blyth}

Stachyris nigriceps Blyth.

Grey-throated Babbler.

[Stachyris nigriceps nigriceps Blyth.

Stachyris nigriceps Blyth, Journ. Asiat. Soc. Bengal, xiii, I844, p. 378 : Nepal.

Disir.-Extra-limital.]

\section{Stachyris nigriceps davisoni Sharpe.}

Stachyris davisoni Sharpe, Bull. Brit. Orn, Cl. i, I892, p. 7 : Pahang. Stachyris nigriceps dipora Oberb., Smiths. Misc. Coll. Lxxiv, 2, 192z, 1). 7: Trang, Peninsular Siam.

Stachyris natunensis Hart, Nov, Zool. i, 1894 , D. 470 : Bunguran Island, North Natuna Islands.

Distr.-Malay Peninsula. North Natuma Islands.

Stachyris nigriceps tionis Rob. and Kloss.

Stachyris nigriceps lionis Robinson and Kloss, Journ. Fed. Malay States Mus, xiii, 1926, p. 2 II : Tiontan Island.

Distr.-Tionan Island, east coast, Malay Peninsula.

Stachyris nigriceps borneensis Slarpe.

Stachyris borneensis Sharpe, Ibis, 1887, p. 449: Mit. Kinabalu, North Borneo.

Stachyris larvatn vermiculatat Farrisson and Hartley, Bull. B. O. Cl. liv, 1934, p. 553 : Mt. Dulit, Sarawak.

Distr.-Borneo (not West Sarawak).

Stachyris nigriceps hartleyi Chas.

Stachyris nigriceps harlleyi Clasen, Bull. Raffles Mus. IO, I935, p. 43 : Mt. Poi, West Sarawak.

Distr.-West Sarawak (Mts. Penrissen and Poi), Borneo.

Stachyris nigriceps larvata (Bp.).

Timalia larvala Bonaparte, Consp. Gen. Avium, IS50, p. 217 : Sunatra.

Distr.-Sumatra; Lingga Archipelago.

1 Eleven topotypes of "vermiculata" seem absolutely inseparable from thirty new skins of borneensis from Mt. Kinabalu. As in other birds of this group new skins are slightly more olive and rusty in colour than older specimens, and very old skins are useless for comparison. Some dated 1899 are altered beyond all subspecific recognition. 
Stachyris poliocephala (Temm.).

Grey-headed Babbler.

Stachyris poliocephala poliocephala (Temm.).

Timalia poliocephala Temminck, P1. Col. 593, fig. 2, IS36: Bencoolen, West Sumatra.

Stachyris poliocephala diluta Robinson and Kloss, Ibis, Iop 18, p. 597 : Taiping, Perak, Malay States.

Distr.-Malay Peninsula.

Sumatra (part).

Borneo.

Stachyris poliocephala pulla Kloss.

Stachyris poliocephala pulla Kloss, Treubia, xiii, I931, D. 348 :

Deli, North-east Sumatra.

Distr.-Sttmatra (north-east).

Stachyris grammiceps (Temm.). Javan White-breasted Babbler. Myiothera grammiceps Temninck, Pl. Col. 44S, fig. 3, ISzS: West Java.

Timalia grammicephala Bp., Consp. Gen. Av. 1850 , D. 217 : West Java. Distr.-Java (west).

Stachyris nigricollis (Temm.).

Black-necked Babbler-

Stachyris nigricollis nigricollis (Temm.).

Timalia nigricollis Temminck, P1. Col. 594, fig. 2, 1836 : Borneo.

Distr.-Borneo.

Sumatra.'

Stachyris nigricollis erythronotus (Blyth).

Timalia erythronotus Blyth, Journ. Asiat. Soc. Bengal, xi, IS42, p. 793 : Singapore Island.

Brachypteryx nigrogularis Eyton, Ann. and Mag. Nat. Hist. xvi. 1845. j. 228: Malacca.

Distr.-Malay Peninsula.

Stachyris leucotis (Strichl.).

White-eared Babbler.

Stachyris leucotis leucotis (Strickl.).

Timalia lencotis Strichland, Contr. Orn. 1848, p. 63, pl. 12 : Malacca.

Distr.-Malay Peninsula.

Stachyris leucotis goodsoni Hart.

Stachyris leucotis goodsoni Hartert, Bull. Brit. Orn. Cl. xxxvi, 1915, p. 7: Mt. Mulu, Sarawak.

Distr._Borneo.

1 Racial identity of the Sumatran form not absolutely certain. 
Stachyris maculata (Temun.).

Red-rtumped Babbler.

Stachyris maculata maculata (Temm.).

Timalia maculata Temuinck, Pl. Col. 593, fig. I, I\$ 36 : Borneo.

Distr.-Borneo.

Stachyris maculata pectoralis Blytl.,

Timalia pectoralis Blyth, Journ. Asiat. Soc. Bengal, xi, IS42, p. 793: Singapore Island.

Malacopteran squamatm Eyton, Ann. Mag. Nat. Hist. xvi, 1845, p. 228 : Malacca.

Distr.-Malay Peninsula.

Sumatra; Rhio Archipelago.

* Stachyris maculata banjakensis Richm.

Stachyris banjakensis Richmond, Proc. Biol. Soc. Wash. xv, 1902, [. 190: Tuangku Island, Banjak Islands.

Distr.-Banjak Islands, West Suntatra.

* Stachyris maculata hypopyrrha Oberh.

Stachyris maculata hypopyrha Oberholser, Suitls. Misc. Coll. Ix, No. 7, 1912, p. 9: Pinie Island, Batu Islands.

Distr.-Batu Islands, West Sumatra.

Stachyris striolata (S. Müll.).

Spotted Babbler.

Stachyris striolata striolata (S. Mïll.).

Timalia striolata Sal. Müller, Tijo. Nat. Gesch. Phys. IS38, p. 346 :

Padang Residencies, West Sumatra.

Distr.-Sunatra (west).

Stachyris striolata umbrosa (Kloss).

Thringorhina striolata umbrosa K̈loss, Jottrn. Fed. Mal, States Mus. x, 1921, p. 212: Deli, North-east Sumatra.

Distr.-Sunatra (north-east).

Stachyris striolata guttata (Blyth).

Turdinus gutiatus Blyth, Journ. Asiat. Soc. Bengal, Xxviii, IS59, p. 4 I4: Tenasserin.

Distr.-Peninsular Sian.

Stachyris thoracica (Tellnt1.).

White-collared Babbler.

Stachyris thoracica thoracica (Temmin.).

Pilla thoracica Temninck, P1. Col, 76, 1S22: West Java.

Distr.-Sumatra.

Java (west).

When birds from the Malay Peninsula and Borneo are compared, the differences are so slight that they are scarcely worth recognition, but specimens from the Rhio Archipelago and Sumatra average very dark on the ear-coverts. No exact topotypes from Singapore have been examined. 


\section{Stachyris thoracica orientalis Rob.}

Stachyris orientalis Robinson, Journ. Fed. Mal. States Mus. vii, 1915, 13. 236: Idjen Massif, East Java.

Distr.-Java (east).

Stachyris chrysæa Blyth.

Golden-lieaded Babber.

[Stachyris chrysaea chrysaea Blyth.

Stachyris chrysaa Blyth, Journ. Asiat. Soc. Bengal, xiii, 1844 , p. 379: Nepal.

Distr.-Extra-limital.]

Stachyris chrysaea chrysops Richm.

Slachyris chrysops Richnond, Proc. Biol. Soc. Wash. xv, 1902, p.

I57: Trang, Peniustular Siam.

Distr.-Malay Peniustula.

Stachyris chryszea frigida (Hartl.)."

Zosterops (Heleia) frigida Hartlaub, Journ. f. Ornith. I865, p. 27 : West Sumatra.

Stachyris bocagei Salvad., Ann. Mus. Civ. Gen. xiv, 4879, p. 223: Padang Highlands, West Sumatra.

Distr.-Sumatra.

Genus CYANODERMA Salvadori.

Cyanoderma erythroptera (Blyth). Red-winged Babbler.

Cyanoderma erythroptera erythroptera (Blyth).

Timalia erylhroplera Blyth, Journ. Asiat. Soc. Bengal, xi, $1 S_{42}$, p. 794 : Singapore Island.

Brachypteryx acutirostris Eyton, Ann. Mag. Nat. Hist. xvi, 1845, p, 228 : Malacca.

Timalia pirrhoptera Bp., Consp. Gen. Av. i, 1850, p. 217: nom. nov.

Cyanoderma erythopteri neocara oberh., Bull. U.S. Nat. Mus. 159. 1032, p. 66 : Bunguran Island, North Natuna Islands.

Distr.-Malay Pentusula; Tionuan Island.

Sunatra (southeru half).

North Natuna Islands.

Cyanoderma erythroptera pyrrophea (Hartl.).

- Timalia pyrrophaa Hartlatsb, Rev. Mag. Zool. rS44, p: 402: Sumatra (Rob. and filoss, ig2o).

Cyanoderma erythoptera eripella Oberh., Smiths. Misc. Coll. 1xxiv, 1922, 1\%. 2: Upper Siuk River, East Sumatra.

Disir.-Sunatra (nothern half).

z Cyanoderma e. sordida Baker, Buli. B. O. Cl. xxxvii, 1017 , p. to from Patiyu, S. W. Siam appears inseparable.

i Cf. Delacour, Bull. B. O. Cl. $1 v, 1034,1 \% 1^{2} 1$. 
*Cyanoderma exythroptera apega Oberl.

Cyanoderma erythroptera apega Oberh., Smiths. Misc. Coll. Ixxiv, 2, 1922, p. 8: Banka Island.

Distr.-Banka; Billiton.

Cyanoderma erythroptera bicolor (Blyth).

Timalia bicolor Blyth, Ibis, 1865, p. 46: Labuan.

Cyanoderma labuanensis Collin and Hartert, Nov. Zool. xxxiv, 1927, p. \$1 : nom. nov.

Distr.-Borneo (not south); Banguey Island, North Borneo.

Cyanoderma erythroptera rufa Chas. and Kloss.

Cyanoderma erythoptera rufa Chasen and Kloss, Bull. Brit. Orn.

C1. xlviii, 1927, P. 47 : Sampit, South Borneo.

Distr.-Borneo (sonth).

* Cyanoderma erythroptera fulviventris Richm.

Cyanoderma fulviventris Riclmond, Proc. U.S. Nat. Mus. xxvi, Ig03, p. 507: Tuangku Island, Banjak Islands.

Distr.-Banjak Islands, West Simatra.

* Cyanoderma erythroptera pella Oberh.

Cyanoderma erythropterum pellum Oberholser, Sniths. Misc. Coll.

1x, No. 7, 1912, p. 9: Tana Masa Island, Batu Islands.

Distr.-Batu Islands, West Sumatra.

Cyanoderma melanothorax (Temm\}).

Pearl-cheeked Babbler.

Cyanoderma melanothorax melanothorax (Temm.).

Myothera melanothorax Temminck, P1. Col. 185, I\$23: Mt. Gede, West Java.

Distr.-Java (Mts. Gedeh and Pangerano, West Java).

Cyanoderma melanothorax albigula Stres.

Cyanoderma melanothorax albigula Stresemann, Orn. Monatsbr. xxxviii, 1930, p. I4S: Mt. Papandajan, West Java.

Distr,-Java (Mt. Papandajan, West Java).

Cyanoderma melanothorax mendeni Naum.

Cyanoderma melanothorax mendeni Naumann, Bull. Brit. Orn. Cl. lv, 1935, p. 137: Indromaju, north coast of West-central Java.

Distr.-West-central Java.

'An unnecessary renaming as Lafresnaye's combination is French and not binomial.

2I cannot trace Timalia poliopsis Vord., 1886, given by Kuroda (1933) as a synosym. 
Cyanoderma melanothorax intermedia (Rob.).

Stachyris melanothorax intermedia Robinson, Journ. Fed. Malay States Mus. vii, r9rS, p. 236 : Mt. Idjen, East Java.

Distr.-Java (east).

Cyanoderma melanothorax baliensis Hart.

Cyanoderma melanolhorax baliensis Hartert, Bull. Brit. Orn. Cl. xxxvi, 1915, P. 2: Bali.

Distr. - Bali.

Cyanoderma rufifrons (Himne).

Hume's Babbler.

[Cyanoderma rufifrons rufifrons (Hume).

Slachyris mufrous Hume, Stray Feathers, i, 1873, p. 479: Pegu.

Distr.-Extra-linital.]

Cyanoderma rufifrons poliogaster (Huule).

Stachyris poliogaster Hune, Stray Feathers, ix, is8o, D. II6:

Johore, Malay Peninstila.

Distr.-Malay States.

Sumatra.

Borneo.

Gents MIXORNIS Biyth.

Mixornis gularis (Horsf.)."2

Yellow-breasted Babbler.

Mixornis gularis gularis (Horsf.).

Timalia gularis Horsfield, Zool. Res, in Java, IS22: Sumatra.

Prinia pileata Blyth, Journ. Asiat, Soc. Bengal, xi, 1847, p. 204: Malacca.

Mixornis sumatrana $\mathrm{B} p$, Consp. Gen. Av. I\$50, p. 217 : Sumatra.

Distr.-Malay Peninsula (north to Patani).

Stumatra; Rhio and Lingga Archipelagos.

Mixornis gularis connectens Kloss.

Mixomis rubricapilla connectens Kloss, Ibis, I9IS, p. 207:

Peninsular Sian, Lat. $10^{\circ} \mathrm{N}$.

Mixomis gularis chersonesophila Oberholser, Smiths. Misc. Coll. lxxiv, 2. 1922, p. 3: Trang, Peninsular Siam.

Distr.-Malay Peninsula (south to about Penang).

Mixornis gularis zophera Oberh.

Mixomis pileata zophera Oberholser, Bull. U.S. Nat. Mus. 98 , I9I7,

p. 49 : Telaga Island, Anamba Islands.

Distr.-Anamba Islands.

This race, or $S$. r. obscura Baker (if distinct) from Peninsular Siam, Lat $11^{\circ}$ N. may occur in Malaysia.

a Cf. Rob., Journ. F.M.S. Mus, vii, 1917 , p. 176; Oberb., Bulh. U.S.

Nat. Mus. 159, 1932, p. 71 ; Delacour, Bull. Brit. Orn. Cl. Iv, 1935, B. 99. 
Mixornis gularis everetti Hart.

Mixornis evereti Hartert, Nov. Zool. i, I894, D. 472: Bunguran Island, North Natuna Islands.

Distr.-Nortl Natuna Islauds.

-Mixornis gularis zaperissa Oberh.

Mixornis rubricapilla zaperissa Oberholser, Bull. U.S. Nat. Mus. 159, 1932, p. 68: Lingung Island, North Natuma Islands.

Distr._Laut, Kombeh and Lingung Islands, North Natuna Islands.

*Mixornis gularis zarhabdota Oberh.

Mixornis pileata zarhabdota Oberholser, Suiths. Misc. Coll. Lx, I91 2, p. 9: Bangkaru Island, Banjak Istands.

Distr.-Banjak Islands, West Sumatra.

* Mixornis pileata zaptera Oberh.

Mixornis pileata zaptera Oberlı, Smiths. Misc. Coll. lx, No. 7 , 1912, p. 9: Tana Masa Island, Batu Islands.

Distr.-Batu Islands, West Sunatra.

Mixornis gularis bornensis $\mathrm{B} p$.

Mixomis bornensis Bonaparte, Consp. Gen. Avium, I850, p. 217 : Borneo.

Mixornis similis: Blyth. Ibis, $1865, p_{*} 47$ : Borneo.

Distr.-Borneo (not north).

\section{Mixornis gularis montana Sharpe.}

Mixornis monlana Sharpe, Ibis, 1887 , p. 448 : Mt. Kinabalt, North Borneo.

Distr.-Borneo (north).:

Mixornis gularis argentea Chas, and Kloss.

Mixomis gularis argentea Clusen and Kloss, Bull. Raflles Mus. 4, I930, p. 82: Mallewallé Island, North Borneo.

Distr.-Norti Bornean Islands.

* Mixornis gularis pontia Oberh.

Mixornis bornensis pontia Oberholser, Smiths. Misc. Coll. 1xxiv, z, I922, p. 6 : Pulau Laut, South-east Borneo.

Disir._Pulau Laut, South-east Borneo.

x The white throat shows that this is bornensis, not gularis.

- Not really a mountain form. The type came from an altitude of about 1,000 feet and the subspecies occurs in the lowlands of North Borneo, near the sea. 


\section{* Mixornis gularis ruficoma Oberh.}

Mixornis bornensis ruficoma Olerholser, Suiths. Misc. Coll. 1xxiv, 2, I922, p. 6: Banka Island.

Distr.-Banka Island; Billiton.

\section{Mixornis gularis javanica Cab.}

Mixornis jasanica Cabanis, Mus. Hein. i, I850, p. 77, footnote: Java.

\section{Distr.-Java.}

Mixornis favicollis Bp. Bonaparte's Yellow-breasted Babbler. Mixornis flavicollis flavicollis $\mathrm{Bp}$.

Mixornis flavicollis Bonaparte, Consp. Gen, Avium, IS50, p. 217 : Java,

Distr.-Java.

\section{Mixornis flavicollis prillwitzi Hart.}

Mixomis prillwitzi Hartert, Bull. Brit. Orn. Cl. xii, Igor, p. 32 : Kangean Island, Java Sea.

Distr.-Kangean Islands.

\section{Genus KENOPIA Blyth.}

Kenopia striata (Blyth).

Striped Babbler.

Timalia striala Blyth, Journ. Asiat. Soc. Bengal, xi, I\$42, p. 793 : Singapore Island.

Brachypteryx maculatus Eyton, Ann. Mag. Nat. Hist. xvi, 1845, p. 228 : Malacca.

Turdirostris leucostigma Bp., Consp. Gen. Avium, 1850, p. 218 : Borneo (nom. nud.).

Distr.-Malay Peninsula.

Sumatra.

Borneo.

Genus MACRONUS Jardine and Selby.

Macronus ptilosus Jard, and Selb.

Fluffy-backed Babbler.

Macronus ptilosus ptilosus Jard, and Selb.

Macronus plilesus Jardine and Selby, Ill. Orn. I835, pl. I50: Malacca.

Timalia trichorrhos Temm., Pl. Col. 504, 1836 : Sumatra (Hartert, 1015).

Distr.-Malay Peninsula.

Stmatra; Banka.

Macronus ptilosus reclusus Hart.

Macronus ptilostis reclusus Hartert, Bull. Brit. Orn. Cl. xxxvi, 1915, 13. 36: Sarawak.

Distr.-Borneo; Billiton. 
Gentis HETEROPHASIA Blyth.

Heterophasia picaoides (Hodgs.).

Long-taited Sibia.

[Heterophasia picaoides picaoïdes (Horlgs.).

Sibia Pieaoides (sic) Hodgson, Journ. Asiat. Soc. Bengal, viii, I\$39. p. 38 : Nepal.

Distr._Extra-limital.]

Heterophasia picaoìdes wrayi (0.-Grant\}.

Sibia zrayi Ogilvie-Grant, Bull. Brit. Orn. Cl. xxv, rgto, p. 98 :

Batang Padang Mountains, Perak.

Dislr.-Malay States.

Heterophasia picaoides simillima Salvad.

Helerophasia simillima Salvadori, Ann. Mus. Civ. Gen. xiv, 1879. p. 232 : Padang Highlands, West Sumatra.

Distr.-Sumatra.

\section{Genus STAPHIDA Swinhoe.}

Staphida castaniceps (Horsf, and Moore). Chestnut-headed Babbler. [Staphida castaniceps castaniceps (Horsf. and Moore).

Ixulus caslaniceps Horsfield and Moore, Cat. Birds Mus. East Ind. Coy. i, I854, 1) 4 II: Afghanistan error $=$ Khasia Hills.

Distr.-Extra-limital.]

Staphida castaniceps everetti Sharpe.

Staphidia everetti Sharpe, Ibis, 1887, p. 447 : Mt. Kinabalu, North Borneo.

Distr.-Borneo.

\section{Genus SIVA Hodgson.}

Siva strigula Hodgs."

Chestnut-tailed Siva.

[Siva strigula strigula Hodgs.

Siva strigula Hodgson, Ind. Review, I $838, \mathrm{p}, 8_{9}$ : Nepal.

Disir._Extra-limital.]

Siva strigula malayana Hart.

Siva slrigula malayana Hartert, Nov. Zool. iv, 1902, P. 567: Mt. Tahan, Pahang.

Siza strigula omissa Roths., Nov. Zool. xxviii, 1921, p. 40: Mt. Kerbau, Perak.

Distr.-Malay States.

' Cf. Meinertz, Bull. B. O. C. xlvi, rg26, p. 128 .

[228] 
Siva cyanouroptera Hodgs.

Dull Siva.

[Siva cyanouroptera cyanouroptera Hodgs.

Siva cyanouroplera Hodgson, Ind. Review, $8_{3} 8$, p. 88: Nepal.

Distr.-Extra-limital.]

Siva cyanouroptera sordidior Sharpe.

Sita sordidior Sharpe, Proc. Zool. Soc. I888, p. 276: Batang Padang Mts., Perak.

Distr.-Malay Peninsula.

\section{Genus ERPORNIS Blyth.}

Erpornis zantholeuca Blytll.

White-bellied Babbler.

[Erpornis zantholeuca zantholeuca Blytli.

Erpornis zantholeuca Blyth. Journ. Asiat. Soc. Bengal, xiii, I844, p. 3So: Nepal.

Distr._Extra-limital.]

Erpornis zantholeuca interposita Hart.

Herpornis xantholeuca interposita Hartert, Bull. Brit. Orts. Cl. xxxviii, I917, p. 20: Upper Perak.

Distr.-Malay Peninsula.

Erpornis zantholeuca brunnescens Sharpe.

Herpornis brunescens Sharpe, Ibis, I876, p. 41: Sarawak.

Distr.-Borneo.

\section{Genus CUTIA Hodgson.}

Cutia nipalensis Hodgs.

Cutia.

[Cutia nipalensis nipalensis Hodgs.

Cúlia Nipalensis Hodgson, Journ. Asiat. Soc. Bengal, v, $18_{\mathfrak{\jmath}} 6$, p. 773: Nepal.

Distr.-Eixtra-limital. ]

Cutia nipalensis cervinicrissa Sharpe.

Culia cervinicrissa Sharpe, Proc. Zool. Soc. I888, p. 276: Mt. Batu Putel, Perak.

Distr.-Malay States.

\section{Genus PTERUTHIUS Swainson.}

Pteruthius flaviscapis (Temm.).

Greater Shrike-Babbler.

Pteruthius flaviscapis flaviscapis (Temm.).

Allotrius flariscapis Temminck, P1. Col. 589, I836: Java.

Distr.-Java. 
Pteruthius flaviscapis aralatus Tick.

Pteruthius aralatus Tickell, Journ. Asiat. Soc. Bengal, xxiv, I855, p. 267: Tenasserim.

Distr.-Malay Peninsula.'

Pteruthius flaviscapis cameranoi Salvad.

Pteruthius cameranoi Salvadori, Ann. Mus. Civ. Gen. xiv, 1879, p. 232 : Padaug Highlands, Sumatra.

Distr, Sumatra.

Pteruthius fleviscapis robinsoni Chas. and Kloss.

Pteruthius flavicapis robinsoni Chasen and Kloss, Bull. Raffles Mus. 5, I93r, p. 86: Mt. Kinabalu, North Borneo.

Distr.-Borneo.

Pteruthius anobarbus (Temm.).

Lesser Shrike-Babbler.

Pteruthius tenobarbus nobarbus (Temm.).

Allotrius anobarbus Temminck, P1. Col. $589,18_{3} 6$ : West Java.

Distr.-Sumatra. ${ }^{2}$

Java (west).

Pteruthius anobarbus tahanensis Hart.

Pteruthius tahanensis Hartert, Nov. Zool, ix, rgo2, p. 576: Mt. Tahan, Pahang.

Distr.-Malay States.

Mesia argentauris Hodgs.

Genus MESIA Hodgson.

[Mesia argentauris argentauris Hodgs.

Mesia argentauris Hodgson, Indian Review, I838, p. 88: Nepal.

Distr.-Extra-linital.]

Mesia argentauris tahanensis Yen.3

Mesia argentauris tahanensis "Robinson", Yen, Science Journ.

(Sun Yat-Sen University) vi, 2, I934, p. 63: Mt. Tahan, Malay

Peninsula.

Distr.-Malay Peninsula.

Mesia argentauris laurinae (Salvad.).

Letolhrit laurinae Salvadori, Ann. Mus. Civ. Gen. xiv, 1879, p. 23I: Padang Highlands, Sumatra.

Distr. Sumatra,

- Some specimens from the Malay States are eralatus $>$ cameranoi: a female from the Lampongs, S. Sumatra (Buit. Mus.) is much paler below than Korinchi birds.

- I have some doubts as to whether this species really occurs in Sumatra.

3 Many years ago Hartert noticed the characters of this form. Robinson wrote the above name on the box of Malayan specimens in the British Museum, but never published the name. Mr. Yen in his "Etude des Genres du Groupe Siva-Leiothrix" (original publication not seen by me, but details supplied by Mr. N. B. Kinnear) has, apparently unconsciously, described the bird. 


\section{Family TROGLODYTID在.}

\section{Genus PNOEPYGA Hodgson.}

Pnoepyga pusilla Hodgs.

Scaly-breasted Wren.

Pnoepyga pusilla pusilla Hodgs.

Pnoepyga pusilla Hodgson, Proc. Zool. Soc. 1845, p. 25 : Nepal.

Distr.-Peninsular Siam."

Pnoepyga pusilla harterti Rob. and Kloss.

Pnoepyga pusilla harterti Robinson and Kloss, Journ. Fed. Malay

States Mus. viii, pt. 2, I9I8, 205 : Mt. Ijau, Perak.

Distr.-Malay States.

\section{Pnoepyga pusilla lepida Salvad.}

Pnoepyga lepida Salvadori, Ann. Mus. Civ, Gen, xiv, 1879, p. 227 : Padang Highlands, West Sunatra.

Distr.-Sumatra.

Pnoepyga pusilla rufa Sharpe.

Pnoctyga rufa Sharpe, Cat. Birds Brit. Mus. vi, 1881, p. 304 : Java. Distr._Java.

\section{Genus TESIA Hodgson.}

Tesia cyaniventer Hodgs. Slaty-bellied Wren.

[Tesia cyaniventer cyaniventer Hodgs.

Tesia cyaniventer Hodgson, Journ. Asiat. Soc. Bengal, vi, I837, p. Ior: Nepal.

Disir._Extra-limital.]

Tesia cyaniventer superciliaris (Bp.).

Microura superciliaris Bonaparte, Consp. Gert. Avium, i, 1850, p. 258: Java.

Brachypteryx lepturus Gray, Handlist Birds, i, 1869, p. 312: Java (nom. nud.).

Distr.-Java.

Tesia whiteheadi (Sharpe)."

Whiteliead's Wren.

Orthnocichla whiteheadi Sharpe, Ibis, I888, p. 478 : Mt. Kinabalu, North Borneo.

Distr,-Borneo.

- The form occurring in the mountains of Peninsular Siam seems not to be harterti of the Malay States. It is, no doubt, separable irom $P$. pusilla, but the available material is too poot to justify further study. form.

- Nearer to $T$. strbulata of Timor and Flores, than to any continental 


\section{Family TURDIDÆ.' \\ Genus BRACHYPTERYX 2 Horsfield.}

Brachypteryx leucophris ('Temm.).

Lesser Short-wing.

Brachypteryx leucophris leucophris (Temm.).

Myiothera leucophris Temminck, P1. Col. 448, i827 : Java.

Distr.-Peninsular Siam.

Sumatra.

Java; Bali.

Brachypteryx leucophris wrayi O.-Grant..

Brachypteryx wayi O.-Grant, Bull. Brit. Orn. Cl. xix, Igo6, 1). 10: Mt. Batu Puteh, Perak.

Distr.-Malay States,

Brachypteryx montana Horsf.

Greater Short-wing.

Brachypteryx montana montana Horsf.

Brachypteryx montana Horsfield, Trans. I,inn. Soc. xiii, I\$2I, p. 157: Java.

Distr,-Java.

Brachypteryx montana saturata Salvad.

Brachypteryx salurata Salvadori, Ann. Mus. Civ. Gen. xiv, I\$79, p. 225: Padang Highlands, Sttmatra.

Distr.-Suniatra.

Brachypteryx montana erythrogyna Sharpe.

Brachypteryx erythrogyna Sharpe, Ibis, I 888, p. 389 : Mt. Kinabalu, North Borneo.

Distr.-Borneo.

Genus LUSCINIA Forster.

Luscinia cyane (Pall.).

Siberian Blue Chat.

Luscinia cyane cyane (Pall.).

Molacilla Cyane Pallas, Reise Prov. Russ. Reichs., iii, 1776, p. 697: Eist Siberia.

Distr.-Malay Peninsula.

Simatra.

Borneo.

I In the absence of an authenticated specimen Phoenicura letcoplera Blyth (Journ. As. Soc. Bengal, xii, 1843, p. 962) is not accepted for the Malay Peninsula.

- What is Brachypteryx favivertris Salvad., Ann. Mus. Civ. Gen. xiv, 1879, p. 226, based on a single female from Mt. Singgalang, Padang Highlands, Sumatra? 
Genus SAXICOLA Bechstein.

Saxicola torquata (Linn.).

Stone-Chat.

[Saxicola torquata torquata (Linn.).

Muscicapa torquata Linn., Syst. Nat, I2th. ed. i, I766, p. 328 :

Cape of Good Hope.

Distr.-Extra-limital.]

Saxicola torquata stejnegeri (Parrot).

Pratincola rubicola stejnegeri Parrot, Verh. Orn. Ges. Bayern, viii, Igo8, P. I24: North Japan.

Distr, - Malay Peninstula.

Borneo.

Saxicola caprata (Linn.).

Black Stone-Chat. ${ }^{1}$

[Saxicola caprata caprata (Linn.).

Motacilla Caprata Limn., Syst. Nat. I2th. ed. I, 1766, p. 335 : Luzon, Philippine Islands.

Distr.—Extra-linital.]

Saxicola caprata pyrrhonota (Vieill.).

Oenanthe pyrrhonota Vieillot, Nov. Dict. d'Hist. Nat, ed. 2, xxi, 18IS, P. 428 : Timor.

Saxicola fruticola Horsf., Trans. Linn, Soc. xiii, p. 157, I821 : Java.

Distr.-Borneo (? subsp.). ${ }^{2}$

Java; Bali.

\section{Gents ENICURUS Temminck.}

Enicurus leschenaulti (Vieill.).

White-crowned Forktail.

Enicurus leschenaulti leschenaulti (Vieill.).

Turdus Leschenaulti Vieillot, Nov. Dict. d'Hist. Nat. ed. 2, $\mathrm{xx}$, I8I8, p. 269 : Java.

Enicurus coronatus Temm., Pl. Col. 113,1824 : Java.

Motacilla speciosa Horsf., Trans. Linn. Soc, xiii, 1820, p. 155: Java.

Distr._Java; Bali.

Enicurus leschenaulti frontalis Blyth.

Enicurus frontalis Blyth, Journ, Asiat. Soc. Bengal, xvi, I847, p. I56: Malacca.

Distr._Malay Peniusula.

Sumatra; Nias Island; West Sumatra.

Borneo.

' Revision: Stres., Nov. Zool. xix, 1912, p. 319.

2 Blasius has recorded caprata from Borneo: there is no reliable record of any form from the Malay Peninsula. 
Enicurus leschenaulti borneensis Sharpe.

Henicurus borneensis Sharpe, Ibis, 1889, p. 277 : Mt. Kinabalu, North Borneo.

Distr.-Borneo (mountains).

Enicurus ruficapillus Temm.

Chestnut-backed Forktai].

Enicurus ruficapillus Temminck, P1. Col. 534, I832: Palembang, Sumatra.

Enicurus diadematus S. Müll., Tijd. Nat. Gesch. Phys. 1835, p. 346 : Sumatra.

Henicurus rufidorsalis Sharpe, Ibis, 1879, p. 255 : Lawas River, Sarawak. Distr.-Malay Peninsula.

Sumatra.

Borneo.

Enicurus schistaceus Hodgs.

Slaty-backed Forktatl.

Enicurus schistaceus Hodgson, Asiat. Res. xix, 1836, p. i 89 : Nepal.

Distr.-Malay States.

Enicurus velatus Temm.

Small Forktail.

Enicurus velatus velatus Temm.

Enicurus velatus Temminck, P1. Col. I60, I823: Java.

Distr,-Java.

Enicurus velatus sumatranu, Rob. and Kloss.

Henicurus velalus sumatranus Robinson and Kloss, Journ. Fed. Malay States Mus. xi, I923, p. 56: Korinchi, Sunzatra.

Distr.-Sumatra.

\section{Genus MUSCISYLVIA Hodgson.}

Muscisylvia leucura Hodgs.

White-tailed Blue Robin.

Muscisylvia leucura leucura Hodgs.

Muscisylvia leucura Hodgson, Proc. Zool. Soc. I\$45, p. 27 : Nepal. Distr._Malay States."

Muscisylvia diana (Less.).

Sunda Island Blue Robin.

Muscisylvia diana diana (Less.).

Lanius (Notodela) diana Lesson, in Bélanger, Voy. Inde, Zool., I834, p. 246, Oiseaux pl. 3: Pegu error=Java.

Brachypleryx albifrons Bp., Consp. Gen. Avium, 1850, p. 257: Java.

Brachyptery $x$ salaccensis Vorderm. Nat. Tijdschr. Ned, Ind. xliv, t 886 , p. 336 : Java.

Distr.-Java,

- Perhaps separable from the typical race on account of small size. 
Muscisylvia diana sumatrana (Rob. and Kloss).

Notodela diana sumatrana Robinson and Kloss, Journ. Fed. Malay States Mus. viii, pt. 2, I9I8, p. 2I5: Korinchi, Sumatra.

Distr.-Sumatra.

\section{Genus COPSYCHUS Wagler.}

\section{Copsychus saularis (Linn.). ${ }^{\mathrm{T}}$}

Straits Robin.

[Copsychus saularis saularis (Linnt.).

Gracula Saularis Linn., Syst. Nat. Ioth, ed. 1758, p. I09: Bengal. Distr.-Extra-limital.]

Copsychus saularis musicus (Raffl).

Lanitus musicus Raffles, Trans. Linn. Soc. xiii, 1822, p. 307 : Bencoolen, West Sumatra.

Copsychus saularis ephalus Oberholser, Smiths. Misc. Coll, Ixxvi, No. 6, 1923, p. 2: Tarussan Bay, North-west Sumatra.

Distr.-Malay Peninsula; ${ }^{z}$ Tioman Island.

Sumatra; Rhio Archipelago, Billiton.

*Copsychus saularis nesiotes Oberh.

Copsychus saularis nesiotes Oberholser, Smiths. Misc. Coll. Ixxvi, No. $6,19 \mathrm{r} 8$, p. 3 : Banka Istand.

Distr,-Banka Island.

* Copsychus saularis zacnecus Oberh.

Copsychus saularis zacnecus Oberholser, Smiths. Misc. Coll. $1 \mathrm{x}$, No. 7 , 1912, p. I2: Simalur Island.

Distr.-Simalur Island, West Sumatra.

* Copsychus saularis nesiarchus Oberh.

Copsychus saularis nesiarchus Oberholser, Smiths. Misc. Coll. 1xxvi,

No. 6 , 1912, p. 3 : Nias Island.

Distr.-Nias Island, West Sumatra.

Copsychus saularis pagiensis Richm.

Copsychus saularis pagiensis Richmond, Proc. Biol. Soc. Wash. xxv, 1912, p. 105: North Pagi Island, West Sumatra.

Distr.—Siberut, Sipora and Pagi Islands, West Sunatra.

Copsychus saularis problematicus Sharpe.

Copsychus problematicus Sharpe, Ibis, 1876, p. 36 : Sibu, Sarawak. Distr.-Borneo (west).

' Cf. Stres., Journ. f. Orn. I924, p. 252; Chas. and Kl., Bull. Raff. Mus. 4. 1930, p. 84 .

3 The Malayan bird is not strictly identical with musicus, but is saularis ? musicus although even the form occurring in Peninsular Siam is nearer to musicus than to saularis. 
Copaychus saularis niger Ward1. Rams.

Copsychus niger Wardlaw Ramsay, Proc. Zool. Soc. I\$86, p. I23: Sandakan, Nortl Borneo.

Distr.-Borneo (north).

\section{Copaychus saularis pluto Bp.}

Copsychus pluto Bonaparte, Consp. Gen. Aviun, i, 1850, P. 267 : Samarinda, Fast Borneo.

Distr.-Borneo (east); Maratua Islands, East Borneo (? subsp.).

Copsychus saularis javensis Chas, and Kloss.

Copsychus saularis javensis Chasen and Kloss, Bull. Raffles Mus. 4, 1930, p.p. 87 and 89: Wynkoops Bay, South-west Java.

Distr.-Java (west).

Copsychus saularis amcenus (Horsf.).'

Turdus amanus Horsfield, Trans. Linn. Soc. xiii, 1821, p. 147: Java.

Gryllivora brevirostra Swains, Anim. in Menag, 1837, p. 292: Java.

Distr._Java (east); Bali.

Genus KITTACINCLA Gould.

Kittacincla malabarica $($ Scop.).2

Shama.

[Kittacincla malabarica malabarica (Scop.).

Muscicapa malabarica Scopoli, Del. Faun, et Flor. Insubr. ii, 1786, p. 96 : Malabar, South-west India,

Distr._Extra-linital.]

Kittacincla malabarica interposita Rob. and Kloss.

Kittacincla malabarica interposita Robinson aud Kँloss, Journ. Fed. Malay States Mus. x, I922, p. 262 : Daban, Sonth Annam.

Distr,-Peninsular Sian (part).

Kittacincla malabarica mallopercna Oberh.

Killacincla malabarica mallopercna Oberltolser, Smiths. Misc. Coll. lxxvi, 6, I923, p. 5: Singkep Island.

Distr.-Malay Peninstila.

Rhio and Lingga Archipelagos.

The distribution of the various forms of Copsychus in Borneo and Java is complicated and the bird is unstable in several large areas: we have tried to appreciate the subspecific ranges in Bull. Raff. Mus, 4, 1930, pi. 84-9o.

2Revision: Oberh., Bull. U.S. Nat. Mus. 159, 1932, p. 82.

3 Oberholser restricts the range of interposita considerably and considers that at least the northern and middle portions of the Malay Peninsula are occupied by pellogyna Oberh. described from Tenasserim.

$$
\text { [ } 236]
$$




\section{Kittacincla malabarica tricolor (Vieill.).}

Turdus tricolor Vieillot, Nov. Dict. d'Hist. Nat, xxx, ISI8, p. 291 : Isles de la mer du Sud = West Java $($ Rob. and Kloss, 192t).

Distr. S Sumatra.

Karimata Islands.

Java (west).

Kittacincla malabarica javana Kloss.

Killacincla malabarica javuna Kloss, Jouru. Fed. Malay States Mus. $\mathrm{x}$, I92I, D. 2 Io: Karanglolong, mid Java.

Distr.-Java (central).

\section{Kittacincla malabarica omissa Hart.}

Kitlacincla macrurus omissa Hartert, Nov. Zool. ix, 1902, p. 572 : Lawang, East Java.

Distr.-Java (east).

Kittacincla malabarica ochroptila Obeth.

Kitlacincla malabarica ochroptila Oberholser, Bull. U.S. Nat. Mus. 9 S, 1917, [. 51: Siantan Island, Anamba Islands.

Distr.-Anamba Islands (part).

*Kittacincla malabarica heterogyna Oberh.

Kittacincla malabarica heterogyna Oberholser, Bull. U.S. Nat. Mus. 98,1917, P. 53: Riabu Island, Anamba Islands.

Distr,-Ananba Islands (part).

Kittacincla malabarica eumesa Oberh.

Kitlacincla malabarica eumesa Oberlolser, Bull. U.S. Nat. Mus. I59, I932, p. $S_{1}$ : Bunguran Island, Natuna Islands.

Distr.-North Natuna Islands.

Kittacincla malabarica suavis (Scl.).

Copsychus suaris P. L. Sclater, Proc. Zool. Soc. 1S61, p. I\$5: Bandjermasin, South Borneo.

Kitucincla malabarica zaphotina Oberholser, Smiths. Misc. Coll. Ixxvi, I023, D. 6: Central Borneo.

Distr.-Borneo (not north).

*Kittacincla malabarica abbotti Oberh.

Kitlacincla malabarica abbolti Oberholser, Smiths. Misc. Coll. Ixxvi, No. 6, 1923, p. 5: Banka Island.

Distr.-Banka Island; Billiton Island.

+ This race merges into interposita and we have used the name mallofercna for birds from an intermediate area in which the racial characters ate ill-defined. 
Kittacincla malabarica opisthisa Oherl.

Killacincla malabarica opisthisa Oberholser, Sniths. Misc. Coll. 1x

No. 7, I9I2, P. I3: Tuanku Island, Banjak Islands.

Distr,-Banjak Islands, West Sumatra.

-Kittacincla malabarica opisthopela Oberh.

Killacincla malabarica opisthopela Oberholser, Sniths. Misc. Coll.

Ix, No. 7, I9I2, p. I3: Tana Bala Island, Batu Islands.

Distr,-Batu Islands, West Sumatra

Kittacincla malabarica melanura Salvad.

Cillocincla melanura Salvadori, Ann. Mus. Civ. Gen. (2) iv, I887, p. 549, pl. 8 , fig. I : Nias Island.

Kitlacincla melanura hypoliza Oberh., Smiths. Misc. Coll. Ix, No. 7. 1912, p. 13: Simalur Island.

Kittacincla melanura opisthochra Oberh., Smiths. Misc. Coll. 1x, No. 7, 1912, p. 13: Lasia Island.

Kittacincla melamma pagensis Oberh., Smiths. Misc. Coll. 1xxvi, No. 6, 1923, p. 3: North Pagi Island.

Distr.-West Sumatran Islands of Simalır, Lasia, Babi, Nias, Siberut, Sipora and Pagi.

Kittacincla malabarica nigricauda Vord.

Cillacincla nigricauda. Vorderman, Nat. Tijdschr. Ned. Ind. xlii, IS93, P. I97: Kangean Island.

Distr.-Kangean Islands; Mata Siri Island, Java Sea.

Kittacincla stricklandii (Motl. and Dillw.). White-crowned Shana.

Kittacincla stricklandii stricklandii (Motl. and Dillw.).

Copsychus Stricklandii Motley and Dillwyn, Nat. Hist. Labuan, 1855 , p. 20, pl. 4 : Labtian Island.

Distr.-Borneo (north).

Kittacincla stricklandii barbouri Bangs and Petrs.

Kittacincla barbouri Bangs and Peters, Occ. Pap. Boston Soc. Nat. Hist. v, 1927, p. 239: Maratua Island.

Distr.-Maratua Island, East Borneo.

\section{Genus TRICHIXOS Lesson.}

Trichixos pyrropyga Less.

Orange-tailed Thrush.

Trichixos pyrropyga Lesson, Rev. Mag. Zool. 1839, p. I67: Sumatra.

Turdinus? superciliaris A. Hay, Madr. Journ. xiii, pt, 2, 1844, p. 163: Malacca.

Myiolestes pyrhonota Bp., Consp. Gen. Avium, i, 1850, p. 358 : Borneo.

Distr.--Malay States.

Sunnatra.

Borneo. 


\section{Genus COCHOA Hodgson,}

Cochoa azurea (Temm.).

Blue Thrush.

Cochoa azurea azurea (Temm.).

Turdus azureus Temminck, Pl. Col. 274, IS24 : Java.

Distr.-Java.

\section{Cochoa azurea beccarii Salvad.}

Cochoa beccarii Salvadori, Ann. Mus. Civ. Gen. xiv, iS79, p. 228 : Padang Highlands, Sunatra.

Distr.-Sumatra.

Genus MYOPHONUS' 'Tenuninck.

Myophonus glaucinus (Temm.).

Javan Whistling Thrusl.

Pitta glaucina Temminck, P1. Col. J94, I\$23: Java.

Turdus cyaneus Horsf, (not Müll. 1776) Trans. Linn. Soc. xiii, 1821 , p. 149: Java.

Distr. - Java.

Myophonus castaneus Wardl. Rams. Brown-winged Whistling Thrush.

Myiophoneus castancus Wardlaw Ransay, Proc. Zool. Soc. 1880, p. 16: West Sumatra.

Distr. Sumatra.

Myophonus borneensis Slater.

Bornean Whistling Thrush.

Myiophoneus borneensis H. H. Slater, Ibis, $1 \$ 85$, p. I23: Sarawak. Dislr.-Borneo.

Myophonus melanurus (Salvad.). Sumatran Whistling Thrush. Arrenga melanura Salvadori, Ann. Mus. Civ. Gen. xiv, I $879, \mathrm{p}$. 227: Padang Highlands, Sumatra.

Distr.-Sumatra.

Myophonus robinsoni O.-Grant. Malayan Whistling Thrush. Myiophoneus robinsoni Ogilvie-Grant, Bull. Brit. Orn. Cl. xv, 1905, p. 69 : Mt. Mengkuang Lebar, Selangor, Malay States.

\section{Distr.-Malay States.}

- We have left most of the forms of this genus under a specific name as in the present state of our knowledge it seems impossible to link them up any furtber. A recent occurrence of crassirostris in South-east Siam, within the range of engenei, destroys our former conception of the grouping of the large yellow-bilted forms. Robinson has united the yellow-billed robinsoni to glaucina (=cyanets) a black-billed form of the "Arrenga" section, but this action is open to criticism and we now feel more than doubtful about melanura and cyaneus being forms of one species: the Sumatran form of cyaneus is more likely to be castaneus. 
Myophonus flavirostris (Horsf.).

Larger Whistling Thrush.

Myophonus flavirostris flavirostris (Horsf.).

Turdus flavirostris Horsfield, Trans. Linn. Soc. xiii, IS2I, P. I49: Java.

Myophomus metallicus Temm., P1. Col. 170,1823 : Java.

Distr.-Java.

Myophonus flavirostris dicrorhynchus Salvai.

Myophonus dicrorhynchus Salvadori, Ann. Mus. Civ. Gen. xiv, 1879, p. 227 : Padang Highlands, West Sumatra.

Distr.-Malay Peninsula (north to Patani).

Sumatra.

Myophonus flavirostris crassirostris Rob.

Mylophoneus crassirostris Robinson, Bull, Brit. Oru. Cl. xxv, Igro, 1. 99: Trang, Peninsular Siam.

Distr.-Malay Peninsula (south to Langkawi Islancis).

\section{Gents TURDUS Linn.}

Turdus javanicus Horsf.

Horsfield's 'Thrush.

Turdus javanicus javanicus Horsf.

Turdus Javanicus Horsfield, Trans. Linn. Soc. xiii, I82 I, p. I4S: Central Java, restr. Mt. Tjerimai.

Turdus migricrissus Bp., Compt. Rend, xxxviti, 1854, p. 6: Java.

Distr.-Java (central).

Turdus javanicus fumidus $S$. Müll.

Turdus (Merula) funidus S. Müller, Verh. Nat. Gesch. Land en Volk. 1843, p. $20 \mathrm{I}$ : Mt. Gede, West Java.

Turdus hypopyrrhus Hartl., Verz. Brem. Samml. 1844, p. 43: Java.

Turdus vulcanus Pelz., Reis Novara, Vögel, 1865, p. 70: Java.

Distr.-Java (west, pt.).

Turdus javanicus biesenbachi Stresm.

Turdus javanicus biesenbachi Stresemann, Orn. Monatsbr. xxxviii, 1930, P. 149: Mt. Paprancajan, West Java.

Distr.-Java (west, pt.).

Turdus javanicus whiteheadi (Seeb.).

Merula whitcheadi Seeboh111, Bull. Brit. Or1. C1. i, 1893, p. 25: Tosari, East Java.

Distr. - Java (east). 
Turdus javanicus indrapurae Rob. and Kloss.

Turdus indrapurae Robinson and Kloss, Journ. Straits Branch, Roy. Asiat. Soc. 73, 19r6, p. 277: Mt. Korinchi, Sunatra.

Distr. - Sumatra.

Turdus javanicus seebohmi (Sharpe).

Merula seebohmi Sliarpe, Ibis, I888, p. 386: Mt. Kinabalı, North Borneo.

Distr.-Borneo.

Turdus javanicus erythropleurus Sharpe.

Turdus erythropleurus Sharpe, Proc. Zool. Soc, I887, p. 515: Christmas Island.

Distr.-Christnas Island, Indian Ocean; Cocos-Keeling Islands (introduced).

Turdus obscurus Ginel.

Dusky 'Thrush.

Turdus obscurus obscurus Gin.

Turdus obscurus Gmelin, Syst. Nat. I, 1789, p. 8r6: Lake Baikal. Turdus rufulus Draj,. Dict. Class. Hist, Nat, $x, 1826$, p. 443: Java.

Turdus modestus Eyton, Proc. Zool. Soc. 1839, p. 103: Malacca.

Distr.-Malay Peninsula.

Sumatra; Billiton.

Boriteo.

Java.

Genus GEOKICHLA S. Müller.

Geokichla sibirica (Pall.).

Siberian Ground-Thrush.

Geokichla sibirica sibirica (Pall.).

Turdus sibiricus Pallas, Retse Russ, Reichs, iii, 1776, p. 694 * Dauria.

Distr.-Malay States.

Sumatra; Nias Island, West Sumatra.

Borneo.

Java.

Geokichla sibirica davisoni (Hume).

Turdulus davisoni Hume, Stray Featlers, v, $1877,1,63$ : Mt. Muleyit, Tenasserin.

Distr.-Malay States.

Sumatra. 
Geokichla interpres (Tensm.). Temminck's Ground-'Thrush. Geokichla interpres interpres (Temm.).

Turdus interpres Temminck, P1. Col. 458 , I $\delta_{2} \delta$; Java.

Dists.-Malay Peninsula.

Sumatra.

Borneo.

Java.

*Geokichla interpres leucolaema Salvad.

Geocichla leucolaema Salvadori, Ann. Mus. Civ, Gen. (2), xii, I892, p. 135 : Engano Island.

Distr.-Engano Island, West Sumatra.

Geokichla citrina (Latl,.). Grey-and-Orange Ground-Thrush.

Geokichla citrina citrine (Lath.).

Turdus citrinus Latham, Index Orn, i, 1790, p. 350: Cachar.

Geocichla innotota (sic) Blyth, Journ. Asiat. Soc. Bengal, xv, t84f, p. 370 : Malay Peninsula (South).

Dist. - Malay Peninsula.

Simatra.

Geokichla citrina aurata Sharpe.

Geocichla aurata Sharpe, Ibis, 1888, p. 478: Mt. Kinabahu, North Borneo.

Distr.-Borneo.

Geokichla citrina rubecula Gould.

Geocichla rubecula Gould, Proc. Zool. Soc. 1836 , p. 7 : Java.

Distr.-Java; Bali.

Geokichla everetti Sharpe. Everett's Ground-Thrush.

Geocichla everetti Sharpe, Ibis, 1892, p. 323 : Mt. Dulit, North Sarawak.

Distr.-Borreo.

Genus ZOOTHERA Vigors.

Zoothera andromedæ (Temm.).

Hyothera androneda Temminck, P1. Col. 392, IS26: Java.

Distr.-Sumatra; Engano Island, West Sumatra. Java (not east). 
Genus OREOCINCLA Gould.

Oreocincla dauma (Lath.).

Mountain-Thrush.

Oreocincla dauma dauma (Lath.).

Turdus Dauma Latham, Ind. Orn, I, I790, p. 362 : India.

Distr.-Malay States.

Oreocincla dauma horsfieldi Bp.

Oreocincla horsfieldi Bonaparte, Rev. et Mag. Zool. I857, p. 205 : Java.

Oreocincla horsfieldi affris Richmond, Proc. Biol. Soc. Wash. xv, 1902, p. 158: Trang, Peninsular Siam.

Distr.-Peninsulat Sian.

Sumatra,

Java.

Genus MONTICOLA Boie.

Monticola solitaria (Linn.).

Blue Rock-Thrush.

[Monticola solitaria solitaria (Linn.).

Turdus solilarius Linn., Syst. Nat. Ioth. ed. 1758, p. I70: Italy.

Distr.-Extra-limital.]

Monticola solitaria pandoo (Sykes).

Petrocincla pandoo Sykes, Proc. Zool. Soc. I\$32, p. $8_{7}$ : Ghauts, India.

Distr.-Malay Peninsula.

Sumatra.

Monticola solitaria philippensist (Y. L. S. Müll.).

Turdus Philippensis P. L. S. Müller, Natursystem, Anlang, 1776 , p. 142 : Philippine Islands.

Distr. -Malay Peninsula.

Borneo; North Natuna Islands.

Monticola gularis (Swinh.).

Swinhoe's Rock-Thrush.

Monticola gularis (Swinh.).

Oracetes gularis Swinhoe, Proc. Zool, Soc. I862, p. 3I8: Peking. Distr.-Malay Peninsula.

t We have used philippensis for those Malaysian birds with chestnut in the plumage, but all are "a/hnis" Blyth if this latter form is good (the "racial hybrids" of Lord Rothschild). The locality "Java" needs confrmation. 


\section{Family SYLVIIDÆ.}

\section{Genus LOCUSTELLA Kaup.}

Locustella ochotensis (Midd.). Middendorff's Grasshopper-Warbler.

*Locustella ochotensis ochotensis (Midd.).

Sylvia (Locuslclla) ochotensis Middendorff, Siberische Reise, ii, pt. $2,18_{53}$, p. $I 8_{5}:$ Siberia.

Distr.-Borneo.

Locustella certhiola (Pall.).' Pallas's Grasshopper-Warbler.

Locustella certhiola subspp.

Motacilla certhiola Pallas, Zoogr. Rosso-Asiat. i, IS27, p. 509 : Lake Baikal.

Calamodyta Doriae Salvad., Att. R. Accad. Sci. Torino, iii, 1868, p. 531: Sarawak.

Distr,-Malay Peninsula,

Sumatra.

Bormeo; South Natuna Islands.

Java (west).

Locustella lanceolata (Temm.). Streaked Grasshopper-Warbler. Sylvia lanceolata Temminck, Man. d'Orn. ed. 2, iv, 1840, p. 614: Russia.

Dist.r.-Malay Peninsula.

Sumatra.

Borneo.

Java (west).

Genus ACROCEPHALUS Natmann.

Acrocephalus stentoreus (Hempr. \& Ehrenb.) ${ }^{2}$ Great Reed-Warbler. [Acrocephalus stentoreus stentoreus (Hempr, and Ehrenb.).

Curruca stentorea Hemprich and Ehrenberg, Synb. Phys, Aves, fol. bb. ${ }_{1833}$ : Egypt.

Distr.-_[ixtra-limital.]

1 The only Malaysian specimens examined are from the Peninsula and the Natuna Islands. Dr. Stresemann has seen these and is tempted to call all minor (L. minor, Day, and Oust., Ois. Chine, 1877, p. 250: Peking) except one Malayan certhiola, Meise refers a Javan bird to $L$. $C$. sparsimstriata Meise (Abh. Mus. f. Tierk. Völk. Dres. xviij, 1934, 2, p. 39. West Siberia) and Sushkin records $L$. $c$. rubescens Blyth (Calcutta) from Borneo. Until author's are agreed on differentiation in the breeding areas we cannot go further.

: C 7 . Stres., Orn. Monatsber, xxxii, 1924, p. 167. 
Acrocephalus stentoreus orientalis (Temm. and Schleg.).

Salicaria turdina orientalis Temminck and Schlegel, Fauna Japon., Aves, I 847, p. 50 : Japan.

Distr.-Malay Peninsula; Anamba Islands.

Stmatra.

Borneo.

Java (west); Karimon-Java Islands.

*Acrocephalus stentoreus siebersi (SaI.).

Acrocephalus stentoreus siebersi Salomonson, Orn. Monatsbr. xxxvi, 1928 , 1). I 19 : West Java.

Distr.-Java (west).

Acrocephalus bistrigiceps Swinh.

Schrenck's Reed-Warbler. Acrocephalus bistrigiceps Swinhoe, Ibis, 1860, p. 51: Amoy.

Distr.-Peninsular Sian.

Genus ORTHOTOMUS Horsfield.

Orthotomus sutorius (Penn.).

Long-tailed Tailor-Bird.

[Orthotomus sutorius sutorius (I'enn.).

Molacilla sutoria Pennant, Indian Zool. 1769, pl. 7 : Cevlon.

Distr.-Eixtra-limital.]

Orthotomus sutorius maculicollis Moore.

Orthotomus maculicollis Moore, Proc. Zool. Soc. IS54, P. 309 : Malacca.

Orthotomus huegelit Pelz., Sitz. k. Akad. Wien, xxiv, 1857, P. 360 (fide Cat. Bds. : not seen).

Distr.-Malay Peninsula."

Orthotomus sutorius edela Temm.

Orthotoma edela Temminck, P1. Col. 599, fig. 2, 1836: Java.

Distr.-Java. ${ }^{2}$

Orthotomus atrogularis ${ }^{3}$ Temm.

Black-necked Tailor-Bird.

Orthotomus atrogularis atrogularis Temm.

Ortholomus atrogularis Temminck, P1. Col., livr. IOI, I836, text: Malacca,

Orthotomus Aavoviridis Moore, Proc. Zool. Soc. 1854, p. 79: Malacca.

Distr.-Malay Peninsula.*

Sumatra; Rlio Archipelago; Billiton.

Borneo (not north); North Natuna Islands.

- Birds from Peninsular Siam are $O$. sutorius $>$ maculicollis.

- Has been recorded from Sumatra, but we think in error.

$3 \mathrm{~A}$ form of $O$. atrogularis is said to occur in the Banjak Islands, West Sumatra, but no specimens seem to have been obtained.

+ Birds from the north of the Malay Peninsula are perhaps very slightly yellower above than those from the Malay States: they are alroguluris $>$ nitidus Hume (Tenasserim). 
Orthotomus atrogularis humphreysi Chas. and Kloss.

Orthotomus atrogularis humplireysi Chasen and Kloss, Journ. f. Ornith. I929, Band 2, p. I20: Bettotan near Sandakan, North Borneo.

Disir,-Borneo (norti1).

Orthotomus atrogularis major Chas. and Kloss.

Orthotonus atrigularis major Chasen and Kloss, Journ. Malay. Br. Roy. Asiat. Soc. vi, pt. 3, I928, p. 60 : Siantan Island, Anamba Islands.

Distr.-Tionan Istand; Anamba Islands.

*Orthotomus atrogularis eumelas Oberh.

Orthotomus atrogularis eumelas Oberholser, Suniths. Misc. Coll. 1xxvi, I923, p. 6 : Banka Island.

Distr. Banka Island.

Orthotomus sericeus Temm. ${ }^{1}$

Red-headed Tailor-Bird.

Orthotomus sericeus sericeus Tenm.

Orthotomus scriceus Temminck, Pl. Col. livr. ror, 1836 , text: Borneo.

Distr.-Borneo.

Orthotomus sericeus hesperius Oberh.

Orthotomus sericeus hesperius Oberholser, Bull. U.S. Nat. Mus. I59, I932, p. 89 : Lingga Island.

Distr.-Malay Peninstula.

Sumatra; Rhio and Lingga Archipelagos; Billiton.

Orthotomus sericeus rubicundulus Chas. and Kloss.

Orthotomus ruficeps rubicundulus Chasen and Kloss, Nov. Zool. Xxxvi, 1931, p. 279: Sirhassen Island, Soutl Natuna Islands. Distr,-South Natuna Islands.

Orthotomus sepium Horsf.

Ashy Tailor-Bird.

Orthotomus sepium sepium Horsf.

Orthotomus sepium Horsfield, Trans. Linn. Soc. xiii, I82I, p. I66: Java.

Distr.-Java; Bali.

r For this unfortunate, but necessary, change of name from $O$. rufceps which must be transferred to the bird formerly well-known as $O$. cineraceus, see Oberholser, Bull. U.S. Nat. Mus. 159, 1932, p. 87.

$$
\text { [246] }
$$


Orthotomus sepium ruficeps (L.ess.).

- Edela ruficeps Lesson, Traité d'Urt. I831, p. 309: Australia, error = Sumatra (Rob. and Kloss, 1923).

Orthotomus cineracens Blyth, Journ. Asiat. Soc. Bengal, xiv, 1845. p. 589 : Malacca.

Distr.-Malay Peninsula.

Sumatra; Rlio Archipelago; Banka.

Java (part); Bawean Island; Kangean Islands.

Orthotomus sepium palliolatusi Chas, and Kloss.

Orthotomus sepium palliolatus Clasen and Kloss, Treubia, xiv, I933, p. 170: Karimon-Java Island.

Disir.-Karinon-Java Islands.

Orthotomus sepium borneonensis Salvad.

Orthotomus borneonensis Salvadori, Ann. Mus. Civ. Gen. v, 1874 , p. 247 : Sarawak.

Distr.-Borneo; Billiton (? sulbsp.).

*Orthotomus sepium baeus Oberh.

Orthotomus cineraceus bacus Oberholser, Smitls. Misc. Coll. Ix, No. 7 , I91 2, p. 13: Nias Island.

Disir._Nias Island, West Sumatra.

Orthotomus sepium concinnus Riley.

Orthotomus sepium concinnus Riley, Proc. Biol. Soc. Wash. x1, 1927, p. 96 : Sipora Island.

Distr.-Siberut and Sipora Islancls, West Sumatra.

Orthotomus sepium ochrommatus Oberh.

Orthotomus cincraceus ochrommalus Oberholser, Smiths. Misc. Coll. 1x, No. 7, 1912, p. 13: Nortl Pagi Island.

Distr.-Pagi Islands, West Sumatra,

\section{Genus PHYLLERGATES Sharpe.}

Phyllergates cucullatus ('Temun.)."

Ashy-naped Warbler.

Phyllergates cucullatus cucullatus (Temm.).

Orthotomus cuculalus (sic) Temminck, P1. Col. 599, 1836: Java.

Phyllergates sumatranus Salvad., Ann. Mus. Civ. Gen. (2) xii, 1891 , p. 67: Sumatra.

Disir.-Sumatra.

Java; Bali.

- Bartels and Stresemann (Igzo) consider that the lowlands of the north coast of Java are occupied by "cineracers" $(=$ rufteeps): we have seen no specimens, but the race is not improbably palliolatus. Birds from Bawean and Kangean Islands also require re-examination.

a $C f$. Stres., Nov, Zool, xix, 1912, p. 341 ; Rob. and Kloss, Journ. F.M.S. Mus, viii (pt. 2), 1918, p. 219 . 
Phyllergates cucullatus cinereicollis Sharpe.t

Phyllergates cinereicollis Sharpe, Ibis, I888, p. 479: Mt. Kinabalu, North Borneo.

Distr.-Malay States.

Borneo.

Phyllergates cucullatus thais Rob. and Kloss.

Phyllergates cucullatws thais Robinson and Kloss, Journ. Fed. Mal. States Mus, xi, 1923, p. 56: Nakon Sritamarat, Peninsular Sian.

Distr,-Peninsular Siam.

\section{Genus CISTICOLA Kaup. ${ }^{2}$}

Cisticola juncidis (Rafin.). Streaked Fantail Warbler.

[Cisticola juncidis juncidis (Rafin.).

Sylvia juncidis Rafinesquue, Caratteri Anim. d. Scilia, ISIO, 1.6

Sicily.

Distr.-Eixtra-limital.]

Cisticola juncidis malaya Lỹues.

Cisticola juncidis malaya Lynes, Ibis, I930, suppl. p. 92: Klang, Malay Peninsula.

Distr.-Malay Peninsula,

Sumatra; Billiton; Simalur Island, West Sunatra.

Java (west).

Cisticola juncidis fuscicapilla Wall.

Cisticola fuscicapilla Wallace, Proc, Zool. Soc. 1863, p. 489 : Tinor. Distr._Java (east); Bali; Kangean Islands.

Cisticola exilis (Vig. and Horsf.). Red-headed Fantail Warbler. [Cisticola exilis exilis (Vig. and Horsf.).

Malturus exilis Vigors and Horsfield, Trans. Linn. Soc. xv, I\$27, p. 223 : New South Wales.

Distr.-Extra-limital.]

Cisticola exilis lineocapilla Gould.

Cysticola lineocapilla Gould, Proc. Zool. Soc. 1847, p. I : Northeru Territory, Australia.

Cisticola delicalula Blyth, Ibi5, 1870, p. 170: Java,

Distr.-Java (east); Bali.

- Purely from a geographical point of view the division of the southern forms of this species here proposed is a little unsatisfactory, but the skins examined seem to fall into the above arrangement, cinereicollis being very slightly paler on the grey hind-neck and the green mantle than cucullatus. The distinctions, however, are very fine.

Revision, Lynes, Ibis, 1030, suppl. no. 
Genus FRANKLINIA Jerdon.

Franklinia rufescens (Blyth).

Wren-Warbler.

Franklinia rufescens rufescens (Blytis).

Prinia rufescens Blyth, Journ, Asiat. Soc. Bengal, xvi, r847, 1). 456: Arracan.

Distr.-Malay Peninsula.

\section{Genus MEGALURUS Horsfield.}

Megalurus palustris Horsf.

Striated Marsh-Warbler.

Megalurus palustris palustris Horsf.

Megalurus palustris Horsfield, Trans. Linn. Soc, xiii, 1821, p. 159 : Java.

Malurus marginalis "Reinw."-Temm. Pl. Col. 65, fig. 2, 1822: Java. Megalurus citrinus Gray, Gen. Birds, i, 1848, p. 162, pl. 48: Java.

Turdus takko Horsfield and Moore, Cat. Bds. Mus. E. I. Coy., i, 1854 , p. $330:$ nom. nud. as syn.

Distr,-Java; Bali.

\section{Genus BRADYPTERUS Swainson.}

Bradypterus montis (Hart.).

Doherty's Warbler.

Stasiasticus montis Hartert, Nov. Zool, iii, I896, p. 540: Mt. Ardjuno, East Java.

Distr,-Java.

\section{Genus PHRAGAMATICOLA Jerdon.}

Phragamaticola aëdon (Pall.).

Thick-billed Warbler.

[Phragamaticola aëdon aëdon (Pall.).

Muscicapa Aëdon Pallas, Reise Prov. Russ, Reichs. iii, 1776, p. 695 : Dauria.

Distr.-Extra-limital.]

Phragamaticola aëdon rufescens Steg.

Pliragamaticola aëdon rufescens Stegmann, Journ. f. Orn. Ixxvii, 1929, P. 230 : East Siberia.

Distr.-Peninsular Siant.'

- Birds examined from various parts of Siam are all this small form. 


\section{Genus PHYLLOSCOPUS Boie.}

Phylloscopus borealis (Blas.).

Arctic Willow-Warbler,

Phylloscopus borealis borealis (Blas.).

Phyllopneuste borealis Blasius, Nammannia, 1858, p. 313: Lake Ochotska.

Distr,-Malay Peninsula.

Sunatra; Rhio Archipelago; Anamba Islands; Nias Island, West Sumatra.

Borneo; Natuna Islands.

Java; Karimon-Java Islands.

\section{Phylloscopus borealis examinandusi Stres.}

Phylloscopus borealis examinandus Stresemann, Nov. Zool. xx, r913, p. 353 : Bali.

Distr.-Bali.

\section{Phylloscopus borealis xanthodryas Swinh.z}

Phyllopneuste xanthodryas Swinhoe, Proc. Zool. Soc. I863, P. 296 ;

Anoy.

Distr.-? Sumatra.
Borneo.
Java.

Phylloscopus nitidus Blyth.

Green Willow-Warbler.

[Phylloscopus nitidus nitidus Blyth.

Phylloscopus nitidus Blyth, Journ. Asiat. Soc. Bengal, xii, r 843 , p. 965: Calcutta.

Distr._Extra-linital.]

\section{Phylloscopus nitidus plumbeitarsus Swinl.}

Phylloscopus plumbeitarsus Swinhoe, Ibis, I86r, p. 330: between Taku and Peking.

Distr.-Peninsular Sian.

Phylloscopus tenellipes Swinh. ${ }^{3}$

Pale-legged Willow-Warbler.

Phylloscopus tenellipes Swinloe, Ibis, I860, p. 53: Amoy.

Distr.-Peninsular Sian.

1 C. B. Ticehurst (in litt.) "to me not recognizable".

Some of our own previous records of xanthodryas from various parts of Malaysia, and those of some other authors, we now consider as very doubtful, for it appears that the character of a relatively large first primary will not always separate it from $b$. borealis. We have, however, seen birds from Borneo quite clearly belonging to zanthodryas.

3 Hume has recorded $P$. magnirostris Blyth from near Malacca, but as the species has not again been obtained we have omitted it from the list pending confirmation. 
Phylloscopus occipitalis (Blyth). Larger Crowned Willow-Warbler. [Phylloscopus occipitalis occipitalis (Blyth).

Phyllopneuste occipitalis Blyt 1 , Journ. As. Soc. Beng. xiv, 1845 , p. 593: South India.

Distr.-Extra-linital.]

Phylloscopus occipitalis coronatus (Temm, and Schleg.).

Ficedula coronata Tentminck and Schlegel, Fauna Japon., Aves, 1847, p. 48, pl. 18: Japan.

Distr.-Malay Peninsula.

Sumatra.

Java.

Phylloscopus inornatus (Blyth).

Crowned Willow-Warbler.

Phylloseopus inornatus inornatus (Blyth).

Regulus inornatus Blyth, Journ. Asiat. Soc. Bengal, xi, J842, 1 . I91 : India.

Distr.-Malay Peninsula.

\section{Gents SEICERCUS Swainson.}

Seicercus trivirgatus (Strick1.). Green Flycatcher-Warbler.

Seicercus trivirgatus trivirgatus (Strick1.).t

Phylloscopus trivirgatus Strickland, Contrib. Orn. 1849 , p. I23, tab. 34, p. I 23 : Java.

Distr.-Sumatra.

Borneo.

Java; Bali.

Seicercus trivirgata parvirostris (Stres.).

Phylloscopus trivirgatus parvirostris Stresemann, Nov. Zool. xix, Igr2, p. 322 : Mt. Tahan, Malay Peninsula.

Distr.-Malay States.

Seicercus trivirgatus kinabaluensis (Sharpe) :

Cryplolopha kinabaluensis Sharpe, Bull. Brit. Ornith Cl. ii, Igot, p. 60: Mt. Kinabalu, North Borneo.

Distr.-Mt. Kinabalı, North Borneo.

* We have very large serjes of this bird from Malaysia but are still at little doubtful about the name of the Sumatran race : it is really intermediate with a leaning to the Javan form on balance of characters. In Sarawak a form very near to trivirgatus and quite distinct from kinabaluensis occurs. It seems likely that further material will show it to be rather brighter than typical trivirgatus.

a Diverse opinions have been published about this form. I have now seen about one-hundred birds from Mt. Kinabalu. Normally, kimbaluensis is a rather dull, olive-green race, much duller when in worn plumage and always very different from typical trivirgatus. At the top of the mountain I collected birds quite grey in colour, without a trace of yellow, or green in the plumage. The question is complicated by the fact that rare mutants (not merely birds in very fresh plumage) occur which in colour are inseparable from $S$. $t$. tirvirgatus. 
Seicercus castaniceps (Blyth). Chestnut-headed Flycatcher-Warbler. [Seicercus castaniceps castaniceps (Blyth).

Abrornis castaniceps Blyti, Journ. Asiat. Soc. Beng, xiv, pt. Z, r845. p. 593 : Nepal.

Distr._Extra-linital.]

Seicercus castaniceps youngi (Rob.).

Cryplolopha youngi Robinson, Journ. Fed. Mal. States Mus. r, 1915. P. 100: Bandon, Peninsular Star1.

Distr.-Peninsular Siam.

Seicercus castaniceps muelleri Rob. and Kloss.

Cryptolopha muelleri Robinson and Kloss, Journ. Straits Branch, Roy. Asiat. Soc. No. 73, 1916, p. 278 : Korinchi, West Sumatra.

Distr.-Sumatra.

Seicercus castaniceps butleri (Hart.).

Cryplolopha butleri Hartert, Bull. Brit. Orı. Cl. vii, 1898, p. 50 : Mt. Ijaı, Perak.

Distr.-Malay States.

Seicercus grammiceps (Strickl.). Sunda-Island Flycatcher-Warbler.

Seicercus grammiceps grammiceps (Strickt.).

Pycnosphrys grammiceps Strickland, Contr. Or1k. 1849, p. I24: Java.

Sylvia lencorrhoa Blyth, Lhis, 1870, p. 160: Java.

Distr.-Java; Bali.

Seicercus grammiceps sumatrensis (Rob. and Kkloss).

Cryplolopha sumalrensis Robinson and Kloss, Jotrn. Straits Branch, Roy. Asiat. Soc. No. 73, 1916, p. 277 : Korinchi, West Sumatra. Distr.-Stumatra.

Seicercus montis (Sharpe). Yellow-breasted Flycatcher-Warbler. Seicercus montis montis (Sharpe).

Cryplolopha montis Slarpe, Ibis, I 887 , p. 442 : Mt. Kinabalu, North Borneo.

Distr.-Borneo.

Seicercus montis davisoni (Sharpe).

Chryptolopha davisoni Sharpe, Proc. Zool. Soc. IS88, p. 271 : Perak, Malay Peninsula.

Disir.-Malay States. 
Seicercus montis inornata (Rob. and Kloss).

Cryptolopha montis inomata Robinson and Kloss, Journ. Straits Br.

Roy. Asiat. Soc. No. Si, 1920, p. 99 : Korinchi, West Stumatra. Distr.-Simatra.

\section{Genus ABROSCOPUS Stuart Baker.}

Abroscopus superciliaris (Blyth). Bamboo Flycatcher-Warbler. Abroscopus superciliaris superciliaris (Blytli).

Abrornis superciliaris Blyth, Journ. Asiat. Soc. Beng. xxviii, IS59, p. 414: Tenasserin.

Distr.-Peninsular Siann."

\section{Abroscopus superciliaris schwaneri (Blyth).}

Abrornis schwaneri Blyth, Ibis, I870, p. $169:$ Borneo.

Abronis sakaionum Stresm., Bull. Brit. Orn. Cl. xxxi, 1912, p. 27 : Perak -Pahang boundary, Malay States.

Distr.-Malay States.

Sumatra.

Borneo.

Abroscopus superciliaris vordermani (Büttik.).

Cryptolopha Vordermani Büttikofer, Notes Leyd. Mus. xv, 1893 . p. 260 : East Java.

Distr.-Java.

\section{Genus CETTIA Bonaparte.}

Cettia montana (Horsf.).

Bush-Watbler.

Cettia montana montana (Horsf.).

Sylvia monlana Horsfield, Trans. Linn. Soc, xiii, IS2I, p. I56: Java,

Sylvia vulcania Blyth, Ibis, 1870, p. 170: Java (nom. nud.).

Distr.-Java; Bali.

Cettia montana sumatrana 0.-Grant.

Cettia sumatrana Ogilvie-Grant, Bull. Brit. Orn. Cl. xxxvi, I9ı6, p. 66 : Korinchi, West Sumatra.

Distr.-Suntatra (not north).

Cettia montana sepiaria Kloss.

Cettia montana sepiaria Kloss, Tretbia, xiii, I931, p. 352 : Acheen, North Sumatra.

Distr.-Sumatra (nortli).

- The birds I here call $A, s$, superciliaris are like schwaneri in colour but larger: they are therefore perbajs not true superciliaris, but intermediate. or an undescribed race. 
Cettia montana oreophila Sharpe.

Cettia oreophila Sharpe, Ibis, I888, p. 387 : Mt. Kinabalı, Nortl Borneo.

Distr.-Borneo (part).

Cettia montanus banksi Chas.

Cellia montanus banksi Chasen, Ornith. Monatsber. 1935, P. 147 :

Mt. Mulu, N. Sarawak.

Distr.-Borneo (part).

\section{Genus UROSPHENA Swinhoe.}

Urosphena squameiceps (Swinh.). Swinhoe's Reed-Warbler. Urosphena squameiceps squameiceps (Swith.).

Tribura squaneiceps Swinhoe, Proc. Zool, Soc. 1863, p. 292 : Canton.

Distr.-Peninsular Siam.

\section{Genus SUYA Hodgson.}

Suya atrogularis Horsf. and Moore.

Hill-Warbler.

[Suya atrogularis atrogularis Horsf. and Moore,

Suya alrogularis Horsfield and Moore, Cat. Birds Mus. East Ind. Coy. i, I854, p. 326 : Darjeeling.

Distr.-Extra-limital.]

Suya atrogularis albogularis Hume.

Suya albogularis Hume, Stray Feathers, i, I873, P. 459: Acheen, Sumatra.

* ? Burnesia dysancrita Oberh., Smiths. Misc. Coll. 1x, No. 7, 1912, P. 14 * Loh Sidoh Bay, North-west Sumatra.

Distr.-Sumatra.

Suya atrogularis waterstradti Hart.

Suya waterstradti Hartert, Nov. Zool. ix, 1902, D. 568: Mt. Tahan, Malay States.

Distr.-Malay States.

\section{Genus PRINIA Horsfield.}

Prinia inornata Sykes.

Wren-Warbler.

[Prinia inornata inornata Sykes.

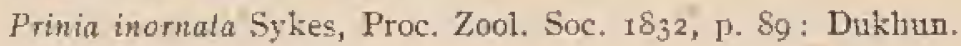

Distr.-Extra-limital.] 
Prinia inornata blythi (Bp.).

Suya blythi Bonaparte, Consp. Gen. Aviun, i, I850, p. $28 \mathrm{r}$ : Java. Distr,-Java.

Prinia polychroa ('Temm.). Javan Wren-Warbler. Malurus polychrous Temminck, Pi. Col. 466, IS28: Java.

Distr.-Java.

\section{Prinia familiaris Horsf.}

Bar-winged Wren-Warbler.

Prinia familiaris familiaris Horsf.

Prinia familiaris Horsfield, Trans. Linn. Soc. xiii, I821, p. 165 : Java, restr. Province of Besoeki (Kloss, I93I).

Orthotomus prinia Temm., P1. Col. text to pl, 599, 1836 , Java.

Distr._Java (east); Bali.

Prinia familiaris olivacea (Raft.).

Motacilla olivacea Raftes, Trans. Linu. Soc. xiii, $\mathrm{I}_{22}$, p. $3 \mathrm{I}_{3}$ : Sumatra.

Distr.-Stumatra. Java (west); ? Karimon-Java Islands.

Prinia flaviventris (Deless.). Yellow-bellied Wret-Warbler. [Prinia flaviventris flaviventris (Deless.).

Orthotomus flavinentris Delessert, Rev. Mag. Zool. I840, p. IOI : Bhutan.

Distr.—Extra-limital.]

\section{Prinia flaviventris rafflesi Tweed.}

Prinia raffesi Tweeddale, Ibis, 1877, p. 3II, pl. vi, fig. I : Lampongs, South Sumatra.

Prinia Aypoxantha Salvad., Ann. Mus. Civ. Gen. :siv, 1879 , p. 235 : Sumatra.

Distr.-Malay Peninsula.

Sumatra. Java.

\section{Prinia flaviventris supereiliaris Salvad.}

Prinia superciliaris Salvadori, Ann. Mus. Civ, Gen. v, IS74, p. 249 : Sarawak.

Distr.---Borneo.

Prinia flaviventris halistona (Oberh.).

Burnesia dysancrita halistona Oberholser, Smitlis. Misc. Coll. Ix, No. 7,1912, p. I4: Nias Island.

Distr.-Nias Island, West Sunatra. 


\section{Family ARTAMID $Æ$.}

Genus ARTAMUS Vieillot.

Artamus leucorhynchus (Lil11.)."

Swallow-Shrike.

Artamus leucorhynchus leucorhynchus (Litni.).

Lanius leucoryn. Linn., Mant. Plant. 1771, p. 524: Manila, Philippine Islands.

Artamus leucoryn. macroterus Oberholser, Butl. U.S, Nat, Mus, 159, 1932, D. 53 : Sirbassen Island, Natuna Islands.

Distr.-Borneo; North Bornean Islands; Maratua Islands; Mantanani Island; Natma Islands.

Artamus leucorhynchus amydrus Oberh.

Arlanus leucoryn. amydrus Oberholser, Proc. U.S. Nat. Mus. liv, 1917, p. I85: Solombo Besar Island, Java Sea.

Distr.-Sumatra; Banka; Billiton; Mendanao.

Java; Bali; Kangean Islands; Solombo Besar Island; Katinon-Java Islands.

\section{Family LANIID正. \\ Gemts HEMIPUS Hodgson.}

Hemipus hirundinaceus (Temm.). Black-winged Pied Shrike. Muscicapa hirutdinacea "Reinw." 'Temminck, Pl. Col. II9, 1823: Java.

Muscicapa obscura Horsf. (not Gmelin, 17\&g), Trans. Linn. Soc. xiii, 1821, p. 146 : Java.

Distr.-Malay Peninsula.

Sumatra; Rhio and Lingga Archipelagos; Nias; Billiton.

Borneo; Banguey Island.

Java; Bali.

Hemipus picatus (Sykes).

Bar-winged Pied Shrike.

Hemipus picatus picatus (Sykes).

Muscicapa picala Sykes, Proc. Zool. Soc. $18_{32}$, p. 85 : Dukhun.

Hemipus intermedius Salvad, Ann. Mus. Civ. Gen. xiv, 1879, p. 209: Sumatra.

Distr.-Malay Peninsula.

Sumatra.

Borneo.

'Ct. Stres, Nov, Zool. xx, 1913, p. 289. 


\section{Genus TEPHRODORNIS Swainson.}

Tephrodornis gularis (Raff.).

Wood-Slrike.

Tephrodornis gularis gularis (Raffl.).

Lanius gularis Raffles, 'Trans. Limn. Soc. xiii, I822, p. 304 : Bencoolew, West Sumatra.

Lanius virgatus Temm., P!. Col. 256 , fig. i, 1824 : Java.

bistr.-Sumatra (sonth-west coastlands).

Java.

Tephrodornis gularis fretensis Rub. ant kiloss.

Tephrodornis gularis fretensis Robinson and Kloss, Journ. Straits Branch koyal Asiat. Soc. 81, I920, p. sog: Negri Sentbilan, Malay States.

Wistr.-Malay states."

Sumatra (except south-west).

Tephrodornis gularis annectens Rob. and Kloss.

Tephrodornis pelvica annectens Robinson and Kiloss, Journ. F.M.S. Mus. viii, pt. 2, I918, [1, 222: 'Trang, Peninsular Sian.

Distr.-Peninsular Sian.

Tephrodornis gularis frenatus Bïttik.

Teprodomis frenalus Büttikofer, Notes Leyd. Mus. ix, 1887, p. 53 : Botneo.

Distr.-Borneo.

Genus PLATYLOPHUS Swainson.

Platylophus galericulatus (Cuv.).

Jay-Shrike.

Platylophus galericulatus galericulatus (Cuv.).

Lanius galoriculalus Cuvier, Règne Anim. i, I\$工7, p. 399: Java.

Lanius scapulatus Licht, Verz. Doubl. 1823, p. 49 : Java.

Vanga cristata Griff., An. Kingd, Birds, i, pl, to p. 486, 1829 : Java,

Distr.-Java.

Platylophus galericulatus ardesiacus (Bp.).

Lophocilla ardesiaca Bonaparte, Consp. Avium, i, 1850, p. 374 :

"Java?" error = Malacca (Robinson and Kloss, 1924).

Distr.-Malay Penizsula.

The form found in Patani, Soutb Peninsular Siam is perhaps nearer to fretensis than to annecters. 
Platylophus galericulatus coronatus (Kaftl.).

Lanius coronalus Raffles, Trans. Linn, Soc. xiii, 1822, p. 306 : Stuntatra.

Lophocitha histrionica Bp., Proc. ZooT. Soc. 1850, D. 79 : Sumatra.

Garrulus rufulus Schl., Handl. Dierk. i, 1857, p. 327 : Sumatra.

Dislr. - Sunatra.

Borneo (not north).

Platylophus galericulatus lemprieri Nich.

Plalylophus lemprieri Nicholson, Ibis, ISS3, p. 88 : Sandakan Bay, North Borneo.

Distr,-Borneo (north).

\section{Genus PITYRIASIS Lesson.}

Pityriasis gymnocephala ('Temun.).

Bald Slirike.

Barita gymnocephala Tentuninck, P1. Col. 572, IS35: Borneo.

Distr.-Borneo.

\section{Genus CROCIAS Temminck.}

Crocias guttatus Te1mits.

Spotted Sbrike.

Crocias gullatus Temminck, P1. Col. 592, I836: Java.

Laniellus lewcogrammicus Swains., in Rich., F. Bor.-Amer. (2), 1832, p. 481 : nom. nud.

Distr.-Java.

\section{Genus LANIUS Lintn.}

\section{Lanius tigrinus Drap.}

Thick-billed Shrike.

Lanius tigrinus Drapiez, Dict. Class. Hist. Nat. xiii, IS28, P. 523 : Java.

Lanius ferox Drap., Dict. Class. Hist. Nat. xiii, I828, p. 523 : Java.

Lanius strigalus Eyton, Proc. Zool. Soc. 1839, p. 103: Malacca.

Enneoclonts crassirostris Bp., Consp. Gen. Avium, 18550, p. 362 : Java (nom. nud.).

Distr-Malay Peninsula; Ananba Islands.

Sunatra; Rhio Archipelayo; Mentavi Islands and Simalur, West Sumatra.

Borneo.

Java. 
Lanius cristatus ${ }^{3}$ Linn.

Brown Shrike.

Lanius cristatus cristatus Linn.

Lanius cristatus Linn, Syst. Nat. Ioth. ed. 1758, 1. 93 : Bengal.

Distr.—Malay Peninsula; Anaruba Islands.

? Stumatra.

13ot 11eo.

Lanius cristatus lucionensis I,inn.

Lanius lucionensis Linn., Syst. Nat. Izth, ed, i, 1766, 1) 135: Lizon, Philippine Islands.

Enneoctonus schwanteri Bp., Consj. Gen, Avium, i, $1850,12.363$ : Borneo.

Distr-Malay States.

Sumatra.

Borneo.

\section{Lanius cristatus superciliosus Lath.}

Lanius superciliosus Lathatn, Ind. Om, Supp]. 180r, p. 20: Java.

Distr.-Malay Peninsula; Ananba Islands.

Sumatra.

Borneo.

Java; Bali; Karimon-Java Islanls.

\section{Lanius cristatus confusus Stegm.}

Lanius cristatus confusus Stegmann, Journ. f. Orn, 1929, p. 248 : Anurland.

Distr.-Malay Peninsula.

Lanius schach Linin.

Grey-lacked Shrike.

[Lanius schach schach Linn.

Lanius schach Linn., Syst. Nat, Ioth. ed. 1758, 1. 94 : China.

Dislr.-Eixtra-linital.]

\section{Lanius schach bentet Horst.}

Lauius Bentet Horsfield, Trans. Linn. Soc. xiii, IS2I, p. I44 : Java.

Lanius pyrrhonotus Vieill, and Oudart, Gal. des Ois. i, 1825 , p. 219 , pl. 135: "Grandes Indes" = Java (Mathezus, 1930).

Lanius macrourus Cuv., fide Less., Traité d'Orn. 1831, p. 372: Java.

Lanius schach tosariensis Kuroda, Tori, vi, 1930, p. 76: Tosari, East Java.

Distr,-Malay states.

Sumatra.

Java; Bali; Kangean Islands.

Keview : Steg., Orn, Monatsber. xxxviii, 1930, p. 115. Borneo.

- There seems to be no reliable record of Lanius schach bentet from 
Lanius nasutus (Scop.).

Philiprine Grey-backed Shrike.

Lanius nasutus nasutus Scop.

Lanius nasulus Scopoli, Del. Flor. Faun. Itısubr, ii, 17 $\$ 6$, p. $S_{5}$ : Panay, Philippine Islands.

Distr.-Borneo (north).

\section{Geun PACHYCEPHALA Vigors.}

Pachycephala pectoralis (Lath.). Thick-liead, or Golden Whistler. [Pachycephala pectoralis pectoralis (Lath.).

Muscicapa pectoralis Latham, Index Orn. Suppl. ISUI, p. 5I : New Soutl Wales.

Distr.-Extra-linnital.]

Pachycephala pectoralis javana Hart.

Pachycephala pectoralis javana Hartert, Bull. Brit. Ort1. Cl. xlviii, 1928, p. 88: Mt. Ardjuno, East Java.

Distr,-Java (east); Bali.'

Pachycephala cinerea (Blyth) : Mangrove Flycatcher-Shrike.

[Pachycephala cinerea cinerea (1ilyth) .

Muscilrea cinerea Blytl, Journ. Asiat. Soc. Bengal, xvi, IS47, p. 122: Island of Ranuree, Arracan.

Disir.-Extra-linuital.]

Pachycephala cinerea butaloides Stres.

Pachycephala grisola bulaloides Stresemantn, Treubia, xi, 1929, p. 135: Bolang, West Java.

Muscicapa philomela Cab., Arch. f. Naturg., ii, 1847, P. 322: Java (nom. nud.).

Distr.-Malay Peninsula.

Sumatra; Rhio Archipelago; Mentawi Islands, W. Sunatra. Borneo (not north); Karimata Islands.

Java; Bali; Kangean Islands; Karimon-Java Islands.

Pachycephala cinerea secedens Stres.

Pachycephala grisola secedens Stresemann, Now. Zool. xx, Igr3, p. 355 : Sirhassen Island, South Natuna Islands.

Distr.-Borneu (north); Nortl Bornean Islands; Maratua Islands; Natuna Islands; Karimata Islauds.

- Rensch considers that the Bali bird is P. p. javana fulwotincta Wall. (Flores).

a This is a most difficult bird to understand and in spite of good series in out possession the racial divisions here adopted can only be regarded as tentative: $c f$. Chas, and KI., Bull. Raff. Mus. 4, 1030, p. 50.

3 We do not see how Tephrodornis grisola Blyth (Calcutta) can be applied to this bird. 
*Pachycephala cinerea nesiotis (Oberts.).

Muscitrea grisola nesiotis Oberholser, Smiths. Misc. Coll. Ix, no. 7 , I912, P, I I : Simalur Islaud.

Dislr.-Simalur Island, West Sumatra.

*Pachycephala cinerea vandepolli Finsch.

Pachycephala Vandepolli Finsch, Notes Leyden Mus. xx, ISg9,

p. 224 : 'Tello Island, Batu Islands.

Distr.-Batu Islands, West Sumatra.

Pachycephala hypoxantha (Sharpe). Kinabalu Filycatcher-Shrike.

Pachycephala hypoxantha hypoxantha (Shatpe).

Hylolerpe hypoxantha Sharpe, Ibis, IS87, p. 45I: Mt. Kinabalu, North Borneo.

Distr.-Borneo (part).

Pachycephala hypoxantha sarawacensis Chas.

Pachycephala hypotantha sarazacensis Chasen, Bull. Raffles Mus.

10, 1935, 1. 44 : Mt. Poi, Sarawak.

Dislr.-Borneo (mountains of West Sarawak).

Family PARIDA.

\section{Geuus MELANOCHLORA Lessoll.}

Melanochlora sultanea (Hodgs.).

Sultan-Tit.

[Melanochlora suitanea sultanea (Hodgs,).

Panus sultaneus Hodgson, Indian Review, 1836, [. 31: Nepal.

Distr.-Lixtra-Linital.]

Melanochlora sultanea flavocristata (Lafr.).

Parus Alasocristatus Lafresnaye, Mag. Zool. C1. 2, I\&37, [. ISo:

"Isles de la Sonde" = Sumatra (Rob. and Kloss, 191S).

Melanochlora sumatrana Less., Rev. Mag. Zoot. I\$39, p. 42: Sumatra. Distr.-Malay Peninsula.

Stunsatra.

\section{Gent1s PARUS Lintı.}

Parus major Linu.

Great Tit.

[Parus major major Lint.

Parus major Linn., Syst. Nat. Iotlı. ed. I758, p. IS9: Swederl, Eturope.

Distr.- Extra-limital.]

Parus major cinereus Vieill.

Parus cinereus Vieillot, Nov. Dict. d'Hist. Nat. ed. 2, $\mathbf{x x}$, I8I8, p. 316 : Batavia, West Java.

Parus atriceps Horsf., Trans, Linn. Soc, xiit, 182:, p. 160: Javi.

Distr.-Javi; Bali. 
Parus major ambiguus (Raffs.).

Turdus ambiguns Raffies, 'Trans. Linn, Soc. xiii, IS2I, p. 313: Sulnatra.

Parus major malayorum Robinson and Kloss, Journ. Fed. Malay States Mus. viii, pt. 2, 1918, p. 226: Korinchi, West Sumatra.

Distr,-Malay Peninstla. Sunatra.

Parus major einerascens Slater.

Parus cinerascens H. H. Slater, Ibis, 1885 , p. 122, pl. 4: Sarawak. Parus saranacensis H. H. Slater, Ibis, 1885, p. 327 : new name only. Distr.-Borneo.

Genus PSALTRIA 'Temminck.

Psaltria exilis Tennun. Pygny Tit.

Psallria exilis Temminck, P1. Col. 600, 1836 : Java.

Distr.-Java.

\section{Family SITTID必.}

\section{Genus SITTA Linn, ${ }^{1}$}

Sitta frontalis (Swains.).

Velvet-fronted Nuthatch.

Sitta frontalis frontalis (Swains.).

Orthorhynchus frontalis Swainson, Zool. I11, i, 1820, pl. 2: Ceylon. Sitta frontalis Hotsf., Trans. Linn. Soc. xiii, $182 \mathrm{r}$, p. 162 : Java.

Sitta velata Temm., Pl. Col. $72,182 \mathrm{I}$ : Java.

Sitta frontalis hageni Parrot, Abh. Kon. Bay. Akad. Wiss. xxiv, 1907, p. 244 : Banka.

Distr.-Peninsular Sian (north). Sinnatra (south); Lingga; Banka. Java.

Sitta frontalis saturatior Hart.

Silta fromlalis saluratior Hartert, Nov, Zool. ix, 1902, p. 573: Mt, 'Tahan, Malay States.

Disir.-Peninsular Sian (south); Malay States. Sunatra (north).

Sitte frontalis corallipes (Sharpe).

Dendrophila corallipes Sharpe, Ibis, 1888, p. 479: Mt. Kinabalu, North Borreo.

Distr--Borneo; Maratua Islands, Last Borneo.

" Sitta longirostra Latb., Index Orn. 1790, p. 264 (Batavia) is a puzzle. It may be the earliest name for the bird now known as Sitta frontalis, but the description, taken from a drawing, is not good and the size foo large. The description fits Tephrodornis gularis fairly well, but Latham surely knew a nuthatch! 
Sitta azurea I.ess.

Bhue Nuthateh.

Sitta azurea azurea Less.

Sitta azurea Lesson, Traité d'Orn. $1 \$_{30}$, 1. 316: Java (Hart., I9 I4; restr. Last Java, Mt. Ardjuno, Rob, and Kloss, 1010).

Dendrophila Aavipes Swains, Anim. in Menag. ${ }^{8} 8_{37}$, p. 323: India, errot $=$ Java, $^{1}$

Dendrophila gymuophrys Reichnb., Handb. Scans. 1853. 1. 154: Java.

Distr.-Java (east).

Sitta azurea nigriventer (Rob. ancl Kloss).

Poliosilta azurea nigriventer Robinson and Kloss, Bull. Brit. Orn. Cl. $\mathrm{xl}$, rg19, p. I7 : Mt. Gede, West Java.

Distr.-Java (west).

Sitta azurea expectata (Hart.).

Callisilla azurea expectala Hartert, Bull. Brit. Orn. Cl., xxxv, r9I4, p. 34: Pahang, Malay States.

Distr. -Malay States.

Sunnatra.

\section{Family ZOSTEROPIDA.}

Genus ZOSTEROPS Vigors and Horsfield. ${ }^{3}$

\section{Zosterops palpebrosa ('Temini.).4}

White-eye.

[Zosterops palpebrosa palpebrosa ('Temm.).

Sylvia palpebrosa Temminck, P1. Col, 293, fig. 3, 1824: India.

Distr._Extra-limital.]

Zosterops palpebrosa williamsoni Rob. and Kloss.5

Zosterops palpebrosa williamsoni Robiuson and Kloss, Jouru. Nat.

Hist, Soc, Siam, iii, 1919, p. 445: Meklong, Central Sian.

Distr.-Malay Peninsula (lowlands).

Rhio Archipelago.

- Mathews, 1030, restricts the type locality to Timor, but the species seems nat to occur there.

${ }^{3}$ Revision: Stres., Mitt, Zool. Mus. Berl. xvii, 193!, P. 201.

3 In addition to the species listed Zosterops japonica simplex Swinh., occurs on Singavore Island : we have handled locally shot specimens in the flesh and have also seen it in the Botanic Gardens. We regard these-birds as introduced sjecimens. The species is imported in large numbers.

4 There seems no reason why the Malaysian forms of Zosterops, variously referred to the "species" palpebrosa and aureivenler should not be grouped in a species. Broadly speaking, the grey-bellied "aureiventer" birds are mountain forms, whereas the pale-bellied palpebrosa forms live in the lowiands.

$s$ It is just possible that birds from the south of the Malay Peninsula should be separated from true williamsoni. 
Zosterops palpebrosa aureiventer Hume.

Zosterops aurcivenler Hume, Stray Feathers, vi, 1878, p. 519: Tavoy.

Zosterops tahanensis O. Grant, Bull. Brit. Orn. Cl. xix, 1906, p. 10: Mt. Tahan, Malay States.

Distr.-Malay Peninsula (nountains).

Zosterops palpebrosa sumatrana Kloss.

Zosterops palpebrosa sumatrana Kloss, T'renbia, xiii, I93 I, p. 369 :

Korinchi Valley, West Sumatra.

Distr.-Sinnatra.

Zosterops palpebrosa media Rob. and Kloss.

Zosterops aureiventer media Robinson and Kloss, Ibis, I923, p. 152 : Samarahan, Sarawak.

Zosterops aureiventer paryus Hachisuka, Bull. Brit. Orn. Cl. xlvii, 1026, p. 56 : Mt. Kinabalu, North Borneo.

Distr.-Borneo.

Zosterops palpebrosa erwini Chas.

Zosterops palpebrosa eraini Clasen, Bull. Raftles Mus. no. 9, I934,

1. 96 : Pulau Panjang, South Natuna Islands.

Dislr.-South Natuna Islands.

Zosterops palpebrosa buxtoni Nich.

Zosterops burtoni Nicholson, Ibis, 1379, p. 167: West Java.

Zosterops lateralis Hartl. (not Lath. 1801) Journ. f. Orn. 1865, p.15: Java.

Distr.-Borneo (part). ${ }^{1}$

Java (west and central).

*Zosterops palpebrosa salvadorii Mey. and Wigl.

Zosterops salvadorit A. B. Meyer and Wiglesworth, Journ. f. Orn. ISg4, p. 1 I5: Engano Island.

Zosterops incerta Salvad. (not Meyer), Ann. Mus. Civ. Gen. (2) xxxii, 1892, p. 133 : Engano Island.

Distr.-lingano Island, West Suntratra.

Zosterops natalis ${ }^{2}$ Lister.

Christuas Island White-eye.

Zosterops natalis Lister, Proc. Zool. Soc. I8SS, I. 5I8, pl. 27 : Christmas Island.

Distr.-Christunas Island, Indian Ocean; Cocos-Keeling Islands (introduced).

- Stresemann (1931) seems to be the first to recognize this, or a closely allied form of Zosterops in Borneo, and refers some skins from the mountains of West Sarawak to buxtoni. We have recently examined some skins from the lowlands near Kuching. The specimens are old and faded. They are, clearly, not media, but seem nearer to williamsoni than to buxloni. Here, for the moment, the matter must rest.

- The affinities of this form seem to be with Zosterops citrinella lvide Chasen, Bull. Raffes Mus. \$, 1933, p. 84). 
Zosterops atricapilla Salvad.

Black-capped White-eye.

Zosterops alricapilla Salvadori, Ann. Mus. Civ. Gen. xiv, 1879, p.

2 r 5 : Padang Highlands, West Stunatra.

Zosterops clara Sharpe, Ibis, 1888 , P. 479: Mr. Kinabalu, North Borneo. Disir.-Sunatra.

Borneo.

Zosterops flava (Horsf.).

Yellow White-eye.

Zosterops flava flava (Horsf.).

Diсаиm flavum Horsfield, Trans. Linn. Soc, xili, i821, p. izo: Java.

Zosterops parvule Hombron and Jacquinot, Voy. Pôle sud. Atlas, i 844 , pl. 19: South-east Borneo.

Distr-Borneo (south).

Java (west); North Watcher Islands.

Zosterops melanura Hartl.

Small Bridled White-eve.

*Zosterops melanura melanura Hartlanb.

Zosterops melanura Hartaub, Journ. f. Orn. I865, p. I5 : Pontianak, South-west Borneo.

Distr.-Borneo (south).

Zosterops melanura gallio Sharpe.

Zosterops gallio Sharpe, Cat, Bds. Brit. Mt1s. ix, I884, p. I85: East Java.

Distr.-Java.

Zosterops chlorates Hartl.

Greater Zosterops.

Zosterops chlorates chlorates Hartl.'

Zosterops chlorates Hartlaub, Joum. f. Orn. 1865, p. 23: Morotai Island, North Moluccas ertot=Suniatra.

Zosterops chlorates korinchi Kloss, Treubia, xiii, 1931, p. 360: Mt. Korinchi, South-west Sumatra.

Distr.-Sunatra (part).

*Zosterops chlorates difficilis? Rob, and Kloss.

Zosterops difficilis Robinson and Kloss, Journ. Fed. Malay States Mus. viii, pt. 2, I9IS, p. 250: Mt. Deupo, South Sumatra,

Distr.-Sumatra (part).

' Cf. Kloss, Treubia, xiii, t031, p. 360 , where Morotai Island is accepted as the type locality of $Z$. chlorates.

2 Zosterops montana $\mathrm{Bp}$, 1850 , may be this bird, but it seems also possible that it is a Sumatran form of Z. Rava. 
Zosterops chlorates sindorensis Siebers.

Zosterops palpebrosa sindorensis Siebers, Treubia, xi, 1929, p. I5I :

Mt. Tjerimai, West Java.

Disir.-Java (west and central).

Zosterops chlorates neglecta Seebolim.

Zosterops neglecla Seebolm, Bull. Brit. Orn. Cl. i, 1893, p. 26 : Fast Java.

Distr,-Java (east); Bali.

Zosterops chloris $\mathrm{Bp}$. Large Bridled White-eye.

[Zesterops chloris chloris Bp.

Zosterops chloris Bonaparte, Consp. Gen. Avium, i, 1850, p. 398 : Banda Island, Moluccas.

Distr.-Eixtra-limital.]

\section{Zosterops chloris maxi Finsch.}

Zosterops Maxi Finsch, Journ. f. Orn. 1v, 1907, p. 302: Thousand Islands, West Java.

Distr.-Thousand Islands, Java Sea; Karinon-Java Islands.

*Zosterops chloris solombensis Oberh.

Zosterops solombensis Oberholser, Proc. U. S. Nat. Mus. liv, 1917. 1. ISS: Solonbo Besar Island.

Distr._Solombo Besar Island, Java Sea; Billiton.

*Zosterops chloris zachlora Oberh.

Zosterops solombensis zachlora Oberliolser, Proc. U.S. Nat. Mus. liv, I917, p. I99: Kalambau Island.

Distr-Kalambau Island, Java Sea.

Zosterops javanica (Horsf.).

Javan Zosterops.

Zosterops javanica javanica (Horsf.).

Sylvia javanica Horsfield, Trans. Linn. Soc. xiii, I8zI, P. I56: Java (central).

Distr.Java (central).

Zosterops javanica frontalis Reichenb.

Zosterops fronlalis Reichenbach, Handb., Merop. I 52 , P. 94, pl. 463 : Java (west).

Zosterops fallax Sharpe, Cat, Bds, Brit. Mus, ix, 1854, p. 197 : West Java.

Distr. - Java (west). 
Zosterops javanica elongata (Stres.).

Oreosterops jazanica elongala Stresemann, Nov. Zool. sx, Igr3, p. 336: Bali.

Dislr.-Java (east); Bali.

Zosterops squamifrons Sharpe.

Pygmy Zosterops.

Zosterops squamifrons Sharpe, Ibis, 1892, P. 323: Mt. Kinabalu, North Borneo.

Dislr.-Borneo.

Gents CHLOROCHARIS Sharpe.

Chlorocharis emilize Sharpe.

Sharpe's White-eye.

Chlorocharis emilia emiliae Sliarpe.

Chlorocharis emilia Slarpe, Ibis, I888, p. 392: Mt. Kinabalt, North Borneo.

Dislr.-Borneo (north).

Chlorocharis emilize moultoni Chas, and Kloss.

Chlorocharis emiliae moultoni Chasen and Kloss, Butl. Brit. Oru.

Cl. xlviii, 1927, 1. 47: Mt. Poi, South-west Sarawak.

Distr.-Borneo (mountains of Sarawak).

\section{Family DICÆIDÆ.}

\section{Genus DICAEUM Cuvier.}

Diczum trochileum (Sparru.). Scarlet-breasted Flower-pecher.

Dicaum trochileum trochileum (Sparrm.).

Certhia irochilea Spamman, Mus. Carls, iv, 110. So, 1789: West Java (Strese., ro23).

Motacilla fammeum Sparrm., Mus. Carls. iv, 1789. No. 98: Java.

Dicaetom rubescens Vieill., Nov. Dict, d' Hist. Nat. ed. 2, ix, ist7, p. 408 : Java.

Nectarinia rubro-cana Temm., Pl. Col. 108, 1822: Java.

Dicaeum rubrocosum Cuv., Rẻgne Anim. i, i 829 , D. 433: Java.

Distr.-Borneo (south).

Java; Bali;' Bawean Island; Kangean Islands; Karinon-Java Islands.

Dicæum cruentatum (Linn.).

Scarlet-backed Flower-pecker.

[Dicæum cruentatum cruentatum (Linn.).

Certhia cruenlata Linn., Syst. Nat. roth. ed. I758, p. II9: Bengal.

Distr.-Extra-linital.]

- Rensch considers that the Bali bird is $D$. t. trochileum $\rangle\langle$ stresemanni Rensch (Lombok). 
Dicaum cruentatum siamensis Kloss.

Dicaeum cruentatum siamensis Kloss, Ibis, 19rS, p. 216 : East Siam. Distr,-Peninsular Siam (north).

Diczeum eruentatum ignitum (Begb.).'

Nectarinea ignila Begbie, Malay. Pen. 18,4, P. 518 : Malacca.

Distr.-Malay Peninsula.

Diczum eruentatum nigrimentum Salvad.

Diccun nigrimentum Salvadori, Ann. Mus. Civ. Gen, v, I874, p. 165 : Sarawak.

Dicaeum pryeri Sharpe, Proc. Zool. Soc. 1881, p. 795 : Sandakan, North Borneo.

Distr.-Borneo; North Bornean Islands; Karimata Islands (subsp. incert.).

Diceum cruentatum sumatranum Cab.

Dicacum sumatranum Cabanis, Journ. f. Orn. I878, p. 10I: Sinuatra.

Distr.-Sumatra; Nias Island.

Dicaeum eruentatum batuense Riclnn.

Dicacum sumatranum batuense Richmond, Proc. Biol. Soc. Wash. xxy, IgI2, p. 104: Pinie Island, Batı Islands.

Distr.-West Sumatran Islands of Batu group, Sipora and South Pagi.

Dicaum sanguinolentum 'Tentrn.

Fire-lyreasted Flower-pecker.

Diceum sanguinolentum sanguinolentum 'lemm. 2

Dicacum sanguinolenlum 'Tennunck, P1. Col. 478, I829: West Java (restr. Robinson and Kloss, ro23).

Distr. Java (west).

Dicreum sanguinolentum ablutum Rob, and Kloss.

Dicaeum sanguinolentum ablutum Robinson and Kloss, Jonrn. Fed.

Malay States Mus. xi, 1923, D. 57: Liast Java.

Distr.-Java (east); Bali.

Dicaum sanguinolentum beccarii Rob, and Kloss.

Dicacum beccarii Robinson and Kloss, Journ. Straits Brancl, Roy. Asiat. Soc, no. 73, 1916, 1, 278 : Korincli, Sumitra.

Dicaeum wanheysti Robinson and Kloss, Journ. Fed. Malay States Mus. vii, 1918, p. 239 : Brastagi, North Sumatra.

Distr.-Sumatra.

1 There is a visual record, probably of this subsprecies, from the Rhio Arehipelago.

2 Has also been recorded from Sumatra, but confirmation is needed. 
Dicaum sanguinolentum ignipectus (Hodgs.).

Mranthe ignipectus Hodgson, Journ. Asiat. Soc. Bengal, xii, i 843 , p. $9 S_{3}$ : Nepal.

Distr.-Malay States.

Dicaum sanguinolentum monticolum Sharpe.

Dicaum monticolum Sharje, Ibis, T887, 1\%. 452 : Mt. Kinabalı, N. Borneo.

Dicneum sulaense sila' Harr. and Hart., Bull. B, O. Cl. !iv, 1934, D. 159 : Mt. Dulit, Sarawak.

Distr.-Borneo.

Dicæum trigonostigmum (Scop.)..$^{2}$ (Trange-hellied Flower-pecker.

Dicaeum trigonostigmum trigonostigmum (Scop.).

Certhia trigonostigna Scopoli, Del. Flor. et Fatun. Insubr, ii, I786, P. 91: Malacca.

Certhia cantillans Lath. Ind. Orn. i, 1700, p. 200 : "China" = Malacca, Dicaeum croceoventre Vigors, Mem. Raffles, i 830, p. 673: Sumatra.

Dieneum trigonostigma hypochloum Oberh., Bull, U.S. Nat. Mus. gs, 1917, p. 67 : Siantan Island, Anamba Islands.

Distr.-Malay Peninsula; Anauba Islands.

Sunatra; Rlio and Lingga Archipelagos; Billiton; Banka. Karimata Islands.

Dicreum trigonostigmum rubropygium Baker.

Dicaum trigonostigna rubropygin Sturt Baker, Bul1. Brit. Orn. Cl. xli, 1921, p. 108 : Mergui.

Distr-Peninsular Sian.

Dicaum trigonostigmum dayakanum Chas, and Kloss.

Dicaum trigonostigna dayakana Chasen and K゙loss, Jouru. f. Ornitl. I929, Bd. 2, D. $12 \mathrm{I}$ : near Sandakan, North Borneo.

Distr.-Borneo; North Bornean Islands.

Dicaum trigonostigmum megastomum Hartert.

Dicaw trigonostigma negastoma Hartert, Bull. Brit. Orn. Cl. xxxviii, I9x 8, p. 74 : Great Natnna Island.

Distr.-North Natuna Islands.

Dicaum trigonostigmum flaviclune Hartert.

Diecum trigonostigma flarichuis Hartert, Bull. Brit. Orı. C1. xxxviii, I9I\$,, 75 : Karangholong, Java.

Distr.-Java; Bali.

"After an examjnation of the material on which "sita" is based I believe that the differences noted between birds from $\mathrm{Mt}$. Finabalu and $\mathrm{Mt}$. Dulit are due to individual variation, and the uneclual condition of the skins from the two localities.

Cf. Oberh., Bull. U.S. Nat. Mus. 159. 1932, 13. 119. 
*Dicæum trigonostigmum antioproctum Oberł.

Dicacum trigonostigma antioproctum Oberholser. Smiths. Misc.

Coll. Ix, 170. 7, 1912, D. 21 : Simalur Island.

Dicaenm trigonostigma melanthe Oberholser, Smiths. Misc. Coll. Ix, No. 7, 1912, 1. 21: Lasia Island, West Sumatra.

Distr.-West Simmatran Islands of Sinulur and Lasia.

-Dicæum trigonostigmum lyprum Oberì.

Dicaeum trigonosligma lyprum Oberholser, Smiths. Misc. Coll. Ix, no. 7 , 1912, P. 21 : Nias Island.

Distr.-Nias and Batu islands, West Sumatra.

Dicaum trigonostigmum pagense Oberl..

Dicaum trigonostignum pagense Oberholser, Journ. Wash. Acad. Sci, xvi, 1926, P. 520 : Soutli Pagi Island.

Distr.-Mentawi Islands.

Dicaum chrysorrheum Tentm.

Yellow-vented Flower-pecker.

Dicaum chrysorrheum chrysorrheum Temnt

Dicaeum chrysorneum Temuninck, P1. Col. 478, I829: Java.

Disir.-Malay Peninsula.

Sumatra.

Borneo.

Java (west).

Dicaum concolor Jerd.

Plain-coloured Flower-pecker.

[Dicaum concolor concolor Jerd.

Dicaeum concolor Jerdon, Madr, Journ. Lit. Sici, xi, r\$4⿻, p. 227: Malabar Coast.

Disl. -Eixtra-limital.]

\section{Dicaum concolor borneanum Lönn.}

Diccum minullum borneanum Lönnberg, Sarawak Mus. Journ. iii, 1925, p. I : Mt. Murud, N. Sarawak.

Distr._Malay States.

Stimatra.

Borneo; North Natuna Islands."

Diczeum concolor sollicitans Hart.

Dicaeum sollicitans Hattert, Nov. Zool, viii, rgor, p. 52 : Mt. Gede, West Java.

Dislr.-Java; Bali.

I A specimen from Bunguran Jsland, collected by Everett has been examined. 


\section{Genus ANAIMOS Reiclienlyaclı."}

Anaimos percussus (Temm.). Crinson-breasted Flower-pecker.

Anaimos pereussus percussus ('lemm.).

Pardalolus periusius Temminck, Pl. Col. 394, I826: Java.

Distr.-Java.

Anaimos percussus ignicapillus (Eytort).

Dicaum ignicapilla Eyton, Proc. Zool. Soc. 1\$39, P. I05: Malacca.

Disir.-Malay Peninsula.

Sunlatra; Rhio Archipelago; Billiton; Banka.

Borneo; North Natuma Islands.

Anaimos xanthopygius (Salvad.). Vellow-rumped Flower-pecker.

Anaimos xanthopygius xanthopygius (Salvad.).

Prionochilus xanlhopygius Salvadori, Att. R. Accad. Sci. Torino, iii, 1868, D. 416: Sarawak.

Distr.-Borneo; Nortl Natuna Islands.

Anaimos thoracicus ('T"emu.). Scarlet-breasted Flower-pecker. Pardalolus thoracicus Temminck, P1. Col. 600, r $\$_{3} 6$ : Borneo.

Distr.-Malay States.

Billiton Island.

Boriteo.

Anaimos maculatus (Temur). Vellow-throated Flower-pecker.

Anaimos maculatus maculatus (Temm.).

Pardalotus maculatus Tenminck, P1. Cot. 600, 18,36: Borneo.

Distr,-Malay Peuinsula.

Sumatra; Billiton.

Bormeo.

Anaimos maculatus septentrionalis (Rob. ant Kloss).

Prionochilus naculatus seplentrionalis Robiuson and Kioss, Journ.

Fed. Malay States Mus. x, I921, D. 206: Chumporn, Peninsular Siam.

Distr.-Peninsular Siam.

Anaimos maculatus natunensis (Chas.).

Charitociris maculata nalunensis Chasen, Bull. Raffles Mus., 9, I935,

v. 95 : Bunguran Island, North Natuna Islands.

Distr.-North Natuna Islands.

' Ct. Oberh., Ohio Journ. Sci. xxiii, 1923, p. $2 S_{7}$. 
*Anaimos maculatus opistatus (Oberir.).

Anaimos moculalus opislalus Oherholser, Sniths. Misc. Coll. Ix, no. 7,1917, p. 22 : Nias Island.

Distr.-Nias Island, West Sunatra.

\section{Genus PIPRISOMA Blyth.}

Piprisoma modestum (Hutne).

Hume's Flower-pecker.

Piprisoma modestum modestum (Hume).

Prionochilus modestus Hume, Stray Featlers, iii, IS 75, P. 298 : Soutlı Tenasserim.

Distr.-Peninsular Siam.

Piprisoma modestum finschii (Bartels).

Dicaeum Finschii Bartels, Orn. Monatsbr. xxii, igr4, ए. I25: Wynkoops Bay, West Java,

Piprisoma modestum remotum Robinson and Kloss, Journ. Fed, Malay States Mus, vi, 1915, 1 . 20: Negri Sembilan.

Distr.-Malay States. Java (west).

Piprisoma agile (Tick.). Thick-billed Flower-pecker.

[Piprisoma agile agile (Tick.).

Fringilla Agilis Tickell, Journ. Asiat. Soc. Bengal, ii, I833, p. 578 : Borabhum.

Distr.-Eixtra-linital.]

Piprisoma agile sordidum Rob. and Kloss.

Piprisoma sordidun Robinson and Kloss, Journ. Fed. Malay States Mus, vii, IotS, p. 239: Selangor, Malay States.

Distr.-Malay States.

Rhio Archipelago.

Piprisoma agile everetti (Sliarpe).

Prionochilus everelfi Sharpe, Ihis, 1577 , p, 16 : Labuan Island.

Distr.-Borneo.

\section{Piprisoma agile bungurense Chas.}

Piprisoma agile bungurense Chasen, Bull. Raftles Mus. 9, I934, p. 96: Bunguran Island, North Natuna Island.

Distr,-North Natuna Islands.

This species is confused with $P$. agile in Faun. Brit. Ind., Bds. (and ed.), vii, 1930, p. 294 . 


\section{Family NECTARINIIDÆ.}

\section{Genus CHALCOSTETHA Cabanis.}

Chalcostetha calcostetha (Jard.).

Macklot's Sumbird,

Chalcostetha calcostetha calcostetha (Jard.).

Nectarinia calcostetha Jardine, Nat. Libr. Ornitl. xiii, I843, 0. 263 : Java $($ tom. cil, p. 274 ).

Nectarinia pectoralis (not Horsf, 1821) Temm., P1. Col. 138, 1822 : Java.

Nectarinia insignis Jard, Nat. Libr. Ora. xiis, 1843. 1. 274; (new name for pectoralis Temm. \}.

Cinmyis macklolii Bp., Consp. Gen. Av, i, 1850, 1). 408: (new name for pectoralis Temm.).

Distr.-Malay Peniusula.

Sumatra; Rlijo and I,ingga Archipelagos; Billiton.

Borneo; Karimata Islands.

Java; Karimon-Java Islantis.

Chalcostetha calcostetha proxima Riley.

Chalcostetha calcostelha proxima Riley, Proc. Biol. Soc. Wash. xl, I927, P. I4I: Derawan Isłand, North-east Borneo.

Chaleostetha calcostetha halitypa Oberh.. Bull. U.S. Nat. Mus. 15g, 1932, D. I10: Brian Island, South Natuna Islands.

Distr,-Maratua Islands, Fast Borteo; Natuna Islands.

Chalcostetha calcostetha pagicola Oberll.

Chalcostetha calcostetha pagicola Oberholser, Smiths. Misc. Coll. lx, no. 7,1912, p. 17 : North Pagi Island.

Chalcostetha calcostetha sibiru Chasen and Kloss, Ibis, IQ26, P. 297 : Siberut Jsland, West Sumatra.

Distr.-West Sumatran islands of Nias, Batu group, and Mentawi group.

* Chalcostetha calcostetha heliomarpta Oberli.

Chalcostetha calcostetha heliomarpta Oberholser, Jour11. Wash. Acad. Sci. xiii, 1923, p. 229 : Simalur Island.

Dislr-Simalur Island, West Sumatra.

\section{Genus AETHOPYGA Cabanis.}

Aethopyga saturata (Hodgs.).

Black-breasted Sunbird.

(Aethopyga saturata saturata (Horlgs.).

Cinnyris salurala Hodgson, Ind. Rev, ii, 1837, 0. 273: Nepal.

Dislr. -Extra-linital.] 
Aethopyga saturata anomala Richm.

Aethopyga anomala Riclunond, Proc. U.S. Nat. Mus. xxii, rgoo, p. 319: Trang, Peninsular Sian.

Distr.-Peninsular Siant.

Aethopyga saturata wrayi Sharpe.

Acthopyga itrayi Sliarpe, Proc, Zool, Soc, $1587, \mathrm{p}, 440, \mathrm{pl}, 38$, fig. 2: Perak, Malay States.

Distr.-Malay States.

Aethopyga mystacalis (Tenmu.).'

Scarlet Sumbird.

Aethopyga mystacalis mystacalis (Temm.).

Nectarinia mystacalis "I'emminck, P1, Col, J26, 1822 : Java. Aethopyga Lodoisin Salvad, Ibis, 1865, p. 548 : Java.

Distr.-Java,

Aethopyga mystacalis temmincki (S, Miill.).

Neclarinia temmincki Sal. Müller, Veth. Nat. Gesct. Iand-en Volk. $1843, p .173$, note $=$ Sumatra.

Aethopyg mystacalis perretiz Harrison and Hartey, Bull. B. O. Cl. liv, 1934, D. 158: Mt. Dulit, Sarawak.

Distr.-Malny Peninsula.

Stmatra,

Borneo.

Aethopyga eximia (Horsf.)."

Kinl1]'s Sunbird.

Nectarinia eximia Horsfield, 'Trans. I,mut, soc. Lond, xiii, I\$2 T, p. 168 : Java.

Nectarinia kuhlii Temm., Pl. Col. 376, 1825 : Java.

Distr.-Java.

In the present state of our knowledge these scarlet sunbirds are difficult to arrange in species. In some respects mystacalis and siparaja seem closer than do mystacalis and temminchi, but mystacalis and siparain occur together in West Java.

2 Good series of this bird are available for comparison, from Sumatra, Borneo (including topotypes of "perretti") and the Malay Peninsula and I cannot make any separation, in either sex, on colour, or size. In old skins the red parts of the plumage are usually paler than in recently collectly specimens. In the British Museum, old skins from Mt. Dulit are paler than the newer material collected by Messrs. Harrisson and Hartley.

$\$$ This is a very distinct species. Its nearest relative 15 , perhaps, $A$. nipalensis. 
Aethopyga siparaja (Raffles).

Kaffles' Sunbird.

Aethopyga siparaja siparaja (Raffles).

Cerlhia siparaja Raffles, Trans. Linn. Soc. xili, I\$2 r, P, 299 : Sumatra.

Aethopyge eupogon Cab., Mus. Hein. i, 1850 , p. 103 : Borneo.

Aithopyga chalcopogon Reichenb., Handi. Scans. 1854, p. 303, pl. 586 : Borneo.

Acthopyga siparaja tinoptila Oberh., Smiths. Misc. Coll. 1x, No. 7, $19 t 2$, p. I 8: Siumut Island, West Sumatra.

Aethopyg $a$ siparaja melanetra Oberh., Smiths. Misc. Coll. 1x, No. 7 , 1012,1 , I8: Lasia Island, West Sumatra.

Aethopyga siparaja ochropyrrha Oberh., Bull. U.S. Nat. Mus, 08,1017, 1. 63: Rittan Island, Anamba Islands.

Aethopyga siparaja heliophiletica Oberh., Journ. Wash. Ac. Sci, xiii, 1023, p. 231 : Bangkaru Island, Banjak Islands, West Sumatra.

Disir.-Malay Peninsula; Ananba Islands.

Sumatra; Rhio Archipelago; Biliton; West Sumatran islands of Simalur, Babi, Lasia, and Banjak group.

Boneo; Banguey Island; Maratta Islands; Karimata Islands.

\section{Aethopyga siparaja cara Hume.}

Aethopyga cara Hutue, Stray Feathers, ii, I875, D. 473: South of Moulnein, Tenasserin.

Distr.-Peninsular Sian.

\section{Aethopyga siparaja niasensis Hart.}

Aelhopyga siparaja niasensis Hartert, Orn. Monatsbr, vi, rSoS, p. 92 : Nias Island.

Distr-Nias Island, West Sunatra.

\section{Aethopyga siparaja photina Obert.}

Aethopyga siparaja photina Oberholser, Smiths. Misc. Coll. Ix, no. 7 , r 12, p. 18: North Pagi Island.

Aelhopyga siparaja sibert Chasen and Kloss, Ibis, 1926, p. 208: Siberut Island, West Sumatra.

Distr.-West Sumatra islands of Siberut, Sipora, North and South Pagi.

\section{*Aethopyga siparaja heliogona Obeth.}

Aethopyga siparaja heliogona Oberholser, Journ. Wash. Acad. Sci. xili, 1933, p. 232 : Java.

Distr.-Java (west).

\section{Aethopyga siparaja natunæ Chas.}

Aethopyga siparaja natuna Chasen, Bull. Raffles Mus. 9+ 1934, D. $97:$ Butguran Island, North Natuna Islands.

Distr.-Nortl Natuna Islands. 
Aethopyga nipalensis (Hodgs.). Yellow-backed Sunbird.

[Aethopyga nipalensis nipalensis (Hndgs.).

Cinnyris nipalensis Hodgson, Ind. Rev, ii, iS 37, p. 273: Nepal. Distr,-Extra-limital.]

Aethopyga nipalensis australis Rob. and Kloss.

Aethopyga nipalensis australis Robinson and Kloss, Bull. Brit. Orr.

Cl. xliv, I923, P. I4: Nakon Sritamarat, Peninsular Siam.

Distr.-Peninsular Sinus.

\section{Genus LEPTOCOMA Cahanis.}

Leptocoma brasiliana (Cimel.)."

Van Hasselt's Sumbird.

Leptocoma brasiliana brasiliana (Gmel.).

Cerlhia brasiliana Gmelin, Syst. Nat. i, pt. I, T-88, P. 474 : Brasil ertor= Java (Oberh., rol2).

Nectarinia hasseltii Temn., P1. Col. 376,1825 : Java.

Cinntris ruber Less. Traite d'Orn, $1830,0.206$ : Java.

Convyis brasiliana hypolampis Oberh,, Smiths. Misc. Coll. Ix, No. 7, 1012. 1) in: South Pagi Island, West Sumatra.

Distr.-Malay Peniusula; Tionnan Island.

Stmuatra; Billiton; Banka; Rhio and I ingga Arclipelagos;

West Sumatran islands of Siberut, Sipora and North and South Pagi.

Borneo,

Java.

Leptocoma brasiliana axantha (Oberh. .

Cimnris brasiliana axantha Oberlolser, Bnll. U.S. Nat. Mus. I59, 1032, P. I14: Natuna Islands.

Distr.-Natuna Islands.

*eptocoma brasiliana eumecis (Oberl,.).

Cinnyris brasiliana cumecis Oberlolser, Bull. U.S. Nat. Mus. 98, $1917,0,63$ : Siantan Island, Anamba Islands.

Distr.-Anamba Islands.

-Leptocoma brasiliana mecynorhyncha (Oberh.).

Cinnyis brasiliana necynorhyncha Oberholser, Smiths. Misc. Coll.

1x, no. 7, 19:2, p. 19: Simahur Island.

Dish.-Simalur Istand, West Sumatra.

* Leptocona brasiliana oenopa (Oberlı.).

Cinnyris brasiliana oenopa Oberholser, Sulths. Misc. Coll. 1x, no.

7 , Igt2, P. IS: Nias Island.

Distr.-Nias Island, West Sumatra.

, Ct. Oberh., Bull. U.S. Nat. Mus. 159, 1932, p. 116. 
Léptocoma brasiliana sperata (I.imn). Philippine Sumbird.

Cerlhia sperata I,int., Syst. Nat, 12tl, ed, 1-66, P. I86: Philippine Istands.

Distr.-Maratua Island, Fast Borneo.

Leptocoma jugularis (I.inn.).'

Yellow-breasted Sunbird.

[Leptocoma jugularis jugularis (I.inn.).

Cethia jugularis Lint, Syst. Nat. I2tlt. ed. т766, p. I 85 : Ihilippine Islands.

Disir.-Extra-liujital.]

Leptocoma jugularis pectoralis (Horsf.).

Neclarina pectoralis Horsfield, Itrats. Ifinn, soc, xiii, IS2 I, p. I67 : Java,

Cimnyis ormatus Less., Dict. Sci. Nat, i, I\$27, p. 15: Java.

Nectarinia eximia (not Horsf. 1 \$21) Temm., Pl. Col. 138, 1822: Java.

Cinnyris luleoventer Less., Traité d'Orn. 1830, 1). 208 : Sunda Islands= Java.

Distr,-Java; Bali; Kangean Islankls; Banean Island.

Leptocoma jugularis flammaxillaris (Blytli).

Nectarinia fammaxillaris Blyth, Journ. Asiat. Soc. Bengal, xiv, I 845, 1. 557 (nonu. for descrip. tonn. cit. [1. 979) : 'Tenasseriu.

Distr.-Malay Peninsula (soutli to Penang). ${ }^{2}$

Leptocoma jugularis microleuca (Oberh.).

Cinnyris ormala microlewa Oberlnolser, Proc. IT.S. Nat. M[us. Iv, 1919, 1. 273: Taya Island, South-east Sumatra.

Cinnyris ornata hetiobleta Oberh., Journ. Nash. Acad. Sci, xiii, 1923. P. 230 : Trengganu, Malay Peninsula.

Cinnyris ornata heliomanis Oberh., Journ. Wash. Acad. Sci. xiii, 1923, 13. 231 : East Borneo.

Cinnyris ornata zapega Oberh., Bull, U.S. Nat. Mus. 159, I932, B. II2: Subi Island, South Natuna Islands.

Disfr,--Malay States; Tioman Island.

Sumatra; Taya Island, S.E. Simutra; Rhio and Lingga Archipelagos.

Iorneo; North Bornean Islands; Natuma Islands; Mangalum Island; Karimata Islands.

*Leptocoma jugularis heliozeteta (Oberln.).

Cinnyis ornata heliozeteta Oberholser, Jourı, Wash. Acad. Sci. xiii, I923. j). 23 I : Barka Island.

Distr.-Banka Island.

, Cf. Oberh., Bull. U.S. Nat. Mus. 159, 1932, p. 113.

The division between fammaxillaris and microlevica in the north of the Malay Peninsula is rather vague. 
*Leptocoma jugularis polyclysta (Obeth.).

Cinnyris ornata polyclysta Oberlsolser, Smiths. Misc. Coll. 1x, no. 7 , 1912, p. 18 : Engano Island.

Distr.-Engano Island, West Sunatra.

\section{Gentı ANTHREPTES Swainson.}

Anthreptes macularia Blyth.

Purple-naped Sunbird.

Anthreptes macularia macularia Blyth.

Anthreples macularia Blyth, Journ. Asiat. Soc. Bengal, xi, I842, p. 106 : Malacca.

Awtheptes nuchalis Blyth, Journ. Asiat. Soc. Bengal, xii, 1843. p. 980 : Singapore.

Distr.-Malay Peninsula.

Anthreptes macularia hypogrammica (S. Müll.).

Neclarinia hypogrammica S. Müller, Verh. Nat. Gesch. Land en Volk. I 843 , p. r73: Sumatra.

Anthreptes hypogrammica intensior Hart., Bull. B. O. Cl. xxxviii, 1917, p. 27: Sarawak.

Distr. - Sumatra.

Borneo.

Anthreptes macularia natunensis Clras.

Anthreptes macularia natumensis Chasen, Bull. Raffles Mus. x, r935, p. 43 : Bunguran Island, North Natuna Islands.

Distr.-North Natuna Islands.

Anthreptes simplex (S. Mü1l.). ${ }^{3}$

Plain-coloured Sunbird.

Anthreptes simplex simplex (S. Müll.).

Nectarinia simplex S. Müller, Verh. Nat. Gesch. Land en Volk. I843, p. 173: Sumatra.

Nectarinia frontalis Blyth, Journ. Asiat. Soc. Bengal, xiv, 1845, D. $55^{8}$ : Singapore.

Anthreptes simplex simplicior Oberh., Journ. Wash. Acad. Sci. xiii, I923, p. 220 : Central Borneo.

Anthreptes simplex enthapsimus Oberh., Bull, U.S. Nat. Mus. ${ }^{59}$, I $_{32}$, P. 107 : Bunguran Islands, Natuna Islands.

Distr.-Malay Peninsula.

Sumatra; Nias.

Borneo; North Natuna Islands.

I C/. Oberk., Bull. U.S. Nat. Mus, 150, 1932, p. roS. 
Anthreptes malacensis (Scop).).

Brown-throated Sunbird.

Anthreptes malacensis malacensis (Scop.).

Cerlhia malacensis Scopoli, Del. Flor. et Faum. Instubr. ii, I7S6, p. 9 r: Malacca.

Neclarimia jawmior Horef., 'Trane. Linn. Sor xiii, 1821, p. 167: Java, Anthreples malacensis anumbut Oberliolser, Bull. U.S. Nat. Mus. 28 , I917, P. 61: Mobur Island, Anamba Islands.

Antheptes malacensis nesaews Oberh., Smiths. Misc, Coll. Ix, No. 7, 1912, p. 20: Pagi Jslands, West Sumatra.

Distr.-Malay Peninsula; Tioman Island; Anamba Islands.

Sumatra; Rhio and Lingea Archipelagos; Billiton; Banka; Mentawi Islands, West Sumatra.

Borneo (not north); Karimata Islands.

Java; Bali; Kangean Islands.

\section{Anthreptes malacensis bornensis Riley.}

Anthreptes malacensis bornenstis Riley, Proc. Biol. Soc. Wash. xxxiii, 1920, 3. 55: Santakan, North Borneo.

Distr.-Borneo (north); North Bornean Islands; Mangalun Island.

Anthreptes malacensis exixanthus Oberb.

Anhreptes malacensis evixanthus Oberholser, Bull. U.S. Nat. Mus. 1.59, 1932, 1). J09: Bunguran Island, Natuna Islands.

Distr.-Natuna Islands.

Anthreptes malacensis mjobergi Bangs and Petrs.

Anthreples malacensis mjobergi Bangs and Peters, (ce. Pan. Boston Soc. Nat. Hist, v, 1927, ए. 240 : Maratua 1sland, Fast Borneo.

Distr.-Maratua Islankls, Jast Jorneo.

Anthreptes malacensis baweanus Oberh.

Anlhreples malaconsis baifanus Oberholser, Proc. U.S. Nat. Mus. lii, J9r7, p. 196: Bawean Island.

Dists - Bawean Islank; Karinon-Java Islands.

*Anthreptes malacensis pelloptilus Oberl.

Anthreptes malacensis pelloptilus Oherholser, Smiths. Misc. Coll. Ix, 110.7, 1912, 1. 20: Simalur Istand.

Distr.-Simalur Island, West Sumatia.

*Anthreptes malacensis pollostus Oberli.

Anheples malecensis pollostus Oberhọlser, Sniths. Misc. Coll. 1x, no. $7,19: 2,1 \%, 21$ : Nias Islaud.

Distr.-Nias Island, West Simutra.

- There is certainly a tendency for birds from the Anamba and Mentawi Islands to run heavy in the bilh, but this is a very plastic organ in most sunbirds. Similar heavy-billed birds also occur on coasta] islands in Malaysin and it is difficult to see how they can be regarded as a "race" for the colous and remeral size seem fairly constant. 
Anthreptes rhodolema silelley.

Silelley's Sunbird.

Anthreptes rhodolema rhodolæema Shelley.

Anthreptes thodolama. Shelley, Monogr. Nectarinitae, r 878 , p. $3 \mathrm{r} 3$, pl. tot: Malacca.

Anthreptes rhodolaema aemea Riley, Proc. Biol. Soc. Wash. xlvii, r934, p. I16: Labuan Kiambu, Dutch East Bomeo. ${ }^{1}$

Distr,_Malay- Peninsula.

Suntatra.

Borneo.

\section{Genus CHALCOPARIA Cabanis.}

Chalcoparia singalensis (Cimel.)."

Ruby-cheek.

Chalcoparia singalensis singalensis (Gmel.).

Motacilla singalensis Guelin, Syst. Nat. i, I785, p. 964: Ceylon, error = Malacca (Oberholser, ror $)$.

Distr.-Malay Peninsula.

Chalcoparia singalensis interposita Roh. and Kloss.

Chalcoparia singalensis inlerposila Robinson and Kloss, Journ. Fed. Malay States Muts., x, I02I, p. 200: Takuapa, west coast of Peninsular Siani..

Distr.-Peniusular Siann.

Chalcoparia singalensis sumatrana Kloss.

Chalcoparia singalensis sumalrana Kloss, Journ. Fed. Malay States

Mus. x, 1921, D. 209: Ophir district, Central Sumatra.

Distr.-Sumatra; Billiton; Nias (? subsp.), West Stmatra.

\section{Chalcoparia singalensis borneana Kloss.}

Chalcoparia singalensis borneana Kloss, Journ. Fed. Malay States Mus. x, r92I, p. 209: Samaratan, Sarawak.

Distr.-Borneo (perhaps not the west): Banguey Island.

Chalcoparia singalensis'pallida Chas.

Chalcoparia singalensis pallida Chasen, Bull. Raftles Mus. 9, I934, p. 95: Bunguran Island, North Natuna Islands.

Distr.-North Natuna Islands.

Chalcoparia singalensis phonicotis (Ten111.).

Nectarinia phonicolis Tenminck, P1. Col. IoS, 1822: Java.

Distr.-Java.

- I cannot separate birds from the Malay Peninsula, Sumatra, Sarawak and British North Borneo.

2Cf. Kloss, Journ. F.M.S. Mus. x, 1921, p. 200 and Bull. B. O. C. 1, 1930, D. 69 . 
* Chalcoparia singalensis panopsia Oberh.

Chalcoparia singalensis panopsia Oberholser, Sniths. Misc. Coll. Ix, no. 7, I9I2, 1. 2J: Tuangkn Island; Banjak Islands, West Simuatra.

Distr.-Banjak Islands, West Sumatra.

Gents ARACHNOTHERA 'T'enninck.

Arachnothera longirostra (Latls.)." Little Silici-hunter.

Arachnothera longirostra longirostra (1,atl.).

Certhia longirostra Lathan, Ind. Orn. i, 1790, 1). 299: Bengal.

Cinwyris cinereicollis Vieill, Nov. Dist. Hist. Nat. $x x x i, 1819$, p. joz: Malacca.

Arachnothera longirostris melanchima Oberli., Smith. Misc. Col]. Ix, No. 7,1912, p. $19:$ : East Sumatra.

Arachnothera longirostra exochra Oberh., Smiths. Misc. Coll. Ix, No. 7, 1912, 13. 19: South Pagi Island.

Arachnothera longirosira hypochra Oberh., Smiths. Misc. Coll. $1 \mathrm{x}$, No. 7 , 1912, p. 19: North Pagi Island.

Aracknothera longirostris antelia Oberh., Journ. Wash. Acad. Sci. xiii, 1923, p. 227 : Trang, Peninsular Siam.

Arachnothera longirostris heliocrita Oberh., Journ. Wash. Acad. Sci. siii, 1923, p. 228 : Singapore Island.

Distr.-Malay Peninsula.

Sinnatra; Lingga; Banka; Billiton; Mentawi Islands.

Arachnothera longirostra büttikoferi van Dort.

Arachnolhera longiroslra büllikoferi van Oort, Notes Leyden Mus. xxxii, I910, p. r95: Borneo.

Distr.-Borneo.

\section{Arachnothera longirostra prillwitzi Hart.}

Arachnothera longirostra prillwilzi Hartert, Nov. Zool. viit, 1901, 1. 51 : Java.

Distr,-Java.

Arachnothera longirostra rothschildi van Oort.

Arachnothera longirostra rolhschildi van Oort, Notes Leyden Mus. xxxii, I9I0, p. 195: Bunguran Island, North Natura Islands.

Distr. - North Natuma Islands.

*Arachnothera longirostra atita Oberh.

Arachnothera longirostra atita Uberholser, Bull. U.S. Nat. Mus. 159, 1932, 1) 104: Sirhassen Island, South Natuwa Islands.

Distr.-South Natuma Islands,

I Cf. Oberh., Buil. U.S. Nat. Mus. 159,1932 , p. 106. 
*Arachnothera longirostra zarhina Oberh.

Arachnothera longirosira zarhina Oberholser, Stnitlis. Misc. Coll.

$1 \mathrm{x}$, 10. 7, 1912, p. Ig: Bangkaru Island, Banjak Islands.

Distr.-Banjak Islands, West simmatra.

Arachnothera longirostra niasensis vall Oort.

Arachnolhera longirostra niasensis vall Oort, Notes Leydeu Mus. xxxii, 1910, p. 195: Nias Island.

Distr.-Nias Island, West Sunnatra.

Arachnothera magna (Hodgs.).

Large Streaked Spider-lıunter.

Arachnothera magna magna (Hodgs.)."

Cinnyris magna Hodgson, Ind. Rev. ii, I837, D. 272: Nepal.

Distr.-Malay States.

Arachnothera affinis (Horsf.). Grey-breasted Sivider-hntnter.

Arachnothera affinis affinis (Horst.).

Cinnyris affuis Horsfield, Trans. Lintu. Soc. xiii, IS21, ए. 166: Java.

Nectarinia inernata (sic) Temm., Pl. Col, $\$ 4$, fig. 2, 1821: Java.

Distr.-Java; Bali.

Arachnothera affinis modesta (Eyton).

Anthreples modesta Lyton, Proc. Zool. Soc. I\$39, p. 105 : Matacca. Arachnothera latirostris Blyth, Journ. Asiat. Soc. Beng. xii, 1843, 1). $98 z$ : Singapore.

Arachnolhera concolor Schleg., Veth's Midden-Sumatra, Aves, P!, I : Sumatra.

Arachnothera affitis heliophilns Oberh., Journ. Wash. Acad. Sci. xiii, 1923, 0. 226 : North-west Sumatra.

Distr.-Malay Peninsula.

Sumatra.

Burueo (part).

Arachnothera affinis everetti (Sliarpe).*

Arachnoraphis everelti Sharpe, Ibis, 1893, p. 56I: Mt. Kinabalu, North Borneo.

Distr.-Borneo (part).

It is noteworthy that the Malayan form seems not to be A, m. aurata Blyth, Journ. Asiat, Soc. Bengal, xxiv, 1855, 15. 478 (Pegu).

While 1 think that evereti is only a subspecies of 4 . affnis the ranges of A. a. modesta and A. a. everetli seem curiously complicated in Bomeo: the latter seems to be a mountain form descending into the lowlands in the northern part of the istand. 
Arachnothera chrysogenys (Temu11.). Vellow-eared Spider-hunter. Arachnothera chrysogenys chrysogenys (Temm.).

Nectarinia chrysogenys Temminck, Pl. Col. 388, 1S26: Java.

Cinnyris langirostra Horsf., Trans. Linn. Soc. xiil, 1821, p. 167: Java. Arachnothera favigenis Swains., Ann. Birds, ii, 1837 , p. 320 : nom. nov. Arachnothera chrysogenys copha Oberh., Smiths. Misc, Coll. 1x, No. 7. 1912, p. 20: North-west Sumatra.

Arachnothera chrysogeny's isopega Oberh., Smiths. Misc. Coll. 1x, No, 7, 1012 , p. 20 : Pagi Islands, West Sumatra.

Distr.-Malay Peninsula.

Sumatra; Rhio Archipelago; Mentawi Islands, West Sumatra. Borneo.

Java.

*Arachnothera chrysogenys pleoxantha Oberl.

Arachnothera chrysogenys pleoxantha Oberholser, Sunths. Misc. Coll. Ix, no. $7,1912,17.20$ : Nias Istand.

Distr.-Nias Island, West Sumatra.

Arachnothera juliae Sharpe.

Whitehead's Spider-hunter.

Arachnolhera julice Sharpe, 1bis, ISS7, p. 451, pl. I4: Mt. Kinabalu, Nortl Borneo.

Distr.-Borneo.

Arachnothera robusta Mïll. and Schleg. Long-billed Spider-liunter.

Arachnothera robusta robusta (Miill. and Schleg.).

Arachnolhera robusta Müller and Schlegel, Verh. Nat. Gesch. Zool. (Aves), I\$45, p. 6S, pl. xi, fig. i: West Sumatra.

Distr.-Malay Peninsula.

Sumatra.

Bormeo.

Arachnothera robusta armate 11 üll, and Schleg.

A rachnothera armata Müller and Schlegel, Verh. Nat. Gesch. Zool.

(Aves), IS45, 1\%, 68, pi. xi, fig. 2: Pangerango, West Java.

Arachwothera wropy'gialis Gray, Gen. Birds, i, 1847, pl. 33 : Java, Distr.-Java.

Arachnothera crassirostris (Reichenb,). Thick-billed Spider-hunter. Arachnocesira crassirostris Reichenbach, Handb. Scans. IS54, p. 314, no. 747, pl. 592, fig. 4016: India, error=Sumatra (Rob. and Kloss, 1924).

Arachnothera temmincki Horsf. and Moore, Cat. Bds. Mus. East Ind. Coy., ii, 1856, 13. 728 : Sumatra.

Distr._Malay Peninsula.

Sumatra.

Borneo. 
Arachnothera flavigaster (Eyton). Greater Yellow-eared Spikler-hunter. Anthreples Aarigaster Eyton, Proc. Zool. Soc. IS39, p. ros: Malacca.

Arachnothera latirostris Keichb. Handb. Scans. 1854, 1) 316. No. 747. pl. 593, fig5, 4024-25: Malacca.

Aracknothera eytonii Salvad., Ant. Mus. Civ. Ged, 5, 1\$74, p. $1 \$ 2$ (nom. nov.).

Arachnothera simillima Hume, Str. Featlis. 1 , $1877,13.487$ : Malacca.

Arachothera favizentris Gadow, Cat. Bds. Brit. Mus. ix, $1 \$ 84,1 \% .109$ (nom. nov.).

Distr.-Malay Peninsula.

Sumatra.

Borneo.

\section{Family MELIPHAGIDE.}

Geuus LICHMERA Cabanis.

Lichmera indistincta (Vig. and Horsf.).

[Lichmera indistincta indistincta (Vig. and Hursl.).

Meliphaga indistincta Vigors and Horsfield, 'Trans. Linn. Soc. xv, 1. $315,18_{27}$ : Solth-west Australia.

Distr.-Extra-linital.]

*Lichmera indistincta limbata S. Müll.

Meliphaga limbala S. Müller, Verh. Nat. Gesch. Laud en Volk., 1843, p. 162: 'Timor.

Distr. - Bali.

\section{Family MOTACILLIDE.}

\section{Genus MOTACILLA Linn.}

Motacilla alba Linu.

White Wagtail.

[Motacilla alba alba Linn.

Motacilla alba Limu., Syst. Nit, roth, ed, I758, p. IS 4 : Sweder.

Distr._Eixtra-linital.]

Motacilla alba ocularis Swiull.

Molacilla ocularis Swinloe, Ibis, IS60, p. 55: Annoy.

Distr.-Borneo; Batuguey Island, North Boruto.

Motacilla cinerea Tunst.

Grey Wagtail.

[Motacilla cinerea cinerea Tusst.

Molacilla cinerea Tunstall, Otn. Brit. $\$ 771$, p. z: Great Britain.

Distr.-Extra-limital.]

1 Cf. Stres., Nov, Zool. xix, 1912, p. 342. 


\section{MOTACILLIDE:}

Motacilla cinerea melanope Pall.'

Molacilla Melanope Pallas, Reise Prov. Russ. Reichs, iii, 1776 , p. 696: Siberia.

Molacilla bistrigala Raftles, Trans. Linn. Soc. xiii, 1822 , p. $3^{12}$ : Sumatra. Pallenura javensis Bi., Consp. Av. i, 1850, p. 250 : Java (nom nud.).

Distr.-Malay Peninsula; Ananla Islankis; Tanlelan Islands.

Sumatra; Simalur, Sipora, and Nias Islands, West Sumatra.

Borneo.

Java; Bali; Christmas Island.

Motacilla flava Linn.

\section{[Motacilla flava flava Liun.}

Molacilla Aava Limin., Syst. Nat, 1oth, ed, 1758, If, 185: Swedet1. Distr._E.xtra-Limital.]

Motacilla Hava taivana (Swints.).2

Greeti-headed Wagtail.

Budyles laivanus swiulioe, Proc. Zool. Soc. 1863, p1. 274 and 334 :Formusa.

Budytes metanotis Swinhoe, 1bis, 1844, 12, 422 : Singaive.

Disir.-Malay Peniusula.

Sumatra.

Motacilla flava simillima Hart.3

Hine-heacled Wagtail.

Molacilla llava simillima Hartert, Vög. Mal. Fauma, i, 1905, p. 289 : Sulu Islands.

Distr.-Malay Peninsula; Anauba Istands.

Sunatra; Simalur and Mentawi Islands, West Sumatra;

Billiton.

Borneo; Natuna Islands.

Java; ? Bali; Karimon-Java Islands; Christmas Island.

Motacilla flava macronyx (Stres.).4

Grey-headed Wagtail.

Budytes flavus macronyx Stresentann, Avif. Macedon., 1920, p. 76 :

Vladivostock.

Distr.-Matay Peninsula.

Java.

- See G. L. Bates, Bull. B. O. C1. 1v, 1034, p. 46 wherein a fine point of systematics is raised. We have adopted the name geographically most appropriate.

We have not seen this form from Borneo or Java and can trace no reliable records.

3 This seems to be the common form of $M$. flava in Malaysia, but we have seen few adults: these, however, are apparently simillima and not plexa Thayer and Bangs. M. f. macronyx and laizana are rarer in the Malay Peninsula at least) but the former seems to be more numerous in the north than in the south. We have seen a good series of it from Bangkok, Siam.

4 Only one or two adults are known from the Malay States. Series from Siam seem to be macrony'x rather than thunbergi (trorealis olim). 


\section{Genus DENDRONANTHUS Blyth.}

Dendronanthus indicus (Gine1.).

Fiorest Wagtail,

Motacilla indica Gmelin, Syst. Nat. i, pt. 2, 1759, p. 962: India.

Distr.-Malay Peninsula.

Sumatra; Simalur, Banjak Islands, Nias, and Mentawi Islands, West Simatra.

Borneo.

Java,

Genus ANTHUS Bechstein.

Anthus hodgsoni Richn1.

'Tree-Pipit.

[Anthus hodgsoni hodgsoni Richw.

Anthus hodgsoni Richnond, Publ. Carnegie Inst. Wasl. No. 54, 1907, p. 493: new name for A. maculatus Jerton: Nepal.

Dist.r.-Extra-linital.]

Anthus hodgsoni ? inopinatus Hart. anel Steinb.

Anthus hodgsoni inopinalus Hartert and Steinbacler, Vög. pal.

Faun., Ergänzungsband, Heft 2, 1933, p. 138: Sachalin.

Distr.-Malay Peninsula.

Anthus novaeseelandiae (Ginel.).2

Richard's Pipit.

[Anthus novaeseelandiae novaeseelandiae (Giue].).

Alauda novae Scelandiae Gnelin, Syst. Nat. i, pt. ii, 1789, p. 799 : New Zealand.

Distr.-Eixtra-liuital.]

Anthus novaeseelandiae sinensis $(\mathrm{BP} .)^{\circ} .3$

Corydalla sincnsis Bonaparte, Consp. Gen. Aviuu, i, 1850, p. 247 :

Southern China.

Distr.-Peninsular Siam.

Sumatra.4

Anthus novaeseelandise malayensis Eyton.

Anthus Malayensis Eyton, Proc. Zool. Soc, I\$39, p. 104: Malacca. Anthts enonyx Cab., Mus. Hein. i, 1850, p. 14: Java.

Anthus hasseltii Schleg., Handl. Dierk. i, 1857, p. 263 : Java.

Distr.-Malay Peninsula.

Sumatra; Rhio Archipelago; Nias Island, West Sumatra.

Borneo.

Java.

1'The few winter birds known from the Malay Peninsula are here, tentatively, referred to inopinatus purely on geographic grounds. $A$. hodgsoni is, perhaps, only á subspecies of $A$. trivialis Limn.

Revisions : Stres, Nov, Zool. xix, 1932, p. 315; Meinertz., Ibis, 1921, p. 631 .

$3 A, n$, richardi Vieill. occurs in South-west Siam and therefore will almost certainly be found in our area.

4 New record. 


\section{Anthus novaeseelandiae albidus Stres.}

Anhus richardi albidus Stresentann, Nov. Zool. xix, 1912, 1, 316 : Sontli Flores.

Distr. Bali.

Anthus campestris (Linin,).

Tawny Pipit.

[Anthus campestris campestris (Linn.).

Alauda campestris Linn, Syst. Nat, Ioth, ed. I758, [1. I66: Sweder1. Distr._Eixtra-limital.]

Anthus campestris striolatus Blyth.

Anthes striolatus Blyth, Jouru. Asiat. Soc. Bengal, xvi, 1847, p. 435: Darjiling.

Distr.-C"nistmas Island, Indian Ocean."

Anthus gustavi Siwiul.

Siberian Pipit.

*Anthus gustavi gustavi Swinh.

Anthus gustari Swinhoe, Proc. Zool. Soc. 1863, p. go: Anoy.

Distr.-Borneo.

Anthus rufogularis Brelin.

Red-throated Pipit.

Anthus rujogularis Brelum, Lebrb. Naturg. eur Vög. ii, IS24, p. g6, 3 : Nubia.

Distr.-Peninsular Sian.

\section{Family ALAUDIDÆ.}

Geitus MIRAFRA Horsfield.

Mirafra javanica Horsf.

Bush-Lark.

Mirafra javanica javanica Horsf.'

Mirafra Jananica Horsfield, 'Trans. Lint. Soc, xiii, 1821, p. I59: Java.

Alauda mirafra 'Tenm., PI. Col. 305, 1824: Java.

Mirafra borneeusis Swinh., Ann. Mag. Nit. Hist. (4) vii, 187t, p. 257 : Banjermasin, South Borneo.

Distr.-Borneo (south).

Java; Bali.

1 Mirafra cantillans williamsoni Baker, Bull. Brit. Orn. C1. xxxvi, 1915. p. 9 (Bangkok) is extremely close to this form.

= One specimen only, a puzzling bird and identification not absolutely certaia. Formerly recorded as malayensis.

$$
\left[2 S_{7}\right]
$$




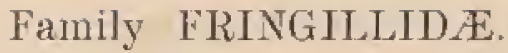

\section{Genus SERINUS Koch.}

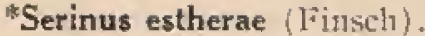

Javan Greenfinch.

Crithagra Ëshcrae Finsch, Notes Leyd. Mus. xxiii, Igoz, P, I5I: West Java.

Disit.-Java (west).

\section{Gemus PASSER Brisson.}

\section{Passer montanus (1.i1111.).}

Tree-Sparrow.

[Passer montanus montanus (Linn.).

Fringilla montan Lisn., Syst. Nat. Ioth. ed. 1758, p. I83: Italy.

Distr.-Eixtra-limital.]

Passer montanus malaccensis: Dub.

Passer malaccensis Dubois, Famna Ill. Vert. Belg., Uis. I, 1885 , p. 572 : Malacca.

Distr.-Malay Peninsula.

Sumatra; Rhio Arclipelago.

Java; Bali.

Passer flaveolus Blyth.

Pegu House-Sparrow.

Passer faveolus Blyth, Jotrin. Asiat, Soc. Bengal, xiii, I\$44, p. 946: Arracan.

Distr,-Peninstlat Sian.

\section{Genus PYRRHULA Brisson.}

\section{Pyrrhula nipalensis Hodgs.}

Bullfinch.

[Pyrrhula nipalensis nipalensis Hodgs.

Pyrrhula nipalcnsis Hodgson, Asiat. Kes. xix, 1836, p. 155 : Nepal. Distr.-Eixtra-linital.]

\section{Pyrrhula nipalensis waterstradti Hart.}

Pyrrhula waterstradt Hartert, Bull. Brit. Orn. C1. xii, I902, p. 69 : Mt. Tahan, Malay States.

Distr.-Malay States.

\section{Genus EMBERIZA Linn.}

Emberiza aureola Pall.

Vellow-breasted Bunting.

Emberiza aureola aureola Pall.:

Emberiza Aureola Pallas, Reise Prov. Russ. Reichs, ii, 1773, 1. 7 II : Irtysh. Distr.-Malay Peninsula.

1 There seems to be no reliable record for Borneo.

2 We have placed our birds under the typical race, but are not absolutely certain of their subspecific identity. 
* Emberiza pusilla Pall.

Little Bunting.

Emberiza pusilla Pallas, Reise Prov. Russ. Reichs. iii, 1776, p. 697 : Daurian Alps.

Distr.-Borneo.

\section{Family PLOCEID无.}

Genus AMANDAVA Blyth.

Amandava amandava (Linn.)."

Annandavat.

[Amandava amandava amandava (Linn.).

Fringilla Amandava Linn., Syst. Nat. Ioth. ed. 1755, p. I80: Bengal.

Distr._Eixtra-1imital.]

Amandava amandava punicea (Horsf.).

Fringilla punicea Horsf., Traus. Lin11. Soc. xiii, IS2I, p. I60 : Java.

Dislr.-Sumatra (introd.). Java; Bali.

Genus PADDA Reichenbach.

Padda oryzivora (Linn.)."

Java Sparrow.

Padda oryzivora oryzivora (Linj.).

Loxia oryzivora Linn., Syst. Nat, roth, ed. 1758, p. 173: "Asia" = Java.

Loxia javensis Sparrm., Mus, Carls. iv, 1789, pl, 89 : Java.

Padda verecunda Reich., Singv. I861, D. 4I, pl. xv, fig. 133: Java.

Oryzivora lewcotis Jerd, Birds Brit. Ind, ii, 1863, p. 359 : China,

Distr.-Malay States.

Sumatra; Rlio Archipelago; Billiton.

Borneo.

Java; Bali; Kangean Islands; Christmas Island; CocosKeeling Islands.

t Various forms of this species bave been imported into Singapore but have died out.

2 This species is now established in so many countries where it is not indigenous that its original natural range is now problematical, although there is reason for supposing that it was restricted to Java and Bali. 


\section{Genus MUNIA Hodgson.}

Munia atricapilla (Vieill.).

Black-headed Munia.

Munia atricapilla atricapilla (Vieill.).

Loxia atricapilla Vieillot, Ois. Chanteurs, I807, P. 84 , pl. 53 : Lower Bengal (restr. Rob, and Kloss, rg23).

Distr.-Peninsular Siam."

\section{Munia atricapilla sinensis Blyth.}

Munia sinensis Blyth, Cat. Bds. Asiat. Soc. Bengal, IS49, D. 337 : Penang.

Munia sumatrensis Bartlett, Monograph of Weaver Birds, 1888, D. 29. pl, 5: Sumatra.

Dislr.-Malay States.

Sumatra (lowlands); Rhio Archipelago.

Munia atricapilla batakana Clas, and Kloss.

Munia alricapilla balakana Chasen and Kloss, Bull. Raffles Mus. if, I929, p. 23 : Brastagi, Battak Highlands, North Sumatra.

Distr.-Sumatra (mountains).

Munia atricapilla ferruginosa (Sparrm.).

Black-throated Munia.

Loxia ferruginosa Sparman, Mus. Carls. iv, I 789 , pls. 9o, oI: Java.

Fringilla majanoide Temm., Pl. Col. 500, 1830 : Java.

Distr.-Java; Bali.

Munia atricapilla minuta: (Mey.).

Fringilla minufa Meyel, Verh. Carol.-Leopold. Akad. d. Naturf. xvi, I\&34, Suppl, 1. \$6 t. I2: Philippine Islands.

Distr.-Borneo; Natuna Islands.

Munia maja (Lin11.).

White-lieaded Munia.

Munia maja maja (Linn.).

Loxia Maja Linn., Syst. Nat. I2th. ed. i, 1766, p. 30I: East Indies = Malacca (restr. Rob. and Kloss, 1924).

Loxia lencocephala Raffles, Trans. Linn. Soc. xiii, 8822, p. 314 : Sumatra. Munia maja zapercma Oberh., Journ. Wash. Acad. Sci. xvi, 1026, p. 521 : Java.

Distr.,Malay Peniusula,

Simutra; Nias.

Java; Bali.

I Perhaps here not quite atricapilla but atricapilla $>$ sinensis.

- A review of this species is needed for the races seem curiously mixed in Malaysia, the Philippines, and Celebes, Birds from Borneo and the Natunas have been referred to brunneiceps Wald., of Macassar, Celebes, but some skins from North Borneo, and from the Natuna Islands are nearer to minuta (syn. jagori). 
* Munia maja simalurensis Oberl.

Munia maja simalurensis Oberholser, Journ. Wash. Acad. Sci. xvi, 1926, p. 521 : Simalur Island.

Disir.-Simalur Island, West Sumatra.

Munia punctulata (I,inn.).'

Spotted Munia.

Munis punctulata punctulata (Limn.).

Munia punctulata Linn., Syst. Nat. Ioth. ed. 1758, p. 173: Asia= Java (Gmel., 1789).

Loxia undulata P. L. S. Müller, Syst. Nat. Anhang, 1776, p. 131 : Java.

Loxia punctularia Gmel., Syst. Nat. i, pt. 2,1789, p. 851 : Java.

Munia nisoria Temm., Pl, Col. 500, fig. 2, IS30: Java.

Distr.-Java; Bali; Bawean Island.

Munia punctulata fretensis Kloss.

Wunia punctulata fretensis Kloss, Treubia, xiii, 1931, p. 363: Kivala Lumpur, Selangor, Malay States.

Distr.-Malay States. ${ }^{3}$

Sumatra; Nias Island.

Munia striata (Linn.).

Shatp-tailed Munia.

[Munia striata striata (Linn.).

Loxia striata Liun., Syst. Nat. 12tlı. ed. i, 1766, 1). 306: Ceylon.]

Munia striata subsquamicollis (Baker).

Uroloncha striata subsquamicollis Stuart Baker, Bull. Brit. Orn. Cl., xlv, 1925, p. 59: South Tenasserin.

Uroloncha acuticatuda lepidota Oberh., Journ. Wash. Acad. xvi, t926, D. 520: Trang, Peninsular Siam.

Distr.-Malay Peninsula.

Sumatra; Banka.

Munia leucogastra (Blytlı).

White-bellied Munia.

Munia leucogastra leucogastra (Blyth).

Amandina leucogastra Blyth, Journ. Asiat. Soc. Bengal, xv, I846, p. 286 : Malacca.

Mnvia chrysura Blyth, 1bis, 1870, p. 172: nom. nud.

Distr.-Malay Peninsula.

Siumatra.

Borneo. 4

${ }^{2}$ Revision: Stres., Nov, Zool, xix, 1912, p. 317

I Reference to the two works indicates that Gmelin's punctularia is the same thing as puctulata Linn.

3 The form occurring at Koh Lak in Soutb-west Siam is subundulatiz Godw. Aust., but unfortunately we have never seen birds from Peninsular Siam.

* Apparently not common, but it occurs in the neighbourhood of Kuching in Sarawak. 


\section{PLOCEIDE.}

Munia leucogastra leucogastroides Horsf. and Moore.

Munia leucogaströdes Horsfield and Moore, Cat. Bds, Mus. East Ind. Coy. ii, 1856, p. 510 : Java.

Trichogramoptila melanopygia Reichenb., Singv. 186t, pp. 39, 48. Taf, 17, figs. 153, 154 : Sumatra.

Distr.-Sumatra. ${ }^{\text {. }}$

Java; Bali.

Munia fuscans (Cass.).

Brown Munia.

Spermestes fuscans Cassin, Proc. Phil. Acad. vi, 1852, p. 185 : Borneo.

Fringilla nigerrima Cass., Proc. Phil. Acad, vi, 1852, p. $185:$ nom. nud. Fringilla aterrima Cass., Proc. Phil. Acad. vi, 1852, p. 185: nom. nud. Distr.-Borneo; Banguey Island; Natuna Islands.

Munia molucca (Limn).

Moluccan Mumia.

[Munia molucea molucca (Linn.).

Loxia molncca Linn., Syst. Nat. I 2 th ed. i, I766, P. 302 : Amboina. Distr.-Extra-limital.]

*Munia molucca propinqua (Slarpe).

Uroloncha propinqua Sharpe, Cat. Birds, xiii, 1890, p. 368: Flores.

Uroloncha kangeangenis Vorderm., Nat. Tijds. Ned. Ind. 1893, lii, p. Ig9: Kangean Island.

Distr.-Kangean Islands.

\section{Genus ERYTHURA Swainson.?}

Erythura prasina (Sparrm.).

Long-tailed Munia.

Erythura prasina prasina (Sparrm.).

Loxia prasina Sparman, Mus. Carls. I788, pls. 72, 73 : Java.

Emberisa quadricolor Gmel., Syst. Nat. i, pt. 2, 1789 , p. 886 : Java.

Emberisa cyanopis Gmel., Syst. Nat. i, pt. 2, 1780 , p. 886 : Java.

Fringilla sthecura Temm., P1. Col. 96, 1822: Java.

Fringilla sphenura Swains. in Jard. Nat, Libr., Birds West Africa, i, 1837 , p. 204 : nom. nov.

Erythura viridis Swain5. in Jard. Nat. Libr., Birds West Africa, i, 1837 , p. 204, footnote: nom. nov.

Distr.-Malay Peninsula.

Sumatra.

Java.

- Both leucogastra and leucogastroides occur in Soutb Sumatra: the latter is probably introduced.

a Cf. Mayr, Amer. Mus. Nov. 193I, p. 489, p. I. 
Erythura prasina coelica Baker.

Erythrura parasina calica Stuart Baker, Bull. Brit. Orn. Cl., xlv, 1925, p. 84 : Borneo.

Distr.-Borneo.

Erythura hyperythra (Reichenb.).

Bamboo-Munia.

Erythura hyperythra hyperythra (Reichenb.).

Chloruta hyperythra Reichenbach, Singvög. I862, p. 33, tab. xi, fig. 97 : New Guinea error= Java.

Distr.-Java (west).

Erythura hyperythra malayana (Rob.).

Chlortura hyperythra malayana Robinson, Bull. Brit. Orn. Cl., xlviii, 1928, p. 72 : Cameron's Highlands, Malay States.

Distr.-Malay States.

Erythura hyperythra borneensis (Sharpe).

Chlorura borncensis Sharpe, Ann. Mag. Nat. Hist. (6) iii, 188g, p. 424 : Mt. Kinabalu, North Borneo.

Distr.-Borneo.

\section{Genus PLOCEELLA Hume.}

Ploceëlla hypoxantha (Sparrm.). ${ }^{1}$

Golden Weaver-Finch.

Ploceëlla hypoxantha hypoxantha (Sparrm.).:

Loxia hypoxantha Sparrman, Mus. Carls, iii, J788, tab. 7 I : Sumatra.

Loxia javanensis Less., Traité d’Orn. $183 \mathrm{I}$, p. 446 : Java.

Distr.-Sumatra.

Java.

\section{Gents PLOCEUS Ctuvier.}

Ploceus philippinus (Linn.).

Weaver-Finch.

[Ploceus philippinus philippinus (Linn.).

Loxia philippina Linn., Syst. Nat. 12th. ed. i, 1766, p. 3015:

Philippines error $=$ Ceylon .

Distr._Extra-limital.]

The Continental form of this species seems separable and is $P . h$. chrysea Hume (Teдasserim).

- At one time introduced birds were apparently well-established and breeding in the Cocos-Keeling Islands. 
Ploceus philippinus infortunatus Hart."

Ploceus passerinus infortunatus Hartert, Nov. Zool. ix, r902, p. 578 : Pahang, Malay States.

Distr.-Malay Peninsula.

Sumatra; Nias Island.

Java.

Ploceus manyar (Horsf.)..$^{2}$

Streaked Weaver-Finch.

Ploceus manyar manyar (Horsf.).

Fringilla Manyar Horsfield, Trans. Linn. Soc. xiii, I82 I, D. I60: Java.

Nelicurvius emberizinus Reichenb., Singv, 1861, p. 76 , t. 34 , figs. $269-272:$ Ceram $=$ Java.

Distr.-Java; Bali; Bawean Island.

\section{Family STURNIDE.}

\section{Gents STURNOPASTOR Blyth,}

Sturnopastor contra (Linn.).

Rose-coloured Starling.

[Sturnopastor contra contra (Linn.).

Sturnus Contra Linn., syst. Nat. Ioth. ed. I758, p. I67 : India.

Distr.-Extra-linital.]

Sturnopaster contra jalla (Horsf.).

Pastor Jalla Horsfield, Traus. Linn. Soc. xiii, IS21, p. I55: Java.

Pastor oricularins Drap., Dict. Class, vii, I841, D. 153: Java.

Distr.-Sunatra.

Java; Bali.

\section{Gentus STURNIA Lesson.}

Sturnia sinensis (Gmel.).

Chinese Starling.

Oriolus sinensis Gmelin, Syst. Nat. i, pt. i, 1788, p. 394 : China.

Distr.-Malay Peninsula.

*Sturnia philippensis (Forst.).

Violet Starling.

Molacilla philippensis Forster, Ind. Zool, 1781, 1. 4I: Philippine Islands.

Distr.-Borneo.

'Cf. Ticehurst, Bull. B. O. C. lii, 1932, p. 104.

2 Stres, Nov, Zool, xix, igiz, p. 318. 
Sturnia sturnina (Pall.).

Daurian Starling.

Gracula sturnina Pallas, Reise Prov. Russ. Reichs. iii, 1776, p. 695 : Dautia.

Turdus strige Raffles, Trans. Linn. Soc. xiii, 1822, p. 311 : Sumatra.

Pastor malayensis Eyton, Proc. Zool. Soc. 1839, p. 103 : Malacca.

Disir.-Malay Peninsula.

Sumatra; Simalur aud Sipora Islands, West Sumatra. Java.

\section{Genus AMPELICEPS Blyth.}

Ampeliceps coronatus Blytl.

Gold-crested Myna.

Ampeliceps coronatus Blyth, Journ. Asiat. Soc. Bengal, xi, 1842 , P. I94: Tenasserin.

Disir.-Peninsular Siam.

\section{Genus GRACUPICA Lesson. ${ }^{\prime}$}

Gracupica melanoptera (Daud.). Javan Myna,

Gracupica melanoptera melanoptera (Datid.),

Gracula melanoplera Daudiı, Traité d'Orn. ii, I80o, p. 286: West Java.

Distr.-Java (west).

Gracupica melanoptera tricolor (Horsf.).

Pastor iricolor Horsfield, Trans, Limn, Soc, xiii, IS2I, P. I55: Java (restr., liast Java, Rob. and Kloss, 1024).

Distr.-Java (east).

Gracupica melanoptera tertia Hart.

Graculipica tertia Hartert, Nov, Zool, iii, I896, P. 547: Bali.

Distr.-Bali.

GeuLs ACRIDOTHERES Vieillot.

Acridotheres tristis (Linn.).

Common Myna.

Acridotheres tristis tristis (Liun.).

Paradisea tristis Limn., Syst. Nat. 12th. ed. i, 1766 , p. I67: Philippines, error $=$ Calcutta $($ Baker $)$.

Distr.-Peninsular Siam. ${ }^{3}$

- Gracupica leucocephala has been recorded from Perak, but we do not believe the bird exists there in a wildstate.

- Has been introduced into Singapore but seems to bave died out.

3 Introduced birds are established in several places in the Malay States, notably in Perak and Province Wellesley. There are a few pairs on Singapore Island. 
Genus AETHIOPSAR Oates.

\section{Athiopsar fuscus (Wagl.).}

[Fthiopsar fuscus fuscus (Wagl.).

Pastor fuscus Wagler, Syst. Avinm, I827, Pastor, sp. 6: India $=$ Nepal (restr., Rob, and Kloss, 1024).

AEthiopsar fuscus torquatus (Davison).

Buffalo Mỹua,

Acridotheres torqualus Davison, Ibis, I892, p. I02: Pahang.

Disir.-Malay Peninsula.

Athiopsar grandis (Horsf. and Moore).

Jungle Myna.

[Aethiopsar grandis grandis (Horsf. and Moore).

Acridotheres grandis Horsfield and Moore, Cat. Bds. Mus, East India

Coy. ii, I858, p. 537: Sumatra, error=Tenasserin (Baker).

Distr.-Extra-linital.]

Ethiopsar grandis javanicus (Cab.).

Acridotheres javanicus Cabanis, Mus. Hein. i, 1850, p. 205 : Java.

Pastor grisetes (not of Wagl. 1800 ) Horsf., Trans. Linn, Soc. xiii, 1821.

p. 154: Java.

Distr.-Java; Christmas Island (introd.).

Sumatra (introd.).

Genus LEUCOPSAR Stresemann.

Leucopsar rothschildi Stres.

Leucopsar rothschildi Stresemann, Bull, Brit. Orn, Cl. xxxi, Igr2, p. 4: Bali.

Distr.-Bali.

Family GRACULID Æ.

Genus GRACULA Linn.

Gracula religiosa Linn. ${ }^{1}$

Grackle.

Gracula religiosa religiosa Linn.2

Gracula religiosa Linn., Syst. Nat. Ioth. ed. I, I758, p. Io8: Java. Pastor musicus Temm., Man. d'Orn. 2nd. ed. i, I820, pl. 55 : Java.

Eulabes javanus Cuv., Règne Anim. 2nd. ed. i, 1829, p. 377 : Java.

Mainatus sumatranus Less., Traité d'Orn. 1830 , p. 357 : Sumatra.

Gracula javanensis Blyth, Journ. Asiat. Soc. Bengal, xv, 1846, p. 31 : Malacca.

Distr.-Malay Peninsula (north to Trang).

Sumatra; Rhio Archipelago; Banka; Billitot,

Borueo; Natura Islands; Karimata Islands.

Java; Bali; Kangean Islands; Christınas Island (introd.).

' Revisions: Stres., Nov. Zool. xix, 19!2, 1). 313; Oberh., Bull. U.S. Nat. Mus. I 59,1932 , p. 92 .

- Oberholser (1932) also quotes as a synonym :-Gracula mintor Forst., Ind. Zool. 1781 ; based on Edwards, Nat, hist. uncommon birds $i, 1776$, pl. 17, p. 17 (Borneo). 
Gracula religiosa intermedia $\mathrm{A}$. Hay.

Gracula intermedius A. Hay, Madr. Journ. Lit. Sci. xiii, pt. 2, I844, p. I57 : Cachar.

Distr.-Peninsular Sian.

Gracula religiosa prasiocara Oberl..

Gracula javana prasiocara Oberholser, Bull. U.S. Nat. Mus. 98, 1917, p. 55: Pulau Piling, Anamba Islands.

Distr.-Tionan Island; Anamba and Tambelan Islands.

* Gracula religiosa baweana Obert.

Gracula javensis baweana Oberholser, Proc. U.S. Nat. Mus. lii, 1917, p. 195: Bawean Island.

Distr.-Bawean Island, Java Sea.

Gracula religiosa miotera Oberh.

Gracula javanensis miotera Oberholser, Smiths. Misc. Coll. 1x, no. 7, I9I2, p. I6: Simalur Island,

Distr.-Sinalur Islaud, West Sumatra.

* Gracula religiosa ophellochlora Oberh.

Gracula javanensis ophellochlora Oberholser, Smiths. Misc. Coll, 1x, no. 7, 1912, p. I7: Tuanku Island, Baujak Islands.

Distr-Banjak Islands, West Sumatra,

Gracula religiosa robusta Salvad.

Gracula robusta Salvadori, Ann. Mus, Civ. Gen. (2) iv, 1887, p. 554 : Nias Island.

Distr.-Nias and Babi Islands, West Sumatra.

Gracula religiosa batuensis Finsch.

Gracula batuensis Finsch, Notes Leyden Mus. xxi, I899, p. I4: Tello Island, Batu Islands.

Distr,-Batu and Mentawi Islands, West Sunatra.

Gracula religiosa enganensis Salvad.

Gracula enganensis Salvadori, Ann. Mus. Civ. Gen. (2) xii, 1892, p. I37: Engano Island.

Distr.-Engano Island, West Sumatra,

Genus APLONIS Gould.

Aplonis panayensis (Scop.). .

Tree-Starling.

[Aplonis panayensis panayensis (Scop.).

Muscicapa panayensis Scopoli, Del Flor. et Faun. Insubr, ii, I783, p. 96 : Philippine Islands.

Distr._Extra-linital.]

I Revisions: Stres, Zool. Nov, xx, 1913, p. 375 : Oberh., Bull. U.S. Nat. Mus. I 59, 1932, p. 94 . 
Aplonis panayensis strigatus (Horsf.).

Turdus strigatus Horsfield, Trans, Linn. Soc. xiii, I82I, p. I4S: Java.

Turdus chalybeus Horsf., Trans. Linn. Soc. xiii, 1821, p. 148: Java.

Lanius insidiator Raffles, Trans. Linn. Soc. xiii, 1822, p. 307: Sumatra.

Lamprocorax panayensis eustathis Oberb., Journ. Wast. Acad. Sci. xvi, 1926, p. 516 : East Borneo.

Lamprocorax panayensis halictypus Oberh., Journ. Wash. Acad. Sci. xvi, I926, p. 516,: Telibon Island, Trang, Peninsular Siam.

Distr._- Malay Peninstrla. ${ }^{\text {}}$

Sumatra; Banka; Billiton.

Borneo.

Java.

Aplonis panayensis gusti Stres.

Aplonis panayensis gusti Stresemann, Nov. Zool. xx, 1913, p. 375 : Bali.

Distr.-Bali.

Aplonis panayensis heterochlorus (Oberh.).

Lamprocorax panayensis heterochlorus Oberholser, Bull. U.S. Nat.

Mus, 98, 1917, p. 57: Mobur Island, Anamba Islands.

Distr.-Aor and Tioman Islands; Rhio Archipelago; Anamba Islands; Tambelan Islands.

Natuna Islands; Mantanani Islands; North Bornean Islands; Karitnata Islands.

*Aplonis panayensis richmondi (Oberh.).

Lamprocorax panayensis richmondi Oberholser, Proc. U.S. Nat. Mus. 1v, I919, P. 27z: Taya Island, South-east Sumatra.

Distr._Taya Island, South-east Sumatra.

Aplonis panayensis alipodis (Oherl.).

Lamprocorax panayensis alipodis Oberholser, Journ. Wash. Acad. Sci, xvi, 1926, p. 516: Panjang Island, near Maratua Island, East Borneo.

Aplonis panayensis suggrandis Bangs and Peters, Occ. Pap. Bost. Soc.

Nat. Hist. v, 1927, 12. $24 t$ : Maratua Island, East Borneo.

Distr._Maratua Islands, East Borneo.

Aplonis panayensis altirostris (Salvad.).

Calonis allirostris Salvadori, Ann. Mus. Civ. Gen. (2) iv, ISS7, p. $553, \mathrm{pl}$. 9, fig. I : Nias Island.

*Lamprocorax chalybeus rhadinorhynchus Oberb., Smiths. Mise. Coll. $1 \pi$, No. 7,1912, p. 17 : Simalur Island.

* Lamprocorax panayensis nesodramus Oberh., Journ. Wash. Acad. Sci. xvi, 1926 , p. 516: Babi Island.

Distr. - West Sumatran islands of Simalur, Babi and Nias.

* Birds from the Malay Peninsula are really A. p. strigatus $>$ aftnis Blyth. While it seems clear that the northern part of Sumatra is occupied by a small race (strigatus), I have recently been puzzled by the receipt of three very large birds with wings measuring $108-115 \mathrm{~mm}$. Perhaps they are migrants from elsewhere. 
*Aplonis panayensis leptorrhynchus Stres.

Aplonis panayensis leplorrhynchus Stresemann, Nov. Zool. xx, 1913,

p. 377 : Pinie Island, West Sumatra.

Dislr.-Batu Islands, West Simatra.

Aplonis panayensis pachistorhinus (Oberli.).

Lamprocorax chalybeus pachislorhinus Oberholser, Smiths. Misc.

Coll. Ix, no. 7, 1912, p. 17 : South Pagi Island.

Distr.-Mentawi Islands (Siberut, Sipora, Pagi), West Sumatra.

-Aplonis panayensis enganensis (Salvad.).

Calomis enganensis Salvadori, Anu. Mus, Civ, Gen. (2) xii, r892, p. 137: Engano Island.

Distr.-Engano Island, West Sutnatra.

Aplonis minor $\left(\mathrm{B}_{\mathrm{p}}\right.$.).

Sinall Tree-Starling.

Aplonis minor minor (Bp.).

Lamprolornis minor Bonaparte, Consp. Gen. Avium i, $1 S_{50}$, p. 417 :

Timor.

Distr.-Java; Bali.

\section{Family ORIOLIDÆ.}

\section{Gentis ORIOLUS Linn."}

Oriolus chinensis (Limn.).

Black-naped Oriole.

[Oriolus chinensis chinensis Linn.

Oriolus chinensis Limn, Syst. Nat. 12th. ed. i, r766, p. 160: China error = Manila (restr. Meinertzhagen, 1023).

Distr._Extra-linital.]

Oriolus chinensis diffusus Sharpe.

Oriolus diffusus Sharpe, Cat. Bds. Brit. Mus. iii, 1877, p. 197 : Malabar.

Distr.-Malay Peniusula.

Oriolus chinensis maculatus Vieill.

Oriolus maculatus Vieillot, Nov. Dict. d'Hist. Nat. ed. 2, xviii, ISr7, p. r94: Java.

Oriolus coronatus Swains., Anim. in Menag. 1838 , p. 342: Java.

Oriolus horsfieldi Bp., Consp. Gen. Av. i, 1850, p. 348: "Malaiasia" = Java.

Distr.-Sunatra; Billiton; Banka; Nias (? subsp.).

Borneo.

Java; Bali.

- Review : Meinertz., Ibis, 1923, p. 52. 
ORIOLIDE.

*Oriolus chinensis insularis Vorderm.

Oriolus insularis Vorderman, Nat. Tijds. Ned. Ind. lii, I893, p. 200: Kangean Island.

Distr.-Kangean Islands; islands of Sapudi and Raas, east of Madura.

*Oriolus chinensis lamprochryseus Oberh.

Oriolus maculatus lamprochryseus Obetholser, Proc. U.S. Nat. Mus. liv, 1917, p. 186: Solombo Besar Island, Java Sea.

Distr.-Solombo Besar and Arends Island, Java Sea.

Orialus chinensis mundus Riclim.

Oriolus mundts Riclimond, Proc. U.S. Nat. Mus. xxvi, 1903, p. 517 : Simalur Island.

Distr.-Simalur Island, West Sumatra.

Oriolus chinensis siberu Chas. and Kloss.

Oriolus chinensis siberu Chasen and Kloss, Ibis, I926, p. 294 : Siberut Island.

Distr.-Sibertut Island, West Sumatra.

Oriolus chinensis sipora Chas, and Kloss.

Oriolus chinensis sipora Chasen and Kloss, Ibis, 1926, p. 294 : Sipora Island.

Distr.-Sipora Island, West Sumatra.

*Oriolus chinensis richmondi Oberh.

Oriolus maculatus richmondi Oberholser, Smiths. Inst. Misc. Coll. $1 \mathrm{x}, 110,7$, I912, p. I6: North Pagi Island.

Distr,-Pagi Islands, West Sunatra.

Oriolus xanthornus (Linn.). Indian Black-headed Oriole.

[Orio]us xanthornus xanthornus (Linn.).

Coracias xanthornus Linu., Syst. Nat. Ioth. ed. 1758, p. roS: Bengal.

Distr.-Extra-limital.]

Oriolus xanthornus thaiocous Hart.

Oriolus luteolus thaiocous Hartert, Bull. Brit. Orn. Cl. xxxviii, 1918, p. 63: Koh Lak, South-west Siam.

Distr._Peninsular Sian."

Sumatra.

- More precisely, south to the Langkawi Islands. 
ORIOLIDA.

*Oriolus xanthornus tanakae Kuroda,"

Oriolus xanthornus lanakae Kuroda, Tori, iv, I9, I925, P. 3 : Tawao, east coast of British North Borneo.

Distr.-Borneo; Maratua Islands.

Oriolus xanthonotus Horsf.

Black-lseaded Oriole.

Oriolus xanthonotus xanthonotus Horsf.

Oriolus Xanthonolus Horsfield, Trans. Linn. Soc. xiii, $x \mathrm{~S}_{21}$, P. I52: Java.

Xanthonotus lewcogaster Temm., Pl. Col, 214,1823 : Java.

Oriolus castanopterws Blyth, Joura. As. Soc. Bengal, xi, 1842, p. 796 : Singapore.

Distr.-Malay Peninsula.

Sumatra; Banka.

Borneo (west).

Java.

Oriolus xanthonotus consobrinus Wardl, Rams.

Oriolus consobrinus Wardlaw Ramsay, Proc. Zool. Soc. I879, p. 709: Sandakan, North Borneo.

Distr,-Borneo (north); North Bornean Islands.

Oriolus xanthonotus mentawi Chas. and Kloss.

Oriolus xanthonolus menlawi Chasen and Kloss, Ibis, I926, p. 295 : Siberut Island.

Distr.-Siberut and Sipora Islands, West Sumatra.

Oriolus hosii Sharpe.

Oriolus hosii Sharpe, Bull. Brit. Orn. C1, i, IS92, P. 4: Mt. Dulit, Sarawak, Borneo.

Distr.-Borneo.

Oriolus cruentus (Wagl.).

Black-and-Crimson Oriole.

Oriolus cruentus cruentus (Wagl.).

Leptopleryx cruentus Wagler, Syst. Av,, I827, addit. Leptopleryx, sp. 9: Java.

Ocypterus sanguinolentus Temm., Pl. Col. 499, 1830, pl. 499: Java.

Erythrolane rubropectus Less., Rev, Mag. Zool. 1840, p. 274 : new name only (nud.).

Distr.-Java.

Oriolus cruentus consanguineus (Ward̉. Rams.).

Analcipus consanguineus Wardlaw Ransay, Ibis, I88I, p. 33, pl. I, figs. 2, 3: Sumatra.

Distr.-Sumatra.

- The recent discovery of an, apparently, resident race of this Oriole in Borneo, where its range must be very restricted, is remarkable. 
Oriolus cruentus malayanus Rob. and Kloss.

Oriolus crucntus malayanus Robinson and Kloss, Joutn. Fed. Malay

States Mus. xi, 1923, 13. 56 : Semangko Pass, Malay States.

Distr.-Malay States.

Oriolus cruentus vulneratus Sharpe.

Oriolus tulneralus Sharpe, Ibis, $ז S S_{7}$, p. 437 : Mt. Kinabalu, Nortlt Borneo.

Distr.-Borneo.

\section{Family DICRURIDÆ.}

\section{GentIs DICRURUS Vieillot.}

Dicrurus annectans (Hodgs.).'

Crow-billed Drongo.

Dicrurus annectans annectane (Hodgs.).

Bhuchanga anneclans Hodgson, Ind. Rev. i, $1 S_{37}$, p. 326 : Nepal.

Edolius afhnis Blyth, Joura. Asiat. Soc. Bengal, xi, I 842, p. 174: Malacca.

Distr.-Malay Peninsula.

Sumatra.

Borneo.

Java (west).

Dicrurus macrocercus Vieill."

"Dicrurus macrocercus macrocercus' Vieill.

Black Drongo.

Dicrurus macrocercus Vieillot, Nov. Dict. d'Hist. Nat. ed. 2, ix, I 8 I 7, ए. 5 SS: Pentinsular India.

Distr.-Extra-limital.]

Dicrurus macrocercus cathoecus Swint.

Dicrurus calhacus Swinlioe, Proc. Zool. Soc. 1871, p. 377 : South China.

Distr.-Peninsular Siant.

Dicrurus macrocercus javanus Kloss.*

Dicrurus macrocercus javanus Kloss, Journ. Fed. Malay States Mus. $x, 1921, p .208$ : East Java.

Distr.-Java; Bali.

' Revision, Baker, Nov. Zool. xxvi, I019, p. 41.

? Revision, Baker, Nov, Zool. xxv, 1918, p. 296 ; Kloss, Journ. F.M.S. Mus. X, 1921, p. 207; Ticehurst, Bull. B. O. C. liii, 1932, p. 20.

${ }^{3}$ Stephen's selection of "India" for the type locality of $D$. indicus (1826). based on "Le Drongolon" of Levaillant is earlier than Cabanis' fixation of "Java" for $D$. bilobus ( 1850$)$ based on the same plate.

"We suggest that "Buchanga atra Herm." Richm., Proc. U.S. Nat. Mus. xxvi, 1903, p. 516 is Dicrurus annectens. 
Dicrurus longicaudatus Jerd.

Grey Drongo.

[Dicrurus longicaudatus longicaudatus Jerd.

Dicrurus longicandatus Jerdon, Madras Journ. Lit. Sci. xiii, pt. 2, IS45, p. I 2 I : Nilgiris.

Distr,-Extra-linital.]

Dicrurus longicaudatus intermedius Blytl.

Dicrums intermedius Blyth, Journ. Asiat. Soc. Bengal, xv, I846, p. 29 S: Penang.

Distr.-Malay Peninsula (south to Perak).

Dicrurus leucophaeus Vieill. ${ }^{1}$

Ashy Drongo.

Dicrurus leucophaeus leucophaeus Vieill.

Dicrurus leucophaeus Vieillot, Nov. Dict. d'Hist. Nat., ed. 2, ix, I 8 I $7, p, 587$ : Ceylon error= Java (Tweeddale, $1 \$ 78$ ).

Edolius cineraceus Horsf., Trans. Linn. Soc, xiii, $1821, \mathrm{p} .145$ : Java.

Dicrurus ceylonensis Stephs., in Shaw's Gen. Zool. (Aves) xiii, (2) 1826, p. 140: Java.

Dicrurus cinerens Swains, Class Bds, $\mathrm{ii}_{1} 1837, \mathrm{p}, 223$ : Java,

Distr.-Java; Bali.

Dicrurus leucophaeus phaedrus Reichn.

Buchanga stigmatops phacdra Reichenow, Wissensch. Eirgebn. Deutsch Tiefsee Exped. vii, 1904, p. 356: West Suntatra.

Buchanga leucophaea batakensis Rob. and Kloss, Journ. Straits Branch Roy. Asiat. Soc. 1xxx, 1919, P. 125: North-east Sumatra.

Distr.-Sumatra.

Dicrurus leucophaeus stigmatops Sharpe.

Buchanga stigmatops Sharpe, Proc. Zool. Soc. 1S79, 1. 247 : Northwest Borneo.

Buchanga leucophaea penrissenensis Harrisson and Hartley, Bull. B. O. Cl. liv, 1934, p. 157: Mt. Penrissen, Sarawak.

Buchanga lencophea dulitensis Harr. and Hart., Bull. B. O. Cl. liv. 1934, p. I 58 : Mt. Dulit, Sarawak.

Distr.-Borneo.

Dicrurus leucophaeus leucogenis (Wald.).

Buchanga leucogenis Walden, Aun. Mag. Nat. Hist. (4), v, I870, p. 219: Japan, error = Yunnan (restr. Kloss, I931).

Dicrurus lencogenys var. salangensis Reichn., Nomenc. Mus. Hein. 1800, p. 69 : Junk Seylon.

Distr.-Malay Peninsula.

- Revisions: Baker, Nov. Zool. xxv, 1918, p. 291 ; Stres., Orn. Monatsber. xxxviij, 1030, p. 58 ; Kloss, Treubia, xiii, I931, p. 358.

a We have not seen this reference but the name is, no doubt, founded on a migrant and may therefore antedate $D$. $l$. cerissatus. 
Dicrurus leucophaeus cerussatus (Bangs and Phillips).

Buchanga leucogenys cerussala Bangs and Phillips, Bull. Mus.

Comp. Zool. Harvard, lviii, rgr4, p. 302: Hupeh, China.

Distr.-Peninsular Siant.

- Dicrurus leucophaeus celaenus Oberh.

Dicrurus cineraceus celaenus Oberholser, Sniths. Misc. Coll. lx, no. 7 , I912, р. 15: Simalur Island.

Distr.-Simalur Island, West Sumatra.

Dicrurus leucophaeus siberu Chas. and Kloss.

Dicrurus leucogenis siberu Clasen and Kloss, Ibis, 1926, p. 294 : Siberut Island.

Distr.-Siberut Island, West Sumatra.

Dicrurus leucophaeus periophthalmicus Salvad.

Buchanga periophthalmica Salvadori, Ann. Mus. Genov. (2) xiv, r\$94, p. 594 : Sipora Island.

Dicrurus lencogenis diporus Oberholser, Smiths. Misc. Coll. $1 x$, No. 7 , 1912, p. 15: North Pagi Island, West Sumatra.

Distr.-Sipora and Pagi Islands, West Sumatra.

Dicrurus hottentottus (Linn.). ${ }^{1}$

Hait-crested Drongo.

[Dicrurus hottentottus hottentottus (Linn.).

Corvus hottentottus Linn., Syst. Nat. I2th. ed. I, I766, p. I55: Sikkim (Baker, Igrg).

Dist. -Extra-limital.]

Dicrurus hottentottus sumatranus Wardl. Rams.

Dicrurus sumatranus Wardlaw Ramsay, Proc. Zool. Soc. I880, p. I5: West Sumatra.

Disir.-Sumatra.

Dicrurus hottentottus borneensis (Sharpe).

Dicruropsis borneensis Sharpe, Proc. Zool. Soc. I879, p. 246 : North-west Borneo.

Distr.-Borneo.

* Dicrurus hottentottus termeuleni (Finsch).

Chibia ler Meuleni Finsch, Joum. f. Orn. liv, I907, p. 302: Thousand Islands, Java Sea (off West Java).

Distr. -Thousand Islands, West Java,

'Revision: Baker, Nov, Zool. Xxvi, 1919, p. 44; Kloss, Journ, F.M.S. Mu5. X, I92 r, p. 221. 
Dicrurus hottentottus jentinki (Vorderm.).

Chibia jenlinki Vorderman, Nat. Tijdschr. Ned. Ind. lii, 1893, p. I94: Kangean Island.

Distr, -Java (east); Bali; Kangean Island.

*Dicrurus hottentottus sirensis (Oberh.).

Dicruropsis pectoralis sirensis Oberholser, Proc. U.S. Nat. Mus. liv, ror7, p. rgs: Pulau Mata Siri, Java Sea.

Distr, -Mata Siri Island, Java Sea.

*Dicrurus hottentottus solombensis (Oberh.).

Dicruropsis pecloralis solombensis Oberholser, Proc. U.S. Nat. Mus. liv, 1917, p. 184: Solombo Besar Island, Java Sea.

Distr.-Solombo Besar Island, Java Sea.

Dicrurus hottentottus viridinitens (Salvad.).

Dicruropsis viridinitens Salvadori, Ann. Mus. Civ. Gen. (2) xiv, I894, p. 593 : Sipora Island.

Distr.-Mentawi Islands (Sihent, Sipora, Pagi), West Sumatra.

\section{Dicrurus hottentottus suluensis Hart.}

Dicrurus suluensis Hartert, Nov. Zool. ix, 1902, p. 441 : Sulu Islands.

Distr.-Maratua Islands, East Borneo.

\section{Genus CHAPTIA Hodgson."}

Chaptia aenea (Vieill.).

Bronzed Drongo.

[Chaptia aenea aenea (Vieill.).

Dicrurts aeneus Vieillot, Nov. Dict, d'Hist. Nat., ed. 2, ix, $18_{17}$, p. $586:$ Bengal.

Distr.-Extra-limital.]

[Chaptia aenea malayensis Blytl.

Chaptia malayensis "A. Hay", Blyth, Journ. Asiat. Soc. Bengal, Xv, I 846, D. 294 : Malacca.

Edolius picinus Bp., Consp. Gen. Av. 1850, p. 352 : Sumatra.

Dist.r.-Malay Peninsula.

Stumatra.

Borneo.

- Revisions: Baker, Nov. Zool. xxv, 1918, p. 303; Chas. and Kloss, Bull. Raff, Mus. 4, 1930, p. 96. 


\section{Genus BHRINGA Hodgson.'}

Bhringa remifer ('Temm.).

Lesser Raçuet-tailed Drongo.

Bhringa remifer remifer (Temm.).

Edolius remifer Temminck, P1. Col. 178, I823: Java.

Bhringa remifer sumatrana Hachisuka, Bull. Brit. Orn. Cl, xlvii, 1926, p. 57 : Korinchi, West Sumatra.

Distr.-Sumatra.

Java.

Bhringa remifer peracensis Baket.

Bhringa remifer peracensis Stuart Baker, Bul1. Brit. Orn. Cl. xxxix, I9IS, p. IS: Mountains of Perak.

Bhringa remifer attenuata Rob. and Kloss, Journ. Fed. Malay States Mus. viii, pt. 21, 1918, 1. 235: Malay States.

Distr.-Malay States".

\section{Genus DISSEMURUS Gloger.}

Dissemurus paradiseus (Linn.). ${ }^{3}$ Large Racquet-tailed Drongo. [Dissemurus paradiseus paradiseus (Litn.).

Cuculus paradiseus I,inn., Syst. Nat. 12tl1. ed. i, I766, p. 172 : Sian (restr. Bangkok to Ayuthia, Kloss, 1910).

Distr,-Extra-limital.]

Dissemurus paradiseus malayensis (Blyth).

Edolius malayensis Blyth, Journ. Asiat. Soc, Bengal, xxviii, I859, P. 272: Penang (restr. Kloss, 1918).

Dissemurus paradiseus hypoballus Oberh., Journ. Wash. Acad. Sci. xvi, 1026, p. 518: Trang, Peninsular Siam.

Distr.-Malay Peninsula (south to Lat. $4^{\circ} \mathrm{N}$.).

Dissemurus paradiseus platurus (Vieill.).

Dicrurus platurus Vieillot, Nov. Dict. d'Hist. Nat. ed. 2, ix, I8I7, p. 5SS: Malacca (restr. Rob, and Kloss, 1920).

Dissemurus paradiseus messatius Oberh., Journ. Wash. Acad. Sci. xvi, 1926, p. 519: Singapore Island.

Dissemurus paradiseus siakensis Oberh., Journ. Wash. Acad. Sci. xvi, 1926, p. 510 : Siak River, East Sumatra.

Dissemurus paradiselss colpiotes Oberh., Journ. Wash. Acad. Sci, xvi, 1916, p. 520 : North-west Sumatra.

Distr.-Malay States (south of Lat. $4^{\circ} \mathrm{N}$.).

Sumatra; Rhio and Lingga Archipelagos; Banka.

- Cf. Baker, Nov. Zool. xxvi, IgI9, p. 42.

- Not yet actually recorded from Peninsular Siam, but almost certain to occur there.

3 Revisions: Rob., Journ. F.M.S. Mus, vii, 1017, p. 187: Baker, Nov. Zool. xxv, 1918, p. 299 ; Oberh., Bull. U.S. Nat. Mus. 159, 1932, p. 99. 
Dissemurus paradiseus microlophus Oberh."

Dissemurus paradiseus microlophus Oberholser, Bull. U.S. Nat. Mus. 98, 1917, p. 59 : Pulau Jimaja, Anamba Islands.

Dissemurus paradiseus endomychas Oberh., Bull. U.S. Nat. Mus. ${ }_{39}$, 1932, p. 98: Pulau Lingung, North Natuna Islands.

Distr.-Anamba Islands; Tioman Archipelago.

Nortl Natuma Islands.

Dissemurus paradiseus brachyphorus (Bp.).

Edolius brachyphorus Bonaparte, Consp. Gen. Avium, i, I850, p. 35I : Soutl Borneo.

Dissemurus paradisens insularis Hachisuka, Bull. Brit. Orn. Cl. xlvii, 1926, D. 58 : Sarawak.

Distr.-Borneo.

Dissemurus paradiseus banguey Chas, and Kloss.

Dissemurus paradiseus banguey Clasen and Kloss, Journ. f. Ornith. 1929, ii, p. 120: Banguey Island, North Borneo.

Dislr.-North Bornean islands of Banguey and Balatubangan.

Dissemurus paradiseus formosus Cab.

Dissemurus formosus Cabanis, Mus. Hein. i, 1850, p. III : Java.

Dissemurus paradisens wallacei Hachisuka, Bull. Brit. Orn, Cl. xlvii, 1026, D. 58 : East Java.

Distr.-Java.

*Dissemurus paradiseus olizurus Oberli.

Dissemurus paradisens olizurus Oberholser, Smiths. Misc. Coll. $1 x$, No. 7,1912, p. 15 : Simalur Island.

Distr.-Simalur Island, West Sumıtra.

*Dissemurus paradiseus pachistus Oberh.

Dissemurus paradiseus pachistus Oberholser, Stuiths. Misc. Coll. $1 \mathrm{x}$, No. 7 , I9r2, p. I6 : Lasia Island.

Distr.-Lasia Island, West Sumatra.

-Dissemurus paradiseus elassopterus Oberls.

Dissemurus paradiseus classopterus Oberholser, Stniths. Misc. Coll.

lx, No. 7, 1912, p. I6: Babi Island.

Distr.-Babi Island, West Stumatra.

*Dissemurus paradiseus adelphus Oberh.

Dissenurus paradiseus adelphus Oberholser, Stniths. Misc. Coll. Ix, No. 7, 1912, p. I5: Nias Island.

Distr.-Nias Island, West Stmatra.

- The races are curiously mixed in the Tioman Archipelago and some birds could be equally well referred to flaturtes. 


\section{Family CORVID止.}

\section{Genus CORVUS Linn. ${ }^{1}$}

Corvus macrorhynchos Wagl.

Large-billed Crow.

Corvus macrorhynchos macrorhynchos Wagl.

Corvus macrorhynchos Wagler, Syst. Av., I827, Corvus sp. 3 : Java.

Distr.-Malay Peninsula.

Sumatra; Nias Island.

? Borneo. ${ }^{2}$

Java; Bali; Kangean Islands.

Corvus splendens Vieill.

House-Crow.

[Corvus splendens splendens Viei]].

Corvus splendens Vieillot, Nov, Dict. d'Hist. Nat, ed. 2, viii, I817, p. 44 : Bengal.

Distr._Extra-limital.]

Corvus splendens protegatus Madar.

Corvus splendens protegatus Madarasz, Orn. Monatsber. xii, Igo4, p. I95: Ceylon.

Distr._Malay States (introduced).

Corvus enca (Horsf.).

Slender-billed Crow.

Corvus enca enca (Horsf.).

Fregilus enca Horsfield, Trans. Linn. Soc. xiii, I821, p. I64: Java.

Distr.-Mentawi Islands, West Sumatra. Java; Bali.

Corvus enca compilator Richm.

Corvus compilator Richmond, Proc. U.S. Nat. Mus. xxvi, Igo3, p. 518. New name for Corvus tenuirostris Horsf. and Moore (not Brelum, I855) Cat. Bds. Mus. East India Coy. ii, 1858 , p. 558: Bombay, error = Malacca $(B l y t h, 1863)$.

Distr.-Malay States.

Sumatra; Rhio Archipelago; West Sumatran islands of Sinnalur and Nias.

Borneo.

- Revisions: Stres, Verh. Orn. Gesell. Bay. xii, 19:6, p. 277; Kloss,

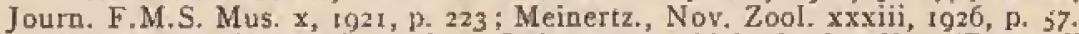

"We seen the specimen from Labuan on which the locality "Borneo" rests and feel a little doubtful about its identity : it is not quite normal. 
Genus DENDROCITTA Gould.

Dendrocitta occipitalis (S. Müll.).

Malaysian Tree-pie.

Dendrocitta occipitalis occipitalis (S. Müll.).

Glaucopis occipitalis Sal. Müller, Tijd. Nat. Ges. Phys. ii, $18_{35}$, p. 343, pl. v : Sumatra.

Dendracitta rufigaster Gould, Proc. Zool. Soc. 1837 , p. 80 : Sumatra.

Distr.-Sumatra.

Dendrocitta occipitalis cinerascens Sharpe,

Dendrocilla cinerascens Sharpe, Ibis, I879, p. 250, pl. 8: Mt. Kinabalu, North Borneo.

Dendrocitta sinensis tuckeri Harrisson and Hartley, Bull. Brit. Orn. Cl. liv, 1934, D, 156: Mt. Dulit, Sarawak.t

Distr.-Borneo.

Genus CRYPSIRINA Vieillot.

Crypsirina temia (Daud.).

Racquet-tailed Magpie.

Corvus temia Daudin, Traite Elem. Orı. ISoo, p. 244 : Africa error = Java (Stuart Baker, 1930).

Corvus varians Lath., Index Orn. Suppl. 180r, p. 26 : Java.

Corvus candatus Shaw and Nodder, Nat. Misc. xiv, 1803, pl. 56r: Java.

Temia vaillanlii Schinz, Cuv.'s Thierréich, i, 1821, p. 614: Java.

Distr,-Peninsular Siam.

Stumatra.

Java; Bali.

\section{Genus KITTA Temminck."}

Kitta chinensis (Bodd.). Hunting-Crow, or Green Magpie.

[Kitta chinensis chinensis (Bodd.).

Coracias chinensis Boddaert, Tabl. P1. Enlum. $178_{3}$, p. $8_{3}$ : China error = South Sian (Rob. and Kloss, 1922).

Distr.-Extra-liuital.]

"Eighteen "tuckeri" from Mc. Dulit seem inseparable on size, or colour from sixteen exact topotypes of cinerascens. The degree of distinctness of the grey cap is a variable feature and 1 cannot appreciate even an average difference between the two series. The British Museum series, at first sight, does seem to indicate that in birds from Dulit the cap is relatively more sharply defined, but this is a character often influenced by the make-up of the skin, the cap being most obvious in birds in which the head is bent back with the bill in line with the tail. Measured from the base of the two central rectrices the maximum tail-length in the serjes before me is about $255 \mathrm{~mm}$. in females, and $265 \mathrm{~mm}$. in males from both localities.

awenty-four skins from all parts of Siam including some from the type locality of "longipennis" give a wing-range of $112-120 \mathrm{~mm}$, against $113-$ $119 \mathrm{~mm}$, in a series from Java: c. f. Naumann, Bull. Brit. Orn. Cl. lv, I935, p. 136 .

3 Revision: Delacour, Rev. Hist. Nat. $x, 1929$, p. 2. 
Kitta chinensis robinsoni (O.-Grant).

Cissa robinsoni Ogilvie-Crant, Bull. Brit, Orn. Cl. xix, r9o6, 1. 9: Mt. Tahan, Pahang, Malay States.

Distr.-Malay States.

Kitta chinensis minor (Cab.).

Cissa minor Cabanis, Mus. Hein. i, 185 I, p. 86, note: Sumatra.

Distr, - Stumatra.

Borneo (lowlands).

Kitta chinensis jefferyi (Sharpe).

Cissa jefferyi Sharpe, Ibis, 1885, P. 383 : Mt. Kinabalu, North Borneo.

Distr,--Borneo (mountains).

Kitta chinensis thalassina 'Temm.

Killa thalassina Temuninck, P1. Col. 401, I\$26: Java.

Distr.-Java.

\section{Gentus PLATYSMURUS Reichenbach."}

Platysmurus leucopterus (Tenm.).

Platysmurus leucopterus leucopterus (Temtn.). Wing-winged Jay.

Glaucopis leucoplerus 'Temninck, Pl. Col. 265, I824: Sumatra.

Distr.-Malay Peninsula.

Smatra.

Platysmurus leucopterus aterrimus (Tem11.).

Black Jay:

Glancopis aterrimus Temuninck, Pl. Col. 1825 , live. 57: Borneo.

Platysmurus schlegeli Pelzeln, Verh. Ges. Wien. xxix, 1880, p. 520 : Sumatra error $=$ Borneo.

Distr.—Borneo.

In view of Reichenbach's excellent plate we can see no reason for dropping this well-established name in favour of Glenargus Cab. (c.f. Stuart Baker, 1930). 


\section{INDEX.}

aลgaardj, Bubo ketupu, $8_{3}$.

abbottj, Butorides javanicus, 58.

abbotti, Dendrophassi vernans, I4.

abbotti, Hirundo tahitica, 6 I.

abbotti, Hypothymis azurea, 173.

abbotti, Hypurolepis javanica, 161 .

abbott, Kakatoe parvulus, 02 .

abbotto Kakatoe sulphurea, 92.

abbotti, Kittacincla malabarica, 237.

abbotti, Malacocincla, 210.

abbotti, Microtarsus melanocephalos, 195.

abbotti, Phodilus badius, 91 .

abboti, Psittinus cyanurus, 94.

abbotti, Spilornis cheela, 76 .

abbotti, Sula, 65.

ablutum, Dicaeum sanguinolentum, 268 .

abnormis, Picumnus, 152.

abnormis, Sasia, 152 .

Abrornis castaniceps, 252.

Abromis sakaiorum, 253.

Abrornis schwaneri, 253.

Abrornis superciliaris, 253 .

Abroscopus, 253.

Abroscopus superciliaris, 253.

Abroscopus superciliaris schwaneri. 253.

Abroscopus superciliaris vorder. mari. 253.

Acanthylis coracinus, it 8.

Acanthylis leucopygialis, 18.

accentor, Androphilus, 208.

Accipiter, 70.

Accipiter badius, 71 .

Accipiter badius poliopsis, 7 .

Accipiter fasciatus, 71 .

Accipiter fasciatus natalis, 71 .

Accipiter nisoides, 72 .

Accipiter nisus, 72

Accipiter nisus nisosimilis, 72 .

Accipiter rufotibialis, 72 .

Accipiter soloensis, 71 .

Accipiter trivirgatus, 70 .

Accipiter trivirgatus indicus, $7 t$,

Accipiter virgatus, 72.

Accipiter virgatus gularis, 72.

acheenensis, Centrococcyx, 120 .

Acomus inornatus, 6.

Acridotheres, 205.

Acridotheres grandis, 206.
Acridotheres javanicus, 206.

Acridotheres torquatus, 296

Acridotheres tristis, 295.

Acrocephalus, 244.

Acrocejlalus bistrigiceps, 245.

Acrocephalus stentoreus, 244 .

Acrocephalus stentoreus orientalis. 245.

Acrocephalus stentoreus siebersi, 245.

actophilus, Butorides striatus, 58 .

acuminata, Erolia, 41.

acuminatus, Totanus, $4 \mathrm{I}$.

acuta, Anas, 63.

acutirostris, Brachypteryx, 223.

adelphus, Dissemurus paradiseus, 307.

adina, Dendrophassa vernans, 15.

adjna, Treron vernans, 15.

adspersus, Batrachostomus, 96.

aedon, Muscicaja, 240.

aedon, Phraganaticola, 249 .

Aegialitis dealbatus, 34 .

Aegialitis jerdoni, 34 .

Aegithina, I8S.

Aegithina tiphia, 188 .

Aegithina tiphia damicra, 180 .

Aegithina tiphia borizoptera, 189 .

Aegithina tiphia micromelaena, ISS.

Aegithina tiphia scapularis, 180 .

Aegithina tiphia singapurensis, I88.

Aegithina tiphia viridis, 189 .

Aegithina tiphia zophonota, ISO.

Aegithina viridissima, 188.

Aegithina viridissima nesjotica, 188.

Aegthina viridissima thapsina, 188 .

Aegrpiidae, 60 .

aenea, Anthreptes rhodolaema, 280.

aenea, Carpophaga, 17.

aenea, Chaptia, 305.

aenea, Columba, 17.

aenea, Ducula, 17

aeneicaudus, Phoenicophoeus, 133.

aeneicaudus, Phoenicophaus curvi-

tostris, I33.

aeneus, Dicrurus, 305.

nenobarbus, Allotrius, 230.

nenobarbus, Pteruthius, 230.

aenothorax, Carpophaga, is.

aeralatus, Pteruthius flaviscapis, 230. 
aeria, Hypothymis azurea, 173. aerophila, Collocalia francica, 115. aerugionsus, Circus, 70 . aeruginosus, Falco, 70 . aeruginosus, Perdix, 4 . aethalea, Aethostoma rostrata, 212. wethereus, Phacthon, 68.

aethiopicus, Tantalus, 51 .

aethiopicus, Threskiornis, 5.I.

Aethiopsar, 206.

Aethopsar fuscus, 296.

Aethiopsar fuscus torquatus, 206 .

Aethionsar grandis, 206.

Aethiopsar grandis javanicus, 206.

Aethopyga, 273.

Aethojyga eupogon, 275 .

Aethopyga eximia, 274 .

Aethopyga lodoisia, 274 .

Aethopyga mystacalis, 274 .

Aethopyga mystacalis perretti, 274.

Aethopyga mystacalis temmincki, 274 .

Aethopyga nipalensis, 274,276 .

Aethopyga nipalensis australis, 276 .

Aethopyga saturata, 273.

Aethopyga saturata anomala, 274.

Aethopyga saturata wrayi, 274.

Aethopyga siparaja, 275.

Aethopyga siparaja cara, 275.

Aethopyga siparaja heliogona, 275.

Aethopyga siparaja heliophiletica, 275.

Aethopyga siparaja melanetra, 275.

Aethopyga siparaja natunae, 275.

Aethopyga siparaja niasensis, 275.

Aethopyga siparaja ochropyrrha, 275.

Aethopyga siparaja photina, 275.

Aethopyga siparaja siberu, 275 .

Aethopyga siparaja tinoptila, 275.

Aethorhynchus, I 8 .

Aethorhynchus lafresnayei, 189.

Aethostoma, 2r I.

Aethostoma pyrrhogenys, $21 z$.

Aethostoma pyrthogenys besuki, 2 I2.

Aethostoma pyrrhogenys buttikoferi, ziz.

Aethostoma pyrrhogenys canicapillum, 212.

Aethostoma pyrrhogenys erythrote, 212.

Aethostoma pyrrhogenys longstaffi, 212.

Aethostoma rostrata aethalea, 212.

Aethostoma rostrata paganica, 212.

Aethostoma rostratum, 211.

Aethostoma rostratum macropterum, 2 I 2.

Aethostoma witmeri, 2 I2.

affinis, Arachnothera, 282. affinis, Ardea, 59.

affinis, Batrachostomus, 96 .

affinis, Caprimulgus, iI.

aftinis, Centropus, 130.

affinis, Cinnyris, 282 .

affinis, Coracias benghalensis, 06.

affinis, Cuculus, 124.

affinis, Cypselus, I 10 .

aftinis, Edolius, 302.

atinis, Gelochelidon nilatica, 45.

affine, Malacopteron, 215.

affinis, Mictopus, IIO.

affinis, Myiothera, 159 .

affinis, Oreocincla horsfieldi, 243 .

affinis, Picus, 140.

affinis, Pitta guajana, 159.

affinis, Sterna, 45.

affinis, Tclitrea, 170

aftinis, Terpsiplione paradisi, $I 76$.

aflinis, Totanus, 43.

affine, Trichastoma, 215.

agile, Piprisoma, 272.

agilis, Fringilla, 272.

Aithopyga chalcopogon, 275.

Alauda campestris, 287 .

Alauda mirafra, 287 .

A]auda novaeseelandiae, 286 .

Alaudidae, 287.

alba, Ardea, \$5.

alba, Crocethia, 39.

alba, Egretta, 55 .

a]ba, Fulica alba, 28 .

a]ba, Gygis, 40.

alba, Motacilla, 284 .

alba, Sterna, 49.

alba, Strix, go.

alba, Tringa, 39.

alba, Tyto, 00.

albicilla, Muscicapa, 163.

albicilla, Siphia parva, 163 .

albicollis, Platyrhynchos, 174 .

albicollis, Rhipidura, 174.

albidus, Anthus novaeseelandiae, 287.

albidus, Antbus richardi, 287.

albifrons, Brachypteryx, 234.

albifrons, Sterna, 48 .

albigula, Cyanoderma melano. thorax, 224 .

albigularis, Setaria, 215.

abipennis, Lophura sumatrana, 6. albiventer, Cyoinis caerulata, 167. albocinctus, Ptilinopus cinctus, I6. albogularis, Brachypteryx, 213 . albogularis, Ophrydornis, 215 .

albogularis, Suya atrogularis, 254. alboniger, Nisaetus, 74 .

alboniger, Spizaetus ripalensis, 74 . albopunctulatus, Cuculus, 122.

albostriatus, Rimator malacoptilus, 218. 
albus, Porphyrio, 28.

Alcedinidae, 97 .

Alcedo, 99 .

Alcedo atthis, 09 .

Alcedo atthis bengalensis, 99 .

Alcedo atthis floresiana, 99.

Alcedo bengalensis, 99 .

Alcedo beryllina, 10r.

Alcedo biru, 101.

Alcedo capensis, 97 .

Alcedo chloris, 103.

Alcedo coerulescens, ror.

Alcedo coromanda, $\mathrm{IOz}^{2}$.

Alcedo coromanda minor, ${ }^{02}$.

Alcedo cryzona, 90.

Alcedo cyanoventris, 103 .

Alcedo erithaca, 10:

Alcedo euryzona, 99.

Alcedo euryzona nigricans, too.

Alcedo fusca, 103.

Alcedo javana, 99 .

Alcedo jаvanica, 99.

Alcedo leucocephala, 99.

Alcedo megarbynchus, 100 .

Alcedo melanoptera, 103.

Alcedo meninting, too.

Alcedo meninting callima, too.

Alcedo meninting proxima, 100.

Alcedo meninting subviridis, 100 .

Alcedo meninting verrauxii, 100.

Alcedo omnicolor, $\mathrm{I}_{3}$.

Alcedo pileata, 103 .

Alcedo smyrnensis, 103.

Alcedo verreauxii, Ioo.

alcinus, Machaerhamphus, 79.

Alcippe, 218

Alcippe cantori, 215.

Alcippe cinerea, 210.

Alcippe cinerea hypocneca, 210.

Alcippe cinereocapilla, 215.

Alcippe dumetoria, 2 to.

Alcippe magnirostris, 215 .

Alcippe nipalensis, 218 .

Alcippe nipalensis peracensis, 218.

Alcinpe nectoralis, 170 .

Alcippe phaeocephala davisoni, 218 .

Alcippe poioicephala, 218 .

Alcippe poioicephala davisoni, 288 .

Alcippe poioicephala pyrrhoptera, 210.

Alcippe solitaria, 219.

Alcippornis brunneicauda eriphaed, 210.

Alcippornis brunneicauda eripolia, 210.

Alcurus, 108

Alcurus leucogrammicus, 198.

aldrovandrii, Falco, $8 \mathrm{r}$.

Alectrophasis personatus, 6.
Alectrophasis pyronotus, 6 .

alexandri, Psittacus, 92.

alexandri, Psittacula, 92.

alexandrinus, Charadrius, 34.

alipodis, Aplonis panayensis, 298 .

alipodis, Lamprocorax panayensis, 208.

Allocotops calvus, 206.

Allotrius aenobarbus, 230 .

Allotrius faviscapis, 220 .

Alophoixus, 197 .

Alophoixus phaeocephalus, 197.

Alophoixus phaeocephalus connectens, 197.

Alophoixus phaeocephalus diardi, 197.

Alophoixus phaeocephalus medius, 107.

aljina, Erolia, 4 r.

alpina, Tringa, $4 \mathrm{I}$.

Alseonax, 163.

Alseonax latirostris, ${ }^{6} 6_{3}$.

altirostris, Aplonis panayensis, 20 .

altirostris, Calornis, 298.

amabjlis, Carcineutes, 102.

amabilis, Lacedo pulchella, 102.

Amandava, 280 .

Amandava amandava, 280 .

amandava, Amandava, 280 .

Amandava amandava punicea, 280.

amandava, Fringilla, $\mathbf{z 8 9 .}$

Amandina leucogastra, $29 \mathrm{~J}$.

amauroptera, Ramphalcyon, 97.

amauropterus, Halcyon, 97.

Amaturornis, 27.

Antarornis phoenicura, 27.

Amaurornis phoenicurus, 27.

Amaurornis phoenicurus chinensis, 27.

Amaurornis phoenicurus javanicus, 27.

ambiguus, Cajrimulgus macrurus, 112.

ambiguus, Parus major, 262.

ambiguus, Turdus, 262.

amechana, Collocalia francica, II4. amelis, Hypothymis azurea, 174.

amicta, Nyctiormis, 110.

amictus, Merops, t to.

amictus, Tiga, 144 .

amoenus, Copsychus saularis, 236 .

amoenus, Turdus, 236 .

ampala, Chotorea mystacophanes, 135.

Ampeliceps, 295.

Ampeliceps coronatus, 205.

amphiala, Culicicapa ceylonensis, I80.

amphiryta, Sauropatis chloris, 104. amurensis, Ardea virescens, 58.

amurensis, Butorides striatus. 58 . 
amydrus, Artamus leucorhynchus, 236.

amydrus, Artamus leucoryn, 256.

Anadoenus ruficauda, 132 .

anaethetus, Sterna, 47 .

Anaimos, 27 I.

Anaimos maculatus, 271 .

Anaimos maculatus natunensis, 27 I.

Anaimos maculatus opistatus, 272.

Anaimos maculatus septentrionalis, 271 .

Anaimos percussus, 271.

Anaimos percussus ignicapillus, 271.

Anaimos thoracicus, $27 \mathrm{t}$.

Anaimos xanthopygius, 271 .

anak, Cyornis, 165.

Analcipus consanguineus, $30 t$.

analis, Dryobates, 144 .

analis, Picus, 144.

analis, Pycnonotus goiavier, 190.

analis, Turdus, 109.

anambae, Anthreptes malacensis, 270 .

anamesus, Caprimulgus macrurus, 1 [ 2.

Anas, 62.

Anas acuta, 63.

Anas arcuata, $6 z$.

Anas clypeata, 63.

Anas coromandeliana, 61 .

Anas crecca, 62

Anas fuligula, 63.

Anas gibberifrons, 63 .

Anas gibberiftons, 63 .

Anas (Querquedula) humeralis, 62.

Anas javanica, 6r.

Anas penelope, 62.

Anas querquedula, 62 .

Anas nlatyrhynchos, 62 .

Anas scutulata, 6I.

Aras superciliosa, 62 .

Anas superciliosa percna, 62 .

Anatidae, 6r.

anceps, Astur, 70.

andrewsi, Eulabeornis philippensis, 25.

andrewsi, Fregata, 66 .

andrewsi, Rallus philippensis, 25.

andromedae, Myiothera, 242.

andromedae, Zoothera, 242

Androphilus, 208.

Androphilus accentor, 208 .

angetinae, Otus spilocephalus, 85.

angelinae, Pisorhina, 85.

Anhinga, 64

Anhinga rufa, 64.

Anbinga rufa melanogaster, 65

Anhingidae. 64 .

annectans, Bhuchanga, 302.

annectan 5, Dicrurus, 302. annectens, Tephrodornis gularis, 257.

annecteas, Tephrodorais pelvica, 257.

annulatus, Buceros, 108.

anochra, Hemiprocne longipennis, 1 I 3.

anomala, Aethopyga saturata, 274.

Anorthinus, 108.

Anorthinus galeritus, 108.

Anous, 40 .

Anous minutus, 49 .

Anous minutus worcesteri, 49 .

Anous stolidus, 40.

Anous stolidus pileatus, 49 .

antarctica, Sterna anaethetus, 47.

antelia, Arachnothera longirostris $28 \mathrm{r}$.

antelia, Cyornis elegans, 165.

Anthipes, 160 .

Anthipes solitaris, 160 .

Anthipes solitaris malayana, 170 .

Anthipes solitaris submoniliger. $1>0$.

Anthracoceros, 105 .

Anthracoceros coronatus, 106.

Anthracoceros coronatus barussen. sis, 106.

Anthracoceros coronatus convexus. Io6.

Anthracoceros coronatus leucogas. ter. 106.

Anthracoceros coronatus zamelaenus, 106.

Anthracoceros malayanus, 106.

Anthreptes, 278 .

Anthreptes flavigaster, 284 .

Anthreptes bypogrammica intensior, 278 .

Anthreptes macularia, 278 .

Anthreptes macularia hypogrammica, 278 .

Anthreptes macularia natunezsis. 278 .

Anthreptes malacensi5, 270 .

Anthreptes malacensis anambae. 270 .

Anthreptes malacensis baweanus, 270.

Anthreptes malacensis bornensis, 270 .

Anthreptes malacensis erixanthus, 270.

Anthreptes malacensis mjobergi. 270 .

Anthreptes malacensis nesaeus, 270 .

Anthreptes malacensis pelloptilus, 270.

Anthreptes malacensis pollostus, 270.

Anthreptes modesta, 282 . 
Anthreptes nuchalis, $27 \mathrm{~S}$.

Anthreptes rhodolaema, 280 .

Anthreptes rhodolaema aenea, 280 .

Anthreptes simplex, 278 .

Anthreptes simplex euthapsinus, 278 .

Anthreptes simplex simplicior, 278 .

Anthus, 286.

Anthus campestris, $28 \%$.

Anthus campestris striolatus, 287 .

Anthus euonyx, 286.

Anthus gustavi, 287.

Anthus hasseltii, 286.

Anthus hodgsoni, 286 .

Anthus hodgsoni inopinatus, 286.

Anthus novaeseelandiae, 286.

Anthus novaeseelandiae albidus. $2 \$ 7$.

Anthus novaeseelandiac malayensis, 286

Anthus novaeseelandiae sinensis, 286.

Anthus richardi albidus, 287 .

Anthus rufogularis, 287.

antigone, Ardea, 20 .

antigone, Grus, 20.

antioproctum, Dicaeum trigono. stigma, 270.

antioproctum, Dicaeum trigonostigmum, 270.

antioxantha, Culicicapa cevlonensis, 180.

antracicus, Buceros, 106.

Anuropsis, 2 r6.

Anuropsis malaccensis, 216.

Antropsis malaccensis docima, 216 .

Anuropsis malaccensis driophila, 216 .

Anuropsis malaccensis drymodrama, 216.

Anuropsis malaccensis exsanguis, 217.

Anuropsis malaccensis feriatus, 217 .

Anuropsis malaccensis nesitis, 217 .

Anuropsis malaccensis poliogenis, 216.

Anuropsis malaccensis saturata, 216.

Anuropsis malaccensis sordidus, 216.

apega, Cyanoderma erythroptera, 224.

apivorus, Falco, 79.

apivorus, Pernis, 79

Aplonis, 207.

Allonis minor, 200.

Aplonis panayensis, 207.

Aplonis panavensis alipodis, 208.

Aplonis panavensis altirostris, $20 \mathrm{~S}$.

Aplonis panavensis enganensis, zno.

Aplonis panayensis gusti, 208.
Aplonis panayensis heterochlorus, 208.

Aplonis panayensis leptorrhynchus, 200.

Aplonis panayensis pachistorhinus 200 .

Aplonis panayensis richmondi, 208.

Aplonis panayensis strigatus, 208 .

Aplonis panayensis suggrandis, 208.

ag)ricarius, Charadrius, 33 .

Arachnocestra crassirostris, 283 .

Arachnoraphis everetti, 282 .

Arachnothera, $28 \mathrm{t}$.

Arachnothera affinis, $28 z$.

Arachnothera affinis everetti, 282 .

Arachnothera affinis heliophilus, 282 .

Arachnothera affinis modesta, 282.

Arachnothera aurata, 282 .

Arachnothera chrysogenys, 283.

Arachnothera chrysogenys copha. 283.

Arachnothera chrysogenys isopega, $2 \delta_{3}$.

Arachnothera chrysogenys pleoxantha, $\geq 83$.

Arachnothera concolor, 282.

Arachnothera crassirostris, 283 .

Arachnothera evtonii, 284 .

Arachnothera flavigaster, $2 \mathrm{~S}_{4}$.

Arachnothera favigenis, 283 .

Arachnothera flaviventris, 284.

Arachnothera juliae, 283 .

Arachnothera latirostris, 282.

Arachnothera Iatirostris, 284.

Arachnothera longirostra, 281 .

Araclinothera longirostra atita, 281.

Arachnothera longirostra buttikoferi, $28:$

Arachnothera longirostra exochra, $28 \mathrm{t}$.

Arachnothera longirostra hypochra, $28 \mathrm{r}$.

Arachnothera longirostra niasensis, 282 .

Arachnothera longirostra prillwitzi, $28 \mathrm{t}$.

Arachnothera longirostra rothschildi, 281.

Arachnothera longirostra zarhina, $28 z$.

Arachnothera longirostris antelia, $28 \mathrm{r}$.

Arachnothera longirostris heliocrita, 281 .

Arachnothera longirostris melanchima, $28:$

Arachnothera magna, 282 .

Arachnothera robusta, 283 .

Arachnothera robusta armata, 283 . 
Arachnothera simillima, $2 \&_{4}$.

Arachnothera temmincki, 283 .

Arachnothera uropygialis, 283 .

Arboricola brunneopectus, 2.

Arboricola campbelli, z.

Arboricola graydoni, 3 .

Arboricola rolli, 2,

Arborophila, 2.

Arborophila brunneopectus, 2.

Arborophila brunneopectus bartelsi, 2.

Arborophila brunneopectus campbelli, 2.

Arborophila brunneopectus erythrophrys, 3.

Arbotophila brunneopectus hyperythra, 3.

Arborophila brunneopectus javanica, 2.

Arborophila brunneopectus orientalis, 3.

Arborophila brunneopectus rolli, 2.

Arborophila brunneopectus sumatrana, 2

Arborophila charltonii, 3 .

Arborophila charltoni graydoni, 3 .

Arborophila javanica bartelsi, 2.

Arborophila rubrirostris, 3.

archetes, Cecropis, 16r.

archipelagicus, Indicator, 133.

arcuata, Anas, 62.

arcuata, Dendrocygna javanica, 62 .

arcuata, Pitta, 150.

Ardea, 54.

Ardea affinis, 50 .

Ardea alba, 55.

Ardea antigone, 20.

Ardea bilineata, 60 .

Ardea caledonica, 57.

Ardea cinerea, 55 .

Ardea cinerea rectirostris, 55.

Ardea cinnamomea, 60.

Ardea episcopus, 53.

Ardea garzetta, 56 .

Ardea ibis, 50.

Ardea intermedia, 55.

Ardea javanica, 58 .

Ardea lepida, 60.

Ardea malaccensis, 50 .

Ardea melanolopha, 57 .

Arder melanopus, 55.

Ardea modesta, 56.

Ardea nebulosa, 60 .

Ardea nigripes, 56.

Ardea nycticorax, 57

Ardea picata, 60 .

Ardea purpurea, 54.

Ardea purpurea manillensis, 54.

Ardea ralloides, 59.

Ardea ralloides bacchus, 59 .

Ardea robusta, 55 .
Ardea sacra, 56.

Ardea sinensis, 60.

Ardea speciosa, 50 .

Ardea stellaris, 61.

Ardea striata, 58.

Ardea sumatrana, 54.

Ardea typhon, 54 .

Ardea virescens amurensis, 58.

Ardeidae, 54.

ardens, Phaenicornis, 185.

Ardeola, 50.

ardeola, Dromas, 44.

Ardeola ibis, 50 .

Arcleola ibis coromanda, 59.

Ardeola ralloides, 59 .

Ardeola ralloides speciosa, 59 . ardesiaca, Lophocitta, 257.

ardesiacus, Platylopus

galeri-

culatus, 257

Ardetta eurhythma, 60.

Arenaria, 30.

Arenaria interpres, 30.

argentina, Columba, 19.

argentauris. Mesia, 23o.

argentea, Mixornis gularis, 226 .

argus, Argusianus, \&.

Argus giganteus, 8.

Argus gravi, 8.

Argus ocellatus, 9 .

Argus paeoninus, 8.

Argus pavoninus, 8.

Argus pavonius, 8.

argus, Phasianus, 8

Argusianus, 8.

Argusianus argus, 8.

Argusianus argus gravi, 8 .

arhadius, Muscadivores aeneus, 17. ariel, Atagen, 67 .

ariel, Fregata, 67.

arignota, Ramphalcyon capensis, 08.

arismicra, Dendrophaşsa olax, I 5.

arixutbus. Phodilus badius, gr.

armata, Arachnothera robusta, 283 . armillaris, Bucco, $\mathrm{\jmath} 36$.

armillaris. Cyanops, 136 .

arquata, Numenius. 35.

arquata, Pitta (Phaenicocichla), 150.

arquata, Scolopax, 35 .

Arrenga melanura, 239.

Artamidne, 256.

Artamides kannegieteri, I82.

Artamides larutensis, 18I.

Artamides sumatrensis calopolius, 182.

Artamides sumatrensis halistephis, 182.

Artamides sumatrensis messeris, $18 \mathrm{r}$. 
Airtamides sumatrensis nesiarchus, 181 .

Artamus, 256.

Artamus leucorhynchus, 256.

Artamus leucorhynchus amydrus, 256.

Artamus leucoryn. amydrus, 256 .

Artamus leucoryn macroterus, 256. arundinaceus, Caprimulgus, il .

Asarcornis, 61.

Asarcornis scutulata, $6 \mathrm{r}$.

asiaticus, Charadrius, 34.

asiatica, Cyanops, 136 .

asiatica, Mycteria, 54.

asiaticus, Trogon, 136 ,

asiaticus, Xenorhynchus, 54 .

Asto, 83 .

Asio flammeus, 83 .

assimilis, Botaurus, 60.

assimilis, Cuculus, 124 .

Astur anceps, 70.

Astur barbatus, 76 .

Astur fasciatus, $7 \mathrm{t}$.

Astur (Nisus) gularis, 72 .

Astur indicus, $7 \mathrm{I}$.

Astur kienerii, 73 .

Atagen ariel, 67 .

aterrima, Fringilla, 292.

Athene borneensis, 88 .

Athene malacensis, 88 .

Athene sylvatica, no.

Athenoptera spilocephalus stresemanni. 85 .

atita, Arachnothera longirostra, $28 \mathrm{r}$. atra, Fulica, 20.

atrata, Rhipidura albicollis, 174 .

atricapilla, Loxia, 200.

atricapilla, Munia, 200.

atricajilla, Napothera, 215.

atricapilla, Zosterops, 265.

atriceps, Brachypodius, 194.

atriceps, Parus, 261.

atriceps, Turdus, 104.

atrifrons, Charadrius mongolus, 33.

atrigularis, Cacopitta, 213 .

atrigularis, Turdinus macrodacty-

lus, 253.

atrocaudata, Muscipeta, 177.

atrocaudata, Terpsiphone, 177.

atrogularis, Hemipodius, 9 .

atrogularis. Orthotomus, 245.

atrogularis, Suva, 254 .

atrogularis, Turnix suscitator, 0 .

atronuchalis, Lobivanellus indicus, 32.

attenuata, Bhringa remifer, 306.

atthis, Alcedo, 00 .

atthis, Gracula, 00 .

aurata, Arachnothera, 282.

aurata, Geocichla, 242.

aurata, Geokichla citrina, 242. aurantiifrons, Cyanops mystacophanes, 135.

aurantiventris, Dryobates hardwickij, 145.

aurantitventris, Picus (Boeopipo), hardwickii, 145.

aureiventer, Zosterops palpebrosa, 264.

aureola, Emberiza, 288.

aureum, Malacopteron, 202.

aurifrons, Chloropsis, $19 \mathrm{t}$.

aurifrons, Phyllornis, Igr.

aurigaster, Pyenonotus, 204.

aurigaster, Turdus, 204.

auritus, Batrachostomus, 95 .

auritus, Dryobates bardwickii, 145 .

auritus, Porargus, 95.

auritus, Tripsurus, 145.

australe, Pellorneum tickelli, zog.

australis, Aethopyga nipalensis, 276 .

australis, Bucco, 137.

australis, Cyanops, 137 .

australis, Drymocataphus tickelli. 200 .

australis, Squatarola helvetica, 32. australis, Squatarola squatarola, 32 . australis, Terpsiphone paradisi, 176.

Aviceda, 70 .

Aviceda jerdoni, so.

Aviceda jerdoni borneensis, 80 .

Aviceda leuphotes, 79.

Aviceda sumatrensis, 80 .

axantha, Cinnyris brasiliana, 276.

axantha, Leptocoma brasiliana, 276 .

axanthizus, Pycnonotus simplex, 201 .

azaleus, Meiglyptes tukki, 147.

azela, Halcyon chloris, 104.

azela, Sauropatis chloris, 104.

azurea, Cochoa, 230 .

azurea, Hypothymis, 172 .

azurea, Muscicapa, 172.

azurea, Sitta, 263.

azureus, Turdus, 239.

babiensis, Coracina sumatrensis, 182 .

babiensis, Ducula aenea, 17 .

babiensis, Graucalus, 182 .

babiensis, Muscadivores consobrina, 17.

bacchus, Ardeola ralloides, 59 .

bacchus. Buphus, 50.

bacha, Falco, 75 .

badia, Cecropis, 16 .

badia, Columba, rg. 
badia, Ducula, 20.

badia, Hirundo datrica, 161.

badia, Strix, 9t.

badiosus, Meiglyptes, 148.

badiosus, Microptemus brachyurus, 148.

badius, Accipiter, 71.

badius, Falco, 7 .

badius, Micropternus brachyurus. 148.

badius, Phodilus, 01.

badius, Picus, 148.

baeus, Orthotomus cineraceus, 247.

baeus, Orthotomus sepium, 247.

bakeri, Turdinus macrodactylus. $2 \mathrm{~s} 3$.

bakkamoena, Otus, 86

balasiensis, Cyp., 118 .

balicus, Criniger gularis, 106.

balicus, Criniger tephrogenys, 106.

baliensis, Cyanoderma melanothorax, 225.

baliensis, Cvanops armillaris, 136 .

baliensis, Ptilinopus albocinctus, 16.

baliensis, Rhinomyias pectoralis, 178 .

Bambusicola erythrophys, 3 .

Bambusicola hyperythra, 3 .

bangkana, Pitta sordida, 158.

bangsi, Sterna dougallii, 46 .

banguey, Discemurus paradiseus, 307.

bangueyensis, Chalcites xanthorhynchus, 127.

bangueyensis, Ptilinopus melanocephalus, 17.

banjakensis. Stachyris maculata, $2 \pm 2$

bankiva, Callus gallus, 7.

banksi, Cettia montanus, 254.

bantamensis, Columba, 23.

banvumas, Cyornis, t66.

banyumas, Muscicaja, 166.

baramensis, Treron fulvicollis, I4.

barat, Pycnonotus bimaculatus, ing.

barbata, Bessethera, 200.

barbatulatus, Psittacus, 93.

barbatus, Astur, 76 .

barbouri, Kittacincla stricklandii, 235 .

Barita gymnocephala, 258.

bartelsi, Arborophila brunneopectus, 2.

bartelsi, Arborophila javanica, 2 .

bartelsi, Caprimulgus pulchellus, i11.

bartelsi, Collocalia francica, 114.

bartelsi, Criniger balicus, 196.

bartelsi, Criniger tephrogenys, 196. bartelsi, Spizatus ripalensis, 74. bartelsi, Strix leptogrammica, 80 . bartelsi, Syrnium, 80.

bartelsorum, Turnjx sy]vatica, I0. barussa, Macropygia phasianella, 21.

barussana, Malacocincla sepiaria, 211.

barussarum, Surniculus lugubris, 122.

barussensis, Hydrocissa convexa, Iob.

barussensis, Anthracoceros coronatus, 106.

basalis, Chalcites, 128 .

basalis, Cuculus, 128 .

bassus, Falco, 75 .

bassus, Spilornis cheela, 75 .

batakana, Munia atricapilla, 200.

batakensis, Buchanga leucophaea, 303.

batasiensis, Cypsiurus, 118.

Batrachostomus, 95 .

Batrachostomus adspersus, 00 .

Batrachostomus atfinis, 06 .

Batrachostomus auritus, 95.

Batrachostomus harterti, 95.

Batrachostomus javensis, 06 .

Batrachostomus mixtus, 96.

Batrachostomus poliolophus, 95.

Batrachostomus stellatus, 06 .

Batrachostomus stictopterus, 96.

batuense, Dicaeum cruențatum, 268.

batuensis, Gracula religiosa, 207.

butudi, Pita, $t 60$.

baueri, limosa lapponica, 37 .

baweana, Gracula javensis, 207.

baweana, Gracula religiosa, 297

baweana, Malacocincla abtotti, 210.

baweana, Strjx orientalis, So.

baweanus, Anthreptes malacensis, 279.

baweanus, Brachypodius atriceps, 195.

baweanus, Spilornis bassus, 75.

Baza bormeensis, 80.

beccariana, Siphia, 165 .

beccarii, Cochoa azurea, 230.

beccarii, Dicaeum sanguinolentum, 268 .

bedouti, Sula dactylatra, 65 .

bemmeleni, Criniger, 197.

bemmeleni, Porphyrio, 20.

bengalensis, Alcedo, go.

bengalensis, Alcedo atthis, 99 .

bengalensis, Centropus, 120.

bengalensis, Cuculus, 120.

bengalensis, Pseudogyps, 60 . 
bengalensis, Sterna, 46. bengalensis, Vultur, 69. benghalensis, Coracias, 96. benghalensis, Corvus, 96 . bengbalensis, Rallus, 31. benghalensis, Rostratula, 3 t. bentet, Lanius schach, 259. Berenicornis, 108.

Berenicornis comatus, 108 . bergii, Sterna, 46. bertae, Pitta, 158. beryllina, Alcedo, ror.

Bessethera barbata, 200 .

besuki, Aethostoma pyrrhogenys, 212.

Bhringa, 300.

Bhringa remifer, 306.

Bhringa remifer attenuata, 306.

Bhringa remifer peracensis, 306.

Bhringa remifer sumatrana, 306.

Bhuchanga annectans, 302.

bicalcaratus, Pavo, 7 .

bicalcaratum, Polyplectron, 7 .

bicincta, Treron, 14.

bicincta, Vinago, 14.

bicolor, Brachypteryx, $2: 3$.

bicolor, Buceros, 106.

bicolor, Columba, เo

bicolor, Cyanoderma erythoptera, 224 .

bicolor, Erythrocichla, $2[3$.

bicolor, Garrulax leucolophus, 205. bicolor, Ichthyaetus, $z \gamma$.

bicolor, Myristicivora, 10.

bicolor, Porzana, 27.

bicolor, Timalia, 224 .

bicornis, Buceros, to5.

bicornis, Dichoceros, 103.

bido, $\mathrm{F}$ alco, 75.

bido, Spilornis cheela, $7 \overline{\text {. }}$

biesenbachi, Turdus javanicus, 240 .

bilineata, Ardea, 60.

billitonis, Chatorhea rafflesii, 135.

billitonis, Eurylaimus javanicus, 155 -

billitonis, Pycnonotus plumosus, 200.

bilobus, Dicrurus, 302

bimaculatus, Caprimulgus macrurus, Iтz.

bimaculatus, Psittacus, 02.

bimaculatus, Pycnonotus, 199.

bimaculatus, Turdus, 190.

birw, Alcedo, 101.

bisignatus, Cajrimulgus, I I.

bistrigata, Motacilla, 285.

bistrigiceps, Acrocephalus, 245 .

bitorquata, Columba, 22.

bitorquata, Streptopelia, 22. blanfordi, Pycnonotus, 200.

blanfordi, Turnix tanki, ıo.

bithi, Prinia inornata, 255.

blythi, Suya, 255 .

Blythipicus, 146.

Blythipicus pyrrhotis, 146.

Blythipicus mrrhotis cameroni, 146.

Blythipicus rubiginosus, 146.

Blythipicus rubiginosus parvus, 140.

bocakei, Stachyris, 233.

bocki, Hierococcyx sparterioides, 123.

Bombycistomas fullertonii, 95 .

borealis, Motacilla, 285.

borealis, Phyllopheuste, 250.

borealis, Phylloscopus, 250.

borneana, Chalcoparia singalensis. 280 .

borneana, Halcion concreta, I04.

borneanum, Dicaeum concolor, 270 .

borneanum, Dicaeum minulium, 270 .

borneense, Glaucidium brodiei, 0. borneensis, Athene, 88.

borneensis, Aviceda jerdoni, So.

borneensis, Baza, 80.

borneensis, Caloperdix oculea, 4 .

borneensis, Campephaga, 184 .

borneensis, Caprimulgus, 111 .

borneensis, Chlorura, 203.

borneensis, Chotorea rattlesii, 136 .

borneensis, Chotorea versicolor, 136.

borneensis, Dicruropsis, 304.

borneensis, Dicrurus hottentottus, 304.

borneensis, Enicurus leschenaulti, 234.

bormeensis, Erythura hyperythra, 203 .

borneensis, Eupetes macrocerus, 204.

borneensis, Henicurus, 234.

borneensis, Macropsigia emiliana, 21.

borneensis, Macropygia phasianella, 8:.

borneensis, Melanoperdix nigra, 4. borneensis, Mirafra, 287 .

borneensis, Myiophoneus, 230.

bormeensis, Myophonus, 230.

borneensis, Ninox scutulata. 88 .

borneensis, Phoenicophaus cur. virostris, 132.

borneensis, Pitta granatina, 130.

borneensis. Psarisomus dalhousiae, 154. 
borneensis, Rhamphococcyx erythrognathus, 132 .

borneensis, Rhojodytes diardi, $13 \mathrm{I}$. borneensis, Stachyris nigriceps, 220

borneensis, Tchitrea patadisi, 176. borneensis, Terpsiphone paradisi, 176.

bornensis, Anthreptes malacensis, 270.

bornensis, Mixornis gularis, 226.

bornensis, Peristera, 24.

bornensis, Pomatorhinus montanus, 207.

borneoensis, Buceros rhinoceros, 105.

borneonensis, Chloropicoides rafflesii, 144.

bormeonensis, Megalaema duvauceli, 138 .

borneonensis, Orchotomus sepium, 247.

borneonensis, Spizaetus, 74 .

borneonensis, Tiga, 149 .

borneonensis, Gauropicoides rafflesi, 144 .

boschii, Pitta, 150.

Botaurus, 61.

Botaurus assimilis, 60 .

Botaurus stellaris, 61 .

Brachylophus chlorolophus vanheysti, 142 .

Brachylophus puniceus continentis, 142.

brachyphorus, Dissemurus paradiseus, 307.

brachyphorus, Edolius, 307.

Brachypodius, 104.

Brachypodius atriceps, 104.

Brachypodius atriceps baweanus, 195.

Brachypodius atriceps chrysophorus, 194.

Brachypodius atriceps hodiernus, 195.

Brachypodius atriceps hyperemnus, 195.

Brachypodius criniger, $10 \$$.

Brachypodius immaculatus, 194.

Brachypodius tristis, 194.

Brachypteryx, 231 .

Brachypteryx acutirostris, 223 .

Brachypteryx albifrons, 234.

Brachypteryx albogularis, 213 .

Brachypteryx bicolor, 213

Brabypteryx buxtoni, 211 .

Brachypteryv flaviventris, 232.

Brachypteryx lepturus, $23 \mathrm{I}$.

Brachypteryx leucophoris wrayi, 231 .
Brachypteryx macroptera, 212.

Brachyptery $x$ maculatus, 227.

Brachypteryx malaccensis, 216.

Brachypteryx montana, 231.

Brachypteryx montana erythrogyna, 231.

Brachypteryx montana saturata, 231.

Brachypteryx nigrocapitata, 209 .

Brachypteryx nigrogularis, 221.

Brachypteryx poliogenis, 216.

Brachyjteryx salaccensis, 234.

Brachypteryx sepiaria, 210.

Brachypus chrysorrhoeus, 204.

Brachypus eutilotus, 194.

Brachypus poliopsis, 202.

Brachypus tigus, 203.

Brachypus tympanistrigus, 194.

Brachypus urostictus, 194.

Brachypus vidua, 105.

brachyrbyncha, Cyanops armillaris, 136.

brachyrhyncha, Cyanops henricii, 130.

brachyurus, Corvus, 157.

Brachyurus davisoni, 156.

brachyurus, Micropternus, 148.

brachyurus, Picus, 148 .

brachyura, Pitta, 157.

brachyurus, Surniculus lugubris, 122.

Bradypterus, 249.

Bradypterus montis, 249.

brasiliana, Certhia, 276.

brasiliana, Leptocoma, 276 .

brevicaudata, Napothera, 217.

brevicaudatus, Turdinus, 217 .

brevipennis, Dendrocophus, 144.

brevipes, Totanus, 43.

brevipes, Tringa incana, 43.

brevirostra, Gryllivora, 236.

brodiei, Glaucidium, go.

brodiei, Noctua, 00.

brookeanus, Hemicercus, 150.

brookei, Eurylaimus javanicus, I55.

brookii, Otus, 87.

brookii, Scops, 87 .

brunneata, Rhinomyias, 178.

brunneata, Siphia, 178 .

brunneicauda, Hyloterpe, 178, 219.

brunneiceps, Munia, zoo.

brunneopectus, Arboricola, 2.

brunneopectus, Arborophila, 2.

brunnescens, Corydon sumatranus, 156.

brunnescens, Erpornis zantholeuca, 220 .

brunnescens, Herpornis, 220.

brunnescens, Lalage nigra, 184. 
INDEX.

brunnescens, Trichophoras, 193. brunneus, Pycnonotus, 20l. brunneus, Hemicircus, 147. brunnicephalus, Larus, 50.

Bubo, 83 .

Bubo coromandus, 84 .

Bubo coromandus klossii, 84 .

Bubo ketupu, 83.

Bubo ketupu aagaardi, 83 .

Bubo ketupu buttikoferi, 84 .

Bubo ketupu pageli, 83 .

Bubo orientalis minor, 84 .

Bubo zeylonensis, 83 .

Bubo zeylonensis leschenault, 83 .

bubutus, Centropus sinensis, 129.

Bubutus duvaucelii, 132.

Bubutus isidori, 132 .

Bucco armillaris, 136 .

Bucco australis, 137.

Bucco chrysopogon, I34.

Bucco corvinus, 134.

Bucco cyanocephalus, 137 .

Bucco duvaucelii, 138 .

Bucco franklinii, 137 .

Bucco frontalis, $13 \mathrm{~s}$.

Bucco fuscicapillus, 139.

Bucco hayi, I34.

Bucco henricii, 136 .

Bucco hoemacephalus, 139 .

Bucco indicu5, 139.

Bucco javensis, 135.

Bucco kotorea, 135.

Bucco lathami, 134.

Bucco malaccensis, 136 .

Bucco mystacaphanos, 135.

Bucco oorti, 137.

Bucco quadricolor, 135.

Bucco rafflesii, 135 .

Bucco roseus, 140 .

Bucco tristis, 135

Bucco versicolor, 135.

Bucco zeylanicus, 139.

buccoides, Dacelo, 102.

Buccotrogon torquatus, 140.

Buceros, 105.

Buceros annulatus, 108 .

Buceros antracicus, 106.

Buceros bicolor, to6.

Buceros bicornis, 105.

Buceros carinatus, 108 .

Buceros comatus, 108.

Buceros convexus, 106

Buceros coronatus, to6.

Buceros corrugatus, 107.

Buceros cristatus, 105.

Buceros diadematus, 105.

Buceros elliotti, 107.

Buceros galeatus, 108 .

Buceros galeritus, 108.

Buceros gracilis, to7.
Buceros indica, 105.

Buceros intermedius, 106.

Buceros javanica, 105.

Buceros javanicus, 108.

Buceros leucogaster, 106.

Buceros lugubris, 108.

Buceros lunatus, to5.

Buceros malaynus, 106.

Buceros niger, 108.

Buceros nigrirostris, 107.

Buceros plicatus, to7.

Buceros rhinoceroides, 105.

Buceros rhinoceros, 105 .

Buceros rhinoceros borneoensis, 105.

Buceros rhinoceros silvestris, 105.

Buceros rugosus, 107.

Buceros scutatus, to8.

Buceros sublunatus, 105.

Buceros subruficollis, 107.

Buceros sumatrana, 105.

Buceros undulatus, 108 .

Buceros vigil, 108 .

Bucerotidae, 105.

Buchanga leucogenis, 303 -

Buchanga leucogenys cerussata, 304.

Buchanga leucophaea batakensis, 303.

Buchanga leucophaea dulitensis, 303.

Buchanga leucophaea penrissenensis, 303.

Buchanga periophthalmica, 304 .

Buchanga stigmatops, $3 \mathrm{O}_{3}$.

Buchanga stigmatops phaedra, 303.

Budytes flavus macronyx, 285 .

Budytes melanotis, 285 .

Budytes taivanus, 285 .

bulweri, Lobiophasis, 7 .

bungurense, Malacopteron cinereum, 2 I 4 .

bungurense, Piprisoma agile, 272.

bungurensis, Coracina sumatrensis, 182 .

bungurensis, Graucalus, 182.

Buphus bacchus, 50 .

Buphus pseudo-ralloides, 50.

Burhinidae, 30.

burmanicus, Buteo, 72 .

burnanica, Ninox scutulata, 88 .

Burnesia dysancrita, 254 .

Burnesia dysancrita halistona, 255.

Burnesia flaviventris rafflesi, 255 .

butaloides, Pachycephala cinerea. 260.

butaloides, Pachycephala grisola, 260 .

Butastur, 76 . 
Butastur indicus, 76 .

Butastur liventer, 76 .

Buteo, 72.

Buteo burmanicus, 72 .

Buteo (Butastur) fasciatus, 76 .

Buteo cristatus, 82.

butleri, Cryptolopha, 252.

butleri, Seicercus castaniceps, 252.

Butorides, 58.

Butorides javanicus abbotti, 58 .

Butorides favanicus carcinophonus, 58.

Butorides striatus, 58.

Butorides striatus actophilus, 58 .

Butorides striatus amurensis, 58 .

Butorides striatus icasopterus, 58 .

Butorides striatus javanicus, 58 .

Butorides striatus sipora, 58 .

Butreron, It.

Butreron capellei, I:

Butreron capellei magnirostris, 11.

Butreron capellei messopora, it.

Butreron capellei panochra, $1 \mathrm{I}$.

Butreron capellei passorhina, it.

buttikoferi, Aethostma pyrrhogenys, 212 .

buttikoferi, Arachnothera Jongirostra, $28 \mathrm{r}$.

buttikoferi, Bubo ketupu, 84 .

buttikoferi, Malacocincla abbotti, 210.

buttikoferi, Thriponax javensis, 153.

buttikoferi, Trichostoma, 2Iz.

buxtoni, Brachypteryx, 2ii.

buxtoni, Zosterops palpebrosa, 264.

Cacomantis, 126 .

Cacomantis dysonymus, 126 .

Cacomantis merulinus, 126 .

Cacomantis merulinus lanceolatus, 126.

Cacomantis merulinus querulus, 126.

Cacomantis merulinus subpallidus. 126.

Cacomantis merulinus threnodes, 126.

Cacomantis variolosus, 127 .

Cacomantis variolosus seputcralis, 127.

Cacopitta atrigularis, 213.

Cacopitta lepidopleura, 213 .

Cacopitta leucogrammica, 216.

Cacopitta perspicillata, 211 .

caeligenus, Merops sumatranus, 110.

caerulata, Cyornis, 167. caerulea, Pitta, 150.

caerulescens, Falco, So.

caerulescens, Microhierax, 80.

caeruleus, Elanus, 78.

caeruleus, Falco, 78 .

caerulifrons, Cyornis magnirostris, 167.

caesia, Drymophila velata, 177 .

caesia, Monarcha, 177.

cala, Psittacula alexandri, 93.

Calamodyta doriae, 244 .

calceuticus, Meiglyptes tukki, 147

calcostetha, Chalcostetha, 273 .

calcostetha, Nectarinia, 273.

caledonica, Ardea, 57.

caledonicus, Nycticorax, 57.

Calidris, 30 .

Calidris canutus, 39.

Calidris tenuirostris, 30.

calidus, Falco peregrinus, 81 .

caligatus, Falco, 74 .

callima, Alcedo meninting, 100.

Callisitta azurea expectata, 263 .

Callocalia esculenta cyanoptila, 116.

Callolophus, 142.

Callolophus miniaceus, 142 .

Callolophus miniaceus dayak, 142 .

Callolophus miniaceus malaccensis, I42.

Callolophus miniaceus niasensis, 143.

Callolophus miniatus dayak, 142.

Calobates radiceus, 133.

calocephala, Cyornis banyumas, 166.

Caloenas, 24.

Caloenas nicobarica, 24 .

calonyx, Eurystomus orientalis, 97.

Caloperdix, 3 .

Caloperdix oculea, 3 .

Caloperdix oculea borneensis, 4 .

Caloperdix oculea ocellata, 4 .

Caloperdix sumatrana, 4 .

calopolius, Artamides sumatrensis, I 82.

Caloramphus sanguinolentus, 134 .

Calorhamphus, 134 .

Calorhamphus fuliginosus, 134 .

Calorhamphus fuliginosus fuliginosus, 134 .

Calorhamphus fuliginosus tertius, 134.

Calornis altirostris, 208 .

Calornis enganensis, 200

calus, Conurus fasciatus, 93 .

calvus, Allocotops, 206.

calvus, Melanocichla lugubris, 206. calvus, Torgus, 60 .

calvus, Vultur, 60 . 
INDEX.

Calyptimena, 153.

Calyptomina raftiesia, 1,53 .

Calyptomena viridis continentis, 153.

Calyptomena hosii, 153.

Calyptomean viridis, 153.

Calyptomena viridis siberu, 153.

Calyptomena whiteheadi, 153.

Calyptomina caudacuta, 153.

cameroni Blythipicus phrrhotis, 146.

cameranoi, Pteruthius fiaviscapis, 230.

campbelli, Arboricola, 2.

campbelli, Arborophila brunneopectus, 2.

Campeghagidae, $18 \mathrm{r}$.

Campeghage compta, 184.

Campephaga borneensis, I $\mathrm{S}_{4}$.

campestris, Alauda, 287 .

campestris, Anthus, 287.

Campophaga minor, 184 .

Cancroma leucoptera, 50.

canente, Hemicircus, 150.

canente, Picus, I50.

canicapillum, Aethostoma pyrrhogenys, 212.

canicapillus, Dryobates hatdwickii, 145.

canicapillus, Picus, 145.

canicapillus, Turdinus, 212.

caniceps, Phaenicophaus, 132.

caniceps, Trichophorus, 197.

canoroides, Cuculus, 124.

canorus, Cuculus, I 24.

cantatrix, Cyornis banyumas, 167.

cantatrix, Muscicapa, 167.

cantillans, Certhia, 260 .

cantonensis, Pericrocotus roseus, 187.

cantori, Alcippe, 2 I5.

cantori, Criniger, 197

cantori, Microtarsus, 104.

canus, Picus, 141.

canutus, Calidris, 39.

canutus, Tringa, 39.

Capella, 38

Capella nuegala, 38 .

Capella gallinago, 38 .

Capella stenura, 38.

capellei, Butreron, 1 t.

capellei, Columba, I I

capensis, Alcedo, 97.

capensis, Ramphalcyon, 97.

capiliata, Ciconia, 54.

capistrata, Columba, 19.

capistrata, Myiothera, 200.

capistratoides, Goldana, 200.

capistratoides, Pellorneum capistratum, 200. capistratum, Pellorneum, 200.

Capito lineatus, 130

Capito rosaceicollis, 140.

Capitonidae, 134 .

caprata, Motacilla, 233.

cajprata, Saxicola, 233.

Caprimulgidae, 1 o.

Caprimulgus, $1 \mathrm{t}$.

Caprimulgus affinis, 111.

Caprimulgus arundinaceus, III.

Caprimulgus, bisignatus, 111 .

Caprimulgus borneensis, II t .

Caprimulgus concretus, 11 .

Caprimulgus faberi, 11.

Caprimulgus indicus, II2.

Caprimulgus indicus innominatus, 112.

Caprimulgus indicus jotaka, I I2.

Caprimulgus innominata, 112.

Caprimulgus macrurus, it2.

Caprimulgus macrurus ambiguus, II 2.

Caprimulgus macrurus anamesus, 112.

Caprimulgus macrurus bimaculatus, II2.

Caprimulgus mirificus, I I I.

Caprimulgus pulchellus, 11 .

Caprimulgus pulchellus bartelsi, III.

Caprimulgus pulcher, $11 \mathrm{t}$.

Caprimulgus salvadorii, 112

cara, Aethopyga siparaja, 275.

Carbo javanica, 64 .

Carbo melanoleucos melvillensis, 64.

carbo, Pelecanus, 63.

carbo, Phalacrocorax, 63.

Carbo sulcirostris, 64 .

Carcineutes amabilis, Io2.

carcinophonus, Butorides javanicus, 58.

carinatus, Buceros, 108.

Carpococcyx, I/33.

Carpococcyx radiatus, 133.

Carpococcyx radiatus viridis, 133 .

Carpophaga aenea, 17 .

Carpophaga aenothorax, 18.

Carpophaga consobrina, 18 .

Carpophaga everetti, 18.

Carpophaga pickeringii, 18.

Carpophaga vandepolli, 18 .

Carpophaga whartoni, 18.

Carpophaga williami, 10.

carulescens, Excalfactoria chinensis, 5.

castaneicaudatus, Lobiopbasis, 7 .

castaneiceps, Minla, 210.

castaneicejs, Pseudominla, 2 ro.

castaneum, Philentoma, 177. 
castaneus, Myiophoneus, 230.

castaneus, Myophonus, 239.

castaneus, Phasianus, 6.

castaniceps, Abrornis, 252.

castaniceps, Ixulus, 228 .

castaniceps, Seicercus, 252.

castanjceps, Staphida, 228.

castanoptera, Strix, 90.

castanopterum, Glaucidium cuculoides, go.

castanopterus, Oriolus, 301.

cathoecus, Dicrurus macrocercus, 302.

caudacuta, Calyptomina, 153.

caudacuta, Chaetura, 17.

caudacuta, Hirundo, 117.

caudatus, Corvus, 300.

Ceblephyris fimbriatus, 183.

Ceblephyris striga, 184 .

Ceblepyris culminatus, 184 .

Ceblepyris javensis, 181 .

Ceblepyris Jarvata, 183 .

Ceblepyris larvata, 183 .

Ceblepyris melanoptera, 183.

Ceblpyris sumatrensis, $13 \mathrm{r}$.

Cecropis archetes, 161 .

Cecropis badia, $16 \mathrm{r}$.

celaenephis, Micropternus brachyurus, 148.

celaenephis, Micropternus phaioceps, 148 .

celaenus, Dicrurus cineraceu5, 304. celaenus, Dicrurus leucophaeus, 304.

celebensis, Centropus, 130.

celebensis, Chaetura, 118.

celebensis, var. Chaetura gigantea, I 18

cenchroides, Falco, 82.

Centrococcyx acheenensis, 120 .

Centrococcyx intermedius, 120.

Centropus, 120.

Centropus affinis, I3o.

Centropus bengalensis, 120 .

Centropus bengalensis javanicus, 130.

Centropus celebensis, 130.

Centropus celebensis kangeangensis, 130.

Centropus lepidus, I 30.

Centropus nigrorufus, 130 .

Centropus philippensis javanica, 129.

Centropus pumilus, 130 .

Centropus purpureus, 130.

Centropus rectunguis, 120 .

Centroptis sinensis, 120.

Centropus sinensis bubutus, 129.

Centropus sinensis eurycercus, 120.

Centropus sinensis intermedius, 129 .
Cerchneis moluccensis microbalia, 82.

Certhia brasiliana, 276 .

Certhia cantillans, 260 .

Certhia cruentata, 267.

Certhia jugularis, $27 \%$.

Certhia longirostra, $28 \mathrm{I}$.

Certhia malacensis, 270 .

Certhia siparaja, 275 .

Certhia sperata, 277.

Certhia trigonostigma, 260 .

Certhia trochilea, 267.

certhiola, Locustella, 244 .

certhiola, Motacilla, 244.

cerussata, Buchanga leucogenys, 304 .

cerussatus, Dicrurus leucophaeus, 304.

cerviniceps, Lyncornis, I10.

cervinicrissa, Cutia nipalensis, 229.

cerviniventris, Stoparola, I8I.

cerviniventris, Stoporala indigo, 181.

Cettia, 253.

Cettia montana, 253.

Cettia montana oreophila, 254

Cettia montana sepiaria, 253 .

Cettia montana sumatrana, 253 .

Cettia, montanus banksi, 254 .

ceylonensis, Culicicapa, 180.

ceylonensis, Dicrurus, 303.

ceylonensis, Platyrhynchus, I80.

Ceyx, ror.

Ceyx dillwynni, rot.

Ceyx enopopycius, 10I.

Ceyx erithacus, 10I.

Ceyx erithacus motleyi, 101.

Cevx euerythra, roı.

Ceyx innominatus, I0I.

Cevx rufidorsa, soI.

Ceyx rufidorsus, 101 .

Ceyx rufidorsus robusta, tot.

Ceyx sharpei, ror.

Chaetura, II7.

Chaetura caudacuta, 117.

Chaetura caudacuta cochinchinensis, 317.

Chaetura caudacuta nudipes, 117.

Chaetura celebensis, $1 \mathrm{f} 8$.

Chaetura celebensis ernsti, 118 .

Chaetura gigantea, 117.

Chaetura gigantea, var. celebensis, 118.

Chaetura gigantea indica, 177 .

Chaetura leucopygialis, 118 .

Chaitaris grandis, 170 .

Chalcites, 127.

Chalcites basalis, 128 .

chalcites, Cuculus, 128.

Chalcites limborgi, 127 
Chalcites maculatus, 127.

Chalcites malayanus, 128 .

Chalcites xanthorhynchus, I27.

Chalcites xanthorhynchus bangueyensis, 127.

Chalcites xanthorhynchus bangueyensis, 127.

chalcocephalus, Ixos, 194.

Chalcoparia, 280.

Chalcoparia singalensis, 280 .

Chalcoparia singalensis borneana, 280.

Chalcoparia singalensis interposita, 280 .

Chalcoparia singalensis pallida, 280.

Chalcoparia singalensis panopsia, 281.

Chalcoparia singalensis phoenicotis. 280.

Chalcoparia singalensis sumatrana, 280.

Chalcophaps, 23 .

Chalcophaps indica, 23.

Chalcophaps indica natalis, 24.

chalcopogon, Aithopyga, 275.

Chalcostetha, 273 .

Chalcostetha calcosterha, 273.

Chalcostetha calcostetha halitypa, 273.

Chalcostetha calcostetha heliomarpta, 273.

Chalcostetha calcostetha pagicola, 273.

Chalcostetha calcostetha proxima, 273.

Chalcostetha calcostetha sibiru, 273.

chalcurum, Polyplectron, 8.

Chalcurus inopinatus, 8.

chalepa, Lalage nigra, 185.

chaleja, Perissolalage, 185.

chalybeus, Turdus, 208.

Chaptia, 305.

Chaptia aenea, 305.

Chaptia aenea malayensis, 305 .

Charadriidae, 31.

Charadrius, 33 .

Charadrius alexandrinus, 34 .

Charadrius alexandrinus dealbatus, 34.

Charadrius alexandrinus peronii, 35.

Charadrius alexandrinus seebohmi, 35.

Charadrius apricarius, 33.

Charadrius apricarius fulvus, 33 .

Charadrius asiaticus, 34.

Charadrius asiaticus veredus, 34 .

Charadrius cirrhepidesmos, 33 .
Charadrius dubius, 34 .

Charadrius dubius curonucus, 34 .

Charadrius dubius jerdoni, 34 .

Charadrius himantopus, 35 .

Charadrus leschenaultii, 33.

Charadrius mongolus, 33 .

Charadrius mongolus atrifrons, 33 .

Charadrius pusillus, 34 .

Charadrius sanguineus, 33 .

Charadrius spinosus, 31 .

Charitociris maculata natunensis, 271.

charlottae, Criniger, 193.

charlottae, Iole olivacea, 193.

charitonit, Arborophila, 3.

charłtonii, Perdix, 3.

chaseni, Harpactes erythrocephalus, t20.

cheela, Spilornis, 75 .

Chelidon dasypus, 160.

chersonesophila, Mixornis gularis, 225.

chersonesus, Chrysocolajtes Jucidus, 149 .

chersonesus, Chrysocolaptes strictus, 149.

chersonesus, Cyanops asiatica, 136 .

chersonites, Cyornis rubeculoides, 165.

Chibia jentinki, 305.

Chibia termeuleni, 304.

chinensis, Amaurornis phoenicurus, 27.

chinensis, Columba, 22.

chinensis, Coracias, 300 .

chinensis, Excalfactoria, 5 .

chinensis, Fulica, 27.

chinensis, Kitta, 300.

chinensis, Oriolus, 209.

chinensis, Streptopelia, 22.

chinensis, Tetrao, 5.

chiroplethis, Pycnonotus plumosus, $20 \mathrm{I}$.

chirurgus, Hydrophasianus, 30.

chirurgus, Tringa, 30,

Chlamydochera, 183 .

Chlamydochera jefferyi, 183.

Chlidonias, 44.

Chlidonias hybrida, 45.

Chlidonias hybrida javanica, 45 .

Chlidonias leucoptera, 44 .

Chlidonias leucoptera grisea, 45.

chloeodis, Pycnonotus olivaceus, 201.

chlorates, Zosterops, 265.

chloris, Alcedo, 103.

chloris, Halcyon, 103.

chloris, Zosterops, 266.

chlorocenhalus, Phaenicophaus, 132. 
Chlorocharis, 267.

ChJorocharis emiliae, 267.

Chlorocharis emiliae moultoni, 267.

chlorolophus, Picus, I4I.

chlorophaea, Rhinortha, 132.

chlorophaeus, Cuculus, 132 .

Chloropicoides, I44.

Chloropicoides rafflesii, ${ }^{144 .}$

Chloropicuides rafflesil borneonensis, 144.

Chloropicoides rafflesii peninsularis, 144.

Chloropsis, 180.

Chloropsis aurifrons, 191.

Chloropsis aurifrons media, Igr.

Chloropsis cochinchinensis, 190.

Chloropsis cochinchinensis flavocincta, 191 .

Chloropsis cochinchinensis icterocephala, ıo.

Chloropsis cochinchinensis nigricollis, 100.

Chloropsis cochinchinensis viridinucha, 190.

Chloropsis connerati, 190.

Chloropsis cyanopogon, 191.

Chloropsis cyanopogon septentrionalis, Jgl.

Chloropsis hardwickii, 180 .

Chloropsis hardwickii malayana, 180 .

Chloropsis kinabaluensis, ıgI.

Chloropsis mysticalis, $19 t$.

Chloropsis sonnerati parvirostris, 190.

Chloropsis sonnerati zosterops, igo.

Chloropsis venusta, 180 .

Chloropsis viridis viriditectus, 190.

Chloropsis zosterops parvirsotris, 190.

chloroptera, Halcyon chloris, 104.

chloroptera, Iora, 188.

chotoptera, Sauropatis chloris, ro4.

chloropus, Fulica, 28.

chloropus, Gallinula, 28.

Chlorura borneensis, 203 .

Chlorura hyperythra, 293.

Chlorura hyperythra malayana, 203.

Chotorea, 134.

Chotorea chrysopogon, $t 34$.

Chotorea chrysopogon chrysopsis, 135.

Chotorea cbrysopogon lacta, 134.

Chotorea corvina, 134 .

Chotorea javensis, 135 .

Chotorea mystacophanes, I35.

Chotorea mystacophanes ampala, 135.
Chotorea mystacophanes humei 135.

Chotorea rafflesii, 135.

Chotorea rafflesii borneensis, 136 .

Chotorea versicolor borneensis, 136.

Chotorhea chrysopogon laetus, 134 .

Chotorhea raffiesii billitonis, 135 .

Chotorhea rafflesii malayensis, 135 .

Chryptolopha davisoni, 252.

chrysaea, Stachyris, 223.

chrysea, Ploceus, 293.

Chrysocolaptes, 149 .

Chrysocolaptes gutticristatus indomalayicus, 149.

Chrysocolaptes lucidus, 149 .

Chrysocolaptes lucidus chersonesus, 149.

Chrysocolaptes lucidus gutticristatus, 149.

Chrysocolaptes lucidus strictus, 150.

Chrysocolaptes strictus chersonesus, 149.

Chrysocolaptes validus, 150.

Chrysocolaptes validus xanthopygius, 150 .

chrysogaster, Piaya, 130.

chrysogenys, Arachnothera, 283.

chrysogenys, Nectarinia, 283 .

Chrysonotus tridactylus, 148 .

Chrysophlegma, 143.

Chrysophlegma flavinticha, 143 .

Chrysophlegrna flavinucha mystacale, 143 .

Chrysophlegma flavinucha wrayi, 143.

Chrysophlegma mentale, 143.

Chrysophlegma mentale humii, 143 .

Chrysophlegma mentale saba, I43.

Chrysophlegma mystacalis, 143 .

Chrysophlegma niasense, 143.

chrysophorus, Brachypodius atriceps, 194.

chrysopogon, Bucco, 134.

chrysopogon, Chotorea, 134 .

chrysops, Stachyris chrysaea, 223.

chrysopsis, Chotorea chrysopogon, 135.

chrysopsis, Megalaima, 135.

chrysorrheum, Dicaeum, 270.

chrysorrhoeus, Brachypus, 204.

chrysura, Munia, 201.

Ciccaba myrtha, 80.

Ciconia capillatà, 54 .

Ciconia javanica, 54.

Ciconitidae, 53.

Cinclosoma erythrocephalum, 205.

cincta, Columba, 16.

cinctus, Ptilinopus, 16.

cineraceus, Edolius, 303. 
cineraceus, Orthotomus, 247.

cinerascens, Dendrocitta occipitalis. 300.

cinerascens, Parus major, 262.

cinerea, Alcippe, 219.

cinerea, Ardea, 55.

cinerea, Fulica, 28.

cinerea, Gallicrex, 28.

cinerea, Iole, 102.

cinerea, Motacilla, 284.

cinerea, Muscitrea, 260.

cinerea, Pachycephala, z6o.

cinerea, Porzana, 26.

cinerea, Scolopax, 42.

cinereicollis Cinnyris, $28 \mathrm{I}$.

cinereicollis, Phyllergates cucullatus, 248.

cinereigula, Pericrocotus montanus, 186.

cinereocapilla, Alcippe, 215.

cinereocapillum,

Malacopteron

magnirostre, 215

cinererceps, Hemichelidon, 163.

cinereum, Malacopteron, 214.

cinereus, Dicrurus, 303.

cinereus, Ibis, 53 .

cinereus, Ixos, 102.

cinereus, Malacopteron, 214.

cinereus, Parus major, 261 .

cinereus, Porphyrio, 26.

cinereus, Tantalus, 53.

cinereus, Xenus, 42.

cinnamomea, Ardea, 60.

cinnamomea, Macropygia phasianella, 21 .

cinnamomea, Motacilla, $18 \%$.

cinnamomeoventris, Iole olivacea, I03.

cinnamomeoventris, Iole virescens, 593.

cinnamomeus, Ixobrychus, 60.

cinnamomeus, Pericrocotus, 187.

Cinnyris affinis, 282 .

Cinnyris brasiliana axantha, 276 .

Cinnyris brasiliana eumecis, 276.

Cinnyris brasiliana hypolampis, 276.

Cinnyris brasiliana mecynorhyncha, 276.

Cinnyris brasiliana oenopa, 276.

Cinnyris cinerejcollis, $28 \mathrm{r}$.

Cinnyris longirostra, 283 .

Cinnyris luteoventer, 277.

Cinnyris macklotii, 273 .

Cinnyris magna, 282.

Cinnyris nipalensis, 276 .

Cinnyris ornata heliomanis, 277 .

Cinnyris ornata heliopleta, 277.

Cinnyris ornata heliozeteta, 277.

Cinnyris ornata microleuca, 277.
Cinnyris ornata polyclysta, 278 .

Cinnyris ornata zapega, 277.

Cinnyris ornatus, 277.

Cinnyris ruber, 276 .

Cinnyris saturata, 273 .

Circaetus, 74 .

Circaetus gallicus, 74 .

Circus, 70 .

Circus teruginosus, 70.

Circus melanoleucus, 70 .

Circus spilonotus, 70.

cirrhatus, Falco, 73 .

cirrhatus, Spizaetus, 73.

cirrhepidesmos, Charadrius, 33.

Cisticola, 248.

Cisticola delicatula, 248 .

Cisticola exilis, 248 .

Cisticola exilis lineocapilla, 248 .

Cisticola juncidis, 248 .

Cisticola juncidis fuscicapilla, $24 \mathrm{~s}$.

Cisticola juncidis malaya, 248 .

citrina, Geokichla, 242.

citrinella, Zosterops, 264.

citrinus, Megalurus, 249.

citrinus, Turdus, 242.

Cittocincla melanura, 238 .

Cittacincla nigricauda, 238 .

clara, Zosterops, 265.

Clamator, 122.

Clamator coromandus, I22.

Clivicola riparia ijimae, 162 .

clypeata, Anas, 63.

clypeata, Spatula, 63 .

coccinea, Pitta granatina, I 59.

coccometopus, Hemicercus concretus, Iso.

Coccyzus rubirostris, 130.

cochinchinensis, Chaetura caudacuta, 117.

cochinchinensis, Chloropsis, ıoo.

cochinchinensis, Turdus, 190.

Cochoa, 230.

Cochoa azurea, 239.

Cochoa azurea beccarii, 239.

coelica, Erythura prasina, 293.

coerulata, Schwaneria, 167.

coerulea, Myiothera, I 56.

coerulescens, Alcedo, Ior.

coerulifrons, Cyornis banyumas, I67.

Collocalia, I I 4 .

Collocalia brevirostris tichelmani, I 16.

Collocalia brevirostris vulcanorum, 116.

Collocalia esculenta, 116.

Collocalia esculenta dodgei, is.

Collocalia esculenta linchi, 116.

Collocalia esculenta natalis, i17.

Collocalia esculenta oberholseri. I 16. 
Collocalia francica, 114 .

Collocalia francica aerophila, 115. Collocalia francica amechana, 114. Collocalia francica bartelsi, it 4 . Collicalia francica germani, 144 . Coltocalia francica javensis, II 4 . Collocalia francica natunae, II5. Collocalia francica perplexa, 114. Collocalia fuciphaga, 115.

Collocalia fuciphaga innominata, 115.

Collocalia gigas, I 16.

Collocalia linchi cyanoptila, I I6.

Collocalia linchi oberholseri, $1: 6$.

Collocalia lowi, Its.

Collocalia lowi robinsoni, I 5.

Collocalia lowi tichelmani, 116.

Collocalia lowi vulcanorum, 116 .

Collocalia nidifica, IIs.

Collocalia vestita, IIs.

Collocalia vestita maratua, I15.

colpiotes, Dissemurus paradiseus, 306.

Columba, 19.

Columba aenea, 17 .

Columba argentina, Io.

Columba badia, 19 .

Columba bantamensis, 23 .

Columba bicolor, 19.

Columba bitorquata $\mathbf{2 2 .}$

Columba capellei, II.

Columba capistrata, I 9 .

Columba chinensis, 22.

Columba cincta, 16.

Columba chinensis, 22.

Columba cincta, 16.

Columba cinnamomea, 13.

Columba cristata, 4.

Columba curvirostra, I 2.

Columba dusumieri, 22.

Columba ferruginea, I3.

Columba fulvicollis, I3.

Columba indica, 16 .

Columba indica, 23 .

Columba jambu, 16.

Columba javanensis, 23.

Columba javanica, 24 .

Columba lacernulata, 19.

Columba leptogrammica, 20 .

Columba malaccensis, 23.

Columba melanocephala, I6.

Colomba Moluccensis, i7.

Columba nicobarica, 24 .

Columba olax, 15.

Columba oxyura, ro.

Columba phasianella, 20.

Columba phasma, 20.

Columba pompadora, 13.

Columba porphyrea, 16.

Columba punicea, 20.

Columba purpurea, I 5.
Columba rosacea, 18 .

Columba roseicollis, 16 .

Columba ruficeps, $2 \mathrm{I}$.

Colymbus ruficollis, 50 .

Columba striata, 23 -

Columba tigrina, 23.

Columba vernans, 14.

Columba vitiensis, 20 .

Columba vitiensis griseogulatis, 20.

Columbidae, so.

comatus, Berenicornis, 108.

comatus, Buceros, 108.

comatus, Cypselus, 113.

compilator, Corvus enca, 308.

compta, Campephaga, 184 .

comata, Hemiprocne, 113 .

compta, Lalage fimbriata, 184.

concinnus, Orthotomus sepium, 247.

concolor, Arachnothera, 282 .

concolor, Dieaeum, 270.

concolor, Ducula, 10.

concreta, Cyornis, 164.

concreta, Dacelo, 104.

concreta, Halcyon, ro4.

concreta, Malacocincla, 210.

concreta, Muscicapa, 164.

concretus, Caprimulgus, 111 .

concretus, Cuculus micropterus.

124.

concretus, Graucalus, 181.

concretus, Hemicercus, 150.

concretus, Picus, t 50.

condea, Trogon, 119.

condorensis, Otus bakkamoena, 86.

confusus, Lanius cristatus, 259.

connectens, Alophoixus phaeocephatus, 197.

connectens, Eurystomus orientalis, 97.

connectens, Grecinus vittatus, $14 \%$. connectens, Hemixus, 102.

connectens, Ixos cinereus, I.92.

connectens, Mixornis gularis, 225.

connectens, Mixornis rubricapilla,

225 .

connectens, Picus vittatus, 141 .

consanguineus, Analcipus, 301.

consanguineus, Oriolus cruentus, $30 \mathrm{~T}$

consobrina, Carpophaga, 18.

consobrina, Ducula aenea, 18.

consobrina, Hypothymis azurea, 173.

consobrinus, Oriolus xanthonotus, 301 .

continentis, Brachylophus puniceus, 142.

continents, Calyptomena viridis, 153.

continentis, Picus puniceus, 142.

contra, Sturnopastor, 204. 
contra, Sturnus, 294.

Conurus erythrogenys, 93.

Conurus fasciatus calus, 93.

Conurus fasciatus perioncus, 93.

convexus, Anthracoceros coronatus. 106.

convexus, Buceros, io6.

cooki, Cypselus pacificus, tio.

cooki, Micropus pacificus, 119.

copha, Arachnothera chrysogeny", 283.

Copsychus, 235 .

Copsychus saularis, 235.

Copsychus saularis amoenus, 236 .

Copsychus saularis ephalus, 235 .

Copsychus saularis javensis, 236 .

Copsychus saularis musicus, 235 .

Copsychus saularis nesiarchus, 235.

Copsychus saularis nesiotes, 235.

Copsychus saularis niger, 236 .

Copsychus saularis pagiensis, 235.

Copsychus saularis pluto, 236 .

Copsychus saularis problematicus, 235.

Copsychus saujaris zacnecus, 235.

Copsychus stricklandii, 238 .

Copsychus suavis, 237.

Coracias, 06.

Coracias benghalensis, 96.

Coracias benghalensis affinis, 06 .

Coracias chinensis, 300 .

Coracias orientalis, 97 .

Coracias puella, 191.

Coracias sumatranus, 156.

Coracias xanthornus, 300.

Coraciidae, 96 .

Coracina, 181 .

Coracina javensis, 181 .

Coracina javensis larutensis, 181 .

Coracina personata, 183.

Coracina personata larvata, 183 .

Coracina personata melanocephala, 183.

Coracina personata normand, 183.

Coracina sumatrensis, 181 .

Coracina sumatrensis babiensis, I 82.

Coracina sumatrensis bungurensis, 182.

Coracina sumatrensis crissalis, I 82

Coracina sumatrensis enganensis, 182.

Coracina sumatrensis kannegieteri. 182.

Coracina sumatrensis simalurensis, 182.

Coracina sumatrensis vordermani, I 82.

coracinus, Acanthylis, I 8.

corallipes, Dendrophila, 262.

corallipes, Sitta frontalis, 262 . comutus, Podargus, 96.

coromanda, Alcedo, 102.

coromanda, Ardeola ibis, 59 .

coromanda, Halcyon, 102.

coromanda, Strix, 84.

coromandeliana, Anas, 61.

coromandelianus, Nettapus, 61.

coromandus, Bubo, 84 .

coromandus, Clamator, I22.

coromandus, Cuculus, 122.

coronata, Ficedula, 251.

coronata, Napothera, 214.

coronata, Perdix 4.

coronatus, Ampeliceps, 295.

coronatus, Anthracoceros, 106.

coronatus, Buceros, 106.

coronatus, Enicurus, 233.

coronatus, Lanius, 258.

coronatus, Oriolus, zog.

coronatus, Phylloscopus occipitalis, 251.

coronatus, Platylophus galericula-

tus, 258

corrugatus, Buceros, 107.

corrugatus, Cranorrhinus, 107.

Corvidae, 308 .

corvina, Chotorea, 134.

corvinus, Bucco, 134 .

Corvus, 308.

Corvus benghalensis, 06

Corvus brachyurus, 157.

Corvus caudatus, 300 .

Corvus enca, 308 .

Corvus enca compilator, 308 .

Corvus hottentottus, 304 .

Corvus leucolophus, 205.

Corvus macrorhynchos, 308 .

Corvus paradisi, 176.

Corvus splendens, 308 .

Corvus splendens protegatus, 308 .

Corvus temia, 300 .

Corvus varians, 309 .

Corydalla sinensis, 286 .

Corydon, 156.

corydon, Eurylaimus, I56.

Corydon sumatranus, i 56 .

Corydon sumatranus brunnencens, I 56.

Corydon temminckii, I 56.

Coryllis javanica, 95 .

Corythocichla brevicaudata herberti, 217.

Corythocichla crassa, 217.

Corythocichla leucosticta, 217.

Coturnix spadicea, 7 .

Cranorrhinus, 107.

Cranorrhinus corrugatus, 107.

Cranorrbinus corrugatus megistus, 107.

crassa, Corythocichla, 217. 
crassa, Napothera brevicaudata, 217. crassirostris, Arachnocestra, 283 crassirostris, Arachnothera, 283 . crassirostris, Enncoctonus, 258. crassirostris, Myiophoneus, 240. crassirostris, Myophonus flavirosttis, 240.

crecca, Anas, 62.

Criniger, 195.

Criniger balicus bartelsi, 196 .

Criniger bemmeleai, I97.

criniger, Brachypodius, 108.

Criniger cantori, 197.

Criniger charlottae, 193.

Criniger diardi, 197.

Criniger finschii, I96.

Criniger gularis balicus, 196.

Criniger gutturalis, 195.

Criniger gutturalis gutturalis, ı06.

Criniger gutturalis ochraceus, 195.

Criniger gutturalis ruficrissus, $\mathbf{1 0 6 .}$

Criniger gutturalis sacculatus, 106. Criniger gutturalis sumatranus, 196. criniger, Irena puella, Igz.

Criniger ochraceus sacculatus, to6. criniger, Setornis, 197.

Criniger sordidus, 195.

Criniger tephrogenys, 196.

Criniger tephrogenys balicus, I06.

Criniger tephrogenys bartelsi, I 06.

Criniger theiodes, ig6.

criniger, Tricholestes, I98.

crispiceps, Tricophorus, 198 .

crissalis, Coracina sumatrensis, 182

crissalis, Graucalu5, - 82 ,

cristata, Columba, 4 .

cristata, Sterna bergii, 47.

cristata, Vanga, 257.

cristatus, Buceros, Ios.

cristatus, Buteo, 82 .

cristatus, Lanius, 250.

cristatus, Pandion haliaetus, 82.

cristatus, Phasianus, 4 .

cristatus, Sterna, 47 .

Crithagra estherae, 288.

croceoventre, Dicaeum, 269 .

Crocethia, 30.

Crocethia alba, 30.

croceus, Pericrocotus, 186.

Crocias, 258

Crocias guttatus, 258 .

Crocopsis bimaculatus tenggerensis, iog.

crocorrhous, Pycnonotus, 204.

cruentata, Certhia, 267 .

cruentatum, Dicaeum, 267.

cruentus, Leptopteryx, 301.

cruentus, Oriolus, 301.

Crypsirina, 300.

Crypsirina temia, 300 . crypta, Iole olivacea, 193.

Cryptolopha butlert, 252.

Cryptolopha kinabaluensis, 25 .

Cryptolopha montis, 252.

Cryptolopha montis inornata, 253.

Cryptolopha muelleri, 252.

Cryptolopha sumatrensis, 252.

Cryptolopha vordermani, 253.

Cryptolopha youngi, 252.

Cryptonyx dussumieri, 4.

Cryptonyx ferrugineus, 4 .

Cryptonyx niger, 4 .

cryzona, Alcedo, 99.

Cuculidae, I 22.

cucullata, Pitta sordida, 158 .

cucullatus, Eurylaimus, I 55.

cucullatus, Phyllergates, 247.

cucullatus, Vanellus, 32.

cuculoides, Falco, 71 .

cuculoides, Glaucidium, go.

cuculoides, Noctua, go.

Cuculus, 124.

Cuculus affinis, I 24.

Cuculus albopunctulatus, 122.

Cuculus assimilis, 124.

Cuculus basalis, 28.

Cuculus bengalensis, 129 .

Cuculus canoroides, 124.

Cuculus canorus, 124.

Cuculus canorus telephonus, 124 .

Cuculus chalcites, 128.

Cuculus chlorophaeus, 132.

Cuculus coromandus, $\llbracket 22$.

Cuculus curvirostris, 132.

Cuculus fasciolatus, 126.

Cuculus fugax, 123.

Cuculus horsfieldi, 125.

Cuculus hyperythrus, 123.

Cuculus intermedius insulindae, 125.

Cuculus javanicus, I 30 .

Cuculus lugubris, I 22.

Cuctulus malayanus, I 28 .

Cuculus merulinus, 126.

Cuculus micropterus, 124.

Cuculus micropterus concretus, 124.

Cuculus musicus, 126 .

Cuculus neglectus, 128 .

Cuculus nigrorufus, 130 .

Cuculus nisicolor, 123 .

Cuculus optatus, 125.

Cuculus paradiseus, 306 .

Cuculus poliocephalus, 125.

Cuculus poliocephalus insulindae, 125.

Cuculus poliocephalus lepidus, 125.

Cuculus pravata, 126.

Cuculus rufo-vittatus, $: 26$.

Cuculus scolopaceus, 128 .

Cuculus sepulcralis, 127. 
Cuculus sonneratii, 125.

Cuculus sparverioides, 123.

Cuculus striatus, 124.

Cuculus sumatranus, I31.

Cuculus sumatrensis, 132 .

Cuculus vagans, 124 .

Cuculus variolosus, 127.

Cuculus xanthorhynchus, 127 .

Culicicapa, 180.

Culicicapa ceylonensis, 180 .

Culicicapa ceylonensis amphiala, 180.

Culicicapa ceylonensis antioxantha, 180 ,

Culicicapa ceylonensis eophila, 180.

Culicicapa ceylonensis meridionalis, 180 .

Culicicapa ceylonensis pellonota, 180.

Culcicapa ceylonensis pellopira, 480.

Culicicapa ceylonensis percnocara, 180.

culminata, Lalage fimbriata, I84.

culminatus, Ceblepyris, 184 .

cumatilis, Cyanoptila cyanomelana, 172.

cumingij, Megapodius reinwardt, J.

curonicus, Charadrius dubius, 34.

Curruca stentorea, 244.

curvirostra, Columba, 12.

curvirostra, Treron, 12.

curvirostris, Cuculus, 132.

curvirostris, Phoenicophaus, 132.

curvirostris, Tetrao, 1.

Cutia, 220.

Cutia nipalensis, 220.

Cutia nipalensis cervidicrissa, 220.

cyane, Luscinia, 232.

cyane, Motacilla, 232.

cyanea, Cyanops australis, 138.

cyanea, Muscicapa, 101.

cyaneopileata, Psittacula, 95.

cyanescens. Halcyon chloris, 104.

cyanescens, Sauropatis chloris, JO4. cyaneus, Mesobucco duvaucelii, 138 .

cyaneus, Turdus, 239.

cyaniventer, Tesia, 231.

cyaniventris, Pycnonotus, 202.

cyaniventris, Pycnonotus, 202.

cyanocephalus, Bucco, 137.

cyanochrus, Pycnonotus erythropthalmos, 202.

Cyanoderma, 223.

Cyanoderma erythroptera, 223.

Cyanoderma erytbroptera apega, 224.

Cyanoderma erythroptera bicolor, 224.
Cyanoderna erythroptera eripella, 223.

Cyanoderma erythroptera fulviventris, 224.

Cyanoderma erythroptera neocara, 223.

Cyanoderma erythroptera pella, 224.

Cyanoderma erythroptera pyrrophaea, 223.

Cyanoderma erythroptera rufa, 224.

Cyanoderma erythropterum pellum, 224.

Cyanoderma labuanensis, 224 .

Cyanoderma melanothorax, 224 .

Cyanoderma melanothorax albigula, 224.

Cyanoderma melanothorax baliensis, 225 .

Cyanoderma melanothorax inter. mediá, 225 .

Cyanoderma melanothorax mendeni, 224

Cyanoderma rufifrons, 225.

Cyanoderma rufifrons obscura, 225.

Cyanoderma rufifrons poliogaster, 225.

Cyanoderma sordida, 223.

cyanomelana, Cyanoptila, 172.

cyanomelana, Muscicapa, 172.

cyanopis, Emberiza, 292.

cyanopogon, Chloropsis, 191.

cyanopogon, Phyllornis, IgI.

Cyanops, 136

Cyanons armillaris, 136 .

Cyanops armillaris baliensis, 136.

Cyanops armillaris brachyrhyncha, 136.

Cyanops armillaris henricij, I36.

Cyanops armillaris pulcherrima, 137.

Cyanops asiatica, 336 .

Cyanops asiatica chersonesus, 136 .

Cyanops australis, 137.

Cyanops au:tralis cyanea, 138.

Cyanops australis duvacelii, 138

Cyanops australis eximia, 138 .

Cyanops australis gigantorhina, 138 .

Cyanops australis bebereri, 138 .

Cyanops australis stuarti, 138 .

Cyanops australis tanamassae, 138 .

Cyanops duvaceli robinsoni, 138 .

Cyanops franklinii, 137.

Cyanops franklinij minor, 137.

Cyanops franklinit trangensis, 137.

Cyanops henricii brachyrhyncha, 136.

Cyanops mystacophanes aurantiifrons, 135.

Cyanops oorti, 137.

Cyanops oorti monticola, 137 . 
cyanoptera, Pitta brachyura, I57. cyanopteryx, Ramphalcyon capensis, 98 .

Cyanoptila, 172 .

cyanoptila, Collocalia esculenta, I 16.

cyanoptila, Collocalia linchi, 116.

Cyanoptila cyanomelana, 172.

Cyanoptila cyanomelana cumatilis, 172.

Cyanoptila cyanomelana intermedia, 172 .

cyanopygius, Merops, ito.

cyanouroptera, Siva, 220.

cyanoventris, Alcedo, 103.

cyanoventris, Halcyon, 103.

cyanurus, Psittacus, 94.

cyanurus, Psittinus, 94.

cyanurus, Turdus, 159.

Cymbirhynchus, I 56 .

Cymbirbynchus macrorhynchos, 156.

Cymbirhynchus macrorhynchos malaccensis, 156.

Cymborhynchus malaccensis, 156.

Cyornis, 163.

Cyornis anak, 165 .

Cyornis banyumas, 166.

Cyornis banyumas calocephala, I 66 .

Cyornis banyumas cantatrix, 167.

Cyornis banyumas coerulifrons, 167.

Cyormis banyumas karimatensis, 166.

Cyornis banyumas lampra, I 66 .

Cyornis banyumas limitans, I 66 .

Cyornis banyumas magnirostris, 167.

Cyornis banyumas montana, 167 .

Cyornis caerulata, 167 .

Cyornis caerulata albiventer, $₫ 67$.

Cyornis caerulata rufifrons, 167 .

Cyornis concreta, 164.

Cyornis concreta everetti, 164.

Cyornis elegans antelia, ${ }^{6} 6$.

Cyornis elegans rupatensis, 165.

Cyornis erythrogaster, 166.

Cyornis frenatus, I66.

Cyornis hosei, I66.

Cyornis magnirostris caerulifrons. 167.

Cyornis nigrigularis, 167.

Cyornis olivaceus, 178 .

Cyornis peninsularis, 164 .

Cyornis rubeculoides, 165.

Cyornis rubeculoides chersonites, 165.

Cyornis rubeculoides dialilaema, 165.

Cyornis rubeculoides glaucicomans, 165 .
Cyornis ruecki, 164 .

Cyornis rufigastra, 165.

Cyornis rufigastra karimatensis, 166.

Cyornis rufigastra lampta, 166 .

Cyornis rufigastra longipennis, ז 66.

Cyornis rufigastra rhizophorae, 166 .

Cyornis rufigastra sumatrensis, 166 .

Cyornis superba, 167 .

Cyornis tickelliae glaucicomans, 165.

Cyornis turcosa, $\mathbf{6} 65$.

Cyornis turcosa rupatensis, 165 .

Cyornis, unicolor, I64.

Cyornis unicolor harterti, I64.

Cyornis unicolor infuscata, 164.

Cyornis vanheysti, 164 .

Cyornis vivida, 163 .

Cyornis vivida sumatrana, 164.

Cyornis whitei montana, 167 .

Cypselus affinis, 110 .

Cypselus comatus, II3.

Cypselus giganteus, II7.

Cypselus infumatus, 118 .

Cypselus leucopygialus, 119.

Cypselus lowi, 115 .

Cypselus pacificus cooki, tro.

Cypselus subfurcatus, ito.

Cyp. balașiensis, 118 .

Cypsiurus, iा 8.

Cypsiurus barasiensis, 118 .

Cypsiurus batasiensis infumatus, It 8.

Cysticola lineocapilla, 248 .

Dacelo buccoides, 102.

Dacelo concreta, 104.

Dacelo pulchella, Iо2.

dactylatra, Sula, 65.

dalhousiae, Eurylaimus, 153.

dalhousiae, Psarisomus, 153.

damacensis, Totanus, 40 .

damicra, Aegithina tiphia, 189.

dammermani, Psittacula alexandri, 93-

damnata, Rhinocichla mitrata, 206.

dasyjus, Chelidon, I6o.

dasypus, Delichon urbica, 160 .

dauma, Oreocincla, 243.

dauma, Turdus, 243

daurica, Hirundo, 16 .

davișoni, Alcippe phaeocephala, 218.

davisoni, Alcippe noioicephala, 218.

davisoni, Brachyurus, 156.

davisoni, Cryptolopha, 252.

davisoni, Geokichla sibirica, 241.

davisoni,Geronticus, 52. 
davisoni, Pseudibis papillosa, 52. davisoni, Seicercus montis, 252.

davisoni, Stachyris nigriceps, 220. davisoni, Turdulus, 24I.

dayak, Callolophus miniaceus, 142. dayak, Callolophus miniatus 142 .

dayakana, Dicaeum trigonostigma, 260 .

dayakanum, Dicaeum trigonostigmum, 260 .

dealbatus, Aegialitis, 34 .

dealbatus, Charadrius alexandrinus, 34

decipiens, Niltava grandis, 170.

dedemi, Gecinus, I4I.

dedemi, Picus canus, I4I.

defontainei, Psittacula longicauda, 93.

delacouri, Lophura sumatrana, 6

delica, Megalaema baemacephala, 139.

delica, Xantholaema haemacephala, 139.

delicatula, Cisticola, 248 .

Delichon, 160.

Delichon urbica, 160.

Delichon urbica dasypus, 160.

Demigrett3, 56.

Demigretta sacra, 56 .

Dendrobiastes, 160 .

Dendrobiastes hyperythra, I60.

Dendrobiastes hyperytbra malay. ana, Ifog.

Dendrobiastes hyperythra mjo. bergi, i6n.

Dendrobiastes hyjerythrus mjo. bergi, 160 .

Dendrobiastes hyperythrus sumatranus, 160 .

Dendrobiastes hyperythra vulcani, 169 .

Dendrocitta, 309 .

Dendrocitta occipitalis, 300 .

Dendrocitta occipitalis cinerascens, 300 .

Dendrocitta rufigaster, 309 .

Dendrocitta sinensis tuckeri, 300 .

Dendrocophus brevipennis, 144 .

Dendrocygna, $6 \mathrm{r}$.

Dendrocygna javanica, 61 .

Dendrocygna javanica arcuata, 62.

Dendronanthus, 286 .

Dendronanthus indicus, 286.

Dendrophassa fulvicollis melopogenys, 13.

Dendrophassa olax arismicra, I5.

Dendrophassa vernans abbotti, 14 .

Dendrophassa vernans adina, 15.

Dendrophassa vernans mesochloa. 15.

Dendrophassa vernans miza, 15 .
Dendrophassa vernans pellochlora, 15.

Dendrophassa vernans polioptila, 15.

Dendrophila corallipes, 262.

Dendrophila flavipes, 263.

Dendrophila gymnophrys, 263.

Dendropicus sordidus, 150 .

deningeri, Phoenicophaes curvirostris, 132

deningeri, Phoenicophaus curvirostris, 132.

diadematus, Buceros, 105.

cliadematus, Enicurus, 234.

dialilaema, Cyomis rubeculoides 165.

diana, Lanius, 234.

diana, Muscisylvia, 234.

diardi, Alophoixus phaęocephalus. 197.

diardi, Criniger, 197.

diardi, Melias, 13I.

diardi, Rhopodytes, $13 \mathrm{I}$.

diardii, Harpactes, I 19.

diardii, Houppifer, 5.

diardii, Trogon, I Io.

diatropurus, Muscadivores aeneus, 17.

Dicaedae, $26 \%$.

Dicaeum, 267 .

Dicaeum chrysorrheum, 270 .

Dicaeum concolor, 270 .

Dicaeum concolor borneanum, 270 .

Dicaeum concolor sollicitans, 270 .

Dicaeum croceoventre, 269 .

Dicaeum cruentatum, 267 .

Dicaeum cruentatum batuense, 268 .

Dicaesm cruentatum ignitum, 268

Dicaeum cruentatum nigrimentum 268.

Dicaeum cruentatum siamensis, 268.

Dicaeum cruentatum sumatranum. 268.

Dicaeum finschii, 272 ,

Dicaeum flavum, 265 .

Dicaeum ignicapilla, 271 .

Dicaeum minullum borneanum, 270.

Dicaeum pryeri, 268.

Dicaeum rubescen5, 267.

Dicaeum rubrocosum, 267.

Dicaeum sanguinolentum, 268.

Dicaetum sanguinolentum ablutum. 268 .

Dicaeum sanguinolentum beccarii, 268.

Dicaéum sanguinolentum ignipectus, 268 .

Dicaeum sanguinolentum monticolum, 260 .

Dicaeum stresemanni, 267. 
Dicaeum sulaense zita, 269 .

Dicaeum trigonostigma antioproctum, 270 .

Dicaeum trigonostigma dayakana, 260 .

Dicaeum trigonostigma flaviclunis, 260 .

Dicaeum trigonostigma hypocbloum, 260.

Dicaeum trigonostigma lyprum, 270.

Dicaeum trigonostigma megastoma, 260.

Dicaeum trigonostigma melanthe, 270 .

Dicaeum trigonostigma rubropy= gium, 260 .

Dicaeum trigonostigmum, 260.

Dicaeum trigonostigmum antioproctum, 270 .

Dicaeum trigonostigmum dayakanum, 260.

Dicaeum trigonostigmum flaviclune, 260.

Dicaeum trigonostigmum lyprum, 270.

Dicaeum trigonostigmum megastomum, $\neq 60$.

Dicaeum trigonostigmum pagense, 270.

Dicaeum trigonostigmum rubropygium, 269 .

Dicaeum trochileum, 267.

Dicaeum vanheysti, 268 .

Dichoceros, 105.

Dichoceros bicornis, 105.

dicrorhynchus, Myophonus flavirostris, 240.

Dicruridae, 302 .

dicruroides, Pseudornis, 122.

dicruroides, Surniculus lugubris, 122.

Dicruropsis borneensis, 304.

Dicruropsis pectoralis solombensis, 305.

Dicruropsis viridinitens, 305.

Dicrurupsis pectoralis sirensis, 305 .

Dicrurus, 302 .

Dicrurus aeneus, 305 .

Dicrurus annectans, 302 .

Dicrurus bilobus, 302 .

Dicrurus ceylonensis, 303 .

Dicrurus cineraceus celaenus, 304

Dicrurus cinereus, 303.

Dicrurus hottentottus, 304.

Dicrurus hottentottus borneensis, 304.

Dicrurus hottentottus jentinki, 305.

Dicrurus hottentottus sirensis, 305.

Dicrurus hottentottus solombensis, 305.
Dicrurus hottentottus suluensis, 305.

Dicrurus hottentotus sumatranus, 304.

Dicrurus bottentottas termeuleni. 304.

Dicrurus hottentottus viridinitens, 305.

Dicrurus leucogenis diporus, 304.

Dicrurus leucogenis siberu, 304.

Dicrurus leucogenis siberu, 304.

Dicrurus leucogenys salangensis, 303.

Dicrurus leucophaeus, 303.

Dicrurus leucophaeus celaenus, 304.

Dicrurus leucophaeus cerussatus, 304.

Dicrurus leucophaeus leucogenis, 303.

Dicrurus leucophacus periophthalmjcus, 304 .

Dicrurus leucophaeus phaedrus, 303.

Dicrurus leucophaeus siberu, 304.

Dicrurus leucophaeus stigmatops, 303.

Dicrurus longicaudatus, 303.

Dicrurus longicaudatus intermedius, 303.

Dicrurus macrocercus, 302.

Dicrurus macrocercus cathoecus, 302.

Dicrurus macrocercus javanus, 302.

Dicrurus platurus, 306.

difficilis, Zosterops chlorates, 265.

diffusus, Oriolus chinensis, 209.

Digenea malayana, 170.

dillwynni, Ceyx, ror.

diluta, Napothera epilepidota, 217.

diluta, Stacbyris poliocephala, 22 r.

dilutus, Turdinulus epilepidotus, 217.

dimidiatus, Falco, 77 .

dimidiatus, Picus, 140.

Dimorpha superciliaris, 160 .

Dinopium, 148 .

Dinopium javanense, 148.

Dinopium javanense borneonensis, 149.

Dinopium javanense exsul, 149.

Dinopium javanense intermedium. I. 49 .

Dinopium javanense palmarum, 148.

Dinopium javanense raveni, 149.

dipora, Stachyris nigriceps, 220.

diporus, Dicrurus leucogenis, 304.

dispar, Pycnonotus, 203.

dispar, Turdus, 203.

Dissemurus, 306.

Dissemurus paradiseus, 306. 
INDEX.

Dissemurus paradiseus adelphus, 307.

Dissemurus paradiseus banguey. 307.

Dissemurus paradiseus brachyphorus, 307 .

Dissemurus paradiseus colpiotes, 306.

Dissemurus paradiseus elassopterus, 307.

Dissemurus maradiseus endomychus, 307.

Dissemurus paradiseus formosus. 307.

Dissemurus paradiseus hypoballus. 306 .

Dissemurus paradiseus insularis, 307.

Dissemurus paradiseus malayensis, 306.

Dissemurus paradiseus messatius, 306.

Dissemurus paradiseus microlophus, 307.

Dissemurus paradiseus olizurus, 307.

Dissemurus paradiseus pachistus, 307.

Dissemurus paradiseus platurus, 306 .

Dissemurus paradiseus siakensis, 306.

Dissemurus paradiseus wallacei, 307.

Dissoura, 53.

Dissoura episcopus, 53 .

Dissoura neglecta, 53 .

Dissoura stormi, 53 .

Dissura mortoni, 53.

divaricatus, Lanius, 187.

divaricatus, Pericrocotus roseus, 187.

docima, Anuropsis malaccensis, 2 r 6 .

dodgei, Collocalia esculenta, II

dolichopterus, Loriculus galgulus,

95.
doriae, Calamodyta, $2 \$ 4$.

dougallii, Sterna, 46.

driophila, Anuropsis malaccensis. $2 \mathrm{t} 6$.

Dromadidae, 44 .

Dromas, 44 .

Dromas ardeola, 44

Drymocataphus nigrocapitatus nyctilampis, 200 .

Drymocataphus tickelli australis, 200.

drymodrama, Anuropsis malaccensis, 216.

Drymonax niger, 184 .
Drymophila, 177 .

Drymophila pyrhoptera, 177

Drymophila pyrhoptera dubia, 178 .

Drymophila velata, 177 .

Drymophila velata caesia, 177.

Dryobates, 144.

Dryobates analis, 144 .

Dryobates analis montis, 145.

Dryobates canicapillus suffusus, 145.

Dryobates canicapillus volzi, 145 .

Dryobates hardwickii, 145.

Dryobates hardwickii aurantiventris, 145.

Dryobates hardwickii auritus, 145.

Dryobates hardwickii canicapillus, 145.

Dryobates hardwickit volzi, 145.

Dryobates moltuccensis, $\mathbf{1 4 5}$.

Dryobates moluccensis tantulus, 146.

Dryocopus, I51.

Dryocopus javensis, 151

Dryocopus javensis buttikoferi, I5I.

Dryocopus javensis parvus, $15 \mathrm{I}$.

dubia, Drymophila pyrhoptera, 178 .

dubium, Philentoma, 178.

dubius, Charadrius, 34.

Ducula, 17.

Ducula aenea, 17.

Ducula aenea babiensis, 17.

Ducula aenea consobrina, 18 .

Ducula aenea mista, 17.

Ducula aenea oenothorax, I8.

Ducula aenea palawanensis, 17.

Ducula aenea vicina, 18 .

Ducula badia, to.

Ducula badia lacernulata, 19 .

Ducula badia williami, I9.

Ducula concolor, ig.

Ducula pickeringii, I 8 .

Ducula rosacea, 18.

Ducula rosacea whartoni, 18

Ducula rosacea zamydra, 18.

dulitensis, Buchanga leucophnea, 303.

dulitensis, Harpactes oreskios, 121. dulitensis, Rhizothera longirostris, 2.

dumetoria, Alcippe, 219.

dumetoria, Oreicola, 170 .

dumetoria, Saxicola, $₫ 70$.

Dupetor, 60 .

Dupetor flavicollis, 60.

dusumieri, Columba, 22.

dusumieri, Streptopelia bitorquata, 22.

duvaucelii, Bubutus, 132.

duvaucelii, Bucco, 138 .

dussumieri, Cryptonyx, 4 .

duvaucelii, Cyanops australis, 138 . 
duvaucelii, Harpactes, 120 . duvaucelii, Hoplopterus spinosus, 31 .

duvaucelii, Trogon, 120.

dysancrita, Burnesia, 254.

dysonymus, Cacomantis, 126.

eclipsis, Rhinomyias umbratilis, 179 .

edela, Orthotomus sutorius, 245.

Edela ruficeps, 247.

Edolius affinis, 302 .

Edolius brachyphorus, 307.

Edolius cineraceus, 303.

Edolius malayensis, 306 .

Edolius picinus, 305.

Edolius remifer, 306 .

edwardsi, Sterna bergii, 46 .

edwardsi, Thalasseus bergii, 46 .

Egretta, 55.

Egretta alba, 55

Egretta alba modesta, 56 .

Egretta eulophotes, 56.

Egretta garzetta, 56.

Egretta garzetta nigripes, 56.

Egretta intermedia, 55.

Elanus, 78.

Elanus caeruleus 78 .

Elanus caeruleus hypoleucus, 78 .

Elanus intermedius, 78 .

elassa, Macropygía emiliana, 21.

elassa, Macropygia phasianella, 21. elassopterus Dissemurus paradiseus, 307.

elegans, Muscicapa, 165 .

elliotti, Buceros, 107.

elongata, Oreosterops javanica, 267.

elongata, Zosterops javanica, 267.

elongatus, Phoenicophaus, 131 .

elongatus, Rhopodytes tristis, \$3I.

elopurensis, Siphia, 170.

Emberiza, 288.

Emberiza aureola, 288.

Emberiza cyanopis, 202.

Emberiza pusilla, 280 .

Emberiza quadricolor, 202.

emberizinus, Nelicurvius, 204 .

emiliae, Chlorocharis, 267.

emiliana, Macropygia phasianella, 21 .

empheris, Lalage nigra, 185.

enca, Corvus, 308.

trica, Fregilus, 308.

endomychus, Dissemurus paradiseus, $30 \%$.

enganensis, Aplonis panayensis, 299. enganensis, Cajornis, 200.

enganensis, Coracina sumatrensis, 182.

enganensis, Graucalus, 182.

enganensis, Gracula religiosa, 207.

enganensis, Otus umbra, 86.

Enicurus, 233.

Enicurus coronatus, 233.

Enicurus diadematus, 234.

Enicurus leschenaulti, 233 .

Enicurus leschenaulti borneensis, 234.

Enicurus leschenaulti frontalis, 233.

Enicurus ruficapillus, 234 .

Enicurus schistaceus, 234 .

Enicurus velatus, 234 .

Enicurus velatus stumatranus, 234.

Enneoctonus crassirostris, 253 .

Enneoctonus schwaneri, 259.

enopopygius, Ceyx, Ior.

Entomophora coromanda neophora, I02.

Entomophora coromanda pagana, 102.

eophila, Culicicapa ceylonensis, 180.

ephalus, Copsychus saularis, 235 .

Ephialtes sagittatus, 87 .

Ephialtes spilocephalus, 85.

epilepidota, Myiothera, 217.

epilepidota, Napothera, 217.

episcopus, Ardea, 53.

episcopus, Dissoura, 53.

epos, Upupa, Iog.

epops, Upupa, tog.

eriphaea, Alcippornis brunneicauda, 210.

eripella, Cyanoderma erythroptera, 223.

eritora, Malacocincla abbotti, 2 Io. erimaca, Treron curvirostra, I 3. ernsti, Chaetura celebensis, 118 . ernesti, Falco peregrinus, 8t. eripolia, Alcippornis brunneicauda, 210.

erithaca, Alcedo, 101.

erithacus, Ceyx, rot.

erixanthus, Anthreptes malacensis, 270.

Erolia, 40.

Erolia acuminata, 41 .

Erolia alpina, 4I.

Erolia alpina sakhatina, 4r.

Erolia minutilla, 40.

Erolia minutilla subminuta, 41 .

Erolia ruficollis, 40.

Erolia temmincki, 41 .

Erolia testacen, 41 .

Erolla naseca, I 56 .

Erpornis, 229.

Erpornis zantholeuca, 220. 
Erpornis zantholeuca brunnescens, 220.

Erpornis zantholeuca interposita, 220.

erubescens, Psittacus, 93.

erwini, Zosterops palpebrosa, 264. erythaca, Siphia, I68.

erythrocephalum, Cinclosoma, 205.

erythrocephalus, Garrulax, 205.

erythrocephalus, Harpactes, 120.

erythrocephalus, Ptilinopus, I6.

erythrocephalus, Trogon, 120.

Erythrocichla, 213.

Erythrocichla bicolor, 213.

Erythrocichla bicolor whiteheadi, 213.

erythrogaster, Cyornis, 166.

erythrogenys, Conurus, 93.

erythrogenys, Palaeornis, 04.

erythrognathus, Phaenicopbaeus, 133 .

erythrogyna, Brachypteryx montana, 232.

Erythrolane rubropectus, 301.

erythronota, Mixornis, z10.

erythronotus, Stachyris nigricollis,

$22 \mathrm{l}$.

erythronotus, Timalia, 221.

erythrophthalmus, Houppifer, 5.

erythrophthalmus, Phasianus, 5

erythrophrys, Arborophila brunneopectus, 3.

erythrophrys. Bambusicola, 3.

erythropleurus, Turdus javanicus, 241.

erythroptera, Cyanoderma, 223.

erythroptera, Timalia, 223.

erythropthalmos, Ixos, 202.

erythropthalmos, Pycnonotus, 202. erythrorhyncha, Piaya, 130.

erythrote, Aethostoma pyrrhogenys, 212.

erythrote, Malacopterum, 2 Iz.

erythrothorax, Gallinula, 26.

erythrothorax, Porzana fusca, 26.

erythrotis, Ixos, 204.

erythrotis, Pycnonotus jocosus, 204.

Erythura, 202.

Erythura hyperythra, 203.

Erythura hyperythra borneensis, 203.

Erythura hyperythra malayana, 203.

Erythura prasina, 202.

Erythura prasina coelica, 203.

Erythura viridis, 202.

Esacus, 30 .

Esacus magnirostris, 30.

Esacus magnirostris scommophorus, 31.

esculenta, Collocalia, 116. esculenta, Hirundo, 116.

estherae, Crithagra, 288 .

estherae, Serinus, 288.

etorques, Sphenocercus, II.

euculatus, Orthotomus, 247.

Eudynamis malayana, 128 .

Eudynamys, iz8.

Eudynamys scolopaceus, 128 .

Eudynamys scolopaceus malayanus, 128.

Eulabeornis philippensis andrewsi, 25.

Eulabes javanus, 296.

eulophotes, Egretta, 56.

eulophotes, Herodias, 56.

eumecis, Cinnyris brasiliana, 276 .

eumecis, Leptocoma brasiliana, 276 .

eumelas, Orthotomus atrogularis, 246.

eumesa, Kittacincla malabarica, 237.

euonyx, Anthus, 286.

Eupetes, 204.

Eupetes macrocerus, 204.

Eupetes macrocerus borneensis, 204.

Eupetes macrocercus griseiventris, 204.

Eupetes macrocercus subrufus, 204.

Euplocamus nobilis, 6.

Euplocamus stmatranus, 6.

Euplocamus vieitloti, 6.

eupogon, Aethopyga, 275.

Euptilosus, rg4.

Euptilosus eutilotus, 194.

Euptilosus urostictus, 104.

Euptilosus urostictus nieuwenhuisii, 104.

euthinus, Totanus totanus, 42 .

eurhinus, Tringa totanus, 42.

eurhythma, Ardetta, 60.

eurhythmu5, Ixobrychus, 60.

eurizoma Gallinula, 25.

eurizonoides, Gallinula, 25.

eurizonoides, Rallus, 25.

eurycercus, Centropus sinensis, 129 .

Eurylaididae, I53.

Eurylaimus, 154.

Eurylaimus corydon, 156.

Eurylaimus cucullatus, 155 .

Eurylaimus dalhousiae, i 53.

Eurylaimus horsfieldii, 154.

Eurylaimus javanicus, 154.

Eurylaimus javanicus billitonis, 155.

Eury]aimus javanicus brookei, 155 .

Eurylaimus javanjcus harterti, I55.

Eurylaimus javanicus pallidus, is5.

Eurylaimus lemniscatus, 156.

Eurylaimus lunatus, 154.

Eurylaimus ochromalus, 155. 
Eurylaimus ochromalus kalamantan, 155.

Eurylaimus ochromalus mecistus, 155.

Eurylaimus psittacinus, 154 .

Eurylaemus rafflesii, 155 .

Eurystomus, 97.

Eurystomus fuscicapillus, 97.

Eurystomus orientalis, 97.

Eurystomus orientalis calonyx, 97.

Eurystomus orientalis connectens, 97.

eurythra, Ceyx, ios.

euryura, Rhipidura perlata, 175.

euryzona, Alcedo, 99.

eustathis, Lamprocorax panayensis, 208.

etthapsinus, Anthreptes simplex. 278.

eutilotus, Brachypus, 194.

eutilotus, Euptilosus, 194.

everetti, Arachnoraphis, 282.

everetti, Arachnothera affinis, 282 .

everetti, Carpophaga, 18.

everetti, Cyornis concreta, 164 .

everetti, Geocichla, 242.

everetti, Geokichla, 242 .

everetti, Mixornis gularis, 226.

everetti, Prionochilus, 272.

everetti, Piprisoma agile, 272.

everetti, Sasia, 152.

everetti, Siphia, 164.

everetti, Staphida castaniceps, 228 .

examinandus, Phylloscopus borea-

Jis, 250 .

Excalfactoria, 5.

Excalfactoria chinensis, 5.

Excalfactoria chinensis carules. cens, 5.

Excalfactoria chinensis lineata, 5. Excalfactoria chinensis palmeri, 5. exilis, Cisticola, 248.

exilis, Malurus, 248.

exilis, Psaltria, 262.

eximia, Aethopyga, 274 .

eximia, Cyanops australis, 138 .

eximia, Nectarinia, 274

eximia, Nectarinia, 277

eximius, Mesobucco, 138.

exochra, Arachnothera longirostra, 281.

expectata, Callisitta azurea, 263.

expectata, Sitta azurea, 263.

exsanguis, Anuropsis malaccensis, 217.

exsul, Dinopium javanense, 149.

exsul, Napothera epilepidota, 218.

exsul, Tiga javanensis, 149.

exsul, Turdinulus, 2I8.

eytonii, Aracbnothera, 284 . taberi, Caprimulgus, int.

Falco, so.

Falco aeruginosus, 70 ,

Falco aldrovandrii, 81 .

Falco apivorus, 79 .

Falco bacha, 75 .

Falco badius, $7 \mathrm{t}$.

Falco bassus, 75 .

Falco bido, 75 .

Falco caeruleus, 78 .

Falco caerulescens, 80 .

Falco caligatus, 74.

Falco cenchroides, 82 .

Falco cirrhatus, 73 .

Falco cuculoides, 7 :

Falco dimidiatus, 77.

Falco fringillarius, 80.

Falco gallicus, 74 .

Falco haliaetus, $8 z$.

Falco humilis, 77.

Falco ichthyaetus, 77 .

Falco indicus, 76 .

Falco indus, 77 .

Falco leucogaster, 77.

Falco leuphotes, 79.

Falco limnaeetus, 74 .

Falco liventer, 76.

Falco malayensis, 73 .

Falco melanoleucus, 70 .

Falco migrans, 78 .

Falco moluccensis, 82.

Falco nisosimilis, 72 .

Falco nisus, 72.

Falco niveus, 74 .

Falco pallidus, 76 .

Falco pennatus, 73 .

Falco peregrinus, 80 .

Falco peregrinus calidus, $8 \mathrm{I}$.

Falco peregrinus ernesti, 8I.

Falco ptilorhyncus, 79.

Falco severus, 81.

Falco soloensis, $7 \mathrm{r}$.

Falco subbuteo, $8 \mathrm{t}$.

Falco tinnunculus, $8 \mathrm{r}$.

Falco tinnunculus japonensis, 8I.

Falco tinnunculus saturatus, 82 .

Falco trivirgatus, 70 .

Falco virgatus, 72.

falcinellus, Limicola, 40.

falcinellus, Plegadis, 52.

falcineltus, Scolopax, 40.

falcinellus, Tantalus, $\mathbf{5 2}$.

Falconidae, 70 .

fallax, Zosterops, 266.

familiaris, Prinia, 255.

fasciata, Psittacula alexandri, 93.

fasciatus, Accipiter, 71.

fasciatus, Astur, 71 .

fasciatus, Buteo, 76 .

fasciatus, Psittacus, 93.

fasciatus, Rallus, 25. 
fasciolatus, Cuculus, 126 .

fasciolatus, Penthoceryx sonneratii, 126.

fastidiosus, Pomatorhinus schisticeps, 207.

feriatus, Anuropsis malaccensis, 217.

ferox, Lanius, 258

ferruginea, Columba, 13.

ferruginea, Hemichelidon, 163.

ferrugineus, Cryptonyx, 4.

ferruginosa, Loxia, 200.

ferruginosa, Munia atricapilla, 200.

ferruginosum, Malacopteron, 213.

Ficedula coronata, $25 \%$.

fimbriata, Lalage, 183 .

fimbriatus, Ceblephyris, 183 .

fialaysoni, Pycnonotus, Igo.

finschii, Criniger, 106.

finschii, Dicaeum, 272

finschii, Piprisoma modestum, 272.

fischeri, Hemilophus, I5I

flagrans, Harpactes erythrocephalus, 120.

flagrans, Pericrocotus, I 86.

flagrans, Trogon, 120 .

flammaxillaris, Leptocoma jugularis, 277 .

flammaxillaris, Nectarinia, 277.

flammea, Muscicapa, 185.

flammea, Strix, 83

flammeum, Motacilla, 267.

flammeus, Asio, 83.

flammeus, Pericrocotus, I 85.

flammifer, Pericrocotus flammeus, 185.

flava, Motacilla, 285 .

flava, Zosterops, 265

flaveolus, Passer, 288.

flavescens, Xanthixus, 200.

flaviclune, Dicaeum trigonostig. mum, 260.

flaviclunis, Dicaeum trigonostigma, 260 .

flavicollis, Dupetor, 60

flavicollis, Mixornis, 227.

flavigaster, Anthreptes, 284.

flavigaster, Arachnothera, 284 .

flavigenis, Arachnothera, 283.

flavinucha, Chrysophlegma, 143.

flavinucha, Picus, 143.

flavipes, Dendrophila, 263 .

flavirostris, Myophonus, 240.

flavirostris, Turdus, 240.

flaviscapis, Allotrius, 220.

Alaviscapis, Pterutbius, 220.

Alaviventris, Arachnothera, 284 .

fiaviventris, Brachypteryx, 232.

flaviventris, Orthotomus, 255.

flaviventris, Prinia, 255.

flaviventris, Vanga, 203. flaviventris, Pycnonotus, 203.

flavo-aurantius, Phaeton, 68.

flavocincta, Chloropsis cochinchinensis, Jo1.

flavocristata, Melanochlora sultanea, $26 \mathrm{t}$

flavocristatus, Parus, 261.

flavoviridis, Orthotomus, 245.

flavum, Dicaeum, 265.

flavum, Malacopteron magnirostre, 215.

floresiana, Alcedo atthis, 99.

floresiana, Ramphalcyon capensis, 99 .

florisiana, Pelargopsis, 99.

forbesi, Ninox, 88.

formosus, Dissemurus paradiseus, 307.

formosus, Hieraaetus kienerii, 73.

formosus, Hierraetus kieneri, 73.

francica, Collocalia, It4.

francica, Hirundo. I 14 .

Franklinia, 240.

Franklinia rufescens, 249.

franklinii, Bucco, 137.

franklinii, Cyanops, 337.

fraseri, Palaeornis, 94.

fraseri, Pelargopsis, 97.

frater, Iyngipicus, 146.

Fregata, 66.

Fregata andrewsi, 66 .

Fregata ariel, 67.

Fregata minor, 67.

Fregata minor listeri, 67.

Fregatidae, 66.

Fregilus enca, 308 .

frenatus, Cyornis, I66.

frenatus, Tephrodornis gularis, 257.

fretensis, Muna punctulata, 201.

fretensis, Tephrodornis gularis, 257.

frigida, Stachyris chrysaea, 223.

frigida, Zosterops, 223.

Fringilla agilis, 272 .

Fringilla amandava, $28 \mathrm{~g}$.

Fringilla aterrima, 202.

Fringilla majanoide, 200.

Friggilla manyar, 204.

Fringilla minuta, 200 .

Fringilla montana, 288.

Fringilla nigerrima, 202.

Fringilla jusicea, 280 .

Fringilla sphecura, 202.

Fringilla sphenura, 292.

fringillarius, Falco, 80.

fringillarius, Micropierax caerulesm cens, 80.

Fringillidae, 288 .

frontalis, Bucco, I 38 . 
INDEX.

frontalis, Enicurus leschenaulti, 233 .

frontalis, Nectarinia, 278.

frontalis, Orthorhynchus, 262.

frontalis, Sitta, 262.

frontalis, Sitta, 262.

frontalis, Zosterops javanica, 266.

frontata, Gallinula tenebrosa, 27.

fruticola, Saxicola, 233.

fuciphaga, Collocalia, 115 .

fuciphaga, Hirundo, I15.

fugax, Cuculus, 123 .

fugax, Hierococcyx, 123.

Fulica, 20 .

Fulica alba alba, 28 ,

Fulica atra, 20.

Fulica chinensis, 27.

Fulica chloropus, 28

Fulica cinerea, 28 .

Fulica lugubris, 29.

fuliginosi, Hemichelidon sibirica, 162.

fuliginosus, Calorhampbus, 134.

fuliginosus, Calorhamphus fuligi. nosus, 134.

fuliginosus, Mycropogon, 134 .

fuligula, Anas, 63.

fuligula, Nyroca, 63 .

fullertonii, Bombycistomas, 95.

fulvicollis, Columba, 13 .

fulvicollis, Treron, 13 .

fulvigaster, Picus, 151 .

fulvis, Charadrius apricarius, 33.

fulviventer, Haliaetus, 77 .

fulviventris, Cyanoderma erythroptera, 224 .

fulvotincta, Pachycephala, 260 .

fulvus, Phaethon, 68 .

fulvus, Phaethon lepturus, 68.

fumidus, Turdus, 240 .

fumidus, Turdus javanicus, 240.

furcatus, Gallus, 7.

fusca, Alcedo, 103.

fusca, Gerygone, 171.

fusca, Halcyon smyrnensis, 103.

fusca, Porzana, $z 6$.

fuscans, Munia, 202.

fuscans, Spermestes, 292.

fuscata, Sterna, 47.

fuscicapilla, Cisticola juncidis, 248 .

fuscicapillus, Bucco, 130.

fuscicapillus, Eurystomus, 97.

fuscigularis, Rhinortha chlorophaea, 132.

fusco-albidus, Iyngipicus, 146 .

fuscus, Aethiopsar, 296.

fuscus, Meiglyptes, 147.

fuscus, Pastor, 206.

fuscus, Psitopus, $17 \mathrm{r}$.

fuscus, Rallus, 76 . galeatus, Buceros, Ios.

galericulatus, Lanius, 257

galericulatus, Platylophus, 257

galeritus, Anorrbinus, 108.

galeritus, Buceros, 108.

galgulus, Loriculus, 95 .

galgulus, Psittacus, 95.

Gallicrex, 28

Gallicrex cinerea, 28 .

Gallicrex cinerea plumbea, 28.

gallicus, Circaetus, 74.

gallicus, Falco, 74 .

gallinácea, Irediparra, 30.

gallinacea, Parra, 30.

gallinago, Capella, 38

Gallinago megala, 38 .

gallinago, Scolopax, 38 .

Gallinula, 27.

Gallinula chloropus, 28 .

Gallinula chlorapus orientalis, 28.

Gallinula erythrothorax, 26.

Gallinula eurizoma, 25.

Gallinula eurizonoides, 25.

Gallinula gularis, 28 .

Gallinula javanica, 27 .

Gallinula lugubris, 28.

Gallinula phoenicurus, 27.

Gallinula plumbea, 28.

Gatlinula sumatranus, 27.

Gallinula teaebrosa, 27.

Gallinula tenebrosa frontata, 27.

gallio, Zosterops maelanura, 265.

Gallus, 7 .

Gallus furcatus, 7 .

gallus, Gallus, 7.

Gallus gallus, 7 .

Gallus gallus bankiva, 7 .

Gallus gallus robinsoni, 7 .

Gallus javanicus, 7.

Gallus macartneyi, 6

gallus, Phasianus, 7.

Gallus varius. 7

Gampsorhynchus, 208.

Gampsorhynchus rufulus, 208.

Gampsorhynchus rufulus saturatior, 208.

Garrulax, 205.

Garrulax erythrocephalus, 205.

Garrulax erythrocephalus penin. sulae, 205.

Garrulax leucolophus, 205.

Garrulax leucolophus bicolor, 205.

Garrulax palliatus, 205.

Garrulax palliatus schistochlamys, 205.

Garrulax rufifrons, 205.

Garrulax rufifrons slamatensis, 205

Garrulax vittatus, 206.

Garrulus rufulus, 258.

garzetta, Ardea, 56.

garzetta, Egretta, 56. 
Gauropicoides rafflesi borneonensis, 144.

Gauropicoides rafflesi peninsularis, 144.

Gecinulus, 144

Gecinulus grantia, 144 .

Gecinulus grantia robinsoni, 144.

Gecinulus viridis robinsoni, 144 .

Gecinus dedemi, 141.

Gecinus puniceus observandus, 142 .

Gecinus robinsoni, 141.

Gecinus rodgeri, 141 .

Gecinus vittatus connectens, 141.

Gecinus weberi, 141 .

Gecinus xanthopygius, 140 ,

Gelochelidon, 45.

Gelochelidon nilotica, 45.

Gelochelidon nilotica affinis, 45 .

Geocichla aruata, 242.

Geocichla everetti, 242 .

Geocichla innotota, 242.

Geocichla leucolaema, 242.

Geocichla rubecula, 242.

Geokichla, 24I.

Geokichla citrina, 242 .

Geokichla citrina aurata, 242.

Geokichla citrina rubecula, 242 .

Geokichla everetti, 242.

Geokichla interpres, 242.

Geokichla interpres leucolaena. 242.

Geokichla sibirica, 241 .

Geokichla sibirica davisoni, 24i.

Geopelia, 23.

Geopelia lineata, 23.

Geopelia striata, 23.

germani, Collicalia francica, 114.

Geronticus davisoni, 52.

Gerygone, 171.

Gerygone fusca, 171 .

Gerygone fusca sulphurea, 17 .

Gerygone modiglianii, 171.

Gerygone modiglianii jacobsoni, I7 I.

Gerygone modiglianii muscicapa, I7 I.

Gerygone pectoralis, I7I.

Gerygone salvadorii, iz

Gerygone sulphurea plesseni, $17 \mathrm{I}$.

gibberifrons, Anas, 63.

gibberifrons, Anas, 63.

gigantea, Chaetura, เ 77

gigantea, Ibis, 52.

gigantea, Thaumatibis, 52 .

giganteus, Argus, 8.

giganteus, Cypselus, 117

gigantoptera, Hypothymis azurea, 173.

gigantorhina, Cyanops australis, 138. gigantorhinus, Mezobucco duvat: celii, 138.

gigas, Collocalia, 116 .

gigas, Pitta, 156.

ginginianus, Neophron percnopterus, 70 .

ginginianus, Vultur, 70

Glareola, 44

Glareola isabe!la, 44.

Glareola maldivarum, 44.

Glareola orientalis, 44 .

Glareola pratincola, 44

glareola, Tringa, 43 .

Glareolidae, 44.

glaucicomans, Cyorsis rubeculoides, 165 .

glaucicomans, Cyornis tickekkiae, 165.

Glaucidium, 00 .

Glaucidium brodiei, go.

Glaucidium brodiei borneense, go.

Glaucidium brodiei sylvaticum, go.

Glaucidium brodiei tubiger, 90.

Glaucidium cuculoides, go.

Glaucidium cuculoides castanopterum, $\infty$.

glaucina, Pitta, 239.

glaucinus, Myophonus, 239.

Glaucomyias thalassoides, $18 \mathrm{r}$.

Glauconympha cyanea megacyanea, In) 2 .

Glaucopis occipitalis, 300.

Glenargus, 3 เo.

goiavier, Muscicapa, 109.

goiavier, Pycnonotus, 109.

Goisakius, 57.

Goisakius melanolopus, 57.

Goldana capistratoides, 209.

goodsoni, Stachyris leucotis, $22 \mathrm{I}$.

gouldii, Harpactes, 121 .

gourdinii, Ixos, 200.

gourdinii. Pycnonotus goiavier, 200

govinda, Milvus migrans, 78 .

gracilis, Buceros, 107.

Gracula, 206.

Gracula atthis, on.

Gracula intermedius, 207.

Gracula javensis baweana, 207.

Gracula javanensis, 206 .

Gracula javensis baweana, 297.

Gracula javanensis miotera, zo7.

Gracula javanensis ophellochlora, 297.

Gracula melanoptera, 295.

Gracula minor, 206.

Gracula religiosa, 206.

Gracula religiosa batuensis, 207.

Gracula religiosa baweana, 297.

Gracula religiosa enganensis, 207.

Gracula religiosa intermedia, 207.

Gracula religiosa miotera, 297. 
Gracuta religiosa ophellochlora, 297.

Gracula religiosa prasiocara, 297.

Gracula religiosa robusta, 297.

Gracula saularis, 235.

Gracula sturnina, 295.

Graculidae, 296 .

Graculipica tertia, 295.

Gracupica, 295.

Gracupica leucocephala, 295.

Gracupica melanoptera, 205.

Gracupica melanoptera tertia, 295.

Gracupica melanoptera tricolor, 295.

grammicephala, Timalia, 221.

grammiceps, Myiothera, 224 .

grammiceps, Pycnosphrys, 252.

grammiceps, Seicercus, 252.

grammiceps, Stachyris, 221.

grammithorax, Meiglyptes tristis, I 47.

grammithorax, Phaiopicus, 147.

grandis, Acridotheres, 206.

grandis, Aethiopsar, 206.

grandis, Chaitaris, 170.

grandis, Niltava, 170 .

granti, Napothera epilepidota, 218 .

granti, Turdinulus, 218 .

grantia, Gecinulus, 144.

grantia, Picus, 144.

granatina, Pitta, 159 -

Graucalus babiensis, 182 .

Graucalus bungurensis, 182 .

Graucalus concretus, 181 .

Graucalus crissalis, 182.

Graucalus enganensis, 182.

Graucalus melanocephalus, 183 -

Graucalus normani, 183.

Graucalus simalurensis, 182 .

Graucalus vordermani, 182.

graydoni, Arboricola, 3 .

graydoni, Arborophila charltoni, 3 . grayi, Argus, 8.

grayi, Argusianus argus, 8.

grisea, Carpophaga, ig.

grisea, Chlidonias leucoptera, 45 .

grisea, Scolopax, 38 .

grisea, Sterna, 45 .

griseicapilla, Treron vernans, 14.

griseisticta, Hemichelidon, Ióz.

griseiventris, Eupetes macrocercus, 204.

griseogularis, Ianthoenas, 20. griseogulatis, Columba vitiensis, 20.

griseus, Limnodromus, $3^{8}$

griseus, Pastor, 206.

grisola, Tephrodornis, 260.

Gruidae, 29.

Grus, 20.
Grus antigone, 29.

Grus antigone sharpii, 20.

Gryllivora brevirostra, 236 . guajana, Pitta, 159.

guajanus, Turdus, 159 .

gularis, Accipiter virgatus, 72.

gularis, Astur (Nisus), 72.

gularis, Gallinula, 28.

gularis, Lanius, 257.

gularis, Mixornis, 225.

gularis, Monticola, 243.

gularis, Oroecetes, 243 .

gularis, Picus, 142.

gularis, Rallus striatus, 24.

gularis, Rhinomyias, 179 .

gularis, Tephrodornis, 257.

gularis, Timalia, 225.

gularis, Turdus, 196.

gurneyi, Pitta, 160.

gustavi, Anthus, 287.

gusti, Aplonis panayensis, 298.

guttacristatus, Picus, 149.

guttata, Rhipidura, 175 .

guttata, Stachyris striolata, 222.

guttatus, Crocias, 258.

guttatus, Turdinus, 222.

gutticristatus, Chrysocolaptes Iuci-

dus, 149.

guttifer, Totanus, 43 .

guttifer, Tringa, 43.

gutturalis, Criniger gutturalis, 196.

gutturalis, Hirundo rustica, I6o.

gutturalis. Picus, 151.

gutturalis, Trichophorus, Ig6.

Gygis, 49.

Gygis alba monte, so.

gymnocephala, Barita, 258.

gymnocephala, Pityriasis, 258 .

gymnophrys, Dendrophila, 263.

Gygis alba, 49 .

Gyps, 60.

Gyps indicus, 69 .

Gyps indicus nudiceps, 69 .

haemacephala: Xantholaema, 1,39.

Haemataena richmondena, 16.

Haematorty $x, 5$.

Haematortyx sanguiniceps, 5.

hageni, Osmotreron olax, 15.

hageni, Sitta frontalis, 262.

Halcyon, 102.

Halcyon amauropterus, 97.

Halcyon chloris, 103.

Halcyon chloris azela, I04

Halcyon chloris chloroptera, 104.

Halcyon chloris cyanescens, 10.4 .

Halcyon chloris humii, 104. 
Halcyon concreta, 104.

Halcyon concreta borneana, 104.

Halcyon coromanda, 102.

Halcyon coromanda minor, 102.

Halcyon cyanoventrís, 103.

Halcyon melanops, 102.

Halcyon perpulchra, 103.

Halcyon pileata, 103.

Halcyon sancta, 103 .

Halcyon sanctus, 103

Halcyon smytmensis, 103.

Halcyon smyrnensis fusca, 103.

Halcyon varia, 104.

Haliaectus, 77 .

Halizeetus leucogaster, 77 .

haliaetus, Falco, 82 .

Haliaetus fulviventer, 77.

Haliaetus lineatus, 78 .

baliaetus, Pandion, 82.

Haliastur, $77^{\circ}$.

Haliastur indus, 77 .

Haliastur indus indus, 77

Haliastur indus intermedius, 78 .

halictypus, Lamprocorax panayensis, 298 .

haliploa, Treron curvirostra, 12.

halistephis, Artamides sumatrensis, 182 ,

halistona, Prinia flaviventris, 255.

halistona, Burnesia dysancrita, 255.

halitypa, Chalcostetha calcostetha, 273.

halizonus, Pycnonotus simplex, 201.

halodrama, Sterna bergii, 47 .

balodramus, Thalasseus bergii, 47.

hardwickii, Chloropsis, 180 .

hardwickii, Dryobates, 145.

hardwickii, Picus, 145.

hardwickii, Polyplectron, 8.

Harpactes, 119.

Harpactes diardii sumatranus, I 10.

Harpactes diardii, 1 ig.

Harpactes duvaucelii, 120.

Harpactes erythrocephalus, 120.

Harpactes erythrocephalus chaseni, 120.

Harpactes erythrocephalus flagrans, 120

Harpactes gouldii, 121.

Harpactes kastumba, 1 I 9 .

Harpactes kasumba impavidus. 120.

Harpactes oreskjos, 121.

Harpactes oreskios dulitensis, $12 \mathrm{I}$.

Harpactes oreskios uniformis, $12 t$.

Harpactes orrhophaeus, 121.

Harpactes orrhophaeus vidua, I 2 I.

Harpactes reinwardtii, I21.
Harpactes reinwardtii mackloti, I 21.

Harpactes whiteheadi, 120.

harterti, Batrachostomus, 95.

harterti, Cyornis unicolor, 164.

barterti, Eurylaimus javanicus, I55.

harterti, Hemiprocne longipennis, I 13.

harterti, Malacocincla sepiaria, $2 \mathrm{I}$.

harterti, Pnocpyga ptsilla, 231.

harterti, Treron curvirostra, 12.

harterti, Treron nipalensis, 12.

hartleyj, Stachyris nigriceps, 220.

hasselti, Muscicapa, I68.

hasselti, Muscicapula melanoleuca, I68.

hasseltii, Anthus, 286.

hasseltii, Nectarinia, 276 .

hayi, Bucco, I34.

hebereri, Cyanops australis, $\mathbf{I}_{3} 8$.

hebereri, Xantholaema australis, 138.

heliobleta, Cinnyris ornata, 277.

heliocrita, Arachnothera longirostris, 281 .

heliogona, Aethopyga siparaja, 275.

heliomanis, Cinnyris ornata, 277.

heliomarpta, Chalcostetha calcostetha, 273 .

Heliopais, 20.

Heliopais personata, 20.

heliophiletica, Aethopyga siparaja, 275.

heliophilus, Arachnothera affinis, 282

Heliornithidae, 20.

heliozeteta, Cinnyris ornata, 277.

heliozeteta, Leptocoma jugularis, 277.

Hemichelidon, 162.

Hemichelidon cineterceps, 163.

Hemichelidon ferruginea, 163 .

Hemichelidon griseisticta, 162 .

Hemichelidon incerta, 162 .

Hemichelidon sibirica, 162.

Hemichelidon sibirica fuliginosa, 162 .

Hemicircus, 1.50 .

Hemicercus brookeanus, 150.

Hemicircus brunneus, 147.

Hemicircus canente, 150.

Hemicercus concretus, 150.

Hemicercus concretus coccometopus, 150.

Hemicitcus concretus sordius, 150.

Hernicircus rubiginosus, 146.

Hemilophus fischeri, 151 .

Hemilophus mulleri, is I. 
Hemipodius atrogularis, 9.

Hemipodius pugnax, 9.

Hemiprocne, 113.

Hemiprocne comata, II 3 .

Hemiprocne longipennis, 113 .

Hemiprocne longipennis anochra, 113.

Hemiprocne longipennis barterti, 13.

Hemiprocne longipennis ocyptera, 113.

Hemiprocne longipennis perlonga, 113.

Hemiprocne longipennis thoa, 113.

Hemiprocne salangana, 115.

Hemipus, 256.

Hemipus hirundinaceus, 256 .

Hemipus intermedius, 256 .

Hemipus picatus, 256.

Hemixus connectens, 192.

Hemixus sumatranus, 102.

Henicurus borneensis, 234 .

Henicurus rufidorsalis, 234 .

Henicurus velatus sumatranus, 234.

henricii, Bucco, $\mathbf{r} 36$.

henricii, Cyanops armillaris, 136.

herberti, Corythocichla brevicaudata, 217.

Herodias eulophotes, 56 .

Herporais brunnescens, 220 .

Herpornis xantholeuca interposita, 279.

hesperius, Orthotomus sericeus, 246.

heterochlorus, Aplonis panayensis, 208.

heterochlorus, Lamprocorax panayensis, 298.

heterogyna, Kittacincla malabarica, 237.

Heterophasia, 228 .

Heterophasia picaoides, 228 .

Heterophasia picaoides simillima, 228.

Heterophasia picaoides wrayi, 228 .

Heteroscops vulpes, 85 .

Hieraaetus, 73 .

Hierraetus kieneri formosus, 73 .

Hieraaetus kienerii, 73 .

Hieraaetus kienerii formosus, 73.

Hieraaetus pennatus, 73 .

Hierax malayensis, 80.

Hierococcyx, 123 .

Hierococcyx fugax, 123.

Hierococcyx fugax hyperythrus, 123.

Hierococcyz fugaz nisicolor, 123.

Hierococcyx nanus, 124.
Hierococcyx sparverioides, 123 .

Hierococcyx sparverioides bocki. 123.

Hierococcyx vagans, 124 .

hilli, Nycticorax caledonicus, 57.

Himantopus, 35.

himantopus, Charadrius, 35.

himantopus, Himantopus, 35.

Himantopus himantopus, 35.

Himantopus himantopus leucocephalus, 35.

hirundinacea, Muscicapa, 256.

hirundinaceus, Hernipus, 256.

Hirundinapus klaesii, 117 .

Hirundinidae, 160.

Hirundo, 160.

Hirundo caudacuta, I 17.

Hirundo daurica badia, 161 .

Hirundo daurica, 161 .

Hirundo daurica striolata, 161.

Hirundo esculenta, 116.

Hirundo francica, I 14.

Hirundo fuciphaga, II5.

Hirundo klecho, 113.

Hirundo longipennis, 113.

Hirundo pacifica, 118 .

Hirundo pratincola, 44 .

Hirundo riparia, 162 .

Hirundo rustica, 160.

Hirundo rustica gutturalis, 160.

hirundo, Sterna, 45.

Hirundo tahitica, I6I.

Hirundo tahitica abbotti, 161 .

Hirundo tahitica hypolampra, 161.

Hirundo tahitica javanica, 161 .

Hirundo urbica, 160 .

histrionica, Lophocitta, 258.

hoemacephalus, Bucco, 139 .

hodgsoni, Anthus, 286.

hodgsoni, Muscicapella, 168.

hodgsoni, Nemura, 168.

hodiernus, Brachypodius atriceps, 105.

hodiernu5, Microtarsus, 105.

Hoplopterus, 31.

Hoplopterus spinosus, $3 \mathrm{I}$.

Hoplopterus spinosus duvaucelii, 31.

horizoptera, Aegithina tiphia, 180 .

horrisonus, Tanygnathus lucionensis, 92.

horsfieldi, Cuculus, r 25.

horsfieldii, Eurylaimus, 154.

horsfieldii, Limnaetus, 74 .

horsfieldii, Pandion, 77 .

horsfieldi, Oreocinela dauma, 243 .

horsfieldi, Oriolus, 290.

horsfieldi, Picus, 151 .

horsfieldi, Picus, 151.

hosei, Cyornis, 166 . 
hosei, Pitta caerulea, 151.

hosii, Calyptomena, 153.

hosit, Oriolus, 30 .

hottentottus, Corvus, 304.

hottentottus, Dicrurus, 304.

Houppifer, 5.

Houppifer diardii, 5.

Houppifer erythrophthalmus, 5.

Houppifer erythrophthalmus pyronotus, 6.

Houppifer inornatus, 6.

huegelii, Orthotomus, 245.

Huhua, 84.

Huhua sumatrana, 84 .

Huhua sumatrana strepitans, 84.

humei, Chotorea mystacophanes, 135.

humei, Megalaema, 135.

humei, Turdinulus, 218 .

humeralis, Anas, $6 z$.

humeraloides, Pycnonotus, 184.

humii, Chrysophlegma mentale, 143.

humii, Halcyon chloris, 104.

humilis, Falco, 77 .

humphreysi, Orthotomus atro. gularis, 246.

hybrida, Chlidonias, 45 .

hybrida, Sterna, 45.

Hydrobatidae, 54.

Hydrocissa convexa barussensis, 106.

Hydrocissa convexa zamelaena, ro6.

Hydrocissa migratorizs, 107.

Hydrocorax melanoleucos, 64.

Hydrocorax niger, 64 .

Hvdrophasianus, 30 .

Hydrophasianus chirurgus, 30.

hydrophila, Ramphalcyon capensis, 08.

hylodromus, Meiglyptes tukki, 147.

Hyloterpe brunneicauda, 178,210 .

Hyloterpe hypoxantha, $26 r$.

hyperythra, Arborophila brunneopectus, 3.

hyperythra, Bambusicola, 3 .

hyperythra, Chlorura, 293.

hyperythra, Dendrobiastes, 160.

hyperythra, Erythura, 293.

hyperythra, Muscicapa, 160.

hyperythra, Siphia, 160.

hyperemnus, Brachypodius atriceps, 195.

hyperythrus, Cuculus, I 23.

hyperythrus, Hierococcyx fugax, 123.

hyperemnus, Microtarsus melanocephalos, 195. hypoballus, Dissemurus paradiseus, 306.

hypochloum, Dicaeum trigonostigma, 260 .

hypochra, Arachnothera longira5tra- 28t.

hypocneca, Alcippe cinerea, 219.

hypogrammica, Anthreptes macul. aria, 278 .

hypogrammica, Nectarina, 278.

hypoides, Malacocincla, zio.

hypolampis, Cinnyris brasiliana, 276.

hypolampra, Hirundo tahitica, $16 \mathrm{r}$.

hypolampra, Hypurolepis javanica, 161.

hypoleucos, Orthorhinus, 207.

hypoleucos, Pomatorhinus, 207.

hypoleucos, Tringa, 43.

hypoleucus, Elanus caeruleus, 78 .

bypoliza, Kittacincla melanura, 238 .

hypopercna, Macropygia phasianella, $2 \mathrm{I}$.

hypopyrtha, Stachyris maculata, 222.

hypopyrthus, Turdus, 240.

Hypotaenidia jouyi, 25.

Hypotaenidia striata reliqua, 25.

hypothapsina, Treron curvirostra, 12.

Hypothymis, $\mathbf{I 7 2 .}$

Hypothymis azurea, 172 .

Hypothymis azurea abbotti, 173.

Hypothymis azurea aeria, 173 .

Hypothymis azurea amelis 174 .

Hypothymis azurea consobrina, 173.

Hypothymis azurea gigantoptera, 173.

Hypothymis azurea işocara, I73.

Hypothymis azurea javana, 173.

Hypothymis azurea karimatensis, 173.

Hypothymis azurea leucophila, I74,

Hypothymis azurea opisthocyanea, 173.

Hypothymis azurea ponera, 174 .

Hypothymis azurea prophata, 172.

Hypothymis azurea richmondi, 174 .

Hypothymis azurea sipora, 174.

Hypothymis azurea slyaria, 172 .

Hypothymis azurea symmixta, 173 .

hypoxartha, Hyloterpe, 261.

hypoxantha, Loxia, 293.

hypoxantha, Pachycephala, $26 \mathrm{r}$.

hypoxantha, Ploceclla, 293.

hypoxantha, Prinia, 255.

Hypsipetes malaccensis, 102. 
Hypsipetes McClellandii, I93.

Hypurolepis javanica abbotti, 161 . Hypurolepis javanica hypolampra, I6!.

Hypurolepis javanica mesata, 16 r.

Ianthocincla Jugubris, 206.

Ianthocincla palliata, 205.

Ianthocincla treacheri, 206.

Ianthoenas griseogularis, 20.

Ibis, 53 .

ibis, Ardea, 59.

ibis, Ardeola, 59.

Ibis cinereus, 53.

Ibis gigantea, 52 .

Ibis leucocephalus, 53 .

Ibis leucon, $5 \mathrm{I}$.

Ibis papillosa, 52 .

Ibis peregrina, 52 .

incana, Tringa, 42.

icasopterus, Butorides striatus, 58 .

Ichthyaetus bicolor, 77.

ichthyaetus, Falco, 77.

ichthyaetus, Icthyophaga, $7 \%$

Ichthyaetus nanus, 77

icterocephala, Chloropsis cochinchinensis, 190.

Icthyophaga, 77.

Icthyophaga ichthyaetus, 77.

Icthyophaga nana, 77.

Ictinaetus, 73 .

Ictinaetus malayensis, 73 .

igneus, Pericrocotus, 186.

ignicapilla, Dicaeum, 271 .

ignicapillus, Anaimos percussus, 271.

ignjpectus, Dicaeum sanguinolentum, 268.

ignipectus, Myzanthe, 268.

ignita, Lophura, 6.

ignita, Nectarinea, 268.

ignitum, Dicaeum cruentatum, 268 .

ignitus, Phasianus, 6.

ignitus, Phasianus, 6

ijimae, Riparia riparia, 162.

ijimoe, Clivicola riparia, I62.

immaculatus, Brachypodius, 104.

impavidus, Harpactes kasumba, 120.

impavidus, Pyrotrogon fasciátus, 120.

incana. Scolopax, 42.

incei, Muscipeta, 177.

incei, Terpsiphone paradisi, 177.

incerta, Hemichelidon, 162 .

incerta, Zosterops, 264.

incertus, Psittacus, 04.

indistincta, Meliphaga, 284 .

indica, Buceros, 105. indica, Chaetura gigantea, 157.

indica, Chalcophaps, 23.

indica, Columba, 16.

indica, Columba, 23.

indica, Motacilla, 286.

indica, Parra, 30.

indica, Tringa, 32 .

indica, Xantholaema haemacephala, 139.

Indicator, 133 .

Indicator archipelagicus, 133

Indicator archipelagicus inornatus, 133.

Indicator malayanus, 133.

Indicatoridae, 133.

indicus, Accipiter trivigatus, 71 .

indicus, Astur, 71,

indicus, Bucco, 139.

indicus, Butastur, 76 .

indicus, Caprimulgus, 112.

indicus, Dendronanthus, 286 .

indicus, Falco, 76 .

indicus, Gyps, 60 .

indicus, Lobivanellus, 32 .

indicus, Metopidius, 30 .

indicus, Phaethon aethereus, 68 .

indicus, Porphyrio albus, 29.

indicus, Vultur, 60 .

indigo, Muscicapa, 180.

indigo, Stoporala, $t 80$.

indistincta, Lichmera, 284 .

indochinensis, Tchitrea affinis, 176 .

indochinensis, Terpsiphone paradisi, 176

indo-malayicus, Chrysocolaptes gutticristatus, 149.

indrapurae, Turdus javanicus, 241.

indus, Falco, 77.

indus, Haliastur, 77.

indus, Haliastus indus, 77.

infortunatus, Ploceus passerinus, 204.

infortunatus, Ploceus philippinus, 294 .

infumata, Leucocirca, 175.

infumatus, Cypselus, 118.

infumatus, Cypsiurus batasiensis, 118.

infuscata, Cyornis unicolor, 164 .

infuscata, Muscicapa, 170 .

infuscata, Sterna, 47.

infuscatus, Meiglyptes tukki, 147.

innominata, Caprimulgu5, 112.

innominata, Collocalia, I15.

innominata, Ramphalcyon cajensis, 90.

innominata, Vivia, 152.

innominatus, Caprimulgus indicus,

$1 \mathrm{t} 2$.

innominatus, Ceyx, ror. 
innominatus, Picumnus, 152.

innotota, Geocichla, 242. inocellatus, Polyplectrum, 8. inopinatus, Anthus hodgsoni, 286. inopinatus, Chalcurus, 8. inopinatus, Polyplectron, 8. inornata, Cryptolopha montis, 253. inornata, Metriopelia, 23.

inornata, Prinia, 254.

inornata, Seicercus montis, 253.

inornatus, Acomus, 6.

inornatus, Houppifer, 6.

inornatus, Indicator archipelagicus, 133.

inornatus, Phylloscopus, 251

inornatus, Picnonotus, 200 .

inornatus, Regulus, $25 \mathrm{~T}$.

insidjator, Lanjus, 208.

insignis, Nectarinia, 273.

insularis, Dissemurus paradiseus, 307.

insularis, Oriolus chinensis, 300.

insularis, Pycnonotus plumosus, 200.

insularis, Terpsiphone paradisi, 177.

insulindae, Cuculus intermedius. 125.

insulindae, Cuculus poliocephalus, 125.

intonsior, Anthreptes bypogrammica, 278 .

intensus, Serilophus Junatus, I54.

intermedia, Ardea, 55.

intermedia, Cvanoderma melanothorax, 225.

intermedia, Cyanoptila cyanomelana, 172.

intermedia, Egretta, 55.

intermedia, Gracula religiosa, 297.

intermedia, Muscicapa cyanomelana, 172

intermedia, Stachyris melanothorax, 225.

intermedia, Timalia pileata, 208.

intermedium. Dinopium javanense. 149.

intermedius, Buceros, 106.

intermedius, Centrococcyx, 120 .

intermedius, Centropus sinensis. I 20.

intermedius, Dicrurus longicaudatus, 303.

intermedius, Elanus, 78 .

intermedius, Gracula, 207.

intermedius, Haliastur indus, 78 .

intermedius, Hemipus, 256.

intermedius, Philentoma, 178 .

intermedius, Picus, 149.

intermedius, Thereiceryx lineatus, 130. intermedius, Thereiceryx zeylanicus, 130.

interposita, Chalcoparia singalensis, 280 .

interposita, Erpornis zantholeuca, 229 .

interposita, Herpornis xantholeuca, 220.

interposita, Kittacincla malabarica, 236.

interpres, Arenaria, 39.

interpres, Goekichla, 242.

interpres, Tringa, 39 .

interpres, Turdus, 242 .

interrumpens, Turnix suscitator, Io.

Iole, 193 .

Iole cinerea, 102.

Iole olivacea, 193.

Iole olivacea charlottae, 193.

Iole olivacea cinaamomeoventris, 103.

Iole olivacea crypta, 193 .

Iole terricolor, 192.

Iole tickelli peracensis, I93.

Iole virescens cinnamomeoventris, 103.

Iora chloroptera, 188 .

Iora lafresnayei, I 89.

iora, Phaenicomanos, I 80 .

Iora scapularis, 180 .

Iora viridis, 189.

Iora viridissima, 188

Irediparra, 30.

Irediparra gallinacea, 30 .

Irena, Igl.

irena, Pitta guajana, 150.

Irena puella, $19 \mathrm{r}$

Irena puella criniger, I0z.

Irena puella malayensis, $19 \mathrm{I}$.

Irena puella turcosa, 192.

isabelli, Glareola, 44.

isidori, Bubutus, I32.

isodcara, Hypothymis azurea, 173.

isola, Rhinomyias ruficauda, 179.

isola, Rhinomyias ruficrissa, 170 .

isopega, Arachnothera chrysogenys, 283 .

isoptera, Ramphalcyon capensis, 98.

isus, Pycnonotus erythropthalmos, 202.

Ixidia paroticalis, 202.

Ixidia webberi, 203.

Ixobrychus, 6o.

Ixobrychus cinnamomeus, 60 .

Ixobrychus eurhythmus, 60 .

Ixobrychus sinensis, 60 .

Ixos, 192.

Ixos chalcocepha]us, 194.

Ixos cinereus, t92. 
INDEX.

Ixos cinereus connectens, 192.

Ixos erythropthalmos, 202.

Ixos erythrotis, 204.

Ixos gourdinii, 200 .

Ixos malaccensis, 192 .

Ixos mcclellandii, I93.

Ixos mcclellandii peracensis, 193.

Ixos metallicus, 194.

Ixos phaeocephalus, 197.

Ixos tympanistrigus, 203.

Ixos virescens, 102.

Ixos virescens sumatranus, 192.

Ixulus castaniceps, 228 .

Jacanidae, 30.

jacobsoni, Gerygone modiglianii, 171.

jagori, Munia, 200 .

jalla, Pastor, 294.

jalla, Sturnopaster contra, 294.

jambu, Columba, I6.

jambu, Ptilinopus, 16.

japonensis, Falco tinnunculus, 81.

japonicus, Pernis apivorus, 79.

javana, Alcedo, 90.

javana, Hypothymis azurea, 173.

javana, Kittacincla malabarica, 237.

javana, Pachycephala pectoralis, 260 .

javana, Pitta sordida, 158.

javana, Ramphalcyon capensis, 99 .

javana, Treron bicincta, 14.

javana, Treron bisincta, 14.

javanense, Dinopium, 148 .

javanensis, Columba, 23.

javanensis, Gracula, 206.

javanensis, Ketupa, 83 .

javanensis, Loxia, 203.

javanensis, Ninox scutulata, 88.

javanensis, Picus, 148 .

javanica, Alcedo, 99.

javanica, Anas, 61

javanica, Arborophila brunneo-

pectus, $z$.

javanica, Ardea, 58.

javanica, Buceros, 105.

javanica, Carbo, 64 -

javanica, Centropus philippensis, 120.

javanjca, Chlidonias hybrida, 45.

javanica, Ciconia, 54 -

javanica, Columba, 24.

javanica, Coryllis, 95 .

iavanica Dendrocygna, 61.

javanica, GajFinula, 27.

javanica, Hinundo tahitica, J6t,

javanica, Mirafra, 287.

javanica, Mixornis gularis, 227. javanica Muscicapa, 175. javanica, Nectarinia, 279. javanica, Rhipidura, 175. javanica, Sterna, 45 javanica, Strix, 01 . javanica, Sylvia, 266. javanica, Tetrao, 2. javanica, Turnix, 9 javanica, Tyto alba, 91 . javanica, Zosterops, 266. javanicus, Acridotheres, 206.

iavanicus, Aethiopsar grandis, 206. javanicus, Amauromis phoenicurus, 27.

javanicus, Buceros, 108. javanicus, Butorides striatus, 58 . javanicus, Centropus bengalensis, 130.

javanicus, Cuculus, I30.

javanicus, Eurylaimus, 154.

javanicus, Gallus, 7.

javanicus, Leptoptilos, 54.

javanicus, Merops superciliosus, 110.

javanicus, Pavo, 9 .

javanicus, Pelecanus, 68.

javanicus, Phaenicophaus, 130.

javanicus, Psittacts, 92.

javanicus, Scops, 86 .

javanicus, Totanus, 42 .

javanicus, Turdus, 240.

javanicus, Zanclostomus, 130.

javanus, Dicrurus macrocercus, 302.

javanus, Eulabes, 296.

javensis, Batrachostomus, 96 .

javensis, Bucco, 135.

javensis, Ceblepyris, I $8 \mathrm{r}$.

javensis, Chotorea, 135.

javensis, Collocalia francica, 114 -

javensis, Copsychus saularis, 236 .

javensis, Coracina, 18I.

javensis, Dryocopus, 131.

javensis, Loxia, 289 .

javensis, Meliphaga, 190.

javensis, Pallenura, 285.

javensis, Picus, 15 I.

javersis, Podargus, 96 .

jefferyi, Chlamydochera, 183 .

jentinki, Chibia, 305.

jentinki, Dicrurus hottentottus, 305. jerdoni, Aegialitis, 34 .

jerdoni, Aviceda, 80 .

jerdoni, Charadrius dubius, 34 .

jerdoni, Pernis, 80.

jocosus, Lanius, 204.

jocosus, Pycnonotus, 204.

jotaka, Caprimulgus indicus, 112. jouyi, Hypotaenidia, 25. jouyi, Rallus striatus, 25. jugularis, Certhia, 277. 
jugularis, Leptocoma, 277. juliae, Arachnothera, 283. juncidis, Cisticola, 248 . juncidis, Sylvia, 248 .

Kakatoe, 92.

Kakatoe parvulus abbotti, 92 .

Kakatoe sulphurea, 92.

Kakatoe sulphurea abbotti, 92.

Kakatoeidae, 92 .

kalamantan, Eury]aimus ochromalus, 355.

kalulongae, Turdinus, 2 I 5.

kangeangensis, Uroloncha, 202.

kangeangensis, Centropus celebensis, 130 .

kangeangensis, Rhopodytes tristis, 131 .

kannegieteri, Artamides, 182.

kannegieteri, Coracina sumatrensis, 182 .

karimatensis, Cyornis banyumas, 166.

karimatensis, Cyornis rufigastra, 166.

karimatensis, Hypotbymis azurea, 173.

kasumba, Harpactes, IIg.

kasumba, Trogon, 110.

Kenopia, 227.

Kenopia striata, 227.

ketupu, Bubo, $8_{3}$.

Ketupa jaranensis, 83 .

Ketupa minor, 84 .

ketupu, Strix, 83.

kienerii, Astur, 73.

kienerii, Hieraaetus, 73 .

kjnabaluensis, Chloropsis, I0 I,

kinabaluensis, Cryptolopha, 25 I.

kinabaluensis, Spilornis cheela, 75.

kinabaluensis, Seicercus trjvirgatus, 25 s.

Kitta, 300

Kitta chinensis, 300 .

Kittacincla, 236 .

Kittacincla macrurus omissa, 237.

Kittacincla malabarica, 236 .

Kittacincla malabarica abbotti, 237.

Kittacincla malabarica eumesa, 237

Kittacincla malabarica heterogyna, 237.

Kittacincla malabarica interposita, 236 .

Kittacincla malabarica javana, 237.

Kittacincla malabarica mallopercna, 236.

Kittacincla malabarica melanura. 238.
Kittacincla malabarica nigricauda, 238.

Kittacincla malabarica ochroptila, 237.

Kittacincla malabarica omissa, 237.

Kittacincla malabarica opisthisa. 238 .

Kittacincla malabarica opisthopela. 238.

Kittacincla malabarica suavis, 237.

Kittacincla malabarica tricolor, 237.

Kittacincla malabarica zaphotina, 237.

Kittacincla melanura hypoliza, 238 .

Kittacincla melanura opisthochra, 238 .

Kittacincla melanura pagensts, 238

Kittacincla stricklandii, 238 .

Kittacincla stricklandit barbouri, 238.

klaesii, Hirundinapus, $\{17$.

klecho, Hirundo, 113.

klossij, Bubo coromandus, 84 .

korinchi, Zosterops chlorates, 265.

korthalsi, Sphenocercus, 11.

korthalsi, Sphenurus sphenurus, Ir. kotorea, Bucco, 135.

kuhlii, Nectarinia, 274.

kuhlii, Totanus, 43.

labarum, Picus, 144

labuanensis, Cyanoderma, 224.

labuanensis, Ninox, 88.

Lacedo pulchella, 102.

Lacedo pulchella amabilis, IOz.

Lacedo pulchella melanops, 102.

lacernulata, Columba, 19.

lacernulata, Ducula badia, 19.

lacteus, Tantalus, 53.

laeta, Chotorea chrysopogon, 134.

laetus, Chotorhea chrysopogon, 134 . lafresnayei. Aethorhynchus, 189.

lafresnayei, Iora, 180.

Lalage, 183 .

Lalage fimbriata, 183

Lalage fimbriata compta, 184.

Lalage fimbriata culminata, 184

Lalage fimbriata neglecta, 184 .

Lalage melanoptera, 183.

Lalage nigra, 184 .

Lalage nigra brunnescens, 184 .

Lalage nigra chalepa, 185.

Lalage nigra empheris, 185 .

Lalage nigra schisticens. 185.

Lalage nigra stieurij, 184.

lampra, Cvornis barvumas, 166.

lampra, Cyornis rufigastra, 166. 
lamprochlorus, Loriculus galguius, 95.

lamprochryseus, Oriolus chinensis, 300.

lamprochryseus, Oriolus maculatus, 300.

Lamprocorax chalybeus pachistor. hinus, 290 .

Lamprocotax chalybeus rhadinorhynchus, 298 .

Lamprocorax panayensis alipodis, 208.

Lamprocorax panayensis eustathis, 208.

Lamprocorax panayensis balictypus, 298.

Lamprocorax panayensis heterochlorus, 208.

Lamprocorax panayensis nesodramus, 208.

Lamprocorax panayensis richmondi, 208 .

Lamprotornis minor, 299.

lanceolata, Locustella, 244.

lanceolata, Sylvia, 244.

lanceolatus, Cacomantis merulinus, 126.

Laniellus leucogrammicus, 258 .

Laniidae, 256 .

Lanius, 258.

Lanius coronatus, 258 .

I.anius cristatus, 250 .

Lanius cristatus confusus, 250.

Lanius cristatus lucionensis, 259.

Lanius cristatus superciliosus, 250.

Lanius (Notodela) diana, 234.

Lanius divaricatus, 18.7.

Lanius ferox, 258 .

Lanius galericulatus, 257 .

Lanius gularis, 257

Lanius insidiator, 208.

Lanius jocosus, 204

Lanius leucoryn, 256.

Lanius macrourus, 259.

Lanius melanocephalos, 104.

Lanius musicus, 235.

Lanius nasutus, 260.

Lanius pyrrbonotus, 250.

Lanius scapulatus, 257 .

Lanius schach, 250 .

Lanius schach bentet, 250 .

Lanius schach tosariensis, 259.

Lanius strigatus, 258 .

Lanius tigrinus, 258 .

Lanius virgatus, 257.

Lanius xanthogaster, 185 .

lapponica, Limosa, 37.

lapponica, Scolopax, 37 .

Laridae, 44

Larus, 50.

Larus brunnicephalus, 50.
Larus ridibundus, 50.

larutensis, Artamides, 181 .

larutensis, Coracina javensis, I81.

larvata, Ceblepyris, 183 .

larvata, Ceblepyris, 183.

larvata, Coracina personata, 183 .

larvata, Stachyris nigriceps, 220.

larvata, Timalia, 220.

lateralis, Zosterops, 264.

lathami, Bucco, 134.

latifrons, Microhierax, 30 .

latirostris, Alseonax, $\mathrm{IO}_{3}$ -

latirostris, Arachnothera, 282.

latirostris, Arachnothera, 284.

latirostris, Musicapa, 163 .

laurinae, Leiothrix, 230 .

laurinae, Mesia argentauris, 230.

Leiothrix laurinae, 230 .

lemniscatus, Eurvlaimus, 156.

lempiji, Otus bakkamoena, 86.

lempiji, Strix, 86.

lemprieri, Platylophus galericulatus, 258 .

lepida, Ardea, 60.

lepida, Pnoepyga pusilla, 23 .

lepidocephalus, Malacopteron, 214.

lepidopleura, Cacopitta, 213.

lepidopleurus, Turdinus macrodactylus, 213

lepidota, Uroloncha acuticauda 201.

lepidus, Centropus, 130.

lepidus, Cuculus poliocephalus, 125.

lepta. Pitta moluccensis, 157.

Leptocoma, 276 .

Leptocoma brasiliana, 276 .

Leptocoma brasiliana axantha, 276 .

Leptocoma brasiliana eumecis, 276 .

Leptocoma brasiliana mecynorbyncha, 276 .

Leptocoma brasiliana oenopa, 276 .

Leptocoma brasiliana sperata, 277.

Leptocoma jugularis, 277.

Leptocoma jugularis flammaxil laris, 277 .

Leptocoma jugularis beliozeteta. 277.

Leptocoma jugularis microleuca, 277.

Leptocoma jugularis pectoralis, 277.

Leptocoma jugularis polyclysta. 278.

leptogrammica, Columba, 20.

leptogrammica, Strix, 89.

Leptopteryx cruentus. 301.

Leytoptilos, 54.

Leptoptilos javanicus, 54 .

leptorrhynchus, Aplonis parayensis. zog.

lepturus. Brachyptervx, 231.

lepturus, Phaethon, 68. 
lenturus, phaeton, 68

leschenault, Bubo zeylonensis, 83. leschenault, Strix, 83.

leschenaulti, Enicurus, 233.

leschenaulti, Merops, ion.

leschenaulti, Turdus, 233 .

leschenaultii, Charadrius, 33 .

leucocephala, Alcedo, 99 .

leucocephala, Gracupica, 295.

leucocephala, Loxia, 200 ,

leucocephalus, Himantopus himantopus, 35.

leucocephalus, Ibis, 53 .

leucocephalus, Tanialus, 53.

Leucocerca rhombifer, 175 .

Leucocirca infumata, 175 .

leucogaster, Anthracoceros coronatus, 106.

leucogaster, Buceros, 106.

leucogaster, Falco, 77.

leucogaster, Haliaeetus, 77.

leucogaster, Pelecanus, 65.

leucogaster, Picus, I $5 \mathrm{t}$.

leucogaster, Sula, 65.

leucogaster, Xanthonotus, $30 \mathrm{r}$.

leucogastra, Amandina, 291.

letcogastra, Munia, $20 i$.

leucogastra, Ptilocichla, 211.

leucogastra, Setaria albigularis, 216.

leucogastroides, Munia leucogastra, 202.

leucogenis, Buchanga, 303.

leucogenis, Dicrurus leucophaeus, 303.

leucogrammica, Cacopitta, 216.

leucogrammica, Ptilocichla, 216.

leucogrammicus, Alcurus, 108.

leucogrammicus, Laniellus, 258

leucogrammicus, Pycronotus, 198.

leucolaema, Geocichla, 242.

leucolaema, Geokichla interpres. 242.

leucolophus, Corvus, 205.

leucolophus, Garrulax, 205.

leucomelas, Procellaria, 5 I.

leucomelas, Puffinus, $5 \mathrm{I}$.

leucon, Ibis, 51.

leucophaeus, Dicrurus, 303 .

leucophila, Hypothymis azurea, 174 .

leucophris, Brachypteryx, $23 \mathrm{I}$

leucophris, Myiothera, $23 \mathrm{I}$.

leucoptera. Phoenicura, 232 .

leucops, Oreoctistes, zoo.

leucops, Pyenonotus, 200.

Leticopsat, 296.

Leucopsar rothschildi. 206 .

leucoptera, Cancroma, 50.

leucoptera, Chlidonias, 44

letscoptera, Sterna, $\$ 4$. leucopygialis, Acanthylis, II8.

leucopygialis, Chaetura, 118.

leucopygialus, Cypselus, IIg-

leucorhoa, Procellaria, $5 \mathrm{I}$.

leucorhoa, Oceanodroma, 51.

leucorodia, Platalea, 52.

leucorthoa, Sylviạ, 252 .

leucoryn, Lanius, 256 .

leucorhynchus, Artamus, 256.

leucosticta, Corythocichla, 217.

leucosticta, Napothera brevicaudata, 217.

leucostigma, Turdirostris, 227.

leucotis, Oryzivora, 289 .

leucotis, Stachyris, 221 .

leucotis, Timalia, $22 \mathrm{I}$.

leucura, Muscisylvia, 234.

leuphotes, Aviceda, 79 .

leuphotes, Falco, 70 .

Lichmera, 284.

Lichmera indistincta, 284 .

Lichmera indistincta limbata, 284 .

limbata, Lichmera indistincta, 284 .

limbata, Meliphaga, 284 .

limborgi, Chalcites, 127 .

Limicola, 40.

Limicola falcinellus, 40 .

Limicola falcinellus sibirica, 40 .

Iimitans, Cyornis banyumas, I66.

limitans, Picus vittatus, 140.

limnaeetus, Falco, 74.

limnaeetus, Spizaetus cirrhatus, 74.

Limnaetus horsfieldii, 74 .

Limnodromus, 38 .

Limnodromus griseus, 38 .

Limnodromus grișeus semipalmatus, 38 .

limnophilax, Nycticorax, 57.

Limosa, 36.

Limosa lapponica, 37.

Limosa lapponica baueri, 37.

Limosa limosa, 36 .

limosa, Limosa, 36.

Limosa limosa melanuroides, 37 .

limosa, Scolopax, 36.

linchi, Collocalia esculenta, I 16.

lineata, Excalfactoria chinensis, 5.

lineata, Geopelia, 23.

lineatum, Polyplectron, 8.

lineatus, Capito, 130.

lineatus, Haliaetus, 78 .

lineatus, Milvus migrans, 78 .

lineatus, Oriolus, 5.

lineatus, Thereiceryx zeylanicus. 139.

lineocapilla, Cysticola, 248 .

lineocapilla, Cisticola exilis, 248 .

listeri, Fregata minor, $6 \%$.

liventur, Butastur, 76 .

liventur', Falco, 76 .

lobatus, Phalaropus, 37. 
Lobiopliasis, 7.

Lobiophasis, bulweri, 7.

Lobiophasis castaneicaudatus, 7 .

Lobivanellus, 32.

Lobivanellus indicus, 32 .

Lobivanellus indicus atronuchalis, 32 .

Locustella, 244 .

Locustella certhiola, 244.

Locustella lanceolata, 244.

Locustella ochotensis, 244.

Locustella rubescens, 244.

Locustella sparsimstriata, 244 .

lidoisia, Aethopyga, 274.

longicauda, Psittacula, 93.

longicauda, Psittacus, 93.

longicauda, Rhinidura javanica, 175.

longicaudatus, Dicrurus, 303.

longicaudatus, Phaenicophaeus, I3I.

longicaudatus, Rhopodytes tristis, 131.

longipennis, Cyornis rufigastra, 166.

longipennis, Hemiprocne, $1 \pm 3$.

longipennis, Hirundo, 113

longirostra, Arachnothera, 281 .

longirostra, Certhia, $28 \mathrm{r}$.

longirostra, Cinnyris, 283.

longirostra, Sitta, 262.

longirostris, Perdix,

longirostris, Rhizothera, $\mathrm{t}$.

longirostris, Upupa epops, 100.

longpennis, Sterna hirundo, 45.

longstaffi, Aethostoma pyrrhogenys, 212.

longstaffi, Malacocincla canicapillus, 2 t2,

Lophocitta ardesiaca, 257.

Lophocitta histrionica, 258.

Lophura, 6.

Lophura ignita, 6.

Lophura ignita sumatrana, 6 .

Lonhura rufa, 6.

Lophura sumatrana albipennis. 6 .

Lophura sumatrana delacouri, 6 .

loricata, Myiothera, 213.

Loriculus, 94.

Loriculus galgulus, $0 \%$

Loriculus galgulus dolichopterus, 95.

Loriculus galgulus lamprochlorus, 95.

Loriculus vernalis, 94 .

Loriculus vernalis pusillus, 95.

Loriidae, ol.

lowi, Collocalia, 115.

lowi, Cypselus, 115.

lowii, Megapodius, 1.

Loxia atricapilla, 200 .
L.oxia ferruginosa, 290.

Loxia bypoxantha, 203

Loxla jávanensis, 293.

Loxia javensis, 280 .

Loxia leucocephala, 290 .

Loxia maja, 290.

I.oxa molucca, 292.

Loxia oryzivora, 280 .

Loxia philippina, 293.

Loxia prasina, 202 .

Loxia punctularia, 291.

I oxia striata, 291 .

Loxia undulata, 201.

luciae, Otus spilocephalus, 85 .

luciae, Scops, 85.

lucianae, Palaeornis, 94.

lucidus, Chrysocolaptes, 349 .

lucidus, Phaenicophaus, 132.

lucidus, Picus, 140.

lucionensis, Lanius cristatus, 250.

lucionensis, Psittacus, 92 .

lucionensis, Tanygnathus, 02 .

lugubris, Buceros, 108.

lugubris, Cuculus, I22.

lugubris, Fulica, 29.

lugubris, Gallinula, 28.

lugubris, I anthocincla, 206.

lugubris, Melanocichla, 206.

lugubris, Surniculus, 122.

lunatus, Buceros, 105.

lunatus, Eurvlaimus, 154.

lunatus, Serilophus, 154.

luridus, Picus, 147.

Luscinia, 232.

Luscinia cvane, 232.

luteoventer, Cinnyris, 277.

Lyncornis, 110.

Lyncornis cerviniceps, tro.

Lyncornis temminckii, tu.

lyprum, Dicaeum trigonostigmi, 270.

lyprum, Dicaeum trignonstigmum, 270.

macartneyi, Gallus, 6.

Machaerhamphus, 79 .

Machaerhamphus alcinus. 70.

mackloti, Harpactes reinwardtit, I 2 I .

mackloti, Picus, I5r.

mackloti, Trogon, 121 .

macklotij, Cinnyris, 273.

macrocercus, Dicrurus, 302.

macrocerus, Eupetes, 204.

macrodactylum, Malacopteron, 213

macrodactylus, Turdinus, 233.

Macronus, 227. 
Macronus ptilosus, 227.

Macronus ptilosus reclusus, 227.

macronyx, Budytes flavus, 285 .

macronyx, Motacilla flava, 285 .

macroptera, Brachypteryx, 2 iz.

macropterum, Aethostoma rostratum, 2 2 2.

macropterus, Psittacus, 94.

macropteryx perlonga, I 13.

Macropygia, 20.

Macropygia emiliana borneensis, 21 .

Macropygia emiliana elassa, 2 I.

Macropygia phasianella, 20.

Macropygia phasianella barussa, 2 I.

Macropygia phasianella borneensis. $2 \mathrm{I}$.

Macropygia phasianclla cinnamemea, 21 .

Macropygia phasianella elassa, 2 . Macropygia phasianella emiliana, 21 ,

Macropygia phasianella hypopercna, 21.

Macropygia phasianella megala, 2 r. Macropygia phasianella modig. lianii, $2 \mathrm{I}$.

Macropygia ruficeps, 2 I

Macropygia ruficeps malayana, 22.

Macropygia ruficels nana, 22.

Macropygia ruficeps simalurensis, 22.

Macropygia ruficeps sumatrana, 22. Macropygia ruficeps sumatranus, 22.

Macropygia unchall, 20.

Macropygia walik-mehra, 20.

Macrorhamphus semipalmatus, 38 . macrorhynchos, Corvus, 308.

macrorhynchos, Cymbirhynchus, 156.

macrorbynchos, Todus, 156.

nacroterus, Artamus leucoryn, 756 .

mactourus, Lanius, 250.

macrurus, Caprimulgus, I Iz.

macularia, Anthreptes, 278 .

maculata, Stachyris, 222 .

maculata, Timalia, 222.

maculatus, Anaimos, 271.

maculatus, Brachypteryx, 227

maculatus, Chalcites, 127.

maculatus, Oriolus chinensis, 290.

maculatus, Pardalotus, 271.

maculatus, Trogon, 127

maculicollis, Orthotomus sutorius, 245.

madagascariensis, Numenius, 36. madagascariensis. Scolopax, 36.

Haemataena melanocephala mas. soptera, 16.

magna, Arachnothera, 282. magna, Cinnyris, 282 .

magna, Malacopteron, 214.

magnirostre, Malacopteron, 2I5.

magnirostris, Alcippe, 215.

magnirostris, Butreron capellei, II.

magnirostris, Cyornis banyumas, 167.

magnirostris, Esacus, 3o.

magnirostris, Oedicnemus, 30.

magnirostris, Sasia abnormis, 152.

magnirostris, Treron, I I.

magnum, Malacopteron magnum, 2. 4 .

Mainatus sumatranus, 296.

maingayi, Strix leptogrammica, 8g. maingayi, Syrnium, 89 .

maja, Loxia, 200.

maja, Munia, 290.

majanoide, Fringilla, 200.

inajor, Orthotomus atrogularis, 246.

major, Palneornis, 93.

major, Parus, 261.

major, Psittacula alexandri, 93.

major, Rhinocichla mitrata, 206.

majus, Malacopteron, 214.

malabarica, Kittacincla, 236 .

malabarica, Muscicapa, 236.

malaccensis, Anuropsis, 216

malaccensis, Ardea, 59.

malaccensis, Athene, 88.

malaccensis, Brachypteryx, 216.

malaccensis, Bucco, 136 .

malaccensis, Callolophus miniaceus, $\ 42$.

malaccensis, Columba, 23.

malaccensis, Cymbithychus macrorhynchos, 156.

malaccensis Cymborhynchus, 156.

malaccensis, Hypsipetes, 102.

malaccensis, Ixos, 192.

malaccensis, Ninox scutulata, 88 .

malaccensis Nyctiornis, I10.

malaccensis, Passer montanus, 288.

malaccensis, Pelargopsis, 98.

malaccensis, Picus, I 42.

malaccensis, Pitta, 157.

malaccensis, Pitta, 158.

malaccensis, Psittacus, 93.

malaccensis, Psittacus, 94.

malaccensis, Ramphalcyon capensis, 98 .

malacensis, Anthreptes, 270.

malacensis, Certhia, 270 .

malacensis, Phasianus, 279.

malacensis, Polyplectron bicalcaratum, 8 .

Malacocincla, 2 ro.

Malacocincla abbotti, 210.

Malacocincla abbotti baweana, 210.

Malacocincla abbotti buttikoferi, 210. 
Malacocincla abbolti eritora, 2 to.

Malacocincla abbotti olivacea, 210.

Malacocincla abbotti sirensis, 210.

Malacocincla canicapillus longstafi, 212 .

Malacocincla perspicillatus, $21 \%$.

Malacocincla sepiaria, 2 to.

Malacocincla sepiaria barussana, $2 !$.

Malacocincla sepiaria harterti, 21 .

Malacocincla sepiaria minot, 211.

Malacocincla sepiaria rufiventris, 2 II.

Malacocincla sepiaria tardinata, $21 \mathrm{t}$.

Malacopteron, 214.

Malacopteron affine, 215.

Malacopteron affine notatum, 215.

Malacopteron aureum, 202.

Malacopteron cinereum, 2 IA.

Malacopteron cinereum bungurense, 214.

Malacopteron cinereum rufifrons, 214.

Malacopteron cinereus, $2 \mathrm{t} 4$.

Malacopteron ferruginosum, 213.

Malacopteron macrodactylum, 213 -

Majacopteron magna, 214.

Malacopteron magnirostre, 215 .

Malacopteron magnirostre cinereocapillum, 215.

Malacopteron magnirostre flavum, 215.

Malacopteron magnum magnum, 214.

Malacopteron magnum saba, 2 I4.

Malacopteron majus, 2 I4.

Malacopteron olivaceum, 210.

Malacopteron squamatum, 222.

Malacouterum melanocephalum, 215.

Malacopterum erythrote, 212.

Malacopterus, 2I 4.

malacoptilus, Rimator, 218.

Malacornis, 214.

malaya, Cisticola juncidis, 248 .

malayana, Anthipes solitaris, 170

malayana, Chloropsis hardwickii, 180.

malayana, Chlorura hyperythra, 203.

malayana, Dendrobiastes hypery. thra, 160 .

malayana, Digenea, 170.

malayana, Erytbura hyperythra, 293.

malayana, Macropygia ruficeps, 22 malayana, Muscicapula, I60.

malayana, Siva strigula, 228 . malayanam Eudynamis, 128. malayanus, Chalcites, 128. malayanus, Cuculus, 128. malayanus, Eudynamys scolopaceus, $1 \geq 8$.

malayanus, Indicator, 133.

malayanus, Oriolus cruentus, 302.

malayanus, Otus scops, 85 .

malayanus, Penthocery $\mathrm{x}$ sonneratii, 125.

malavanus, Scops, 85 .

malayensis, Arthus novaeseclandiae, 286.

malayensis, Chaptia aenea, 305.

malayensis, Chotorhea rafflesii, 135.

malayensis, Dissemurus paradiseus. 306.

matayensis, Edolius, 306.

malayensis, Falco, 73 .

malayensis, Hierax, 80.

malayensis, Ictinaetus, 73.

malayensis, Irena puella, 101.

malayensis, Otus rufescens, 87 .

malayensis, Pastor, 205.

nalayensis, Picus maximus, 151. malayensiz, Spilornis cheela, 75.

malayanus, Anthracoceros, 106.

malayanus, Buceros, 106.

nialayorum, Parus major, 262.

nalayorum, Picumnus innominatus, 152.

malayorum, Vivia innominata, 152. maldivarum, Glareola (Pratincola). 44.

nallopercna, Kittacincla malabarica, 236

Malurus exilis, 248 .

Malurus marginalis, 249.

Malurus polychrous, 255.

manillensis, Ardea purpurea, 54. manillensis, Nycticorax caledonicus, 57.

mantananensis, Otus scops, 85 .

mantananensis, Scops, 85.

mantis, Scops, 87 .

manyar, Fringilla, 294.

manyar, Ploceus, 204.

maratua, Collocalia vestita, 115

marginalis, Malurus, 249.

marmoratus, Turdinus, 213.

martini, Pycnonotus aurigaster, 20.4.

massoptera, Haemataena melanocephala, 16 .

massopterus, Ptilinopus melanocephalus, 16.

massorhinus, Surniculus lugubris, 122 ,

maxi, Zosterops chloris, 266

maxwelli, Philentoma, i 8.

McClellandii, Hypsipete5, 193.

mcclellandit, Ixos, 193.

mecistus, Eurylaimus ochromalus, I 55 . 
mecynorhyacha, Cinnyris brasiliana, 276 .

mecynorbyncha, Leptocoma brasiliana, 276 .

medius, Alophoixus phaeocephalus, 107.

media, Chloropsis aurifrons, 191.

media, Phyllornis, 101.

media, Sterna, 46.

media, Zosterops aureiventer, 264 .

media, Zosterops palpebrosa, 264 .

megacyanea, Glauconympha cyanea, 102.

megala, Capella, 38 .

megala, Gallinago, 38 .

megala, Macropygia phasianella, 21.

Megalaema haemacephala delica, 139.

Megalnema humei, 135.

Megalaima chrysonsis, 135 .

Megalaema duvauceli borneonensis, 138.

Megalaema pulcherrima, 137.

Megalarhynchus spinosus, 134

Megalurus, 249.

Megalurus citrinus, 240.

Megalitrus palustris, 249.

Megapodijdae, J.

Megapodius, $\mathbf{I}$.

Megapodius cumingii tolutilis, I.

Megapodius lowii, I*

Megapodius reinwardt, I.

Megapodius reinwardt cumingit, 1. megarhyncha, Pitta, 157.

megarhynchus, Alcedo, 100.

megastoma, Dicaeum trigonostigma, 260 .

megastomum. Dicaeum trigonostigmum, 260.

megistus, Cranorrhinus corrugatus, I07.

Meiglyptes, 146.

Meiglyptes badiosus, 148.

Meiglyptes fuscus, 147.

Meiglyptes grammithorax microterus, 147.

Meiglyptes tristis, 146.

Meiglyptes tristis grammithorax, 147.

Meiglyptes tristis micropterus, 147 .

Meiglyptes tristis microterus, 147.

Mejglyptes tukki, 447.

Meiglyptes tukki azaleus, 147.

Meiglyptes tukki calceuticus, 147.

Meiglyptes tukki hylodromus, 147.

Meiglyptes tukki infuscatus, 147.

Meiglyptes tukki percnerpes, 147.

Meiglyptes tukki pulonis, 147. melanchima, Arachnothera longi. rostris, 281 .

melanetra, Aethopyga siparaja, 275.

melanocephala, Columba, 16.

melanocephala, Coracina personata, 183

melanocephalos, Lanius, I94.

melanocephalum, Malacopterum, 2 I 5.

melanocephalus, Graucalus, 183 .

melanocephalus, Ptilinopus, I 6 .

melanocephalus, Tantalis, 51.

melanocephalus,

aethiopicus, $5 \mathrm{t}$.
Melanochlora, $26 \mathrm{t}$.

Melanochlora sultanea, 26 .

Melanochlora sultanea flavocris. tata, 261.

Melanochlora sumatrana, $26 \mathrm{t}$.

Melanocichla, 206.

Melanocichla lugubris, 206 .

Melanocichla lugubris calvus, 206.

Melanocichla peninsularis, 206.

melanogaster, Anhinga rufa, 65.

melanogaster, Picus, 146.

melanognathus, Phaenicophaus, I32.

melanoleuca, Muscicapula, 168.

melanoleucos, Hydrocorax, 64.

melanoleucos, Microtarsus, 105.

nuelanoleucos, Phalacrocorax, 64.

melanoleucus, Circus, 70.

melanoleuctis, Falco, 70.

melanoloplsa, Ardea, 57.

melanolopus, Goisakius, 57.

melanope, Motacilla cinerea, 285.

Melanopelargus episcopus stormi, 53.

Melanoperdix, 4 -

Melanoperdix nigra, 4.

Melanoperdix nigra borneensis, 4.

melanops, Halcyon, 102.

melanojs, Lacedo pulchella, $10 z$.

melanoptera, Alcedo, 103

melanoptera, Ceblepyris, ${ }_{3}$.

melanoptera, Gracula, 205.

melanoptera, Gracupica, 205.

melanoptera, Lalage, 183.

melanopus, Ardea, 55.

melanopygia, Trichogramoptila, 202 .

melanothorax, Cyanoderma, 224.

melanothorax, Myiothera, 224.

melanotis, Budytes, 285 .

melanthe, Dicaeum trigonostigma, 270.

melanura, Arrenga, 230 .

melanura, Cittocincla, 238 .

melanura, Kittacincla malabarica. 238 . 
melanura, Zosterops, 265.

melanuroides, Limosa limosa, 37.

melanurus, Myophonus, 230.

Melias diardi, $13 \mathrm{I}$.

Melias tristis, 131.

Meliphaga indistincta, 284 .

Meliphaga javensis, 100.

Meljphaga limbata, 284.

Meliphagidae, 284 .

melopogenys, Dendrophassa fulvicollis, 13 .

melopogenys, Treron fulvicollis, 13.

melvillensis, Carbo melanoleucos, 64 .

melvillensis, Phalacrocorax melanoleucos, 64 .

mendeni, Cyanoderma melanothorax, 224

meninting, Alcedo, 100.

mentale, Chrysophlegma, I43.

mentalis, Picus, 143.

mentawi, Oriolus xanthonotus, zor.

mentawi, Otus bakkamoena, 86.

meridianus, Picus viridanus, I4I.

meridionalis, Culicicape ceylonensis, I 80 .

Meropidae, 109.

Merops, 100 .

Merops amictus, 10.

Merops cyanopygius, 110.

Merops leschenaulti, 109.

Merops leschenaulti quinticolor. rog.

Merops sumatranus, $\llbracket 10$.

Merops sumatranus caeligenus, I. 10.

Merops superciljosus, 110.

Merops superciliosus javanicus, 110.

Metops urica, 100 .

Merops viridis, $[10$.

Merula seebohmi, $24 t$.

Merula whiteheadi, 240.

merulinus, Cacomantis, 126 .

merulinus, Cuculus, 126.

mesata, Hyjurolepis javanica, $16 r$.

Mesia, 230.

Mesia argentauris, 230.

Mesia argentauris latinae, 230.

Mesia argentauris tahanensis, 230 .

Mesobucco duvauceli stuarti, ${ }_{3} 8$.

Mesobucco duvauceli tanamassae, 138.

Mesobucco duvaucelii cyaneus. 138.

Mesobucco eximius, 138 .

Mesocarbo ater territori, 64.

mesochloa, Dendrophassa vernans, 15 mesochloa, Treron vernans, 15.

messatius, Dissemurus paradiseus, 306.

messeris, Artamides sumatrensis, I8:

messopora, Butreron capel]ci, 1 .

metallicus, Ixos, 104.

metallicus, Myophonus, 240.

Metopidius, 30.

Metopidius indicus, 30.

Metriopelia inornata, 23.

Mesobucco duvaucelii gigantorhinus, I 38 .

Micranous worcesteri, 40.

microbalis, Cerchneis moluccensis, 82.

Microhierax, 80 .

Microhicrax caerulescens, 80.

Microhierax caerulescens fringillarius, 8o.

Microhierax latifrons, 80.

microleuca, Cinnyris ornata, 277.

microleuca, Leptocoma jugularis. 277.

microlophrs, Dissemurus paradiscus, 307.

micromelaena, Aegitbina tiphia, 188.

Micronisus poliopsis, 7 I.

Micropodidae, II 3 .

Micropternus, 148.

Micropternus brachyurus, 148 .

Micropternus brachyurus badiosus, 148.

Micropternus brachyurus badius, 148.

Micropternus brachyurus celaenephis, 148.

Micropternus brachyurus squamigularis, 143.

Micropternus phaioceps celaenephis, 148 .

mícronterus, Cuculus, 124.

micropterus, Meiglyptes tristis, 1.47.

Micropus, 118.

Micropus affinis, 110 .

Micropus affinis subfurcatus, 119

Micropus pacificus, 118.

Micropus pacificus cooki, ilo.

microrhinus, Phoenicophaes, 132.

Microtarsus, 195.

Microtarsus cantori, 194.

Microtarsus hodiernus, 195.

Microtarsus melanocephalos ab botti, 195.

Microtarsus melanocephalos hy. peremnus, 105.

Microtarsus melanoleucos, 195. 
INDEX.

Microtarsus melanoleucos proximus, 195.

Microtarsus olivaceus, zor.

microterus, Meiglyptes grammithoIax, 147

microterus, Meiglyptes tristis, 147. Microura superciliaris, $23 \mathrm{t}$.

migrans, Falco, 78 .

migrans, Milvus, 78 .

migratorius, Hydrocissa, 107.

Milvus, 78 .

Milvus migrans, 78 .

Milvus migrans govinda, 78 .

Milvus migrans lineatus, 78 .

mineaceus, Picus, 142.

miniaccus, Callolophus, 142.

miniata, Muscicapa, 187.

miniatus, Pericrocotus, 187.

Minla castaneiceps, 219.

Minla soror, 219.

minor, Alcedo coromanda, 102.

minor, Aplonis, 290.

minor, Bubo orientalis, 84 .

minor, Campophaga, 184 .

minor, Cyanops franklinii, 137.

minor, Fregata, 67.

minor, Gracula, 296.

minor, Halcyon coromanda, 102

minor, Ketupa, 84.

minor, Lamprotornis, 290.

minor, Malacocincla sepiaria, 2IJ.

minor, Noctua hirsuta, 88.

minor, Otocompsa flaviventris, 203.

minor, Pelecanus, 67.

minor, Pericrocotus brevirostris, I 86 .

minor, Pycnonotus flaviventris, 203.

minor, Streptopelia chinensis, 23.

minor, Turdinus sepiaria, 21 I.

minor, Turtur tigtinus, 23.

minuta, Fringilla, 200 .

minuta, Munia atricapilla, 290.

minutilla, Erolia, 4o.

minutilla, Tringa, 40.

minutus, Anous, 40.

minutus, Numenius, 36.

minutus, Pericrotus, 186.

minutus, Trichophorus, 108 .

minythomelas, Pericrocotus andamanensis, 186.

minythomelas, Pericrocotus flammeus, 186.

miotera, Gracula javanensis, 297.

miotera, Gracula religiosa, 207.

Mirafra, 287.

mirafra, Alauda, 287 .

Mirafra borneensis, 287 .

Mirafra javanica, 287 .
Mirafra williamsoni, 287.

mirificus, Caprimulgus, [ 1 ].

mista, Ducula aenea, 17.

mistus, Muscadivores aeneus, 17.

mitchelli, Trichoglossus ornatus,

gr.

mitrata, Rhinocichla, 206.

mitrata, Timalia, 206.

Mixornis, 225.

Mixornis bornensis pontia 326 .

Mixomis bormensis ruficoma, 227.

Mixornis erythronota, 210.

Mixornis fiavicollis, 227.

Mixornis flavicollis prillwitzi, 227.

Mixornis gularis, 225.

Mixornis gularis argentea, 226.

Mixornis gularis bornensis, 226 .

Mixornis gularis chersonesophila, 225.

Mixornis gularis connecten, 225 .

Mixornis gularis everetti, 226.

Mixornis gularis javanica, 227.

Mixornis gularis montana, 226 .

Mixornis gularis pontia, 226.

Mixornis gularis ruficoma, 227.

Mixornis gularis zaperissa, 226.

Mixornis gularis zarhabdota, 226.

Mixornis gularis zophera, 225.

Mixornis pileata zaptera, 226 .

Mixornis pileata zarhabdota, 226.

Mixornis pileata zophera, 225.

Mixornis rubricapilla connectens, 225.

Mixornis rubricapilla zaperissa, 226.

Mixornis similis, 226 .

Mixornis stmatrana, 225.

mixtus, Batrachostomus, 96 .

miza, Dendrophassa vernans, 15.

miza, Treron vernans, 15.

mjobergi, Anthreptes malacensis, 270 .

mjobergi, Dendrobiastes bypery* thra, 160 .

mjobergi, Dendrobiastes hyperythrus, 160 .

modesta, Anthreptes, 282 .

modesta, Arachnothera affinis, 282.

modesta, Ardea, 56.

modesta, Egreta alba, 56 .

modesta, Psittacula longicauda, 94.

mociestum, Pijrisoma, 272.

modestus, Palaeornis, 94.

modestus, Pericrocotus, 1 S7.

modestus, Prionochilus, 272.

modestus, Turdus, 241.

modiglianii, Gerygone, 171.

modiglianii, Macropygia phasianella, $2 \mathrm{r}$. 
modiglianii, Pericrocotus flammeus, 185.

molucca, Loxia, 202.

molucca, Munia, 202.

Moluccensis Colomba, 17 .

moluccensis, Dryobates, 145.

moluccensis, Falco, 82 .

moluccensis, Phyllornis, 100.

moluccensis, Picus, I45.

Monarcha caesia, 177.

mongolus, Charadrius, 33 .

monorhis, Oceanodroma leucorhoa,

5 I.

monorbis, Thalassidroma, 51 .

montana, Brachypteryx, 231.

montana, Cettia, 253.

montana, Cyornis banyumas, 167.

montana, Cyornis whitei, 167 .

montana, Fringilla, 288 .

montana, Mixornis gularis, 226.

montana, Sylvia, 253 .

montana, Zosterops, 265.

montanus, Passer, 288.

montanus, Pericrocotus, 186.

montanus, Pomatorhinus, 207.

monte, Gygis alba, 50.

Monticola, 243 .

monticola, Cyanops oorti, 137.

Monticola gularis, 243.

Monticola solitaria, 243.

Monticola solitaria pandoo, 243.

Monticola solitaria philippensis, 243.

monticolum, Dicaeum sanguinolentum, 269.

montis, Bradywterus, 249.

montis, Cryptolopha, 252.

montis, Dryobates analis, 145.

montis, Pycnonotus flaviventris, 203.

montis, Rubigula, 203.

montis, Seicercus, 252.

montis, Stasiasticus, 249 .

morrelli, Pellorneum capistratum, 209.

mortoni, Dissura, 53.

Motacilla, 284.

Motacilla alba, 284 .

Motacilla alba ocularis, 284 .

Motacilla bistrigata, 285 .

Motacilla borealis, 285 .

Motacilla caprata, 233.

Motacilla certhiola, 244.

Motacilla cinerea, 284 .

Motacilla cinerea melanope, 285 .

Motacilla cinnamomea, 187.

Motacilla cyane, 232.

Motacilla flammeum, 267 .

Motacilla flava, 255 .

Motacilla flava macronyx, 285 .
Motacilla flava simillima, 285 .

Motacilla flava taivana, 285.

Motacilla indica, 286 .

Motacilla olivacea, 255.

Motacilla philippensis, 294.

Motacilla plexa, 285 .

Motacilla singalensis, 280.

Motacilla speciosa, 233.

Motacilla thunbergi, 285.

Motacilla sutoria, 245.

Motacilla tiphia, I88.

Motacillidae, $2 S_{4}$.

motleyi, Ceyx erithacus, ıot.

moultoni, Chlorocharis emiliae, 267.

moultoni, Ophrydornis albogularis, 216.

muelleri, Cryptolopha, 252.

muelleri, Muscicapa, 170.

muelleri, Oreicola dumetoria, 170.

muelleri, Seicercus castaniceps, 252.

mugimaki, Muscicapa, 168.

mugimaki, Poliomyias, 168 .

mulleri, Hemilophus, I5I.

mulleri, Pitta sordida, I58.

mulleri, Phyllornis, roo.

Mulleripicus, I 50.

Mulleripicus pulverulentus, I 1 .

mundus, Oriolus chinensis, 300 .

Munia, 200.

Munia atricapilla, 290.

Munia atricapilla batakana, 290.

Munia brunneiceps, 200.

Munia atricapilla batakana, 200 .

Munia atricapilla ferruginosa, 290.

Munia atricapilla sinensis, 200.

Munia chrysura, 201.

Munia fuscans, 292.

Munia jagori, 200.

Munia leucogastra, 201.

Munia leucogastra leucogastroides, 202.

Munia maja, 290.

Munia maja simalurensis, $20 \mathrm{r}$.

Munia maja zapercna, 200.

Munia molucca, 292.

Munia molucca nropinqua, 202.

Munia nisoria, $20 \mathrm{I}$

Munia punctulata, $20 \mathrm{I}$.

Munia punctulata fretensis, $20 \mathrm{l}$.

Munia striata, $20 \mathrm{I}$,

Munia striata subsquamicollis, 291.

Munia subundulata, 291.

Munia sumatrensis, 200.

murina, Myiothera, 217.

Muscadivores aeneus arhadius, 17.

Muscadivores aeneus diatropurus,

17.

Muscadivores aeneus mistus, 17. 
Muscadivores acneus polius, 17 .

Muscadivores aeneus vicinus, I8.

Muscadixores consobrina babiensis, 17.

Muscadivores rosaceus zamydrus, 18.

Muscicapa, 162 ,

Muscicapa aedon, 249.

Muscicapa albicilla, 163.

Muscicapa azurea, 172.

Muscicapa banyumas, 166.

Muscicapa cantatrix, 167 .

Muscicapa concreta, 164 .

Muscicapa cyanea, 191.

Muscicapa cyanomelana, 172 .

Muscicapa cyanomelana intermedia, 172 .

Muscicapa elegans, 165 .

Muscicapa flammea, 185.

muscicapa, Gerygone modiglianii, 171.

Muscicapa goiavier, 100 .

Muscicapa hasselti, 168 .

Muscicapa hirundinacea, 256 .

Muscicapa hyperythra, i6g.

Muscicapa indigo, iso.

Muscicapa infuscata, 179 .

Muscicapa javanica, 175.

Muscicaja latirostris, 163.

Muscicapa malabarica, 236 .

Muscicapula melanoleuca, 168.

Muscicapula melanoleuca westermanni, I68.

Muscicapa miniata, 187.

Muscicapa muelleri, 170.

Muscicapa mugimaki, 168.

Muscicapa narcissina, $17 \mathrm{t}$.

Muscicapa obscura, 256 .

Muscicapa panayensis, 297.

Muscicapa parva, 163.

Muscicapa pectoralis, 177 .

Muscicapa pectoralis, 260.

Muscicapa philomela, 260 .

Muscicapa picata, 256 .

Muscicapa pyrhoptera, 177.

Muscicapa rosea, 187.

Muscicapa rufigastra, 165.

Muscicapa rufigula, 168.

Muscicapa sibirica, 162 .

Muscicapa solitaris, 160.

Muscicapa superciliaris, 160 .

Muscicapa thalassina, 18 .

Muscicapa torquata, 233.

Muscicapa tricolor, 171 .

Muscicapa turcosa, 165.

Muscicapa umbellata, 175.

Muscicapa zanthopygia, 171 .

Muscicapella, 168.

Muscicapella hodgsoni, 168.
Muscicapella hodgsoni sondaica, 168.

- Muscicapidae, 162.

Muscicapula, 168.

Muscicapula malayana, 160 .

Muscicapula melanoleuca hasselti, 168.

Muscipeta atrocaudata, 177.

Muscipeta plumosa, 177.

Muscisylvia, 234.

Muscisylvia diana, 234 .

Muscisylvia diana sumatrana, 235.

Muscisylvia leucura, 234 .

Muscitrea cinerea, 260 .

Muscitrea grisola nesiotis, 261.

musicus, Copsychus saularis, 235.

musicus, Cuculus, 126.

musicus, Lanius, 235.

musicus, Pastor, 206.

musicus, Penthoceryx sonneratii, 126.

muticus, Pavo, 9.

Mycropogan fuliginosus, 134.

Mycteria asiatica, 54 .

Myjolestes pyrrhonota, 238 .

Myiophoneus borneensis, 239 .

Myiophoneus castaneus, 239.

Myiophoneus crassirostris, 240.

Myiophoneus robinsoni, 239.

Myiothera aftinis, 150.

Myiothera andromedae, 242.

Myiothera capistrata, 200.

Myiothera coerulea, 156 .

Myiothera epilepidota, 217.

Myiothera grammiceps, 22x.

Myiothera leucophris, 23I.

Myiothera loricata, 213.

Myiothera melanothorax, 224.

Myiothera murina, $21 \%$.

Myiothera pyrrhogenys, 212.

Myophonus, 230.

Myophonus borneensis, 239 .

Myophonus castaneus, 239 .

Myophonus flavirostris, 240 .

Myophonus flavirostris crassiros. tris, 240.

Myophonus flavirostris dicrorhynchus, 240.

Myophonus glaucinus, 230 .

Myophonus metallicus, 240 ,

Myojhonus melanurus, 239.

Myophonus robinsoni, 239 .

Myristicivora, 19.

Myristicivora bicolor, 19.

Myristicivora grisea, 10.

myrtha, Ciccaba, 80.

myrtha, Strix leptogrammica, 80 .

mystacale, Chrysophlegma flavinucha, 143.

mystacalis, Aethopyga, 274 . 
mysticalis, Chloropsis, 191. mysticalis, Chrysojlılegma, 143. mystacalis, Nectarinia, 274 . mystacophanes, Chotorea, 135. mystacophanos, Bucco, 135. Myzanthe ignipectus, 268 .

nanus, Hierococcyx, 124. nanus, Ichthyaetus, 77 . nana, Icthyophaga, 77. nana, Macropygia ruficeps, 22. nana, Spizaetus, 74.

Napothera, 217.

Napothera atricapilla, 215.

Napothera brevicaudata, 217.

Napothera brevicaudata crassa, 2 i 7.

Napothera brevicaudata leucosticta, 217 ,

Napothera coronata, 214.

Napothera epilepidota, 217.

Napothera epilepidota diluta, 217.

Napothera epilepidota exsu!, 218.

Napothera epilepidota granti, 218.

Napothera pileata, 214.

Napothera pyrthoptera, 219.

Napothera rubicauda, 213 .

narcissina, Muscicapa, 171

narcissina, Zanthopygia, 171 .

nasica, Erolla, 156 .

nasica, Treron, 12.

nasicus, Numenius, 35.

nesiotis, Muscitrea grisola, 26 t.

nesodramus, Lamprocorax panayensis, 208

nasutus, Ianius, 260

nasutus, Todus, 156 .

natalis, Accipiter fasciatus, 7f .

natalis, Chalcophaps indica, 24.

matalis, Collocalja esculenta, 117.

natalis, Ninox forbesi, 88 .

natalis, Zosterops, 264 .

natunae, Aethopyga siparaja, $27 j$.

natunae, Collocalia francica, its.

natumensis, Anaimos maculatus, 27 r.

natunensis, Anthrejtes macularia, 278.

Ratunensis, Charitociris maculata, 271.

natunensis, Spilornis checla, 76 .

natunensis, Stachyris, 220.

natunensis, Zanclostomus java. nicus, $13 \mathrm{~T}$.

nebularia, Scolopax, 43.

nebularia, Tringa, 43 .

nebulosa, Ardea, 60 .

Nectarinea ignita, 268.
Nectarinia calcostetha, 273 .

Nectarinia eximia, 274.

Nectarinia, eximia, 277.

Nectarinia flammaxillaris, 277.

Nectarinia frontalis, $\mathbf{2 7 8}$.

Nectarinia basseltii, 276 .

Nectarinia hypogrammica, 278 .

Nectarinia inornata, $28 z$.

Nectarinia insignis, 273.

Nectarinia javanica, 279 .

Nectarinia kuhlii, 274.

Nectarinja mystacalis, 274.

Nectarinia pectoralis, 277 .

Nectarinia pectoralis, 273

Nectarinia phoenicotis, aSo.

Nectarinia rubro-cana, 267.

Nectarinia simplex, 278 .

Nectarinia temmincki, 274 .

Nectariniidae, 273.

neglecta, Dissoura, 53.

neglecta, Lalage fimbriata, 184.

neglecta, Volvocivora, 184.

neglecta, Zosterops chlorates, 266

neglectus, Cuculus, 128.

neglectus, Pyrotrogen, 1 I 9 .

Nelicurvius emberizinus, 294.

Nemura hodgsoni, 168.

neocara, Cyanoderma erythroptera. 223.

Neophron, 7o.

neophron, Entomophora coromanda, 102.

Neophron percmopterus, 70 .

Neophron percnopterus ginginianus, 70 .

nesacus, Anthreptes malacensis, 270.

nesiarchus, Artamides sumatrensis, I $8 \mathrm{r}$.

nesiarchus, Copsychus saularis, 235.

nesiotes, Copsychus saularis, 235.

nesiotica, Aegithina viridissima, 188.

nesiotis, Pachycephala cinerea, 261.

nesitis, Anuropsis malaccensis, 217. nesoeca, Ramphalcyon capeasis, 08.

Nettapus, 61.

Nettapus coromandelianus, 61.

niasense, Chrysophlegma, 143.

niasense, Syrnium, So.

niasensis, Aethonyga siparaja, 275. niasensis, Arachnothera longirostra, 282 .

niasensis, Callolophus miniaceus, 143.

niasensis, Strix leptogrammica, 80. nicobarica, Caloenas, 24.

nicobarica, Columba, 24. 


\section{INDEX.}

nidifica, Collocalia, 115.

nieuwenhuisii, Euptilosus urostictus, I94.

nieuwenhuisii, Poliolophus, 194.

niger, Buceros, ro8.

niger Copsychus saularis, 236 .

niger, Cry'ptonyx, 4.

niger, Drymonax, 184.

niger, Hydrocurax, 64.

niger, Phalacrocorax, 64.

niger, Turdus, 184 .

nigerrima, Fringilla, 202.

nigra, Lalage, 184.

nigra, Melanoperdix, 4.

nigrescens, Rheinardius ocellatus, 9.

nigricans, Alcedo euryzona, too.

nigricauda, Cittacincla, 238 .

nigricauda, Kittacincla malabarica, 238.

nigriceps, Stachyris, 220,

nigricollis, Chloropsis cochinchinensis, 190 .

nigricollis, Phitemon, 100.

nigricollis, Stachyris, 221 .

nigricollis, Timalia, 221 .

nigricrissus, Turdus, 240 .

nigrigularis, Cyornis, 167 .

nigrimentum, Dicaeum cruentatum, 268.

nigripes, Ardea, 56 .

nigripes, Egretta garzetta, 56 .

nigrirostris, Buceros, 107.

nigriventer, Poliositta azurea, 263 .

nigriventer, Sitta azurea, 263.

nigriventris, Phaenicophaeus, 131 .

nigrocapitata, Brachypteryx, 200.

nigrocapitatum, Pellorneum capis-

tratum, 200.

nigrogularis, Brachypteryx, 221.

nigrolineata, Zapornia, 25.

nigrolineatus, Rallus eurizonoides, 25.

nigrorufus, Centropus, 130.

nigrorufus, Cuculus, 130 .

nilotica, Gelochelidon, 45 .

nilotica, Sterna, 45.

Niltava, J70.

Niltava, grandis, 170.

Niltava grandis decipiens, 170.

Niltava sumatrana, 164 .

Ninox, 87.

Ninox forbesi, 88 .

Ninox forbesi natalis, 88.

Ninox labuanensis, 88 .

Ninox scutulata, 87 .

Ninox scutulata borneensis, 88 .

Ninox scutulata burmanica, 88 .

Ninox scutulata javanensis, 88 .

Ninox scutulata malaccensis, 88 . nipalensis, Aethopyga, 274, 276 .

nipalensis, Alcippe, 218 .

nipalensis, Cinnyris, 276 .

nipalensis, Cutia, 229 .

nipalensis, Nisaetus, 74 .

nipalensis, Paludicola, 157.

nipalensis, Pitta, 157.

nipalensis, Pyrrhula, 288.

nipalensis, Siva, 218 .

nipalensis, Spizaetus, 74 .

nipalensis, Toria, 12.

nipalensis, Treron curvirostra, 12

Nisaetus alboniger, 74 .

Nisaetus nipalensis, 74.

nisicolor, Cuculus, 123 .

nisicolor, Hierococcyx fugax, 123.

nisoides, Accipiter, 72.

nisoria, Munia, 201.

nisosimilis, Accipiter nisus, 72.

nisosimilis, Falco, 72 .

nisus, Accipiter, 72 .

nisus, Falco, 72 .

Nitidula hodgsoni sondaica, 168.

nitidus, Orthotomus, 245.

nitidus, Phylloscopus, 250.

niveus, Falco, 74 .

nobilis, Euplocamus, 6.

Noctua brodici, go.

Noctua cuculoides, go.

Noctua hirsuta minor, 88.

Noctua tubiger, go,

noctula, Strix, 86.

normani, Coracina personata, 183 .

normani, Graucalus, 183 .

notatum, Malacopteron affine, 215 .

Notodela diana sumatrana, 235 .

novaeseelandiae, Anthus, 286 .

nubilosa, Sterna fuscata, 47.

nuchalis, Anthreptes, 278 .

nudiceps, Gyps indicu5, 69.

nudipes, Chaetura caudacuta, 117.

Numenius, 35 .

Numenius arquata, 35.

Numenius arquata orientalis, 35 .

Numenius madagascariensis, 36.

Numenius minutus, 36 .

Numenius nasicus, 35.

Numenius phaeopus, 36 .

Numenius phaeopus variegatus, 36.

Nycticorax, 57 .

nycticorax, Ardea, 57.

Nycticorax caledonicus, 57,

Nycticorax caledonicus hilli, 57.

Nycticorax caledonicus manillensis, 57.

Nycticorax limnophilax, 57.

Nycticorax, nyeticorax, 57.

ny'cticorax, Nycticorax, 57. 


\section{INDEX.}

nyctilampe, Pellorneum capistratım, 200 .

nyctilampis, Drymocataphus nigrocapitatus, 200.

Nyctiornis, Ito.

Nyctiornis amicta, 110.

Nyctiornis malaccensis, 110.

nyctiphasma, Strix leptogrammica, 80.

nympha, Pitta, 158.

Nyroca, 63.

Nyroca fuligula, 63 .

oberholseri, Collocalia esculenta, I 6 .

oberholseri, Collocalia linchi, I 16. oberholseri, Treron fulvicollis, I3. obscura, Cyanoderma rufifrons, 225.

obscura, Muscicapa, 256.

obscura, Siphia, 163.

obscurus, Turdus, 24 I.

observandus, Gecinus puniceus, I 42.

observandus, Picus puniceus, $\mathrm{t}_{42}$. occipitalis, Dendrocitta, 300.

occipitalis, Glaucopis, 300.

occipitalis, Phyllopneuste, $25 \mathrm{I}$.

occipitalis, Phylloscopus, $25 \mathrm{I}$.

occidentalis, Pomatorhinus montanus, 207.

occidentalis, Tinnunculus moluccensis, 82 .

Oceanodroma, 51 .

Oceanodroma leucorhoa, $5 \mathrm{I}$.

Oceanodroma leucorhoa monorhis, $5 \mathrm{I}$.

ocellata, Caloperdix oculea, 4.

ocellata, Rheinartia, 0 .

ocellatus, Argus, o.

ocellatus, Tetrao, 4.

ochotensis, Locustella, 244.

ochotensis, Sylvia, 244.

ochracea, Sasia, I52.

ochraceus, Criniger gutturalis, 195. ochrocephalus, Turdus, 198.

ochromalus, Eurylaimus, I.55.

ochrommatus, Orthotomus cineraceus, 247.

ochrommatus, Orthotomus sepium, 247.

ochroptila, Kittacincla malabarica, 237.

ochropus, Tringa, 42.

ochropyrrba, Aethopyga siparaja, 275.

ocularis, Motacilla alba, 284.

oculea, Caloperdix, 3.

oculea, Perdix, 3. ocyptera, Hemiprocne longipennis, I. 3.

Ocypterus sanguinolentus, 301.

Oedicnemus magnirostris, 30.

Oenanthe pyrrbonota, 233.

oenope, Cinnyris brasiliana, 276.

oenopa, Leptocoma brasiliana, 276 . oenothorax, Ducula aenea, 18.

olax, Columba, 15.

olax, Treron, 15.

Olcyornis poliogenys, 178 .

Olcyornis saturatior, 178 .

olivacea, Iole, J93.

olivacea, Malacocincla abbotti. 210.

olivacea, Motacilla, 255.

olivacea, Prinja familiaris, 255.

olivacea, Rhinomyias, 178 .

olivaceum, Malacopteron, zio.

olivaceus, Cyornis, 178 .

olivaceus, Microtarsus, $20 t$.

olizurus, Dissemurus paradiseus, 307.

omissa, Kittacincla macrurus, 237.

omissa, Kittacincla malabarica, 237.

omissa, Siva strigula, 228 .

omnicolor, Alcedo, 103.

onocrotalus, Pelecanus, 68.

oorti, Bucco, 137.

oorti, Cyanops, 137 .

ophellochlora, Gracula javanensis, 207.

ophellochlora, Gracula religiosa, 297.

Ophrydornis, 215.

Ophrydornis albogularis, 2 I5.

Ophrydornis albogularis moultoni, ? 6 .

opistatus, Anaimos maculatus, 272.

opisthisa, Kittacincla malabarjca, 238.

opisthochra, Kittacincla melanura, 238.

opisthocyanea, Hypothymis azurea, 173.

opisthopela, Kittacincia malabarica, 238 .

optatus, Cuculus, 125.

orantus, Trichoglossus, gr.

Oreicola, I7o.

Oreicola dumetoria, 170 .

Oreicola dumetoria muelleri, 170.

Oreocincla, 243.

Oreocincla dauma, 243

Oreocincla dauma horsfieldi, 243.

Oreocincla borsfieldi affinis, 243 .

Oreoctistes leucops, 200.

oreophila, Cettia montana, 254 .

Oreosterops javanica elongata, 267. 
oreskios, Harpactes, I2t.

oreskios, Trogon, I $2 \mathrm{I}$.

oricularius, Pastor, 204.

orientalis, Acrocephalus stentoreus, 245.

orientalis, Arborophila brunneopectus, 3 .

oriențalis, Coracias, 97.

orientalis, Eurystomus, 97.

orientalis, Glareola, 44.

crientalis, Gallinula chloropus, 28. orientalis, Numenius arquata, 35.

otientalis, Perdix, 3.

crientalis, Rhynchaea, 31.

orientalis, Salicaria turdina,-245.

orientalis, Stachyris thoracica, 22.3.

orientalis, Strix, 84 .

orientalis, Strix, 88.

orientalis, Turdus, 184

Oriolidae, 299.

Oriolus, 299.

Oriolus castanopterus, $30 \mathrm{x}$.

Orjolus chinensis, 200 .

Oriolus chinensis diffusus, 299.

Oriolus chinensis insularis, 300.

Oriolus chinensis lamprochryseus, 300.

Oriolus chinensis maculatus, 290.

Oriolus chinensis mundus, 300.

Oriolus chinensis richmondi, 300.

Oriolus chinensis siberu, 300.

Oriolus chinensis sipora, 300.

Oriolus coronatus, 209.

Oriolus cruentus, 301 .

Oriolus cruentus consanguineus, $30 \mathrm{~T}$.

Oriolus cruentus malayanus, 302 .

Oriolus cruentus vulneratus, 302.

Orjolus horsfieldi, 200 .

Oriolus hosii, 301 .

Oriolus lineates, 5.

Oriolus luteolus thaiocous, 300.

Oriolus maculatus lamprochryseus, 300.

Oriolus maculatus richmondi, 300 .

Oriolus sinensis, 204.

Oriolus xanthonotus, 301.

Oriolus xanthonotus consobrinus, $30 t$.

Oriolus xanthonotus mentawi, 301.

Oriolus xanthornus, 300.

Oriolus xanthornus tanakae, 301.

Oriolus xanthornus thaiocous, 300.

ornatus, Cinnyris, 277 .

ornatus, Psittacus, 91.

Oroecetes gularis, 243 .

orrhophaeus, Harpactes, I2 [.

orrophaeus, Pyrotrogon, 121 .

Orthnocichla whiteheadi, 231.

Orthorhynchus frontalis, 262 .
Orthorhamphus magnirostris scommophorus, 3 I.

Orthorhinus hypoleucos, 207.

Orthotomus, 245 .

Orthotomus atrogularis, 245 .

Orthotomus atrogularis eumelas, 246.

Orthotomus atrogularis humphreysi, 246.

Orthotomus atrogularis major, 246 .

Orthotomus cineraceus, 247.

Orthotomus cineraceus baeus, $24 \%$.

Orthotomus cineraceus ochrommatus, 247.

Orthotomus cuculatus, 247.

Orthotomus flaviventris, 255.

Orthotomus flavoviridis, 245 .

Orthotomus huegelii, 245 .

Orthotomus nitidus, 245 .

Orthotomus prinia, 255.

Orthotomus ruficeps rubicundulus, 246.

Orthotomus sepium, 246.

Orthotomus sepium baeus, 247.

Orthotomus sepium borneonensis. 247.

Orthotomus sepium ochrommatus, 247.

Orthotomus sepium concinnus, 247.

Orthotomus sepium palliolatus, 247 .

Orthotomus sepium ruficeps, 247.

Orthotomus sericeus, 246.

Orthotomus sericeus hesperius, 246 .

Orthotomus sericeus rubicundulus, 246 .

Orthotomus sutorius, 245.

Orthotomus sutorius edela, 245 .

Orthotomus sutorius maculicollis, 245.

Oryzivora leucotis, 280 .

oryzivora, Loxia, 280 .

oryzivora, Padda, 280.

osbeckii, Psittacus, 92.

Osittacus vernalis, 94.

Osmotreron olax hageni, is.

Otocompsa flaviventris minor, 203.

Otocompsa personata, to0.

ottolanderi, Pomatorhinus montanus, 207.

Otus, 84 .

Otus bakkamoena, 86

Otus bakkamoena condorensis, 86 .

Otus bakkamoena lempiji, 86.

Otus bakkamoena mentawi, 86 .

Otus brookii, 87.

Otus brookii solokensis, 87.

Otus luciae siamensis, 85 .

Otus rufescens, 87 .

Otus rufescens malayensis, 87 .

Otus sagittatus, 87 .

Otus scops, 84 . 
Otus scops malayanus, 85 .

Otus scops mantanamensis, 85 .

Otus spilocephalus, 85 .

Otus spilocephalus angelinae, 85 .

Otus spilocephalus luciae, 85 .

Otus spilocephatus siamensis, 85.

Otus spitocephalus stresemanni, 85 .

Otus spilocephalus vulpes, 85.

Otus umbra, 86.

Otus umbra enganensis, 86.

Otus vandewateri, 86.

oxyura, Columba, Io.

oxyurus, Sphenurus, 10.

pachistorhinus, Aplonis panayensis, 200.

pachistorhinus, Lamprocorax chalybeus, 290 .

pachistus, Dissemurus paradiseus, 307.

Pachycephala, 260.

Pachycephala cinerea, 260 .

Pachycephala cinerea butaloides, 260 .

Pachycephala cinerea nesiotis, 261.

Pachycephala cinerea secedens, 260.

Pachycephala cinerea vandepolli, 261.

Pachycephala fulvotincta, 260.

Pachycephala grisola butaloides. 260.

Pachycephala grisola secedens, 260.

Pachycephala hypoxantha, 261 .

Pachycephala hypoxantha sarawacensis, $26 \mathrm{t}$.

Pachycephala pectoralis, 260.

Pachycephala pectoralis javana, 260.

pacifica, Hirundo, 118.

pacificus, Micropus, 118.

Padda, 280.

Padda oryzivora, 280 .

Padda verecunda, 280 .

paeoninus, Argus, 8.

pagana, Entomophora coromanda, 102.

paganica, Aethostoma rostrata, 212. pageli, Bubo ketupu, 83 .

pagense, Dicaeum trigonostigmum, 270 .

pagensis, Kittacincla melanura, 238. pagicola, Chalcostetha calcostetha, 273.

pagiensis, Copsychus saularis, 235.

pagodorum, Strix, 8o.

Palaeornis erythrogenys, 94 .

Palaeornis fraseri, 04.

Palacornis lucianae, 94 .

Palaeornis major, 93 .
Palaeornis modestus, 94.

Palacornis viridimystax, 93 .

palawanensis, Ducula aenea, 17.

Pallenura javensis, 285 .

palliata, Ianthocincla, 205.

palliatus Garrulax, 205 .

pallida, Chalcoparia singalensis, 280.

pallidus, Eurylaimus javanicus, 155 .

pallidus, Falco, 76 .

pallidus, Spilornis, 75.

pallidus, Zanclostomus javanicus, 130.

palliolatus, Orthotomus sepium. 247.

palmarum, Dinopium javanense, 148.

palmeri, Excalfactoria chinensis, 5.

palmeri, Sauropatis chloris, 104.

Dalpebrosa, Svlvia, 263.

palpebrosa, Zosterop5, 263.

Paludicola aipalensis, 157 .

palustris, Megalurus, 249.

pammicrus, Pycnonotus erythropthalmos, 202.

panavensis, Aplonis, 297.

payanensis, Muscicapa, 297

Pandion, 82.

Pandion haliaetus, 82 .

Pandion haliaetus cristatus, 82.

Pandion horsfieldij, 77 .

Pandionidae, 82 .

pandoo, Monticola solitaria, 243.

pandoo, Petrocincla, 243.

panochra, Butreron capellei, It.

panopsia, Chalcoparia singalensis, $28 \mathrm{t}$.

papillosa, Ibis, 52 .

papillosa, Pseudibis, 52.

Paradisea tristis, 295.

paradiseus, Cuculus, 306

paradiseus, Dissemurus, 306.

paradisi, Corvus, 176.

paradisi, Terpsiphone, 176 .

Pardalotus maculatus, 271.

Pardalotus percussus, 271.

Pardalotus thoracicus, $27 \mathrm{I}$.

Paridae, 26t.

paroticalis, Ixidia, $20 z$.

paroticalis, Pycnonotus cyaniventris, 202.

Parra gallinacea, 30 .

Parra indica, 30.

Parra superciliosa, 30.

Parus, 26i.

Parus atricep5, 261.

Parus flavocristatus, $26 \pi$.

Parus majot, 26i.

Parus major ambiguus, 262. 


\section{INDEX.}

Parus major cinerascens, 262.

Parus major cinereus, 261.

Parus major malayorum, 262.

Parus sarawacensis, 262.

Parus sultaneus, 261.

parva, Muscicapa, 163 .

parva, Siphia, 163 .

parva, Treron vernans, 15.

parvirostris, Cbloropsis sonnerati, 100.

parvirostris, Chloropsis zosterops, 100.

parvirostris, Phylloscopus trivirgatus, 251 .

parvirostris, Seicercus trivirgata, 251.

parvule, Zosterops, 265.

parvulus, Podargus, 06.

parvus, Blythipicus rubiginosus, parvus, Dryocopus javensis, I 5 I.

jiarvus, Thriponax, 151.

parvus, Zosterojs aureiventer, 264

Passer, 288.

Passer flaveolus, 288 .

Passer montanus, 288 .

Passer montanus malaccensis, $\mathbf{2 8 8}$

passorhina, Butreron capellei, 11.

Pastor fuscus, 206.

Pastor griseus, 296.

Pastor jalla, 204.

Pastor malayensis, 205.

Pastor musicus, 206.

Pastor oricularius, 294.

Pastor tricolor, 295.

Pavo, 9 .

Pavo bicalcaratus, 7.

Pavo javanicus, 9 .

Pavo muticus, 9 .

pavonius, Argus, 8.

pavoninus, Azgus, 8

paykullij, Rallus, 26.

pectinicauda, Scolopax, 38

pectoralis, Alcippe, 170.

pectoralis, Gerygone, i7i.

pectoralis, Leptocoma jugularis, 277.

pectoralis, Muscicapa, r77.

pectoralis, Muscicapa, 260.

pectoralis, Nectarinia, 277.

pectoralis, Nectarinia, 273

pectoralis, Pachycephala, 260.

pectoralis, Stachyris maculata, 222.

pectoralis, Timalia, 222.

pega, Treron cutvirostra, 12.

Pelargopsis forisiana, no.

Pelargopsis fraseri, 97

Pelargopsis malaccensis, 08 .

Pelargopsis simalurensis, 98 .

Pelargopsis sodalis, 08 .

Pelecanidae, 68.

Pelecanus, 68
Pelecanus carbo, 63.

Pelecanus javanicus, 68 .

Pelecanus leucogaster, 65.

Pelecanus minor, 67.

Pelecanus onocrotatlus, 68 .

Pelecanus plotus, 66.

Pelecanus roseus, 60 .

Pelecanus sinensis, 63 .

Pelecanus sula, 66.

pella, Cyanoderma ervthroptera, 224.

pellochlorả, Dendrophassa vernans, 15.

pelínota, Culicicapa ceylonensis. 180.

pellopira, Culicicapa ceylonensis, 180.

pelloptilus, Anthreptes malacensis, 279.

Pellorneum, 208.

Pellorneum capistratum, 200.

Pellorneum capistratum capistratoides, 209.

Pellorneum capistratum morrelli, 209.

Pellorneum capistratum nigrocapitatum, 209.

Pellorneum capistratum nyctilampe, 209.

Pellorneum ruficeps, 208.

Pellorneum ruficeps subochraceum, 200.

Pellorneum tickelli, 209.

Pellorneum tickelli australe, 209.

pellum, Cyanoderma erythropterum, 224.

Peloperdix rubrirostris, 3 .

penrissenensis, Buchanga leucophaea, 303 .

penelope, Anas, 62.

peninsulae, Garrulax erythrocephalus, 205.

peninsulae, Trochalopterum, 205.

peninsularis, Chloropicoides raf. flesii, 144.

peninsularis, Cyornis, $\$ 64$.

peninsularis, Gauropicoides raffesi, 144.

peninsularis, Melanocichla, 206.

pennatus, Falco, 73 .

pennatus, Hieraaetus, 73

Penthoceryx, 125.

Penthoceryx sonneratii, 125.

Penthoceryx sonneratii fasciolatus. 126.

Penthoceryx sonnerati malayanus. 25.

Penthoceryx sonneratii musicus, 126.

peracensis, Alcippe nipalensis, 218 . peracensis, Bhringa remifer, 306. 
peracensis, Iole tickelli, 103. peracensis, Ixos miclellandii, I93. perena, Anas superciliosa, $6 z$. percnerpes, Meiglyptes tukki, 147. percnocara, Culicicaja ceylonensis, 180.

percnopterus, Neophron, 70.

percussus, Anaimos, $27 \mathrm{t}$

percussus, Pardalotus, 27 I.

Perdix aeruginosus, 4.

Perdix charltonii, 3 .

Perdix coronata, 4 .

Perdix longirostris, $\mathbf{I}$.

Perdix oculea, 3

Perdix orientalis, 3 .

Perdix personata, 3

peregrina, Ibis, 52.

peregrinus, Falco, 80 .

peregrinus, Plegadis falcinellus, 52.

verenopterus, Vultur, 70 .

Pericrocotus, 185.

Pericrocotus andamanensis minythomelas, 186.

Pericrocotus brevirostris minor, 186

Pericrocotus cinnamomeus, 187 .

Pericrocotus cinnamomeus saturatus, 187.

Pericrocotus cinnamomeus vividus, 187.

Pericrocotus croceus, 186.

Pericrocotus flagrans, 186.

Pericrocotus flammeus, 185.

Pericrocotus flammeus flammifer, 185 .

Pericrocotus flammeus minythomelas, 186 .

Pericrocotus flammeus modiglianii, 185.

Pericrocotus fammeus siebersi, 186.

Pericrocotus flammeus xantho. gaster, I85.

Pericrocotus igneus, 186.

Pericrocotus igneus trophis, 186.

Pericrocotus miniatus, 187.

Pericrocotus minutus, 186.

Pericrocotus modestus, 187

Pericrocotus montanus, I 86.

Pericrocotus montanus cjnereigula, I 86.

Pericrocotus peregrinus saturatus, I 87.

Pericrocotus peregrinus vividus, 187.

Pericrocotus roseus, 187.

Pericrocotus roseus cantonensis, 187.

Pericrocotus roseus divaricatus, 187.

Pericrocotus speciosus siebersi, 186.

Pericrocotus subardens, 185 .

Pericrocotus wrayi, 186. perionca, Psittacula alexandri, 93. perioncus, Conurus fasciatus, 93. periophthalmica, Buchanga, 304. periophthalmicus, Dicrurus leucophaeus, 304 .

Perissolalage chalepa, 185.

Peristera bornensis, 24.

perlata, Rhipidura, 175.

perlonga, Hemiprocne longipennis, 113.

perlonga, Macropteryx, 1 I3.

Pernis, 79.

Pernis apivorus, 79.

Pernis apivorus japonicus, 70.

Pernis apivorus ptilorbyncus, 79 .

Pernis apivorus ruficollis, 79 .

Pernis jerdoni, 80 .

Pernis torquata, 70 .

Pernis tweedalii, 70 .

perolivacea, Rhinomyias olivacea, 178.

ретоnii, Charadrius alexandrinus, 35.

perplexa, Collocalia francica, 114. perplexus, Pycnonotus simplex, 201. perpulchra, Halcyon, 103. perretti, Aethopyga mystacalis, 274 . personata, Coracina, 183. personata, Heliopais, 20.

personata, Otocompsa, I99.

personata, Perdix, 3 .

personata, Podica, 20 .

personatus, Alectrophasis, 6.

personatus, Pycnonotus goiavier. 100.

perspicillata, Cacopitta, 21 I.

perspicillatus, Malacocincla, 2II.

perspicillatus, Platyrhynchos, 175.

Petrocincla pandoo, 243.

phaedra, Buchanga stigmatops, 303.

phaedrus, Dicrurus leucophaeus, 303.

Phaentcomanos jora, 189 .

Phaenicophaeus erythrognathus, 133.

Phaenicophaeus longicaudatus, I 3 I.

Pbaenicopbaeus nigriventris, I3I.

phacnicophaus caniceps, I 32.

Phacnicophaus chlorocephalus, 132.

Phaenicophaus javanicus, 130.

Phaenicophaus lucidus, 132 .

Phaenicophaus melanognathus, 132.

Phaenicophaus viridirostris, 132 .

Phaenicophaus viridirufus, 132 .

Phaenicophaus viridis, 132 .

Phaenicophoeus tricolor, 132.

Phaenicornis ardens, 185.

Phaenicura rubeculoides, 165 . phaeocephalus, Alophoixus, 507. phaeocephalus, Ixos, 197. 


\section{INDEX.}

phaeopus, Numenius, 36 .

phaeopus, Scolopax, 36 .

Phaethon, 67.

Phathon aethereus, 68 .

Phaethon aethereus indicus, 68 .

Phaethon fulvus, 68.

Phaethan lepturus, 68.

Phaethon lepturus fulvus, 68 .

Phaethon rubricauda, 67.

Phathon rubricauda westralis, 67 .

Phiethontidae, 67.

Phaeton fiavo-durantius, 68.

Phaeton lepturus, 68.

Phaiopicus grammithorax, 147.

Phalacrocoracidae, 63.

Phalacrocorax, 63 .

Phalacrocorax carbo, 63 .

Phalacrocorax carbo sinensis, 63 .

Phalacrocorax melanoleucos, 64.

Phalacrocorax melanoleucos melvillensis, 64 .

Phalacrocorax niger, 64 .

Phalacrocorax sulcirostris, 64 .

Phalacrocorax sulcirostris territori, 64.

Phalaropus, 37.

Phalaropus lobatus, 37.

phasianella, Columba, 20 .

phasianella, Macropygia, 20.

Phasianidae, 1.

Phasjanus argus, 8 .

Phasiagus castaneus, 6.

Phasianus cristatus, 4.

Phasianus erythrophthalmus, 5.

Plasianus gallus, 7 .

Phasianus ignitus, 6 .

Phasianus ignitus, 6.

Phasianus malacensis, 8.

Phasianus purpureus, 5 .

Phasianus roulroul, 4.

Phasianus rufus, 6 .

Phasianus varius, 7.

phasma, Columba, 20.

Philemon nigricollis, rgo.

Philentoma castaneum 177 .

Philentoma dubium, 178.

Philentoma intermedius, 178 .

Philentoma maxwelli, 178 .

Philentoma saravacense, 178 .

Philentoma unicolor, 177 .

philippensis, Motacilla, 204.

philippensis, Monticola solitaria, 243.

philippensis, Podiceps ruficolli5, 50 . philippensis, Rallus, 25.

philippensis, Sturnia, 294.

philippensis, Turdus, 243.

philippina, Loxia, 203.

philippinus, Ploceus, 203.

Philomachus, 40.

Philomachus pugnax, 40. philomela, Muscicapa, 260.

Phodilus, 91.

Phodilus badius, $9 \mathrm{I}$.

Phodilus badius abbotti, $9 t$.

Phodilus badius arixuthus, 91.

Phoenicophaes curvirostris deningeri, I32.

Phoenicophaes microrhinus, 132.

Phoenicophaus, 132.

Phoenicophaus curvirostris, I32.

Phoenicophaus curvirostris aeneicaudus, 133.

Phoenicophaus curvirostris borneensis, 132.

Phoenicophaus curvirostris eryth. rognathus, 133 .

Phoenicophaus curvirostris deningeri, I32.

Phoenicophaus elongatus, I 31 .

Phoenicophoeus aeneicaudus, 133.

phoenicotis, Chalcoparia sintraJensis, 280 ,

phoenicotis, Nectarinia, 280.

phoenicura, Amaurornis, 27.

phoenicura, Rhipidura, 175.

phoenicurus, Amaurornis, 27.

phoenicurus, Gallinula, 27.

photina, Aethopyga siparaja, 275.

Phragamaticola, 249.

Phragamaticola aedon, 240.

Phragamaticola aedon rufescems. 240.

Phyllergates, 247 .

Phyllergates cucullatus, 247.

Phyllergates cucullatus cinerercollis, 248 .

Phyllergates cucullatus thais, 248 .

Phyllergates sumatranus, 247.

Phyllopneuste borealis, 250 ,

Phyllopneuste occipitalis, 251.

Phyllopneuste xanthodryas, 250.

Phyllornis aurifrons, Igr.

Phyllornis cyanopogon, 19r.

Phyllornis media, I9I.

Phyllornis moluccensis, Igo.

Phyllornis mullerii, ıgo.

Phyllornis venusta, 180 .

Phyllornis viridinucha, Igo.

Phylloscopus, 250.

Phylloscojus borealis, 250.

Phylloscopus borealis examinandus, 250.

Phylloscopus borealis xantbodryas, 250.

Phylloscopus inornatus, $25 \mathrm{I}$.

Phylloscopus magnirostris, 250.

Phylloscopus nitidus, 250.

Phylloscopus nitidus plumbeitarsus, 250.

Phylloscopus occipitalis, $25 \mathrm{I}$. 
Phylloscopus occipitalis coronatus, 251.

Phylloscopus tenellipes, 250.

Phylloscopus trivirgatus, 251

Phylloscopus trivirgatus parvirostris, $25 \mathrm{I}$.

Piaya chrysogaster, 130 -

Piaya erythrorhyncha, 130.

picaoides, Heterophasia, 228.

picata, Ardea, 60.

picata, Muscicapa, 256.

picatus, Hemipus, 256.

picatus, Iулgipicus, 145.

Picidae, I40.

pickeringii, Carpophaga, 18.

pickeringii, Ducula, I8.

picinus, Edolius, 305.

Picnonotus inornatus, 200.

Picunnus abnormis, 152.

Picutnnus innominatus, 152.

Picumnus innominatus malayorum, 152.

Picus, 140.

Picus affinis, 140.

Picus analis, 144.

Picus badius, 148 .

Picus, (Boeopipo) aurantilventris, 145.

Picus brachyurus, 148.

Picus canente, 150.

Picus canicapillus, 145.

Picus canus, 14 ?.

Picus canus dedemi, I4I.

Picus canus robinson, 141 .

Picus chlorolophus, I4:.

Picus chlorolophus rodgeri, 141.

Picus chlorolophus vanheysti, 142 .

Picus concretus, Izo.

Picus (Dendrocopus) hardwickii, 145

Picus dimidiatus, 140.

Picus flavinucha, 143.

Picus fulvigaster, $15 \mathrm{I}$.

Picus grantia, I44.

Picus gularis, 142.

Picus guttacristatus, 149.

Picus gutturalis, $15 \mathrm{I}$.

Picus borsfieldi, 15 I.

Picus horsfieldi, 151 .

Picus javanensis, 148 .

Picus javensis, 151 .

Picus leucogaster, I51.

Picus lucidus, 140.

Picus luridus, 147.

Picus mackloti, I 5 I.

Picus malaccensis, ${ }^{4} 42$.

Picus maximus malayensis, 151 .

Picus melanogaster, 146.

Picus mentalis, 143 .

Picus mineaceus, 142.

Picus moluccensis, 145 .

Picus poicilophus, 146.
Picus pulverulentus, $15 \mathrm{I}$.

Picus puniceus, 142.

Picus puniceus continentis, 142.

Picus puniceus observandus, 142 .

Picus rafflesii, 144.

Pjcus sondaicus, 146.

Picus squamicollis, 143 .

Picus strictus, 150.

Picus tiga, 148.

Picus (Tiga) intermedius, 149.

Picus (Tiga) labarum, 144.

Picus tristus, 146.

Picus tukki, 147.

Picus validus, 150 .

Picus variegatus, 145.

Picus viridanus, 141 .

Picus viridanus meridianus, 141 .

Picus viridanus weberi, 141 .

Picus vittatus, 140 .

Picus vittatus connectens, I41,

Pjcus vittatus limitans, 140 .

picaoides, Sibia, 228.

vileata, Alcedo, 103.

pileata, Halcyon, 103.

pileata, Napothera, 214.

pileata, Prinia, 225.

pileata, Sterna, 49.

pileata, Timalia, 208.

pileatus, Anous stolidus, 49.

Piprisoma, 272.

Piprisoma agile, 272.

Piprisoma agile bungurense, 272.

Piprisoma agile everetti, 272 .

Piprisoma agile sordidum, 272.

Pjprisoma modestum, 272.

Piprisoma modestum finschii, 272.

Pjprisoma modestum remotum, 272.

pirthoptera, Timalia, 223.

Pisorhina angelinae, 85 .

Pisorhina solokensis, 87.

Pisorhina umbra, 86.

Pisorhina vandewateri, 86 .

Fitta, 156.

Pitta arcuata, 159.

Pitta baudi, 560 .

Pitta bertae, 158 .

Pitta boschii, I50.

Pitta brachyura, 157.

Pitta brachyura cyanoptera, 157.

Pitta caerulea, 156.

Pitta caerulea hosei, r 57.

Pitta gigas, 156.

Pitta glaucina, 230.

Fitta granatina, I50.

Pitta granatina borneensis, 150 .

Pitta granatina coccinea, 159.

Pitta granatina vanheurni, 159.

Pitta guajana, 150.

Pitta guajana affinis, 150.

Pitta guajana irena, I 50.

Pitta guajana schwaneri, 159. 
Pitta gurneyi, 160.

Fitta malaccensis, 158 .

Pitta malaccensis, 157.

Pitta megarhyncha, 157.

I'itta moluccensis lepta, 137 .

Pitta nipalensis, 157.

Pitta nipalensis schneideri, I 57.

Pitta nompha, 158.

Pitta (Phaenicocichla) arquata, Ij

Pitta sordida, 158.

Pitta sordida bangkana, 158 .

Pitta sordida cucucllata, 158.

Pitta sordida javana, 158.

Pitta sordida mulleri, 158.

Pitta sordida sumatrana, 158 .

Pitta thoracica, 222

Pitta venusta, 158

Pitta venusta ussheri, 158.

Pittidae, 156.

Pityriasis, 258 .

Pityriasis gymnocephala, 25S.

Platalea, 52.

Platalea leucorodia, 52.

Platalea leucorodia regia, $\$ 3$.

Plataleidae, \$2.

platurus, Dicrurus, 306.

platurus, Dissemurus paradiseus 306.

Platylophus, 257

Platyrhynchos albicollis, 174.

platyrhynchos, Anas, $6 z$.

Platy rhychos perspicillatus, 175.

Platyrunchus ceylonensis, 180.

Platylophus galericulatus, 257 .

platylophus galericulatus ardesiacus, 257.

Platylophus galericulatus coronatus, 258.

Platylophus galericulatus lemprieri. 258.

Plegadidae, 51 .

Plegadis, 52 .

Plegadis falcinellus, 52 .

Plegadis falcinellus peregrinus, 57. pleoxantha, Arachnothera chrysogenys, 283 .

plesseni, Gerygone sulphurea, $17 \mathrm{I}$.

plexa, Motacilla, 285 .

plicatus, Buceros, 107.

plicatus, Rhyticeros, 107.

Ploceella, 203.

ploceella hypoxantha, 293.

Ploceidae, 280 .

Ploceus, 203.

Ploceus chrysea, 203.

Ploceus manyar, 204.

Ploceus passerinus infortunatus, 204.

Ploceus philjppinus, 203.

Ploceus philippinus infortunatus, 294. plotus, Pelecanus, 66.

Plotus rufus, 64

plotus, Sula leucogaster, 66.

plumbea, Gallinula, 28 .

1) lumbea, Gallicrex cinerea, 28 .

plumbeitarsus, Phylloscopus nitidus, 250.

plumosa, Muscipeta, 177.

plumosus, Pycnonotus, 200.

pluto, Copsychus saularis, 236 .

Pnoepyza, 23 L.

pnoepyga pusilla, 231.

Proepyga pusilla harterti, 231 .

Pnoepyga pusilla lepida, 23 .

Pnoepyga pusilla rufa, 231.

Podargidae, 05.

Podargus atritus, 95.

Podargus cornutus, 96.

Podargus javensis, 06.

Podargus parvulus, 96.

Podargus stellatus, 06 .

Podica personata, 29.

Podiceps, 50.

Podiceps ruficollis, 50 .

Podiceps ruficollis philippensis, 50.

Podiceps ruficollis vulcanorum, 50.

Podicipidae, 50 .

poicilophus, Picus, 146.

poioicephala, Alcippe, 218.

poliocephala, Stachyris, 221.

poioicephala, Thimalia, 2 I8.

poliocephala, Timalia, 221.

poliocephalus, Cuculus, 125.

poliogaster, Cyanoderma rufifrons, 225 .

poliogaster, Stachyris, 225.

poliogenis, Anuropsis malaccensis, 216.

poliogenis, Brachypteryx, 2 I6.

poliogenys, Olcyornis, i78.

poliolophus, Batrachostomus, 95.

Poliolophus nieuwenhuisii, 194.

Poljomvias, 168.

Poliomyias mugimaki, 168.

poliopsis, Accipiter badius, 7 I.

poliopsis, Brachypus, 702.

poliopsis, Micronisus, 7i.

poliopsis, Timalia, 224 .

polioptila, Dendrophassa vernans, 15.

Poliositta azurea nigriventer, 263 . polius, Muscadivores aeneus, 17. pollostus, Anthreptes malacensis, 270.

Polophilus sinensis, 129.

polychroa, Primia, 255.

polychrons, Malurus, 255.

polyclysta, Cinnyris ornata, 278 .

polyclysta, Leptocoma jugularis, 278 
Polyplectron, 7 .

Polyplectron bicalcaratum, 7 .

Polyplectron bicalcaratum malacensis, 8.

Polyplectron chalcurum, 8 .

Polyplectron hardwickii, 8 .

Polyplectron inopinatus, 8.

Polyplectron lineatum, 8.

Polyplectron schleiermacheri, 8.

Polyplectrum inocellatus, 8.

Pomatorhinus, 207.

Pomatorhisus bypoleucos, 207.

Pomatorhinus hypoleucos wrayi, 207.

Pomatorhinus montanus, 207.

Pomatorhinus montanus bornenșis, 207.

Pomatorhinus montanus occidentalis, 207.

Pomatorhinus montants ottolanderi, 207.

Pomatorhinus schisticeps, 207.

Pomatorhinus schisticeps fastidiosus, 207.

pompadora, Columba, 13.

pompadora, Treron, 13.

ponera, Hypothymis azurea, I74.

pontia, Mixornis bornensis, 226 .

pontia, Mixornis gularis, 226 .

pontius, Psittinus cyanurus, 94.

porphyrea, Columba, 16.

porphyreus, Ptilinopus, I6.

parjhyreus, Pycnonotus plumosus, 200.

Porphyrio, 28.

Porphyrio albus, 28 .

Porphyrio albus indicus, 29.

Porphyrio albus viridis, $2 \$$.

Porphyrio bemmeleni, 20.

Porphyrio cinereus, 26.

Porphyrio smaragdinus, 29 .

porphyrio, Tetrao, 4.

Porzana, 26.

Porzana bicolor, 27.

Porzana cinerea, 26.

Porzana fusca, 26.

Porzana fusca erythrothorax, 26.

Porzana pusilla, 26.

Porzana rufigenis, 26 .

praetermissa, Treron bicincta, I4.

praetermissa, Treron bisincta, 14.

prasina, Erythura, 202.

prasina, Loxia, 202.

prasiocara, Gracula javana, 297.

prasiocara, Gracula religiosa, 297.

pratincola, Glareola, 44.

pratincola, Hirundo, 44.

Pratincola rubicola stejnegeri, 233.

pravata, Cuculus, Iz6. prillwitzi, Arachnothera longirostra, $28 \mathrm{t}$.

prillwitzi, Mixornis flavicollis, 227.

prillwitzi, Pycnonotus simplex, $20 \%$

Prinia flaviventris, 255 .

Prinia, 254.

Prinia familiaris, 255.

Prinia familiaris olivacea, 255.

Prinia flaviventris halistona, 255.

Prinia flaviventris superciliaris, 255.

Prinia hypoxantha, 255.

Prinia inornata, 254.

Prinia inornata blythi, 255.

prinia, Orthotomus, 255 .

Prinia pileata, 225 .

Prinia polychroa, 255.

Prinia raftlesi, 255.

Prinia rufescens, 249.

Prionochilus everetti, 272.

Prionochilus maculatus septentrionalis, 271.

Prionochilus modestus, 272.

Prionochilus xanthopygius, 271 .

problematicus, Copsychus saularis, 235.

Procellaria leucomelas, 51.

Procellaria leucorhoa, $5 \mathrm{I}$.

Procellariidae, 51.

procera, Tchitrea, 177.

procera, Terpsiphone paradisi, 177.

prophata, Hypothymis azurea, 172. propinqua, Munia molucca, 292.

propinqua, Uroloncha, 292.

protegatus, Corvus splendeas, 308 . proxima, Alcedo meninting, 100.

proxima, Chalcostetha calcostetha, 273.

proximus, Microtarsus melanoleucos, 195 .

pryeri, Dicaeum, 268 .

Psaltria, 262.

Psaltria exilis, 262.

Psarisomus, 153.

Psarisomus dalhousiae, 153.

Psarisomus dalhousiae psittacinus, I54.

Psarisomus dalhousiae borneensis, 154.

Pseudibis, 52.

Pseudibis papillosa, 52.

Pseudibis papillosa davisoni, 52 .

Pseudogyps, 60.

Pseudogyps bengalensis, 69 .

Pseudominla, 2 ro.

Pseudominla castaneiceps, 219. 
Pseudominla castaneiceps soror, 210.

pseudo-ralloides, Buphus, 59.

Pseudornis dicruroides, 122.

Psilopogon, 140.

Psilopogon pyrolophus, 140.

Psilopus fuscus, 171.

Psittacidae, 92.

psittacinus, Eurylaimus, 154.

psittacinus, Psarisomus dalhousiae, 154.

Psittacula, 92.

Psittacula alexandri, 92.

Psittacula alexandri cala, 93.

Psittacula alexandri, dammermani, 93.

Psittacula alexandri, fasciata, 93.

Psittacula alexandri major, 93.

Psittacula alexandri perionca, 93.

Psittacula cyaneopileata, 95.

Psittacula longicauda, 93.

Psittacula longicauda defontainej, 93.

Psittacula longicauda modesta, 94.

Psittacula reticulata, 94 .

Psittacus alexandri, 92.

Psittacus barbatulatus, 93 .

Psittacus bimaculatus, 92 .

Psittacus cyanurus, 94.

Psittacus erubescens, 93.

Psittacus fasciatus, 93.

Psittacus galgulus, 95 .

Psittacus jncertus, 94.

Psittacus javanicus, 92.

Psittacus longicauda, 93.

Psittacus lucionensis, 92.

Psittacus macropterus, 94.

Psittacus malaccensis, 94.

Psittacus malaccensis, 93.

Psittacus ornatus, 9i.

Psittacus osbeckii, 92 .

Psittacus pumilus, 95.

Psittacus sulphureus, g2.

Psittinus, 94.

Psittinus cyanarus, 94.

Psittinus cyanurus abbotti, 94.

Psittinus cyanurus pontius, 94 .

Pteruthius, 220.

Pteruthius aenobarlous, 230.

Pteruthius aenobarbus tahanensis, 230.

Pteruthius flaviscapis, 220.

Pteruthius flaviscapis aeralatus, 230.

Pteruthius flaviscapis cameranoi, 230.

Pteruthius flaviscapis robinsoni, 230.

Ptilinopus, 16.
Ptilinopus albocinctus baliensis, 16.

Ptilinojus cinctus, 16 .

Ptilinopus cinctus albocinctus, 16 .

Ptilinopus erythrocephalus, 16.

Ptilinopus jambu, I6.

Ptilinopus melanocephalus, 16.

Ptilinopus melanocephalus bangueyensis, 17 .

Ptilinopus melanocephalus massopterus, 16.

Ptilinopus porphyreus, I6.

Ptilocichla, 216.

Ptilocichla leucogastra, $2 I$ I.

Ptilocichla leucogrammica, 216.

ptilorhyncus, Falco, 79.

ptilorhyncus, Pernis apivorus, 79.

ptilosus, Macronus, 227.

puella, Coracias, igr.

puella, Irena, $19 \mathrm{t}$.

Puffinus, 51.

Puffinus leucomelas, 5 I.

pugnax, Hemipodius, 9.

pugnax, Philomachus, 40.

pugnax, Tringa, 40 .

pulchelia, Dacelo, 102.

pulchella, Lacedo, 102.

pulchellus, Caprimulgus, 11 .

pulcher, Caprimulgus, III.

pulcherrima, Cyanops armillaris, 137.

pulcherrima, Megalaema, 137.

pulla, Stachyris poliocephala, 221 . pulonis, Meiglyptes tukki, 147.

pulverulenta, Treron pompadora,

13.

pulverulentus, Mulleripicus, 151 .

pulverulentus, Picus, $15 \mathrm{t}$.

pulverulentus, Trichophorus, 192.

pumilus, Centropus, 130.

pumilus, Psittacus, 95.

punctularia, Loxia, 20 r.

punctulata, Munia, 20 i.

puricea, Amandava amandava, 289-

punicea, Columba, 2o,

punicea, Fringilla, 289.

puniceus, Picus, 142.

purpurea, Ardea, 54.

purpurea, Columba, 15.

purpurea, Trerons vernans, 15.

purpureus, Centropus, 130.

purpureus, Phasianus, 5.

pusilla, Emberiza, 280 .

pusilla, pnoepyga, 231 .

pusilla, Porzana, 26.

pusilla, Sterna albifrons, 48 .

pusillus, Charadrius, 34 .

pusillus, Loriculus vernalis, 95. 
pusillus, Pycnonotus, 202.

pusillus, Rallus, 26.

pyca, Malacocincla, 210 .

Pycnonotidae, 188 .

Pycnonotus, 199.

pyronotus, Alectrophasis, 6 .

Pycnonotus aurigaster, 204.

Pycnonotus aurigaster martini. $204:$

Pycnonotus bimaculatus, 190.

Pycnonotus bimaculatus barat, 199.

Pycnonotus bimaculatus snouckaerti, 100.

Pycnonotus blanfordi, 200 .

Pycnonotus blanfordi robinsoni, 200.

Pycmonotus brunneus, 201.

Pycnonotus brunneus zaphaeus, 20 t.

Pycnonotus brunneus zapolius, 202.

Pycnonotus crocorrhous, 204.

Pycnonotus cyaniventris, 202.

Pycnonotus cyaniventris, 202.

Pycnonotus cyaniventris parotical. is, 202.

Pycnonotus dispar, 203.

Pycnonotus erythropthalmos, 202.

Pycnonolus erythorpthalmos cyanochrus, 202.

Pycnonotus erythropthalmos isus, 202.

Pycnonotus erythrojthalmos pammicrus, 202.

Pycnonotus erythropthalmos salva. dorii, 202.

Pycnonotus finlaysoni, I00.

Pycnonotus Aariventris, 203.

Pycnonotus flaviventris minor, 203.

Pycnonotus flaviventris montis, 203.

Pycnonotus goiavier, 199.

Pycnonotus goiavier gourdinil, 200.

Pycnonotus goiavier analis, rgo.

Pycnonotus goiavier personatus, 199.

Pycnonotus humeraloides, 184 .

Pycnonotus jocosus, 204.

Pycnonotus jocosus erythrotis, 204.

Pycnonotus leucogrammicus, I08.

Pycnonotus leucolss, 200.

Pycnonotus olivaceus chloeodis, 20 .

Pycnonotus plumosus, 200.

Pycnonotus plumosus billitonis, 200.

Pycnonotus plumosus chiroplethis, $20 \mathrm{I}$.
Pycnonotus plumosus insularis, 200.

Pycnonotus plumosus porphyreus, 200 .

Pycnonotus pusillus, 202.

Pycnonotus simplex, zor.

Pycnonotus simplex axanthizus, $20 \mathrm{t}$.

Pycnonotus simplex halizonus, $20 \mathrm{I}$,

Pycnonotus simplex perplexus, zol.

Pycnonotus simplex prillwitzi, 201.

Pycnonotus squamatus, 203.

Pycnonotus squamatus webberi, 203.

Pycnonotus tympanistrigus, 203.

Pycnosphtys grammiceps, 252.

pyrhoptera, Drymophila, I77.

pyrhojtera, Muscicapa, 177.

pyrolophus, Psilopogon, 140.

pyronotus, Houppifer ervthrophthalmus, 6.

Pyrotrogon fasciatus impavidus, 120.

Pyrotrogon kasumba usa, 120.

Pyrotrogon neglectus, i 19.

Pytotrogon oreskios uniformis, 121.

Pyrotrogon orrophaeus, 121 .

pyrthogenys, Aethostoma, 212.

pyrrhogenys, Myjothera, 212.

phrrhonota, Myiolestes, 238.

phrrhonota, Oenanthe, 233.

pyrrhonota, Saxicola caprata, 233.

pyrrhonotus, Lanius, 259.

phrrhoptera, Alcippe poioicephala, 219.

pyrrhoptera, Napothera, 210.

pyrrhotis, Blythipicus, 146 .

Pyrrhula, 288.

Pyrrbula nipalensis, 288 .

Pyrrhula nipalensis waterstradti, 288.

pyrrophaea, Cyanoderma erythroptera, 223.

pyrrophaea, Timalia, 223.

pyrropyga, Trichixos, $23 \mathrm{~S}$.

quadricolor, Bucco, 135 .

quadricolor, Emberiza, 291.

quadristrigatus, Ralius, 26.

querquedula, Anas, 6z.

querulus, Cacomantis merulinus, 126.

quinticolor, Merops leschenaulti, I00. 


\section{INDEX}

radiatus, Carpococcyx, I33.

radiceus, Calobates, 133.

rafflesi, Burnesia flaviventris, 255.

raftiesi, Prinia, 255.

rafflesia, Calyptomina, 153.

raftlesii, Bucco, 135 .

raftlesii, Chloropicoides, 144.

rafflesii, Chotorea, 135.

raftlesii, Eurylaemus, I55.

rafflesii, Picus, 144.

raja, Spilornis, 75 .

Rallidae, 24.

Rallina telmatophila, 25 .

Rallina zonaventris, 26

ralloides, Ardea, 50.

ralloides, Ardeola, 59 .

Rallus benghalensis, 31 .

Rallus eurizonoides, 25 .

Rallus eurizonoides nigrolineatus, 25 .

Rallus fasciatus, 25 .

Rallus fuscus, $z 6$.

Rallus paykullit, 26.

Rallus philippensis, 25

Rallus philippensis andrewsi, 25.

Rallus pusillus, 26.

Rallus quadristrigatus, 26.

Rallus rubiginosus, 26 .

Rallus ruficeps, 25.

Rallus striata reliquus, 25 .

Rallus striatus, 24 .

Rallus striatus gularis, 24 .

Rallus striatus jouyi, 25.

Rallus superciliaris, 25.

Ramphalcyon, 97.

Ramphalcyon arnauroptera, 97.

Ramphalcyon capensis, 07 .

Ramphalcyon capensis arignota, 98.

Ramphalcyon capensis cyanoptery, 08.

Ramphalcyon capensis floresiana, 08.

Ramphalcyon capensis hydrophila, 96.

Ramphalcyon capensis innominata, 99.

Ramphalcyon capensis isoptera, 98.

Ramphalcyor capensis javana, 00 .

Ramphalcyon capensis malaccensis, 08 .

Ramphalcyon capensis nesoeca, 98 .

Ramphalcyon capensis simalurensis, 08 .

Ramphalcyon capensis sodalis, 98 raveni, Dinopium javanense, I 40 . reclusus, Macronus ptilosus, 227. rectirostris, Ardea cinerea, 55. rectunguis, Centropus, 120. regia, Platalea leucorodia, 53.

Regulus inornatus, 251 .

reichenowi, Sasia ochracea, 152.

reinwardt, Megapodius, I.

reinwardtii, Harpactes, 121 ,

reinwardtii, 'Trogon, iz1.

religiosa, Gracula, 290.

reliqua, Hypotaenidia striata, 25.

relicuus, Rallus striata, 25.

remifer, Bhringa, 306.

remifer, Edolius, 306.

remifer, Piprisoma modestum, 272.

reticulata, Psittacula, 94.

rhadinorhynchus,
chalybeus, 208.

Rhamphococcs curvirostris singularis, 133 .

Rhamphococcyx erythrognathus borneensis, 132 .

Rheinartia, 0 .

Rheinartia ocellata, 0 .

Rheinartia ocellata nigrescens, 9 .

rhinoceroides, Buceros, 105 .

rhinocerus, Buceros, 105.

Rhinocichla, 206 .

Rhinocichla mitrata, 206.

Rhinocichla mitrata damnata, 206.

Rhinocichla mitrata major, 206.

Rhinocichla mitrata treacheri, 200 . Rhinomyias, 178 .

Rhinomyias brunneata, 178

Rhinomyias gutaris, 179 .

Rhinomyias olivacea, 178 .

Rhinomyias olivacea perolivacea, $1>8$.

Rhinomyias pectoralis baliensis, 178.

Rhinomyias ruficauda isola, 179.

Rhinomyias ruficrissa, 170 .

Rhinomyias ruficrissa isola, 179

Rhinomyias tardus, 178 .

Rhinomyias umbratilis, 179 .

Rhinomyias umbratilis eclinsis, 170.

Rhinomyias umbratilis richmondi, I 79.

Rhingylax, zo8.

Rhinoplax vigil, tos.

Rhinortha, 132.

Rhinortha chlorophaea, I 32.

Rhinotha chlorophaea fuscigularis, 132.

Rhjpidura, 174 .

Rhipidura albicollis, 174.

Rhipidura albicollis atrata, 174

Rhipidura guttata, 175 -

Rhipidura javanica, 175 .

Rhipidura javanica longicauda, 175.

Rhipidura perlata, 175. 
Rhipidura perlata euryura, 175 . Rhipidura phoenicura, 175.

Rhipidura schlegeli, 174

Rhipidura vidua, I74.

rhizophorae, Cyornis rufigastra, 166.

Rhizothera, 1.

Rhizothera longirostris, $t$.

Rhizothera longirostris dulitensis, 2

rhodolaema, Anthreptes, 280.

rhombifer, Leucocerca, 175.

Rhopodytes, 13 I

Rhopodytes diardi, 131.

Rhopodytes diardi borneensis, I31.

Rhopodytes sumatranus, 131.

Rhopodytes tristis, $13 \mathrm{I}$.

Rhopodytes tristis elongatus, 131.

Rhopodytes tristis kangeangensis, 131.

Rhopodytes tristis longicaudatus, 131.

Rhynchaea orientalis, 31 .

Rhyticeros, 107.

Rhyticeros plicatus, 107.

Rhyticeros plicatus subruficollis, to7.

Rhyticeros undulatus, 108.

richardi, Anthus, 286 .

richmondena, Haemataena, 16

richmondi, Aplonis panayensis, 208.

richmondi, Hypothymis azurea, 174.

richmondi, Lamprocorax panayensis, 208.

richmondi, Oriolus chinensis, 300 .

richmondi, Oriolus maculatus, 300 .

richmondi, Rhinomyias umbratilis, 179.

richmondi, Spilornis cheela, 75 .

ridibundus, Larus, 50.

Riparia, 162.

riparia, Hirundo, 162.

riparia, Riparia, 162.

Riparia ripatia, 162 .

Riparia riparia ijimae, 162.

Rimator, 218.

Rimator malacoptilus, $21 \mathrm{~S}$.

Rimator malacoptilus albostriatus, 218.

robinsoni, Collocalia, lowi, II5.

robinsoni, Cyanops duvaceli, 138 .

robinsoni, Gallus gallus, 7 .

robinsoni, Gecinulus grantia, 144.

robinsoni, Gecinulus viridis, 144 .

robinsoni, Gecinus, 141.

robinsoni, Myiophoneus, 239

robinsoni, Myophonus, 239.

robinsoni, Picus canus, I 41 . robinsoni, Pteruthius flaviscapis, 230.

robinsoni, Pycnonotus blanfordi, 200 .

robinsoni, Sphenocercus, ir. robinsoni, Sphenurus sphenurus, t $t$.

robusta, Arachnothera, 283.

robusta, Ardea, 55.

robusta, Ceyx rufidorsus, 101.

robusta, Gracula religiosa, 297.

rodgeri, Gecinus, 141 .

rodgeri, Picus chlorolophus, I4I, rolli, Arboricola, 2.

rolli, Arborophila brunneopectus, 2.

Rollulus, 4.

Rollulus roulroul, 4.

rosacea, Columba, i 8 .

rosacea, Ducula, 18.

rosaceicollis, Capito, 140.

rosea, Muscicapa, 187.

rosea, Xantholaema haemacephala, I 40 ,

roseicollis, Columba, 16 .

roseus, Bucco, 140.

roseus, Pelecanus, 60.

roseus, Pericrocotus, 187.

Rostratula, 3 r.

Rostratula benghalensis, 31 .

Rostratulidae, 31.

rostratum, Aethosoma, 211.

rostratum, Trichastoma, 21 I.

rothschildi, Arachnothera longirostra, $28 \mathrm{r}$.

Tothschildi, Leucopsar, 296.

rothschildi, Serilophus lunatus, 154 .

roulroul, Phasianus, 4.

roulroul, Geocichla, 242.

rubecula, Geokichla citrina, 242.

rubeculoides, Cyornis, 165.

rubeculoides, Phaenicura, 165.

ruber, Cinnyris, 276.

rbescen5, Dicaeum, 267.

rubescens, Locustella, 244.

rubicauda, Napothera, 213 .

rubicundulus, Orthotomus ruficeps, 246.

rubicundulus, Orthotomus sericeus, 246.

rubiginosus, Blythipicus, 146.

rubiginosus, Hemicircus, I 46.

rubiginosus, Rallus, 26.

Rubigula montis, 203 .

rubirostris, Coccyzus, 130.

rubricauda, Phaethon, 67.

rubripes, Sula sula, 66.

rubrirostris, Arborophila, 3. 
INDEX.

rubrirostris, Peloperdix, 3.

rubro-cana, Nectarinia, 267.

rubrocostm, Dicaeura, 267.

rubropectus, Erythrolane, 301.

rubropygium, Dicaeum trigonos. tigma, 269.

rubropygium, Dicaeum trigonostigmum, 260 .

ruecki, Cyornis, 164.

rufa, Anhinga, 64.

rufa, Cyanoderma erythroptera, 224 .

Iufa, Lophura, 6.

rufa, Pnoepyga pusilla, 23I.

rufa, 'Tiga, 148 .

rufescens, Franklinia, 249.

rufescens, Otus, 87 .

rufescens, Phragamaticola aedon, 249.

rufescens, Prinia, 240.

rufescens, Strix, 87.

ruficapilla, Timalia, 214 .

ruficapillus, Enicurus, 234.

ruficauda, Anadoenus, 132.

ruficeps, Columba, $2 I$.

ruficeps, Edela, 247 .

ruficeps, Macropygia, 21 .

ruficeps, Pellorneum, zo8.

ruficeps, Rallus, 25 .

ruficeps, Orthotomus sepium, 247 .

ruficollis, Colymbus, 50 .

ruficollis, Erolia, 40.

ruficollis, Pernis apivorus, 79 .

ruficollis, Podiceps, jo.

ruficollis, Trynga, 40 .

ruficoma, Mixornis bornensis, 227.

ruficoma, Mixornis gularis, 227 .

ruficrissa, Rhinomyias, 179 .

ruficrissa, Stoporala indigo, 130.

ruficrissus, Criniger gutturalis, 196 ;

rufidorsa, Cevx, ror.

rufidorsalis, Henicurus, 234.

rufidorsus, Ceyx, 10I.

rufifrons, Cyanoderma, 225 .

rufifrons, Cyornis caerulata, 167.

rufifrons, Garrulax, 205.

rufifrons, Malacopteron cinereum, 214.

ruffitons, Stachyris, 225.

rufigaster, Dendrocitta, 309.

rufigastra, Cyornis, 165.

rufigastra, Muscicapa, 165.

rufigenis, Porzana, 26.

rufigula, Muscicapa, 168.

rufipectus, Turdinus macrodactylus, 213.

rufiventris, Malacocincla sepiaria, $21 \mathrm{I}$.

rufocaudatus, Pyenonotus, 197 rufogularis, Anthus, 287.

rufotibialis, Accipiter, 72 .

rufo-vittatus, Cuculus, 126.

rufulus, Gampsorhynchus, 208.

rufulus Garrulus, 258.

rufulus, Turdus, 243.

rufus, Phasianus, 6,

rufus, Plotus, 64.

rugosus, Buceros, 107.

rupatensis, Cyornis elegans, 165.

rupatensis, Cyornis turcosa, 165.

rustica, Hirundo, 160.

rusticota, Scolopax, 37.

rutilus, Trogon, 121 .

saba, Chrysophlegma mentale, 143. saba, Malacopteron magnum, 244 .

sacculatus, Criniger gutturalis, 106.

sacculatus, Criniger ochraceus, 106.

sacra, Ardea, 56.

sacra, Demigretta, 56.

sagittatus, Ephialtes, 87.

\$agittatus, Otus, 87

sakaiorum, Abrornis, 253.

sakhalina, Erolia alpina, $4 \mathrm{t}$.

sakhalina, Scolopax, $4 \mathrm{I}$.

salaccensis, Brachypteryx, 234.

salangana, Hemiprocne, 15.

Salangana vestita, 115.

salangensis, Dicrurus leucogenys, 303.

Salicaria turdina orientalis, 245 .

\$alvadorii, Caprimulgus, 112 .

salvadorii, Gerygone, 171.

salvadorii, Pycnonotus erythropthal mos, 202.

salvadorii, Spilomis cheela, 76 .

salvadorii, Tanygnathus, 92 .

salvadorii, Zosterops palpebrosa, 264.

sancta, Halcyon, 103.

sanctus, Halcyon, to3.

sanguineus, Charadrius, 33.

sanguiniceps, Haematortyx, 5 .

sanguinolentum, Dicaeum, 268 .

sanguinolentus, Caloramphus, 134 .

sanguinolentus, Ocypterus, $30 \mathrm{r}$.

saravacense, Philentoma, i78.

sarawacensis, Pachycephala hypoxantha, 261

sarawacensis, Parus, 262.

Sasia, 152.

Sasia abnormis, 152 .

Sasia abnormis magnirostris, 152.

Sasia evertti, I5z. 
Sasiz ochracea, I 52.

Sasia ochracea reichenowi, I52. saturata, Aethopyga, 273.

saturata, Anuropsis malaccensis, 216.

saturata, Brachypteryx montana, 231 .

saturata, Cinnyris, 273.

saturata, Scolopax, 37.

saturata, Upupa epops, 109.

saturatior, Gampsorhynchus rufulus, 208

saturatior, Olcyornis, I78.

saturatior, Sitta frontalis, 262.

saturatior, Tchitrea affinis, 176.

saturatior, Terpsiphone paradisi, 176.

saturatus, Cuculus, 125.

saturatus, Falco tinnunculus, 82.

saturatus, Pericrocotus cinaamomeus, 187 .

saturatus, Pericrocotus peregrinus, 187.

saularis, Copsychus, 235.

saularis Gracula, 235.

saundersi, Sterna albifrons, 48 .

Sauropat is chloris amphiryta, 104.

Sauropatis chloris azela, 104.

Sauropatis chloris chloroptera, 104.

Sauropatis chloris cyanescens, 104.

Sauropatis chloris palmeri, 104.

Saxicola, 233 .

Saxicola caprata, 233.

Saxicola caprata pyrrhonota, 233.

Saxicola dumetoria, 170.

Saxicola fruticola, 23.3 .

Saxicola torquata, 233 .

Saxicola torquata stejnegeri, 233.

scapularis, Aegithina tiphia, I\&0.

scapularis, Iora, 180 .

scapulatus, Lanius, 257.

schach, Lanius, 250 .

schierbrandii, Volvocivora, 184

schistaceus, Enicurus, 234.

schisticeps, Lalage nigra, 185.

schisticeps, Pomatorhinus, 207.

schistochlamys, Garrulax palliatus, 205.

schlegeli, Rhipidura, 174.

schleiermacheri, Polyplectron, 8. schneideri, Pitta nipalensis, 157.

scliwaneri, Abrornis, 253.

schwaneri, Abroscopus superciliaris, 253 .

schwaneri, Enneoctorus, 259.

schwaneri, Malacocincla, 2 ro.

schwaneri, Pitta guajana, 159.

Schwaneria coerulata, 167.

scolopaceus, Eudynamys, 128.

scolopaceus, Cuculus, 128.
Scolopacidae, 35 .

Scolojax, 37.

Scolopax arquata, 35 .

Scolopax cinerea, 42 .

Scolopax falcinellus, 40 .

Scolopax gallinago, 38 .

Scolopax grisea, 38 .

Scolopax incuna, 42 .

Scolopax lapponica, 37 .

Scolopax limosa, 36 .

Scolopax madagascariensis, 36 .

Scolopax nebularia, 43 .

Scolopax pectinicauda, 38 .

Scolopax phaeopus, 36 .

Scolopax rusticola, 37.

Scolopax sakhalina, 4I.

Scolopax saturata, 37 .

Scolopax stenura, 38 .

Scolopax sumatrana, 42 .

Scolopax testacea, 41 .

Scolopax totanus, 42.

scommophorus, Esacus magnirostris, $3 \mathrm{I}$.

scommophorus,

magnirostris, 3I.

Scops brookii, 87 .

Scops javanicus, 86.

Scops lucize, 85 .

Scops malayanus, 85 .

Scops mantananensis, 85 .

Scops mantis, 87.

scops, Otus, 84 .

scops, Strix, 84 .

scutatus, Buceros, 108.

scutulata, Anas, 6 r.

scutulata, Asarcornis, $6 \mathrm{r}$.

scutulata, Ninox, 87.

scutulata, Strix, $\$ 7$.

secedens, Pachycephala cinerea, 260.

secedens, Pachycephala grisola, 260.

secbohmi, Charadrius alexandrius, 35.

seebhomi, Merula, 241.

seebohmi, Turdus javañicus, 24 I.

Seicercus, 251.

Seicercus castaniceps, 252.

Seicercus castaniceps butleri, 252.

Seicercus castaniceps muelleri, 252.

Seicercus castaniceps youngi, 252 .

Seicercus grammiceps, 252.

Seicercus grammiceps sumatrensis, 252.

Seicecus montis, 252 .

Seicercus montis davisoni, 252.

Seicercus montis inornata, 253 .

Seicercus trivirgatus, 25 I.

Seicercus trivirgatus kinabaluensis, 251. 
Seicercus trivirgata parvirostris, 251 .

seimundi, Sphenocercus, 10.

seimundi, Sphenurus, 10.

seloputo, Strix orientalis, 89 .

semipalmatus, Limnodromus grjseus, 38 .

semipalmatus, Macrorhamphus, 38 . semitorquatus, Sphenurus, Io.

sepiaria, Brachypteryx, 210.

sepiaria, Cettia montana, 253

sepiarja, Malacocincla, 210.

sepium, Orthotomus, 246 .

septentrionalis, Anaimos maculatus, 271.

septentrionalis, Chloropsis cyanopogon, 101.

septentrionalis, Prionochilus maculatus, $27 \mathrm{I}$.

sepulcralis, Cacomantis variolosus, 127.

sepulcralis, Cuculus, Iz7.

sericea, Tricholestes criniger, I98.

sericeus, Orthotomus, 246.

Serilophus, 154 .

Serilophus lunatus, I 54.

Serilophus lunatus intensus, 154.

Serilophus lunatus rothschildi, I 54 .

Serilophus lunatus stolidus, I 54 .

Serinus, 288 .

Serinus esthrae, 288 .

Setaria albigularis, 2t5.

Setaria albigularis leucogastra, 216.

Setornis, I07.

Setormis criniger, 107.

severus, Falco, 8. .

sharpii, Grus antigone, 29.

siakensis, Dissemurus paradiseus, 306.

siamensis, Dicaeum cruentatum, 268.

siamensis, Otus luciae, 85 .

siamensis, Otus spilocephalus, 85.

seberu, Calyptomena viridis, 153 .

siberu, Dicrurus leucogenis, 304.

siberu, Dicrurus leucophaeus, 304.

siberu, Oriolus chinensis, 300 .

Sibia picaoides, 228.

Sibia wrayi, 228 .

sibirica, Geokichla, 241.

sibirica, Hemichelidon, $16 z$.

sibirica, Limicala falcinellus, 40.

sibiricus, Turdus, 241 .

sibiru, Cbalcostetha cålcostetha, 273.

sibririca, Muscicapa, 162.

siebersi, Acrocephalus stentoreus, 245 .

siebersi, Pericrocotus flammeus, I 86. siebersi, Pericrocotus speciosus, 186.

silvestris, Buceros rhinoceros, 105. simalurensis, Coracina sumatrensis, 182.

simalurensis, Graucalus, 182 .

simalurensis Macropygia ruficeps, 27.

simalurensis, Munia maja, 201.

simalurensis, Pelargopsis, 98.

simalurensis, Ramphalcyon capensis, 98 .

similis, Mixorais, 226 .

simillima, Arachnothera, 284.

simillima, Heterophasia picaoides, 228 .

simillima, Motacilla flava, 285 .

simplex, Anthreptes, 278.

simplex, Nectarinia, 278.

simplex, Pycnonotus, 201 .

simplex, Zosterops, 263.

simplicior, Anthrejtes simplex, 278 . sindorensis, Zosterops chlorates. 266.

sindorensis, Zosterops palpebrosa, 266.

sinensis, Anthus novaeseelandiae, 286.

sinensis, Ardea, 60.

sinensis, Centropus, 120.

sinensis, Corydalla, 286.

sinensis, Ixobrychus, 60

sinensis, Munia atricapilla, 200

sinensis, Oriolus, 294.

sinensis, Pelecanus, 63.

sinemsis, Phalacrocorax carbo, 63 .

sinensis, Polophilus, 120.

sinensis, Sterna albifrons, 48 .

sinensis, Sturnia, 204.

singalensi5, Chalcoparia, 280.

singalensis, Motacilla, 280.

singaøurensis. Aegithina tiphia, 188.

singularis, Rhamphococcyx curvirostris, 133.

siparaja, Aethopyga, 275.

siparaja, Certhia, 275.

Siphia, 163.

Siphia albo-olivacea, 170 .

Siphia beccariana, 165.

Siphia brunneata, 178 .

Siphia elopurensis, 170.

Siphia erythaca, 168 .

Siphia everetti, 164 .

Siphia hyperythra, I6o.

Siphia abscura, 163 .

Siphia parva, 163.

Siphia parva albicilla, 163 .

Siphia styani, 172 .

Siphia sumatrensis, 166.

Siphia vordermani, 170. 
sipora, Butorides striatus, 58. sipora, Hypothymis azurea, 174. sipora, Oriolus chinensis, 300 , sipora, Spilornis cheela, 76. sipora, Stjilornis elgini, 76. sirensis, Dicruropsis pectoralis, 305 . sirensis, Dicrurus hottentottus, 305. sirensis, Malacocincla abbotti, 210.

Sitta, 262.

Sitta azurea, 263 .

Sitta azurea expectata, 263.

Sitta azurea nigriventer, 263 .

Sitta frontalis, 262 .

Sitta frontalis, 262.

Sitta frontalis corallipes, 262.

Sitta frontalis hageni, 262 .

Sitta frontalis saturatior, 262 .

Sitta longirostra, 262.

Sitta velata, 262 .

Sittidae, 262

Siva, 228 .

Siva cyanouroptera, 220.

Siva cyanouroptera sordidior, 220.

Siva nipalensis, 218.

Siva strigula, 228 .

Siva strigula malayana, 228 .

Siva strigula omissa, 228 .

slamatensis, Garrulax rufifrons, 205.

samaragdinus, Porphyrio, 20 .

smicra, Treron curvirostra, 12.

smyrnensis, Alcedo, 103.

smyrnensis, Halcyon, 503 .

snouckaerti, Prcnonotus bimaculatus, 100 .

sodalis, Pelargopsis, 08.

sodalis, Ramphalcyon capensis, 08 . solitaria, Alcippe, 2 Io.

solitaria, Monticola, 243.

solitaria, Anthipes, 160 .

solitaris, Muscicapa, 169.

solitarius, Turdus, 243.

sollicitans, Dicaeum concolor, 270.

soloensis, Accipiter, 7I.

soloensis, Falco, $7 \mathrm{I}$.

solokensis, Otus brookii, 87

solokensis, Pisorhina, 87.

solombensis, Zosterops cbloris, 266

solombensis, Dicruropsis pectoralis, 305.

solombensis Dicrurus hottentottus. 305.

sondaica, Muscicapella hodgsoni, ז 68.

sondaica, Nitidula hodgsoni, 168.

sondaicus, Picus, r 46.

sonnerati, Chloropsis, igo.

sonneratii, Cuculus, 125.

sonneratii. Penthoceryx, 125.

sordida, Cyanoderma, 223

sordida, Pitta, 158. sordidior, Siva cyanouroptera, 220. sordidum, Piprisoma agile, 272.

sordidus, Anuropsis malaccensis. 216.

sordidus, Criniger, I95.

sordidus, Dendropicus, 150.

sordidus, Hemicircus concretus, 150.

sordidus, Turdus, 158 .

soror, Minla, 210.

soror, Pseudominla castaneiceps. 210.

spadicea, Coturnix, 7.

sparpei, Ceyx, ror.

sparsimstriata, Locustella, 244.

sparverioides, Cuculus, I23.

sparverioides, Hierococcyx, I23.

Spatula, 63.

Spatuta clypeata, 63

speciosa, Ardea, 50 .

speciosa, Ardeola ralloides, 59.

speciosa, Motacilla, 233 .

sperata, Certbia, 277

sperata, Leptocoma brasiliana, $\mathbf{2 7 7}$.

Spermestes fuscans, 292.

sphecura, Fringilla, 292.

Sphenocercus etorques, i r.

Sphenocercus korthalsi, I r.

Sphenocercus robinsoni, II.

Sphenocercus seimundi, 10.

sphenura, Fringilla, 292.

Sphenurus, 10.

Sphenurus oxyurus, 10.

Sphenurus seimundi, 10.

Sphenurus semitorquatus, 10.

Sphenurus 5phenurus, it.

sphenurus, Sphenurus, I1.

Sphenurus sphenurus korthalsi, II.

Sphenurus sphenurus robinsoni, $\mathbf{t}$. sphenura, Vinago, I1.

spilocephalus, Ephialtes, 85.

spilocephalus, Otus, 85 .

spilonotus, Citcus, 70

Spilornis, 75 .

Spilornis cheela, 75 .

Spilornis cheela abbotti, 76 .

Spilornis cheela bassus, 75.

Spilornis cheela baweanus, 75 .

Spilornis cheela bido, 75 .

Spilornis cheela kinabaluensis, 75.

Spilornis cheela malayensis, 75 .

Spilornis cheela natunensis, 76 .

Spilornis cheela richmondi, 75 .

Spilornis cheela salvadorii, 76 .

Spilornis cheela sipora, 76 .

Spilornis elgini sipora, 76 .

Spilornis pallidus, 75 .

Spilornis raja, 75 .

spinosus, Charadrius, 3I.

spinosus, Hoplopterus, $3 x$.

spinosus, Megalorhynchus, 134. 
Spizaetus, 73.

Spizaetus borneonensis, 74 .

Spizaetus cirrbatus, 73 .

Spizaetus cirrhatus limnaeetus, 74 .

Spizaetus nanus, 74 .

Spizaetus nipalensis, 74 .

Spizaetus nipalensis alboniger, 74 .

Spizaetus nipalensis bartelsi, 74 .

splendens, Corvus, 308.

squamatus, Pycnonotus, 203.

squamatum, Malacopteron, 222.

squamatus, Turdus, 203.

squameiceps, Tribura, 254.

squameiceps, Urosphena, 254.

squamicollis, Picus, 143.

squamifrons, Timalia, 214.

squamif rons, Zosterops, 267.

squamigularis, Micropternus brachyurus, 148.

Squatarola, 32.

Squatarola helvetica australis, 32 .

Squatarola squatarola australis, 32. *

Squatarola squatarola, 32 .

squatarola, Tringa, 32 .

Stachyris, 220.

Stachyris bocagei, 223 .

Staphida castaniceps, 228.

Stachyris chrysaea, 223.

Stachyris chrysaea chrysops, 223.

Stachyris chrysaea frigida, 223.

Stachyris grammiceps, $22 \mathrm{~J}$.

Stachyris larvata vermiculata, 220.

Stachyris leucotis, 22I.

Stachyris leucotis goodsoni, 221.

Stachyris maculata, 222.

Stachyris maculata banjakensis, 222.

Stachyris maculata hypopyrtha, 222.

Stachyris maculata pectoralis, 222.

Stachyris melanothorax intermedia, 225.

Stachytis natunonsis, 220 .

Stachyris nigriceps, 220.

Stachyris nigriceps borneensis, 220.

Stachyris nigriceps davisoni, 220 .

Stachyris nigriceps dipora, 220.

Stachyris nigriceps bartleyi, 220 ,

Stachyris nigriceps Iarvata, 220.

Stachyris nigriceps tionis, 220.

Stachyris nigricollis, 221.

Stachyris nigricollis erythronotus, 227.

Stachyris poliocephala, 221 .

Stachyris poliocephala diluta, $22 \mathrm{I}$.

Stachyris poliocephali pulla, $22 \mathrm{i}$.

Stachyris poliogaster, 225.

Stachyris rufifrons, 225 .

Stachyris striolata, 222.
Stachyris striolata guttata, 222 .

Stachyris striolata umbrosa, 222.

Stachyris thoracica, 222.

Stachyris thoracica orientalis, 223.

stagnatilis, Totanus, 42 .

stagnatilis, Tringa, 42 .

Staphida, 228

Staphida castaniceps everetti, 228 .

Stasiasticus montis, 249.

stejnegeri, Pratincola rubicola, 233.

stejnegeri, Saxicola torquata, 233.

stellaris, Ardea, 6I.

stellaris, Botaurus, 6r.

stellatus, Batrachostomus, $q 6$.

stellatus, Podargus, 96.

stentorea, Curruca, 244.

stentoreus, Acrocephalus, 244.

stenura, Capella, 38 .

stenura, Scolopax, 38 .

Sterma, 45 .

Sterna affinis, 45 .

Sterna alba, 40 .

Sterna albifrons, 48 .

Sterna albifrons pusilla, 48 .

Sterna albifrons saundersi, 48 .

Sterna albifrons sinensis, 48.

Sterna anaethetus, 47.

Sterna anaethetus antarctica, 47.

Sterna bengalensis, 46 .

Sterna bergii, 46.

Sterna bergii cristata, 47.

Sterna bergit edwardsi, 46 .

Sterna bergii halodrama, 47 .

Sterna cristatus, 47.

Sterna dougallii, 46 .

Sterna dougallii bangsi, 46 .

Sterna fuscata, 47.

Sterna fuscata nubilosa, 47.

Sterna grisea, 45 .

Sterna hirundo, 45.

Sterna hirundo longpennis, 45 .

Sterna hybrida, 45 .

Sterna infuscata, 47 .

Sterna javanica, 45 .

Sterna leucoptera, 44.

Sterna media, 46.

Sterna nilotica, 45 .

Sterna pileata, 49 .

Sterna sto]ida, 49.

Sterna sumatrana, 48.

stictopterus, Batrachostomus, 96.

stigmatops, Buchanga, 303.

stigmatops, Dicrurus leucophaeus, 303.

stolida, Sterna, 40.

stolidus, Anous, 49.

stolidus, Serilophus lunatus, I 54.

Stoparola cerviniventris, $18 \mathrm{I}$

Stoparola indigo ruficrissa, 180 .

Stoporala, I 80 .

Stoporala indigo, 180. 
Stoporala indigo cerviniventris, $18 \mathrm{t}$.

Stoporala indigo ruficrissa, I80.

Stoporala thalassina, $18 \mathrm{r}$.

Stoporala thalassina thalassoides, ist.

stormi, Dissoura, 53.

stormi, Melanopelargus episcopus, 53 .

strepizans, Huhua sumatrana, 84 .

strepitans, Strix, 84.

Streptopelia, 22 .

Streptopelia bitorquata, 22.

Streptopelia bitorquata dusumieri, 22.

Streptopelia chinensis, 22

Streptopelia chinensis minor, 23.

Streptopelia chinensis tigrina, 23.

stresemanni, Athenoptera spilocephalus, 85.

stresemanni, Dicaeum, 267.

Stresemanni, Otus spilocephalus, 85.

striata, Ardea, 58.

striata, Columba, 23.

striata, Geopelia, 23.

striata, Kenopia, 227.

striata, Loxis, 201.

striata, Munia, 291.

striata, Timlia, 227 .

striatus, Butorides, 58 .

striatus, Cuculus, 124.

striatus, Rallus, 24.

stricklandii, Copsychus, 238

stricklandif, Kittacincla, 238 .

strictus, Chrysocolaptes lucidus, 150.

strictus, Picus, 150.

striga, Ceblephyris, 184.

striga, Turdus, 205.

strigatus, Aplonis panayensis, 208.

strigatus, Lanius, 258.

strigatus, Turdus, 208 .

Strigidae, 83 .

strigula, Siva, 228.

striolata, Hirundo daurica, 161.

striolata, Stachyris, 222.

striolata, Timalia, 222.

striolatus, Anthus campestri5, 287.

striolatus, Trichophorus, 102.

Strix, 88

Strix alba, go.

Strix badia, 91.

Strix castanoptera, 90

Strix coromanda, 84 .

Strix flammea, $8_{3}$.

Strix javanica, 91

Strix ketupu, 83 .

Strix lempiji, s6.

Strix leptogrammica, 80

Strix leptogrammica bartelsi, 89 .
Strix leptogrammica maingayi, 89 .

Strix leptogrammica myrtha, 89 .

Strix leptogrammica niasensi5, 80 .

Strix leptogrammica nyctiphasma, So.

Strix leschenault, 83 .

Strix noctula, 86.

Strix orientalis, 84 .

Strix orientalis, 88 .

Strix orientalis baweana, 80 .

Strix orientalis seloputo, 80 .

Strix pagodorum, 89 .

Strix rufescens, 87 .

Strix scops, 84 .

Strix scutulata, 87 .

Strix strepitans, 84 .

Strix sumatrana, 84 .

Strix zeylonensis, 83 .

stuarti, Cyanops australis, 138 .

stuarti, Mesobucco duvauceli, 138 .

Sturnia, 204.

Sturnia philippensis, 204

Sturnia sinensis, 204.

Sturnia sturnina, 205.

Sturnidae, 204.

sturnina, Gracula, 295.

sturnina, Sturnia, 205.

Sturnopaster contra jalla, 204 .

Sturnopastor, 294.

Sturnopastor, contra, 294.

Sturnus contra, 204.

Sturnus zeylanicus, 108.

styani, Hypothymis azurea, 172.

stỹani, Siphia, 172.

suavis, Copsychus, 237.

suavis, Kittacincla malabarica, 237. subardens, Pericrocotus, I 85 .

subbuteo, Falco, 8ı.

subfurcatus, Cypselus, 1 in.

subfurcatus, Micropus affinis. I19.

sublunatus, Buceros, 105.

subminuta, Erolia, minutilla, $4 t$.

subminuta, Tringa, 41 .

submoniliger, Anthipes solitaris, I 70 .

subochraceum, Pellorneum ruficeps. 200

subpallidus, Cacomantis merulinus, 126.

subruficollis, Buceros, $10 \%$.

subruficollis, Rhyticeros plicatus, 107.

subrufus, Eupetes macrocercus, 204. subsquamicollis, Munia striata, 201. subsquamicollis, Uroloncha striata, 201.

subundulata, Munia, $20 \mathrm{I}$.

subviridis, Alcedo meninting, юо sueuri, Lalage nigra, I\$4.

suerii, Turdus, 184. 
suffusus, Dryobates canicapillus, 145.

suggrandis, Aplonis panayensis, 208.

Sula abbotti, 65 .

Sula dactylatra, 65 .

Sula dactylatra bedouti, 65 .

Sula leucogaster, 65.

Sula leucogaster plotus, 65 .

sula, Pelecanus, 66.

Sula sula, 66.

sula, Suli, 66.

Sula sula rubripes, 66 .

sulcirostris, Carbo, 64 .

sulcirostris, Phalacrocorax, 64 .

Sulidae, 65.

sulphuratus. Trichophorus, 107.

sulphurea, Gerygone fusca, 17 .

sulphurea, Kakatoe, 9 ?

sulphureus, Psittacus, g2.

sultanea, Melanochlora, 26t.

sultaneus, Parus, 261.

suluensis, Dicrurus hottentottus, 305 .

sumatrana, Arborophila brunneopectus, 2 .

sumatrana, Ardea, 54.

sumatrana, Bhringa remifer, 306.

sumatrana, Buceros, I05.

sumatrana, Caloperdix, 4 .

sumatrana, Cettia montana, 253.

sumatrana, Chalcoparia singalensis, 280 .

sumatrana, Cyornis vivida, 164.

sumatrana, Huhua, 84.

sumatrana, Lophura ignita, 6.

sumatrana, Melanochlora, 26 r.

sumatrana, Mixornis, 225.

sumatrana, Muscisylvia diana, 235.

sumatrana, Niltava, 164.

sumatrana, Notodela diana, 235.

sumatrana, Pitta sordida, 158 .

sumatrana, Scolopax, 42 .

sumatrana, Sterna, 48.

sumatrana, Strix, 84 .

sumatrana, Zosterops palpebrosa, 264

sumatrana, Macropygia ruficeps, 22.

sumatranum, Dicaeum cruentatum, 268.

sumatranus, Coracias, I 56.

sumatranus, Corydon, I 56.

sumatranus, Criniger gutturalis, I06.

sumatranus, Cuculus, I 3 I.

sumatranus, Dendrobiastes hyperythrus, 160

sumatranus, Dicrurus hottentottus, 304.

sumatranus, Enicurus velatus, 234. sumatranus, Euplocamus, 6.

sumatranus, Gallinula, 27.

sumatranus, Harpactes diardii, 1 lo.

sumatranus, Hemixus, 192.

sumatranus, Henicurus velatus, 234 .

sumatranus, Ixos virescens, 192.

sumatranus, Macropygia ruficeps, 22.

sumatranus, Mainatus, 206.

sumatranus, Merops, 110.

sumatranus, Phyllergates, 247.

sumatranus, Rhopodytes; $13 \mathrm{I}$.

sumatrensis, Avicedn, 8o,

sumatrensis, Ceblypyris, $18 \mathrm{r}$.

sumatrensis, Coracina, I8t.

sumatrensis, Cryptolopha, 252.

sumatrensis, Cuculus, 132.

sumatrensis, Cyornis rufigastra, 166.

sumatrensis, Munia, 200.

sumatrensis, Seicercus grammiceps, 252 .

sumatrensis, Siphia, I66.

superciliaris, Abrornis, 253.

superciliaris, Abroscopus, 253.

superciliosa, Ana5, 62.

superba, Cyornis, 167.

superciliosus, Lanius cristatus, 250 .

superciliosus, Merops, 1 Io.

superciliaris, Dimorpha, 160 .

superciliaris, Microura, 231.

superciliaris, Muscicapa, 160 .

superciliosa, Parra, 30.

superciliaris, Prinia flaviventris, 255 .

superciliaris, Rallus, 25.

superciliaris, Tesia cyaniventer, $23 \mathrm{I}$.

superciliaris, Turdinus, 238.

Surniculus, 122.

Surniculus lugubris, 122.

Surniculus lugubris dicruroides, 122.

Surniculus lugubris barussarum, 122.

Surniculus lugubris brachyurus, 122.

Surniculus lugubris massorhinus, 122.

susanni, Trichophorus, I04.

suscitator, Tetrao, 9.

suscitator, Turnix, 9 .

sutoria, Motacilla, 245.

sutorius Orthotomus, 245.

Suya, 254.

Suva atrogularis albogularis, 254.

Suya atrogularis waterstradti, 254.

Suva blythi, 255 .

sylvatica, Athene, 00 .

sylvatica, Turnix, 10. 
INDEX.

sylvaticum, Glaudidium brodiei, go.

sylvaticus, Tetrao, 10.

Sylvia javanica, 266.

Sylvia juncudus, 248.

Sylvia lanceolata, 244 .

Sylvia leucorrhoa, 252 .

Sylvia (Locustella) ochotensis, 244.

Sylvia montana, 253.

Sylvia palpebrosa, 263.

Sylvia vulcania, 253 .

Sylviidae, 244-

symmixta, Hypothymis azurea, 173 .

Syrnium bartelsi, 89 .

Syrnium maingayi, 80.

Syrnium niasense, \&9.

tahanensis, Mesia argentauris, 230 . tahanensis, Pteruthius aenobarbus, 230.

tahanensis, Zosterops, 264.

tahitica, Hirundo, tot.

taivana, Motacilla flava, 285 .

taivanus, Budytes, 28 .

takko, Turdus, 240 .

tanakae, Oriolus xanthornus, 301.

tanamassae, Cyanops australis, 138.

tanamasae, Mesobucco duvauceli. 138.

tanki, Turnix, ro.

Tantalus aethiopicus, $5 \mathbf{t}$.

Tantalus cinereus, 53 .

tantulus, Dryobates moluccensis, 146.

Tantalus falcinellus, 52 .

Tantalus lacteus, 53.

Tantalus leucocephalus, 53 .

Tantalus melanocephalus, 5 I.

Tantalus variegatus, 36 .

Tanygnathus, 92 .

Tanygnathus lucionensis, 92 .

Tanygnathus lucionensis horrisonus, 92 .

Tanygnathus salvadorii, 92.

tardinata, Malacocincla sepiaria, 211.

tardus, Rhinomyias, 378 .

Tchitrè affinis, 176 .

Tchitrea affinis indochinensis, 176

Tehitrea affinis saturatior, i 76 .

Tehitrea paradisi borneensis, 176 .

Tehitrea procera, 177.

telephonus, Cuculus canorus, 124 .

telmatophila, Rallina, 25.

temia, Corvus, 309 .

temia, Crypsirina, 300.
Temia vaillantii, 309 .

temmincki, Aethopyga mystacalis, 274.

temmincki, Arachnothera, 283.

temminckii, Corydon, 156 .

temmincki, Erolia, 41 .

temminckii, Lyncornis, 14 .

temminckij, Trogon, I19.

temminckii, Typhon, 55 -

temminckii, Tringa, 4t.

temmincki, Nectarinia, 274.

tenebrosa, Gallinula, 27.

tenellipes, Phylloscopus, 250.

tenggerensis, Crocopsis bimaculatus, 109.

tenuirostre, Treron, 13.

tenuirostris, Calidris, 39.

tenuirostris, Totanus, 39 .

Tephrodornis, 257.

Tephrodornis grisola, 260 .

Tephrodornis gularis, 257.

Tephrodornis gularis annectens, 257.

Tephrodornis gularis frenatus, 257.

Tephrodornis gularis fretensis, 257 .

Tephrodornis pelvica anectens, 257 .

tephrogenys, Criniger, ıg6.

tephrogenys, Trichophorus, 106.

tephrops, Turdinus, 211.

tèrat, Turdus, 184 .

termeuleni, Dicrurus hottentottus, 304.

Terpsiphone, 176.

Terpsiphone atrocaudata, 177 .

Terpsiphone paradisi, 176.

Terpsiphone paradisi affinis, 176 .

Terpsiphone paradisi australis, 176 .

Terpsiphone paradisi borneensis, 176.

Terpsiphone paradisi incei, 177.

Terpsiphone paradisi indochinensis, 176.

Terpsiphone paradisi insularis, 177.

Terpsiphone paradisi procera, 177.

Terpsiphone paradisi satutatior, 176.

terricolor, Iole, 192.

territori, Mesocarbo ater, 64.

territori, Phalacrocorax sulcirostris, 64.

tertia, Graculipica, 295.

tertia, Gracupica malanoptera, 295.

tertius, Calorhamphus fuliginosus, I34.

Tesia, 23 i.

Tesia cyaniventer, 231 .

Tesia cyaniventer superciliaris, $23 \pi$.

Tesia whitebeadi, 23 t. 
testacea, Erolia, $4 \mathrm{I}$.

testacea, Scolojax, 4 .

Tetragonops frantzi, 134.

Tetrao chinensis, 5.

Tetrao curvirostris, I.

Tetrao javanica, 2.

Tetrao ocellatus, 4.

Tetrao porphyrio, 4 .

Tetrao suscitator, 9 .

Tetrao $\$ y^{\prime}$ vaticus, 10.

Tetrao viridis, 4 .

thaiocous, Oriolus luteolus, 300 .

thaiocous, Oriolus xanthornus, 300 ,

thais, Phyllergates cucullatus, 248.

Thalasseus bergii edwardsi, 46.

Thalasseus bergii halodramus, 47.

Thalassidroma monorbis, 51.

thalassina, Muscicapa, I8t.

thalassina, Stoporala, I8t.

thalassoides, Glaucomyias, $18 \mathrm{I}$.

thalassoides, Stoporala thalassina, $18 \mathrm{I}$.

thapsina, Aegithina viridissima, 188.

Thaumatibis, 52 ,

Thaumatibis gigantea, $5 z$.

theiodes, Criniger, 196.

Thereiceryx, $\mathbf{I} 39$.

Thereiceryx lineatus intermedius, 139.

Thereiceryx zeylanicus, 139 .

Thereiceryx zeylanicus intermedius, I 39.

Thereiceryx zeylanicus lineatus, I39.

Thimalia poioicephala, 218 .

thoa, Hemiprocne longipennis, 113.

thoracica, Pitta, 222.

thoracica, Stachyris, 222.

thoracicus, Anaimos, 271.

thoracicus, Pardalotus, 27 I.

threnodes, Cacomantis merulinus, 126.

Threskiornis, $5 \mathrm{t}$.

Threskiornis aethiopicus, 5 I

Threskiornis aethiopicus melanoce. phalus, $5 r$.

Thringorhina striolata umbrosa, 222.

Thriponax parvus, 15 I.

thunbergi, Motacilla, 285 .

tichelmani, Collocalia brevirostris, 116.

tichelmani, Collocalia lowi, is 6.

tickelli, Pellorneum, 200.

Tiga amictus, I44.

Tiga borneonensis, 149 .

'Tiga javanensis exsul, I 49. tiga, Picus, 148.

Tiga rufa, 148 .

tigrina, Columba, 23.

tigrina, Streptopelia chinensis, 23.

tigrinus, Lanius, 258.

tigus, Brachypus, 203.

Timalia, 208.

Timalia bicolor, 224 .

Timalia erythronotus, 22 .

Timalia erythroptera, 223.

Timalia grammicephala, 22 I.

Timalia gularis, 225.

Timalia, larvata, 220.

Timalia leucotis, 221 .

Timalia maculata, 222 .

Timalia mitrata, 206.

Timalia nigricollis, $22 t$.

Timalia pectoralis, 222 .

Timalia pileata, 208 .

Timalia pileata intermedia, 208 .

Timalia pirrhoptera, 223 .

Timalia poliocephala, 2zi.

Timalia poliopsis, 224 .

Timalia pyrrophaea, 223 .

Timalia ruficapilla, 214.

Timalia squamifrons, 2 I 4 .

Timalia striata, 227 .

Timalia striolata, 222 .

Timalia trichorrhos, 227.

Timaliidae, 204.

tinnunculus, Falco, 81.

Tjnnunculus moluccensis occidentalis, 82 .

tinoptila, Aethopyga siparaja, 275. tionis, Stachyris nigriceps, 220.

tiphia, Aegithina, 188.

tiphia, Motacilla, 188.

tobata, Tringa, 37 .

Todus macrorhynchos, 156.

Todus nasutus, I 56.

tolutilis, Megapodius cumingii, I.

Torgos, 60 .

Torgus calvus, 60.

Toria nipalensis, 12.

torquata, Pernis, 79.

torquata, Muscicapa, 233.

torquatus, Acridotheres, 296.

torquata, Saxicola, 233.

torquatus, Aethiopsae fuscus, 206.

torquatus, Buccotrogon, 140.

tosariensis, Lanius schach, 250.

Totanus acuminatus, $4 \mathrm{l}$.

Totanus affinis, 43 .

Totanus brevipes, 43.

Totanus damacensis, 40.

Totanus guttifer, 43 .

Totanus javanicus, 42.

Totanus kuhlii, 43. 
toatanus, Scolopax, 42.

Totanus stagnatilis, 42 .

Totanus tenuirostris, 39 .

Totanus totanus eurhinus, 42 .

totanus, Tringa, 42 .

Trachycomus, 108.

Trachycomus zeylanicus, 108.

trangensis, Cyanops franklinii, 137.

tieacheri, Janthocincla, 206.

treacheri, Rhinocichla mitrata, 206.

Treron, 12 .

Treron bicincta, 14 .

Treron bicincta javana, 14 -

Treron bicincta praetermissa, 14 .

Treron bisincta javana, 14.

Treron bisincta praetermissa, I4.

Treron curvirostra, $\mathbf{t z .}$

Treron curvirostra erimaca, 12.

Treron curvirostra haliploa, 12.

Treron curvirostra harterti, 12.

Treron curvirostra hypothapsina, 12.

Treron curvirostra nipalensis, 12.

Treron curvirostra pega, 12.

Treron curvirostra smicra, $[2$.

Treron fulvicoltis, I3.

Treron fulvicollis baramensis, 14.

Treron tulvicollis melopogenys, 13 .

Treron fulvicollis oberholseri, I3.

Treron magnirostris, 1 .

Treron nasica, 12.

Treron mipalensis harterti, 12.

Treron olax, 15.

Treron pompadora, 13.

Treron pompadora pulverulenta, 13.

Treron pompadora vordermani, 13.

Treron tenuirostre, I3.

Treron vernans, 14.

Treron vernans adiua, I 5.

Treron vernans griseicapilla, 14.

Treron vernans mesochloa, 15.

Treron vernans miza, 15 .

Treron vernans parva, 15.

Trerons vernans purpurea, 15.

Tribura squatneiceps, 254 .

Trichastoma affine, 215.

Trichastoma rostratum, 211 .

Trichixos, 238 .

Trichixos pyrropyga, 238 .

Trichoglossus, $9 i$.

Trichoglossus ornatus, 91 .

Trichoglossus ornatus mitchelli, gt.

Trichogramoptila melanopygia, 292.

Tricholestes, 108.

Tricholestes criniger, 108.

Tricholestes criniger sericea, 108.
Tricholestes criniger virjdis, 108

Trichophoropsis viridis, 108 .

Trichophoropsis typus, 1.07.

Trichophorus brunnescens, 193.

Trichophorus caniceps, 197.

Trichophorus gutturalis, 196.

Trichophorus minutus, 198.

Trichophorus pulverulentus, 192.

Trichophorus strjolatus, 192.

Trichophorus sulphuratus, 197.

Trichophorus susanni, 194.

Trichophorus tephrogenys, 196.

trichorrhoa, Timalia, 227.

Trichostoma buttikoferi, 2 I 2.

Trichostoma umbratile, 370 .

tricolor, Gracupica melanoptera, 295.

tricolor, Kittacincla malabarica, 237.

tricolor, Muscicapa (Muscicapula). [7!.

tricolor, Pastor, 295.

tricolor, Phaenicophaeus, 132.

tricolor, Turdus, 237.

tricolor, Vanellus, 32 .

tricolor, Xiphidiopterus, 32.

Tricophorus crispiceps, 198.

tridactylus, Chrysonotus, 148.

trigonostigma, Certhia, 260.

trigonostigmum, Diczeum, 260 .

Tringa, 42 .

Tringa alba, 39 .

Tringa alpina, $4 \mathrm{I}$.

Tringa canutus, 39 .

Tringa chirurgus, 30.

Tringa glareola, 43 .

Tringa guttifer, 43 .

Tringa hypoleucos, 43 .

Tringa incana, 42 .

Tringa incana brevipes, 43.

Tringa indica, 32.

Tringa interpres, 39 .

Tringa minutilla, 40.

Tringa nebularia, 43 .

Tringa ochropus, 42.

Tringa pugnax, 40 .

Tringa squatarola, 32.

Tringa stagnatilis, 42 .

Tringa subminuta, 41 .

Tringa temminckii, $4 \mathrm{I}$.

Tringa tobata, 37 .

Tringa totants, 42 .

Tringa totanus eurhinus, 42.

Tripsurus auritus, 145.

tristis, Acridotheres, 295.

tristis, Brachypodius, 104.

tristis, Bucco, 135.

tristis, Meiglyptes, 146 . 
INDEX.

tristis, Melias, 131.

tristis, Paradisea, 295.

tristus, Picus, 146.

tristis, Rhopodytes, I3t.

trivirgatus, Accipiter, 70.

trivirgatus, Falco, 70.

trivirgatus, Phylloscopus, $25 \mathrm{I}$.

trivirgatus, Sercercus, 251 .

Trochalopterum peninsulae, 205

trochilea, Certhia, 267.

trochileum, Dicaeum, 267.

Troglodytidae, $23 t$.

Trogon asiaticus, $\$ 36$.

Trogon condea, IIO.

Trogon diardii, 1 Ig.

Trogon duvaucelii, I 20.

Trogon erythrocephalus, 120 .

Trogon flagrans, 120.

Trogon kasumba, 10 .

Trogon mackloti, $12 \mathrm{r}$.

Trogon maculatus, $[27$.

Trogon oreskios 121 .

Trogon reinwardtii, 121.

Trogon rutilus, 121 .

Trogon temminckii, 1 ig.

Trogonidae, 1 io.

trophis, Pericrocotus igneus, 186.

Trynga ruficollis, 40.

tubiger, Glaucidium brodiei, go.

tubiger, Noctua, go.

tuckeri, Dendrocitta sinensis, 309.

tukki, Meiglyptes, 147.

tukki, Picus, 147.

turcosa, Cyornis, 165 .

turcosa, Irena puella, 192.

turcosa, Muscicaja, 165.

Turdidae, 231 .

Turdintus epilepidotus dilutus, 217.

Turdinulus exsul, 218.

Turdinulus granti, 2 t 8 .

Turdinulus humei, 218.

Turdinus, 213 .

Turdinus brevicaudatus, 217 .

Turdinus canicapillus, 212 .

Turdinus guttatus, 222.

Turdinus ka!ulongae, 215.

Turdinus macrodactylus, 213 .

Turdinus macrodactylus atrigularis, 213 .

Turdinus macrodactylus bakeri, 213.

Turdinus macrodactylus lepidopleurus, 2 เ3.

Turdinus macrodactylus rufipectus, 213.

Turdints marmoratus, 213 .

Turdinus sepiaria minor, $26 \mathrm{r}$.

Turdinus superciliaris, 238 .
Turdinus tephrops, $2 t \mathrm{t}$

Turdirostris leucostigma, 227.

Turdulus davisoni, $24 t$.

Turdus, 240.

Turdus ambiguus, 262 .

Turdus amoenus, 236 .

Turdus analis, 199 .

Turdus atriceps, 104.

Turdus aurigaster, 204.

Turdus azureus, 230.

Turdus bimaculatus, 190 .

Turdus chalybeus, 798 .

Turdus citrinus, 242.

Turdus cochinchinensis, ron.

Turdus cyaneus, 239.

Turdus cyanurus, 159

Turdus dauma, 243 .

Turdus dispar, 203.

Turdus flavirostris, 240 .

Turdus fumidus, 240 .

Turdus guajanus, 159

Turdus gularis, 106 .

Turdus hypopytrhus, 240.

Turdus interpres, 242.

Turdus javanicus, 240 .

Turdus javanicus biesenbachi, 240 .

Turdus javanicus erythropleurus, 241.

Turdus javanicus fumidus, 240.

Turdus javanicus indrapurae, 241.

Turdus javanicus seebohmi, 24 I.

Turdus javanicus whiteheadi, 240.

Turdus leschenaulti, 233.

Turdus modestus, 241 .

Turdus niger, 184 .

Turdus nigricrissus, 240.

Turdus obscurus, 241 .

Turdus ochrocephalus, I 08.

Turdus orientalis, 184.

Turdus philippensis, 243 .

Turdus rufulus, $24 \%$.

Turdus sibiricus, 241 .

Turdus solitarius, 243.

Turdus sordidus, 158 .

Turdus squamatus, 203.

Turdus striga, 205.

Turdus strigatus, $20 \$$.

Turdus suerii, 184.

Turdus takko, 240.

Turdus terat, I $S_{4}$.

Turdus tricolor, 237

Turdus viridis, 190

Turdus vulcanus, 240 .

Turnicidae, 9 .

Turnix, 9 .

Turnix juvanica,

Turnix sylvatica bartelsorum, Io.

Turnix suscitator, 9 .

Turnix suscitator atrogularis, 9. 
Turnix suscitator interrumpens, 10. Turnix sylvatica, 10 ,

Turnix tanki, 10 .

Turnix tanki blanfordi, to.

Turtur tigrinus minor, 23.

tweedalii, Pernis, 79 .

tympanistrigus, Brachypus, 194.

tympanistrigus, Ixos, 203.

tympanistrigus, Pycnonotus, 203.

typhon, Ardea, 54.

Typhon temminckii, 55 .

typus, Trichophoropsis, 197.

Tyto, go.

Tyto alba, go.

Tyto alba javanica, 91 .

Tytonidae, go.

umbellata, Muscicapa, $\mathbf{1 7 5}$.

umbra, Otus, 86.

umbra, Pisorbina, 86.

umbratile, Trichostoma, 179 .

umbratilis, Rhinomyias, I79.

umbrosa, Stachyris striolata, 222.

umbrosa, Thringorhina striolata, 222 .

unchall, Macropygia, 20.

undulata, Loxia, 291.

undulatus, Buceros, 108.

undulatus, Rhyticeros, 108.

unicolor, Cyornis, 164.

unicolor, Philentoma, 177.

uniformis, Harpactes oreskios, I2I, uniformis, Pyrotrogon oreskios, 121.

Upupa, 109.

Upupa epops, Iog.

Upupa epos, Iog.

Upupa epops longirostris, Iog.

Upupa epops saturata, sog.

Upupidae, 100.

urbica, Delichon, 160.

urbica, Hirundo, 160.

urjca, Merops, roo.

Uroloncha acuticauda lepidota, 201.

Uroloncha kangeangenis, 292.

Uroloncha propinqua, 202 .

Uroloncha striata subsquamicollis, 201.

uropygialis, Arachnothera, 283.

Urosphena, 254.

Urosphena squameiceps, 254 .

urostictus, Brachypus, 194 .

urostictus, Euptilosus, 194.

usa, Pyrotrogon kasumba, 120.

ussheri, Pitta venusta, 158 . vagans, Cuculus, 124 .

vagans, Hierococcyx, $1 \geq 4$.

vaillantii, Ternia, 300 -

validus, Chrysocolaptes, 150.

validus, Picus, 150.

Vanellus cuculfatus, 32.

Vanellus tricolor, 32 .

vandepolli, Carpophaga, 18.

vandepolli, Pachycephala cinerea, 261 .

vandewateri, Otus, 86.

vandewateri, Pisorhina, S6.

Vanga cristata, 257.

Vanga flaviventris, 203.

varheurni, Pitta granatina, 159.

vanheysti, Brachylophus chlorolophus, 142.

vanheysti, Cyornis, 164.

vanheysti, Dicaeum, 268 .

vanheysti, Picus chlorolophus, 142.

varia, Halcyon, 104.

varians, Corvus, 309 .

yariegatus, Numenius phaeopus, 36.

variegatus, Picus, 145.

variegatus, Tantalus, 36 .

variolosus, Cacomantis, 127.

variolosus, Cuculus, I 27.

varius, Gallus, 7.

varius, Phasianus, 7.

velata, Drvmophila, 177.

velata, Sitta, 262.

velatus, Enicurus, 234.

venusta, Chloropsis, iso.

venusta, Phyllornis, 180 .

venusta, Pitta, 158.

verecunda, Padda, $28 \%$.

yeredus, Charadrius asiaticus, 34.

vermiculata, Stachyris larvata, 220.

vernalis, Loticulus, 94.

vernalis, Psittacus, 94.

vernans, Columba, 14

vernans, Treron, 14.

verrauxii, Alcedo meninting, 100.

verreauxii, Alcedo, roo.

versicolor, Bucco, 135.

vestita, Collocalia, 115.

vestita, Salangana, 115.

vicina, Duculn aenea, 18.

vicinu5, Muscadivores aeneus, 18.

vidua, Brachyus, 195.

vidua, Harpactes orrhophaeus, 121.

vidua, Rhipidura, 174 .

vidua, Volvocivora, 183.

vieilloti, Euplocamus, 6.

vigil, Buceros, to8,

vigit, Rhinoplax, to8.

Vinago bicincta, 14 . 
Vinago sphenura, 11.

virescens, Ixos, 192.

virgatus, Accipiter, 72 .

virgatus, Falco, 72.

virfatus, Lanius, 257.

viridanus, Picus, 141 .

viridimystax, Palacornis, 93.

viridinitens, Dicruropsis, 305.

viridinitens, Dicrurus hottentottus, 305.

viridinucha, Chloropsis cochinchinensis, 100.

viridinucha, Phyllornis, 100.

viridirostris, Phaenicophas, 132 .

viridirufus, Phaenicophaus, - 132.

viridis, Aegithina tiphia, 180.

viridis, Calyptomena, I53.

viridis, Carpococcyx radiatus, 133.

viridis, Erythura, 202.

viridis, Iora, 180 .

viridis, Merops, Ito.

viridis, Phaenicophaus, 132.

viridis, Porphyrio albus, 28.

viridis, Tetrao, 4.

viridis, Tricholestes criniger, I 98.

viridis, Trichophoropsis, 198.

viridis, Turdus, 190.

viridissima, Aegithina, 188.

viridissima, Iora, I88.

viriditectus, Chloropsis, viridis, 190.

vivida, Cyornis, 163.

Vivia innominata, 152 .

Vivia innominata malayorum, 152.

vividus, Perictocotus cinnamomeus, 187.

vividus, Pericrocotus peregrinus, 187.

vitiensis, Columba, 20.

vittatus, Garrulax, 206.

vittatus, Picus, 140 .

Volvocivora neglecta, 184 .

Volvocivora schierbrandii, I84.

Volvocivora vidua, 183 .

volzi, Dryobates canicapillus, 145.

volzi, Dryobates hardwickii, 145.

vordermani, Abroscopus super. ciliaris, 253.

vordermani, Coracina sumatrensis, 182 .

vordermani, Cryptolopha, 253.

vordermani, Graucalus, 182.

vordermani, Siphia, I7o.

vordermani, Treron pompadora, 13.

vulcani, Dendrobiastes hyperythra, 160.

vulcania, Sy]via, 253.

vulcanorum, Collocalia brevirostris, 16. vulcanorum, Collocalia, 116.

vulcanorum, Podiceps ruficollis, 50.

vulcanus, Turdus, 240.

vulneratus, Oriolus cruentus, 302.

Vultur bengalensis, 60 .

Vultur calvus, 60.

Vultur ginginianus, 70 .

vulpes, Heteroscops, 85

Vultur indicus, 60 .

vulpes, Otus spilocephalus, $\$ 5$.

Vultur perenopterus, 70.

walik-mehra, Macropygia, 20.

wallacei, Dissemurus paradiseus, 307.

westermanni, Muscicapula melanoleuca, 168.

waterstradti, Pyrrhula nipalensis, 288.

waterstradti, Suya atrogularis, 254 . webberi, Ixidia, 203.

webberi, Pycnonotus squamatus, 203.

weberi, Grecinus, 14I,

weberi, Picus viridanus, 141.

westralis, Phaethon rubricauda, 67. whartoni, Carpophaga, 18.

whartoni, Ducula rosacea, 18.

whiteheadi, Calyptomena, 153.

whiteheadi, Erythrocichla bicolor, 213.

whiteheadi, Harpactes, 120.

whiteheadi, Merula, 240.

whiteheadi, Orthnocichla, 23 .

whiteheadi, Tesia, 231 .

whiteheadi, Turdus javanicus,

240.

williami, Carpophaga, 19.

williami, Ducula badia, 19.

willamsoni, Mirafra, 287 .

williamsonf, Zosterops palpebrosa, 263.

witmeri, Aethostoma, 212 ,

worcesteri, Anous minutus, 49.

worcesteri, Micranous, 49.

wrayi, Aethopyga saturata, 274.

wrayj, Brachypteryx leucophris.

231.

wrayi, Chrysophlegma flavinucha,

143.

wrayi, Heterophasia picaoides,

228.

wrayj, Pericrocotus, 186.

wrayi, Pomatorbinus hypoleucos, 207.

wrayi, Sibia, $22 \mathrm{~S}$. 
Xanthixus flavescens, 200 .

xanthodryas, Phyllopneuste, 250. xanthodryas, Phylloscopus borealis, 250 .

xanthogaster, Lanius, 185.

xanthogaster, Pericrocotus flammeus, 185 .

Xantholaema, 130.

Xantholaema australis hebereri, 138 .

Xantholaema haemacephala, 139.

Xantholaema haemacephala delica, 139.

Xantholaema haemacephala indica, 130.

Xantholaema haemacephala rosea, 140.

Xanthonotus leucogaster, 30 :

xanthonotus, Oriolus, 301 .

xanthopygius, Anaimos, 271 .

xanthopygius, Chry'socolaptes valjdus, 150.

xanthopygius, Grecinus, 140.

xanthopygius, Prionochilus, $27 \mathrm{I}$.

xanthorhynchus, Chalcites, 127.

xanthorhynchus, Cuculus, 127.

xanthornus, Coracias, 300 .

xanthornus, Oriolus, 300 .

Xenorhynchus, 54.

Xenorbynchus așiaticus, 54 .

Xenus, 42.

Xenus cinereus, 42.

Xiphidiopterus, 32.

Xiphidiopterus tricolor, 32.

youngi, Cryptolopha, 252.

youngi, Seicercus castaniceps, 252.

2achlora, Zosterops chloris, 266.

zachlora, Zosterops, solombensis, 266.

zacnecus, Copsychus saularis, 235.

zamelaena, Hydrocissa convexa, I06.

zamelaenus, Anthracoceros coronatus, to6.

zamydra, Ducula rosacea, 18 .

zamydrus, Muscadivores rosaceus, 18.

Zanclostomus, 130.

Zanclostomus javanicus, 130.

Zanclostomus javanicus natunensis, 131.

Zanclostomus javanicus pallidus, 130.

zantholeuca, Erpornis, 229.
Zanthopygia, 171.

zanthopygia, Muscicapa, 171.

Zanthopygia narcissina, 171.

Zanthopygia narcissina zanthopygia, 171

zanthonygia, Zanthopygia narcissina, 171.

zapega, Cinnyris ornata, 277 .

zapercna, Munia maja, 290.

zaperissa, Mixornis gularis, 226.

zaperissa, Mixornis rubricapilla, 226.

zaphaeus, Pycnonotus brunaleus, 201.

zaphotina, Kittacincla malabarica, 237.

zapolius, Pycnonotus brunneus, 202.

Zapornia nigrolineata, 25 .

zaptera, Mixornis pileata, 220

zarhabdota, Mixornis gularis, 226.

zarhabdota, Mixornis pileata, 226 .

zarhina, Arachnothera longirostra, 282.

zeylanicus, Bucco, 130.

zeylanicus, Thereiceryx, 139 .

zeylanicus, Sturnus, 108.

zeylanicus, Trachycomus, ig8.

zeylonensis, Bubo, 83 .

zeylonensis, Strix, $\$_{3}$.

7ita, Dicaeum sulaense, 260 .

Zoothera, 242.

Zoothera, andromedae, 242.

zophera, Mixornis gularis, 225.

zophera, Mixornis pileata, 225.

zophonota, Aegithina tiphia, I8g.

zonaventris, Rallina (Euryzona), 26.

Zosteropidae, 263.

Zosterops, 263 .

Zosterops atricapilla, 269.

Zosterops aureiventer media, 264 .

Zosterops aurejventer parvus, 264 .

Zosterops chlorates, 265.

Zosterops chlorates difficilis, 265.

Zosterops chlorates korinchi, 265.

Zosterops chlorates neglecta, 266.

Zosterops chlorates sindorensis, 266.

zosterops, Chloropsis sonnerati, 190.

Zosterops chloris, 266.

Zosterops chloris maxi, 266.

Zosterops chloris solombensis, 266 .

Zosterops chloris zachlora, 266.

Zosterops citrinella, 264 .

Zosterops clara, 265 .

Zosterops fallax, 266.

Zosterops flava, 265. 
INDEX .

Zosterops frigida, 223 .

Zosterops incerta, 264

Zosterops javanica, 266.

Zosterops javanica clongata, 267.

Zosterops javanica frontalis, 266 .

Zosterops lateralis, 264.

Zosterops melanura, 265.

Zosterops melanura gallio, 265.

Zosterops montana, 265.

Zosterops natalis, 264 .

Zosterops palpelyrosa, 263 .

Zosterops palpebrosa aureiventer. 264.

Zosterops palpebrosa buxioni, 264.

Zosterops palpebrosa erwini, 264
Zosterops palpebrosa media, 264.

Zosterops palpebrosa salvadorii, 264.

Zosterops yalpebrosa sindorensis, 260.

Zosterops palpebrosa sumatrana, 264.

Zosterops palpebrosa willamsoni, 263.

Zosterops simplex, 263 .

Zosterops parvule, 265.

Zosterops solombensis zachiora, 266.

Zosterojes squamitrons, $\mathbf{2 6 7}$.

Zosterops tahanensis, 264 . 




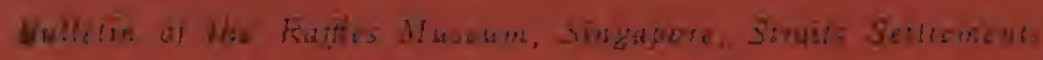

No. 21, Drecentbor, 1039 .

\section{A HANDLIST OF}

\section{MALAYSIAN BIRDS}

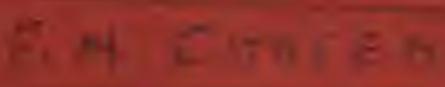

A SYSTEMATIC LIST OF THE BIRDS OF THE MALAY PENINSULA, SUMATRA, BORNEO AND IAVA, INCLUDING THE ADJACENT SMALL ISLANDS

FREDERICK ALTTER CHASEX, U.M.A.S, M.R.R.C.

Direclar, Rogles Mugum, Singepote

\section{Pullithed by indwall}

ISTHURE

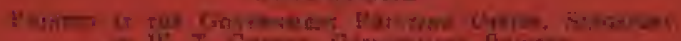

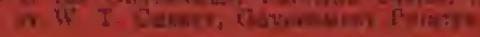

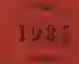

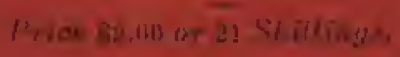


$x_{4}^{4}+4$

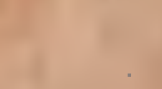

$$
\text { - }
$$

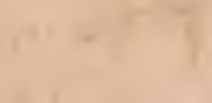$$
\sqrt{2}+2
$$

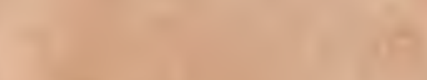

$\sqrt{2}+x^{2}$

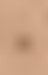

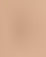

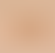

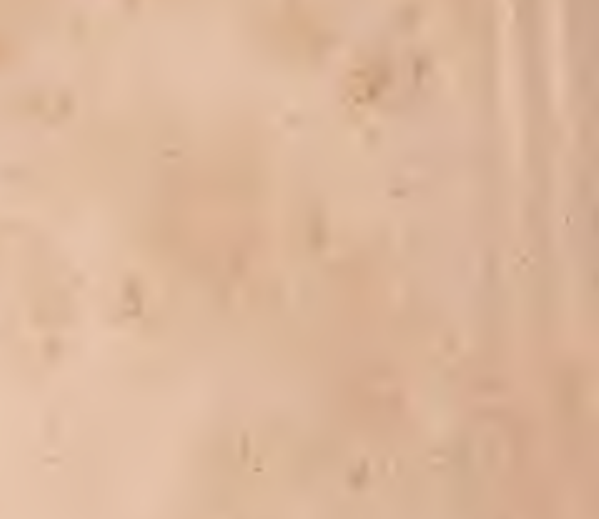





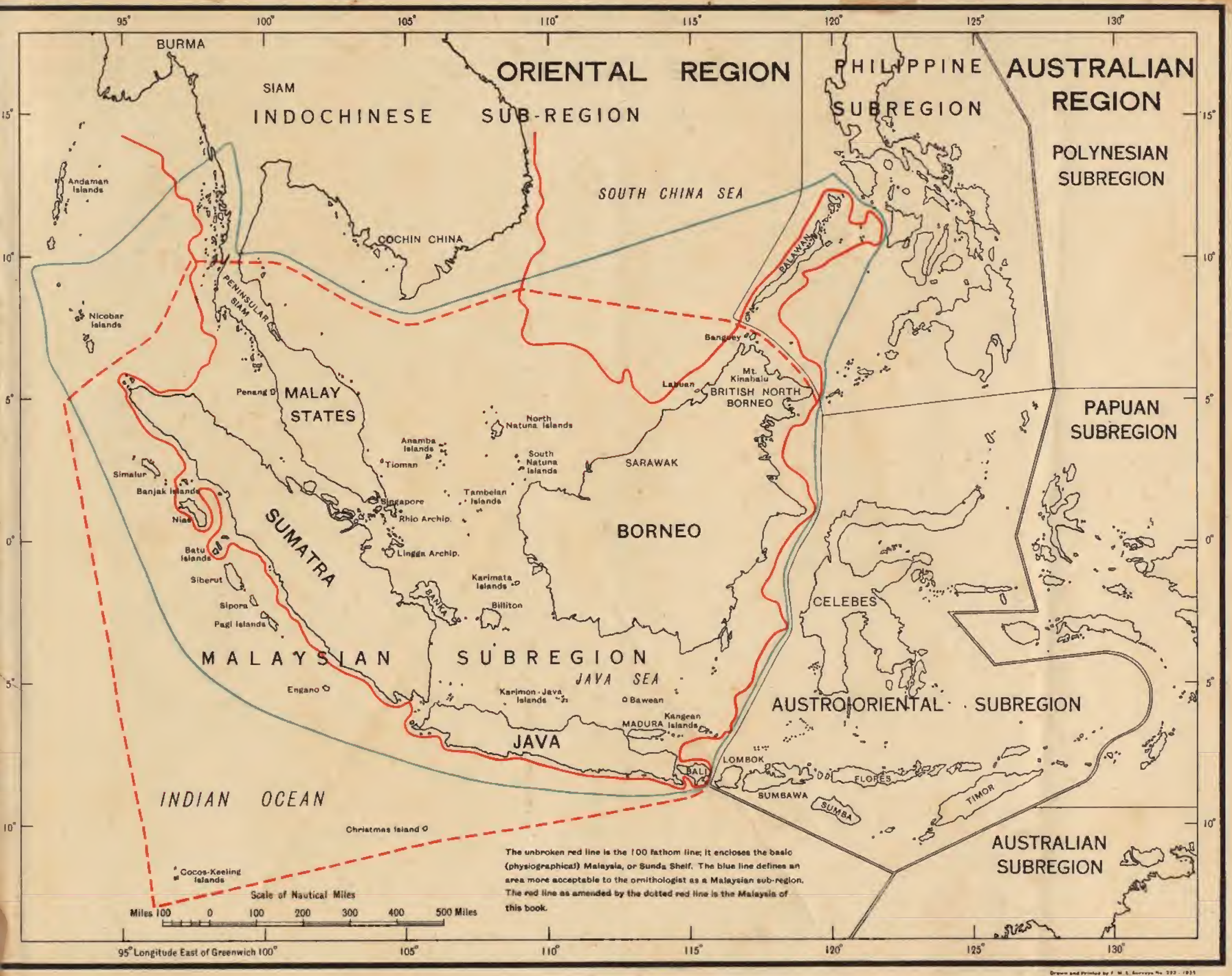

